\title{
DisSERTATION
}

Neutrino Oscillation Parameter Sensitivity in Future Long-Baseline EXPERIMENTS

\author{
Submitted by \\ Matthew Bass \\ Department of Physics
}

In partial fulfillment of the requirements

For the Degree of Doctor of Philosophy

Colorado State University

Fort Collins, Colorado

Fall 2014

Doctoral Committee:

Advisor: Robert J. Wilson

John Harton

Walter Toki

Wen Zhou 
Copyright by Matthew Bass 2014

All Rights Reserved 
ABSTRACT

\section{Neutrino Oscillation Parameter Sensitivity in Future Long-Baseline}

\section{EXPERIMENTS}

The study of neutrino interactions and propagation has produced evidence for physics beyond the standard model and promises to continue to shed light on rare phenomena. Since the discovery of neutrino oscillations in the late 1990s there have been rapid advances in establishing the three flavor paradigm of neutrino oscillations. The 2012 discovery of a large value for the last unmeasured missing angle has opened the way for future experiments to search for charge-parity symmetry violation in the lepton sector.

This thesis presents an analysis of the future sensitivity to neutrino oscillations in the three flavor paradigm for the $\mathrm{T} 2 \mathrm{~K}, \mathrm{NO} \nu \mathrm{A}, \mathrm{LBNE}$, and $\mathrm{T} 2 \mathrm{HK}$ experiments. The theory of the three flavor paradigm is explained and the methods to use these theoretical predictions to design long baseline neutrino experiments are described. The sensitivity to the oscillation parameters for each experiment is presented with a particular focus on the search for $\mathrm{CP}$ violation and the measurement of the neutrino mass hierarchy. The variations of these sensitivities with statistical considerations and experimental design optimizations taken into account are explored. The effects of systematic uncertainties in the neutrino flux, interaction, and detection predictions are also considered by incorporating more advanced simulations inputs from the LBNE experiment. 


\section{TABle of Contents}

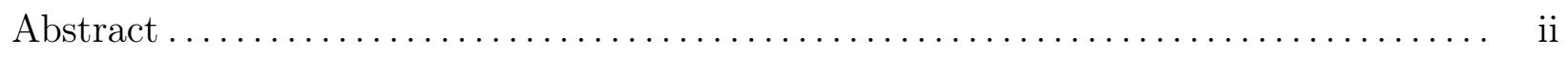

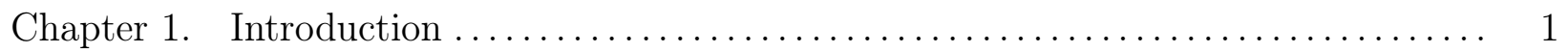

Chapter 2. Neutrino Mixing \& Oscillations........................ 5

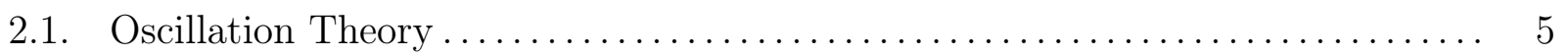

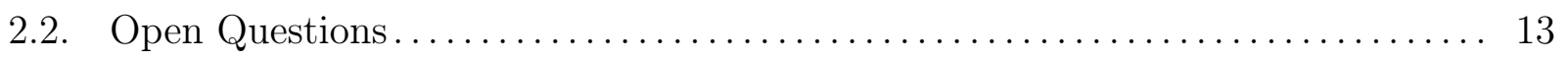

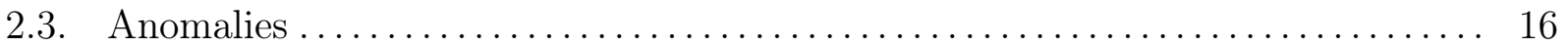

Chapter 3. Current Three-Flavor Experimental Measurements and Global Fit....... 18

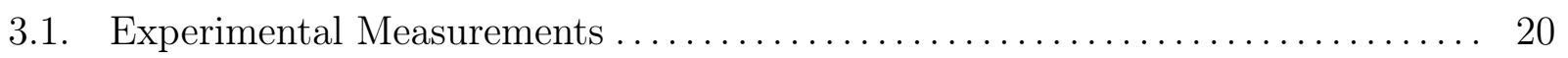

3.2. Experimental Measurements Summary $\ldots \ldots \ldots \ldots \ldots \ldots \ldots \ldots \ldots \ldots \ldots \ldots \ldots 27$

3.3. Global Fit Results .................................... 28

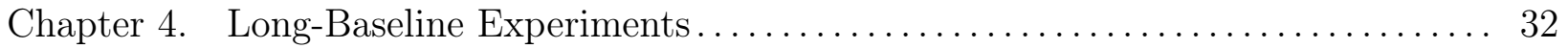

4.1. Components of an LBL Experiment .......................... 33

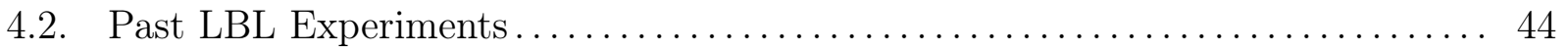

4.3. Current and Future LBL Experiments......................... 45

Chapter 5. Methods for Computing Sensitivity to Neutrino Oscillations .......... 67

5.1. Profile Log-likelihood Method ............................ 67

5.2. Asimov Data Set ................................... 69

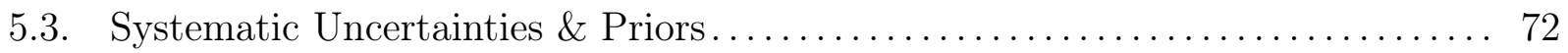

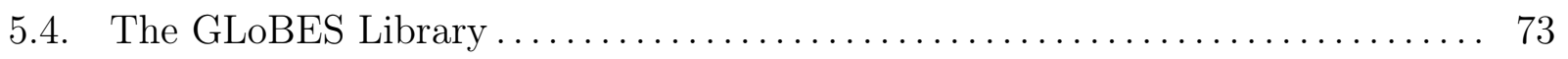

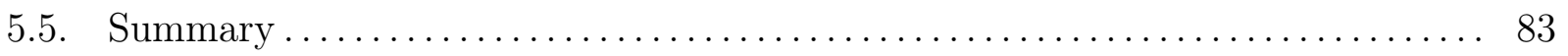

Chapter 6. Neutrino Oscillation Sensitivities in T2K, NO $\nu$ A, LBNE, and T2HK.... 84 
6.1. Event Spectra for Oscillation Parameter Variations ................... 84

6.2. Oscillation Parameter Sensitivities ............................. 107

6.3. Sensitivity to CP Violation, Mass Hierarchy, and $\theta_{\mathbf{2 3}}$ Octant ............ 129

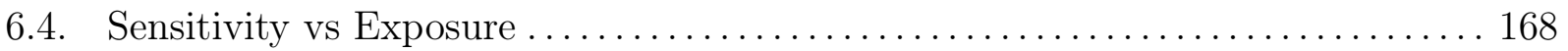

6.5. Concluding Remarks ..................................... 180

Chapter 7. Systematic Uncertainties ................................. 181

7.1. Shape and Rate Contributions to Sensitivity ..................... 184

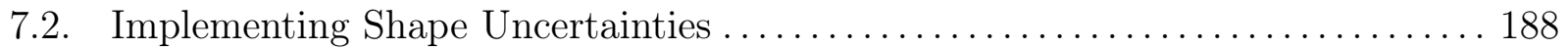

7.3. LBNE Systematic Response Functions . . . . . . . . . . . . . . . . . . . . . 195

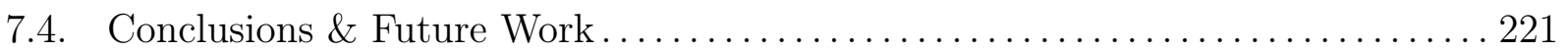

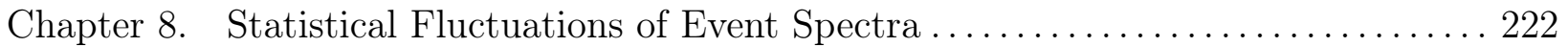

8.1. Mass Hierarchy \& Statistical Fluctuations .............................. 222

8.2. CP Violation \& Statistical Fluctuations ........................... 234

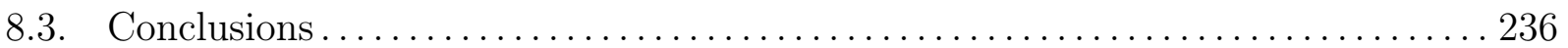

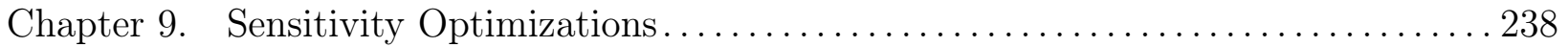

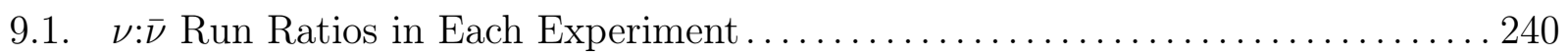

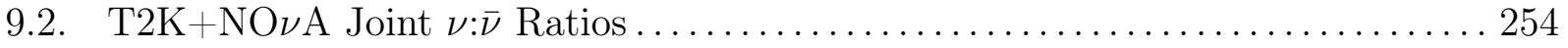

9.3. LBNE Second Oscillation Maximum ........................... 257

Chapter 10. Summary \& Conclusions ................................ 262

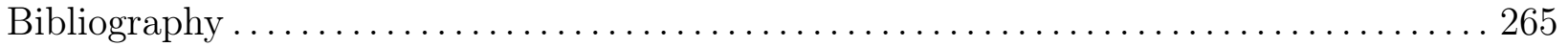

Appendix A. $\quad \mathrm{NO} \nu \mathrm{A}$ Event Rate Reproductions ........................... 271 
Appendix B. Event Spectra for Oscillation Parameter Variations in the Inverted

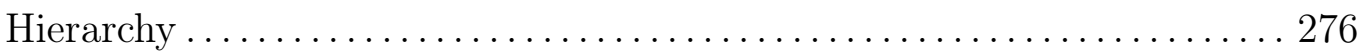

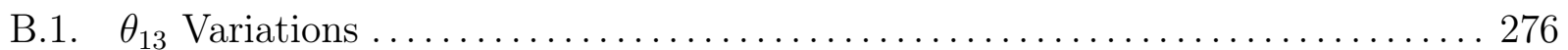

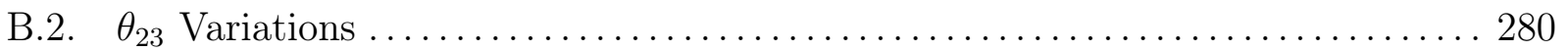

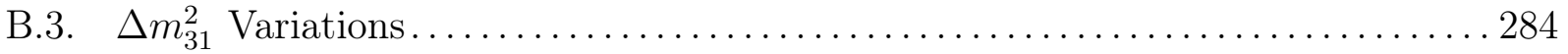

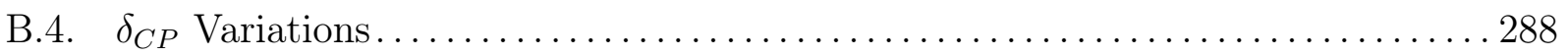

Appendix C. Resolutions on Oscillation Parameters ........................... 292

Appendix D. Computational Resources ............................... 294 


\section{CHAPTER 1}

\section{INTRODUCTION}

Neutrinos are thought to be the most abundant massive particle in the universe. There are hundreds of billions passing through an area the size of a fingernail every second from the sun alone. Given their abundance, it is perhaps surprising that they were not proposed until 1930 and not discovered experimentally until 1957. They are exceedingly difficult to detect because they rarely interact. It is the elusive nature of the neutrino that has inspired many experiments in order to study their properties and learn about a fundamental constituent of the universe.

Though they were initially assumed to be massless, we now know that they have masses that are incredibly small, at least seven orders of magnitude smaller than the next smallest known mass of the electron. The neutrino interaction cross section is also incredibly small and when initial predictions were made it was thought to be virtually impossible to detect.

The neutrino, denoted by the Greek letter $\nu$, underlies a number of different phenomena that are still not completely understood even after nearly a century of efforts. The crux of every experiment involving the detection of neutrinos is the feeble interaction cross section of the particle which is on the order of $10^{-43} \mathrm{~cm}^{2}$ for common lower energy neutrinos. This corresponds to a mean free path in lead of order $10^{16} \mathrm{~m}$, which is well over a light year!

The change of neutrino flavor during propagation, or neutrino oscillations, are a well established phenomenon experimentally. Proposed in analogy to oscillations observed in mesons, neutrino oscillations have now been observed using multiple experimental methods. In the dominant theoretical paradigm there are three neutrino flavors associated with three neutrino masses and this paradigm will be the focus of this thesis. The three flavors are 
comprised of a mix of three different mass states that lead to neutrino flavor oscillations during propagation. In this framework the mixing and oscillations are parameterized by a set of neutrino oscillation parameters that relate the neutrino flavor states to the neutrino mass states via a mixing matrix.

The focus of this thesis is on estimating the ability of future long-baseline neutrino oscillation experiments to constrain the neutrino oscillation parameters. In order to plan future experiments and the running parameters of existing experiments it is important to be able to predict the sensitivity of an experiment as a function of its running parameters. For example, the unknown parameters of the mixing matrix that affect the predicted interaction rates of neutrinos in an experiment need to be carefully considered. Also the accuracy of determining the energies of the detected neutrinos and other systematic uncertainties need to be considered in order to plan a robust experiment. This document outlines the experimental methods used, planned and existing experiments and measurements, and methods for determining the sensitivity of an experiment. Specific estimates for the running conditions, e.g. exposure, that are needed in order to meet specific measurement levels of the oscillation parameters are given. The methods and simulated inputs described here were used for official analyses for both the T2K and LBNE experiments.

Chapter 2 will cover the theory of neutrino oscillations and mixing. It will introduce the neutrino oscillation parameters that describe the oscillation from one neutrino flavor to another in vacuum and in matter. The open questions with respect to the oscillation parameters will also be discussed.

Chapter 3 will describe past and current neutrino oscillation experiments. In particular, a recently published global fit of the neutrino oscillation parameters will be described that 
includes the results from many experiments and the results from these experiments will be discussed.

Chapter 4 will focus on long-baseline neutrino oscillation experiments and the experimental setup that allows oscillations to be observed, in a controlled way, over long baselines and high neutrino energies. The components that make up the experiment will be described and the specific implementations given for the case of the four existing and future experiments that are the focus of this thesis.

Chapter 5 will cover the methods and software library used to compute the sensitivity to the oscillation parameters. It will describe how the outputs from external simulations are combined to create estimates of expected neutrino interaction event spectra in the detectors and how uncertainties in the estimates are incorporated and degrade the sensitivities to the neutrino oscillation parameters.

Chapter 6 will cover the sensitivities to the oscillation parameters for the four experiments. The expected constraints on the oscillation parameters for the nominal experiment configurations will be given for the individual experiments as well as for a combination of their results.

Chapter 7 will include a detailed discussion of the systematic uncertainties that need to be taken into account when estimating the sensitivities. The systematic uncertainties will be expanded to consider neutrino interaction uncertainties and then incorporated into the determination of the sensitivities so that a more detailed sensitivity estimate can be produced.

Chapter 8 will present detailed treatments of statistical issues that arise when the expected Poisson statistical fluctuations of a binned experimental measurement are taken into account. The method described in previous chapters will be modified slightly to allow a toy 
Monte Carlo simulation to be developed in order to explore these fluctuations and a summary of their effects on the sensitivity will be given with a particular focus on $\mathrm{CP}$ violation and the mass hierarchy.

Chapter 9 will discuss optimizations of the experimental running conditions that maximize the sensitivity to the various neutrino oscillation parameters. These optimizations will depend heavily on the assumptions made concerning the other experimental run parameters and oscillation parameters and these caveats will be explored so that the maximal range of parameter phase space is covered.

Chapter 10 will conclude this thesis with a summary of the expected sensitivity and the assumptions that go into these expectations. It will highlight remaining questions that must be addressed by more detailed simulations in the future.

Chapters 6 through 9 are comprised entirely of work by the author of this thesis unless stated otherwise. Though the author has produced many official sensitivities for T2K and LBNE, the sensitivities in this thesis are not official sensitivities for any of the experiments. 


\section{CHAPTER 2}

\section{Neutrino Mixing \& Oscillations}

It has been established experimentally that neutrinos have mass and as a consequence they undergo flavor oscillations. In neutrino flavor oscillations the neutrinos that are produced in one flavor state may oscillate to another flavor state after propagating some distance. The known flavor states are the electron neutrino $\left(\nu_{e}\right)$, muon neutrino $\left(\nu_{\mu}\right)$, and tau neutrino $\left(\nu_{\tau}\right)$ and oscillations have been observed between all three of these flavor states. This process is fundamentally one of quantum interference and is controlled by mixing angles, phases, and differences between the masses of the mass states that are the components of each flavor state. This chapter will briefly outline the phenomenon of neutrino oscillations and discuss the open questions that are being or will be explored by current and future experiments.

\subsection{Oscillation Theory}

The mixing between the neutrino flavors occurs as result of their interacting as flavor eigenstates but propagating as mass eigenstates. A neutrino of a particular flavor is associated with a lepton of the same flavor when it interacts. For example, in a $\nu_{\mu}$ charged current interaction a $\nu_{\mu}$ interacts with a neutron to produce a proton and a muon. The neutrino interaction types will be discussed further in Chapter 4. The flavor eigenstates consists of superpositions of the mass eigenstates and are related via a mixing matrix that will be discussed below.

Schematically, the mass eigenstates are produced with equal energy and can be seen as propagating at different velocities because of their differing masses. The differences in the masses of the mass eigenstates leads to differences in their propagation velocities. These 
add up to generate interfering waves and leads to variations in the probability of detecting a neutrino of a specific flavor.

2.1.1. Mixing Matrix. For three flavor neutrino mixing there is a unitary mixing matrix that describes the mixing between the flavor eigenstates $\left(\left|\nu_{e}\right\rangle,\left|\nu_{\mu}\right\rangle,\left|\nu_{\tau}\right\rangle\right)$ and the mass eigenstates $\left(\left|\nu_{1}\right\rangle,\left|\nu_{2}\right\rangle,\left|\nu_{3}\right\rangle\right)$. This matrix is defined as:

$$
U=\left(\begin{array}{ccc}
U_{e 1} & U_{e 2} & U_{e 3} \\
U_{\mu 1} & U_{\mu 1} & U_{\mu 3} \\
U_{\tau 1} & U_{\tau 2} & U_{\tau 3}
\end{array}\right)
$$

The matrix can be expressed in terms of four physically observable parameters with three mixing angles and one phase[1]. There are also two additional phases that come into the mixing matrix if neutrinos are Majorana particles but these will be left aside for the purpose of this discussion as they do not affect neutrino oscillations. Expressing the matrix in terms of these parameters, it becomes:

$$
\begin{aligned}
U & =\left(\begin{array}{ccc}
1 & 0 & 0 \\
0 & c_{23} & s_{23} \\
0 & -s_{23} & c_{23}
\end{array}\right)\left(\begin{array}{cccc}
c_{13} & 0 & s_{13} e^{-i \delta} \\
0 & 1 & 0 \\
-s_{13} e^{i \delta} & 0 & c_{13}
\end{array}\right)\left(\begin{array}{ccc}
c_{12} & s_{12} & 0 \\
-s_{12} & c_{12} & 0 \\
0 & 0 & 1
\end{array}\right) \\
& =\left(\begin{array}{ccc}
c_{12} c_{13} & s_{12} c_{13} & s_{13} e^{-i \delta} \\
-s_{12} c_{23}-c_{12} s_{23} s_{13} e^{i \delta} & c_{12} c_{23}-s_{12} c_{23} c_{13} e^{i \delta} & s_{23} c_{13} \\
s_{12} s_{23}-c_{12} c_{23} s_{13} e^{i \delta} & -c_{12} s_{23}-s_{12} c_{23} s_{13} e^{i \delta} & c_{23} c_{13}
\end{array}\right)
\end{aligned}
$$

where $s_{i j} \equiv \sin \left(\theta_{i j}\right)$ and $c_{i j} \equiv \cos \left(\theta_{i j}\right)$. This is the standard parameterization of the mixing matrix[1]. 
The matrix describes the relative content of mass eigenstates in each flavor eigenstate, so that

$$
\left|\nu_{\alpha}\right\rangle=\sum_{i=1}^{3} U_{\alpha i}^{*}\left|\nu_{i}\right\rangle
$$

where $\alpha$ is $e, \mu$, or $\tau$ representing the flavor index. For example, the $\left|\nu_{e}\right\rangle$ eigenstate consists of a mixture of the mass eigenstates,

$$
\left|\nu_{e}\right\rangle=c_{12} c_{13}\left|\nu_{1}\right\rangle+s_{12} c_{13}\left|\nu_{2}\right\rangle+s_{13} e^{i \delta}\left|\nu_{3}\right\rangle
$$

This describes the initial mass components that exist when an electron neutrino is created. Similar equations will describe the mass components of a muon or tau neutrino. The neutrinos propagate via the mass eigenstates and this is why the oscillation occurs and an example with two flavor mixing will be given to show this effect.

2.1.2. Two Neutrino Mixing. To simplify the approach to oscillations relevant to long-baseline neutrino oscillation experiments an example that considers only two flavors will be considered here.

In two neutrino mixing there are two flavor and two mass eigenstates described by one mixing angle,

$$
\begin{aligned}
& \left|\nu_{e}\right\rangle=\cos \theta\left|\nu_{1}\right\rangle+\sin \theta\left|\nu_{2}\right\rangle \\
& \left|\nu_{\mu}\right\rangle=-\sin \theta\left|\nu_{1}\right\rangle+\cos \theta\left|\nu_{2}\right\rangle
\end{aligned}
$$

The mass eigenstates $\left|\nu_{1}\right\rangle$ and $\left|\nu_{2}\right\rangle$ each have corresponding masses $m_{1}$ and $m_{2}$. No complex phase is allowed in two neutrino mixing because of the constraints of a unitary mixing matrix. 
The goal is to compute the probability of detecting a $\nu_{e}$ or a $\nu_{\mu}$ after an initial state consisting of only $\nu_{\mu}$ of known energy E is allowed to propagate some distance L. An initial state $\left|\nu_{a}\right\rangle$ is defined as

$$
\left|\nu_{a}\right\rangle=\left|\nu_{\mu}\right\rangle=-\sin \theta\left|\nu_{1}\right\rangle+\cos \theta\left|\nu_{2}\right\rangle
$$

and the final state, $\left|\nu_{b}\right\rangle$, consists of the time evolved mass eigenstates that make up the $\left|\nu_{\mu}\right\rangle$ eigenstate,

$$
\left|\nu_{b}\right\rangle=-\sin \theta e^{-i E_{1} t}\left|\nu_{1}\right\rangle+\cos \theta e^{-i E_{2} t}\left|\nu_{2}\right\rangle
$$

where $E_{1}$ is the energy of $\left|\nu_{1}\right\rangle$ and the $E_{2}$ is the energy of $\left|\nu_{2}\right\rangle$. To get the survival probability, or the probability of detecting $\nu_{\mu}$ when the initial state was $\nu_{\mu}$, the square of the inner product of the initial and final state is taken with the time evolved state.

$$
\begin{aligned}
P_{\nu_{\mu} \rightarrow \nu_{\mu}} & =\left|\left\langle\nu_{a} \mid \nu_{b}\right\rangle\right|^{2} \\
& =\left|\left(-\sin \theta\left\langle\left.\nu_{1}\left|+\cos \theta\left\langle\nu_{2}\right|\right)\left(-\sin \theta e^{-i E_{1} t}\left|\nu_{1}\right\rangle+\cos \theta e^{-i E_{2} t}\left|\nu_{2}\right\rangle\right)\right|^{2}\right.\right.\right. \\
& =\left|\sin ^{2} \theta e^{-i E_{1} t}+\cos ^{2} \theta e^{-i E_{2} t}\right|^{2} \\
& =1-\sin ^{2}(2 \theta) \sin ^{2}\left(\frac{\left(E_{1}-E_{2}\right) t}{2}\right)
\end{aligned}
$$

Using $E_{i}=\sqrt{p_{i}^{2}+m_{i}^{2}}$ and the fact that $p_{i}>>m_{i}^{2}$ because the neutrinos are assumed to be ultra-relativistic the energy for mass eigenstate $\left|\nu_{i}\right\rangle$ can be written as

$$
E_{i}=\sqrt{p_{i}^{2}+m_{i}^{2}}=p_{i} \sqrt{1+\frac{m^{2}}{p_{i}^{2}}}=p_{i}+\frac{m_{i}^{2}}{2 p_{i}}+\ldots
$$


All of the mass eigenstates have the same momentum and $p=E / c$ so that

$$
\begin{aligned}
\sin ^{2}\left(\frac{\left(E_{1}-E_{2}\right) t}{2}\right) & =\sin ^{2}\left(\frac{t}{4 E}\left(m_{1}^{2}-m_{2}^{2}\right)\right) \\
& =\sin ^{2}\left(\frac{L \Delta m_{21}^{2}}{4 E}\right)
\end{aligned}
$$

where factors of $c$ have been suppressed and the replacement $\Delta m^{2}=m_{2}^{2}-m_{1}^{2}$ has been made. The baseline, $L=c t$, is the distance the neutrinos have propagated.

The oscillation probability ${ }^{1}$ for $\nu_{\mu}$ disappearance is then

$$
P_{\nu_{\mu} \rightarrow \nu_{\mu}}=1-\sin ^{2}(2 \theta) \sin ^{2}\left(\frac{L \Delta m^{2}}{4 E}\right) .
$$

The corresponding probability for $P_{\nu_{\mu} \rightarrow \nu_{e}}$, or $\nu_{e}$ appearance is related to this probability via

$$
P_{\nu_{\mu} \rightarrow \nu_{e}=}=1-P_{\nu_{\mu} \rightarrow \nu_{\mu}}=\sin ^{2}(2 \theta) \sin ^{2}\left(\frac{L \Delta m^{2}}{4 E}\right) .
$$

These oscillation probabilities have an amplitude term, $\sin ^{2}(2 \theta)$, and a frequency term, $\sin ^{2}\left(\frac{L \Delta m^{2}}{4 E}\right)$ and so they form an oscillatory pattern when plotted as a function of energy. Figure 2.1 shows this pattern for the $P_{\nu_{\mu} \rightarrow \nu_{\mu}}$ from Equation 13. For an amplitude of $\sin ^{2}(2 \theta)=1$ there are multiple energies where $P_{\nu_{\mu} \rightarrow \nu_{\mu}}=0$ and all of the muon neutrinos have oscillated away. These approximations using two flavors are valid to first order because the oscillations amongst the flavors are decoupled by the existence of two distinct mass squared differences. However, the mixing angles are very different between $P_{\nu_{\mu} \rightarrow \nu_{\mu}}$ and $P_{\nu_{\mu} \rightarrow \nu_{e}}$.

2.1.3. Three Flavor Mixing in Vacuum. In the case of mixing between the three neutrino flavors the oscillations become more complicated. As described above, in three

\footnotetext{
${ }^{1}$ The oscillation frequency, $\frac{L \Delta m^{2}}{4 E}$, can be made unit-less by adding back in the missing factors of $\bar{h}$ and $\mathrm{c}$ to become $\frac{1.2669 \cdot L \Delta m^{2}}{E}$.
} 


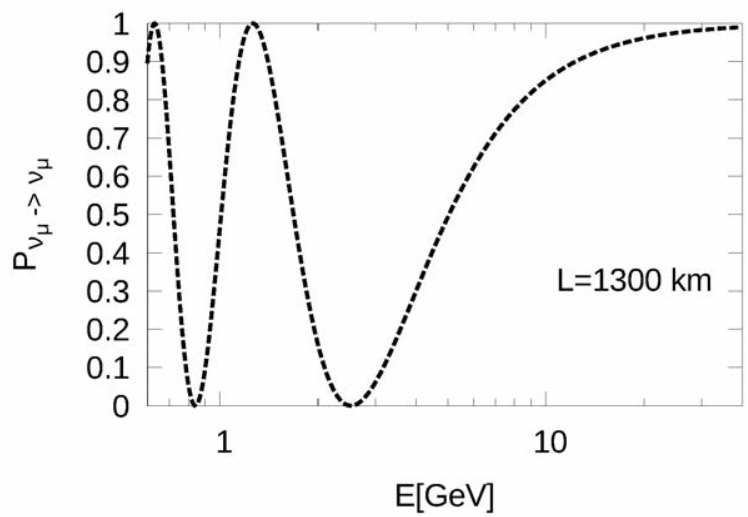

Figure 2.1. The two-flavor oscillation probability $P_{\nu_{\mu} \rightarrow \nu_{\mu}}$ vs Energy for $L=$ $1300 \mathrm{~km}$ and for an amplitude, or $\sin ^{2}(2 \theta)$, of 1 .

neutrino mixing there are three angles, one phase, and three neutrino masses that define the neutrino oscillation probabilities. The $\nu_{e}$ appearance probability, $P\left(\nu_{\mu} \rightarrow \nu_{e}\right)$, is approximately given by Freund in $[2]$ as:

$$
P\left(\nu_{\mu} \rightarrow \nu_{e}\right) \approx P_{0}+P_{\sin \delta}+P_{\cos \delta}+P_{3}
$$

where

$$
\begin{aligned}
P_{0} & =\sin ^{2} \theta_{23} \sin ^{2} 2 \theta_{13} \sin ^{2} \hat{\Delta} \\
P_{\sin \delta} & =\alpha \sin \delta \cos \theta_{13} \sin 2 \theta_{12} \sin 2 \theta_{13} \sin 2 \theta_{23} \sin ^{3} \hat{\Delta} \\
P_{\cos \delta} & =\alpha \cos \delta \cos \theta_{13} \sin 2 \theta_{12} \sin 2 \theta_{13} \sin 2 \theta_{23} \cos \hat{\Delta} \sin ^{2} \hat{\Delta} \\
P_{3} & =\alpha^{2} \cos ^{2} \theta_{23} \sin ^{2} 2 \theta_{12} \sin ^{2} \hat{\Delta}
\end{aligned}
$$

where $\hat{\Delta}=\Delta m_{31}^{2} L /(4 E)$ and the approximation keeps terms up to second order in $\alpha \equiv$ $\Delta m_{21}^{2} / \Delta m_{31}^{2}$. Except for the $\sin ^{2}\left(\theta_{23}\right)$ factor the $P_{0}$ term directly corresponds to the two flavor oscillation probability given in Equation 14. The next set of terms, $P_{\sin \delta}$ and $P_{\cos \delta}$, 
are a factor $\alpha$ smaller $^{2}$ in amplitude than the leading order term and are odd and even in $\delta_{C P}$ variations respectively. The final term is proportional to $\alpha^{2}$ and is therefore a small correction to the other terms.

Figure 2.1 depicts each of the terms in Equation 15 as a function of neutrino energy, E, at a baseline of $1300 \mathrm{~km}$ and for two different values of $\delta_{C P}$. In the case of the $\delta_{C P}=0$ the $P_{\cos \delta}$ acts to increase the oscillation probability at both maxima while in the $\delta_{C P}=\pi / 2$ case the $P_{\sin \delta}$ term increases the probability at the first ${ }^{3}$ maximum but decreases it at the second maximum. In either case the $P_{\sin \delta}$ or $P_{\cos \delta}$ term is roughly $20 \%$ of the total oscillation probability at the peak of the first oscillation maximum.

The values of the mixing angles and mass squared differences for this figure are taken from a 2014 global fit to the data from multiple experiments. This global fit will be described in Chapter 3

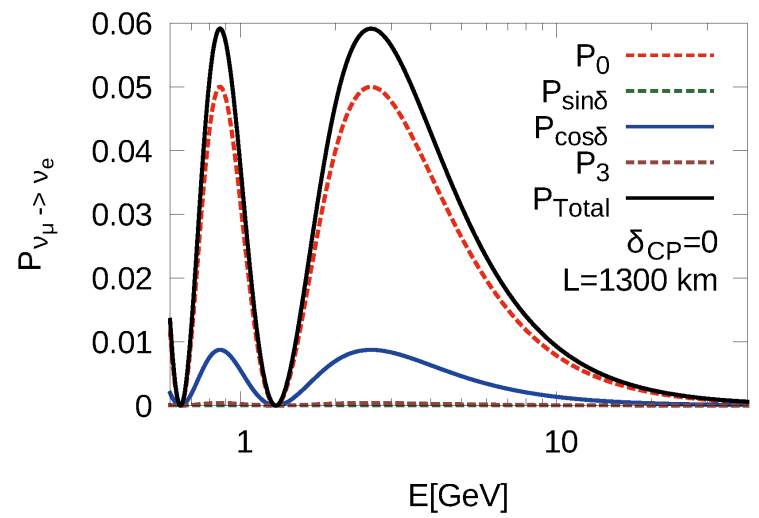

(A) $\delta_{C P}=0$

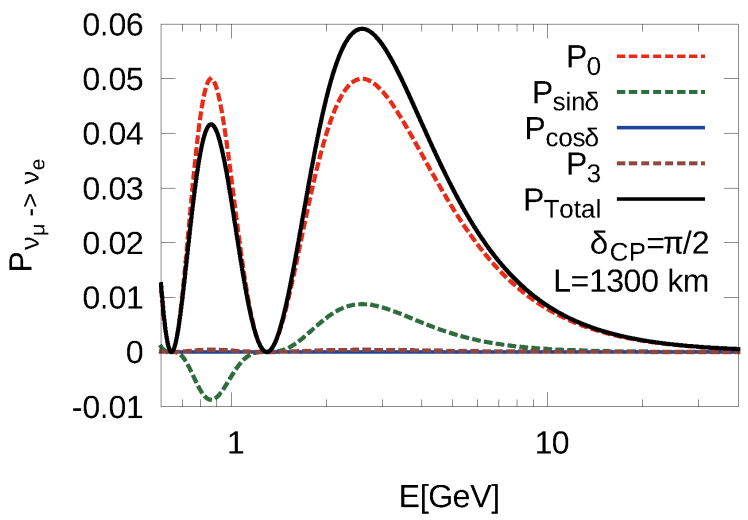

(B) $\delta_{C P}=\pi / 2$

FiguRE 2.2. The oscillation probabilities $P_{\nu_{\mu} \rightarrow \nu_{e}}$ vs Energy for $L=1300$ $\mathrm{km}$ for both $\delta_{C P}=0$ and $\delta_{C P}=\pi / 2$. The values of the mixing angles are taken from a current global fit. The subcomponents that make up the total probability are given in Equation 15

\footnotetext{
${ }^{2}$ Using the current global fit measurements of the parameters: $\alpha=\Delta m_{21}^{2} / \Delta m_{31}^{2} \simeq 32$.

${ }^{3}$ The oscillation maxima are numbered from right to left when the oscillation probability is plotted versus energy. This is because the $\mathrm{E}$ appears in the denominator of the frequency term in the oscillation probability so that increasing energy corresponds to a lower frequency.
} 
2.1.4. Three Flavor Mixing in Matter. When neutrinos pass through matter the $\nu_{e}$ component interacts with the electrons present in matter. This process is known as charged current coherent forward scattering and it modifies the propagation of the neutrinos through matter. Schematically, this modifies the Hamiltonian to include an effective interaction term for the $\nu_{e}$ component thus changing the effective mass eigenstates that make up the $\nu_{e}$ flavor eigenstate. An approximation to the three flavor $\nu_{e}$ appearance probability with matter effects included is also given by $\lfloor 2\rfloor$ as:

$$
P\left(\nu_{\mu} \rightarrow \nu_{e}\right) \approx P_{0}+P_{\sin \delta}+P_{\cos \delta}+P_{3}
$$

where

$$
\begin{aligned}
P_{0} & =\sin ^{2} \theta_{23} \frac{\sin ^{2} 2 \theta_{13}}{(\hat{A}-1)^{2}} \sin ^{2}((\hat{A}-1) \hat{\Delta}) \\
P_{\sin \delta} & =\alpha \frac{\sin \delta \cos \theta_{13} \sin 2 \theta_{12} \sin 2 \theta_{13} \sin 2 \theta_{23}}{\hat{A}(1-\hat{A})} \sin (\hat{\Delta}) \sin (\hat{A} \hat{\Delta}) \sin ((1-\hat{A}) \hat{\Delta}) \\
P_{\cos \delta} & =\alpha \frac{\cos \delta \cos \theta_{13} \sin 2 \theta_{12} \sin 2 \theta_{13} \sin 2 \theta_{23}}{\hat{A}(1-\hat{A})} \cos (\hat{\Delta}) \sin (\hat{A} \hat{\Delta}) \sin ((1-\hat{A}) \hat{\Delta}) \\
P_{3} & =\alpha^{2} \frac{\cos ^{2} \theta_{23} \sin ^{2} 2 \theta_{12}}{\hat{A}^{2}} \sin ^{2}(\hat{A} \hat{\Delta})
\end{aligned}
$$

where $\hat{\Delta}=\Delta m_{31}^{2} L /(4 E)$ and $\hat{A}=2 E \sqrt{2} G_{F} n_{e} / \Delta m_{31}^{2}$. The term $G_{F}$ is the Fermi coupling constant and $n_{e}$ is the electron density in the matter through which the neutrino is passing. This approximation keeps terms up to second order in $\alpha \equiv \Delta m_{21}^{2} / \Delta m_{31}^{2}$ and first order in $\sin ^{2}\left(\theta_{13}\right)$. For $P\left(\bar{\nu}_{\mu} \rightarrow \bar{\nu}_{e}\right)$ the sign of $\hat{A}$ and $P_{\sin \delta}$ are flipped. Figure 2.2 shows a comparison between the $P\left(\nu_{\mu} \rightarrow \nu_{e}\right)$ in vacuum and in matter.

2.1.5. Oscillation Probabilities. It is useful to evaluate approximations to the oscillation equations, as in the preceding sections, to gain an intuitive sense of the oscillation 

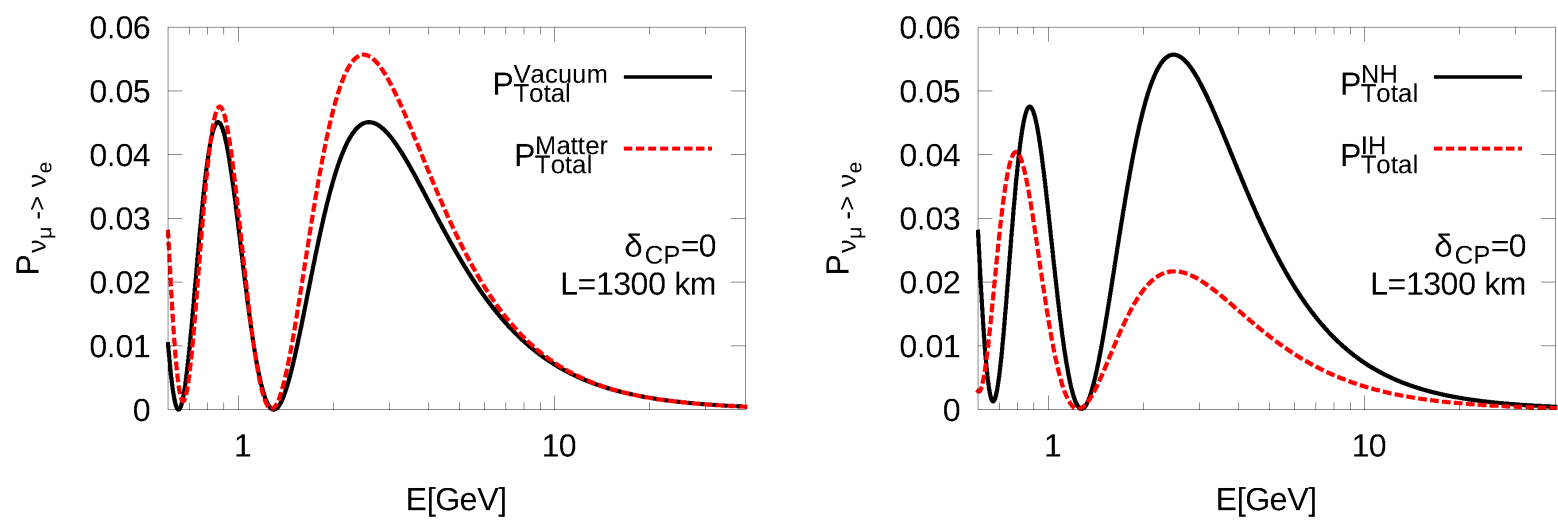

FIgURE 2.3. The oscillation probabilities $P_{\nu_{\mu} \rightarrow \nu_{e}}$ vs Energy for $L=1300$ $\mathrm{km}$ in vacuum vs matter (left) and in the normal hierarchy $(\mathrm{NH})$ vs inverted hierarchy (IH) (right).

effects for particular assumptions. In practice, the neutrino oscillation probabilities are computed numerically and so no approximations need be made for an analysis. The work in this thesis uses the GLoBES library [4]|5] which implements the evolution operator method to numerically compute the oscillation probabilities in matter.

An evaluation of these oscillation probabilities at multiple $L$ values will be given in Chapter 4. All oscillation probabilities for the remainder of this thesis will be computed using the GLoBES library.

\subsection{Open Questions}

For the type of experiment that is of primary interest in this thesis, the long-baseline neutrino oscillation experiments, there are a few questions that make up their primary goals. These questions are the ones that can be addressed by generating an artificial beam of primarily muon neutrinos with reasonably well understood energy (E) spectrum, allowing them to propagate some distance $(\mathrm{L})$, and detecting their interactions in a detector. By looking for $\nu_{e}$ appearance, or the presence of $\nu_{e}$ in a beam where there were initially none (or few) present, the oscillation parameters of the $P\left(\nu_{\mu} \rightarrow \nu_{e}\right)$ equation are measured. Similarly, 
by looking for $\nu_{\mu}$ disappearance, or the absence of $\nu_{\mu}$ in a beam where the initial number is known, the oscillation parameters of the $P\left(\nu_{\mu} \rightarrow \nu_{\mu}\right)$ equation are measured.

2.2.1. CP Violation. The phenomenon of Charge-Parity $(\mathrm{CP})$ violation refers to the joint symmetry of charge, or changing the charge of the particles in an interaction, and parity, or reflecting the interaction in a mirror. In neutrino interactions, applying the CP symmetry to the relevant interactions corresponds to changing from neutrinos to antineutrinos. This means that $P\left(\nu_{\mu} \rightarrow \nu_{e}\right) \neq P\left(\bar{\nu}_{\mu} \rightarrow \bar{\nu}_{e}\right)$ if the CP symmetry is violated.

The existence of $\mathrm{CP}$ violation has important implications for the origins of the matter/antimatter asymmetry in the universe. Along with other requirements affecting the cosmological theory of leptogenesis, CP violation in the lepton sector via neutrinos is an important piece of this matter vs antimatter asymmetry arising in the theory|1]. If neutrinos behave differently from antineutrinos then part of a theoretical explanation for this asymmetry in the universe is supported.

One quantitative measure of the asymmetry between neutrino and antineutrino oscillations due to the effects of $\mathrm{CP}$ violation is the approximation from [6]:

$$
\begin{aligned}
A_{C P} & \equiv \frac{P\left(\nu_{\mu} \rightarrow \nu_{e}\right)-P\left(\bar{\nu}_{\mu} \rightarrow \bar{\nu}_{e}\right)}{P\left(\nu_{\mu} \rightarrow \nu_{e}\right)+P\left(\bar{\nu}_{\mu} \rightarrow \bar{\nu}_{e}\right)} \\
& \simeq \frac{\cos \theta_{23} \sin \left(2 \theta_{12}\right) \sin \delta}{\sin \theta_{23} \sin \theta_{13}}\left(\frac{\Delta m_{21}^{2} L}{4 E_{\nu}}\right)+\text { matter effects }
\end{aligned}
$$

This approximation is valid to first order in the $\Delta m_{21}^{2}$ mass squared difference. The asymmetry decreases with increasing $\sin ^{2}\left(\theta_{23}\right)$, decreases with increasing $\sin ^{2}\left(2 \theta_{13}\right)$ and of course depends directly on the value of $\sin \delta_{C P}$. The size of the asymmetry also depends on the baseline and falls off with increasing energy. 
A value of $\delta_{C P}$ that is not equal to 0 or $\pi$ would mean that $\mathrm{CP}$ violation exists and that neutrinos behave differently from antineutrinos. So in practice $\mathrm{CP}$ violation could be determined by running only neutrinos to isolate the $\delta_{C P}$ terms in the $P\left(\nu_{\mu} \rightarrow \nu_{e}\right)$ equation. However, it is important to compare $\nu$ running to $\bar{\nu}$ running to directly establish that neutrinos oscillate differently than antineutrinos. In addition, as will be shown in Chapter 9 , a combination of the $\nu$ and $\bar{\nu}$ running generally leads to optimal sensitivity to CP violation.

2.2.2. Mass Hierarchy. As described above, the mass hierarchy (MH) refers to the ordering of the masses that make up the neutrino mass eigenstates. Two possible arrangements of the mass states are depicted in Figure 2.4. It is known that $m_{2}^{2}>m_{1}^{2}$ based on observations of solar neutrinos, but their relationship with $m_{3}^{2}$ is not known. One possibility is to have $m_{3}^{2}>m_{2}^{2}>m_{1}^{2}$ and this is referred to as the normal hierarchy (NH) case in analogy with the ordering of quark and charged lepton generations with respect to mass. The other possibility is that $m_{2}^{2}>m_{1}^{2}>m_{3}^{2}$ and this is known as the inverted hierarchy (IH). The matter effect increases(decreases) the $P\left(\nu_{\mu} \rightarrow \nu_{e}\right)$ oscillation probability in the $\mathrm{NH}(\mathrm{IH})$ and has the opposite effect for $P\left(\bar{\nu}_{\mu} \rightarrow \bar{\nu}_{e}\right)$ as seen in Figure 2.3.

The matter effect also causes differences in the oscillations of neutrinos and antineutrinos, making $P\left(\nu_{\mu} \rightarrow \nu_{e}\right) \neq P\left(\bar{\nu}_{\mu} \rightarrow \bar{\nu}_{e}\right)$, due to there being matter rather than antimatter along the path. The matter effects have to be disentangled from the true $\mathrm{CP}$ violating effects caused by $\sin \delta_{C P} \neq 0$. This can be done by either having a much longer baseline to make the matter effect much larger than the effect of $\delta_{C P}$ or by having a shorter baseline where the matter effects are minimized in which case the $\mathrm{MH}$ would remain unresolved. The degeneracies in event rates between matter effects and $\delta_{C P}$ effects and how to separate them will be a common theme throughout this thesis. 


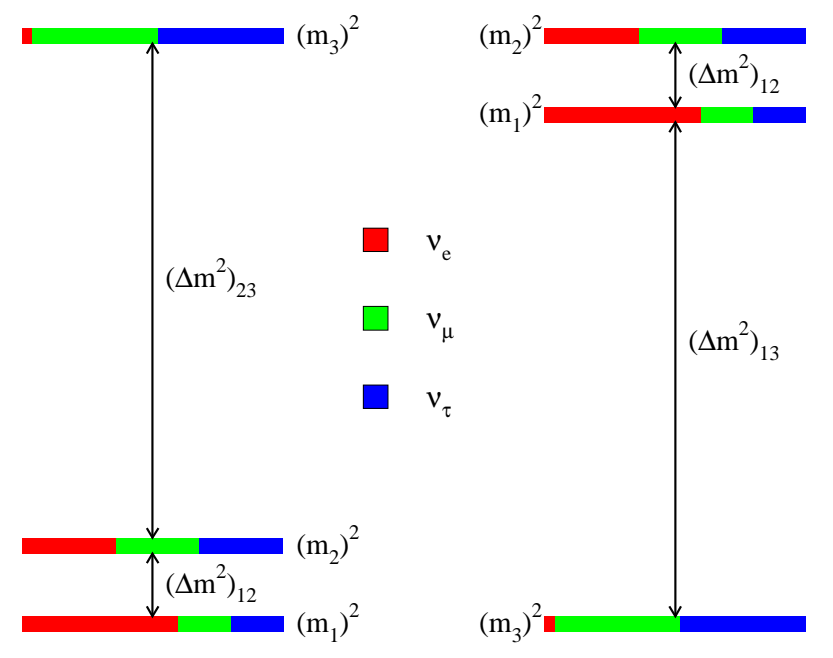

FiguRE 2.4. Figure from [7]. The arrangement of mass states in the normal and inverted hierarchies. In the normal hierarchy $m_{3}^{2}>m_{2}^{2}>m_{1}^{2}$ and in the inverted hierarchy $m_{2}^{2}>m_{1}^{2}>m_{3}^{2}$. The colors in each bar represent the flavor components of each mass state.

2.2.3. $\theta_{23}$ Octant vs Maximal Mixing. The angles $\theta_{12}, \theta_{13}$, and $\theta_{23}$ that relate the neutrino flavor eigenstates to the mass eigenstates are defined to be between 0 and $\pi / 2$. The measured value of $\theta_{13}$ is relatively small and is in the first octant and the value of $\theta_{12}$ is also in the first octant. The octant of $\theta_{23}$ is relatively unconstrained by current measurements and the angle may even be maximal $\left(\theta_{23}=\pi / 4\right)$. A high precision measurement is needed in order to access the octant of $\theta_{23}$.

The combination of the unknown value of $\delta_{C P}$, the mass hierarchy, and the $\theta_{23}$ octant leads to degeneracies, or equal oscillation probabilities, for various value of these parameters. The resolution of these degeneracies is a primary focus of this thesis.

\subsection{Anomalies}

In general the experimental measurements made to date are consistent with the three neutrino hypothesis. However there are a few experimental results that have led to the 
consideration of additional generations of neutrinos beyond three. These are referred to as sterile neutrino hypotheses in which the existence of a third or fourth generation of neutrino is hypothesized along with additional mixing angles and mass differences.

In addition to the sterile neutrino hypotheses, non-standard interactions (NSI) of neutrinos have been proposed. These modify the Hamiltonian for propagation of the neutrinos, beyond the MSW effect described above, in a way that is general and allows for the quantification of effects that do not fit within the standard three flavor paradigm. These are sub-leading effects, below the sub-leading effects of CP violation and the MSW (matter) effect.

The work in this thesis does not consider oscillation models beyond the standard three neutrino mixing model. 


\section{CHAPTER 3}

\section{Current Three-Flavor Experimental Measurements}

\section{AND Global Fit}

There are multiple ways of observing neutrino oscillations experimentally. The general approach is to identify a source, determine the baseline or the distance traveled by the neutrinos, determine the initial flux of neutrinos being detected as a function of energy, and detect the neutrinos interacting in a detector. In practice each of these pieces is complicated by the fact that neutrinos interact so rarely and there are significant backgrounds, or other interactions that look like neutrinos, to keep many of the pieces obscured. By designing an experiment to access different $\mathrm{L} / \mathrm{E}$ ratios, different oscillation parameters can be accessed.

Neutrino oscillation experiments can be divided into the following categories:

- Long-baseline (LBL) accelerator experiments typically have $E / L \approx \Delta m_{31}^{2} \sim 2.5 \times$ $10^{-3} \mathrm{eV}^{2}$ by design. The conventional method is to accelerate protons to high energy and collide them with a fixed target. Pions are produced, magnetically focused, and allowed to decay in flight to yield muon neutrinos. The muon neutrinos propagate through the Earth over baselines of $\mathcal{O}(100-1000 \mathrm{~km})$. LBL experiments are typically sensitive to $\sin ^{2}\left(2 \theta_{23}\right)$ and $\Delta m_{32}^{2}$ through muon neutrino disappearance and $\sin ^{2}\left(2 \theta_{13}\right), \sin ^{2}\left(\theta_{23}\right), \Delta m_{31}^{2}$ and $\delta_{C P}$ through electron neutrino appearance. An LBL experiment is also sensitive to the matter effect that increases with baseline and gives access to the sign of $\Delta m_{31}^{2}$. LBL experiments will be described in greater detail in Chapter 4.

- Short-baseline (SBL) accelerator experiments share much in common with their LBL counterparts, except that their baselines are much shorter, $\mathcal{O}(100 \mathrm{~m})$, to give 
access to very rapid oscillations with $E / L \approx \Delta m^{2} \sim 1 \mathrm{eV}^{2}$. They are sensitive to hypothetical sterile neutrino oscillations where some fraction of the expected signal disappears. These types of oscillations are beyond the three-flavor neutrino model.

- Atmospheric neutrino experiments measure the flux of $\nu_{\mu}$ that result from cosmic rays colliding with the atmosphere. Because they arrive at the detector from all directions, some passing through the Earth, they have L/E ratios ranging from $1-10^{5} \mathrm{~km} / \mathrm{GeV}[9]$. They are predominantly sensitive to $\sin ^{2}\left(2 \theta_{23}\right)$ and $\Delta m_{32}^{2}$, but with more accurate zenith angle reconstruction they are becoming more sensitive to sub-leading effects from $\delta_{C P}, \theta_{13}$, and the mass hierarchy.

- Short-baseline (SBL) and Long-baseline (LBL) Reactor neutrino experiments measure the $\bar{\nu}_{e}$ flux that results from nuclear fission within reactors. Liquid scintillator detectors are placed within $\mathcal{O}(1 \mathrm{~km})$ of the reactor for SBL and $\mathcal{O}(100 \mathrm{~km})$ for LBL and observe inverse beta decay reactions $\left(\bar{\nu}_{e}+p \rightarrow n+e^{+}\right)$. Reactor experiments are primarily sensitive to $\sin ^{2}\left(2 \theta_{13}\right)$ with short baseline oscillations dominated by $\Delta m_{31}^{2}$ and to $\Delta m_{21}^{2}$ and $\theta_{12}$ with long-baseline oscillations.

- Solar neutrino experiments measure the $\nu_{e}$ flux resulting from nuclear fusion in the sun. Solar neutrino oscillations were established by measuring the total neutrino flux to be about three times that of the electron neutrino flux[10]. These experiments are primarily sensitive to $\theta_{12}$.

All of these experiment types have contributed to the constraints on the neutrino oscillation parameters. The focus of this thesis is on LBL neutrino experiments as they have the potential to resolve many of the open questions, but they will rely on the constraints on the neutrino oscillation parameters provided by the other experiments. A global fit is performed to many of these experiments to extract the best possible constraints on all of the 
oscillation parameters and will be discussed below. Not mentioned here are supernova neutrino, geo-neutrino, and neutrinoless double beta decay experiments. These do not currently contribute to the global three-flavor oscillation parameter fits.

\subsection{Experimental Measurements}

The work in this thesis uses the best-fit and uncertainties on the neutrino mixing angles and mass squared differences from a global fit by Capozzi et al. [11] assuming the threeneutrino hypothesis. This section will briefly review experimental results that contribute to this global fit. All of the results presented in this section were used as the inputs for the global fit.
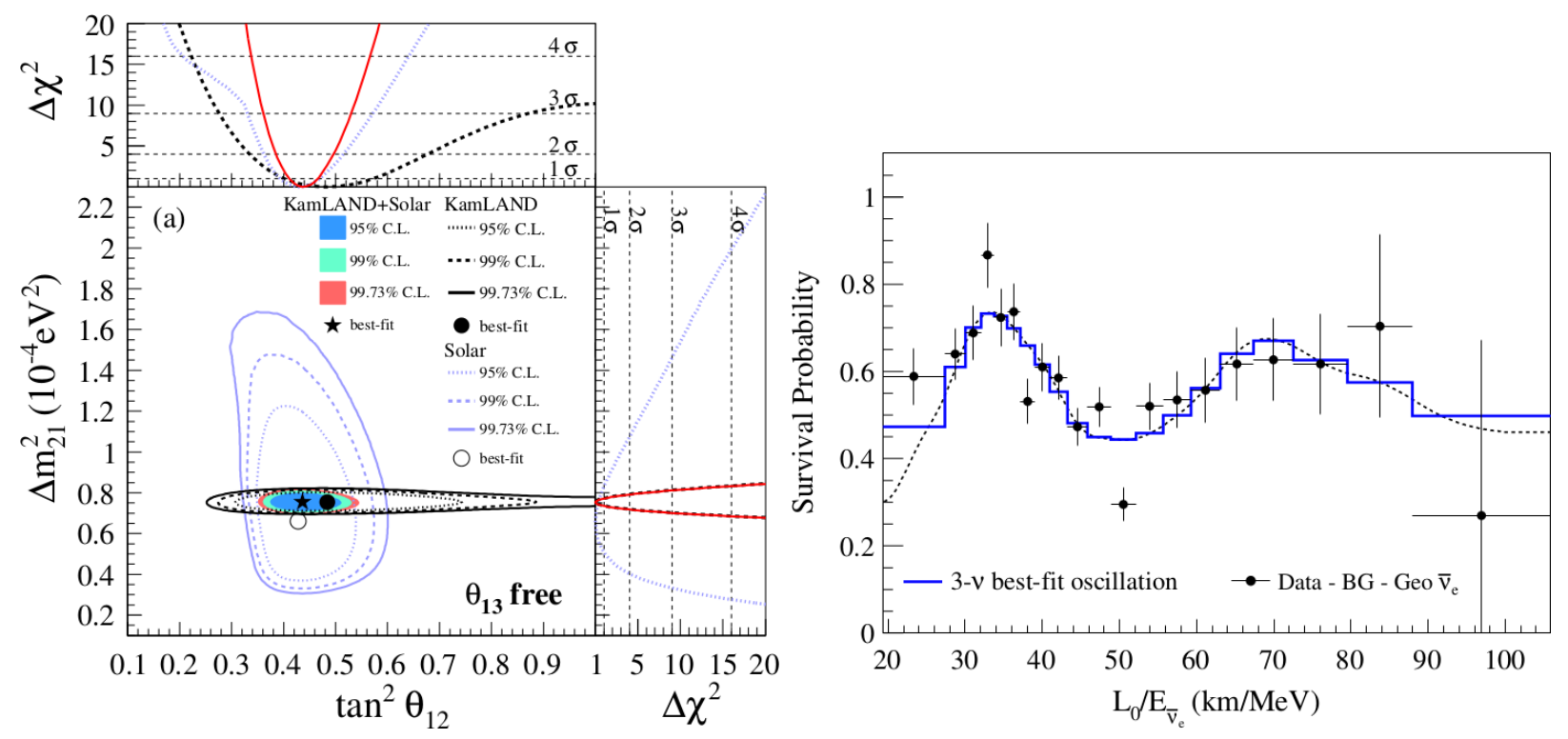

Figure 3.1. The 2013 results from KamLAND[12]. The left figure shows the best-fits and allowed contours in $\Delta m_{31}^{2}$ vs $\tan ^{2} \theta_{12}$ for KamLAND, Solar experiments, and KamLAND+Solar experiments. The right figure shows the expected survival probability vs L/E for the three neutrino best-fit and the KamLAND data. 


\subsubsection{KAmLAND. The Kamioka Liquid scintillator Anti-Neutrino Detector (KamLAND)}

is an LBL reactor neutrino experiment looking for $\bar{\nu}_{e}$ disappearance over multiple longbaselines. It measured the $\bar{\nu}_{e}$ flux from 53 Japanese nuclear reactors at an average baseline of $180 \mathrm{~km}$ by detecting $\bar{\nu}_{e}$ interactions via inverse beta decay. Figure 3.1 shows the 2013 KamLAND+Solar result $\mid 12]$ with measurements of $\Delta m_{21}^{2}=7.53_{-0.18}^{+0.18} \times 10^{-5} \mathrm{eV}^{2}$ and $\tan ^{2}\left(\theta_{12}\right)=0.436_{-0.025}^{+0.029}$. The KamLAND result gives the best measurement on the $\Delta m_{21}^{2}$ mass squared difference and when combined with solar neutrino measurements gives the best constraint on the $\theta_{12}$ mixing angle of any experiment.
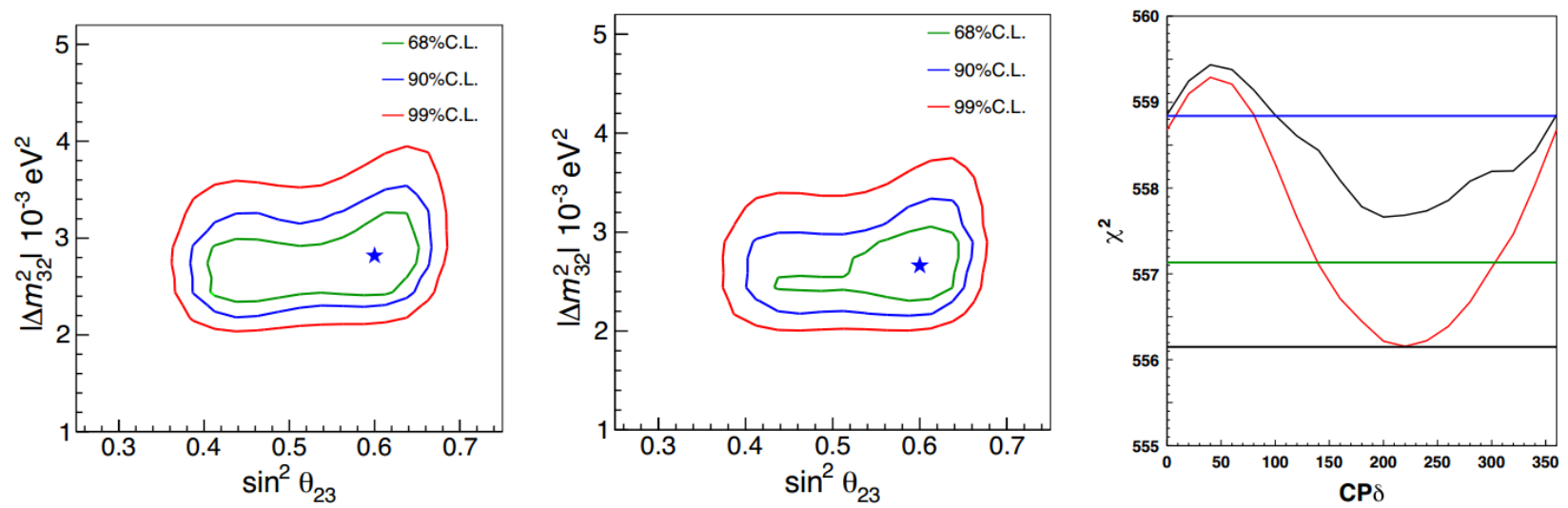

Figure 3.2. The SKI-IV fit three-flavor results from SK $|13|$. The left and center figures show the allowed contours and best-fits in $\left|\Delta m_{32}^{2}\right|$ vs $\sin ^{2}\left(\theta_{23}\right)$ under normal and inverted mass hierarchy assumptions respectively. The right figure shows the $\chi^{2}$ vs $\delta_{C P}$ for normal (black) and inverted (red) hierarchy where the $1 \sigma$ (green) and $2 \sigma$ (blue) $\chi^{2}$ levels are drawn as horizontal lines.

3.1.2. Super-Kamiokande. The Super Kamiokande (SK) experiment|14] is a $50 \mathrm{kt}$ water Cerenkov detector located underground at a depth of 2700 meters-water-equivalent (MWE). An atmospheric neutrino analysis measures the flux of both $\nu_{e}+\bar{\nu}_{e}$ and $\nu_{\mu}+\bar{\nu}_{\mu}$ events ( $\nu$ and $\bar{\nu}$ events are not distinguished) as a function of zenith angle and compares them with Monte Carlo predictions. Figure 3.2 shows the results from an analysis covering run periods SK I-IV. There are slight preferences for the second octant $\left(\sin ^{2}\left(\theta_{23}\right)>0.5\right)$, 
$\delta_{C P}=220^{\circ} \pm 80^{\circ}$, and the inverted hierarchy at $1.2 \sigma$. The best-fits and allowed regions for $\Delta m_{32}^{2}, \sin ^{2}\left(\theta_{23}\right)$, and $\delta_{C P}$ are not currently reported numerically in a published work. The SK results contribute only slightly to the constraints on $\delta_{C P}$ and $\sin ^{2}\left(\theta_{23}\right)$ in the global fit.
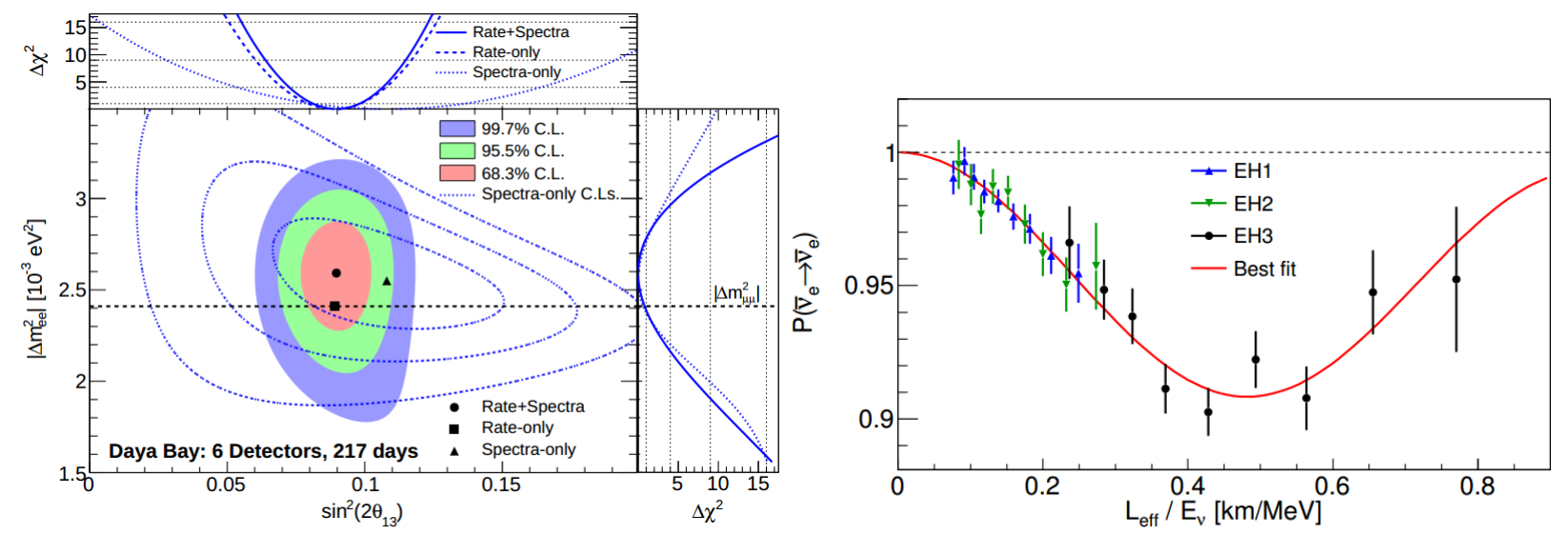

Figure 3.3. The 2013 results from Daya Bay|15]. The left figure shows the best fits in a rate+spectra, rate-only, and spectra-only analysis in the $\left|\Delta m_{e e}^{2}\right|$ vs $\sin ^{2}\left(2 \theta_{13}\right)$ where $\left|\Delta m_{e e}^{2}\right|=\cos ^{2}\left(\theta_{12}\right)\left|\Delta m_{31}^{2}\right|+\sin ^{2}\left(\theta_{12}\right)\left|\Delta m_{32}^{2}\right|$. The best-fit is given by the black dot. The right figure shows $\bar{\nu}_{e}$ disappearance probability vs effective baseline divided by the energy of events at each experimental hall (EH). The best-fit oscillation hypothesis is shown in red.

3.1.3. DAYA BAY. Daya Bay $|15| \mid 16]$ is an SBL reactor neutrino experiment in Daya Bay, China. It consists of six liquid scintillator detectors looking for inverse beta-decay to measure $\bar{\nu}_{e}$ disappearance from the flux produced by six reactor cores. The near detectors are at flux-weighted baselines of $470 \mathrm{~m}$ and $576 \mathrm{~m}$ and the far detectors are at $1648 \mathrm{~m}$. The 2013 Daya Bay result used an analysis of both spectral and rate information from the event spectra at far and near detectors. The spectral shape allowed them to constrain not only $\sin ^{2}\left(2 \theta_{13}\right)$, but also $\Delta m_{e e}^{2}$, which is the effective mass difference influencing $\bar{\nu}_{e}$ disappearance in a reactor experiment|17]. Figure 3.3 shows the 2013 results. The best-fit points are $\sin ^{2}\left(2 \theta_{13}\right)=0.090_{-0.009}^{+0.008}$ and $\left|\Delta m_{e e}^{2}\right|=2.59_{-0.20}^{+0.19} \times 10^{-3} \mathrm{eV}^{2}$. This Daya Bay result provides the strongest constraint on $\sin ^{2}\left(2 \theta_{13}\right)$ via $\bar{\nu}_{e}$ disappearance to date. 

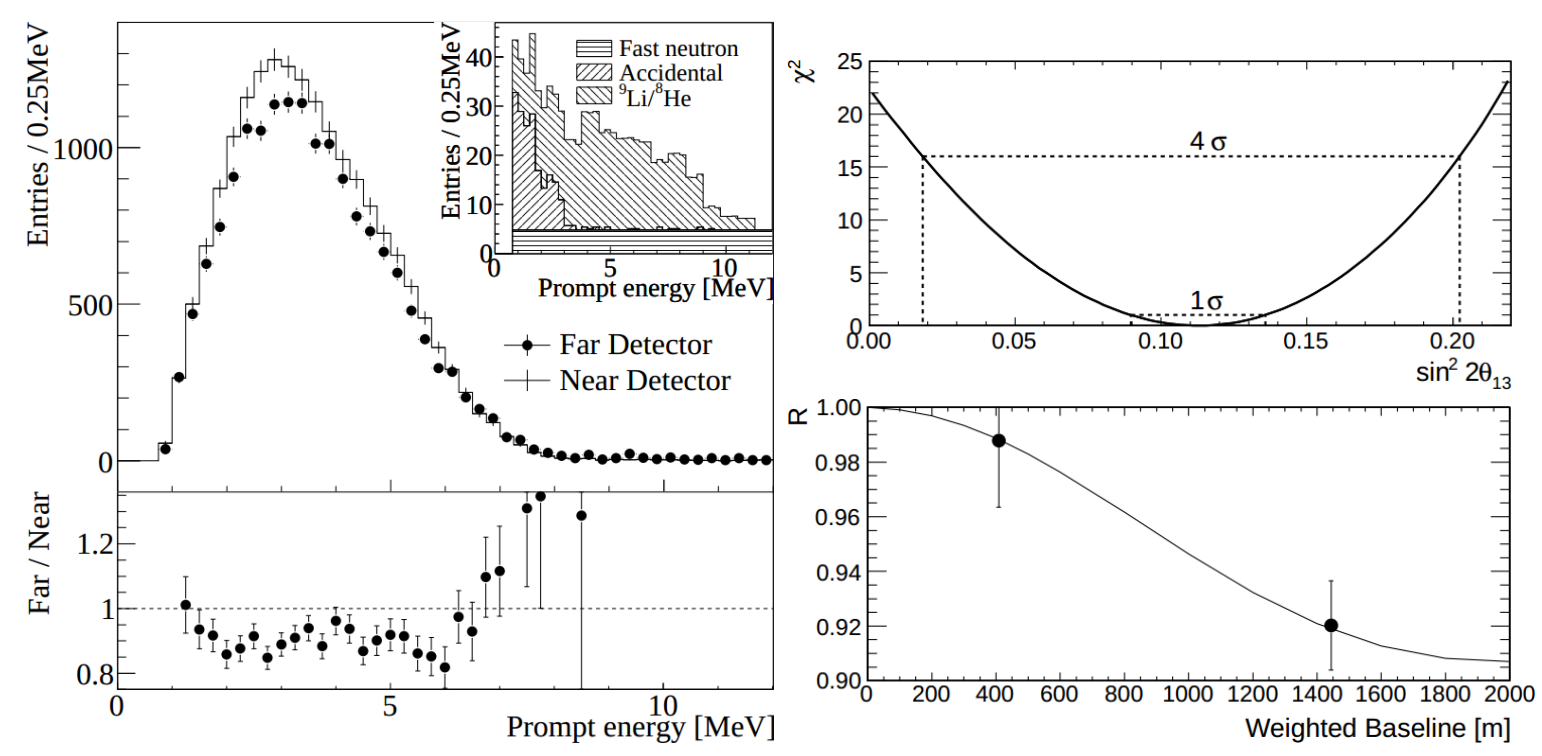

FiguRE 3.4. The 2012 results from Reno[18]. The left figure shows the measured event spectrum at the far detector compared with the near detector and the ratios between them. A clear disappearance effect is seen. The top-right figure shows the $\chi^{2}$ vs $\sin ^{2}\left(2 \theta_{13}\right)$ curve corresponding to the best-fit from the $\bar{\nu}_{e}$ disappearance data. This was a rate-only analysis.

3.1.4. RENO. The Reactor Experiment for Neutrino Oscillation (RENO)[18] is an SBL reactor neutrino experiment in South Korea measuring $\sin ^{2}\left(2 \theta_{13}\right)$ via $\bar{\nu}_{e}$ disappearance. It consists of a near detector at $294 \mathrm{~m}$ and an identical far detector at $1383 \mathrm{~m}$. The rates in the near and far detectors are compared to measure a $\bar{\nu}_{e}$ disappearance effect that is dependent on $\sin ^{2}\left(2 \theta_{13}\right)$. The 2012 results are shown in figure 3.4 and the analysis finds a best-fit of $\sin ^{2}\left(2 \theta_{13}\right)=0.113 \pm 0.013$ (stat.) \pm 0.0019 (syst.) and is a $4.9 \sigma$ determination of $\theta_{13} \neq 0$. The RENO result does not constrain the value of $\sin ^{2}\left(2 \theta_{13}\right)$ as well as the Daya Bay result. The RENO central value is also a bit higher in $\sin ^{2}\left(2 \theta_{13}\right)$ than the Daya Bay central value but is well within $2 \sigma$ so could be a statistical fluctuation. This difference will be interesting to watch in the future as the results from each experiment become more statistically significant.

3.1.5. Double Chooz. Double Chooz|19] is an SBL reactor neutrino experiment located at the Chooz nuclear reactor in France. It is a single $10.3 \mathrm{~m}^{3}$ fiducial volume detector located at $1050 \mathrm{~m}$ from the reactor cores. Figure 3.5 shows the results of the 2012 spectral 


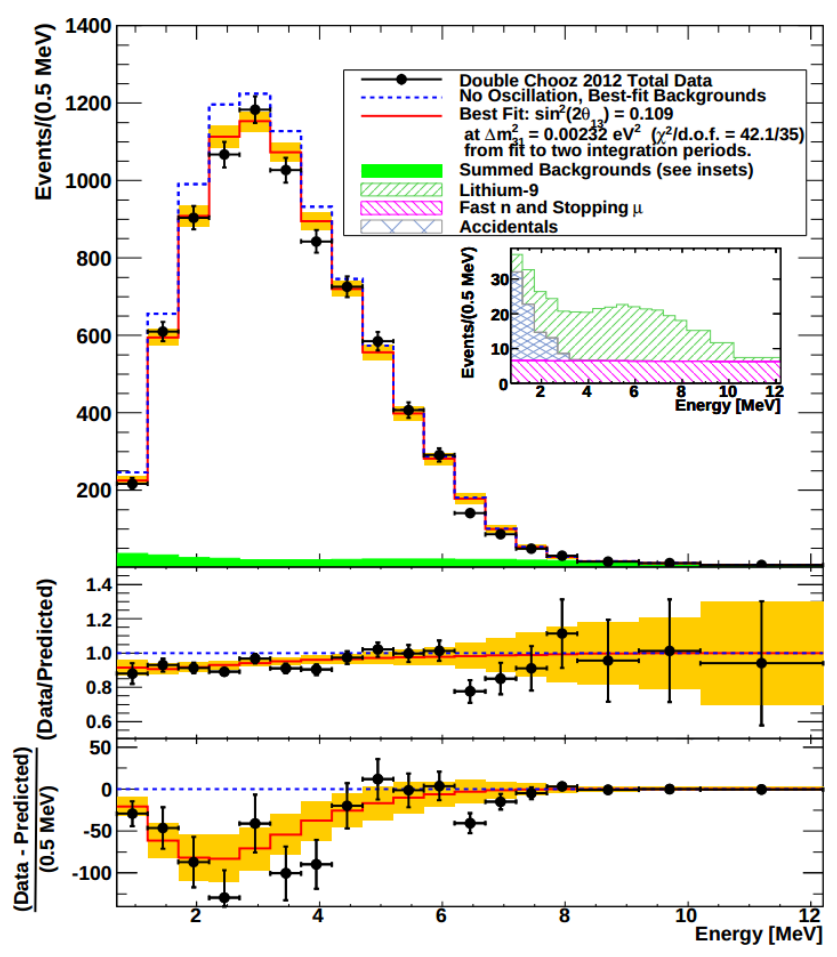

Figure 3.5. The $2012 \bar{\nu}_{e}$ disappearance results from Double Chooz. The black data points show the spectrum of candidate $\bar{\nu}_{e}$ events. The blue and red curves are the expectations with no oscillation and with the best-fit value of $\sin ^{2}\left(2 \theta_{13}\right)=0.143$, respectively. The data vs no oscillation prediction ratio and difference are also shown.

analysis. They observe 8249 candidate $\bar{\nu}_{e}$ events. The expectation with no mixing is 8937 events. The rate and spectral analysis finds $\sin ^{2}\left(2 \theta_{13}\right)=0.109 \pm 0.030$ (stat.) \pm 0.025 (syst.) The Double Chooz result does not constrain $\sin ^{2}\left(2 \theta_{13}\right)$ as well as Daya Bay and RENO but it does agree more closely with the RENO result than with the Daya Bay result.

3.1.6. T2K. Tokai-to-Kamioka $(\mathrm{T} 2 \mathrm{~K})$ is an LBL neutrino experiment with off-axis beam coming from Tokai, Japan and propagating $295 \mathrm{~km}$ to the SK detector described in Section 3.1.2 in the Kamioka mine in Japan. The beam is a primarily $\nu_{\mu}$ beam that is approximately $94 \%$ pure below $1.5 \mathrm{GeV}$ and is optimized to peak at $0.6 \mathrm{GeV}$ where the oscillation probability is maximum for $\nu_{e}$ appearance and $\nu_{\mu}$ disappearance for the $295 \mathrm{~km}$ baseline. There is a near detector, located at $280 \mathrm{~m}$ from the target, used to characterize the beam 
and constrain backgrounds as well as make independent cross-section measurements. Two primary measurements are made: $\nu_{e}$ appearance and $\nu_{\mu}$ disappearance.
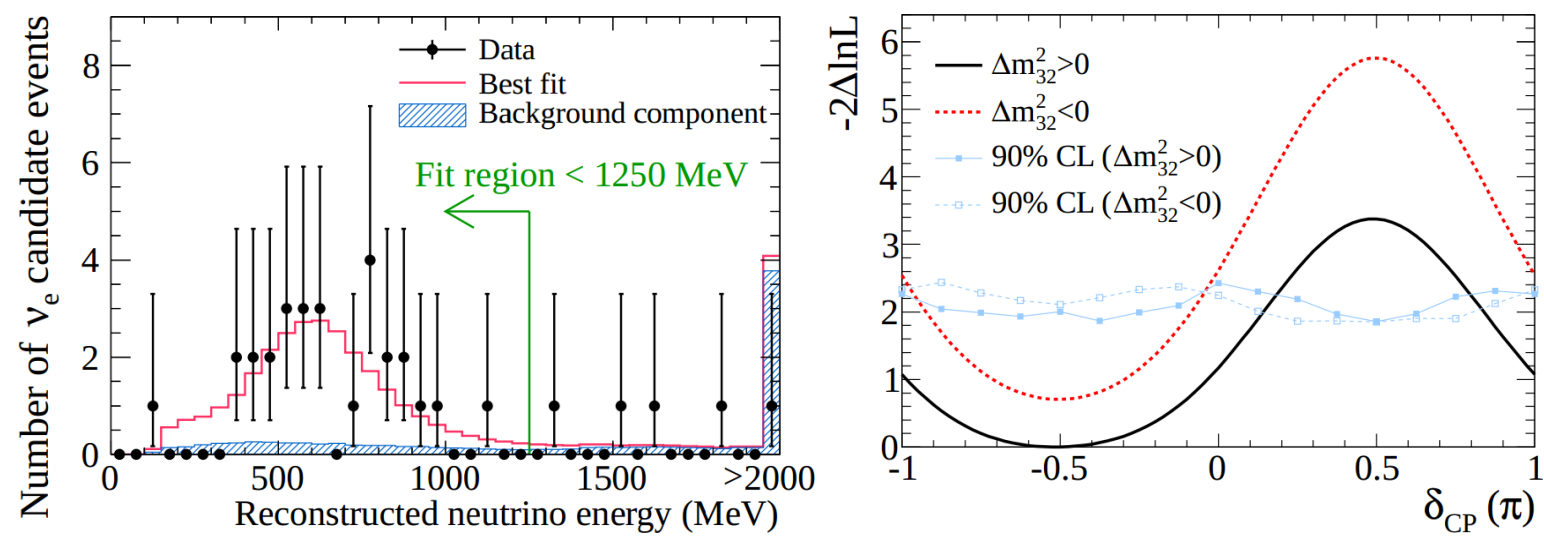

Figure 3.6. The $2013 \nu_{e}$ appearance results from T2K|20]. The left figure shows the measured $\nu_{e}$ appearance event spectrum at SK as a function of reconstructed neutrino energy. The best-fit oscillation curve, with $\sin ^{2}\left(2 \theta_{13}\right)=$ 0.144 , is in red. The right figure shows the $-2 \Delta \ln \mathrm{L}$ value as a function of $\delta_{C P}$ for both the normal (black) and inverted (red) hierarchies. This uses the best-fit values for $\sin ^{2}\left(2 \theta_{13}\right)$ from reactor neutrino data.

Figure 3.6 shows the $2013 \nu_{e}$ appearance analysis results. A total of $28 \nu_{e}$ appearance events were observed with an expected background of 4.92 corresponding to a $\nu_{e}$ appearance significance of $7.3 \sigma$. The best fit values were $\sin ^{2}\left(2 \theta_{13}\right)=0.140_{-0.032}^{+0.038}$ in the normal hierarchy and $\sin ^{2}\left(2 \theta_{13}\right)=0.170_{-0.037}^{+0.045}$ in the inverted hierarchy. If the constraints on $\sin ^{2}\left(2 \theta_{13}\right)$ from reactor measurements are used then the $\mathrm{T} 2 \mathrm{~K}$ data show a preference for $\delta_{C P}=-\pi / 2$ where $\delta_{C P}=\pi / 2$ is excluded at greater than $90 \%$ CL.

Figure 3.7 shows the T2K $2013 \nu_{\mu}$ disappearance analysis results. The disappearance analysis looks for a deficit of muon like events in the SK detector. The analysis found 58 events where $205 \pm 17$ were expected in the absence of oscillations. The three-neutrino fit, assuming the normal hierarchy, finds $\sin ^{2}\left(\theta_{23}\right)=0.514 \pm 0.082$ and $\left|\Delta m_{32}^{2}\right|=2.44_{-0.15}^{+0.17} \times$ $10^{-3} \mathrm{eV}^{2}$ and is consistent with maximal mixing. No fit is given for the inverted hierarchy, 

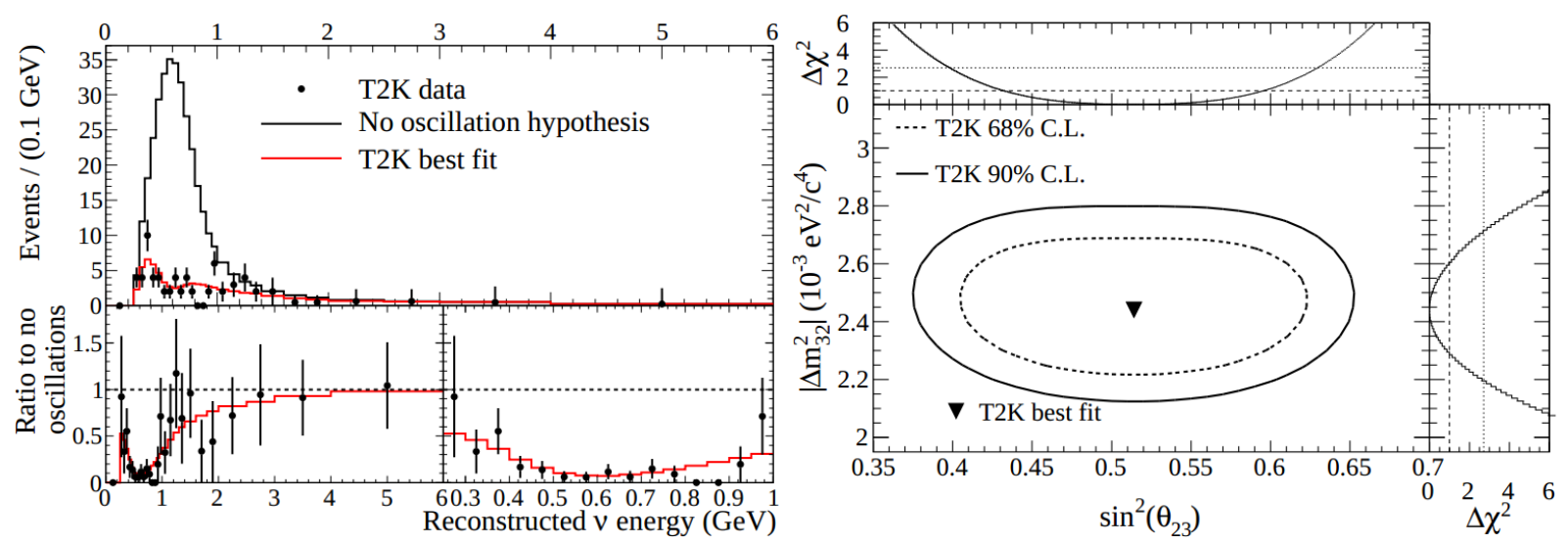

FiguRE 3.7. The $2013 \nu_{\mu}$ disappearance results from T2K|21]. The left figure shows the measured $\nu_{\mu}$ disappearance event spectrum at SK as a function of reconstructed neutrino energy in the top pane. The bottom pane shows the ratio of the event spectrum to the expectation with no oscillations over two different energy ranges. The best-fit oscillation curve, with $\sin ^{2}\left(\theta_{23}\right)=0.514$, is shown in red for both panes. The right figure shows the best-fit and contours in $\left|\Delta m_{32}^{2}\right|$ vs $\sin ^{2}\left(\theta_{23}\right)$.

likely due to the fit with the inverted hierarchy giving the same results as there is very little affect on $\nu_{\mu}$ disappearance from the mass hierarchy.

T2K is an ongoing experiment and will be discussed in more detail in Chapter 4 as input to future sensitivity studies in LBL experiments.

3.1.7. MINOS. The Main Injector Neutrino Oscillation Search (MINOS) $[22\rfloor \mid 23\rfloor \mid 24\rfloor \mid 25\rfloor$ is an LBL neutrino experiment with $\nu_{\mu}$ beam from Fermilab that has an energy spectrum peak at $3 \mathrm{GeV}$. It has a $29 \mathrm{t}$ fiducial mass near detector at $1.04 \mathrm{~km}$ on the Fermilab site. The far detector is $3.8 \mathrm{kt}$ fiducial mass $735 \mathrm{~km}$ away and underground (2100 MWE) in the Soudan mine in Minnesota. Both detectors are magnetized tracking calorimeters so they can distinguish between $\nu$ and $\bar{\nu}$ induced events in their analyses.

Figure 3.8 shows the 2014 analysis from MINOS. The analysis uses $\nu_{e}$ appearance, $\nu_{\mu}$ disappearance, and atmospheric neutrino samples in a joint fit of all MINOS data sets. This is the first combined analysis of $\nu_{e}$ appearance and $\nu_{\mu}$ disappearance in an LBL experiment. The fit shows a slight preference for the first octant, inverted hierarchy, and $\delta_{C P} \neq-\pi / 2$. 

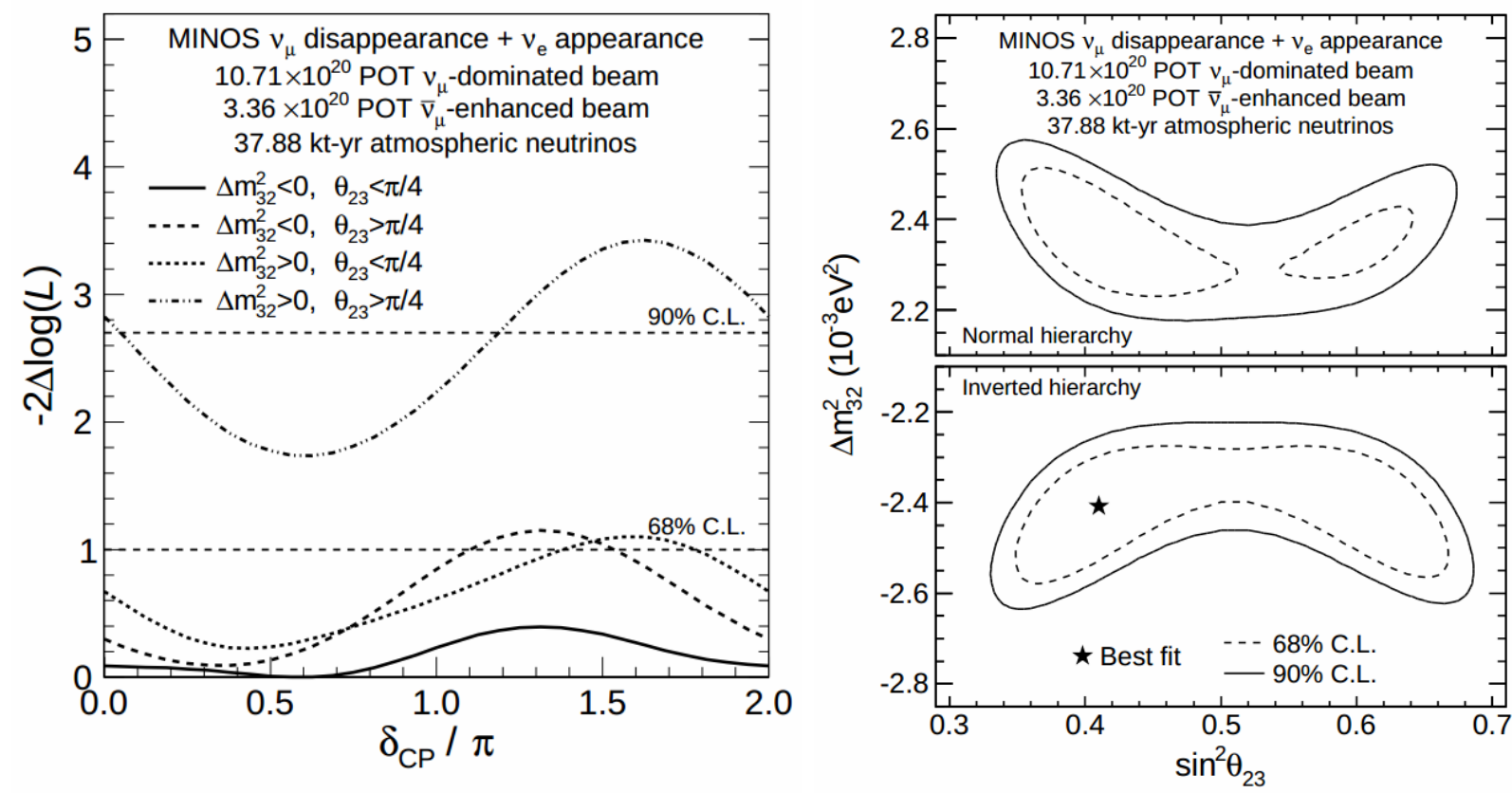

Figure 3.8. The 2014 joint $\nu_{e}$ appearance, $\nu_{\mu}$ disappearance, and atmospheric neutrino analysis results from MINOS[25]. The left figure shows the $-2 \Delta \log (\mathrm{L})$ value as a function of $\delta_{C P}$ for both the normal and inverted hierarchies each with the first and second octant assumptions. This uses the best-fit values and uncertainties on $\sin ^{2}\left(2 \theta_{13}\right)$ from reactor neutrino data. The right figure shows the best-fit and contours in $\Delta m_{32}^{2}$ vs $\sin ^{2}\left(\theta_{23}\right)$.

They report measurements of $\left|\Delta m_{32}^{2}\right|=[2.28-2.46] \times 10^{-3} \mathrm{eV}^{2}$ at the $68 \% \mathrm{CL}$ and $\sin ^{2}\left(\theta_{23}\right)=$ $0.35-0.65$ at the $90 \% \mathrm{CL}$ in the normal hierarchy and $\left|\Delta m_{32}^{2}\right|=[2.32-2.53] \times 10^{-3} \mathrm{eV}^{2}$ at the $68 \% \mathrm{CL}$ and $\sin ^{2}\left(\theta_{23}\right)=0.34-0.67$ at the $90 \% \mathrm{CL}$ in the inverted hierarchy. The preference in the MINOS $\nu_{\mu}$ disappearance data for non-maximal $\theta_{23}$ is more significant|11] than the preference for maximal $\theta_{23}$ in the $\mathrm{T} 2 \mathrm{~K} \nu_{\mu}$ disappearance data. This will change in the near future as T2K is still a running experiment while MINOS has completed.

\subsection{Experimental Measurements Summary}

Table 3.1 summarizes the experimental measurements contributing to the Capozzi et al. global fit. There are a few conflicts among these best-fits, but they are not yet significant. The second octant for $\theta_{23}$ is preferred by SK and T2K, but there is a slight preference 
for the first octant in MINOS. However, they are all still consistent with maximal mixing $\left(\sin ^{2}\left(\theta_{23}\right)=0.5\right)$. The T2K best-fit prefers a value of $\delta_{C P}$ that maximizes the $\nu_{e}$ appearance probability $\left(\delta_{C P}=-\pi / 2\right)$ but the MINOS best-fit prefers a value of $\delta_{C P}$ closer to $\delta_{C P}=\pi / 2$. SK and MINOS both prefer the IH. Notably, MINOS also has the strongest exclusion ability for $\mathrm{NH}$, second octant, and $\left(\delta_{C P} \sim 270^{\circ}\right)$ which is the best fit in T2K. However, none of these conflicts are statistically significant and further measurements are required to resolve these conflicts.

\subsection{Global Fit Results}

The Capozzi et al. global three-neutrino fit|11] uses published and unpublished data from the experiments described above. They are able to extract best-fits by fitting observed data with expected event spectra from each of the experiments. Figure 3.9 and Table 3.2 show the results of the global fit. They adopt the conventions $\Delta m^{2}=m_{3}^{2}-\left(m_{1}^{2}+m_{2}^{2}\right) / 2$ and $\delta m^{2}=\Delta m_{21}^{2}$ so that the mass squared difference is $+\Delta m^{2}$ in the $\mathrm{NH}$ and $-\Delta m^{2}$ in the IH.

The global fit finds a slight preference for the first octant with $\sin ^{2}\left(\theta_{23}\right)<0.5$ at $\sim 95 \%$ CL. The $\nu_{e}$ appearance maximizing value of $\delta_{C P} \simeq 1.5 \pi$ is also preferred. It shows no significant preference for the NH or IH.

The best-fit and $1 \sigma$ range values from this global fit are adopted for this thesis unless stated otherwise. 
TABLE 3.1. Summary of best-fit experimental measurements used as inputs to the Capozzi et al. global fit. Statistical and systematic errors are added in quadrature where applicable. Items marked with " " are estimated from figures.

\begin{tabular}{|c|c|c|}
\hline Experiment & Parameter & Value \\
\hline \multirow[t]{2}{*}{ KamLAND+Solar } & $\tan ^{2}\left(\theta_{12}\right)$ & $0.436_{-0.025}^{+0.029}$ \\
\hline & $\Delta m_{21}^{2}$ & $=7.53_{-0.18}^{+0.18} \times 10^{-5} \mathrm{eV}^{2}$ \\
\hline \multirow[t]{3}{*}{ SK } & $\sin ^{2}\left(\theta_{23}\right)$ & $>0.5$ \\
\hline & $\delta_{C P}$ & $\sim 220 \pm 80^{\circ}$ \\
\hline & $\mathrm{MH}$ & IH Preferred at $1.2 \sigma$ \\
\hline \multirow[t]{2}{*}{ Daya Bay } & $\sin ^{2}\left(2 \theta_{13}\right)$ & $0.090_{-0.009}^{+0.008}$ \\
\hline & $\left|\Delta m_{e e}^{2}\right|$ & $2.59_{-0.20}^{+0.19} \times 10^{-3} \mathrm{eV}^{2}$ \\
\hline Reno & $\sin ^{2}\left(2 \theta_{13}\right)$ & $0.113 \pm 0.013$ \\
\hline Double Chooz & $\sin ^{2}\left(2 \theta_{13}\right)$ & $0.109 \pm 0.039$ \\
\hline \multirow[t]{5}{*}{$\mathrm{T} 2 \mathrm{~K}$} & $\sin ^{2}\left(2 \theta_{13}\right)(\mathrm{NH})$ & $0.140_{-0.032}^{+0.038}$ \\
\hline & $\sin ^{2}\left(\theta_{23}\right)$ & $0.514 \pm 0.082$ \\
\hline & $\left|\Delta m_{32}^{2}\right|$ & $2.44_{-0.15}^{+0.17} \times 10^{-3} \mathrm{eV}^{2}$ \\
\hline & $\delta_{C P}(\mathrm{NH})$ & $\sim 270 \pm 90^{\circ}$ \\
\hline & $\mathrm{MH}$ & $\mathrm{NH}$ \\
\hline \multirow[t]{5}{*}{ MINOS } & $\sin ^{2}\left(2 \theta_{13}\right)$ & 0.095 \\
\hline & $\sin ^{2}\left(\theta_{23}\right)$ & $0.41,0.35-0.65(90 \% \mathrm{CL})$ \\
\hline & $\left|\Delta m_{32}^{2}\right|$ & $2.41_{-0.13}^{+0.03} \times 10^{-3} \mathrm{eV}^{2}$ \\
\hline & $\delta_{C P}$ & 36 \\
\hline & $\mathrm{MH}$ & $\mathrm{IH}$ \\
\hline
\end{tabular}



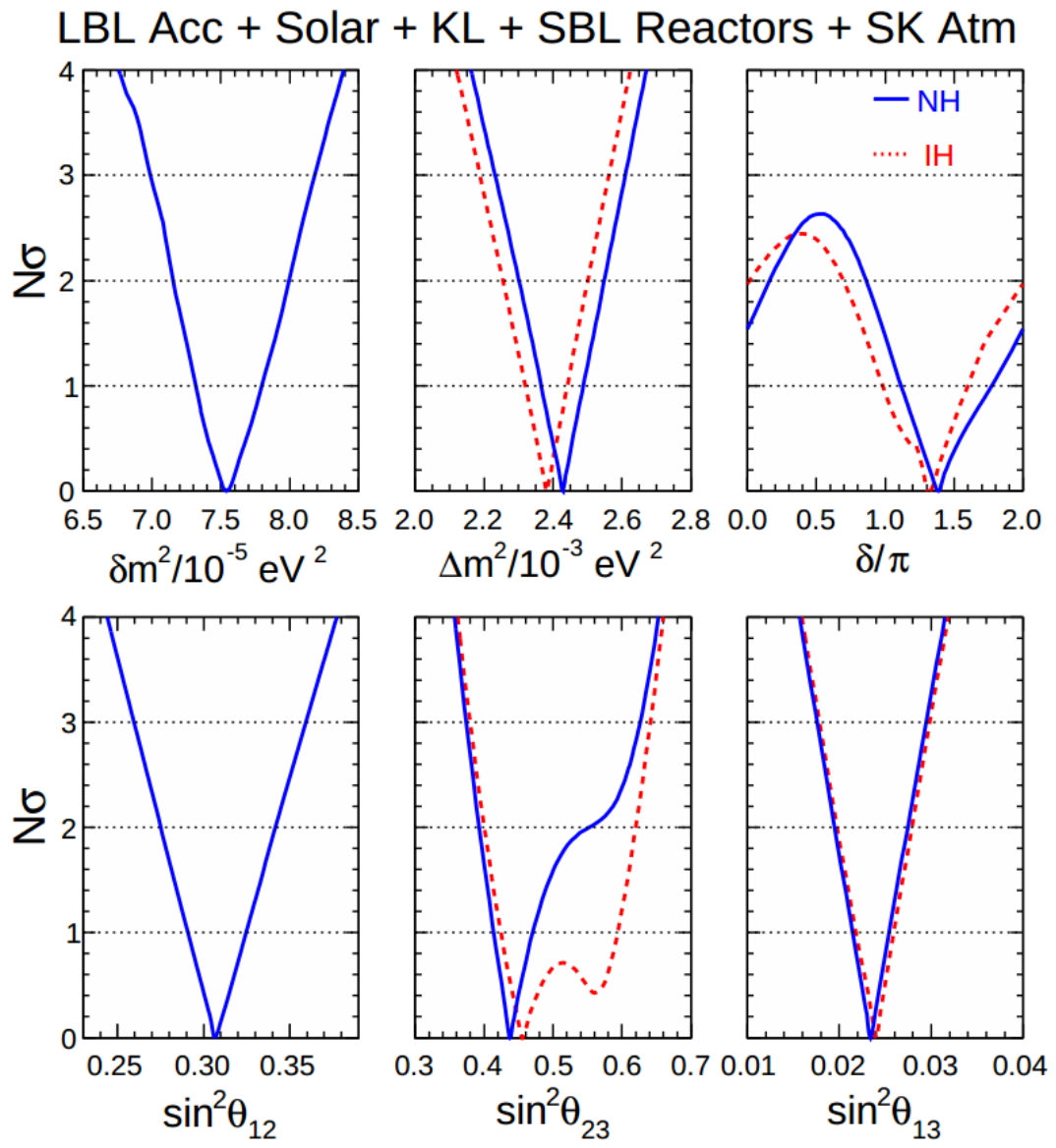

Figure 3.9. The 2014 Capozzi et al. global fit|11] results for LBL, Solar, KamLAND, short-baseline reactor, and SK atmospheric experiments. Separate global fits are performed assuming the $\mathrm{NH}$ (blue) and IH (red) mass hierarchies. 
TABle 3.2. Table of best-fits, $1 \sigma$ ranges, and $3 \sigma$ ranges for the Capozzi et al. global fit.

\begin{tabular}{lcccc} 
Parameter & $\mathrm{MH}$ & Best-fit & $1 \sigma$ range & $3 \sigma$ range \\
\hline$\delta m^{2} / 10^{-5} \mathrm{eV}^{2}$ & $\mathrm{NH}, \mathrm{IH}$ & 7.54 & $7.32-7.80$ & $6.99-8.18$ \\
\hline $\sin ^{2}\left(\theta_{12}\right)$ & $\mathrm{NH}, \mathrm{IH}$ & 0.308 & $0.291-0.325$ & $0.259-0.359$ \\
\hline$\Delta m^{2} / 10^{-3} \mathrm{eV}^{2}$ & $\mathrm{NH}$ & 2.43 & $2.37-2.49$ & $2.23-2.61$ \\
& $\mathrm{IH}$ & 2.38 & $2.32-2.44$ & $2.19-2.56$ \\
\hline $\sin ^{2}\left(\theta_{13}\right)$ & $\mathrm{NH}$ & 0.0234 & $0.0215-0.0254$ & $0.0176-0.0295$ \\
& $\mathrm{IH}$ & 0.0240 & $0.0218-0.0259$ & $0.0178-0.0298$ \\
\hline $\sin ^{2}\left(\theta_{23}\right)$ & $\mathrm{NH}$ & 0.437 & $0.414-0.470$ & $0.374-0.626$ \\
& $\mathrm{IH}$ & 0.455 & $0.424-0.594$ & $0.380-0.641$ \\
\hline$\delta_{C P} / \pi$ & $\mathrm{NH}$ & 1.39 & $1.12-1.77$ & - \\
& $\mathrm{IH}$ & 1.31 & $0.98-1.60$ & -
\end{tabular}




\section{CHAPTER 4}

\section{LONG-BASELINE EXPERIMENTS}

In order to study neutrinos and their weak interactions it is desirable to have control of the timing and kinematics of the neutrino source by producing a beam of neutrinos in a lab setting as opposed to waiting for reactions from reactor, solar, and atmospheric neutrinos. Since the early 1960s, neutrino beams have been made for this purpose by using protons from high-energy proton synchrotron accelerators. They were first used to study weak interactions and to demonstrate the existence of two neutrino flavors in 1962[28].

The question of whether or not neutrinos have mass and undergo flavor oscillations was proposed in the late 1960s. Long-baseline neutrino experiments were proposed to answer this question by Mann and Primakoff in 1977|29] as a "feasible experiment to search for neutrino oscillations using the neutrino beam produced at a high-energy proton accelerator." The proposal, depicted in Figure 4.1, was to have a neutrino beam from Fermi National Laboratory (Fermilab), directed towards a near detector (ND) at some short distance from the beam target and a far detector at a distance $\mathcal{O}\left(10^{3} \mathrm{~km}\right)$ away. This is not too different from the designs of present day LBL experiments, but there have been many improvements in design to increase proton beam cycle rates, focusing of the secondary beam, and detection methods. In particular, with some knowledge of $\Delta m^{2}$, the baseline can be optimized and lower energy neutrinos propagated to produce L/E values that maximize oscillation probabilities and reduce backgrounds from high energy neutrino interactions.

The components of a long-baseline experiment are described in this section: the primary and secondary beam-lines that produce the beam of primarily muon neutrinos, the near detector (ND) used to characterize the beam before it oscillates, and the far detector (FD) that 

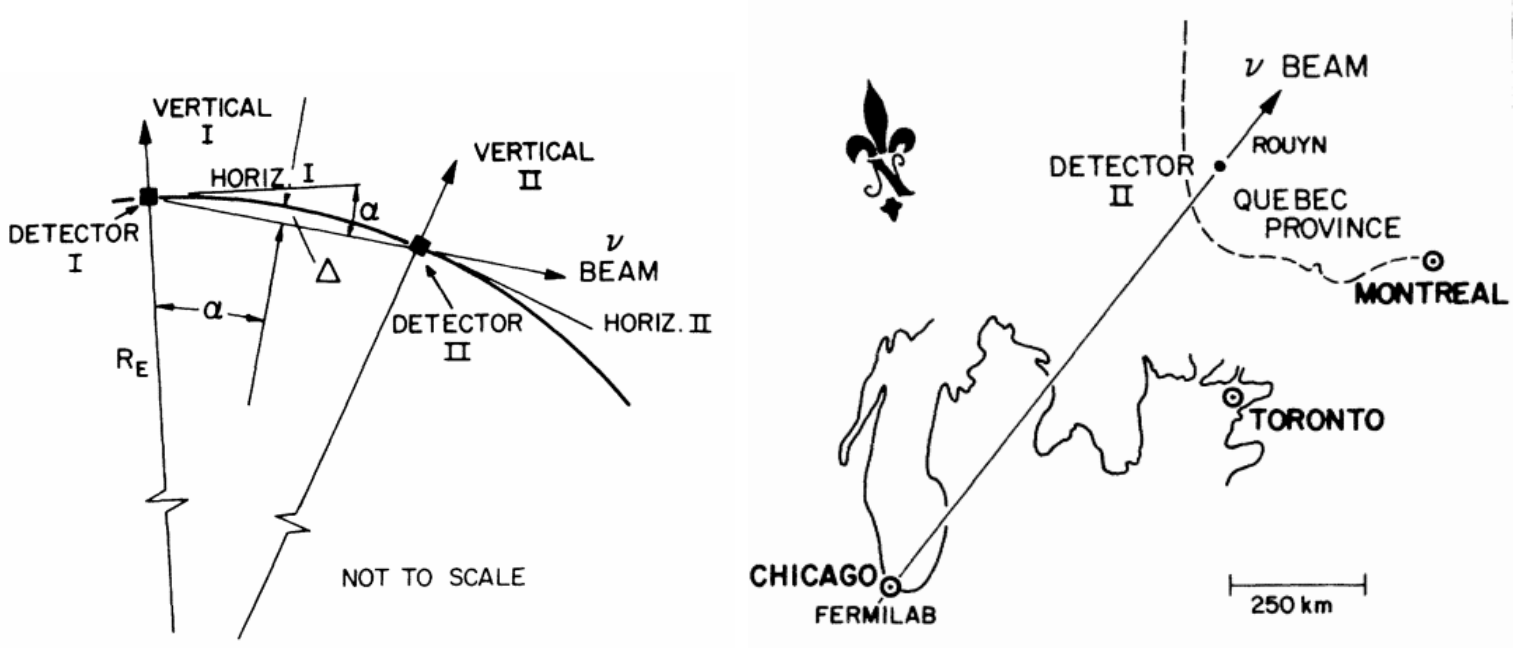

Figure 4.1. Geometry (left) and proposed baseline (right) of an LBL experiment[29] initially proposed by Mann and Primakoff in 1977.

detects the neutrinos after they have oscillated. Past, current, and planned LBL experiments are also described.

Many LBL experiments are multi-purpose and perform measurements outside of LBL neutrino oscillations. The ND can serve as an SBL experiment, looking for oscillations over the short baseline, $\mathcal{O}(0.1-1 \mathrm{~km})$, from the target to the ND. The ND also makes independent neutrino cross-section measurements. The FD is also often used as a detector for atmospheric, supernova, and solar neutrinos. This is true especially in the case where the detector is located underground. These purposes are set aside here to focus on the LBL neutrino oscillation measurements and sensitivities.

\subsection{Components of An LBL Experiment}

This section describes the components of an LBL and the variables relevant to an LBL oscillation analysis.

4.1.1. Primary Beam-Line. The protons and the beam components before the target are referred to as the primary beam-line. Protons are extracted from either a dedicated or 
multi-use accelerator complex. The time it takes to refill and re-accelerate protons in the accelerator is called the cycle time and is around $2-6 \mathrm{~s}$. The cycle time determines the rate at which protons can be delivered to a target, described below. After the accelerator, the protons are delivered to the target in pulses. These pulses are typically a few microseconds long and are made up of smaller bunches of protons. The bunch structure within a pulse can be seen for the T2K neutrino beam in Figure 4.2. The bunch time structure of the beam allows timing cuts to be made in an ND and FD and greatly reduce backgrounds coming primarily from atmospheric muons. The proton energies are typically of energy

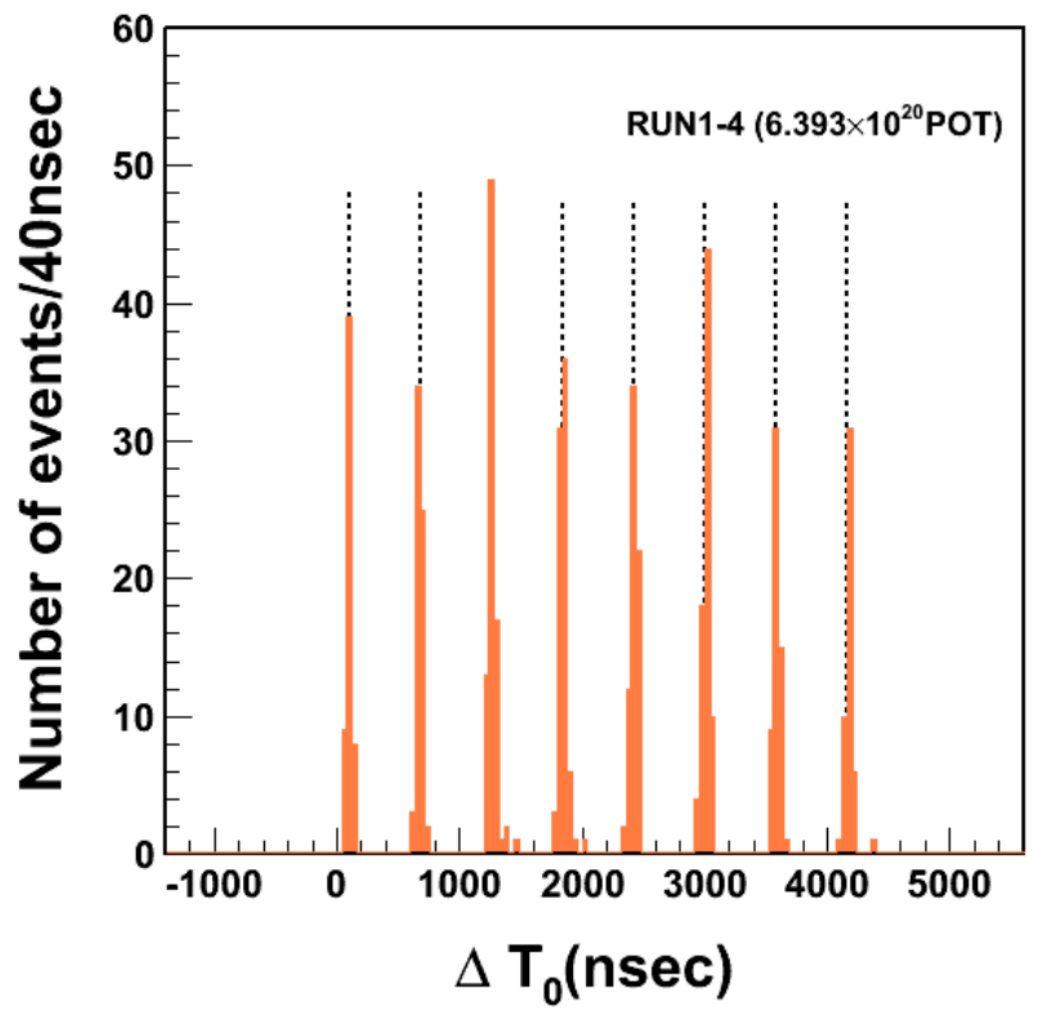

Figure 4.2. Timing of detected events in the T2K far detector (SK) relative to their production time from $[30 \mid$. The bunch structure of the neutrino beam is clearly visible with the T2K beam producing 8 bunches in every pulse.

$\mathcal{O}(1-100 \mathrm{GeV})$ and affect the resulting neutrino energy spectrum. The number of protons delivered in each bunch is $\mathcal{O}\left(10^{13}\right)$. Taking these parameters all together leads to the beam 
power which has been between $5 \mathrm{~kW}$ and $250 \mathrm{~kW}$ in past and current experiments, but this figure is always improving with time. The ultimate goal is to increase the number of protons delivered to the target, or protons on target (POT), per unit time.

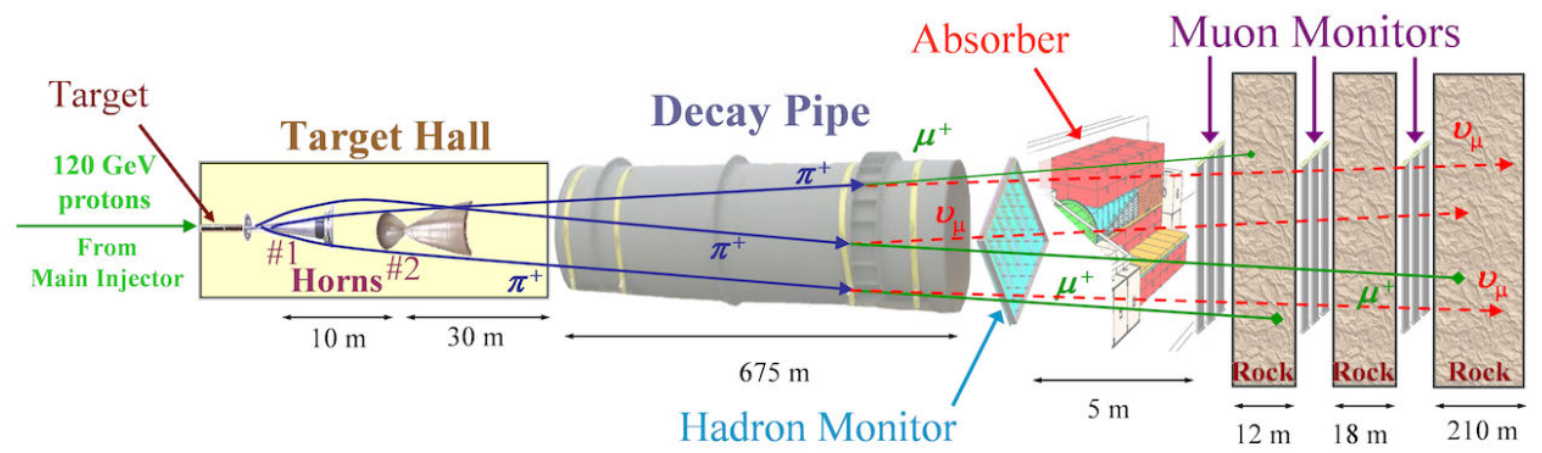

Figure 4.3. Layout of secondary beam-line in NuMI from [8].

4.1.2. Secondary Beam-Line. After the protons are extracted from the primary beamline, they are focused onto a target and from this point the beam-line is referred to as the secondary beam-line. A layout for the Neutrinos in Main Injector (NuMI) beam-line, used by the $\mathrm{NO} \nu \mathrm{A}$ experiment, is shown in figure 4.3. The targets are typically made of aluminum or graphite and are a few centimeters in diameter and on the order of one meter in length.. The length and diameter of the target are optimized to maximize the number of proton interactions and to minimize the number of secondary interactions which complicate modeling of the neutrino flux. The protons interact with the nuclei in the target and primarily produce pions and a smaller amount of kaons. These mesons are focused by a series of two or three magnetic horns. The horns are pulsed with a current that depends on the energy and sign of the pions to be forward focused. The horn is pulsed with positive(negative) current to focus $\pi^{+}\left(\pi^{-}\right)$when running in neutrino(anti-neutrino) mode. The relative positions of the target and focusing horns affect the momentum and angular distribution of the selected 
pions and kaons. Focusing the pions has been shown to increase the neutrino flux at the FD by a factor of about 20|31].

After the target region, the pions and kaons are allowed to enter a decay region. This is typically of length on the order of $100-1000 \mathrm{~m}$ and of radius $2-3 \mathrm{~m}$. The decay region is either evacuated or filled with helium to reduce interactions of the pions and kaons with the medium. Because the pions are highly boosted with respect to the lab frame the decays are in the forward direction. The dominant decay mode for the pion is $\pi^{+} \rightarrow \mu^{+} \nu_{\mu}$ or in the case of negative pion focusing in anti-neutrino mode $\pi^{-} \rightarrow \mu^{-} \bar{\nu}_{\mu}$. Some of the kaons decay to produce $\nu_{e}$ and $\bar{\nu}_{e}$ which is a background to the $\nu_{e}$ appearance measurement. At the end of the decay volume there is a beam dump that stops all hadrons and lower energy muons. The beam direction, profile, and intensity is monitored after the beam stop by measuring the profile and energy of the remaining muons. The neutrinos are not affected and propagate to the near and far detectors.

The resulting neutrino beam has energy typically on the order 1-10 GeV. Understanding the flux, in terms of energy and composition $\left(\nu_{e}, \bar{\nu}_{e}, \nu_{\mu}, \bar{\nu}_{\mu}\right)$, of the neutrino beam is a difficult problem and is crucial to performing an oscillation analysis. Aiming the beam directly at the FD results in a wide range of neutrino energies, known as a wide-band beam. This produces the largest flux at the FD. However, there are many effects, such as backgrounds that feed down from higher energy neutrino interactions and limited accuracy of neutrino energy reconstruction, that produce large systematic uncertainties for the oscillation analysis of a wide-band beam experiment.

A neutrino energy spectrum that is narrow in energy is desirable to avoid uncertainties, but also limits the available oscillation information coming from the shape of the measured 
spectrum. A forward going pion decaying in the decay region will lead to an angular dependence in the neutrino energy spectrum. The flux and energy of the resulting neutrino, $E_{\nu}$ is related to the energy of the pion, $E_{\pi}$, and a small angle, $\theta$, of the outgoing neutrino with respect to the pion direction and are given by[32]

$$
\begin{aligned}
\Phi & \propto\left(\frac{1}{L^{2}}\right)\left(\frac{2 \gamma}{1+\gamma^{2} \theta^{2}}\right)^{2} \\
E_{\nu} & =\frac{0.43 E_{\pi}}{1+\gamma^{2} \theta^{2}}
\end{aligned}
$$

where $\gamma=E_{\pi} / m_{\pi}$. This results in a peaked $E_{\nu}$ spectrum as the off-axis angle is increased. Figure 4.4 shows the off-axis effects in the NuMI and T2K beams.

For an oscillation analysis the signal comes from the $\nu_{\mu}+\bar{\nu}_{\mu}$ flux and the $\nu_{e}+\bar{\nu}_{e}$ flux is a background known as intrinsic, or beam, $\nu_{e}$ background. The beam $\nu_{e}$ backgrounds come from three-body muon and kaon decays (e.g. $K^{+} \rightarrow \pi^{0} e^{+} \nu_{e}, K_{L}^{0} \rightarrow \pi e^{+} \nu_{e}$, and $\mu^{+} \rightarrow e^{+} \bar{\nu}_{\mu} \nu_{e}$ ) that are more widely distributed in energy relative to the two-body $\pi^{+} \rightarrow \mu^{+} \nu_{\mu}$ decays that are the primary source of signal. The off-axis technique reduces beam $\nu_{e}$ backgrounds because the $\nu_{\mu}+\bar{\nu}_{\mu}$ signal events are peaked in energy while the beam $\nu_{e}$ component is widely distributed in energy so that a large reduction in the background is achieved by considering events in a limited energy range.

In order to predict an event spectrum at an FD, the beam is simulated with a multicomponent Monte Carlo (MC) simulation. A beam MC simulation attempts to model all aspects of the beam as accurately as possible incorporating measurements made before the target, at the muon monitors, and other external measurements that constrain hadron production in the target. However, there are large uncertainties, on the order of $10 \%$, associated 

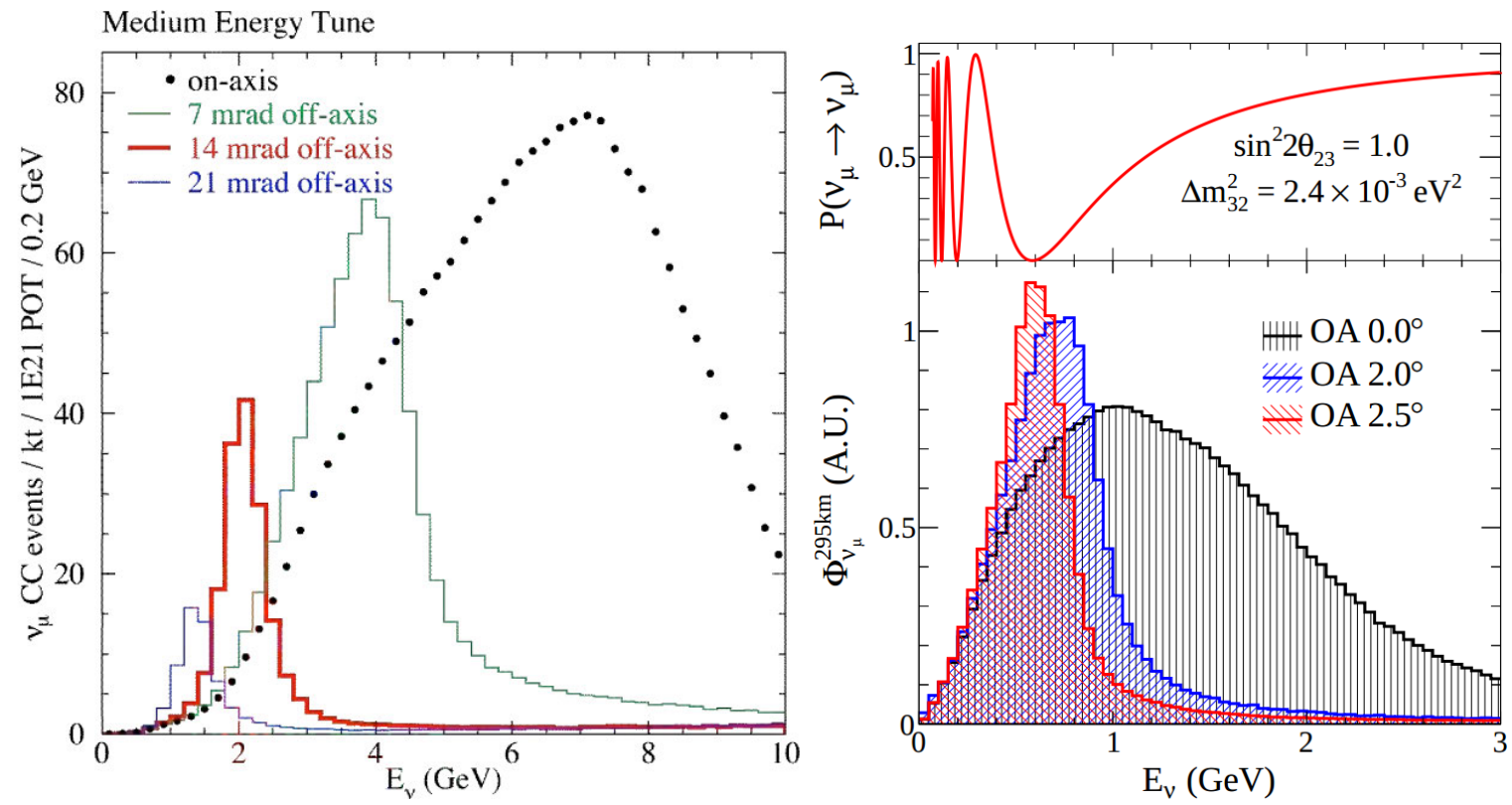

FIgurE 4.4. Off-axis effects in NuMI|32」 (left) and T2K|31」 (right). The left figure shows the predicted unoscillated $\nu_{\mu}$ flux, as a function of $E_{\nu}$, at a baseline of $810 \mathrm{~km}$ for on-axis, $7 \mathrm{mrad}\left(0.4^{\circ}\right), 14 \mathrm{mrad}\left(0.8^{\circ}\right)$, and $21 \operatorname{mrad}\left(1.2^{\circ}\right)$ offaxis angles. The right figures gives the corresponding predicted fluxes in T2K for on-axis, $2^{\circ}$, and $2.5^{\circ}$ off-axis.

with predicting the resulting neutrino beam flux. Significant sources of uncertainty about the flux prediction at the FD come from uncertainties in:

- the number of pions and kaons produced in the target per incident proton,

- secondary interactions in the target that can account for $20 \%|31|$ of the neutrino flux,

- pion production cross-sections,

- the primary beam direction,

- neutrino beam direction,

- target and horn alignment and magnetic field,

- and target degradation. 
The dominant source of uncertainties is from the hadron production uncertainties. A dedicated, ND measurement of a neutrino beam before it oscillates is used to constrain the flux prediction at an FD.

4.1.3. Event Selections in the ND And FD. An ND allows the flux, energy spectrum, and flavor composition of the neutrino beam to be monitored before oscillations occur. This refines the flux prediction at the FD beyond what would be possible with only beam monitoring. It also measures interaction cross-sections, typically on the same material that makes up the FD, which helps to constrain predicted event rates at the far detector. Often a ratio between the measured flux at the FD and the ND is taken to cancel many of the uncertainties, but many uncertainties remain because the detectors are never completely identical. The ND also sees the beam as a line source along the decay region while the FD is located far enough away so that it sees a point source so the two detectors actually measure different fluxes and this must be taken into account when propagating an ND flux measurement to an FD.

A primary role of the ND relevant to LBL physics is the measurement of the $\nu_{\mu}$ content of the beam. In the $\nu_{\mu}$ disappearance measurement the initial flux of $\nu_{\mu}$ is needed to look for a deficit of $\nu_{\mu}$ charged current (CC) like events in the far detector, indicating an oscillation to another flavor. In the $\nu_{e}$ appearance measurement the initial $\nu_{\mu}$ flux is used to predict the number of expected $\nu_{e} \mathrm{CC}$ like events expected in the FD for a given oscillation hypothesis (i.e. $\Delta m^{2}$ and $\sin ^{2}\left(2 \theta_{13}\right)$ values).

One of the measurements used to constrain the $\nu_{\mu}$ beam flux at the ND is the $\nu_{\mu}$ charged current (CC) interaction. Figure 4.5 shows interaction diagrams for three of the most common $\nu_{\mu}$ CC interactions. The charged current quasi-elastic (CCQE) interaction consists of a muon neutrino interacting with a neutron via a $\mathrm{W}$ boson to produce a proton and a muon. 

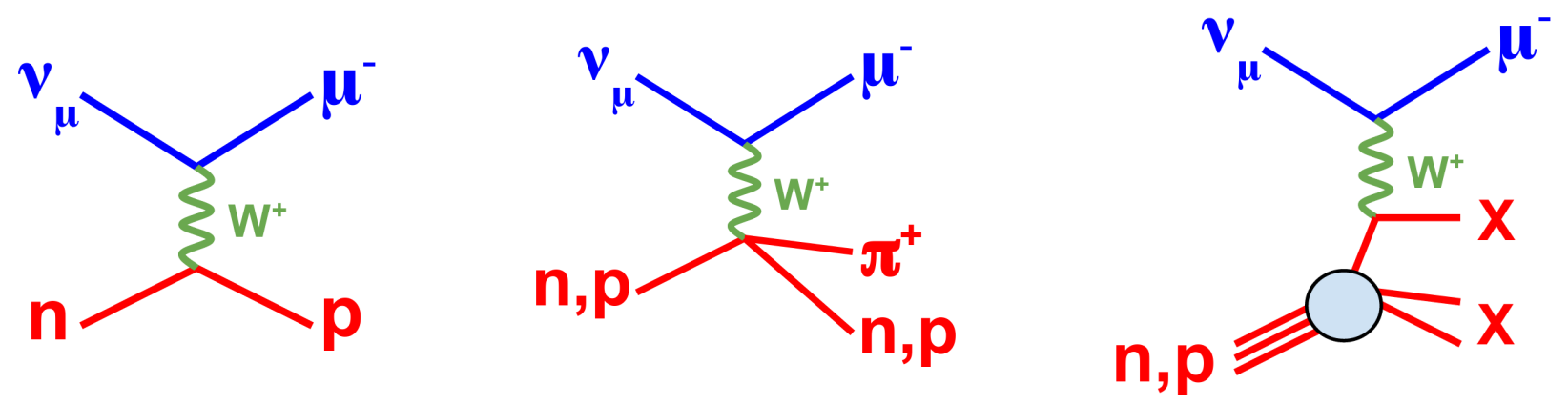

FiguRE 4.5. Diagrams for the charged current processes in each category: charged current quasi-elastic (left), charge current resonant production (center), and charged current deep inelastic scattering (right).

The charged current resonant production (CC Res) interaction is similar but has the nucleon emitting a $\pi^{+}$via resonance. The CC deep-inelastic-scattering (DIS) interaction occurs at higher neutrino energies where the incident neutrino interacts with the constituent quarks in a nucleon and results in many interaction products. These are often taken together to form a $\mathrm{CC}$ inclusive sample because they all involve an outgoing muon and have similar interaction topologies depending on the detector technology.

Each of these processes has an energy regime where it is the dominant process, but there is overlap of the regimes so that an analysis can never be conclusive about which interaction took place if the experiment covers a wide range of neutrino energies. The energy and angle with respect to the neutrino beam direction of an interaction are used to kinematically reconstruct the energy of the incoming neutrino under a quasi-elastic (QE) assumption via

$$
E_{\text {reco }}=\frac{M_{N} E_{l}-m_{l}^{2} / 2}{M_{N}-E_{l}+\left|\vec{p}_{l}\right| \cos \theta}
$$

where $M_{N}$ is the mass of the target nucleon assumed to be at rest, $E_{l}$ is the energy of the outgoing lepton, $m_{l}$ is the mass of the lepton, $\vec{p}_{l}$ is the three-momentum of the lepton, and $\theta$ is the angle between the lepton and beam direction. This method of energy reconstruction 
only requires the energy and angle of the outgoing lepton to be measured, and no information about the nucleon needs to be measured.

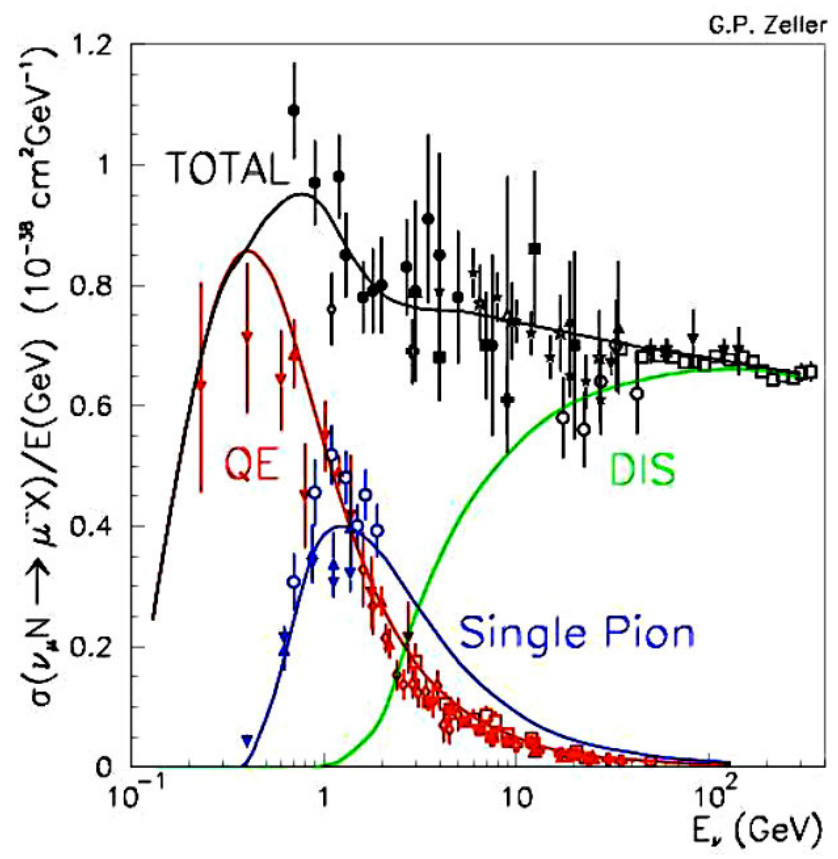

FIGURE 4.6. Summary[33] of charged current cross-section measurements over many experiments overlaid with theoretical predictions.

The QE assumption is an estimate that can perform poorly for neutrinos at higher energies. Figure 4.6 shows the measured and predicted cross-sections for the three CC interaction modes as a function of incident neutrino energy. At energies around $1 \mathrm{GeV}$, enough energy can be transferred to the nucleus for a $\Delta$ resonance to be produced which then decays to a nucleon and and pion. At energies around $3 \mathrm{GeV}$, DIS becomes dominant as the neutrino "sees" the quark content of the nucleus. Care has to be taken in the analysis to select the events that represent the chosen interaction channel or channels. Accurately reconstructing the energy of the neutrino is critical to an oscillation measurement since the predicted oscillation rates, as shown in Chapter 4, are determined by this reconstructed energy. 
The selected target plays an important role in the measurement and reconstruction of $E_{\nu}$. A heavier target may be selected to increase the event rates in the detector. However, there are complicated effects that come into play when nuclear targets are used. A neutrinonucleon interaction in a nucleus heavier than $D_{2}$ may involve re-interactions before the initial reaction products can exit the nucleus. These are referred to as final state interaction (FSI) effects. These interaction products can cause an event selection to reject an event that may have been included without the FSI effects. For example, a $\mathrm{CC} 1 \pi$ event may undergo pion absorption in the nucleus, ejecting only neutrons that are not visible near the interaction vertex so that energy is missing from the reconstructed event. This causes the $E_{\text {reco }}$ to be biased and has to be accounted for when generating MC predictions of event rates in the ND and FD.

Another interaction that is important to characterize in order to predict backgrounds at the far detector are the neutral current $(\mathrm{NC})$ interactions, depicted in Figure 4.7. Here the incoming $\nu$ does not change and no charged lepton is produced in the final state. Since no charged lepton is produced, the flavor of the incoming $\nu$ cannot be determined and so this interaction remains constant with respect to oscillations in the $\mathrm{CC}$ interactions. The $\pi^{0}$ typically decays to two $\gamma \mathrm{s}$ that overlap at the energies of most LBL experiments. The overlapping $\gamma \mathrm{s}$ may look like an electron and mimic a $\mathrm{CC}$ interaction. This makes them a background that must be carefully measured and accounted for in an oscillation analysis.

The typical samples selected as signal are these $\nu_{\mu}$ or $\nu_{e} \mathrm{CC}$ (inclusive) events taken together. Modeling the backgrounds to these selections is critical to understanding the signal, and subsequently any oscillation effects. The signal selections are made to maximize signal while minimizing background, but the backgrounds are typically never completely removed by any selection. The selection quality is quantified by the purity and efficiency of 


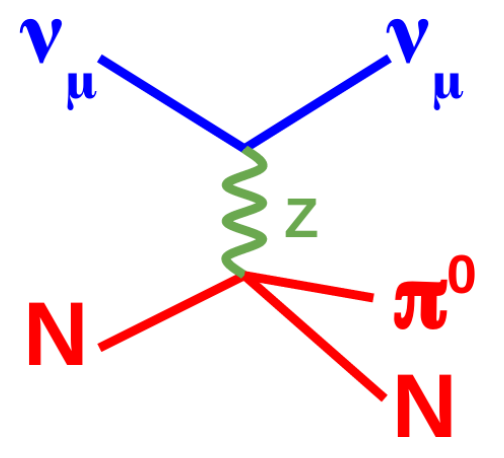

FIGURE 4.7. Diagram for the neutral current interaction where a $\nu_{\mu}$ interacts with a nucleon $(\mathrm{N})$ and produces a $\pi^{0}$ which may be mistaken for an electron during event reconstruction.

the selection computed from Monte Carlo (MC) simulations. The efficiency of a selection is the number of signal events selected divided by the number of actual signal events that were in the MC. The purity of a selection is the number of signal events selected divided by the number of signal plus background events that were selected.

In a typical CC inclusive selection for $\nu_{e}$ appearance or $\bar{\nu}_{e}$ appearance the backgrounds are:

- a neutral current (NC) event containing a $\pi^{0}$ that decays to two gammas that may overlap and look like an electron

- $\nu_{\tau}$ and $\bar{\nu}_{\tau}$ CC interactions that produce $\tau$ leptons that immediately decay to a muon or electron; this is only significant for higher energy beams since the tau production threshold is $\sim 3.5 \mathrm{GeV}$

- $\nu_{e}$ and $\bar{\nu}_{e}$ that are intrinsically in the beam from muon and kaon decays in the decay region

- $\nu_{\mu}$ and $\bar{\nu}_{\mu}$ CC may be mistaken for signal in some rare cases

The $\nu_{e}$ appearance signal selection also includes a small number of $\bar{\nu}_{e}$ appearance events since the far detector is typically not magnetized and so cannot distinguish between positive and negatively charged particles. 
The NC channel is the largest background to $\nu_{e}$ appearance. The interaction does not depend on the flavor of the neutrino and so does not experience oscillations. When a $\pi^{0}$ is produced it very quickly decays to two $\gamma \mathrm{s}$ with a lifetime (in the $\pi^{0}$ rest frame) of $8.4 \pm$ $0.6 \times 10^{-17} s[1]$. These two $\gamma \mathrm{s}$ are very forward going for energetic events and produce an EM shower that looks very similar to a $\nu_{e} \mathrm{CC}$ event with an electron. A very high resolution detector is required to separate these events by distinguishing the electron and $\gamma \mathrm{s}$ before the shower starts.

\subsection{Past LBL Experiments}

4.2.1. K2K. The KEK to Kamioka (K2K) experiment|34] was an on-axis measurement of $\nu_{\mu}$ disappearance that ran from 1999 to 2004. It was in some sense the precursor to the T2K experiment since it traverses Japan to SK. With an average energy of $1.3 \mathrm{GeV}$ the beam operated between June 1999 and November 2004 to deliver $1.049 \times 10^{20}$ POT. The ND was a series of detectors, located at $300 \mathrm{~m}$ from the target, including a $1 \mathrm{kt}$ water Cerenkov (WC) detector and a series of fine-grained detectors. The FD was the SK detector at 250 $\mathrm{km}$, described in more detail below.

The experiment measured[34] a $\nu_{\mu}$ disappearance effect that was inconsistent with a no oscillation hypothesis at a level of $4.3 \sigma$. It also place early constraints on $\sin ^{2}\left(\theta_{23}\right)$ and $\Delta m_{32}^{2}$ that agree with earlier SK atmospheric measurements.

4.2.2. MINOS. MINOS|22], also described in Chapter 3, was an on-axis measurement of $\nu_{e}$ appearance and $\nu_{\mu}$ disappearance. From the NuMI beam, the experiment received a neutrino beam exposure of $10.6 \times 10^{20} \mathrm{POT}$ in $\nu$ mode and $3.6 \times 10^{20} \mathrm{POT}$ in $\bar{\nu}$ mode and the beam was peaked at $3 \mathrm{GeV}$ in the "medium energy" tune. The ND and FD detectors are magnetized tracking calorimeters made up of alternating planes of steel and scintillator bars. 
The ND has a fiducial mass of $29 \mathrm{t}$ located $1.04 \mathrm{~km}$ from the target and the FD a fiducial mass of $3.8 \mathrm{kt}$ at $735 \mathrm{~km}$. Being magnetized, the FD had the unique ability to distinguish between $\nu_{\mu}$ and $\bar{\nu}_{\mu} \mathrm{CC}$ events. The ND and FD detectors were optimized to detect muon neutrinos, but had some ability to distinguish between the EM showers induced by $\nu_{e}$ events and hadronic activity induced by an NC event. The 2014 MINOS three flavor oscillation results were summarized in Chapter 3 .

The NuMI beam has been optimized for use in NO $\nu$ A so that the MINOS experiment no longer receives a beam optimized for a three flavor oscillation measurement. It is now tuned to achieve a higher beam energy for an off-axis measurement at the $\mathrm{NO} \nu \mathrm{A}$ baseline. As such, MINOS will continue as MINOS + which will measure high energy neutrino interactions to look for effects beyond the standard three flavor neutrino oscillation model.

\subsection{Current and Future LBL Experiments}

The experiments listed in this section are the focus of the LBL neutrino oscillation studies in this document. They are described in some detail here and along with the experimental inputs used for the future sensitivity analyses.

4.3.1. T2K. The Tokai-to-Kamioka experiment is an operating experiment with the goal of measuring $\nu_{e}$ appearance and $\nu_{\mu}$ disappearance. It has been running since 2009 and has collected $6.57 \times 10^{20}$ POT. The experiment and its recent results are described in Chapter 3. More detail is given on the beam, ND, and FD here.

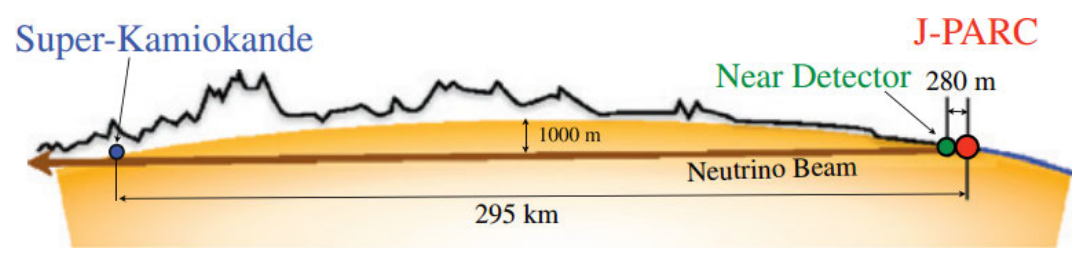

Figure 4.8. Layout of the T2K Experiment|35]. 
The T2K beam is produced at the Japan Proton Accelerator Research Complex (JPARC). The primary beam is from the $30 \mathrm{GeV}$ main ring and has achieved continuous beam power of $220 \mathrm{~kW}$ as of May 2013 with $1.2 \times 10^{14}$ protons per pulse[20]. The $30 \mathrm{GeV}$ protons strike a graphite target that is $26 \mathrm{~mm}$ in diameter and $90 \mathrm{~cm}$ long. This corresponds to 2 interaction lengths and approximately $80 \%$ of the protons interact in the target. Three horns, pulsed with $250 \mathrm{kA}$, magnetically focus the secondary beam of pions and kaons into the $96 \mathrm{~m}$ He-filled decay region. The profile and intensity of muons are monitored via muon monitors (MUMON) after a beam dump at the end of the decay region. The beam is pointed at an angle $2.5^{\circ}$ degrees off-axis from the direction of SK, and is tunable down to an off-axis angle of $2.0^{\circ}$. When viewed at the $2.5^{\circ}$ off-axis angle, the beam energy is peaked at $600 \mathrm{MeV}$.

The ND complex for T2K is located $280 \mathrm{~m}$ downstream from the target. It consists of the Interactive Neutrino GRID (INGRID) on-axis detector and the ND280 off-axis detector. INGRID is made up of 14 identical modules that are arranged in a cross pattern. Each 7.1 ton module is made up of 9 steel plates alternating with 11 scintillator planes (with horizontal and vertical bars). INGRID measures the beam center up to a precision of 10 $\mathrm{cm}$ which corresponds to an angle of $0.4 \mathrm{mrad}$ with respect to the target. It also checks the axial symmetry of the beam and makes cross-section measurements on iron.

Figure 4.9 shows the layout of the ND280 off-axis detector along with an event display of a candidate muon. The inner detector starts with the $\pi^{0}$ detector (P0D) which is designed to measure neutral current interactions on water that are a critical background to the $\nu_{e}$ appearance signal. The P0D is followed by three time-projection chambers (TPC) interleaved with two fine grained detectors (FGD) that allow particle momentum, energy, and charge to be reconstructed. The P0D, TPCs, and FGD are surrounded by an electromagnetic calorimeter $(\mathrm{ECAL})$ in order to detect gamma rays that exit the contained detectors. This 
is all surrounded by a magnet, originally constructed for the UA1 experiment, that has been instrumented with the side muon range detector (SMRD) to measure muons exiting the detector through the sides and serves as a cosmic ray trigger. The magnet provides a 0.2 $\mathrm{T}$ field and allows the momentum and sign of charge particles to be determined by their curvature.
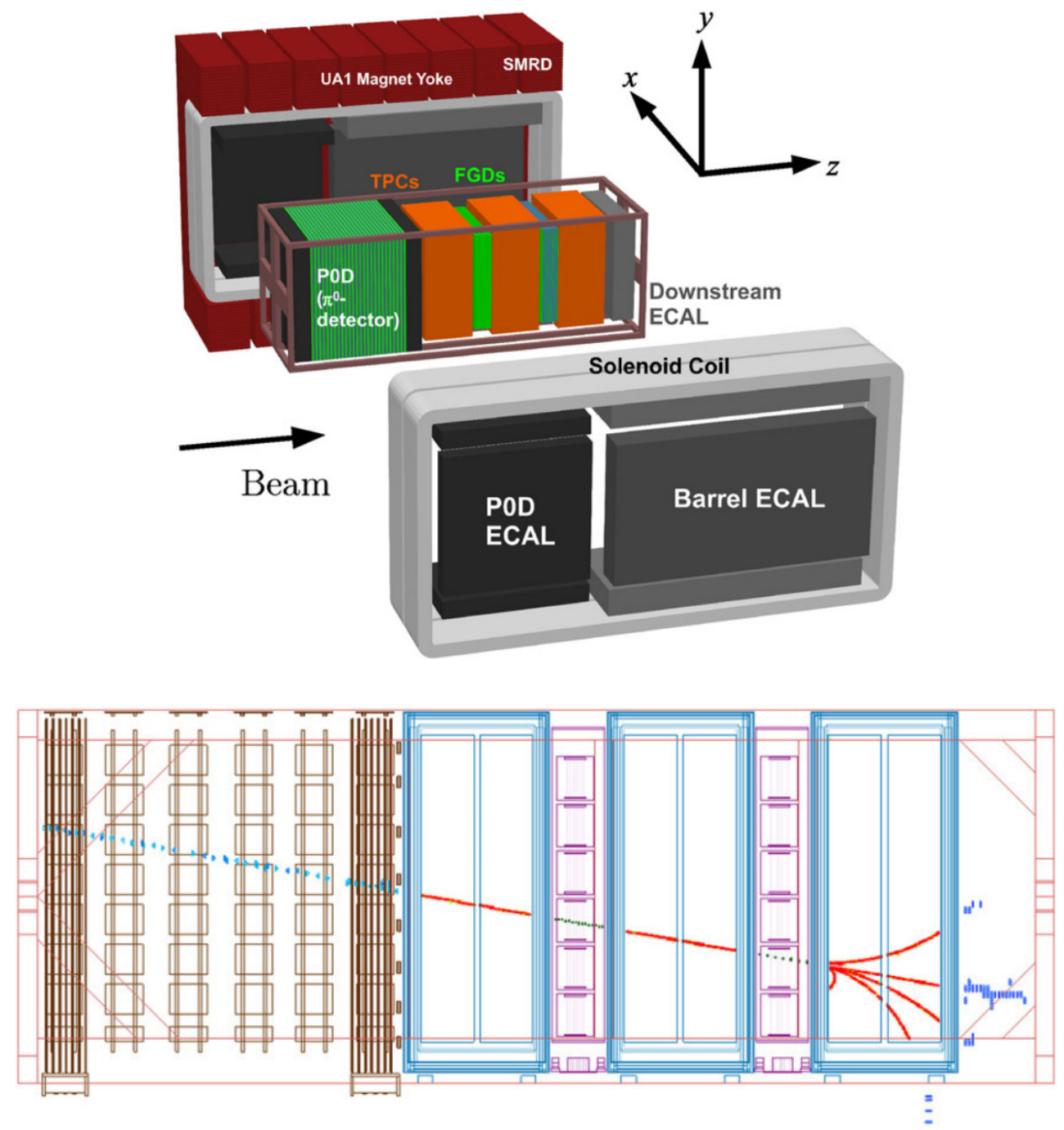

Figure 4.9. Layout of the T2K ND280 (top) and a example event display (bottom)[35]. The event shows a muon starting in the P0D and traversing through the entire detector. In the TPC, the muon produces secondary particles that traverse into the downstream ECAL. 
The FD for T2K is the SK detector, described briefly in Chapter 3, and it is located at $295 \mathrm{~km}^{1}$ from the target. SK is a large underground WC detector and has a total mass of $50 \mathrm{kt}$ (22.5 kt fiducial mass). It is separated into inner and outer detector regions to form a veto against events originating outside of the detector. The detector is instrumented with 11,129 inward facing photomultiplier tubes (PMT) that give $40 \%$ coverage of the inner surface. The outer veto region is instrumented with 1885 PMTs. Charged current events, $\nu_{\mu}$ or $\nu_{e}$, are separated by identifying the Cerenkov radiation from the outgoing lepton of the event. An electron will produce a ring that is fuzzy at the edges as the scattering of the electron slightly redirects the Cerenkov cone of light that reaches the PMTs on the detector wall. A muon will produce a ring with sharp edges since it is heavier and scatters less than an electron and if the event is completely contained a decay electron may be visible. Figure 4.10 shows event displays for these two cases.

The run plan for T2K has the experiment collecting a total of $7.8 \times 10^{21}$ POT over the next 10 years while ramping up to a power of $750 \mathrm{~kW}$. The amount of running to be done in $\nu$ mode and $\bar{\nu}$ mode, the $\nu: \bar{\nu}$ ratio, has not yet been determined for these runs and is the subject of optimization studies in Chapter 9. The author of this thesis has also prepared an internal technical note, as a member of the T2K collaboration, that studies the $\nu: \bar{\nu}$ ratio optimizations in detail.

4.3.1.1. T2K Experimental Inputs. The T2K inputs used for future sensitivity analyses are based on T2K-SK MC. In this MC the simulated neutrino beam flux is constrained by $\nu_{\mu}$ measurements in the ND280 tracker by fitting a set of nuisance parameters affecting flux and cross-section uncertainties from a neutrino event generator. This allows the neutrino beam flux to be extrapolated to the FD while reducing the flux and cross-section uncertainties.

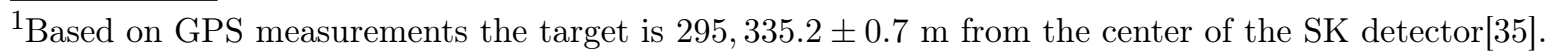




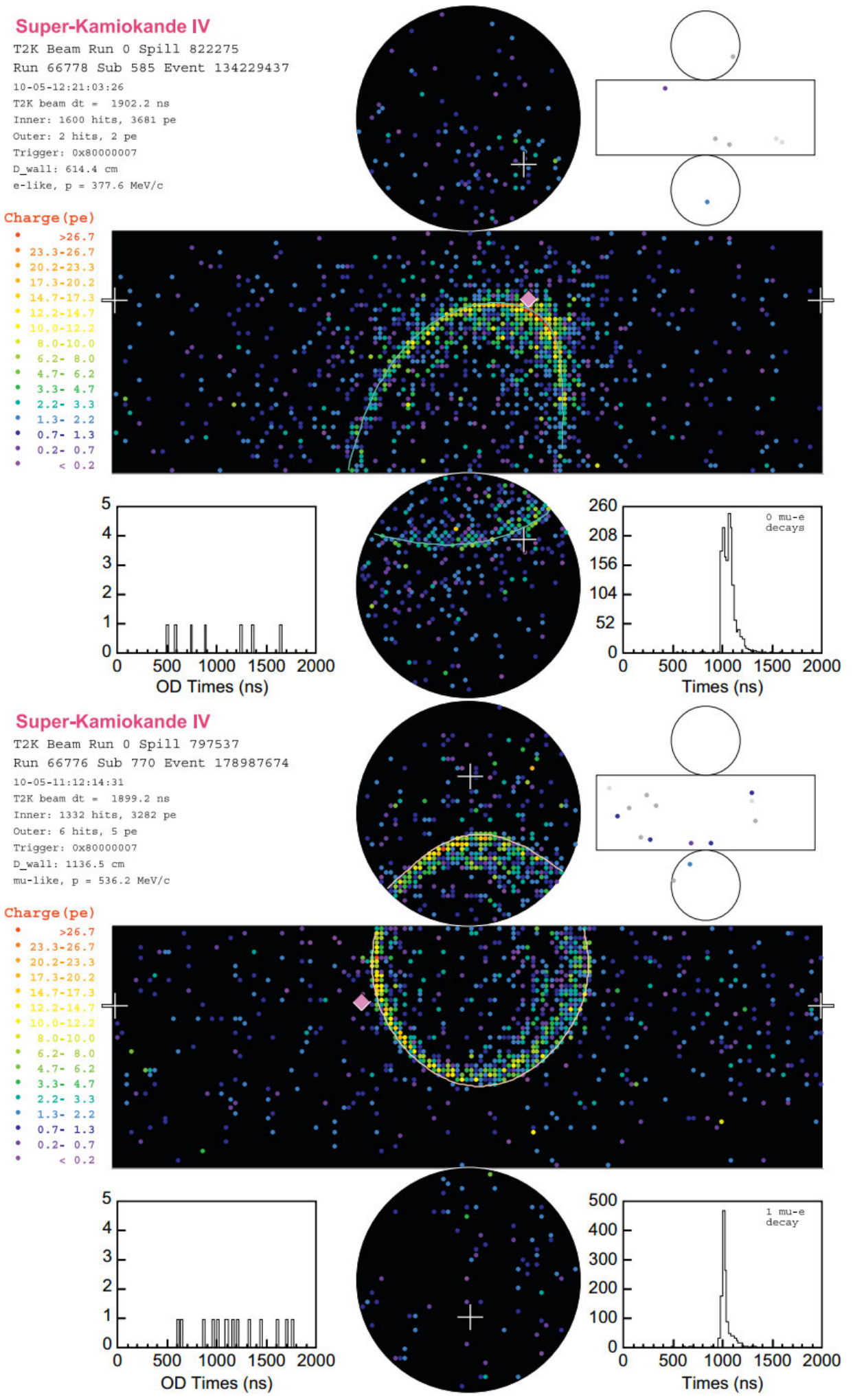

Figure 4.10. Event displays for the SK detector[35] in the T2K beam. The top figure shows an electron-like event with a fuzzy ring and no decay electron present. The bottom figure shows a muon-like event where the ring has sharply defined edges and contains a decay electron. 
TABLE 4.1. Table of of event rates expected in T2K for $7.8 \times 10^{21} \mathrm{POT}$ split evenly between neutrino and antineutrino running.

\begin{tabular}{lcc|ccc} 
& Signal & Bkg & $\nu_{\mu}+\bar{\nu}_{\mu} \operatorname{Bkg}$ & $\nu_{e}+\bar{\nu}_{e}$ Bkg & NC Bkg \\
\hline$\nu_{e}$ appearance & 84.8 & 54.1 & 1.1 & 32.6 & 20.4 \\
$\bar{\nu}_{e}$ appearance & 24.2 & 26.3 & 0.4 & 15.8 & 10.1 \\
$\nu_{\mu}$ disappearance & 612.3 & 43.7 & - & 1.4 & 42.3 \\
$\bar{\nu}_{\mu}$ disappearance & 298.7 & 19.5 & - & 0.6 & 18.9
\end{tabular}

With the flux prediction at SK in hand, the SK MC is used to generate event samples for $\nu_{e}$ appearance, $\bar{\nu}_{e}$ appearance, $\nu_{\mu}$ disappearance, and $\bar{\nu}_{\mu}$ disappearance.

The selections applied in this manner give seven channels for the $\nu_{e}$ appearance and $\bar{\nu}_{e}$ appearance analyses: $\nu_{e}$ and $\bar{\nu}_{e}$ for the signal, and $\nu_{\mu}, \bar{\nu}_{\mu}, \mathrm{NC}$, beam $\nu_{e}$, beam $\bar{\nu}_{e}$ for backgrounds. Seven channels also represent the $\nu_{\mu}$ disappearance and $\bar{\nu}_{\mu}$ disappearance analyses: $\nu_{\mu}$ and $\bar{\nu}_{\mu}$ for the signal, and $\mathrm{NC}$, beam/oscillated $\nu_{e}$, beam/oscillated $\bar{\nu}_{e}$ for backgrounds. The spectra ${ }^{2}$ for the samples are given in Figure 4.11 in terms of signal and background. Table 4.1 breaks down the integrated number of events per channel.

\subsubsection{T2K BAseline And Beam EnERGy. The T2K baseline and neutrino energy were}

chosen to maximize the disappearance probability and to restrict the energy range of events to those energies where CCQE is dominant and so the energy is able to be reconstructed accurately from SK measurements. Figure 4.12 shows the oscillation probabilities for $\nu_{e}$ appearance and $\nu_{\mu}$ disappearance in $\nu$ and $\bar{\nu}$ modes assuming the Capozzi et al. best fit values for the oscillation parameters. The figures also show the expected unoscillated $\nu_{\mu} \mathrm{CC}$ event spectrum in T2K. The original goal of T2K was to establish the phenomenon of $\nu_{e}$ appearance. Having established a relatively large value of $\sin ^{2}\left(2 \theta_{13}\right)$ early in its operation ${ }^{3}$,

\footnotetext{
${ }^{2}$ The spectra in this chapter were generated, by the author of this thesis, using inputs and methods that
} will be described in Chapter 5 . These inputs were used as the basis for $\mathrm{T} 2 \mathrm{~K}+\mathrm{NO} \nu \mathrm{A}$ combined fit sensitivity studies done for the T2K collaboration and published in $[36\rfloor$. The expected total numbers of events predicted for $\mathrm{T} 2 \mathrm{~K}$ were confirmed to match those predicted in official $\mathrm{T} 2 \mathrm{~K}$ oscillation analyses.

${ }^{3}$ The latest $\nu_{e}$ appearance result from T2K 20$\rfloor$ obtains a best-fit value of $\sin ^{2}\left(2 \theta_{13}\right)=0.140_{-0.032}^{+0.038}$ in the $\mathrm{NH}$ and $\sin ^{2}\left(2 \theta_{13}\right)=0.170_{-0.037}^{+0.045}$ in the IH with $\delta_{C P}=0$ and $\sin ^{2}\left(\theta_{23}\right)=0.5$ assumed. This is a $7.3 \sigma$ determination of $\theta_{13} \neq 0$. 


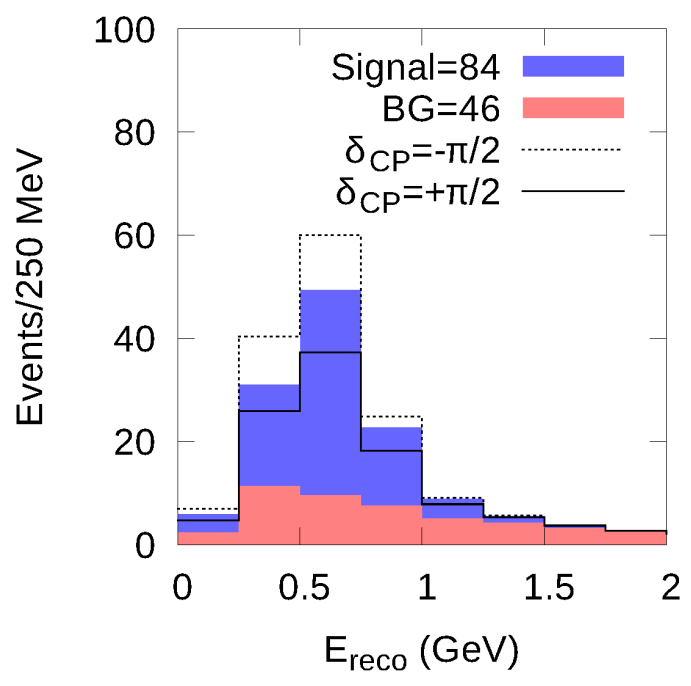

(A) $\nu_{e}$ appearance

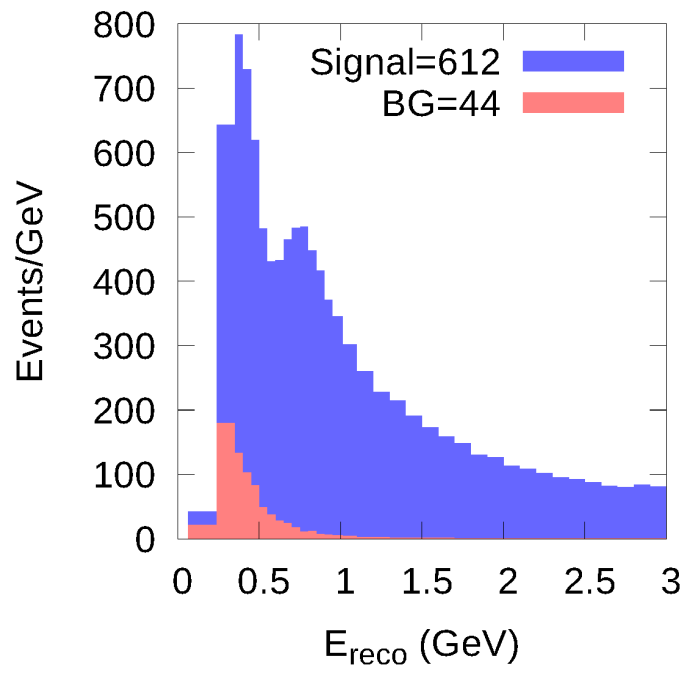

(C) $\nu_{\mu}$ disappearance

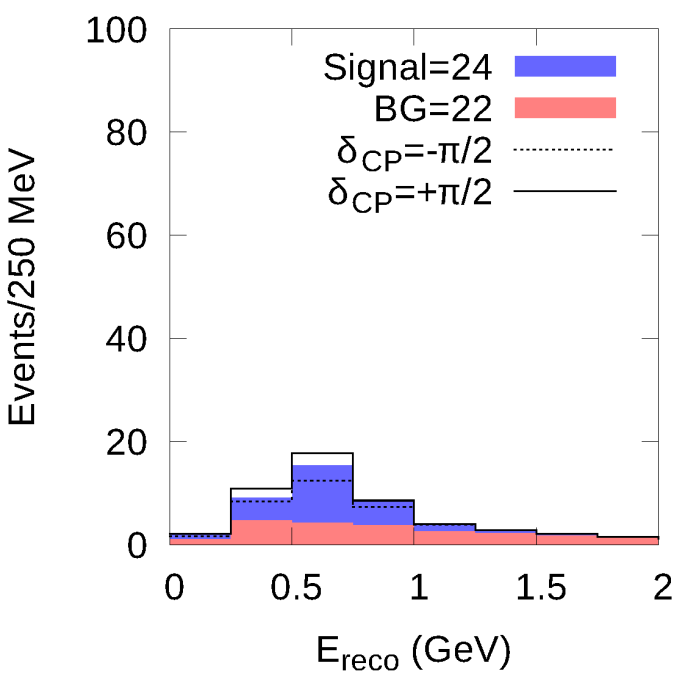

(B) $\bar{\nu}_{e}$ appearance

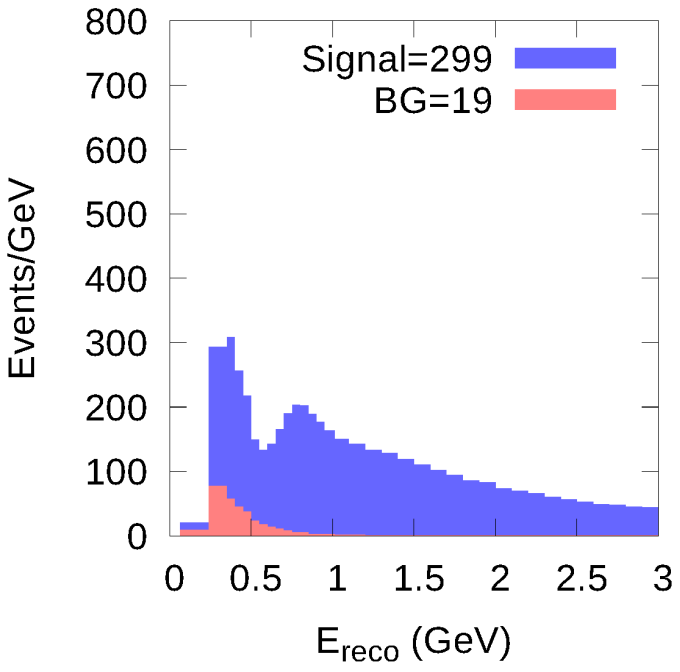

(D) $\bar{\nu}_{\mu}$ disappearance

Figure 4.11. The expected event spectra in the T2K FD, computed from $\mathrm{T} 2 \mathrm{~K} \mathrm{MC}$, for the four samples. Each assume a 1:1 $\nu: \bar{\nu}$ ratio and a total of $7.8 \times 10^{21}$ POT. Each figure shows the integrated number of events for the energy range for that figure. The Capozzi et al. set of oscillation parameters are assumed.

$\mathrm{T} 2 \mathrm{~K}$ is now focusing on constraining $\delta_{C P}$. The figures show that, in $\nu_{e}$ appearance, T2K as some ability to distinguish between different values of $\delta_{C P}$. This will be discussed further in later sections. 


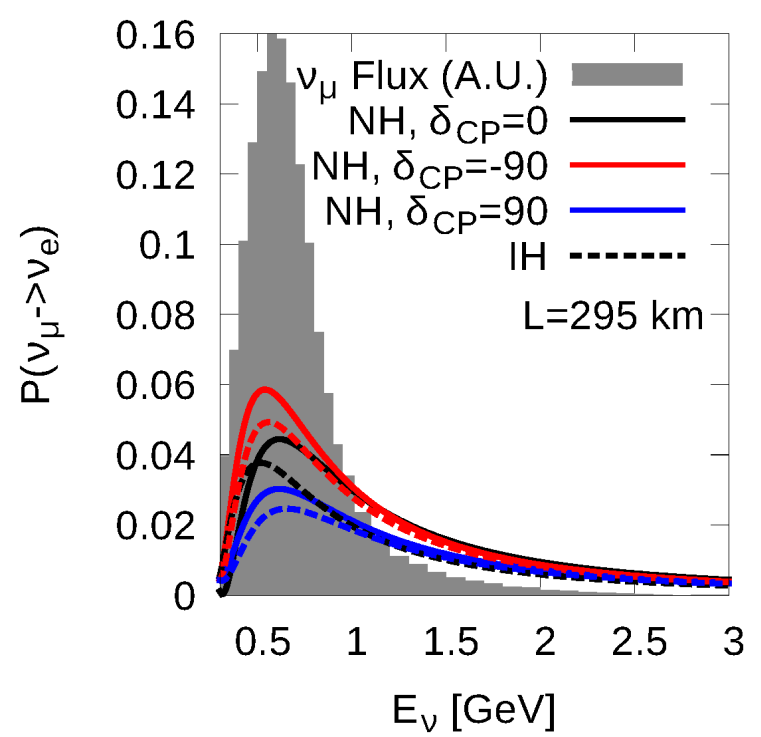

(A) $\nu_{e}$ appearance

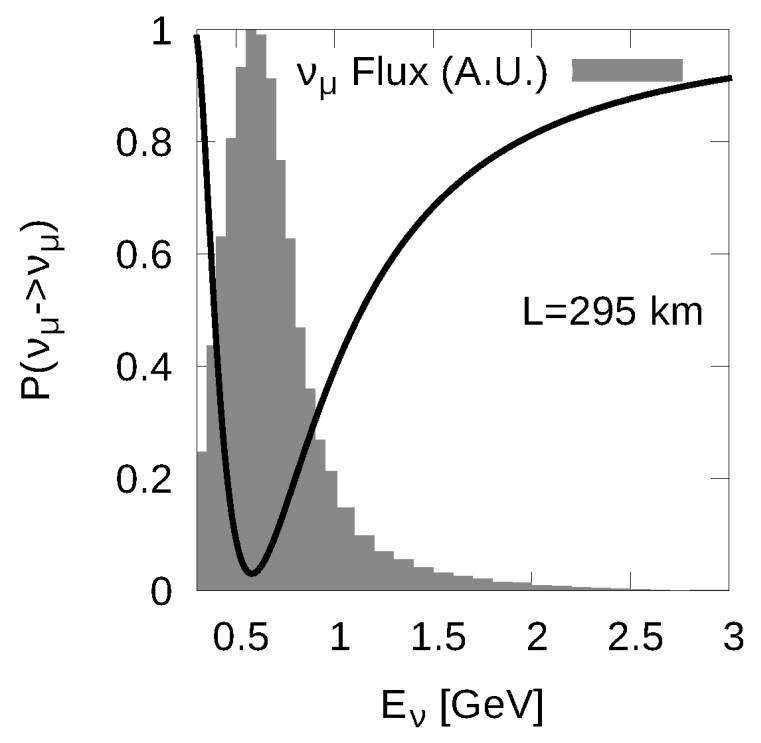

(C) $\nu_{\mu}$ disappearance

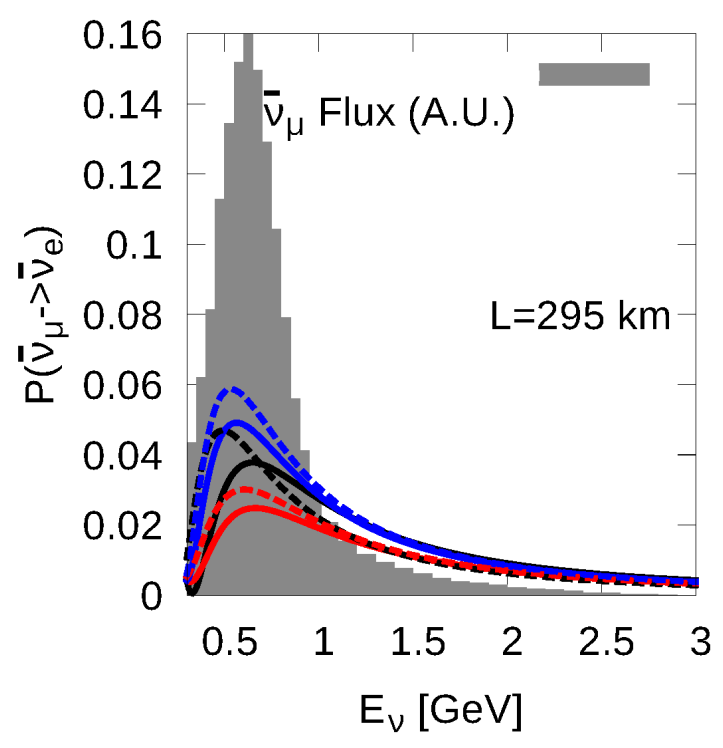

(B) $\bar{\nu}_{e}$ appearance

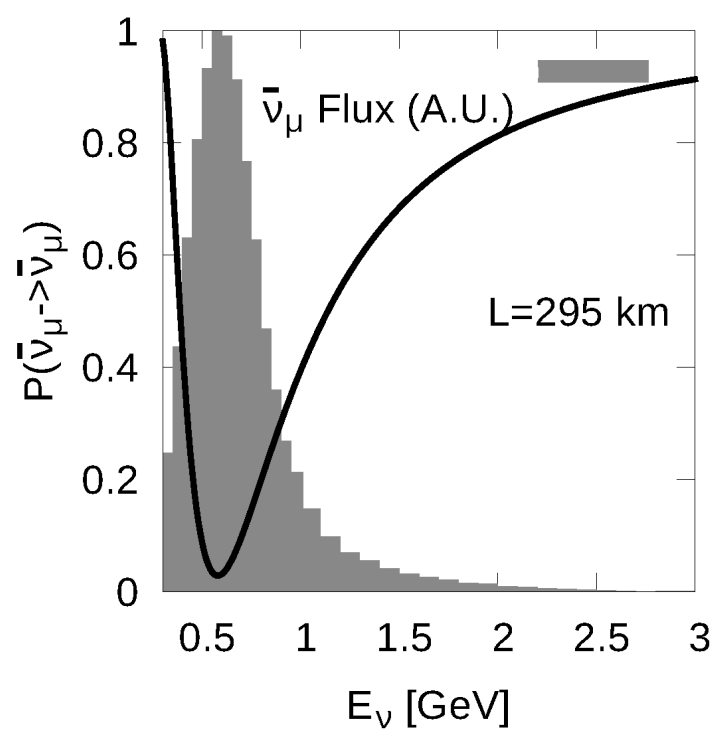

(D) $\bar{\nu}_{\mu}$ disappearance

FIGURE 4.12. Oscillation probabilities for $\nu_{e}$ appearance and $\nu_{\mu}$ disappearance in $\nu$ and $\bar{\nu}$ modes at $295 \mathrm{~km}$. The unoscillated $\nu_{\mu}\left(\bar{\nu}_{\mu}\right) \mathrm{CC}$ spectrum in $\mathrm{T} 2 \mathrm{~K}$ is plotted in gray.

4.3.3. $\mathrm{NO} \nu \mathrm{A}$. $\mathrm{NO} \nu \mathrm{A}$ is an off-axis LBL experiment with neutrino beam starting at Fermilab, passing through the ND on-site, and propagating $810 \mathrm{~km}$ to the far detector at Ash River in northern Minnesota. With its long-baseline it is more sensitive than any existing 
LBL experiment to the matter effect and the mass hierarchy. The NO $\nu \mathrm{A}$ experiment completed construction in the Summer of 2014. It is currently taking data with $1.35 \times 10^{20}$ POT taken as of February 2014 at a beam power of $300 \mathrm{~kW} \mathrm{[37].}$

The NuMI beam, which is now primarily the neutrino beam for $\mathrm{NO} \nu \mathrm{A}$, is produced at Fermilab. The primary beam from the Main Injector (MI) is $120 \mathrm{GeV}$ and has achieved $300 \mathrm{~kW}$ as of February 2014 but will be upgraded to produce $700 \mathrm{~kW}$. The protons strike a graphite target that is 2 interaction lengths long. Two magnetic horns pulsed with $185 \mathrm{kA}$ focus the resulting hadrons into a $675 \mathrm{~m}$ long He-filled decay region that is two meters in diameter. The profile and intensity of the beam are monitored by hadron and muon monitors at the end of the decay region and after various levels of absorption. The beam is pointed at an angle $14 \mathrm{mrad}\left(0.8^{\circ}\right)$ off-axis from the near and far detectors to produce a beam energy spectrum peaked at $2 \mathrm{GeV}$.

The $\mathrm{NO} \nu \mathrm{A}$ ND is located $1 \mathrm{~km}$ downstream of the target. Both the ND and FD are totally active tracking calorimeters. They use liquid scintillator contained in extruded PVC cells organized into alternating $\mathrm{X} / \mathrm{Y}$ planes. The ND will have $\sim 18,000$ cells and be $4 \mathrm{~m}$ $\times 4 \mathrm{~m} \times 14 \mathrm{~m}$ with a mass of $0.3 \mathrm{kt}$ and located $105 \mathrm{~m}$ underground. Each of the cells is designed identically to the FD. It will also have a muon ranger downstream to catch muons not contained by the primary detector.

The $\mathrm{NO} \nu \mathrm{A}$ FD is located $810 \mathrm{~km}$ away from the target in Ash River, Minnesota. It is composed of $\sim 344,000$ cells with overall dimensions of $16 \mathrm{~m} \times 16 \mathrm{~m} \times 60 \mathrm{~m}$ and have a mass of $14 \mathrm{kt}$. Charged current $\nu_{\mu}$ events are identified by reconstructing the muon track using either a CCQE or CC Non-QE (RES and DIS events) assumption where the muon is contained and so the energy of the event can be reconstructed with $4 \%$ resolution|37]. For CC $\nu_{e}$ events, the reconstruction algorithm looks for an EM shower starting from the vertex. 
The nominal running plan has the experiment running for 3 years in $\nu$ mode and 3 years in $\bar{\nu}$ mode. At full power, the experiment will receive $6 \times 10^{20} \mathrm{POT} / \mathrm{yr}$.

4.3.3.1. NOעA Experimental Inputs. The $\mathrm{NO} \nu \mathrm{A}$ inputs for this thesis are based on publicly available configurations[38] that use the predicted NuMI beam flux and estimated performance of the far detector. $\mathrm{NO} \nu \mathrm{A}$ beam flux, energy resolutions, and selection efficiencies are implemented in GLoBES files[38] created by the GLoBES developers (GLoBES is described in Chapter 5). These were based on the NO $\nu$ A 2007 Technical Design Report|32]. Updates to the efficiencies were made to match the total number of events in $\nu_{e}$ appearance, $\bar{\nu}_{e}$ appearance, $\nu_{\mu}$ disappearance, and $\bar{\nu}_{\mu}$ disappearance events with those shown publicly by the $\mathrm{NO} \nu \mathrm{A}$ collaboration[37]. These files describe both $\nu_{e}$ appearance and $\nu_{\mu}$ disappearance event selections in both $\nu$ and $\bar{\nu}$ modes at $1.8 \times 10^{21}$ POT in each mode. The oscillation sensitivities produced from these configurations were compared with official $\mathrm{NO} \nu \mathrm{A}$ sensitivities and found to agree well. The comparisons are shown in Appendix A.

In $\nu_{e}$ appearance the signal is defined as $\nu_{e} \mathrm{CC}$ with backgrounds from beam $\nu_{e}, \nu_{\mu} \mathrm{CC}$, and $\mathrm{NC}$ events. Events arising from $\bar{\nu}$ contamination in the beam are considered negligible in $\nu$ mode. In $\nu_{e}$ appearance the signal is defined as $\nu_{e}+\bar{\nu}_{e} \mathrm{CC}$ with backgrounds from beam $\nu_{e}+\bar{\nu}_{e}, \nu_{\mu}+\bar{\nu}_{\mu} \mathrm{CC}$, and NC events. Figure 4.13 shows the event spectra for the $\nu_{e}$ appearance and $\bar{\nu}_{e}$ appearance samples.

In $\nu_{\mu}$ disappearance the samples are broken up into multiple sources to take advantage of the improved energy resolution of CCQE events. The signal samples are CCQE, CC-NonQE, and CC uncontained. CCQE was described previously. CC-NonQE contains RES and DIS and has worse energy resolution, when compared with CCQE, coming from missing or misreconstructed energy. CC uncontained events contain a muon that exits the detector and so the energy is estimated from the vertex activity and from scattering of the muon before 
TABLE 4.2. Table of of event rates expected in $\mathrm{NO} \nu \mathrm{A}$ for $3.6 \times 10^{21} \mathrm{POT}$ split evenly between neutrino and antineutrino running.

\begin{tabular}{lcc|ccc} 
& Signal & Bkg & $\nu_{\mu}+\bar{\nu}_{\mu}$ Bkg & $\nu_{e}+\bar{\nu}_{e}$ Bkg & NC Bkg \\
\hline$\nu_{e}$ appearance & 57.4 & 32.7 & 5.0 & 8.4 & 19.3 \\
$\bar{\nu}_{e}$ appearance & 26.7 & 15.0 & $<1$ & 5.0 & 10.0 \\
$\nu_{\mu}$ disappearance & 508 & 20 & - & - & 20 \\
$\bar{\nu}_{\mu}$ disappearance & 270 & 9 & - & - & 9
\end{tabular}

it exits the detector. Figure 4.14 shows the event spectra for the $\nu_{\mu}$ disappearance and $\bar{\nu}_{\mu}$ disappearance samples.

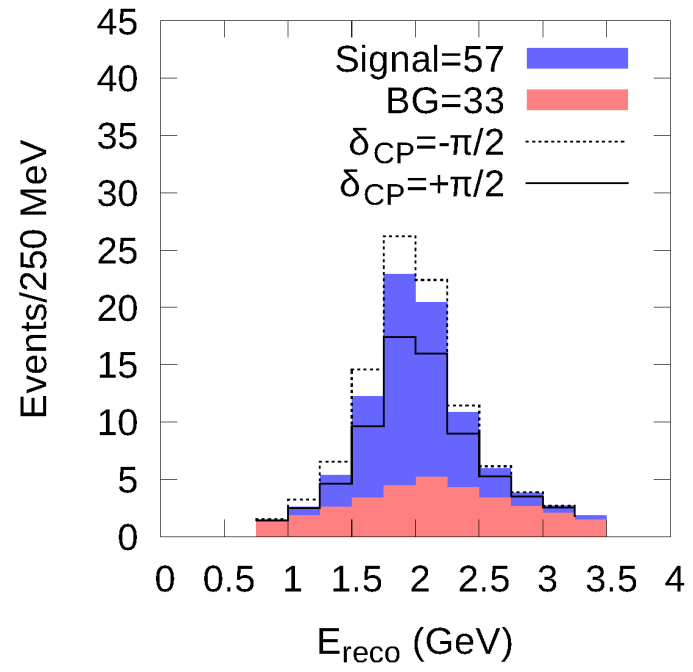

(A) $\nu_{e}$ appearance

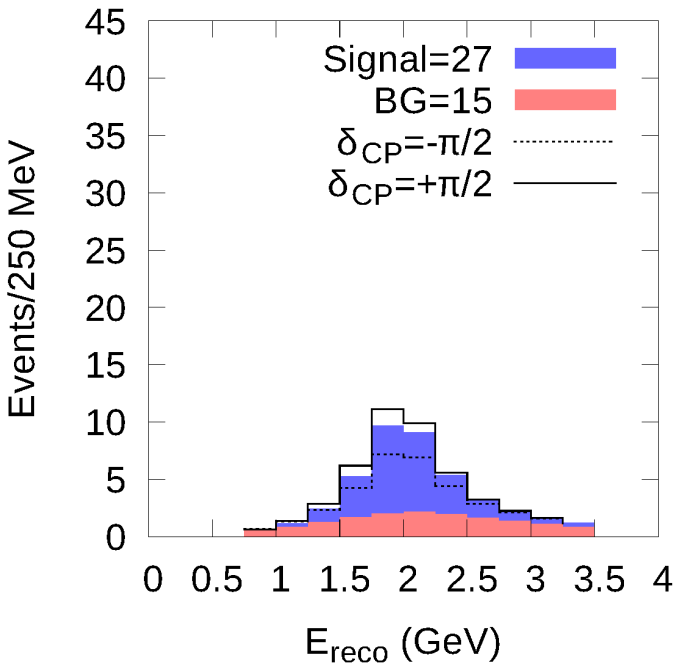

(B) $\bar{\nu}_{e}$ appearance

FiguRE 4.13. The expected event spectra in the NO $\nu \mathrm{A}$ FD for $\nu_{e}$ appearance and $\bar{\nu}_{e}$ appearance analyses. A 1:1 $\nu: \bar{\nu}$ ratio and a total of $3.6 \times 10^{21} \mathrm{POT}$ is assumed. Each figure shows the integrated number of events in the plotted energy range. The Capozzi et al. set of oscillation parameters are assumed.

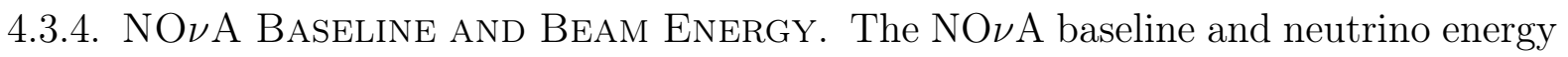
were chosen to maximize the appearance probability, maximize the matter effect, and to maximize statistics due to higher neutrino cross sections at higher energies. Figure 4.15 shows the oscillation probabilities for $\nu_{e}$ appearance and $\nu_{\mu}$ disappearance in $\nu$ and $\bar{\nu}$ modes assuming the Capozzi et al. best fit values for the oscillation parameters. The figures 


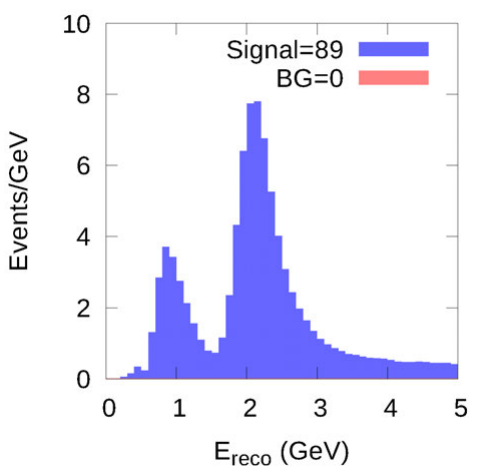

(A) $\nu_{\mu}$ disappearance CC QE

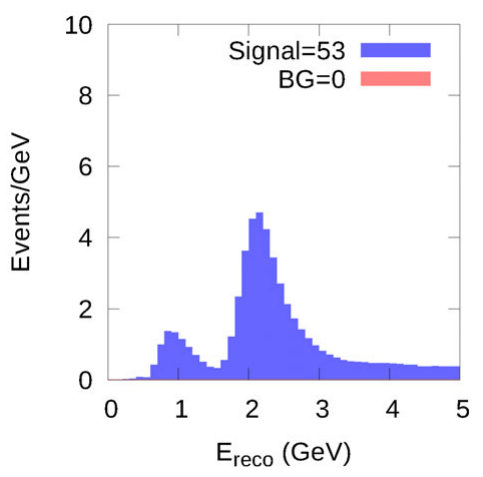

(D) $\bar{\nu}_{\mu}$ disappearance CC $\mathrm{QE}$

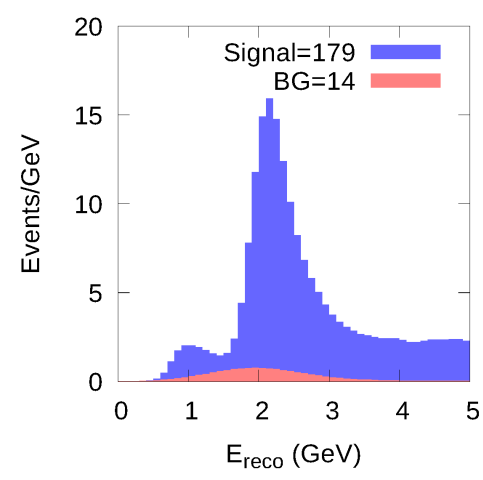

(B) $\nu_{\mu}$ disappearance $\mathrm{CC}$ Non-QE

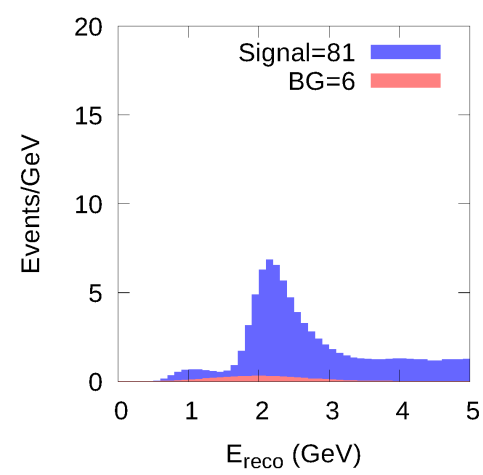

(E) $\bar{\nu}_{\mu}$ disappearance CC Non-QE

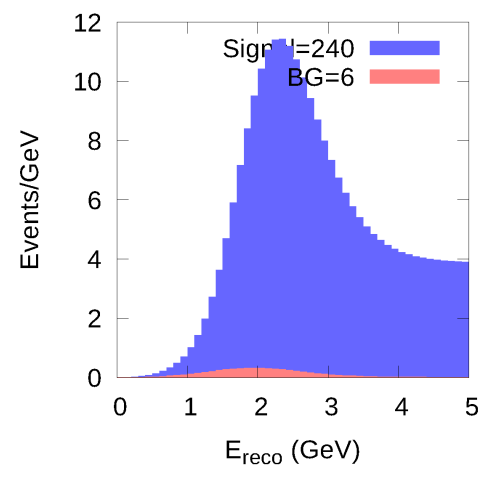

(C) $\nu_{\mu}$ disappearance CC Uncontained

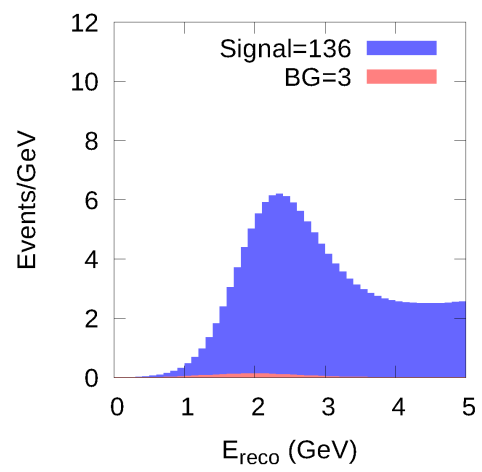

(F) $\bar{\nu}_{\mu}$ disappearance CC Uncontained

FIGURE 4.14. The expected event spectra in the NO $\nu \mathrm{A}$ FD for $\nu_{\mu}$ disappearance and $\bar{\nu}_{\mu}$ disappearance analyses for each sample: CCQE, CC Non-QE, and

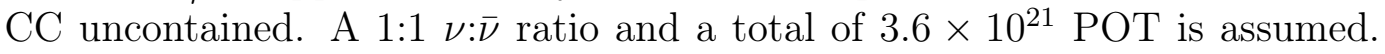
Each figure shows the integrated number of events in the plotted energy range. The Capozzi et al. set of oscillation parameters are assumed.

also show the expected unoscillated $\nu_{\mu} \mathrm{CC}$ event spectrum in $\mathrm{NO} \nu \mathrm{A}$. The $\nu$ flux energy spectrum aligns well to cover most of the first oscillation maximum. In the $\nu_{e}$ appearance $\left(\bar{\nu}_{e}\right.$ appearance $)$ spectrum the oscillation probability for $\delta_{C P}=-90^{\circ}\left(\delta_{C P}=90^{\circ}\right)$ in the $\mathrm{NH}(\mathrm{IH})$ is well separated from the other probabilities, but there is significant overlap for the other probabilities. This forms a degeneracy for these values of $\delta_{C P}$ and the MH. If the true value of $\delta_{C P}$ and $\mathrm{MH}$ are at these values then $\mathrm{NO} \nu \mathrm{A}$ will need to use constraints from another experiment, like T2K, to break the degeneracy. 


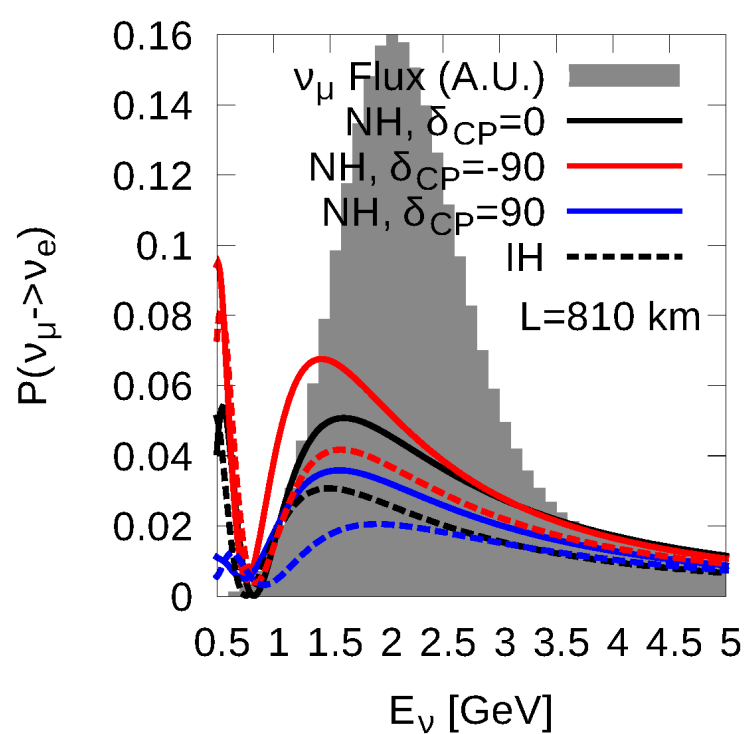

(A) $\nu_{e}$ appearance

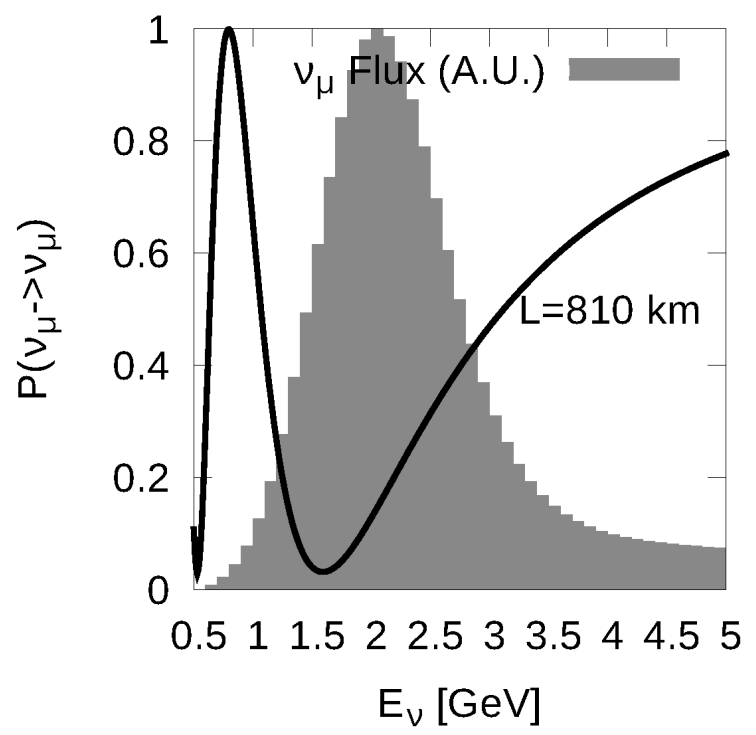

(C) $\nu_{\mu}$ disappearance

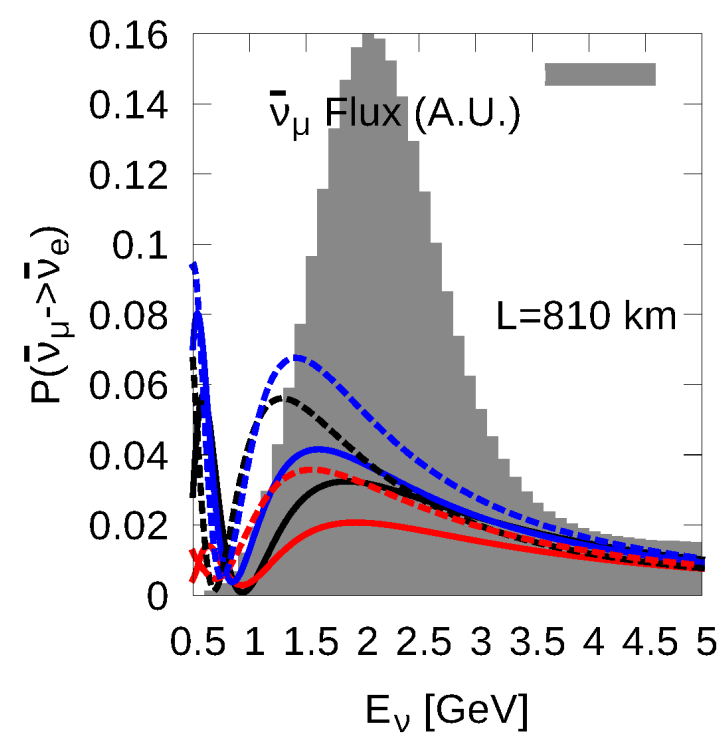

(в) $\bar{\nu}_{e}$ appearance

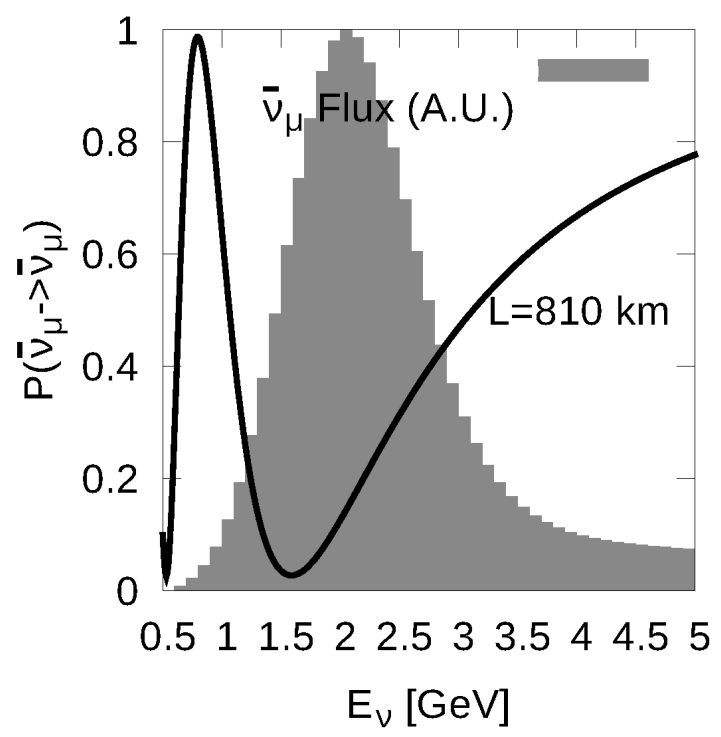

(D) $\bar{\nu}_{\mu}$ disappearance

FIGURE 4.15. Oscillation probabilities for $\nu_{e}$ appearance and $\nu_{\mu}$ disappearance in $\nu$ and $\bar{\nu}$ modes at $810 \mathrm{~km}$. The unoscillated $\nu_{\mu}\left(\bar{\nu}_{\mu}\right) \mathrm{CC}$ spectrum in $\mathrm{NO} \nu \mathrm{A}$ is plotted in gray.

4.3.5. LBNE. The Long-Baseline Neutrino Experiment (LBNE) $39 \mid$ is a planned LBL experiment with the primary goals of measuring $\mathrm{CP}$ violation and determining the mass hierarchy via $\nu_{e}$ appearance. It is projected to start collecting data around 2025. The 
project is being planned in stages that successively lead to improved physics reach. This section will describe the options being considered. The future sensitivity analysis will assume the full program.

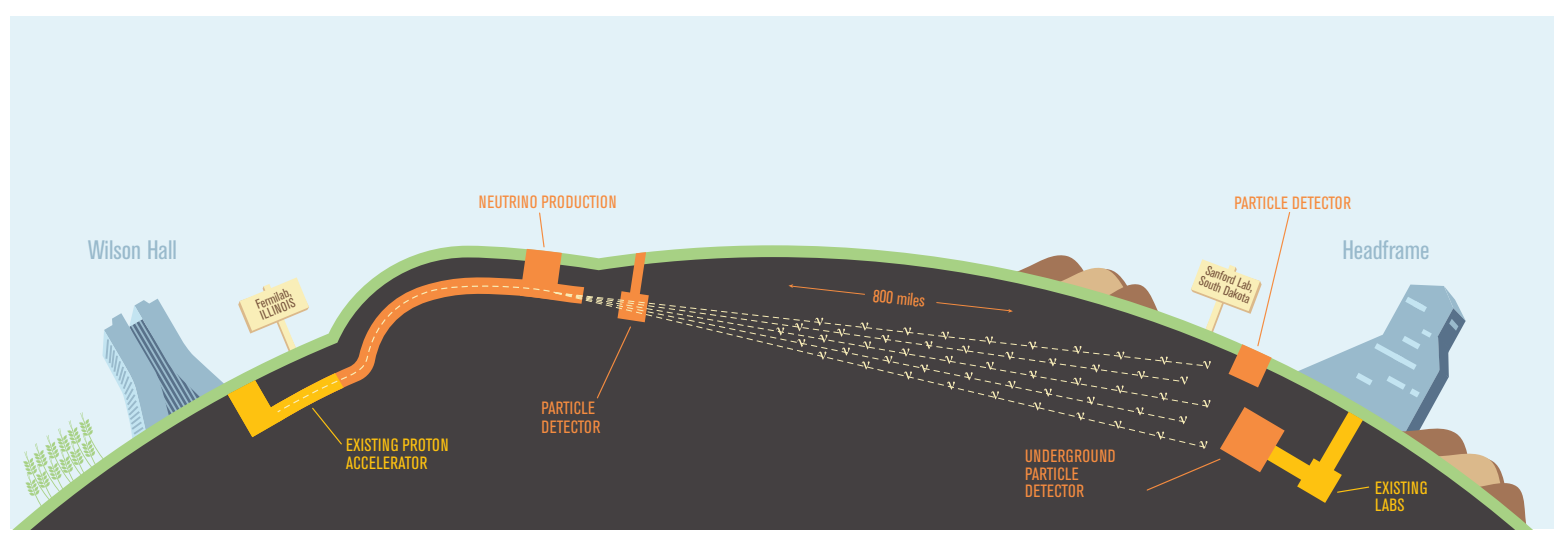

FiguRE 4.16. Layout of the LBNE Experiment[40].

The LBNE beam will be produced at Fermilab and the primary beam will use 60-120 $\mathrm{GeV}$ protons from the Main Injector proton accelerator. The baseline beam design will run at 1.2 MW and will be designed to be upgradeable to 2.3 MW. Protons will strike a graphite target that is $7.4 \mathrm{~mm} \times 15.4 \mathrm{~mm}$ and more than 2 interaction lengths in length. After the target two horns will focus the secondary beam with a pulsed current of between $180 \mathrm{kA}$ and $350 \mathrm{kA}$ into a decay region that is $204 \mathrm{~m}$ in length and air filled.

An ND, located $\sim 500 \mathrm{~m}$ downstream of the target, is planned. The current reference design is a fine-grained tracking detector consisting of a straw tube tracker and an electromagnetic calorimeter inside of a 0.4 T magnetic field. Argon gas, water, and other nuclear targets are placed upstream of the tracking volume to provide nuclear cross section measurements on different materials. It also enables the identification of uncontained muons using resistive plate chambers in the magnet and also before and after the tracker. The ND design is under study by the LBNE collaboration and alternative or additional detectors, such as a LAr TPC, are being considered. 
The LBNE FD will be a large volume LAr Time-Projection Chamber (TPC) $1300 \mathrm{~km}$ downstream of the target and located $\sim 4800$ feet underground at the Homestake mine in South Dakota. The TPC has a modular design which gives the possibility of a phased construction. The full LBNE FD is planned to have a mass of $34 \mathrm{kt}$. The underground nature of the experiment decreases backgrounds and allows it to also search for proton decay and supernova neutrinos.

A simulation, based on lessons learned from current and previous LAr experiments, has been created to study events in LAr detectors. Figure 4.17 shows an example for three types of events that are critical to a LBL physics analysis. The first is a CC $\nu_{\mu}$ event where the muon is clearly visible as a long straight track and is contained in the detector so that the decay electron is visible at the end of the track. The second is a CCQE $\nu_{e}$ event where the proton is visible as a track that deposits a lot of energy over a short distance (high $\mathrm{dE} / \mathrm{dx}$ ) and the electron produces a shower. The third is an $\mathrm{NC}$ event where vertex activity at the interaction point is clearly visible followed by a gap and then two $\gamma$ s converting to start a shower. A LAr TPC has a high resolution and so in many cases is able to distinguish a CCQE $\nu_{e}$ event and $\mathrm{NC}$ event by distinguishing between electrons and $\gamma \mathrm{s}$ before the EM shower begins.

The current design of LBNE has the experiment running for equal amounts in $\nu$ and $\bar{\nu}$ modes with a beam power of 1.2 MW for a total POT of $6.0 \times 10^{21}$ POT over 6 years. The $\nu: \bar{\nu}$ running ratio for LBNE will be discussed further in Section 9.

4.3.5.1. LBNE Experimental Inputs. The LBNE inputs for the future sensitivity analyses in this document are based on outputs from the LBNE Fast Monte Carlo (Fast MC). The Fast MC uses parameterization of expected detector responses as well as LBNE neutrino beam flux predictions and the GENIE event generator to simulate neutrinos interacting in 


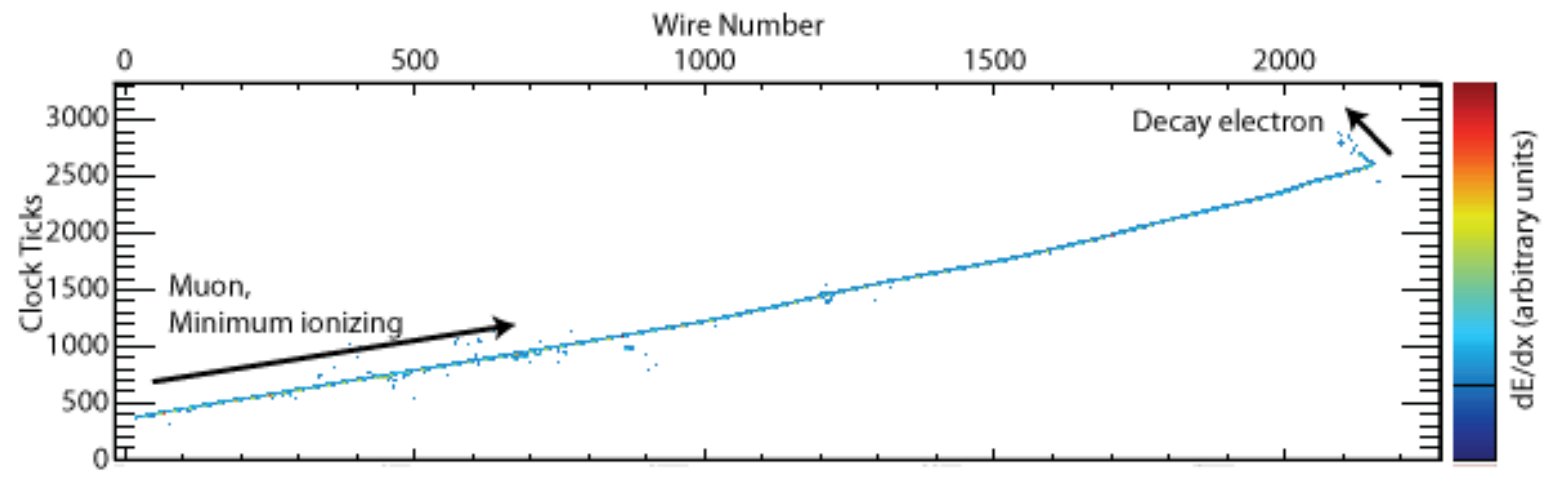

(A) $\mathrm{CC} \nu_{\mu}$ event

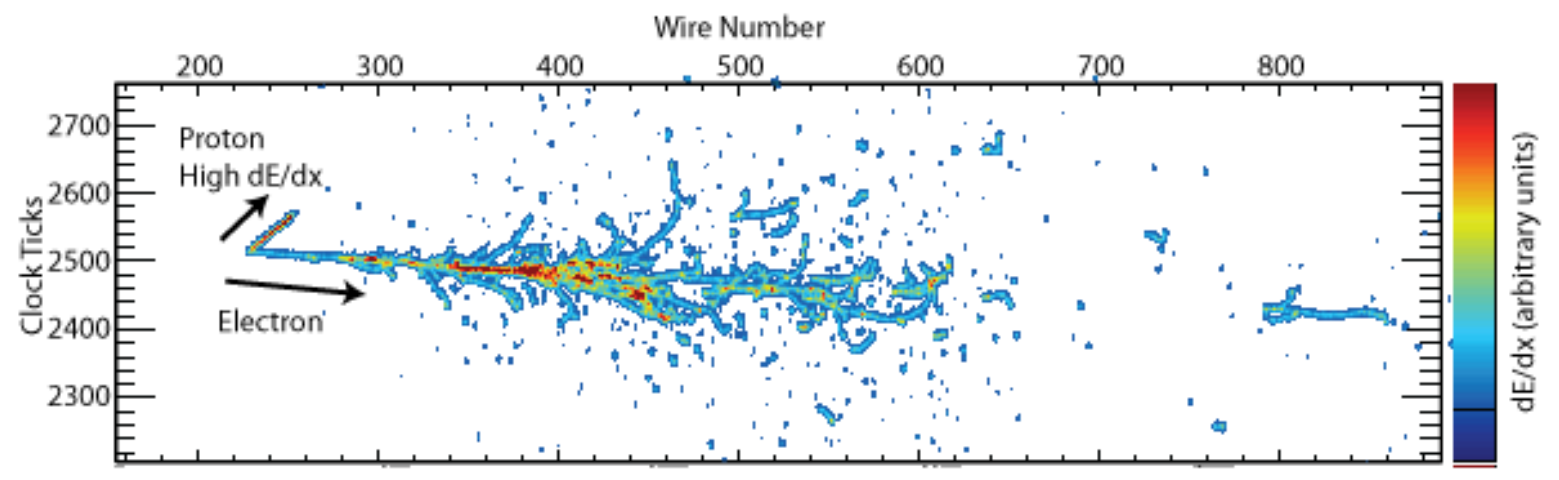

(B) CCQE $\nu_{e}$ event

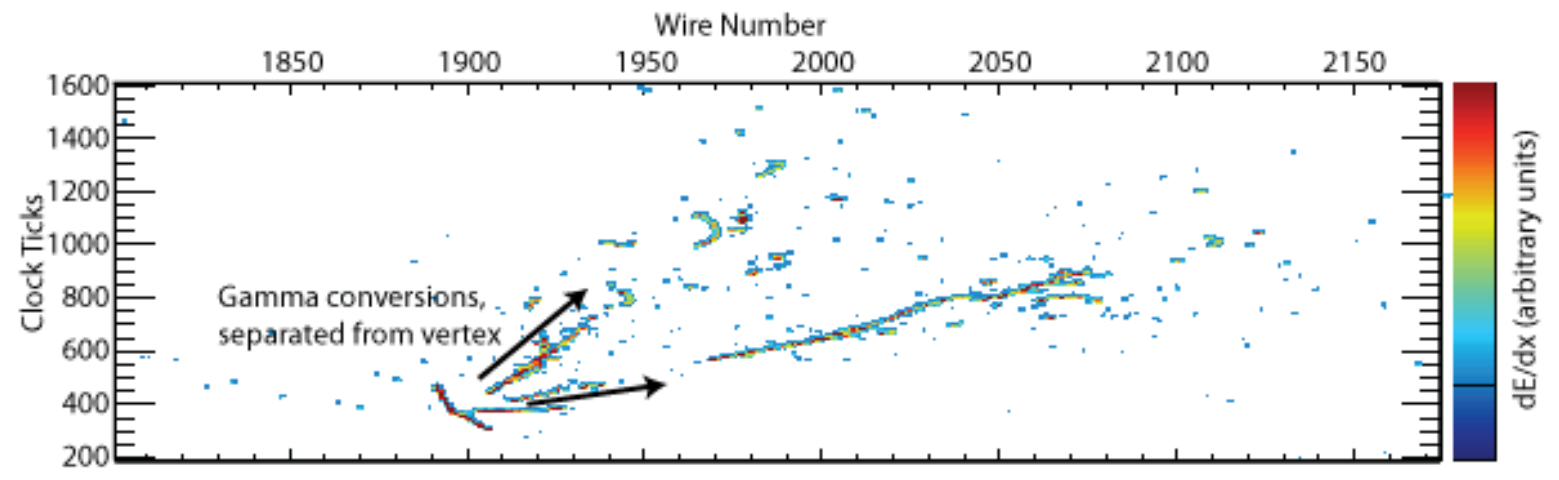

(C) $\mathrm{NC} \pi^{0}$ event

FigURE 4.17. Simulated events in a LAr detector|39].

the FD. Simulated neutrino interactions in the FD are recorded along with the identity and kinematics of the initial neutrino and the final-state particles that will interact in the detector. The detector response parameterization includes energy smearing so that a realistic 
TABLE 4.3. Table of of event rates expected in LBNE for $6.0 \times 10^{21}$ POT split evenly between neutrino and antineutrino running. The Capozzi et al. set of oscillation parameters are assumed

\begin{tabular}{lcc|cccc} 
& Signal & $\mathrm{Bkg}$ & $\nu_{\mu}+\bar{\nu}_{\mu} \mathrm{Bkg}$ & $\nu_{e}+\bar{\nu}_{e} \mathrm{Bkg}$ & $\nu_{\tau}+\bar{\nu}_{\tau} \mathrm{Bkg}$ & $\mathrm{NC} \mathrm{Bkg}$ \\
\hline$\nu_{e}$ appearance & 490 & 251 & 2 & 120 & 94 & 36 \\
$\bar{\nu}_{e}$ appearance & 309 & 178 & 1 & 98 & 57 & 22 \\
$\nu_{\mu}$ disappearance & 4971 & 299 & - & 4 & 140 & 147 \\
$\bar{\nu}_{\mu}$ disappearance & 2889 & 185 & - & 2 & 85 & 94
\end{tabular}

estimation of reconstructed neutrino energy can be made. Using this information, all of the events are classified as either $\mathrm{CC} \nu_{e}, \mathrm{CC} \nu_{\mu}$, or NC.

Using the Fast MC outputs also allows for the impact of systematic uncertainties to be propagated to the analyses. This will be discussed further in Chapter 7. ${ }^{4}$

The event spectra for $\nu_{e}$ appearance, $\bar{\nu}_{e}$ appearance, $\nu_{\mu}$ disappearance, and $\bar{\nu}_{\mu}$ disappearance are built from an analysis of the final states in the simulated events. The selections give nine channels for the $\nu_{e}$ appearance and $\bar{\nu}_{e}$ appearance analyses: $\nu_{e}$ and $\bar{\nu}_{e}$ for the signal, and mis-identified CC $\nu_{\mu}+\bar{\nu}_{\mu}$, mis-identified CC $\nu_{\tau}+\bar{\nu}_{\tau}, \mathrm{NC}$, and beam CC $\nu_{e}+\bar{\nu}_{e}$ for backgrounds. A similar set of nine channels are used for the $\nu_{\mu}$ disappearance and $\bar{\nu}_{\mu}$ disappearance analyses: $\nu_{\mu}$ and $\bar{\nu}_{\mu}$ for the signal, mis-identified CC $\nu_{\tau}+\bar{\nu}_{\tau}, \mathrm{NC}$, and beam $\mathrm{CC} \nu_{e}+\bar{\nu}_{e}$ for backgrounds. Figure 4.18 shows the expected event spectra in terms of signal and background and Table 4.3 gives the integrated number of events per channel. $\mathrm{CC} \nu_{\tau}$ events contribute a significant background due to the higher energy neutrinos required for longer baseline oscillations.

\subsubsection{LBNE Baseline and Beam Energy. The LBNE baseline and neutrino energy} were chosen to maximize the appearance probability, maximize the matter effect, and to

\footnotetext{
${ }^{4}$ The LBNE Fast MC is described further in $[39\rfloor$ which describes the LBNE in detail and also includes sensitivity studies, developed by the author of this thesis, that use parameterized inputs and estimates of detector efficiencies that are independent of the Fast MC. The sensitivities in this thesis are based on the Fast MC outputs and so may differ slightly due to the different assumptions.
} 


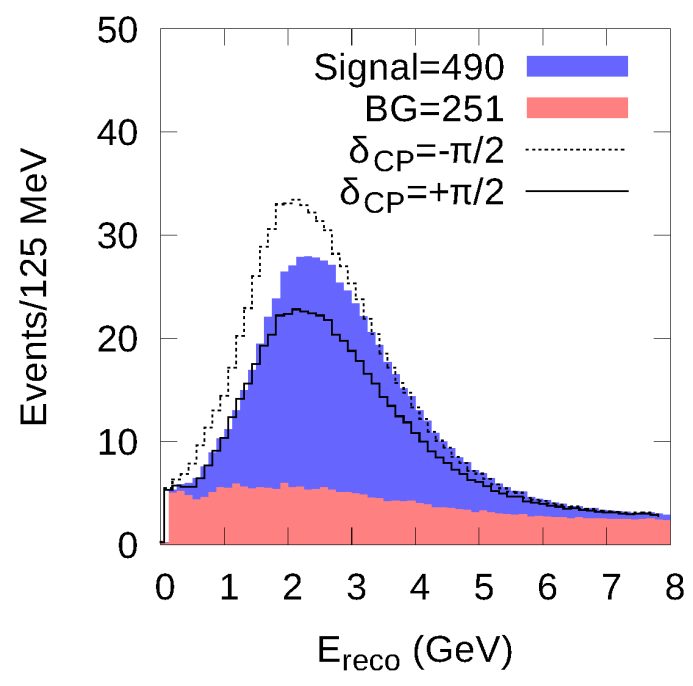

(A) $\nu_{e}$ appearance

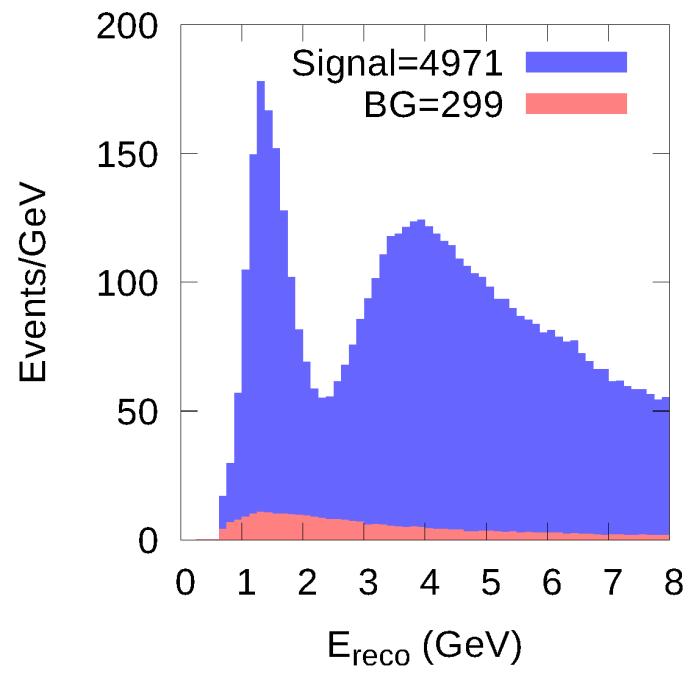

(C) $\nu_{\mu}$ disappearance

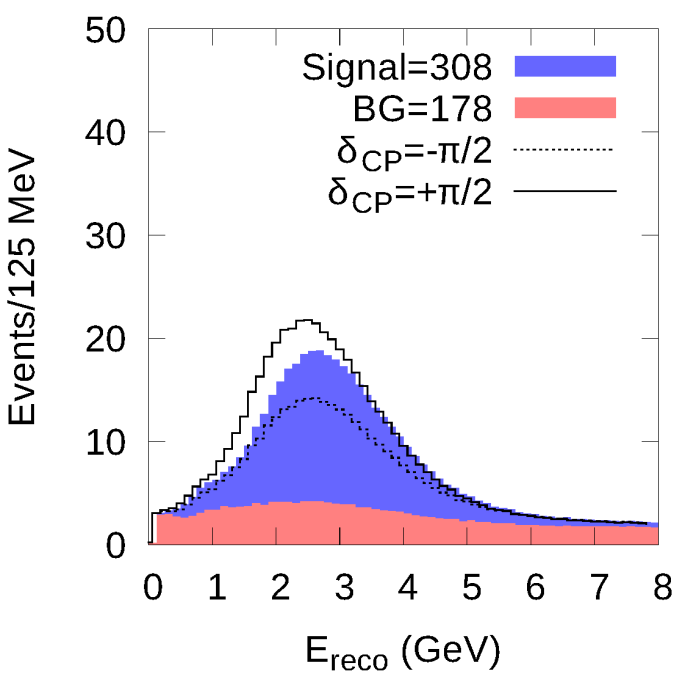

(B) $\bar{\nu}_{e}$ appearance

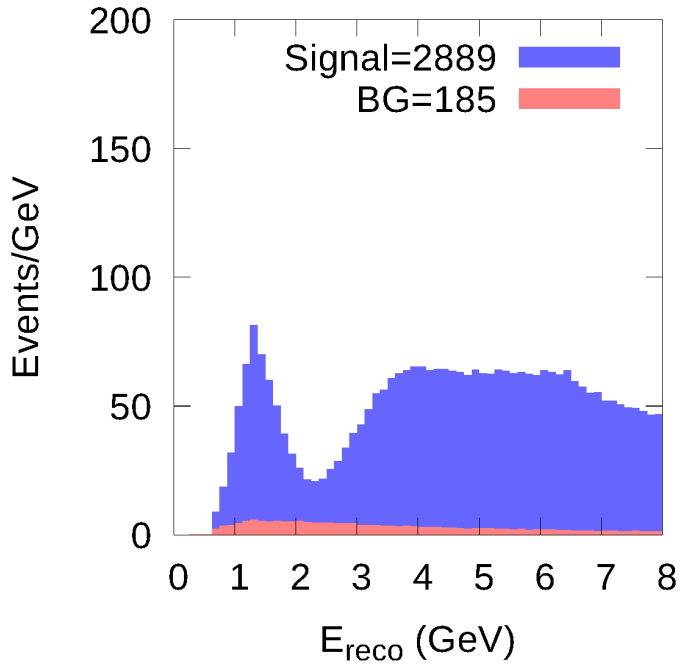

(D) $\bar{\nu}_{\mu}$ disappearance

Figure 4.18. The expected event spectra in the LBNE FD, computed from LBNE Fast MC, for the four samples. Each assume a 1:1 $\nu: \bar{\nu}$ ratio and a total of $6.0 \times 10^{21}$ POT. Each figure shows the integrated number of events for the energy range for that figure. The Capozzi et al. set of oscillation parameters are assumed.

maximize statistics due to higher neutrino cross sections at higher energies. Figure 4.19 shows the oscillation probabilities for $\nu_{e}$ appearance and $\nu_{\mu}$ disappearance in $\nu$ and $\bar{\nu}$ modes 
assuming the Capozzi et al. best fit values for the oscillation parameters. The figures also show the expected unoscillated $\nu_{\mu} \mathrm{CC}$ event spectrum in LBNE.

An extensive study[41] was done by the LBNE collaboration to optimize the sensitivity for $\mathrm{CP}$ violation detection, $\mathrm{MH}$ determination, and for resolution on $\delta_{C P}$. The study chose a set of baselines, optimized the neutrino beam at each baseline, and computed the sensitivity at each baseline. Short baselines suffer from not being able to resolve the second oscillation maximum and so cannot resolve degeneracies between $\delta_{C P}$ and the $\mathrm{MH}$ that occur in the oscillation probabilities. At shorter baselines the energy of the second oscillation maximum is below that which can typically be resolved in typical neutrino oscillation experiments. The second oscillation maximum is visible at the LBNE $1300 \mathrm{~km}$ baseline, as can be seen in Figure 4.19. While the matter effect causes longer baselines to have greater mass hierarchy sensitivity, if the baseline is too long then the experiment will suffer from increased $\nu_{\tau}$ backgrounds and decreased $\nu$ flux. Taking these sensitivity consideration into account, the study finds that a baseline of at least $1000 \mathrm{~km}$ in a wide-band neutrino beam is optimal.

4.3.7. T2HK. Tokai to Hyper-Kamiokande (T2HK) [42] is a proposed LBL experiment with the primary goal of measuring CPV. It is the successor to T2K and consists of major upgrades to the beam and ND and a completely new FD.

The T2HK beam will be produced at J-PARC using the same beam-line as T2K. It is anticipated that the beam will be running at $1.66 \mathrm{MW}$ at that time. The nominal beam for T2HK is assumed to be $2.5^{\circ}$ off-axis from the FD. The ND will be the T2K ND280 detector with possible upgrades.

The proposed FD for T2HK is the Hyper Kamiokande (HK) large WC detector that will also be at a baseline of $295 \mathrm{~km}$ as is SK for T2K. It has a fiducial mass of $560 \mathrm{kt}$, which is about 25 times larger than SK. It is also to be located underground (1750 MWE). By using 


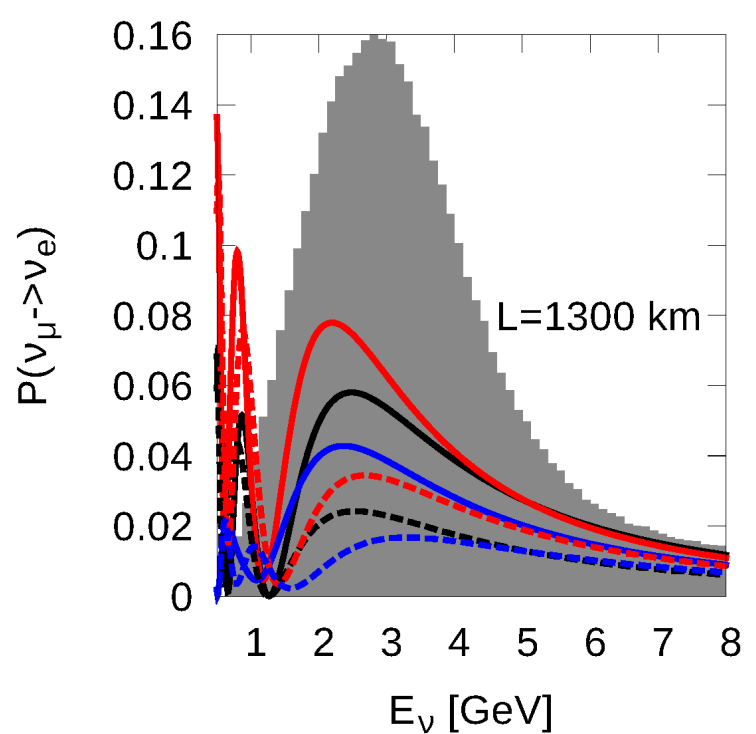

(A) $\nu_{e}$ appearance

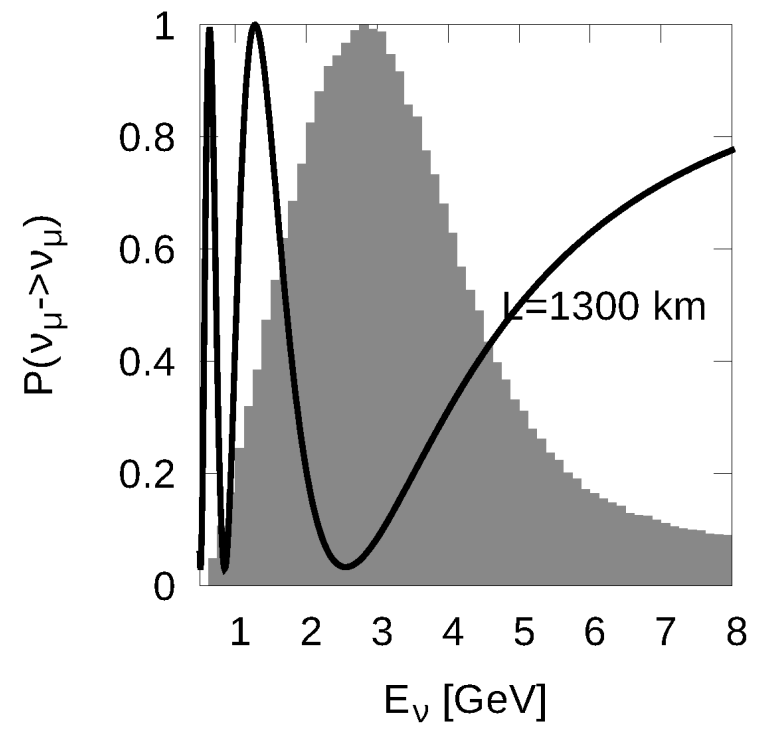

(C) $\nu_{\mu}$ disappearance

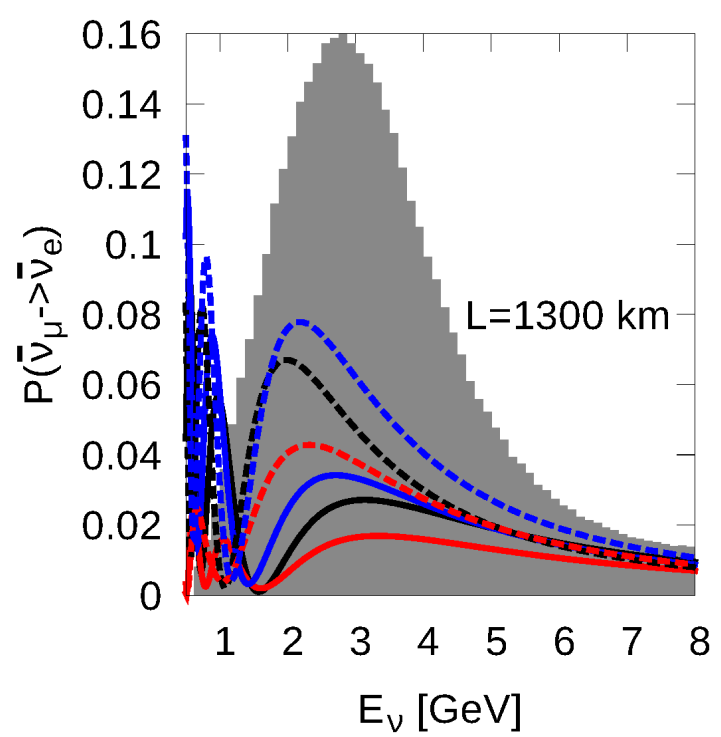

(в) $\bar{\nu}_{e}$ appearance

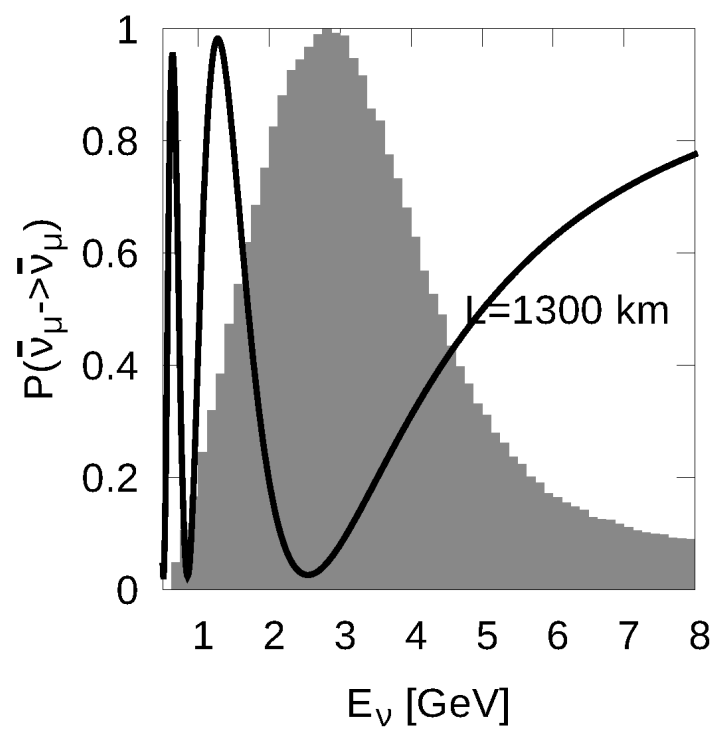

(D) $\bar{\nu}_{\mu}$ disappearance

FIGURE 4.19. Oscillation probabilities for $\nu_{e}$ appearance and $\nu_{\mu}$ disappearance in $\nu$ and $\bar{\nu}$ modes at $1300 \mathrm{~km}$. The unoscillated $\nu_{\mu}\left(\bar{\nu}_{\mu}\right) \mathrm{CC}$ spectrum in LBNE is plotted in gray.

99,000 20 inch photo-multiplier tubes, it will achieve $20 \%$ coverage of the inner detector surface. The mechanism for reconstructing CCQE events from electrons and muons in HK is the same as it is in SK. 
TABLE 4.4. Table of of event rates expected in T2HK for 1.5 years in $\nu$ mode and 3.5 years in $\bar{\nu}$ mode at $1.66 \mathrm{MW}$. The Capozzi et al. set of oscillation parameters are assumed.

\begin{tabular}{lcc|ccc} 
& Signal & Bkg & $\nu_{\mu}+\bar{\nu}_{\mu}$ Bkg & $\nu_{e}+\bar{\nu}_{e}$ Bkg & NC Bkg \\
\hline$\nu_{e}$ appearance & 2111 & 1141 & 24 & 660 & 458 \\
$\bar{\nu}_{e}$ appearance & 1788 & 1615 & 25 & 912 & 678 \\
$\nu_{\mu}$ disappearance & 15224 & 1091 & - & 37 & 1054 \\
$\bar{\nu}_{\mu}$ disappearance & 22306 & 1449 & - & 37 & 1412
\end{tabular}

Current design documents[43] have the experiment running for 1.5 years in $\nu$ mode and 3.5 years in $\bar{\nu}$ mode with a beam power of $1.66 \mathrm{MW}$. These differences in the detector mass are accounted for by scaling the mass of the T2K detector by 24.9. The differences in beam power and total running time are accounted for by doubling the T2HK running time relative to T2K..$^{5}$

4.3.7.1. T2HK Experimental Inputs. Experimental inputs for T2HK are the same as for T2K but with a large scaling applied to account for the increased detector size and beam power. Table 4.4 gives the expected event rates for T2HK and Figure 4.20 gives the expected event spectra for $\nu_{e}$ appearance, $\bar{\nu}_{e}$ appearance, $\nu_{\mu}$ disappearance, and $\bar{\nu}_{\mu}$ disappearance.

4.3.8. T2HK Baseline And Beam Energy. Since T2HK has the same baseline and neutrino beam shape, Figure 4.12 also applies to T2K. T2HK will have a much larger event rate, but since the $\nu_{\mu}$ flux is in arbitrary units, the plot remains the same for T2HK and T2K. Although T2HK has very little sensitivity to the mass hierarchy due to its shorter baseline, it is anticipated that HK itself will make a measurement of the MH via highstatistics atmospheric neutrino measurements.

\footnotetext{
${ }^{5}$ No effort was made to precisely match T2HK predicted event rates published in, for example, $\lfloor 43\rfloor$ or $\lfloor 44\rfloor$. The sensitivities produced for T2HK in this thesis are estimates for an experiment with twice the POT and 25 times the detector mass as T2K. Recent work by the T2HK collaboration 44 ] has updated the official T2HK sensitivities with predictions based on improved event selections in the SK detector simulation. The rates in this recent work for $\nu_{e}$ appearance are roughly $16 \%$ higher than those used in this thesis after accounting for differences in assumed oscillation parameter values.
} 


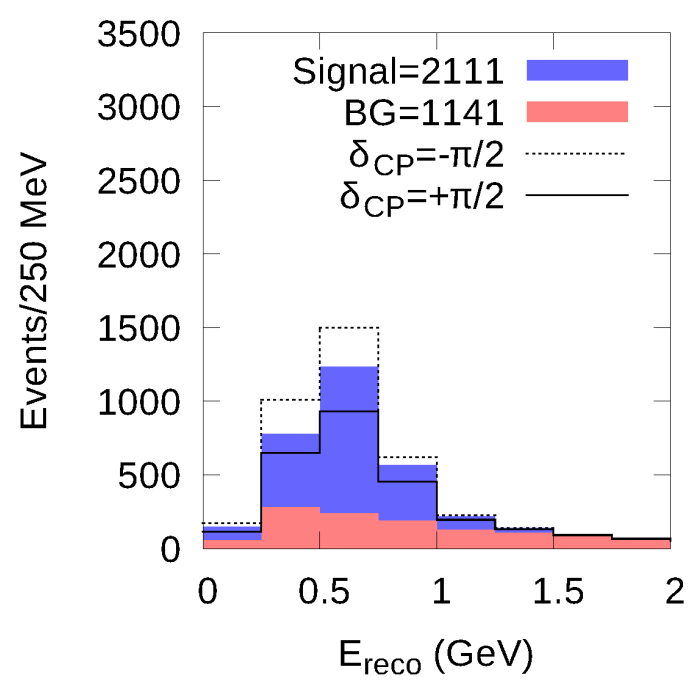

(A) $\nu_{e}$ appearance

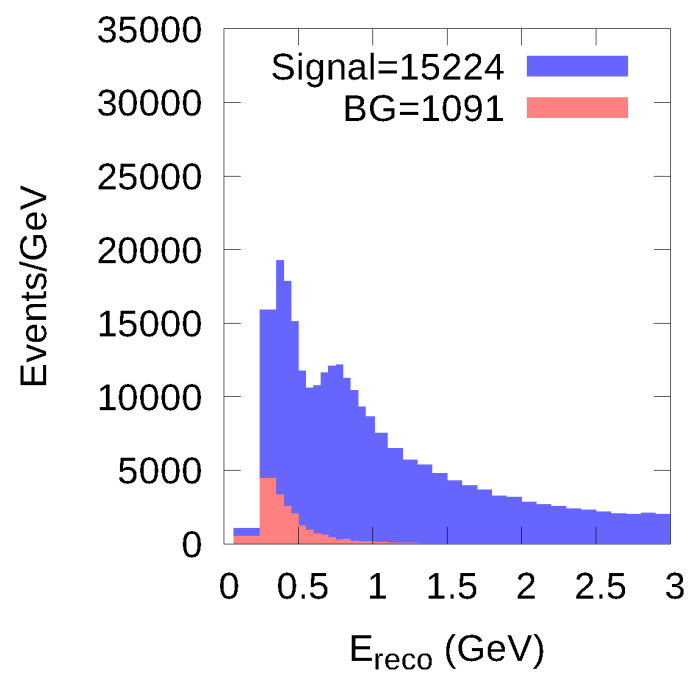

(C) $\nu_{\mu}$ disappearance

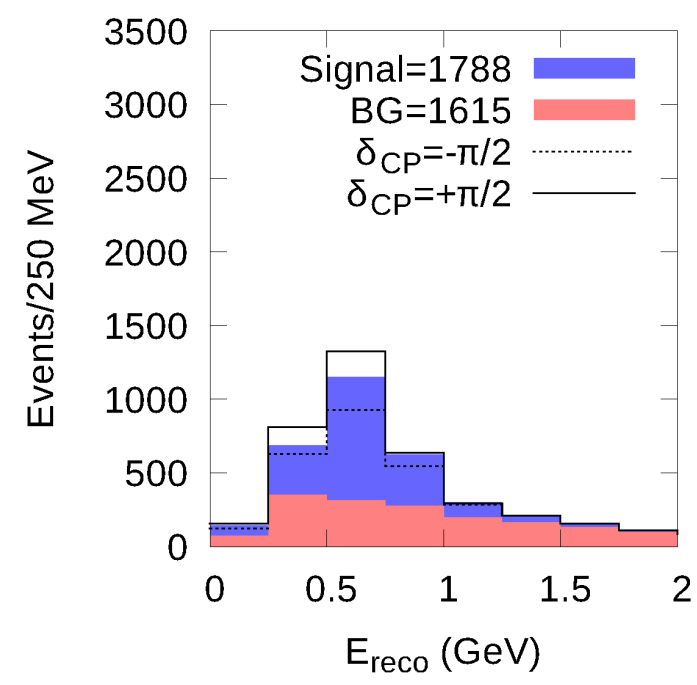

(в) $\bar{\nu}_{e}$ appearance

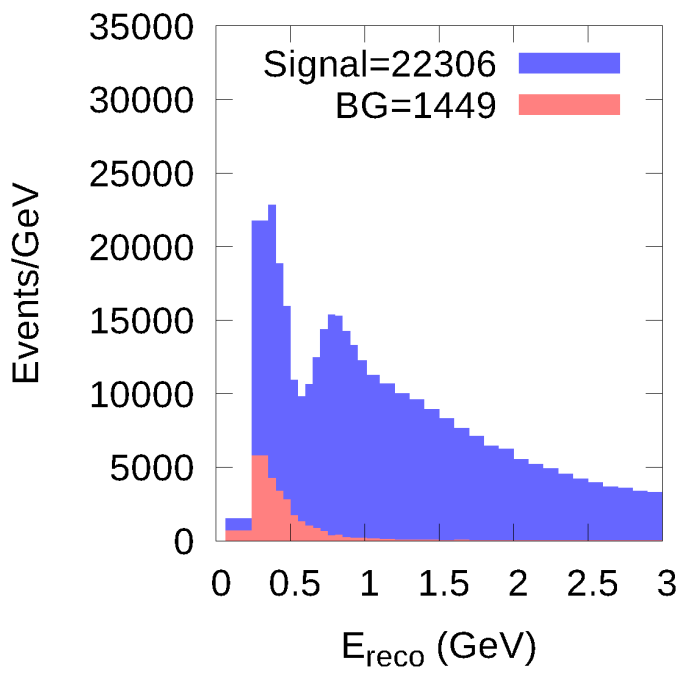

(D) $\bar{\nu}_{\mu}$ disappearance

FiguRE 4.20. The expected event spectra in the T2HK FD. Each assumes 1.5 years in $\nu$ mode and 3.5 years in $\bar{\nu}$ mode at $1.66 \mathrm{MW}$. Each figure shows the integrated number of events for the energy range for that figure. The Capozzi et al. set of oscillation parameters are assumed. 


\section{CHAPTER 5}

\section{Methods for Computing Sensitivity to Neutrino}

\section{OSCILLATions}

In order to predict the sensitivity of current and future experiments it is necessary to have a statistical method for incorporating the experimental inputs described in the previous chapter. This chapter reviews the likelihood ratio test method used to compute the sensitivities and explores the assumptions and approximations that go into it as well as how prior information about the oscillation parameters and systematic uncertainties are propagated to the test hypotheses. It then describes the software library used to perform these tests and how inputs from $\mathrm{T} 2 \mathrm{~K}, \mathrm{NO} \nu \mathrm{A}, \mathrm{LBNE}$, and T2HK, based on simulations, are used with the library.

\subsection{Profile Log-Likelihood Method}

As shown in the previous chapter the expected event rates for a particular oscillation hypothesis can be computed using inputs from each experiment. These are always computed as a function of $E_{\text {reco }}$ and set of oscillation parameters, $\boldsymbol{\theta}$, for the purposes of this work. Details on how the event rates are computed will be given later in this chapter.

The likelihood ratio is a statistical test to compare two different models for consistency with an observation. A null hypothesis, H0, is compared to an alternative hypothesis, H1, in a ratio that grows as the alternative hypothesis further rejects the null hypothesis. This quantity can then be used to determine the p-value, which is the probability of obtaining H1 assuming that $\mathrm{H} 0$ is true. 
In an experiment, the observed number of events, $N^{o b s}$, is compared to an expectation for a given hypothesis, $N^{e x p}$. In a particular time interval, the probability to observe $N^{o b s}$ events when $N^{e x p}$ are expected is given by the Poisson distribution,

$$
f_{\text {Poisson }}\left(N^{o b s} ; N^{e x p}\right)=\frac{\left(N^{e x p}\right)^{N^{o b s}}}{N^{o b s !}} e^{-N^{e x p}} .
$$

The binned Poisson likelihood to observe $N^{\text {obs }}$ events in bin i when $N^{e x p}$ are expected is then

$$
L_{P}\left(N_{i}^{o b s} \mid N_{i}^{e x p}(\boldsymbol{\theta}, \boldsymbol{f})\right)=\prod_{i}^{N_{b i n s}} \frac{\left(N_{i}^{e x p}(\boldsymbol{\theta}, \boldsymbol{f})\right)^{N_{i}^{o b s}}}{N_{i}^{o b s} !} e^{-N_{i}^{e x p}(\boldsymbol{\theta}, \boldsymbol{f})}
$$

where $\boldsymbol{\theta}=\left(\theta_{12}, \theta_{13}, \theta_{23}, \delta_{C P}, \Delta m_{31}^{2}, \Delta m_{32}^{2}\right)$ is a vector of oscillation parameters and $\boldsymbol{f}$ is a vector of nuisance parameters describing, for example, a systematic effect and will be discussed below. The bin index i and total number of bins, $N_{\text {bins }}$, represents the reconstructed energy $\left(E_{\text {reco }}\right)$ bin.

The log of the likelihood ratio (LLR) between $\mathrm{H} 0$ and $\mathrm{H} 1$ is

$$
\begin{aligned}
& \mathcal{L}=-2 \ln \left[\frac{L(\text { data } \mid H 0)}{L(\text { data } \mid H 1)}\right] \\
& =-2 \ln \left[\frac{L\left(N^{o b s} \mid N^{\exp }(\boldsymbol{\theta}, \hat{\hat{\boldsymbol{f}}})\right)}{\left.L\left(N^{o b s} \mid N^{\exp }(\hat{\boldsymbol{\theta}}, \hat{\boldsymbol{f}})\right)\right)}\right] \\
& =-2 \ln \left[\frac{\prod_{i}^{N} \frac{\left(N_{i}^{e x p}(\boldsymbol{\theta}, \hat{\boldsymbol{f}})\right)^{N_{i}^{o b s}}}{N_{i}^{o b s} !} e^{-N_{i}^{e x p}(\boldsymbol{\theta}, \hat{\hat{\boldsymbol{f}}})}}{\prod_{i}^{N} \frac{\left(N_{i}^{\text {exp }}(\hat{\boldsymbol{\theta}}, \hat{\boldsymbol{f}})\right)^{N_{i}^{o b s}}}{N_{i}^{\text {obs }} !} e^{-N_{i}^{e x p}(\hat{\boldsymbol{\theta}}, \hat{\boldsymbol{f}})}}\right] \\
& =2 \sum_{i}^{N}\left[N_{i}^{e x p}(\boldsymbol{\theta}, \hat{\hat{\boldsymbol{f}}})-N_{i}^{\exp }(\hat{\boldsymbol{\theta}}, \hat{\boldsymbol{f}})+N_{i}^{\text {obs }} \ln \frac{N_{i}^{\exp }(\hat{\boldsymbol{\theta}}, \hat{\boldsymbol{f}})}{N_{i}^{\exp }(\boldsymbol{\theta}, \hat{\hat{\boldsymbol{f}}})}\right] .
\end{aligned}
$$

The log of the likelihood ratio is taken because when computing the product of many small numbers a computer may run into machine precision errors so taking the log turns the small numbers into larger negative numbers that can be represented more precisely with floating 
point numbers. The factor of two makes $\mathcal{L}$ equal to a $\chi^{2}$ for large statistics and will be discussed below in the context of interpreting the statistical significance of an experimental measurement or simulated experimental measurement. The ${ }^{\wedge}$, over $\boldsymbol{\theta}$ and $\boldsymbol{f}$ mean that those parameters are to be profiled. In general, profiling a parameter refers to maximizing the likelihood, or minimizing the LLR, with respect to that parameter. The $N_{i}^{\exp }(\hat{\boldsymbol{\theta}}, \hat{\boldsymbol{f}})$ term

will have both $\boldsymbol{\theta}$ and $\boldsymbol{f}$ profiled, while $N_{i}^{\text {exp }}(\boldsymbol{\theta}, \hat{\hat{\boldsymbol{f}}})$ will have only $\boldsymbol{f}$ profiled. The likelihood for $\mathrm{H} 0$ will have the oscillation parameters at fixed values while those of $\mathrm{H} 1$ will be profiled. As a result, the likelihood of $\mathrm{H} 0$ will always be less than or equal to H1, making the ratio fall between zero and one. Profiling the parameters is a numerical approximation to a full integration over the probability distributions for the parameters.

5.1.1. Wilk's Theorem. The LLR can be used to reject the null hypothesis, H0, if it is above some predetermined value. This value is quantified using Wilk's theorem which states that for large sample sizes the LLR is distributed like a $\chi^{2}$ distribution with the degrees of freedom (DOF) at the number of fixed parameters in $\boldsymbol{\theta}_{\text {test }}$ minus the number of fixed parameters in $\boldsymbol{\theta}_{\text {true }}$. When applicable, this theorem means that the LLR test can be interpreted as a $\chi^{2}$ test and with statistical significance of $\sigma=\sqrt{\chi^{2}}$.

Exceptions to the validity of the theorem occur in the limit of low statistics and when H0 is not $\chi^{2}$ distributed. The simple scenario, assuming $\sigma=\sqrt{\chi^{2}}$, is assumed for the majority of studies in this document. Chapter 8 will discuss these statistical issues in more detail in the context of evaluating the experimental sensitivity to $\mathrm{CP}$ violation and the mass hierarchy.

\subsection{Asimov Data Set}

When computing the future sensitivity of an experiment there is no data, or $N^{o b s}$, with which to compare an expectation, $N^{e x p}$. In this case one can assume a "true" set of oscillation 
and nuisance parameter values, $\boldsymbol{\theta}_{\text {true }}$ and $\boldsymbol{f}_{\text {true }}$, and compute

$$
L_{\text {Asimov }}\left(N^{\text {true }}\left(\boldsymbol{\theta}_{\text {true }}, \boldsymbol{f}_{\text {true }}\right) \mid N_{i}^{\text {exp }}(\boldsymbol{\theta}, \boldsymbol{f})\right)=\prod_{i}^{N} \frac{\left(N_{i}^{\text {exp }}\right)^{N_{i}^{\text {true }}}}{N_{i}^{\text {true }} !} e^{-N_{i}^{\text {exp }}}
$$

The data set, $N^{\text {true }}$, produced in this way is the data set that produces the maximum likelihood when $\boldsymbol{\theta}=\boldsymbol{\theta}_{\text {true }}$, also known as the Asimov data set[45]. The Asimov data set does not take into account the statistical fluctuations that would be expected from repeated experimental measurements, but a statistical fluctuation of the Asimov data set will increase or decrease the LLR of a measurement in $50 \%$ of cases so that the median LLR value is given by computing the LLR using the Asimov data set to replace $N^{o b s}$. Statistical fluctuations will be introduced into the sensitivities computed in Chapter 8 .

This likelihood is propagated in a similar way as above and the LLR becomes

$$
\mathcal{L}\left(\boldsymbol{\theta}_{\text {true }}, \boldsymbol{\theta}\right)=-2 \ln \left[\frac{L\left(N^{\text {true }}\left(\boldsymbol{\theta}_{\text {true }}, \boldsymbol{f}_{\text {true }}\right) \mid N^{\exp }(\boldsymbol{\theta}, \hat{\hat{\boldsymbol{f}}})\right)}{\left.L\left(N^{\text {true }}\left(\boldsymbol{\theta}_{\text {true }}, \boldsymbol{f}_{\text {true }}\right) \mid N^{\exp }(\hat{\boldsymbol{\theta}}, \hat{\boldsymbol{f}})\right)\right)}\right]
$$

but the likelihood in the denominator is equal to one because, by definition, the likelihood is maximized when $\boldsymbol{\theta}=\boldsymbol{\theta}_{\text {true }}$ which will happen during the profiling of $\hat{\boldsymbol{\theta}}$ to maximize the likelihood. Taking this into account and using Stirling's approximation $(\ln (n !) \approx n \ln (n)-n)$ 
the LLR can be simplified to

$$
\begin{aligned}
\mathcal{L}\left(\boldsymbol{\theta}_{\text {true }}, \boldsymbol{\theta}\right) & =-2 \ln \left[\frac{L\left(N^{\text {true }}\left(\boldsymbol{\theta}_{\text {true }}, \boldsymbol{f}_{\text {true }}\right) \mid N^{\text {exp }}(\boldsymbol{\theta}, \hat{\hat{\boldsymbol{f}}})\right)}{1}\right] \\
& =-2 \ln \left[\prod_{i}^{N} \frac{\left(N_{i}^{\text {exp }}\right)^{N_{i}^{\text {true }}}}{\left.N_{i}^{\text {true }} e^{-N_{i}^{\text {exp }}}\right]}\right. \\
& =-2 \sum_{i}^{N}\left[\ln \left[\left(N_{i}^{\text {exp }}\right)^{N_{i}^{\text {true }}} e^{-N_{i}^{\text {exp }}}\right]-\ln \left[N_{i}^{\text {true }} !\right]\right] \\
& \approx 2 \sum_{i}^{N}\left[N_{i}^{\text {exp }}-N_{i}^{\text {true }}+N_{i}^{\text {true }} \ln \left[\frac{N_{i}^{\text {true }}}{N_{i}^{\text {exp }}}\right]\right]
\end{aligned}
$$

The LLR is written only as function of $\boldsymbol{\theta}_{\text {true }}$ and $\boldsymbol{\theta}$ because the nuisance parameters in $\boldsymbol{f}$ are profiled and so do not affect the interpretation of the LLR.

The LLR is typically interpreted as a $\Delta \chi^{2}$, so the equivalence $\mathcal{L} \equiv \Delta \chi^{2}$ will be assumed when using the Asimov data set.

5.2.1. Combining Test Statistics. To combine the results from multiple experiments and samples, the test statistics from multiple experiments can be added. For example, the total test statistic for a combined $\mathrm{T} 2 \mathrm{~K}$ and $\mathrm{NO} \nu \mathrm{A}$ analysis would be,

$$
\begin{aligned}
\Delta \chi_{N O \nu A}^{2} & =\Delta \chi_{\nu_{e}, N O \nu A}^{2}+\Delta \chi_{\bar{\nu}_{e}, N O \nu A}^{2}+\Delta \chi_{\nu_{\mu}, N O \nu A}^{2}+\Delta \chi_{\bar{\nu}_{\mu}, N O \nu A}^{2} \\
\Delta \chi_{T 2 K}^{2} & =\Delta \chi_{\nu_{e}, T 2 K}^{2}+\Delta \chi_{\bar{\nu}_{e}, T 2 K}^{2}+\Delta \chi_{\nu_{\mu}, T 2 K}^{2}+\Delta \chi_{\bar{\nu}_{\mu}, T 2 K}^{2} \\
\Delta \chi_{T 2 K+N O \nu A}^{2} & =\Delta \chi_{T 2 K}^{2}+\Delta \chi_{N O \nu A}^{2}
\end{aligned}
$$

Where $\Delta \chi_{\nu_{e}}^{2}, \Delta \chi_{\bar{\nu}_{e}}^{2}, \Delta \chi_{\nu_{\mu}}^{2}$, and $\Delta \chi_{\bar{\nu}_{\mu}}^{2}$ represent the samples for $\nu_{e}$ appearance, $\bar{\nu}_{e}$ appearance, $\nu_{\mu}$ disappearance, and $\bar{\nu}_{\mu}$ disappearance respectively for the indicated experiment. 


\subsection{Systematic UnCertainties \& Priors}

The systematic uncertainties to be incorporated into the $\Delta \chi^{2}$ fall into two categories. First are the oscillation parameter systematics that alter the underlying physical parameters affecting the neutrino oscillation probabilities. The oscillation parameter systematics allow the oscillation parameters to float within their uncertainties in order to minimize the $\Delta \chi^{2}$. These parameters represent the constraints on the oscillation parameters from global fits as described in Section 3. Table 3.2 gives the $1 \sigma$ uncertainties that make up the constraints on the oscillation parameters.

Second are the non-oscillation parameter systematics that alter the event spectra based on uncertainties in the expected event spectra. These represent uncertainties in the flux, neutrino cross-sections, and detector effects. For the first set of sensitivity studies, to be presented in the following Chapter, a simple model of the uncertainties is adopted which allows the total signal and total background to float with an overall normalization. Chapter 7 will explore more sophisticated approaches of incorporating non-oscillation systematic uncertainties. For the sensitivity studies in this document, the level of uncertainty in the signal and background depends on the experiment and the sample under consideration $\left(\nu_{e}\right.$ appearance, $\nu_{\mu}$ disappearance). The levels assumed are:

\section{- LBNE and T2HK}

- $\nu_{e}$ appearance: $1 \%$ on signal, $5 \%$ on background normalization

- $\nu_{\mu}$ disappearance: $5 \%$ on signal, $10 \%$ on background normalization

\section{- T2K and NO $\nu \mathrm{A}$}

- $\nu_{e}$ appearance: $5 \%$ on signal, $10 \%$ on background normalization

- $\nu_{\mu}$ disappearance: $5 \%$ on signal, $10 \%$ on background normalization 
The systematic parameters are allowed to vary in the minimization of the $\Delta \chi^{2}$. The variation of these systematic parameters is constrained by pull terms that implement a Gaussian constraint on the systematic parameters. The likelihood is multiplied by the probability of a particular fluctuation in each parameter so that the likelihood with the prior included is

$$
\begin{aligned}
L_{\text {Prior }}\left(N_{i}^{\text {obs }} \mid N_{i}^{e x p}(\boldsymbol{\theta}, \boldsymbol{f})\right)= & \prod_{i}^{N} \frac{\left(N_{i}^{e x p}(\boldsymbol{\theta}, \boldsymbol{f})\right)^{N_{i}^{\text {obs }}}}{N_{i}^{\text {obs }} !} e^{-N_{i}^{e x p}(\boldsymbol{\theta}, \boldsymbol{f})} \\
& \times \prod_{j}^{N_{\text {systs }}} \exp \left(-\frac{f_{j}^{2}}{2 \sigma_{f_{j}}^{2}}\right) \\
& \times \prod_{k}^{N_{\text {oscs }}} \exp \left(-\frac{\left(\theta_{k}^{\text {nominal }}-\theta_{k}\right)^{2}}{2 \sigma_{\theta_{k}}^{2}}\right) .
\end{aligned}
$$

Where the first line is the same as above, the second line constrains the non-oscillation systematic parameters, and the third line constrains the oscillation parameters. The nominal value for the $\mathrm{k}^{\text {th }}$ oscillation parameter is represented by $\theta_{k}^{\text {nominal }}$ and is given in Table 3.2 . When these terms for the systematic priors are incorporated into the $\Delta \chi^{2}$ it becomes,

$$
\begin{aligned}
\Delta \chi^{2}= & 2 \sum_{i}^{N}\left[N_{i}^{\text {exp }}(\boldsymbol{\theta}, \boldsymbol{f})-N_{i}^{\text {true }}+N_{i}^{\text {true }} \ln \left[\frac{N_{i}^{\text {true }}}{N_{i}^{\text {exp }}(\boldsymbol{\theta}, \boldsymbol{f})}\right]\right] \\
& +\sum_{j}^{N_{\text {systs }}} \frac{f_{j}^{2}}{\sigma_{f_{j}}^{2}}+\sum_{k}^{N_{\text {oscs }}} \frac{\left(\theta_{k}^{\text {nominal }}-\theta_{k}\right)^{2}}{\sigma_{\theta_{k}}^{2}} .
\end{aligned}
$$

This is the $\Delta \chi^{2}$ that is used for all studies in this document except for those in Chapter 8 , where this profile likelihood method using the Asimov data set will be extended beyond the Asimov data set to include statistical fluctuations.

\subsection{The GLoBES LiBraRY}

Sensitivities are computed using the General Long Baseline Experiment Simulator (GLoBES) [4][5] software library. The library facilitates the use of standardized input formats, 
systematics definitions, oscillation parameter uncertainties, and analysis methods among multiple experiments in order to compute sensitivity to the neutrino oscillation parameters. In particular it defines methods that enable the $\Delta \chi^{2}$ to be computed given this set of inputs.

The C library defines a set of methods allowing for the use of specially formatted inputs, described below, in order to compute the expected event spectra at a specified baseline. Figure 5.1 shows the inputs that go into computing the expected event spectra at the FD. The library does not directly compute the sensitivities, but requires the developer to use the library to compute $\Delta \chi^{2}$ for different $N^{\text {true }}\left(\boldsymbol{\theta}_{\text {true }}\right)$ and $N^{\text {exp }}(\boldsymbol{\theta})$ values as defined above. An explicit example is given below for a T2K sensitivity to measuring $\sin ^{2}\left(2 \theta_{13}\right)$ and $\delta_{C P}$ simultaneously.

The GLoBES library also allows the developer to include custom $\Delta \chi^{2}$ implementations to include the effects systematics beyond what the library has by default. This functionality was used to study detailed systematics implementations that will be discussed in Chapter 7 .

5.4.1. GLoBES Event Spectra. All of the inputs to GLoBES for the sensitivity studies considered in the document come from external Monte Carlo simulations and estimates of detector performance. The sources for the T2K, NO $\nu \mathrm{A}$, LBNE, and T2HK GLoBES inputs were described in Chapter 4.

The GLoBES library computes the event spectra using

$$
N_{i}^{c_{\beta}}(\boldsymbol{\theta})=\sum_{j}^{N_{E_{\text {true }}}} n_{j}^{c_{\alpha}} \cdot P_{j}^{c_{\alpha} \rightarrow c_{\beta}}(\boldsymbol{\theta}) \cdot S_{i j}^{c_{\beta}},
$$

where

- the superscript, $c_{\alpha}$, represents the channel being considered before oscillations and is what is produced in the beam 


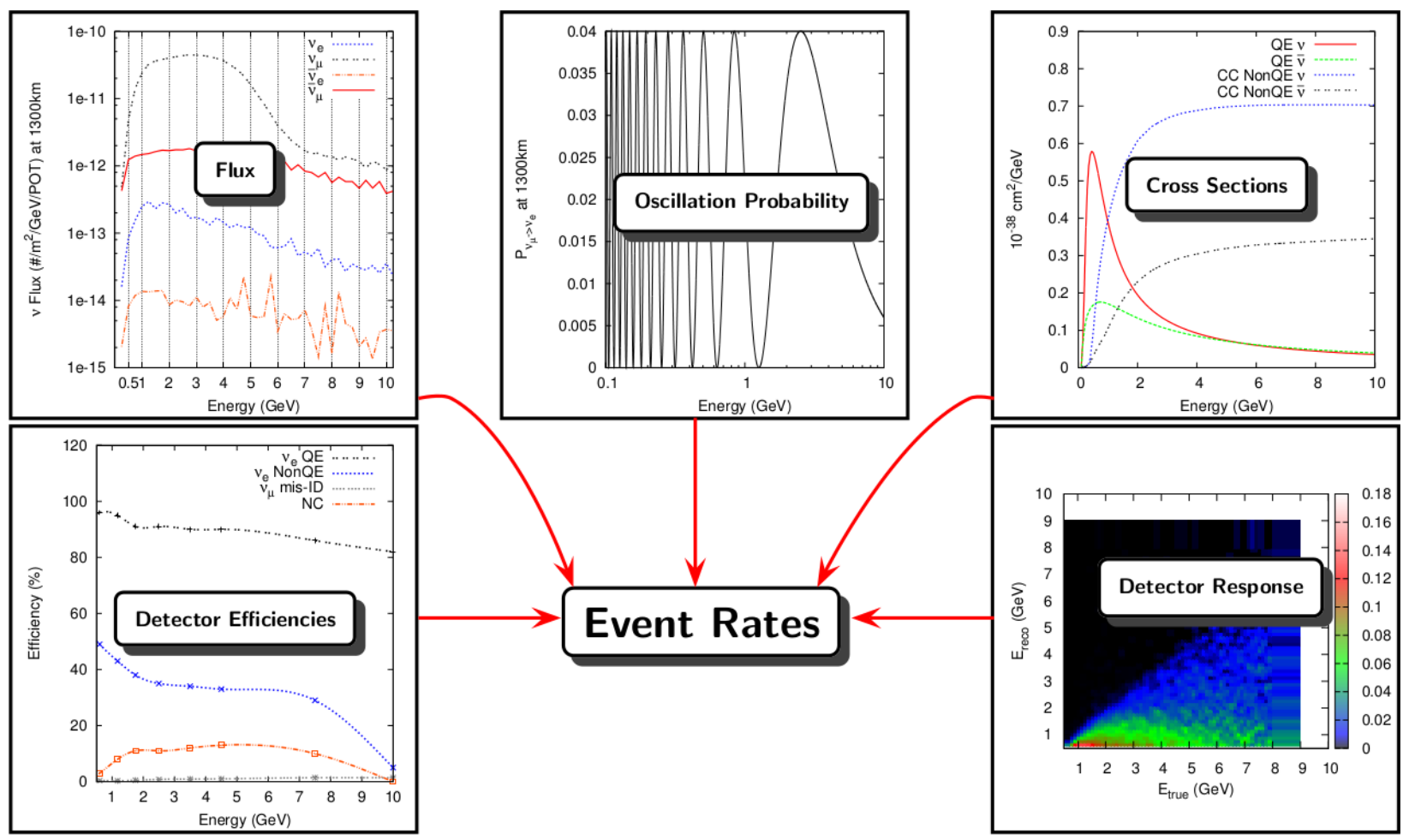

FIGURE 5.1. Schematic representation of the inputs provided to the GLoBES library. The flux, oscillation probability, neutrino interaction cross sections, detector efficiencies and responses are used to generate event rates as a function of energy for a particular experiment.

- the superscript, $c_{\beta}$, represents the channel being considered after oscillation and is what interacts in the FD

- the subscript i represents a reconstructed energy bin

- the subscript j represents a true energy bin

- $N_{E_{\text {true }}}$ is the number of bins in true energy; the binning structure for each of the experiments is depicted in the event spectra in Chapter 4

- $n_{j}^{c_{\alpha}}$ is the expected interaction rate in the far detector for the unoscillated channel $c_{\alpha}$ and includes the neutrino flux, cross sections for the neutrino interactions, and detector efficiencies that are depicted in Figure 5.1 
- $P_{j}^{c_{\alpha} \rightarrow c_{\beta}}(\boldsymbol{\theta})$ is the probability of the appropriate beam flux component, $c_{\alpha}$, oscillating into channel $c_{\beta}$ and is a function of the oscillation parameters including the baseline, true neutrino energy, $\mathrm{j}$, and the matter density and is depicted in Figure 5.1

- $S_{i j}^{c_{\beta}}$ is a matrix that defines the response of the detector to interactions of the type $c_{\beta}$, it maps the true neutrino energy to a PDF of reconstructed neutrino energy and is depicted as the "Detector Response" in Figure 5.1; it is often called a smearing matrix as it smears the true energy out over multiple reconstructed energy bins in the far detector

For example, the primary signal for $\nu_{e}$ appearance is the signal $\nu_{e}$ channel, defined as

$$
N_{i}^{\nu_{e}}(\boldsymbol{\theta})=\sum_{j}^{N_{E_{\text {true }}}} n_{j}^{\nu_{\mu}} \cdot P_{j}^{\nu_{\mu} \rightarrow \nu_{e}}(\boldsymbol{\theta}) \cdot S_{i j}^{\nu_{e}} .
$$

The $N_{i}^{c_{\beta}}(\boldsymbol{\theta})$ is computed for all channels for $\boldsymbol{\theta}_{\text {true }}$ and $\boldsymbol{\theta}$ in order to produce event spectra for $N^{\text {Signal }}$ and $N^{B G}$ as depicted in the event spectra. The complete set of channels and the channels that make up signal and background were defined in Chapter 4 .

5.4.2. GLoBES Sensitivity Example. To illustrate the steps necessary to generate sensitivities using the GLoBES library a set of example sensitivities will be developed here using the T2K GLoBES inputs. As shown in Chapter 3, the oscillation parameters are not all well constrained and have some variability in what the true values might be considered to be. It is useful in evaluating the sensitivity of experiments to compute how well a variable can be constrained by a particular experiment with a well defined set of variables such as exposure, $\nu: \bar{\nu}$ run ratio, systematics, and the set of assumed oscillation parameters. Since $\delta_{C P}$ and $\sin ^{2}\left(2 \theta_{13}\right)$ are not well defined and they are correlated via the $\nu_{e}$ appearance oscillation probability it is informative to evaluate how well an experiment can constrain the 
two variables simultaneously. The $\Delta \chi^{2}$ formula defined above is used to compute the $\Delta \chi^{2}$ at a set of grid points in the $\delta_{C P}, \sin ^{2}\left(2 \theta_{13}\right)$ plane where the vector $\boldsymbol{\theta}_{\text {true }}$ has $\delta_{C P}$ set to 0 and $\sin ^{2}\left(2 \theta_{13}\right)$ set to 0.1 and the vector $\boldsymbol{\theta}$ has $\delta_{C P}$ and $\sin ^{2}\left(2 \theta_{13}\right)$ fixed to the grid point values. The $\Delta \chi^{2}$ is also minimized with respect to the other oscillation parameters, $\sin ^{2}\left(\theta_{23}\right)$ and $\Delta m_{31}^{2}$, and to the non-oscillation systematics that include the effects of normalization uncertainties on the signal and background. These studies do not constrain either $\delta_{C P}$ or $\sin ^{2}\left(2 \theta_{13}\right)$ from external measurements in order to evaluate the experimental ability of T2K alone.

The $\Delta \chi^{2}$ values are interpreted as confidence levels (CL) by taking the square root to get a Gaussian $\sigma$ parameter, $\sigma=\sqrt{\Delta \chi^{2}}$, or the appropriate CL can be interpreted as, for example, a $90 \%$ CL. Since the values of $\delta_{C P}$ and $\sin ^{2}\left(2 \theta_{13}\right)$ are fixed in the minimization, the $\Delta \chi^{2}$ is distributed as a $\chi^{2}$ with 2 DOF and $\Delta \chi^{2}=4.7$ corresponds to the $90 \%$ CL. Contours are drawn at this CL for Figures 5.2, 5.3, 5.4, and 5.5. Values of $\delta_{C P}$ and $\sin ^{2}\left(2 \theta_{13}\right)$ outside of the contours are expected to be excluded at the $90 \%$ CL or higher while values inside the contours are consistent with the true hypothesis $\left(\delta_{C P}=0, \sin ^{2}\left(2 \theta_{13}\right)=0.1\right)$. As the experiment collects more data the regions will become smaller as more of the parameter space is excluded.

Figure 5.2 shows the $90 \%$ projected allowed regions in $\delta_{C P}$ vs $\sin ^{2}\left(2 \theta_{13}\right)$ for true values of $\sin ^{2}\left(2 \theta_{13}\right)=0.1$ and $\delta_{C P}=0$. This includes no systematics but does profile $\sin ^{2}\left(\theta_{23}\right)$ and $\Delta m_{31}^{2}$ as was described in Section 5.1. The nominal ultimate T2K exposure of $7.8 \times 10^{21}$ POT is used. If the true values are $\delta_{C P}=0$ and $\sin ^{2}\left(2 \theta_{13}\right)=0.1$, then $\sin ^{2}\left(2 \theta_{13}\right)$ can be constrained to be between 0.078 and 0.122 (90\% CL). This study also assumes the mass hierarchy is known to be normal and there are no non-oscillation systematic uncertainties included. Disappearance spectra are also fitted to help constrain $\theta_{23}$ and $\Delta m_{32}^{2}$; hence this 
is a joint $\nu_{e}$ appearance and $\nu_{\mu}$ disappearance sensitivity. The curved shape of the contour comes about from the decreased(increased) number of events expected for positive(negative) $\delta_{C P}$ values. The predicted test event spectra become more consistent with the 'true' event spectra for $\delta_{C P}=0$ for larger(smaller) values of $\sin ^{2}\left(2 \theta_{13}\right)$ for positive(negative) $\delta_{C P}$ values. The following plots in this section sequentially apply more assumptions to the study of T2K sensitivity to $\sin ^{2}\left(2 \theta_{13}\right)$ as a function of $\delta_{C P}$.

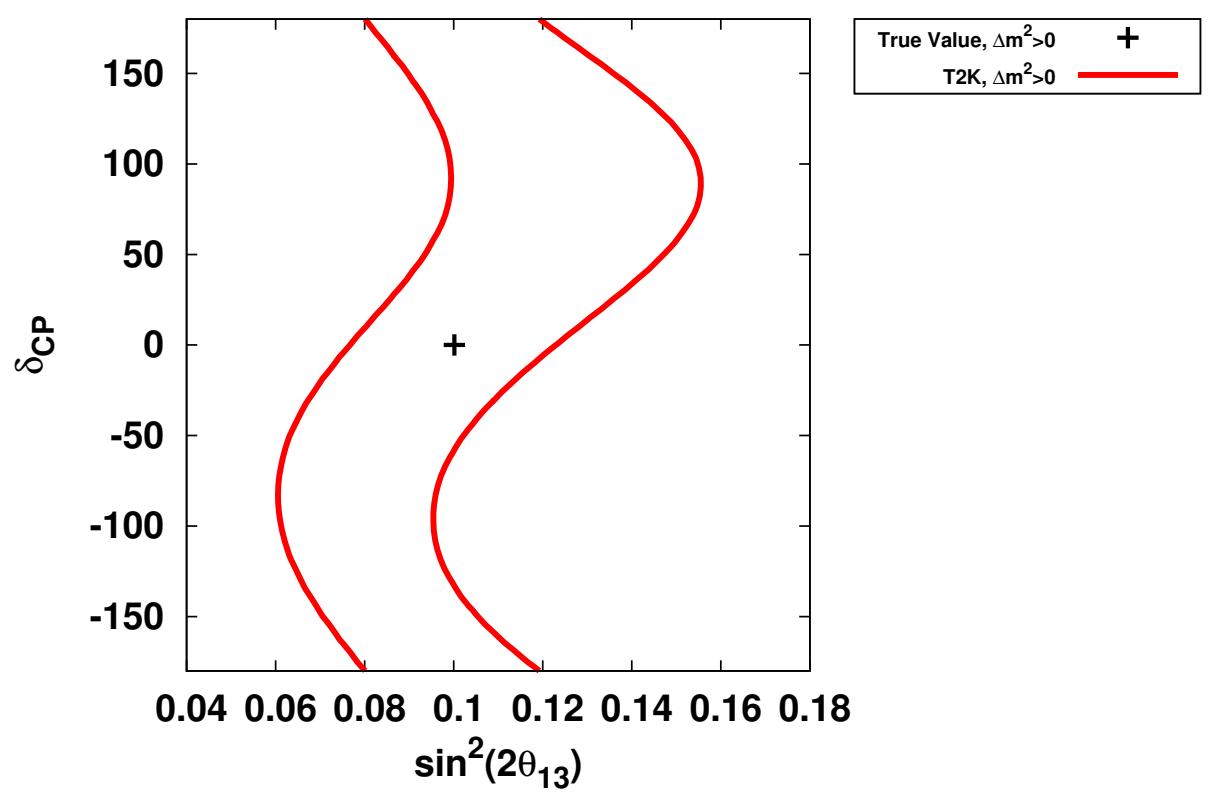

Figure 5.2. T2K 90\% CL allowed regions in $\delta_{C P}$ vs $\sin ^{2}\left(2 \theta_{13}\right)$ for true values of $\sin ^{2}\left(2 \theta_{13}\right)=0.1, \sin ^{2}\left(\theta_{23}\right)=0.5$, and $\delta_{C P}=0$ at $7.8 \times 10^{21}$ POT. The true mass hierarchy is normal and the test spectra is assumed to be normal as well. No systematics are included.

5.4.2.1. Systematics. To estimate non-oscillation systematic uncertainties resulting from simulations of the neutrino fluxes, cross section models and detector effects, a $5 \%$ normalization uncertainty on signal events and a $10 \%$ on normalization uncertainty on background events is adopted for both appearance and disappearance spectra in $\mathrm{T} 2 \mathrm{~K}$, as described in Section 5.3. These are assumed to be uncorrelated for $\nu_{e}$ appearance, $\bar{\nu}_{e}$ appearance, $\nu_{\mu}$ 
disappearance, and $\bar{\nu}_{\mu}$ disappearance which means that there are eight independent systematic parameters for the signal and background normalizations in each sample. Definitions for the signal or background in each sample were given above. Technical details on how the normalization uncertainties contribute to the $\Delta \chi^{2}$ calculation were also described above. Figure 5.3 shows the same contour as Figure 5.2 along with the same contour computed with simple normalization systematics. The increase in the size of the allowed region of the $90 \%$ CL contours is expected because the normalization uncertainties allow for a greater region of the $\sin ^{2}\left(2 \theta_{13}\right)$ vs $\delta_{C P}$ plane to be consistent with the true value for $\sin ^{2}\left(2 \theta_{13}\right)=0.1$ and $\delta_{C P}=0$.

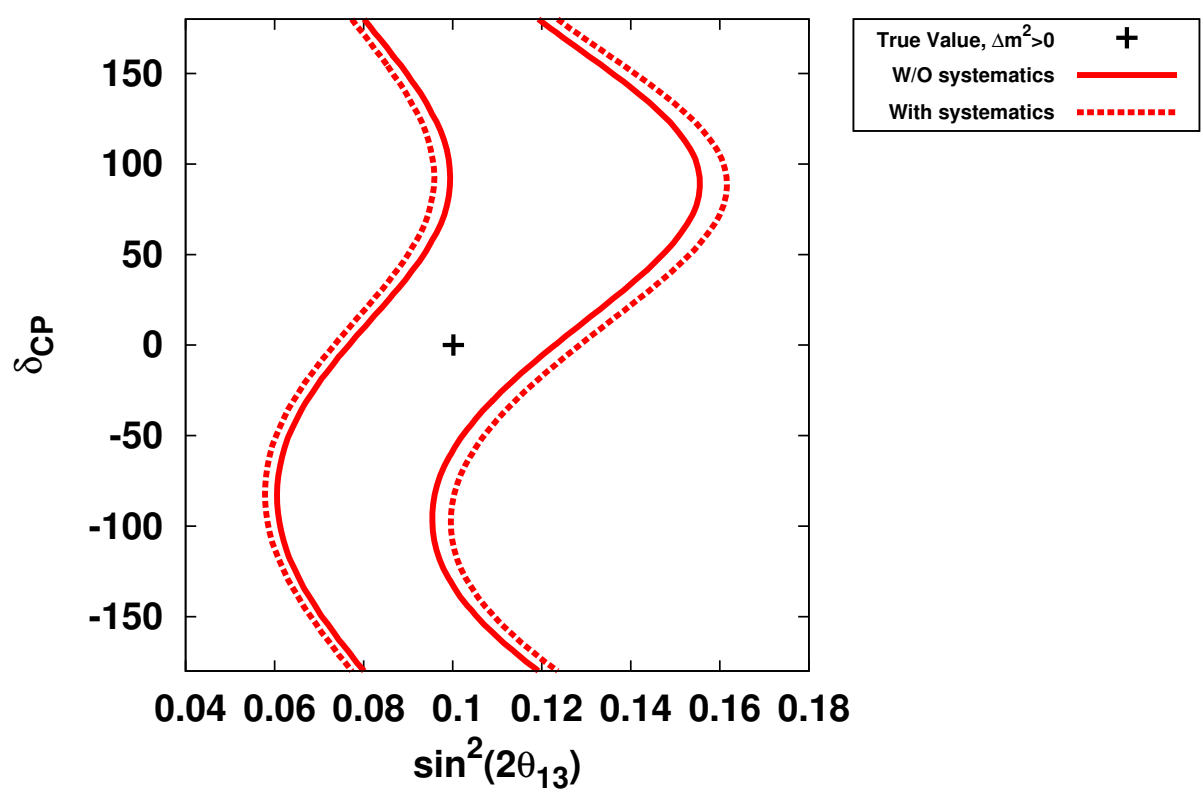

Figure 5.3. T2K 90\% CL allowed regions in $\delta_{C P}$ vs $\sin ^{2}\left(2 \theta_{13}\right)$ for true values of $\sin ^{2}\left(2 \theta_{13}\right)=0.1$ and $\delta_{C P}=0$ at $7.8 \times 10^{21}$ POT. The true mass hierarchy is normal and the test spectra is assumed to be normal as well. A contour with simple systematics (dashed) and without systematics (solid) is shown for comparison. For the simple systematics contours, a 5\% normalization error on signal and $10 \%$ normalization error on background is used. 
5.4.2.2. Mass Hierarchy. Since the true mass hierarchy is not currently known it is necessary to consider multiple test spectra in generating the allowed regions. A mass hierarchy $(\mathrm{MH})$, either normal $(\mathrm{NH})$ or inverted $(\mathrm{IH})$, must be chosen for the true spectra as well as the test spectra. The value of $\Delta m_{31}^{2}$ is different in the inverted hierarchy due to the change in relative positions of the mass splittings. In the inverted hierarchy case it is calculated as

$$
\Delta m_{31, I H}^{2}=-\Delta m_{31, N H}^{2}+\Delta m_{21}^{2} .
$$

The values for each of these terms is given in Table 3.2 .

In Figures 5.2 and 5.3 the $\mathrm{NH}$ was chosen to generate the true spectra and also restrict the test spectra to oscillation hypotheses using only the NH. However, to properly account for the unknown MH it is necessary to check the IH test spectra during the minimization of the $\Delta \chi^{2}$ as well. Figure 5.4 shows both contours. The allowed region for IH is shifted to higher values of $\sin ^{2}\left(2 \theta_{13}\right)$ because the $\nu_{\mu} \rightarrow \nu_{e}$ oscillation probability is lower in the IH relative to the NH. To compensate for this decreased appearance probability in the test spectra the fit will find a higher value of $\sin ^{2}\left(2 \theta_{13}\right)$. A similar set of allowed regions could also be generated for a true IH spectra fitted assuming either $\mathrm{NH}$ or IH for the test spectra. Note that in some cases the MH is not profiled, but separate results are reported for assumptions of either the $\mathrm{NH}$ or IH.

5.4.2.3. $\bar{\nu}$ running. Running in antineutrino mode and performing a combined analysis with $\nu_{e}$ appearance and $\nu_{\mu}$ disappearance in both neutrino and antineutrino modes may help to constrain the oscillation parameters better than just neutrino mode running alone. To study this effect antineutrino mode running is added to the analysis. Figure 5.5 has allowed regions for $100 \% \nu, 100 \% \bar{\nu}$, and $50 \% \nu+50 \% \bar{\nu}$ running. Note that studies where the $\mathrm{MH}$ is unknown choose the lower $\Delta \chi^{2}$ value between the normal and inverted hierarchy 


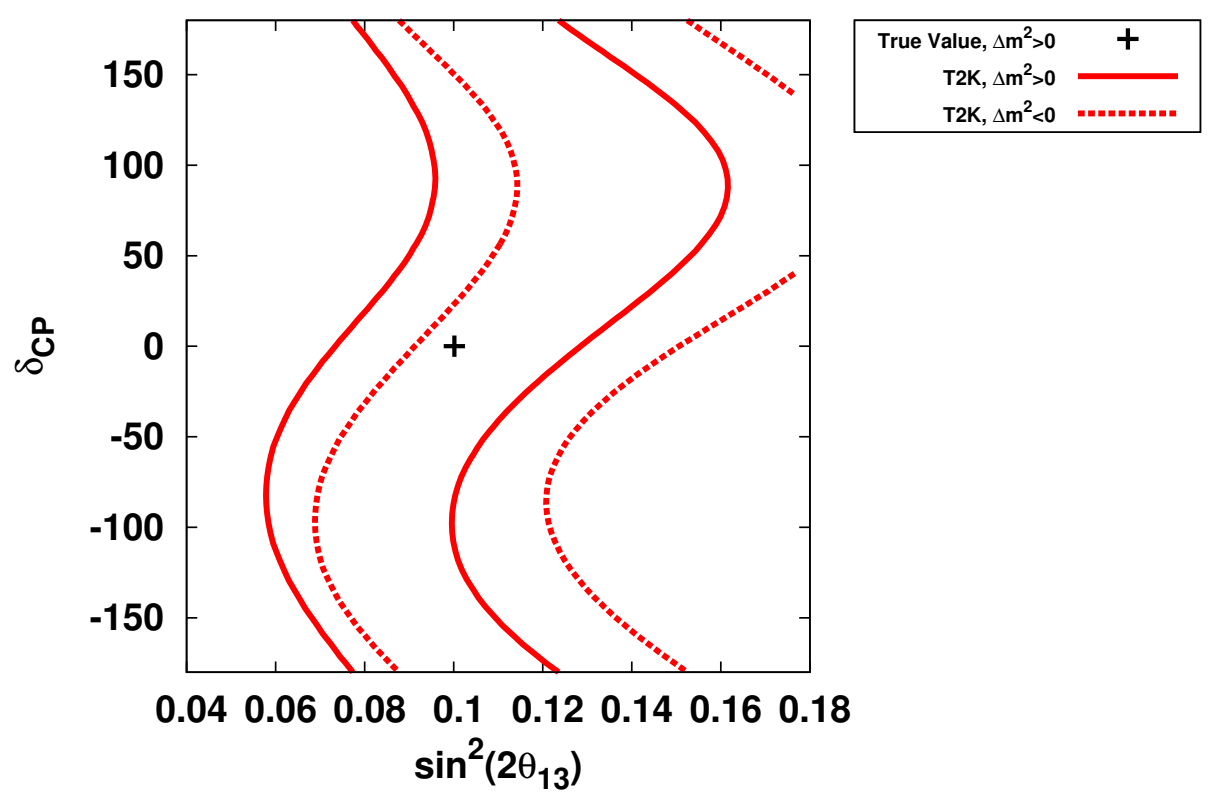

Figure 5.4. T2K 90\% CL allowed regions in $\delta_{C P}$ vs $\sin ^{2}\left(2 \theta_{13}\right)$ for true values of $\sin ^{2}\left(2 \theta_{13}\right)=0.1$ and $\delta_{C P}=0$ at $7.8 \times 10^{21}$ POT. The true mass hierarchy is normal and the test spectra is assumed to be either normal(solid) or inverted hierarchy(dashed). Signal and background normalization systematics are used.

oscillation test hypotheses. This is equivalent to adding the allowed regions that result from the normal and inverted hierarchy test hypotheses. This can be seen by comparing the two outermost curves in Fig. 5.4 with the red curve in Fig. 5.5. The lower(upper) edge of the red contour in Fig. 5.5 corresponds to the $\mathrm{NH}(\mathrm{IH})$ lower(upper) contour in Fig. 5.4. Adding antineutrino running can resolve degeneracies in the $\mathrm{MH}$ for certain regions of the $\sin ^{2}\left(2 \theta_{13}\right)$ vs $\delta_{C P}$ plane. The optimization of the neutrino to antineutrino running ratio $(\nu: \bar{\nu})$ is an interesting problem that will be addressed in a later chapter.

5.4.2.4. External $\theta_{13}$ Constraint. External data can be useful in constraining the allowed parameter space. In this case there is a constraint on $\sin ^{2}\left(2 \theta_{13}\right)$, dominated by the reactor measurements of the $\bar{\nu}_{e} \rightarrow \bar{\nu}_{e}$ survival probability, in the Capozzi et al. 2014 global fit that constrains $\sin ^{2}\left(2 \theta_{13}\right)$ to be $\sin ^{2}\left(2 \theta_{13}\right)=0.092 \pm 0.007$ in the NH and $\sin ^{2}\left(2 \theta_{13}\right)=0.094 \pm 0.007$ in the IH. 


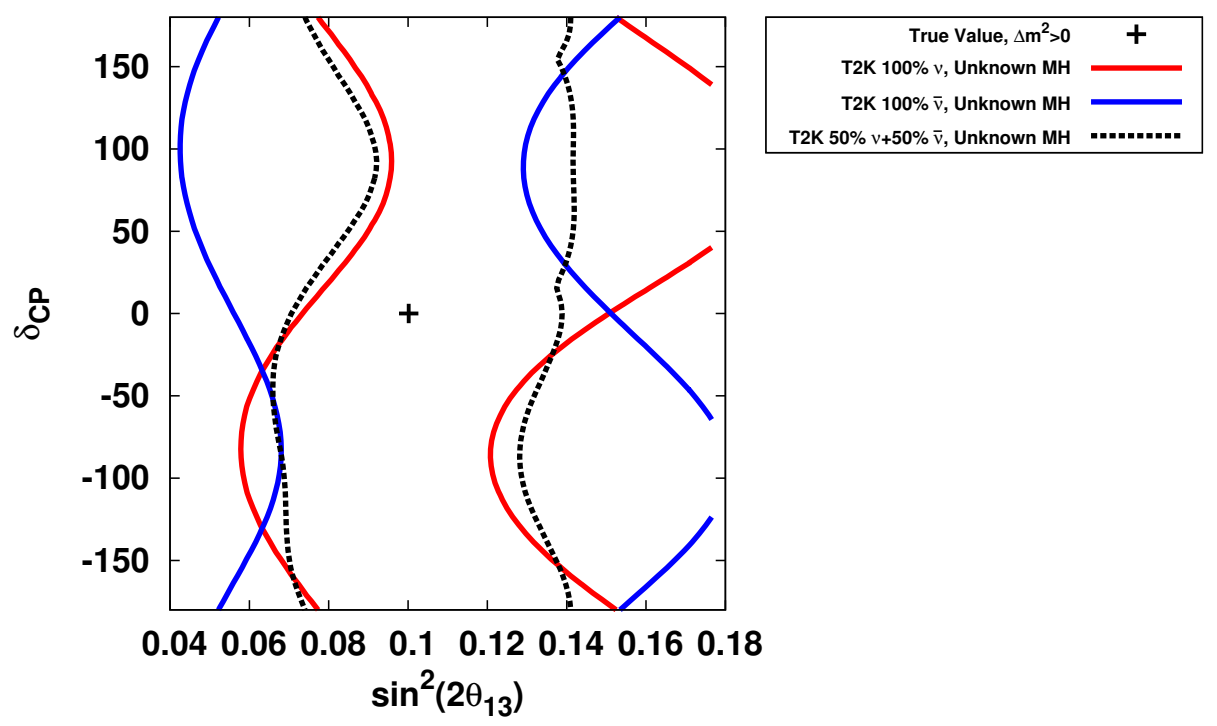

FiguRE 5.5. T2K $90 \% \mathrm{CL}$ allowed regions in $\delta_{C P}$ vs $\sin ^{2}\left(2 \theta_{13}\right)$ for true values of $\sin ^{2}\left(2 \theta_{13}\right)=0.1$ and $\delta_{C P}=0$ at $7.8 \times 10^{21}$ POT. The true mass hierarchy is normal and the test spectra mass hierarchy is unknown (minimum taken between normal and inverted hierarchy $\Delta \chi^{2}$ ). Three variations on $\nu: \bar{\nu}$ run ratios are plotted: $100 \% \nu$ running (red), $100 \% \bar{\nu}$ running (blue), and $50 \% \nu$ $+50 \% \bar{\nu}$ running (black). Signal and background normalization systematics are used.

A penalty term is added to the $\chi^{2}$ formula to include this result in the form

$$
\Delta \chi_{N H}^{2}=\Delta \chi^{2}+\left(\frac{\sin ^{2}\left(2 \theta_{13}\right)-0.092}{0.007}\right)^{2}
$$

in the $\mathrm{NH}$ and similarly in the IH. There is no $\delta_{C P}$ dependence in the $\bar{\nu}_{e} \rightarrow \bar{\nu}_{e}$ survival probability so it provides a strong constraint on $\sin ^{2}\left(2 \theta_{13}\right)$ to be within a vertical region around 0.092 with a $1 \sigma$ width of 0.007 .

Figure 5.6 shows the effects of including this constraint on the $90 \%$ CL allowed regions in $\delta_{C P}$ vs $\sin ^{2}\left(2 \theta_{13}\right)$. The $\mathrm{T} 2 \mathrm{~K}+\mathrm{DB}$ contour (blue) is much smaller than the T2K alone contour and the DB result dominates the constraint on $\sin ^{2}\left(2 \theta_{13}\right)$. Note that this is a different constraint $(0.005)$ applied to a different true value $\left(\sin ^{2}\left(2 \theta_{13}\right)=0.10\right)$, but the same principle will apply for future sections of this thesis. 


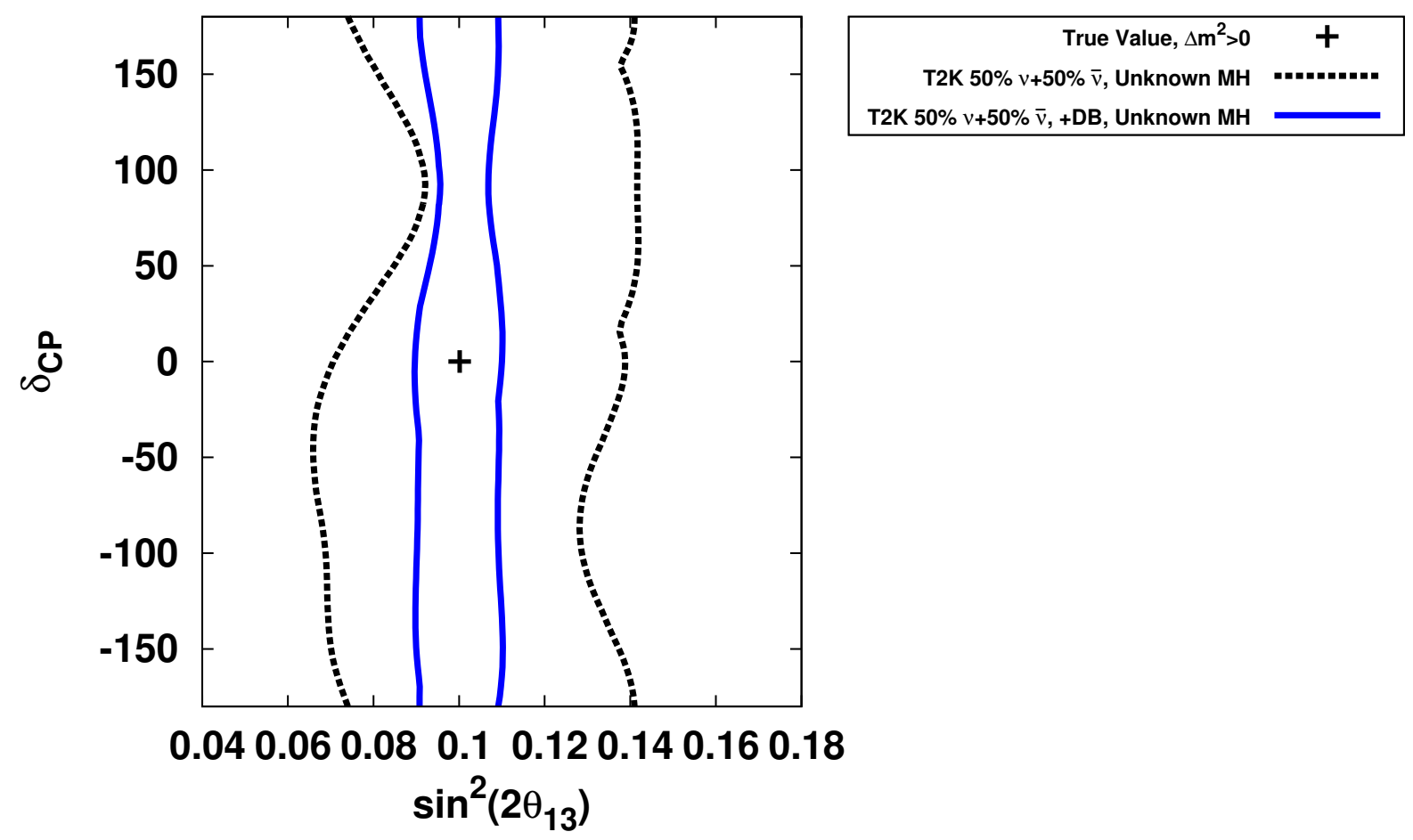

FiguRE 5.6. T2K $90 \%$ CL allowed regions in $\delta_{C P}$ vs $\sin ^{2}\left(2 \theta_{13}\right)$ for true values of $\sin ^{2}\left(2 \theta_{13}\right)=0.1$ and $\delta_{C P}=0$ at $7.8 \times 10^{21}$ POT. The true mass hierarchy is normal and the test spectra mass hierarchy is unknown (minimum taken between normal and inverted hierarchy $\left.\Delta \chi^{2}\right) .50 \% \nu+50 \% \bar{\nu}$ running without (black) and with (blue) the Capozzi et al. $\sin ^{2}\left(2 \theta_{13}\right)$ constraint contours are plotted. Normalization systematics are included.

\subsection{Summary}

This chapter has introduced the LLR and described the statistical interpretation of the LLR as a $\chi^{2}$ distribution. The use of the Asimov data set to describe the median sensitivity of an experiment was introduced and leads to the definition of a $\Delta \chi^{2}$ that incorporates uncertainties in the oscillation parameters and non-oscillation systematics. The GLoBES library is used to take the experimental inputs, primarily from external simulations, and compute the expected event rates and $\Delta \chi^{2}$ comparing a true hypothesis and a test hypothesis. All of the aspects that go into computing these sensitivities will be explored in more detail in the following chapters. 


\section{CHAPTER 6}

\section{Neutrino Oscillation Sensitivities in T2K, NOpA, LBNE, AND T2HK}

The sensitivity to the neutrino oscillation parameters will be explored in this chapter using the methods, inputs, and nominal running conditions outlined in the previous chapters. Systematic uncertainties on signal and background normalizations, $\nu: \bar{\nu}$ running, exposure, and other nominal assumptions made for all of the sensitivities are given in Table 6.1. These parameters will be kept fixed in the chapter unless stated otherwise. Other variations in these parameters will be explored in subsequent chapters.

The sensitivities will be presented in two different categories. First are sensitivities that determine how well the oscillation parameters can be measured, both alone and when correlations among the parameters are considered. Second are sensitivities that check a hypothesis while profiling all of the oscillation parameters in order to evaluate the experiments ability to detect $\mathrm{CP}$ violation, determine the $\mathrm{MH}$, and determine the octant.

These sensitivities will be for the full proposed exposure in $\mathrm{T} 2 \mathrm{~K}, \mathrm{NO} \nu \mathrm{A}, \mathrm{LBNE}$, and T2HK. Where possible a combination of the two current running experiments, T2K and $\mathrm{NO} \nu \mathrm{A}$, will be included. This combination gives an idea of where the constraints on the oscillation parameters from future global fits will be when the future experiments LBNE and T2HK are getting started.

\subsection{Event Spectra for Oscillation Parameter Variations}

To make a connection between the event spectra shown for each experiment in Chapter 4 and the sensitivities to the oscillation parameters, this section will present variations of the 
TABLE 6.1. Table of nominal running parameters for the four experiments.

\begin{tabular}{|c|c|c|c|c|}
\hline $\begin{array}{l}\text { Experiment } \\
\text { (sample) }\end{array}$ & $\begin{array}{c}\text { Signal } \\
\text { Uncertainty }\end{array}$ & $\begin{array}{l}\text { Background } \\
\text { Uncertainty }\end{array}$ & $\nu: \bar{\nu}$ & $\begin{array}{l}\text { Exposure } \\
\text { (POT) }\end{array}$ \\
\hline $\begin{array}{l}\mathbf{T} 2 \mathbf{K} \\
\nu_{e} \text { appearance } \\
\nu_{\mu} \text { disappearance }\end{array}$ & $\begin{array}{l}5 \% \\
5 \% \\
\end{array}$ & $\begin{array}{l}10 \% \\
10 \% \\
\end{array}$ & $1: 1$ & $7.8 \times 10^{21}$ \\
\hline $\begin{array}{l}\mathbf{N O} \nu \mathbf{A} \\
\nu_{e} \text { appearance } \\
\nu_{\mu} \text { disappearance }\end{array}$ & $\begin{array}{l}5 \% \\
5 \%\end{array}$ & $\begin{array}{l}10 \% \\
10 \%\end{array}$ & $1: 1$ & $3.6 \times 10^{21}$ \\
\hline $\begin{array}{l}\text { LBNE } \\
\nu_{e} \text { appearance } \\
\nu_{\mu} \text { disappearance }\end{array}$ & $\begin{array}{l}1 \% \\
5 \% \\
\end{array}$ & $\begin{array}{c}5 \% \\
10 \% \\
\end{array}$ & $1: 1$ & $9 \times 10^{21}$ \\
\hline $\begin{array}{l}\text { T2HK } \\
\nu_{e} \text { appearance } \\
\nu_{\mu} \text { disappearance }\end{array}$ & $\begin{array}{l}1 \% \\
5 \%\end{array}$ & $\begin{array}{l}5 \% \\
10 \%\end{array}$ & $3: 1$ & $15.6 \times 10^{21}$ \\
\hline
\end{tabular}

event spectra that come about from varying the oscillation parameters. Each set of spectra will give the nominal event spectrum overlaid with $\pm 3 \sigma$ variations in $\sin ^{2}\left(2 \theta_{13}\right), \sin ^{2}\left(\theta_{23}\right)$, $\delta_{C P}$, and $\Delta m_{31}^{2}$. These $3 \sigma$ variations for $\sin ^{2}\left(2 \theta_{13}\right), \sin ^{2}\left(\theta_{23}\right)$, and $\Delta m_{31}^{2}$ are taken from the Capozzi et al. 2014 global fit given in Table 3.2. The figures are given for the NH assumption only. The IH versions of the figures are given in Appendix B.

6.1.1. $\theta_{13}$ VARiations. Figures 6.1 through 6.4 show the response in the event spectra for the four experiments in the four samples for $\pm 3 \sigma$ variations in $\theta_{13}$. The red histograms show the signal for the indicated nominal $\theta_{13}$ value with a solid line and variations in $\theta_{13}$ with dashed lines. The solid red histogram also has error bars that indicate the statistical error in each bin. Comparing the statistical errors to the size of the variation that comes from varying $\theta_{13}$ visually indicates how well the experiment can constrain $\theta_{13}$. The blue histogram shows the total backgrounds as described in Chapter 4. Changes in the background are small and so the background is considered constant with respect to changes in $\theta_{13}$. The responses to $\theta_{13}$ variations in the $\nu_{e}$ appearance and $\bar{\nu}_{e}$ appearance spectra are very large as the leading term in the $\nu_{\mu} \rightarrow \nu_{e}$ oscillation probability is directly proportional to $\sin ^{2}\left(2 \theta_{13}\right)$. There is 
very little response to $\theta_{13}$ variations in $\nu_{\mu}$ disappearance and $\bar{\nu}_{\mu}$ disappearance as might be expected since $\theta_{13}$ appears only in sub-leading terms in the $\nu_{\mu} \rightarrow \nu_{\mu}$ survival probability. The sensitivity to $\theta_{13}$ then largely comes from $\nu_{e}$ appearance and $\bar{\nu}_{e}$ appearance, but when the uncertainties in the other oscillation parameters are taken into account including $\nu_{\mu}$ disappearance and $\bar{\nu}_{\mu}$ disappearance in the $\Delta \chi^{2}$ is crucial to achieving the best possible measurement on $\theta_{13}$, largely by constraining $\theta_{23}$.

For T2K, Figure 6.1 shows that the expected signal increases(decreases) by $25 \%(23 \%)$ for a $+(-) 3 \sigma$ change in $\theta_{13}$ in $\nu_{e}$ appearance and 26\%(22\%) in $\nu_{e}$ appearance. While the $\nu_{\mu}$ disappearance and $\bar{\nu}_{\mu}$ disappearance integrated number of events change by less than $1 \%$. Similar changes are seen in the figures for $\mathrm{NO} \nu \mathrm{A}, \mathrm{LBNE}$, and $\mathrm{T} 2 \mathrm{HK}$. 


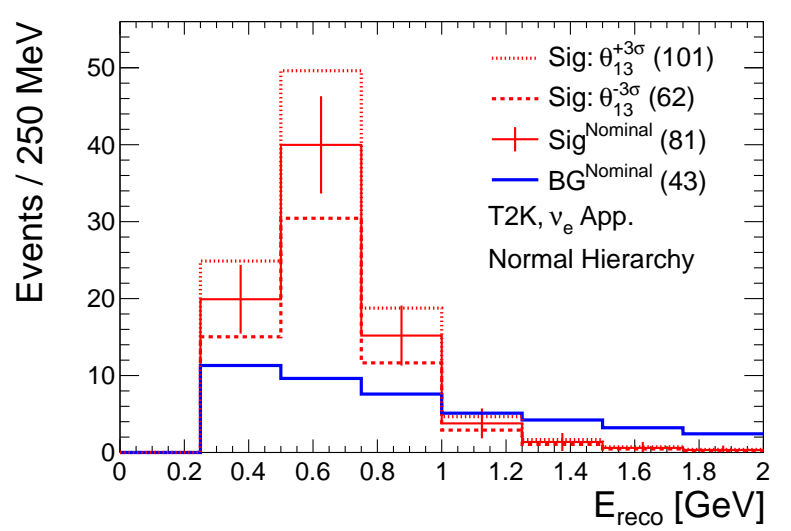

(A) $\nu_{e}$ appearance, True $\mathrm{MH}=\mathrm{NH}$

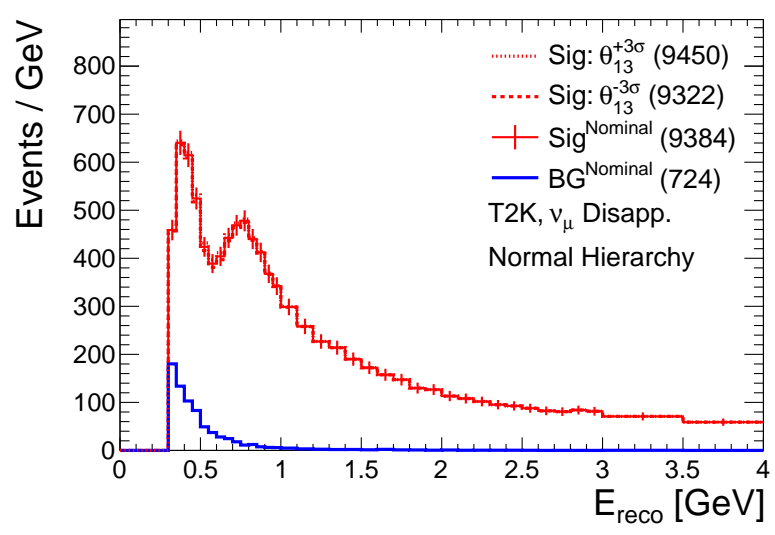

(C) $\nu_{\mu}$ disappearance, True $\mathrm{MH}=\mathrm{NH}$

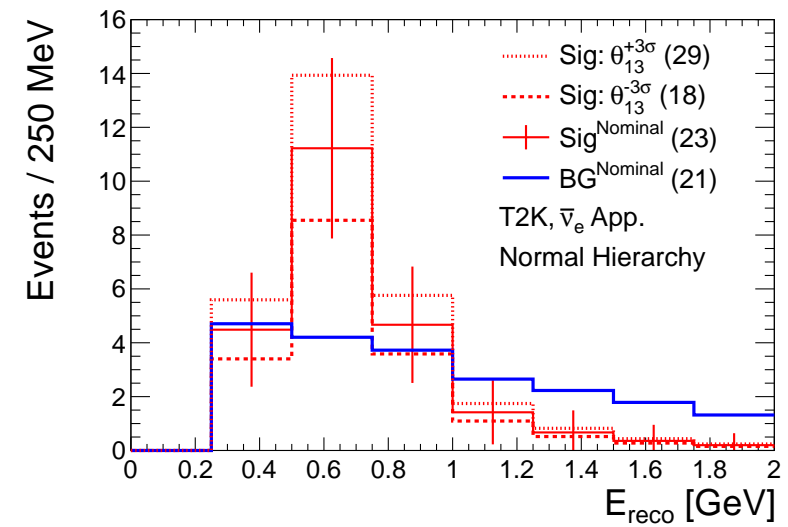

(B) $\bar{\nu}_{e}$ appearance, True $\mathrm{MH}=\mathrm{NH}$

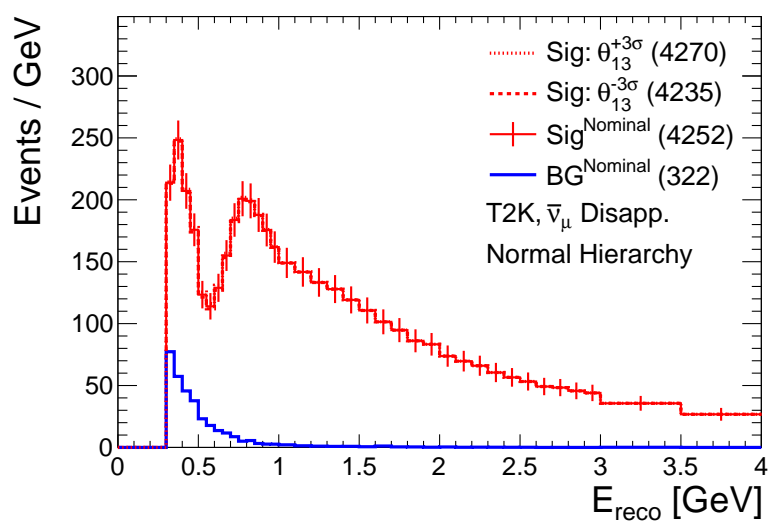

(D) $\bar{\nu}_{\mu}$ disappearance, True $\mathrm{MH}=\mathrm{NH}$

FigurE 6.1. T2K event spectra for $\pm 3 \sigma$ variations in $\theta_{13}$ for $\nu_{e}$ appearance (top left), $\bar{\nu}_{e}$ appearance (top right), $\nu_{\mu}$ disappearance (bottom left), and $\bar{\nu}_{\mu}$ disappearance (bottom right). The histograms are overlaid and not stacked. The nominal $7.8 \times 10^{21}$ POT with $1: 1 \nu: \bar{\nu}$ ratio is assumed. The integrated numbers of events are given in parentheses in the legend. 


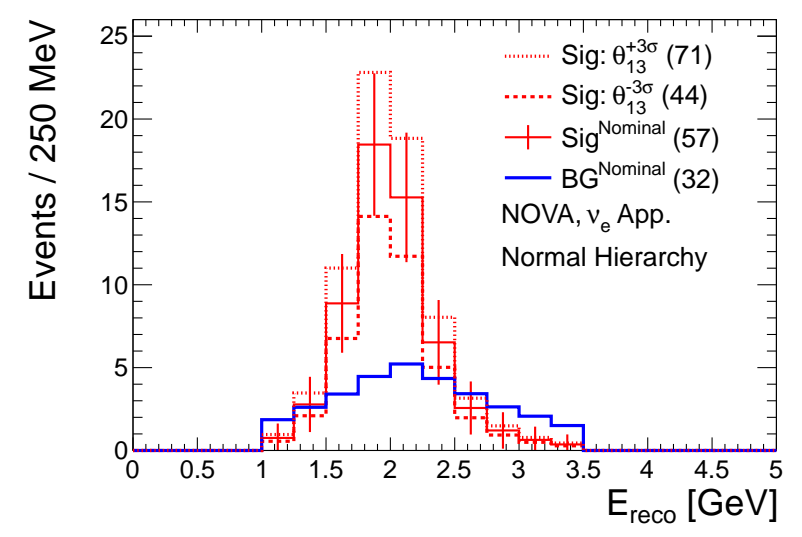

(A) $\nu_{e}$ appearance, True $\mathrm{MH}=\mathrm{NH}$

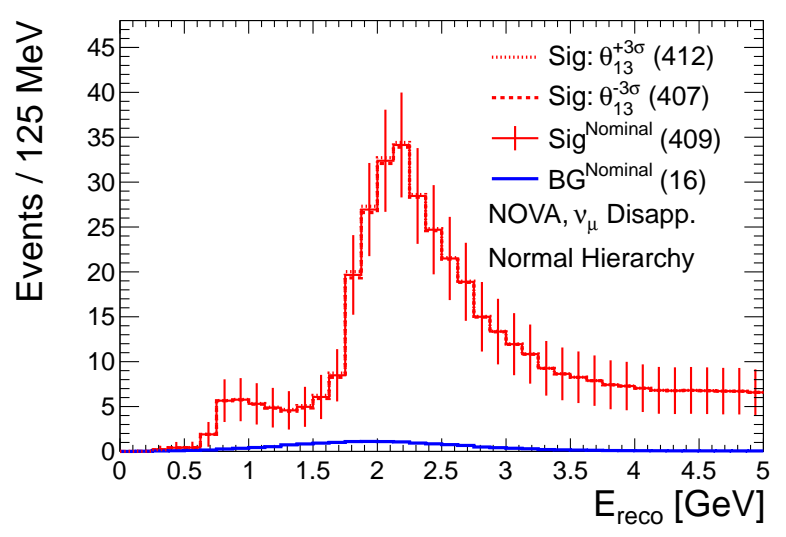

(C) $\nu_{\mu}$ disappearance, True $\mathrm{MH}=\mathrm{NH}$

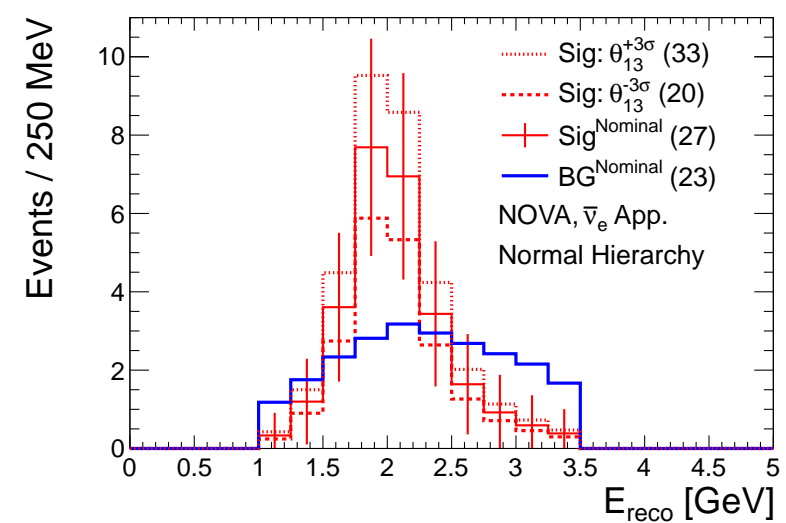

(B) $\bar{\nu}_{e}$ appearance, True $\mathrm{MH}=\mathrm{NH}$

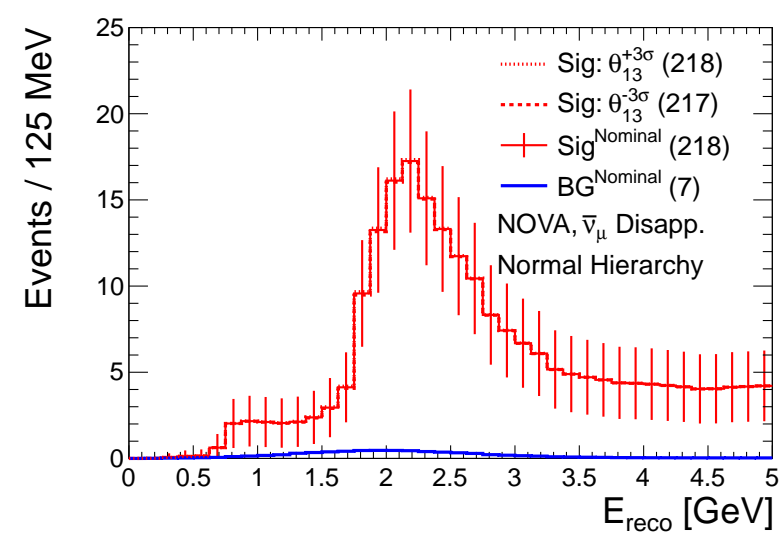

(D) $\bar{\nu}_{\mu}$ disappearance, True $\mathrm{MH}=\mathrm{NH}$

FIGURE 6.2. NO $\nu \mathrm{A}$ event spectra for $\pm 3 \sigma$ variations in $\theta_{13}$ for $\nu_{e}$ appearance (top left), $\bar{\nu}_{e}$ appearance (top right), $\nu_{\mu}$ disappearance (bottom left), and $\bar{\nu}_{\mu}$ disappearance (bottom right). The histograms are overlaid and not stacked. The nominal $3.6 \times 10^{21}$ POT with $1: 1 \nu: \bar{\nu}$ ratio is assumed. The integrated numbers of events are given in parentheses in the legend. 


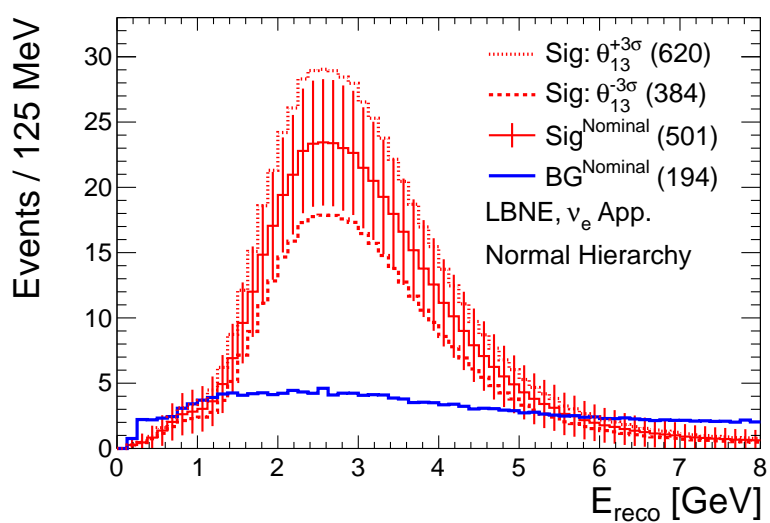

(A) $\nu_{e}$ appearance, True $\mathrm{MH}=\mathrm{NH}$

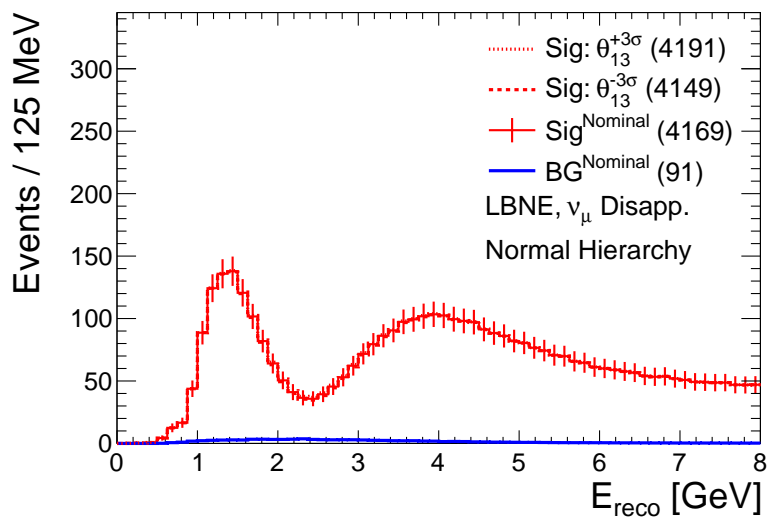

(C) $\nu_{\mu}$ disappearance, True $\mathrm{MH}=\mathrm{NH}$

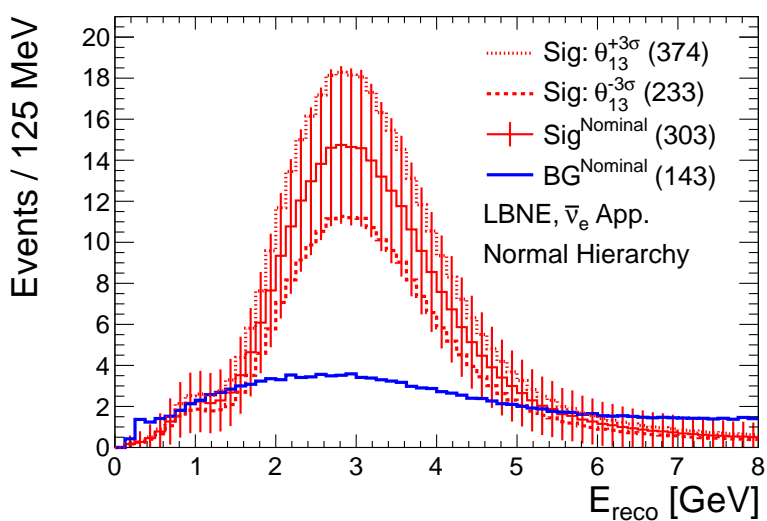

(B) $\bar{\nu}_{e}$ appearance, True $\mathrm{MH}=\mathrm{NH}$

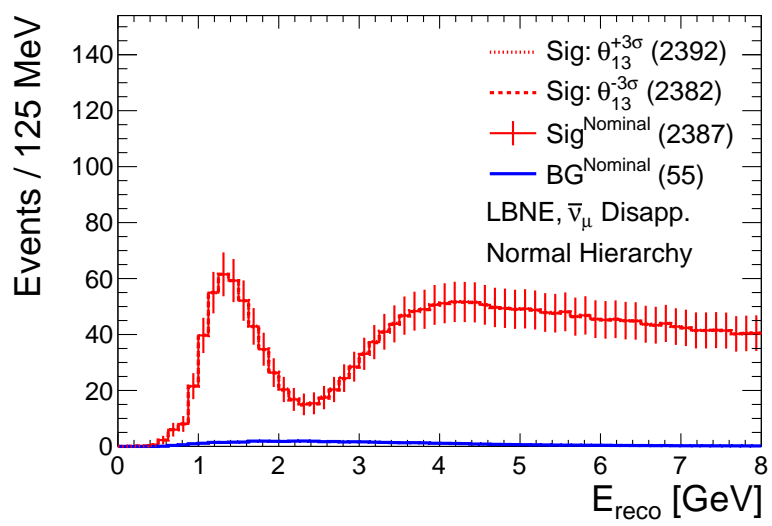

(D) $\bar{\nu}_{\mu}$ disappearance, True $\mathrm{MH}=\mathrm{NH}$

FiguRE 6.3. LBNE event spectra for $\pm 3 \sigma$ variations in $\theta_{13}$ for $\nu_{e}$ appearance (top left), $\bar{\nu}_{e}$ appearance (top right), $\nu_{\mu}$ disappearance (bottom left), and $\bar{\nu}_{\mu}$ disappearance (bottom right). The histograms are overlaid and not stacked. The nominal $6.0 \times 10^{21}$ POT with 1:1 $\nu: \bar{\nu}$ ratio is assumed. The integrated numbers of events are given in parentheses in the legend. 


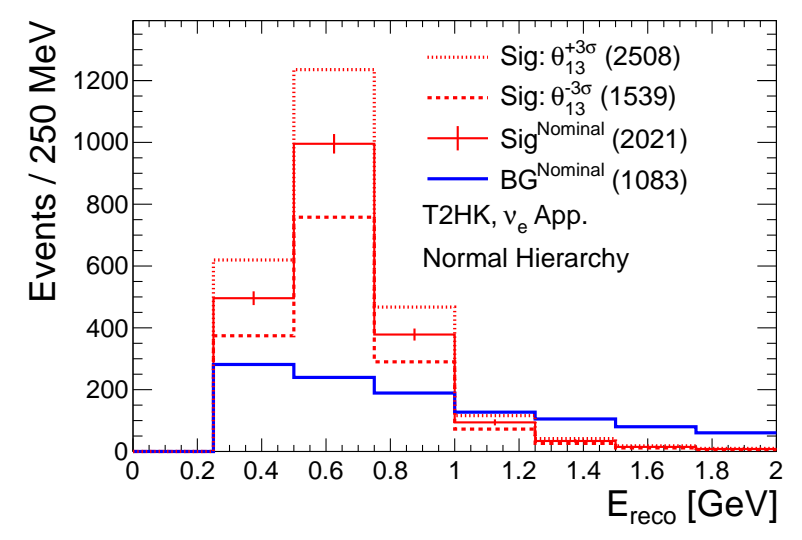

(A) $\nu_{e}$ appearance, True $\mathrm{MH}=\mathrm{NH}$

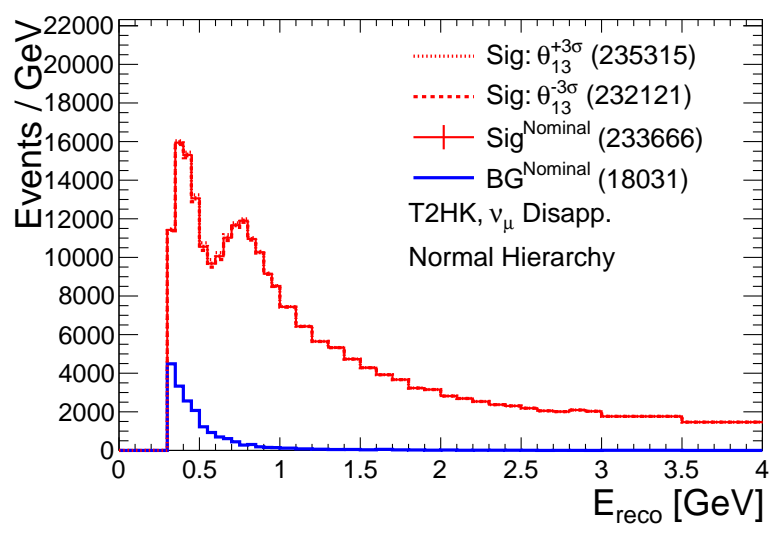

(C) $\nu_{\mu}$ disappearance, True $\mathrm{MH}=\mathrm{NH}$

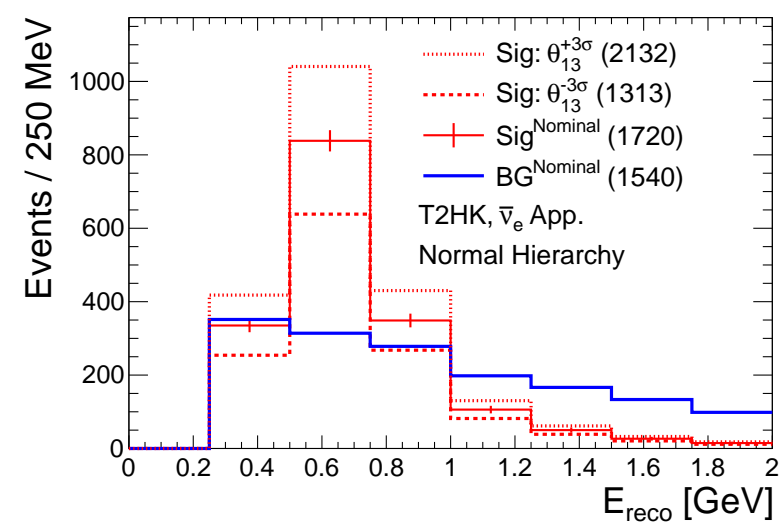

(B) $\bar{\nu}_{e}$ appearance, True $\mathrm{MH}=\mathrm{NH}$

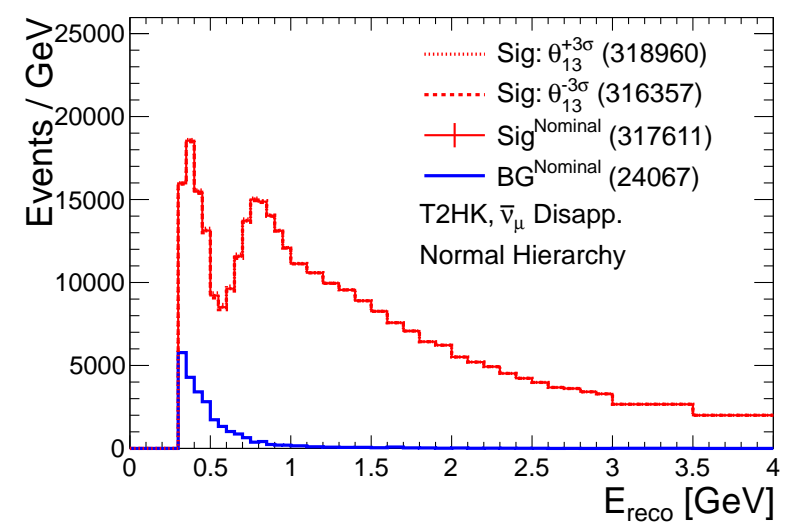

(D) $\bar{\nu}_{\mu}$ disappearance, True $\mathrm{MH}=\mathrm{NH}$

FIGURE 6.4. T2HK event spectra for $\pm 3 \sigma$ variations in $\theta_{13}$ for $\nu_{e}$ appearance (top left), $\bar{\nu}_{e}$ appearance (top right), $\nu_{\mu}$ disappearance (bottom left), and $\bar{\nu}_{\mu}$ disappearance (bottom right). The histograms are overlaid and not stacked. The nominal $15.6 \times 10^{21} \mathrm{POT}$ with $1: 1 \nu: \bar{\nu}$ ratio is assumed. The integrated numbers of events are given in parentheses in the legend. 
6.1.2. $\theta_{23}$ VARIATIONS. Figures 6.5 through 6.8 show the response in the event spectra for the four experiments in the four samples for $\pm 3 \sigma$ variations in $\theta_{23}$. In contrast with $\theta_{13}$, $\theta_{23}$ variations have an effect on both the appearance and disappearance event spectra. For $\nu_{e}$ appearance and $\bar{\nu}_{e}$ appearance the $\theta_{23}$ variations have a similar effect as the $\theta_{13}$ variations because the amplitude of the leading order term for the $\nu_{\mu} \rightarrow \nu_{e}$ oscillation probability is proportional to $\sin ^{2}\left(\theta_{23}\right) \sin ^{2}\left(2 \theta_{13}\right)$. For $\nu_{\mu}$ disappearance and $\bar{\nu}_{\mu}$ disappearance the $\theta_{23}$ variations determine the amplitude of the $\nu_{\mu} \rightarrow \nu_{\mu}$ oscillation probability via a factor of $\sin ^{2}\left(2 \theta_{23}\right)$.

There is some confusion that may arise from the difference between when $\sin ^{2}\left(\theta_{23}\right)$ or $\sin ^{2}\left(2 \theta_{23}\right)$ is used to express the observable parameter. In a two flavor $\nu_{\mu}$ disappearance oscillation probability, as discussed in Section 2.1.2, the probability is expressed as a function of $\sin ^{2}\left(2 \theta_{23}\right)$, and so $\sin ^{2}\left(2 \theta_{23}\right)$ is used for a two-flavor $\nu_{\mu}$ disappearance oscillation analysis. Treated in this way, there is no octant effect as there is no difference in the oscillation probability between the two octants. A full three-flavor $\nu_{\mu}$ disappearance oscillation probability, as given in Equation 17, is a function of $\sin ^{2}\left(\theta_{23}\right)$, as is the $\nu_{e}$ appearance probability. If a joint fit, $\nu_{e}$ appearance and $\nu_{\mu}$ disappearance, is performed for a three-flavor fit then $\sin ^{2}\left(\theta_{23}\right)$ is used and the fit will be sensitive to the octant. In early neutrino oscillation experiments, there was little sensitivity to even the leading order oscillation terms so the approximation provided by the two flavor disappearance probability was sufficient to model the effect of oscillations in those experiments. With the higher statistics, relative to earlier experiments, expected in current and future LBL experiments the full three flavor oscillation effects, including matter effects, can be observed and a new era of precision neutrino oscillation measurements is beginning. 
It is worth pausing here to consider the difference between maximal mixing and maximal disappearance since the distinction comes about via the interplay of appearance and disappearance oscillation probabilities in a three flavor fit. Maximal mixing means that $\theta_{23}=\pi / 4$ or $\sin ^{2}\left(\theta_{23}\right)=0.5$ and this means that there is maximal $\nu_{\mu}-\nu_{\tau}$ mixing. Maximal disappearance is when the disappearance oscillation probability amplitude, $\cos ^{2}\left(\theta_{13}\right) \sin ^{2}\left(2 \theta_{13}\right)$, is equal to zero. For the Capozzi et al. global fit value of $\sin ^{2}\left(2 \theta_{13}\right)=0.092$ in the NH the maximal disappearance probability occurs when $\sin ^{2}\left(\theta_{23}\right)=0.5 / \cos ^{2}\left(\theta_{13}\right)=0.51$. If there is maximal disappearance, then there is a particular $\mathrm{L} / \mathrm{E}$ where there is expected to be no $\nu_{\mu} \rightarrow \nu_{\mu}$ events. In a measurement the events are binned in energy bins that contain a range of energies so that the expectation will not be zero in any one bin in the maximal disappearance case.

A joint fit that includes both appearance and disappearance is critical to constraining $\theta_{23}$ and $\theta_{13}$ simultaneously since the two parameters have very similar effects in $\nu_{e}$ appearance and $\bar{\nu}_{e}$ appearance but very different effects in $\nu_{\mu}$ disappearance and $\bar{\nu}_{\mu}$ disappearance, as can be seen by comparing Figures 6.5 through 6.8 to Figures 6.1 through 6.4. For example, in LBNE the $+3 \sigma \theta_{13}$ change changes the expectation by $24 \%$ in the $\nu_{e}$ appearance sample (Figure 6.3A). The $+3 \sigma \theta_{23}$ also changes the $\nu_{e}$ appearance sample by $44 \%$ (Figure 6.7A). In the $\nu_{\mu}$ disappearance samples, however, the $+3 \sigma \theta_{13}$ variation changes the total number of events by only $0.5 \%$ while the $+3 \sigma \theta_{23}$ variation changes it by $3 \%$. The differences between the responses in the samples are where the increases sensitivity comes from for a combined $\nu_{e}$ appearance and $\nu_{\mu}$ disappearance analysis by further constraining the oscillation parameters. 


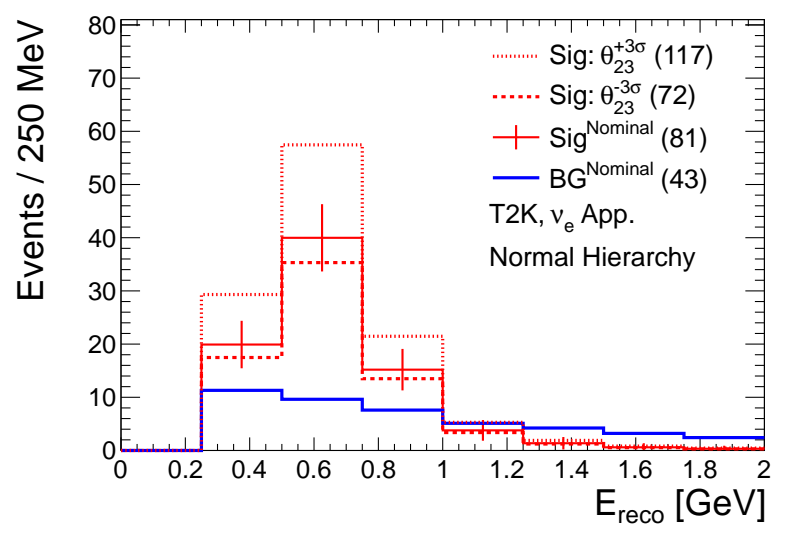

(A) $\nu_{e}$ appearance, True $\mathrm{MH}=\mathrm{NH}$

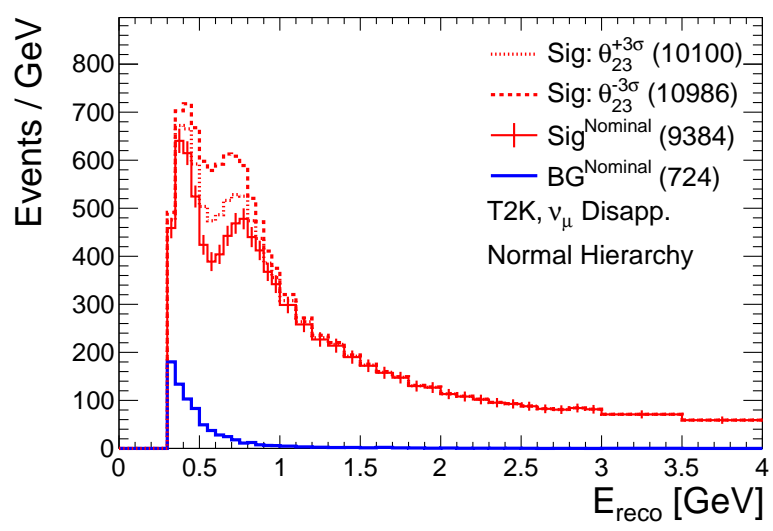

(C) $\nu_{\mu}$ disappearance, True $\mathrm{MH}=\mathrm{NH}$

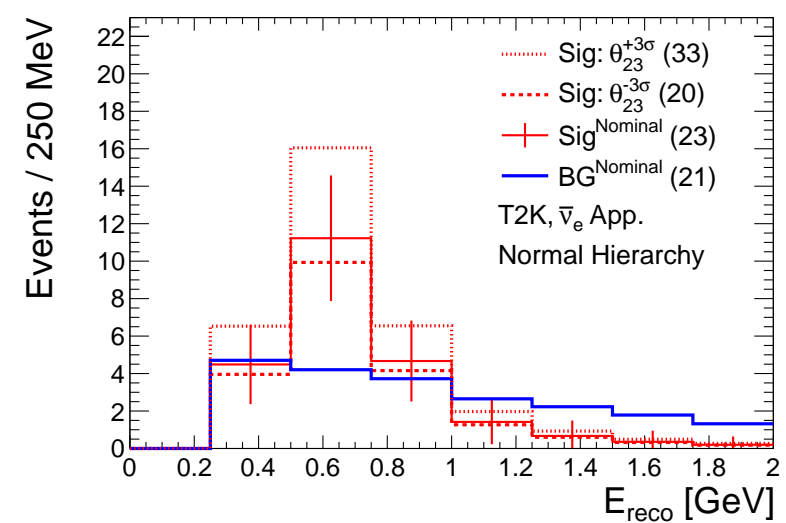

(B) $\bar{\nu}_{e}$ appearance, True $\mathrm{MH}=\mathrm{NH}$

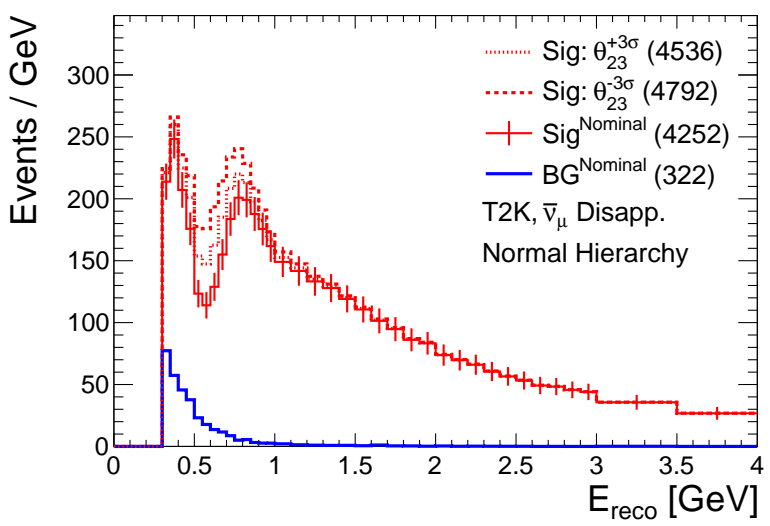

(D) $\bar{\nu}_{\mu}$ disappearance, True $\mathrm{MH}=\mathrm{NH}$

FigurE 6.5. T2K event spectra for $\pm 3 \sigma$ variations in $\theta_{23}$ for $\nu_{e}$ appearance (top left), $\bar{\nu}_{e}$ appearance (top right), $\nu_{\mu}$ disappearance (bottom left), and $\bar{\nu}_{\mu}$ disappearance (bottom right). The histograms are overlaid and not stacked. The nominal $7.8 \times 10^{21}$ POT with $1: 1 \nu: \bar{\nu}$ ratio is assumed. The integrated numbers of events are given in parentheses in the legend. 


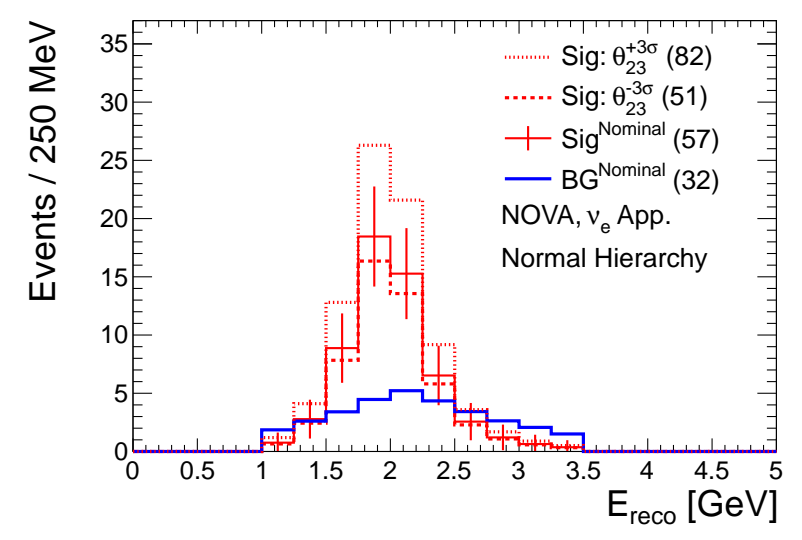

(A) $\nu_{e}$ appearance, True $\mathrm{MH}=\mathrm{NH}$

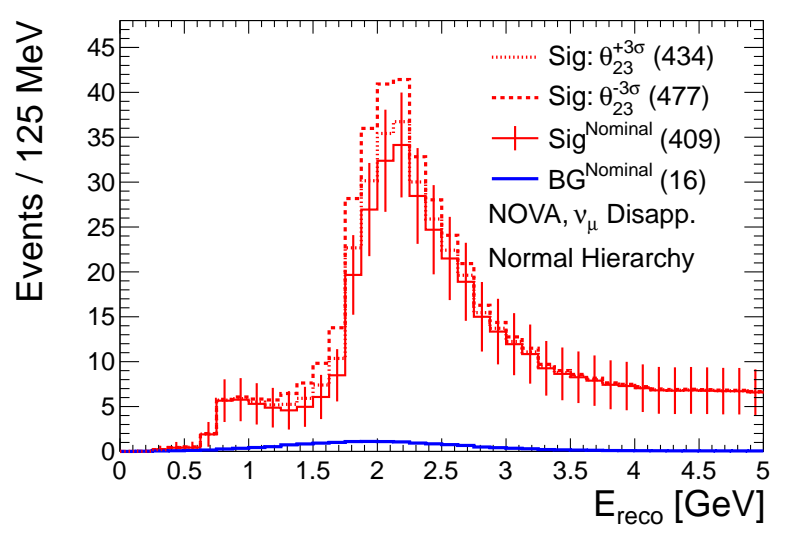

(C) $\nu_{\mu}$ disappearance, True $\mathrm{MH}=\mathrm{NH}$

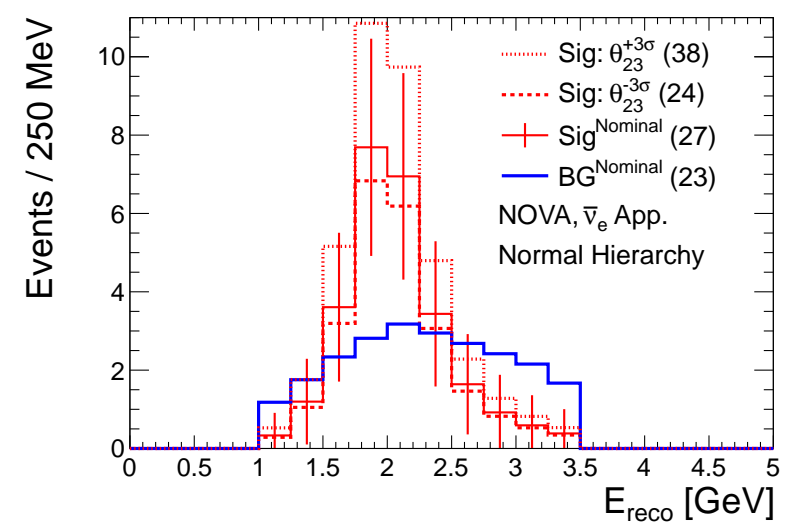

(B) $\bar{\nu}_{e}$ appearance, True $\mathrm{MH}=\mathrm{NH}$

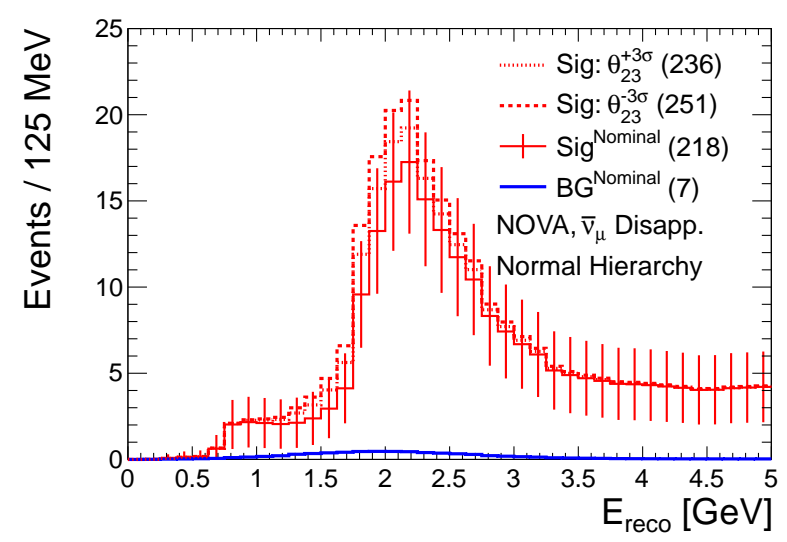

(D) $\bar{\nu}_{\mu}$ disappearance, True $\mathrm{MH}=\mathrm{NH}$

FIGURE 6.6. NO $\nu \mathrm{A}$ event spectra for $\pm 3 \sigma$ variations in $\theta_{23}$ for $\nu_{e}$ appearance (top left), $\bar{\nu}_{e}$ appearance (top right), $\nu_{\mu}$ disappearance (bottom left), and $\bar{\nu}_{\mu}$ disappearance (bottom right). The histograms are overlaid and not stacked. The nominal $3.6 \times 10^{21}$ POT with 1:1 $\nu: \bar{\nu}$ ratio is assumed. The integrated numbers of events are given in parentheses in the legend. 


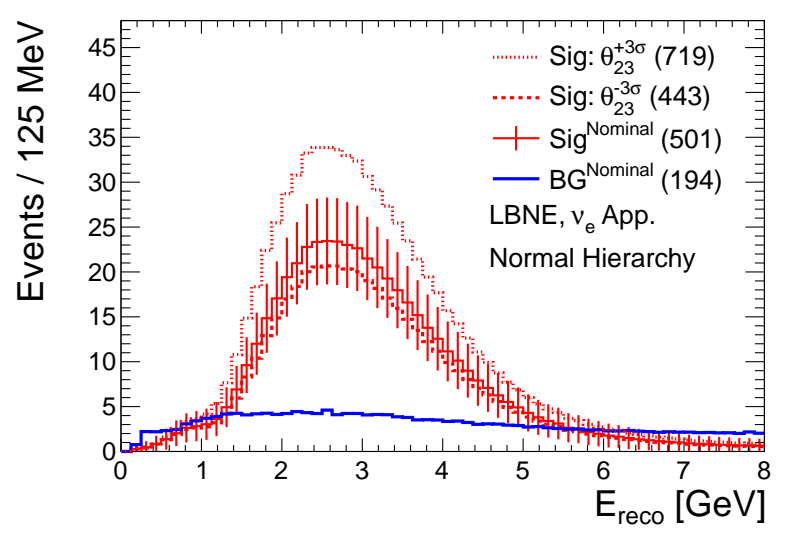

(A) $\nu_{e}$ appearance, True $\mathrm{MH}=\mathrm{NH}$

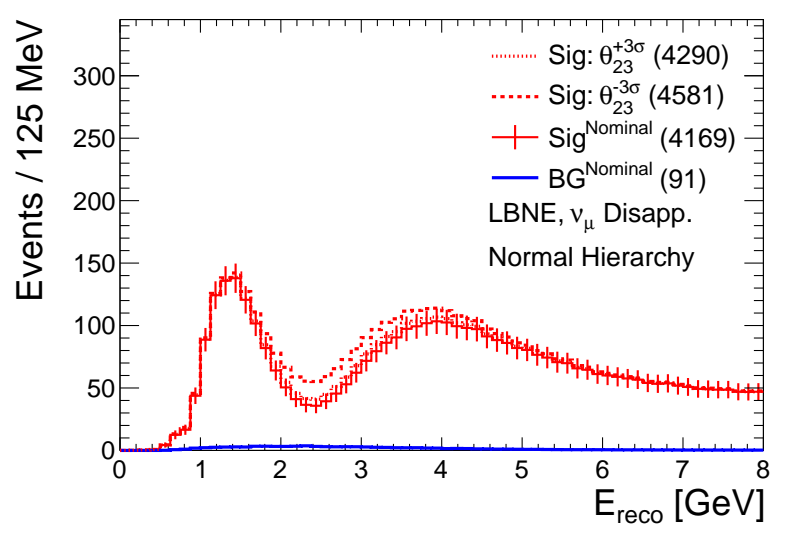

(C) $\nu_{\mu}$ disappearance, True $\mathrm{MH}=\mathrm{NH}$

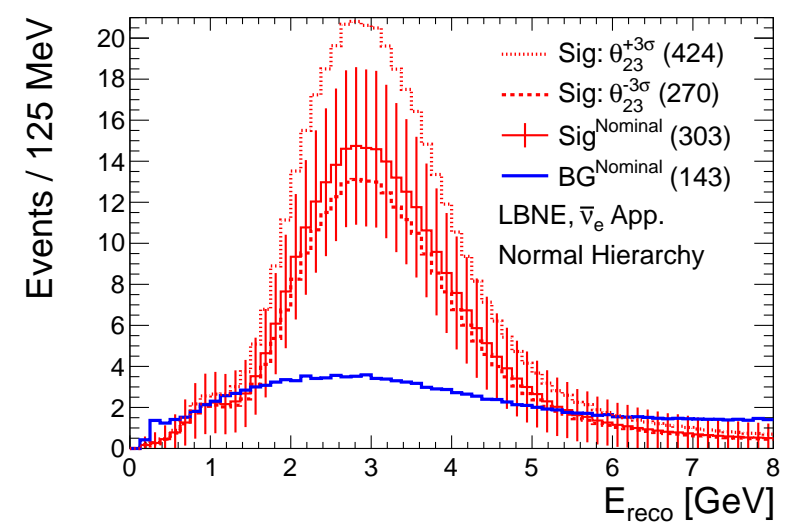

(B) $\bar{\nu}_{e}$ appearance, True $\mathrm{MH}=\mathrm{NH}$

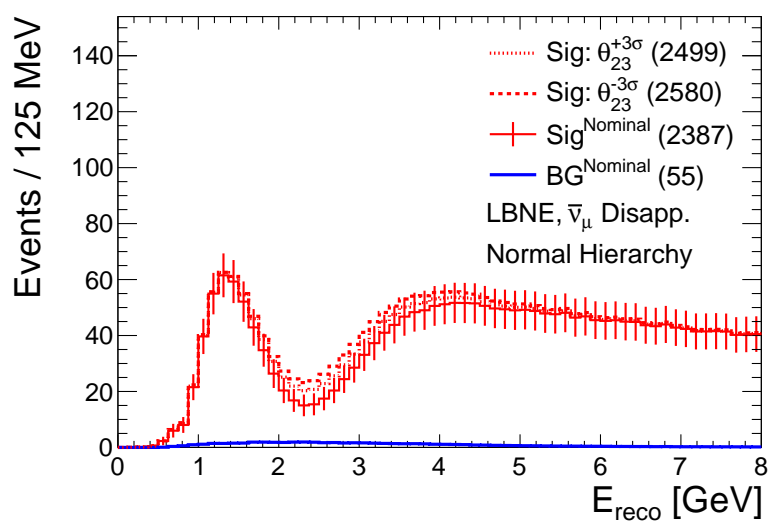

(D) $\bar{\nu}_{\mu}$ disappearance, True $\mathrm{MH}=\mathrm{NH}$

FigURE 6.7. LBNE event spectra for $\pm 3 \sigma$ variations in $\theta_{23}$ for $\nu_{e}$ appearance (top left), $\bar{\nu}_{e}$ appearance (top right), $\nu_{\mu}$ disappearance (bottom left), and $\bar{\nu}_{\mu}$ disappearance (bottom right). The histograms are overlaid and not stacked. The nominal $6.0 \times 10^{21}$ POT with $1: 1 \nu: \bar{\nu}$ ratio is assumed. The integrated numbers of events are given in parentheses in the legend. 


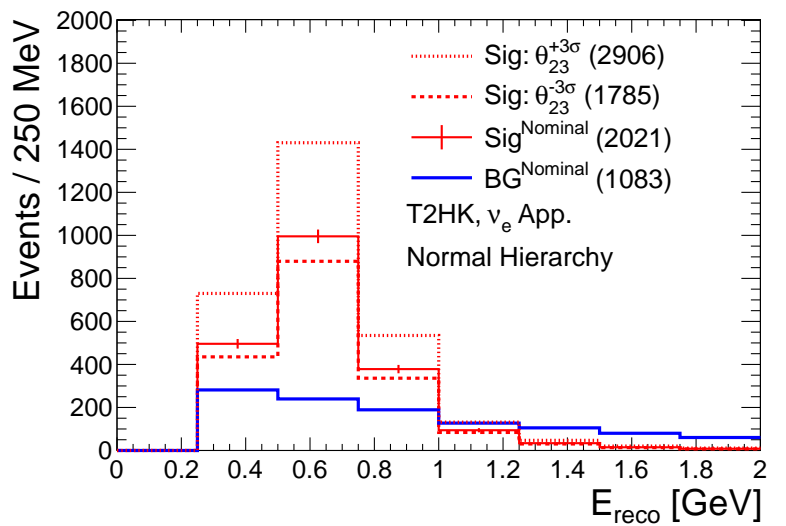

(A) $\nu_{e}$ appearance, True $\mathrm{MH}=\mathrm{NH}$

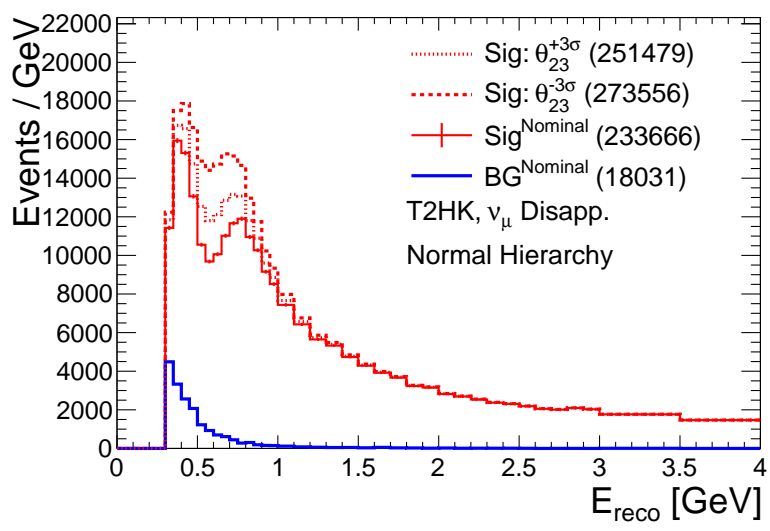

(C) $\nu_{\mu}$ disappearance, True $\mathrm{MH}=\mathrm{NH}$

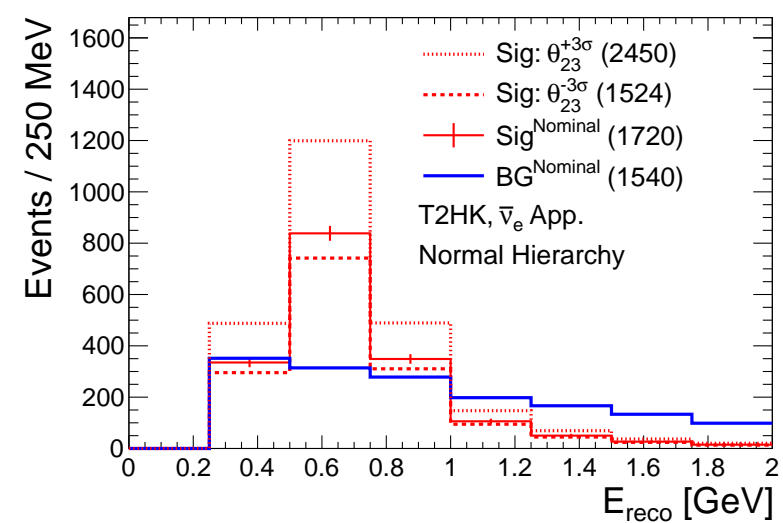

(B) $\bar{\nu}_{e}$ appearance, True $\mathrm{MH}=\mathrm{NH}$

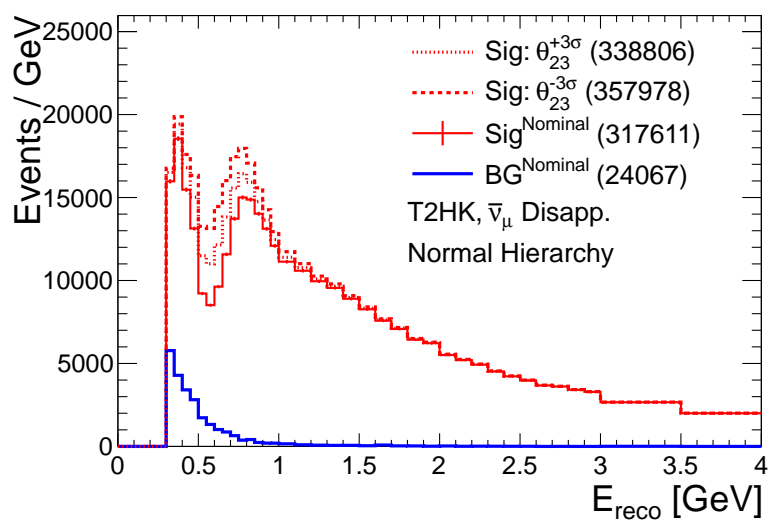

(D) $\bar{\nu}_{\mu}$ disappearance, True $\mathrm{MH}=\mathrm{NH}$

FigurE 6.8. T2HK event spectra for $\pm 3 \sigma$ variations in $\theta_{23}$ for $\nu_{e}$ appearance (top left), $\bar{\nu}_{e}$ appearance (top right), $\nu_{\mu}$ disappearance (bottom left), and $\bar{\nu}_{\mu}$ disappearance (bottom right). The histograms are overlaid and not stacked. The nominal $15.6 \times 10^{21}$ POT with $1: 1 \nu: \bar{\nu}$ ratio is assumed. The integrated numbers of events are given in parentheses in the legend. 
6.1.3. $\Delta m_{31}^{2}$ VARIATIONS. Figures 6.9 through 6.12 show the response in the event spectra for the four experiments in the four samples for $\pm 3 \sigma$ variations in $\Delta m_{31}^{2}$. The effects of $\Delta m_{31}^{2}$ variations are exhibited in all samples as a shift in the oscillation frequency with respect to $\mathrm{L} / \mathrm{E}$, or since the baseline is fixed in each experiment, with respect to $1 / \mathrm{E}$. For example, in Figure 6.9 the bottom-left $\nu_{\mu}$ disappearance event spectra shows the minimum shifting from $0.6 \mathrm{GeV}$ down(up) to closer to $0.5 \mathrm{GeV}(0.7 \mathrm{GeV})$ for a $-3 \sigma(+3 \sigma)$ shift in $\Delta m_{31}^{2}$. A similar shift also occurs in the position of the maximum of the $\nu_{e}$ appearance and $\bar{\nu}_{e}$ appearance spectra. It is critical to have sufficient energy resolution to measure this parameter since it depends on being able to accurately determine the position of the minimum in $\nu_{\mu}$ disappearance and $\bar{\nu}_{\mu}$ disappearance.

For similar reasons if the bin size is too large then the effects of shifting the value of $\Delta m_{31}^{2}$ will be less evident in the event spectra. The binning in the T2K and T2HK $\nu_{\mu}$ and $\bar{\nu}_{\mu}$ samples has been optimized so that the disappearance maximum has fine binning, 50 $\mathrm{MeV}$, while in other regions of $E_{\text {reco }}$ the binning is less fine. The LBNE binning has not been optimized and is constant in bin widths of $125 \mathrm{MeV}$ for both the $\nu_{e}$ appearance and $\nu_{\mu}$ disappearance samples. It is not a simple matter to change these bin widths but future work will need to study the binning structures in order to optimize $\Delta m_{31}^{2}$ sensitivity. 


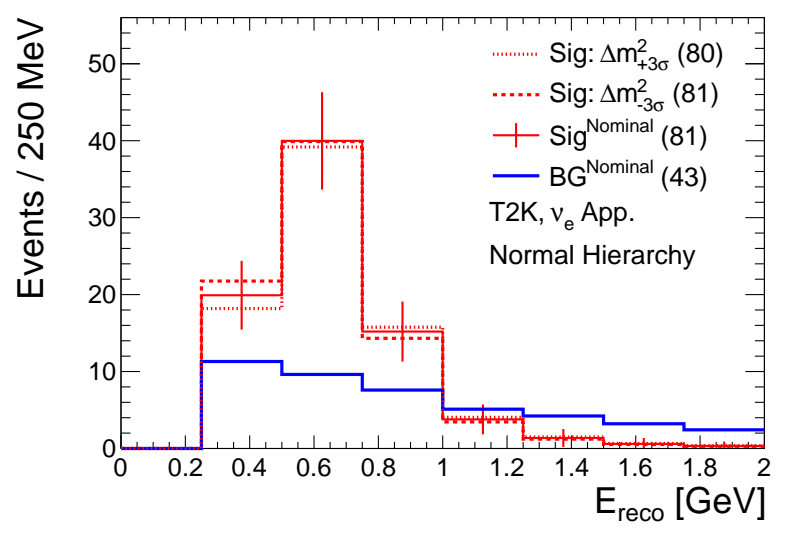

(A) $\nu_{e}$ appearance, True $\mathrm{MH}=\mathrm{NH}$

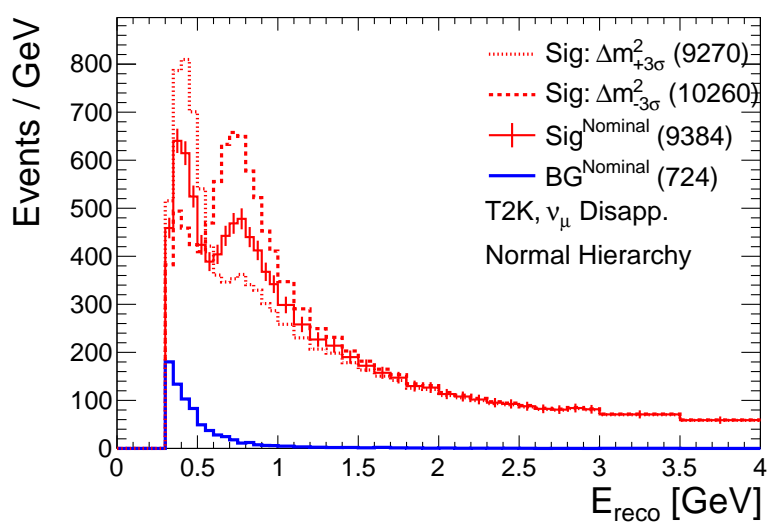

(C) $\nu_{\mu}$ disappearance, True $\mathrm{MH}=\mathrm{NH}$

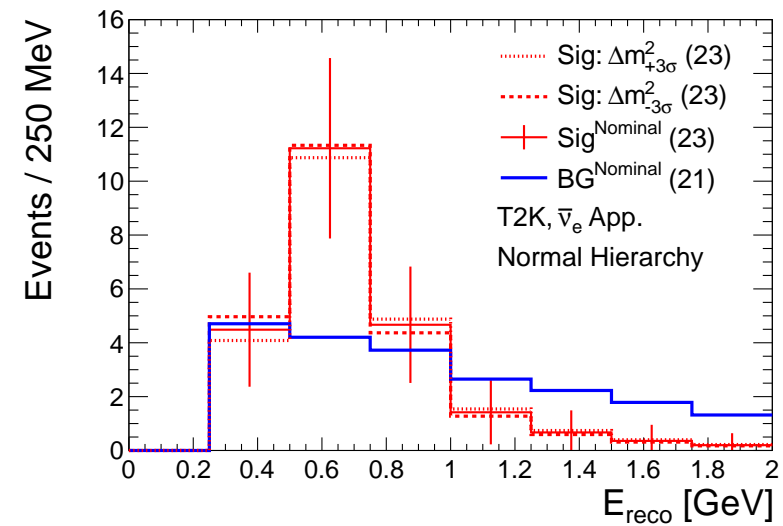

(B) $\bar{\nu}_{e}$ appearance, True $\mathrm{MH}=\mathrm{NH}$

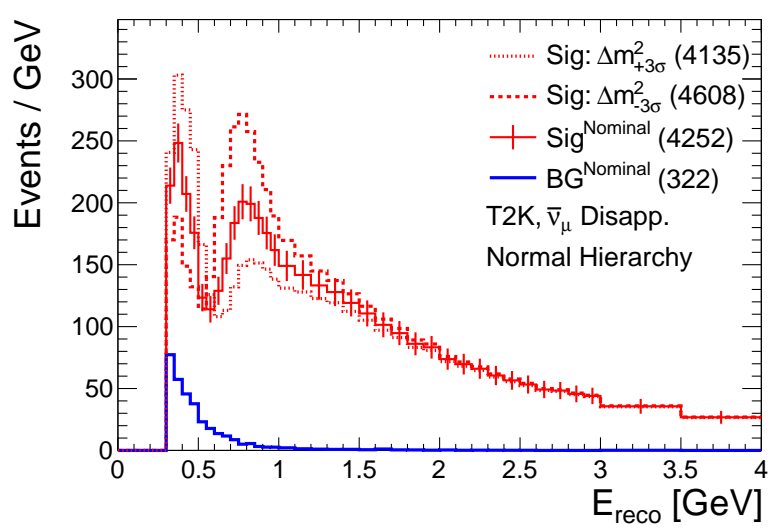

(D) $\bar{\nu}_{\mu}$ disappearance, True $\mathrm{MH}=\mathrm{NH}$

FIGURE 6.9. T2K event spectra for $\pm 3 \sigma$ variations in $\Delta m_{31}^{2}$ for $\nu_{e}$ appearance (top left), $\bar{\nu}_{e}$ appearance (top right), $\nu_{\mu}$ disappearance (bottom left), and $\bar{\nu}_{\mu}$ disappearance (bottom right). The histograms are overlaid and not stacked. The nominal $7.8 \times 10^{21}$ POT with $1: 1 \nu: \bar{\nu}$ ratio is assumed. The integrated numbers of events are given in parentheses in the legend. 


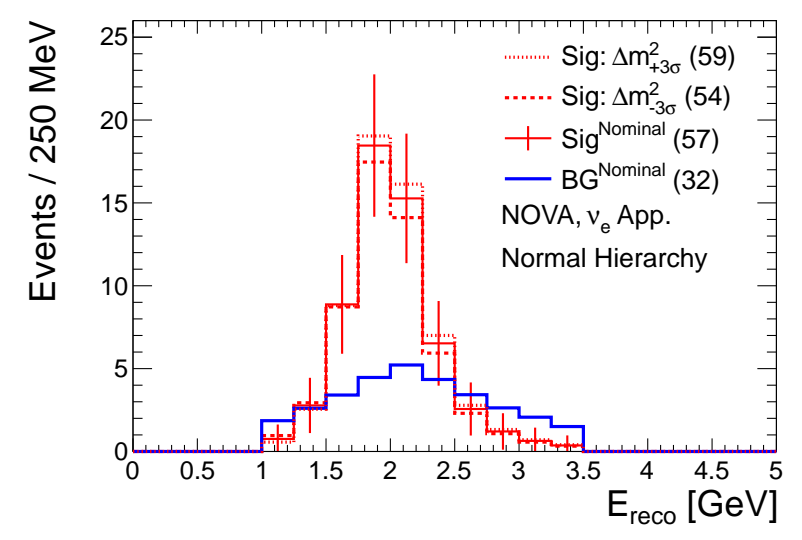

(A) $\nu_{e}$ appearance, True $\mathrm{MH}=\mathrm{NH}$

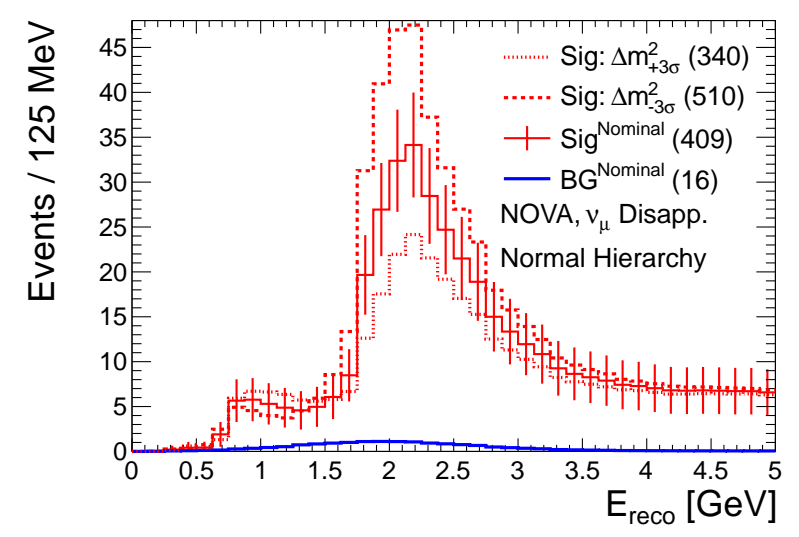

(C) $\nu_{\mu}$ disappearance, True $\mathrm{MH}=\mathrm{NH}$

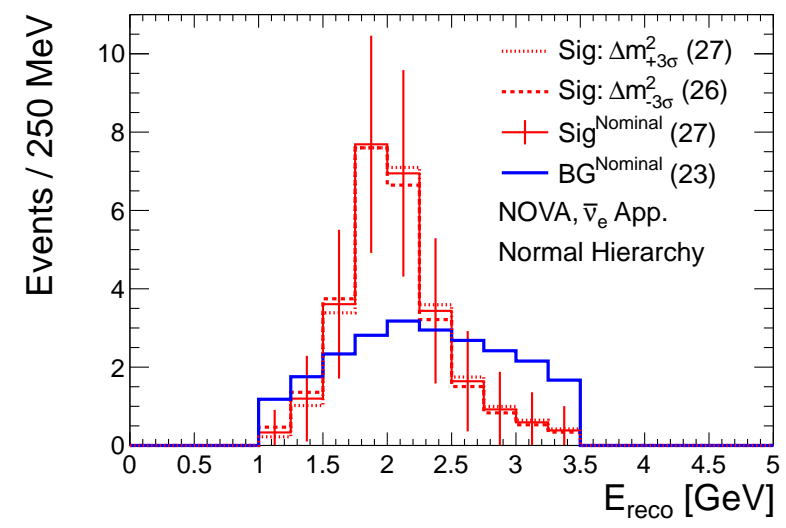

(B) $\bar{\nu}_{e}$ appearance, True $\mathrm{MH}=\mathrm{NH}$

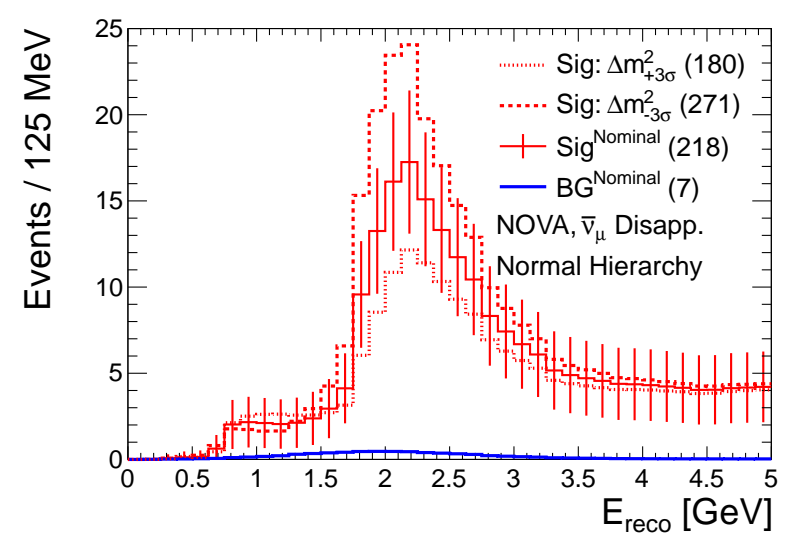

(D) $\bar{\nu}_{\mu}$ disappearance, True $\mathrm{MH}=\mathrm{NH}$

FIGURE 6.10. NO $\nu \mathrm{A}$ event spectra for $\pm 3 \sigma$ variations in $\Delta m_{31}^{2}$ for $\nu_{e}$ appearance (top left), $\bar{\nu}_{e}$ appearance (top right), $\nu_{\mu}$ disappearance (bottom left), and $\bar{\nu}_{\mu}$ disappearance (bottom right). The histograms are overlaid and not stacked. The nominal $3.6 \times 10^{21}$ POT with $1: 1 \nu: \bar{\nu}$ ratio is assumed. The integrated numbers of events are given in parentheses in the legend. 


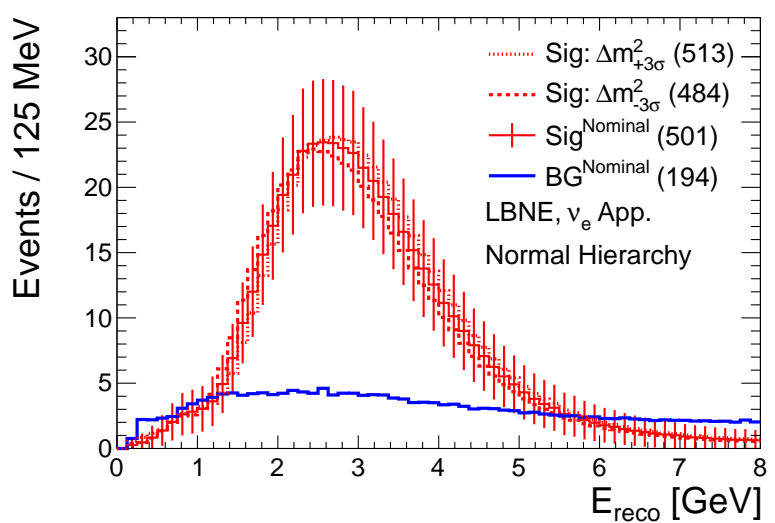

(A) $\nu_{e}$ appearance, True $\mathrm{MH}=\mathrm{NH}$

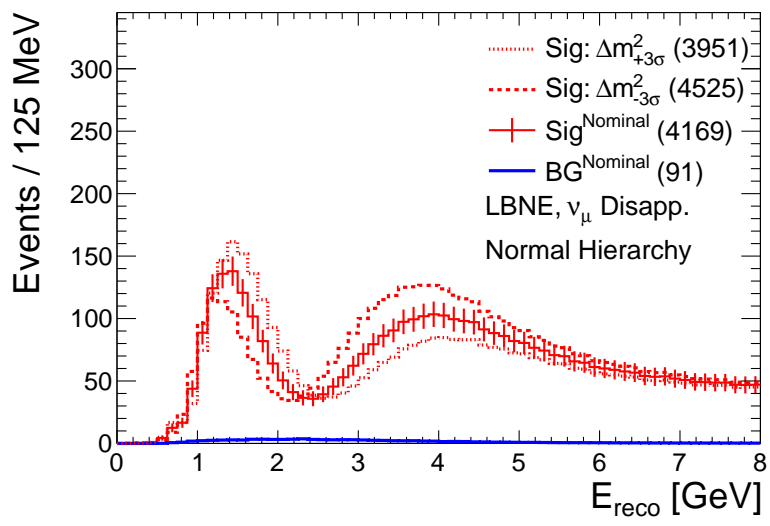

(C) $\nu_{\mu}$ disappearance, True $\mathrm{MH}=\mathrm{NH}$

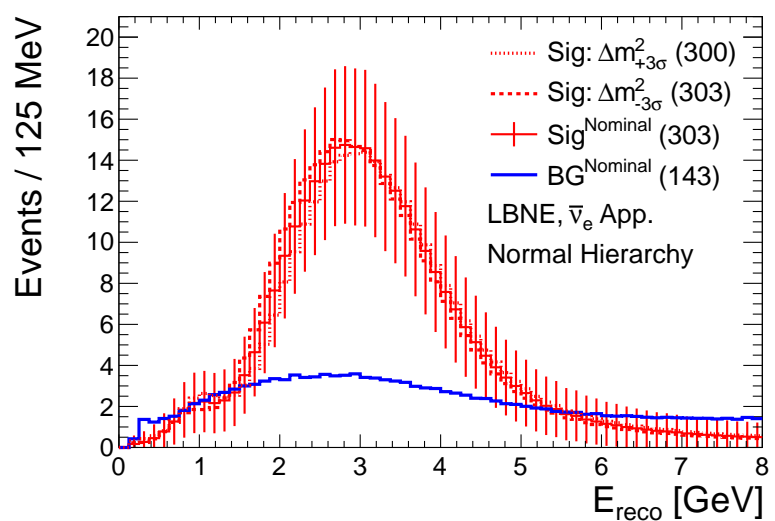

(B) $\bar{\nu}_{e}$ appearance, True $\mathrm{MH}=\mathrm{NH}$

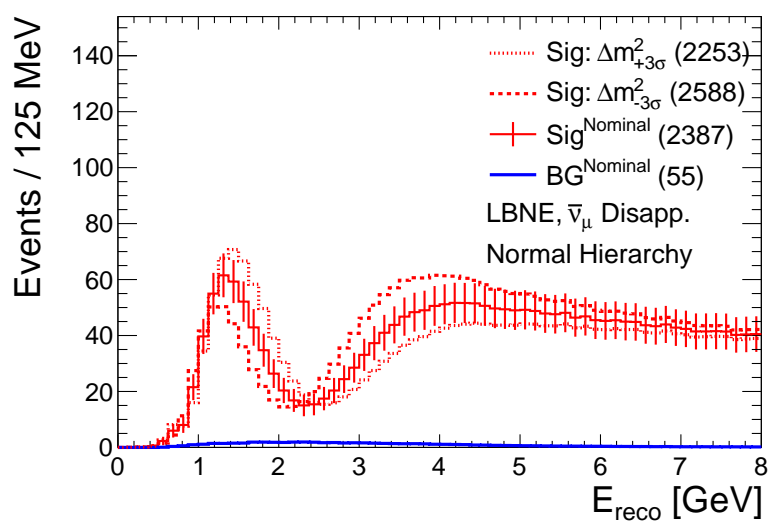

(D) $\bar{\nu}_{\mu}$ disappearance, True $\mathrm{MH}=\mathrm{NH}$

FIGURE 6.11. LBNE event spectra for $\pm 3 \sigma$ variations in $\Delta m_{31}^{2}$ for $\nu_{e}$ appearance (top left), $\bar{\nu}_{e}$ appearance (top right), $\nu_{\mu}$ disappearance (bottom left), and $\bar{\nu}_{\mu}$ disappearance (bottom right). The histograms are overlaid and not stacked. The nominal $6.0 \times 10^{21}$ POT with $1: 1 \nu: \bar{\nu}$ ratio is assumed. The integrated numbers of events are given in parentheses in the legend. 


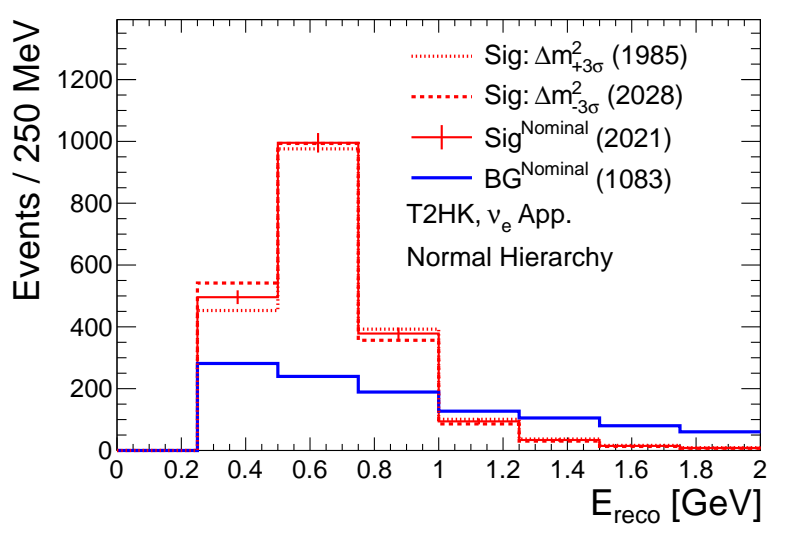

(A) $\nu_{e}$ appearance, True $\mathrm{MH}=\mathrm{NH}$

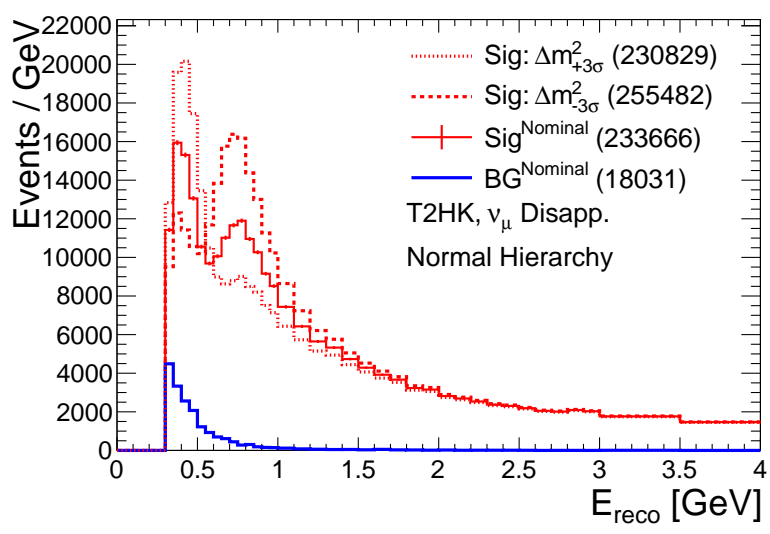

(C) $\nu_{\mu}$ disappearance, True $\mathrm{MH}=\mathrm{NH}$

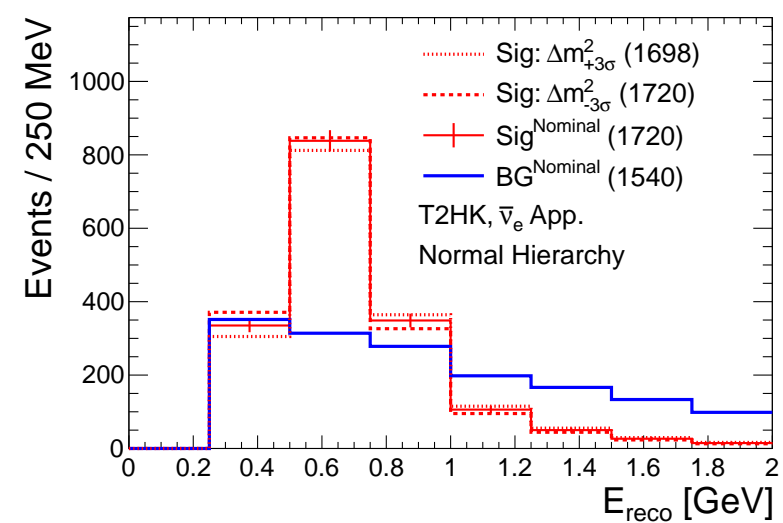

(B) $\bar{\nu}_{e}$ appearance, True $\mathrm{MH}=\mathrm{NH}$

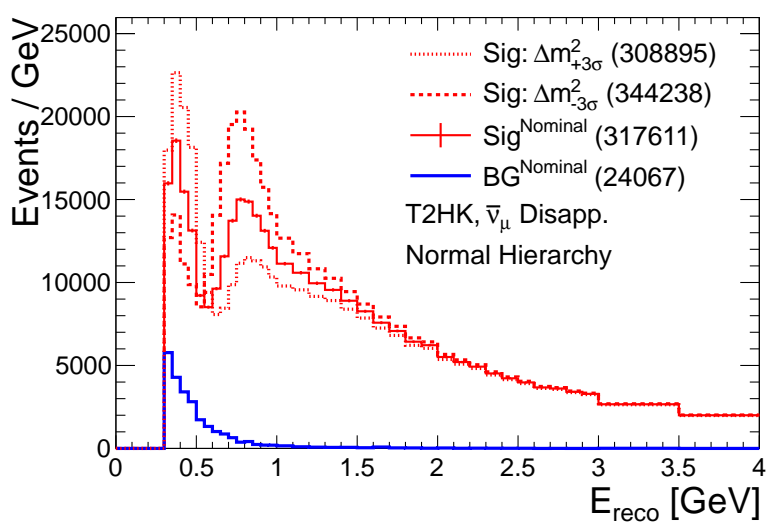

(D) $\bar{\nu}_{\mu}$ disappearance, True $\mathrm{MH}=\mathrm{NH}$

FigurE 6.12. T2HK event spectra for $\pm 3 \sigma$ variations in $\Delta m_{31}^{2}$ for $\nu_{e}$ appearance (top left), $\bar{\nu}_{e}$ appearance (top right), $\nu_{\mu}$ disappearance (bottom left), and $\bar{\nu}_{\mu}$ disappearance (bottom right). The histograms are overlaid and not stacked. The nominal $15.6 \times 10^{21} \mathrm{POT}$ with $1: 1 \nu: \bar{\nu}$ ratio is assumed. The integrated numbers of events are given in parentheses in the legend. 
6.1.4. $\delta_{C P}$ VARIATIONS. Figures 6.13 through 6.16 show the response in the event spectra for the four experiments in the four samples for variations in $\delta_{C P}$. Since there are only weak global-fit constraints on $\delta_{C P}$, relative to the other oscillation parameters, the full range of spectral variation is shown by choosing $\delta_{C P}= \pm \pi / 2$ as the variations and with $\delta_{C P}=0$ as the nominal value.

The $\nu_{e}$ appearance and $\bar{\nu}_{e}$ appearance spectra are changed dramatically by a change in $\delta_{C P}$. The effect depends on whether spectra in neutrino or anti-neutrino mode are being considered. The $\sin \left(\delta_{C P}\right)$ term in the $\nu_{e}$ appearance probability will be negative for neutrinos and positive for anti-neutrinos. It is this difference between the appearance probability of neutrino and anti-neutrinos that makes it critical to run in both modes in order to directly compare the rates relative to expectations.

In a wide-band band beam experiment like LBNE the variations in $\delta_{C P}$, shown in Figure 6.15, create shape distortions that play an important role in distinguishing between changes in the $\mathrm{MH}$ and changes in $\delta_{C P}$. In particular, in the $\nu_{e}$ appearance spectrum the first oscillation maximum is visible around a reconstructed energy of $2.5 \mathrm{GeV}$, but the second oscillation maximum is just barely visible below $1 \mathrm{GeV}$. This effect was discussed in the context of the LBNE baseline in Section 4.3.6 and will be revisited in Chapter 9 in order to evaluate the effects of increasing the $\nu_{\mu}$ flux at the second oscillation maximum.

The disappearance spectra are changed very slightly by the effects of the $\delta_{C P}$ parameter. A small modification comes from the $\cos \left(\delta_{C P}\right)$ term in the $\nu_{\mu}$ disappearance oscillation probability. The variations in the $\nu_{\mu}$ disappearance and $\bar{\nu}_{\mu}$ disappearance spectra are very small so that the differences in the histograms are not visible, but the effect of the $\cos \left(\delta_{C P}\right)$ term can be seen by comparing the event rates as the $\delta_{C P}=+\pi / 2$ and $\delta_{C P}=-\pi / 2$ event spectra integrated numbers of events are both increased with respect to the $\delta_{C P}=0$ numbers 
in each of the experiments. The effect of $\delta_{C P}$ in $\nu_{\mu}$ disappearance and $\bar{\nu}_{\mu}$ disappearance is symmetrical, they both increase with a non-zero value. This is a small change in the disappearance spectra and even with the large event rates in T2HK the effect is $0.2 \%$ as compared with a $\sim 29 \%$ effect in $\nu_{e}$ appearance.

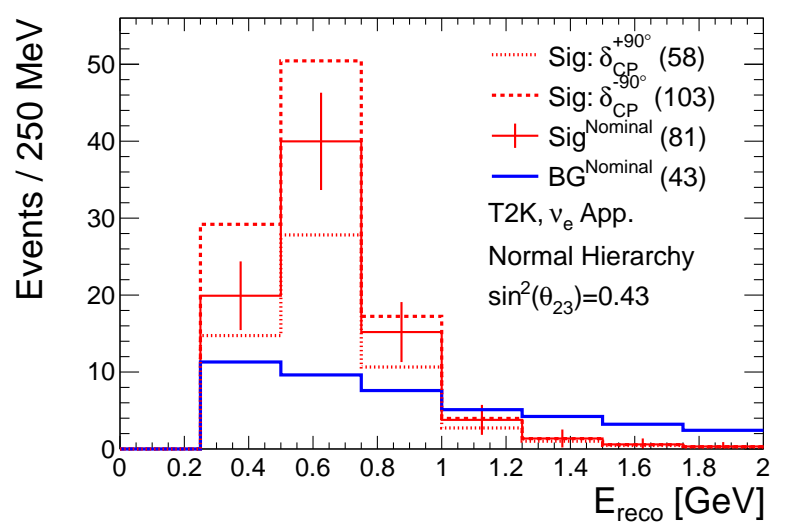

(A) $\nu_{e}$ appearance, True $\mathrm{MH}=\mathrm{NH}$

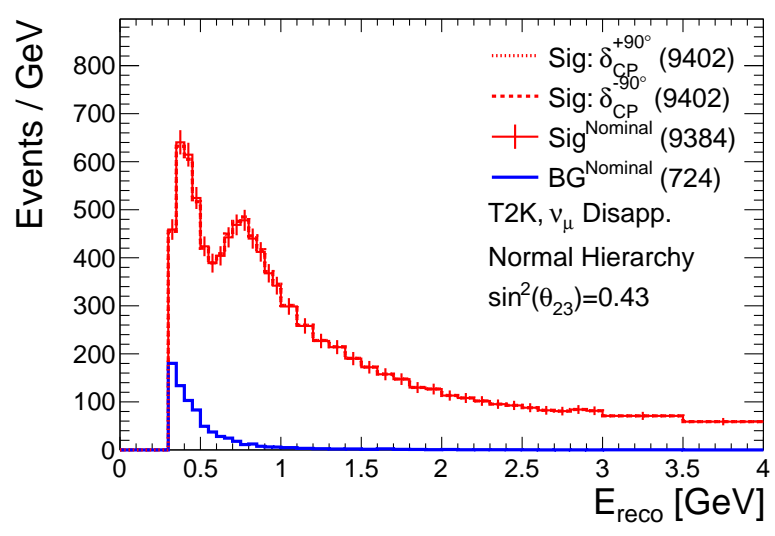

(C) $\nu_{\mu}$ disappearance, True $\mathrm{MH}=\mathrm{NH}$

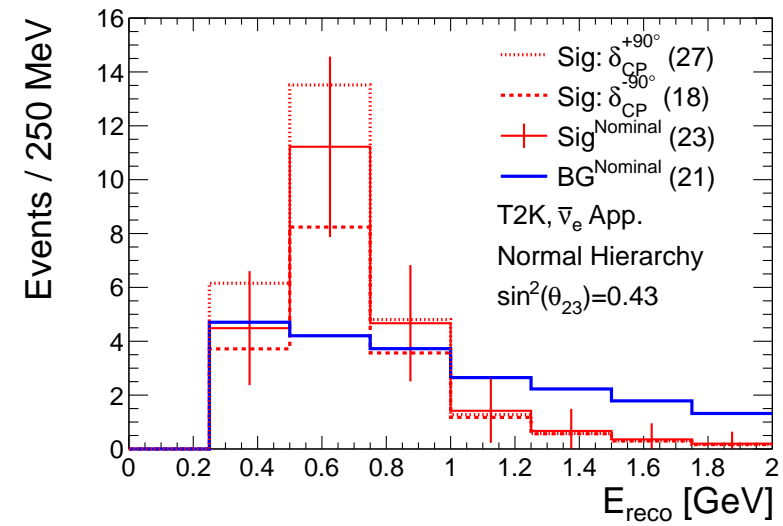

(в) $\bar{\nu}_{e}$ appearance, True $\mathrm{MH}=\mathrm{NH}$

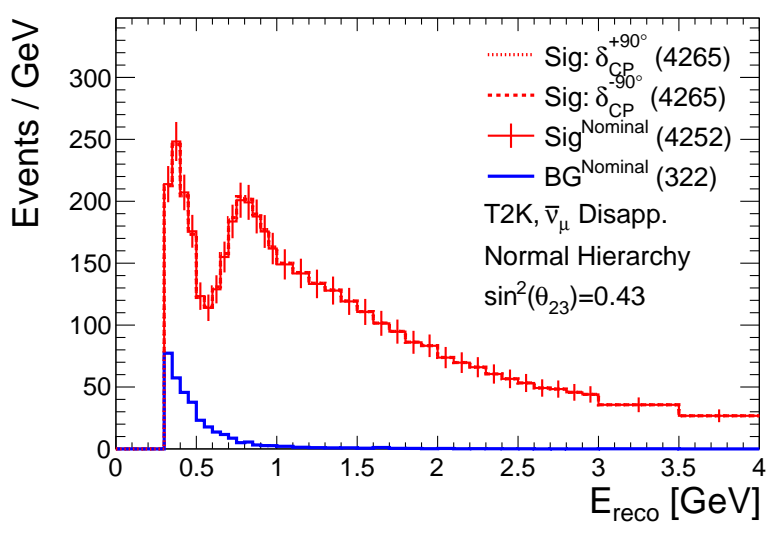

(D) $\bar{\nu}_{\mu}$ disappearance, True $\mathrm{MH}=\mathrm{NH}$

FigURE 6.13. T2K event spectra for $\pm 3 \sigma$ variations in $\delta_{C P}$ for $\nu_{e}$ appearance (top left), $\bar{\nu}_{e}$ appearance (top right), $\nu_{\mu}$ disappearance (bottom left), and $\bar{\nu}_{\mu}$ disappearance (bottom right). The histograms are overlaid and not stacked. The nominal $7.8 \times 10^{21}$ POT with 1:1 $\nu: \bar{\nu}$ ratio is assumed. The integrated numbers of events are given in parentheses in the legend. 


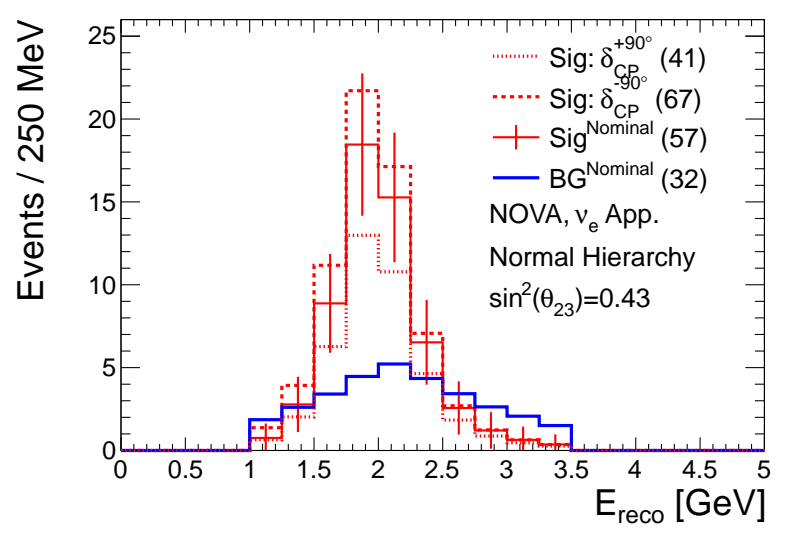

(A) $\nu_{e}$ appearance, True $\mathrm{MH}=\mathrm{NH}$

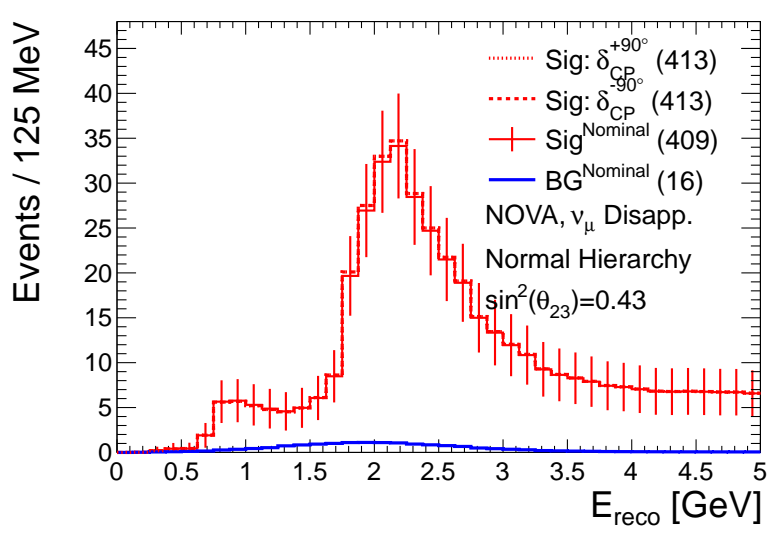

(c) $\nu_{\mu}$ disappearance, True $\mathrm{MH}=\mathrm{NH}$

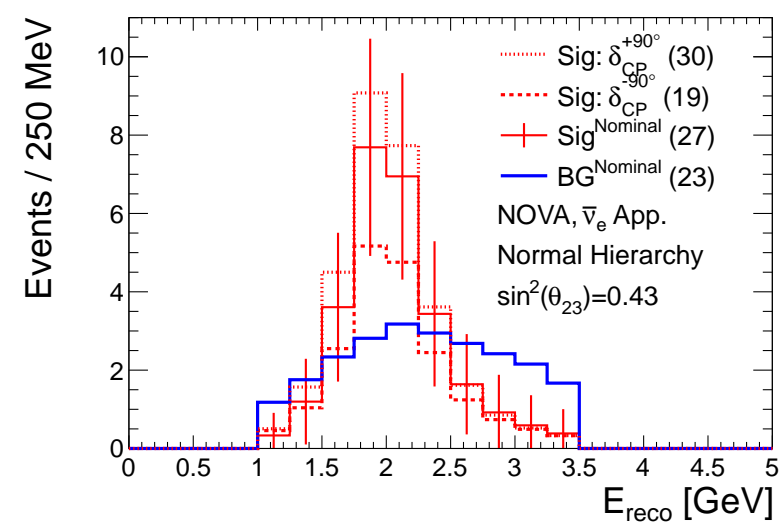

(B) $\bar{\nu}_{e}$ appearance, True $\mathrm{MH}=\mathrm{NH}$

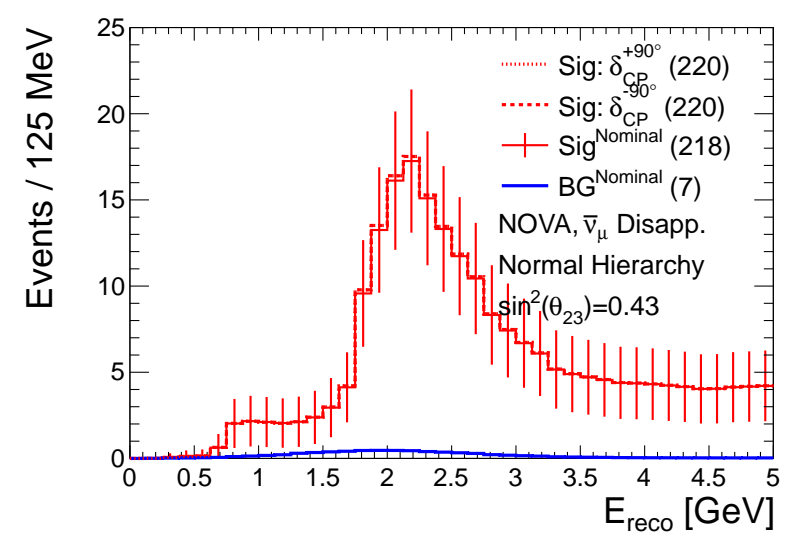

(D) $\bar{\nu}_{\mu}$ disappearance, True $\mathrm{MH}=\mathrm{NH}$

FigURE 6.14. NO $\nu$ A event spectra for $\pm 3 \sigma$ variations in $\delta_{C P}$ for $\nu_{e}$ appearance (top left), $\bar{\nu}_{e}$ appearance (top right), $\nu_{\mu}$ disappearance (bottom left), and $\bar{\nu}_{\mu}$ disappearance (bottom right). The histograms are overlaid and not stacked. The nominal $3.6 \times 10^{21}$ POT with $1: 1 \nu: \bar{\nu}$ ratio is assumed. The integrated numbers of events are given in parentheses in the legend. 


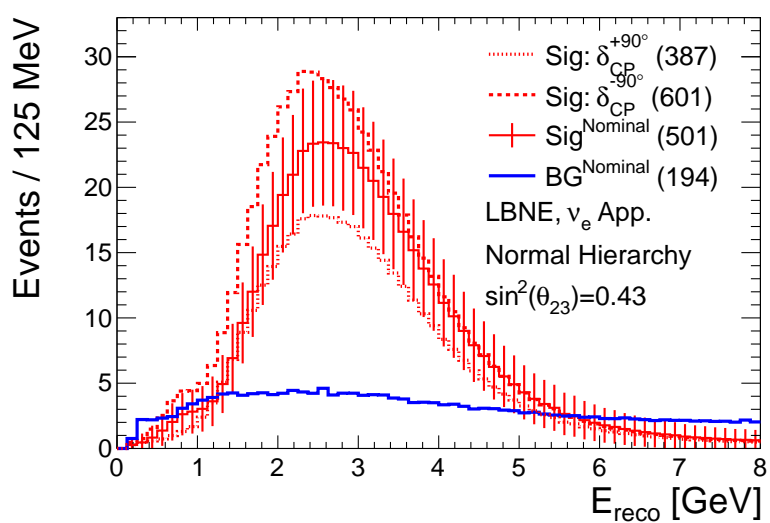

(A) $\nu_{e}$ appearance, True $\mathrm{MH}=\mathrm{NH}$

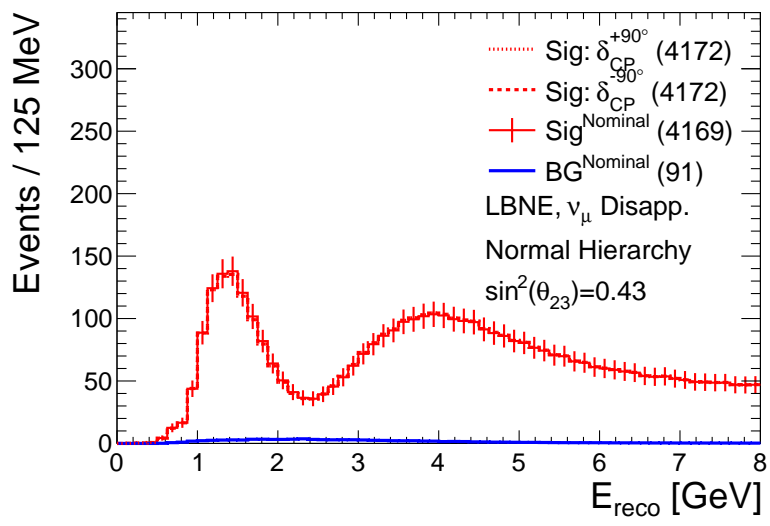

(C) $\nu_{\mu}$ disappearance, True $\mathrm{MH}=\mathrm{NH}$

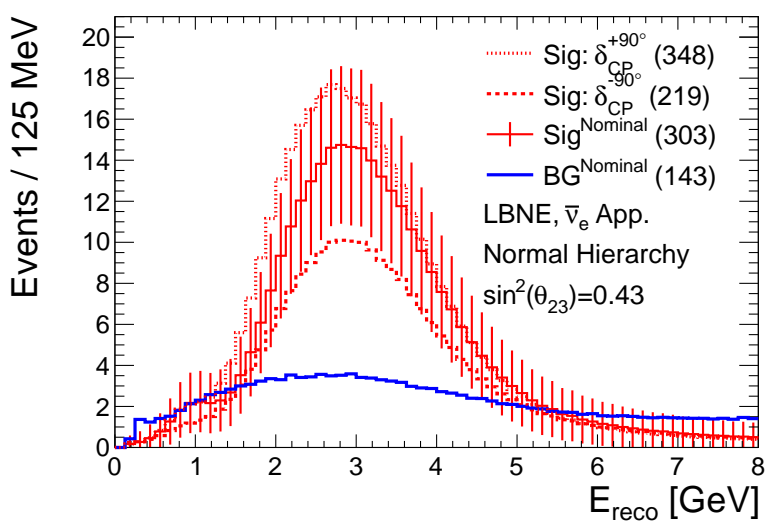

(в) $\bar{\nu}_{e}$ appearance, True $\mathrm{MH}=\mathrm{NH}$

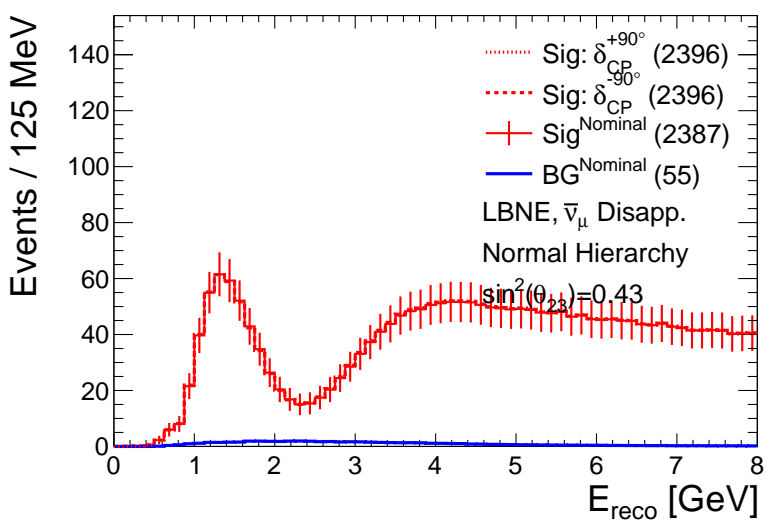

(D) $\bar{\nu}_{\mu}$ disappearance, True $\mathrm{MH}=\mathrm{NH}$

FIGURE 6.15. LBNE event spectra for $\pm 3 \sigma$ variations in $\delta_{C P}$ for $\nu_{e}$ appearance (top left), $\bar{\nu}_{e}$ appearance (top right), $\nu_{\mu}$ disappearance (bottom left), and $\bar{\nu}_{\mu}$ disappearance (bottom right). The histograms are overlaid and not stacked. The nominal $6.0 \times 10^{21}$ POT with $1: 1 \nu: \bar{\nu}$ ratio is assumed. The integrated numbers of events are given in parentheses in the legend. 


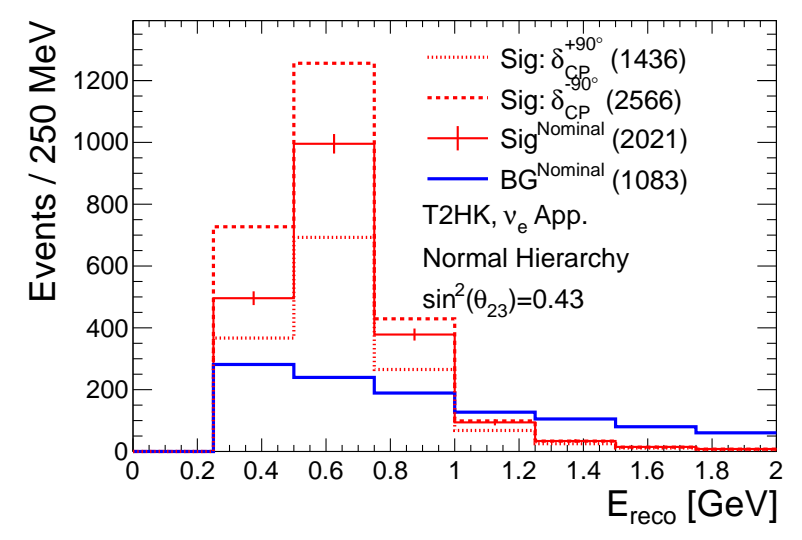

(A) $\nu_{e}$ appearance, True $\mathrm{MH}=\mathrm{NH}$

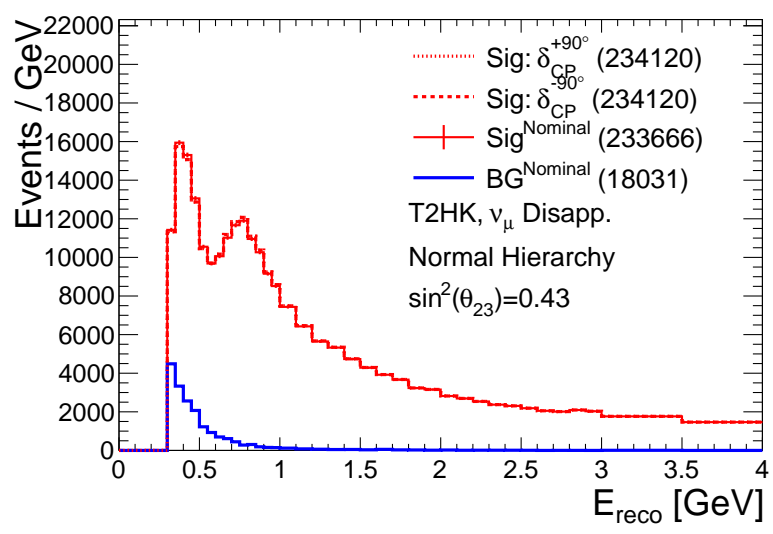

(C) $\nu_{\mu}$ disappearance, True $\mathrm{MH}=\mathrm{NH}$

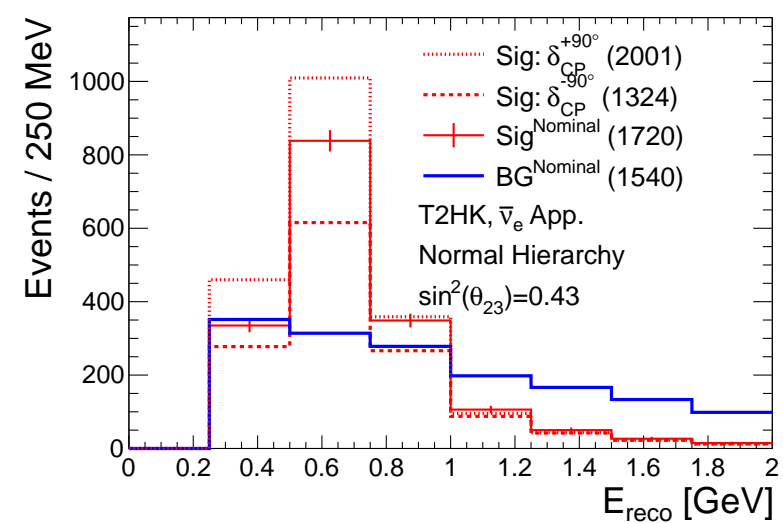

(B) $\bar{\nu}_{e}$ appearance, True $\mathrm{MH}=\mathrm{NH}$

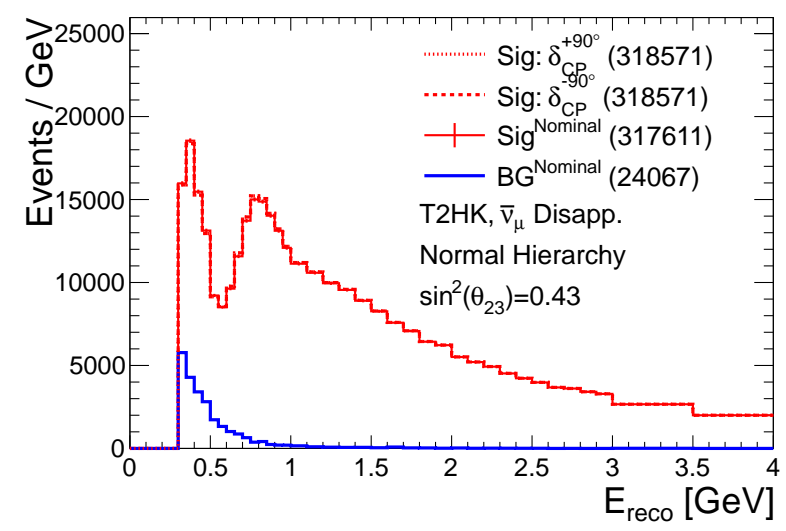

(D) $\bar{\nu}_{\mu}$ disappearance, True $\mathrm{MH}=\mathrm{NH}$

FIGURE 6.16. T2HK event spectra for $\pm 3 \sigma$ variations in $\delta_{C P}$ for $\nu_{e}$ appearance (top left), $\bar{\nu}_{e}$ appearance (top right), $\nu_{\mu}$ disappearance (bottom left), and $\bar{\nu}_{\mu}$ disappearance (bottom right). The histograms are overlaid and not stacked. The nominal $15.6 \times 10^{21} \mathrm{POT}$ with $1: 1 \nu: \bar{\nu}$ ratio is assumed. The integrated numbers of events are given in parentheses in the legend. 


\subsection{Oscillation Parameter Sensitivities}

The predicted resolution on the oscillation parameters quantifies the ability of the experiment to constrain a particular oscillation parameter. This section will revisit the $\Delta \chi^{2}$ used to compute the sensitivity and show one and two dimensional constraints of the parameters assuming the true values are those of the Capozzi et al. global fit.

6.2.1. Revisiting the $\Delta \chi^{2}$. Figures 6.17 and 6.18 show the $\Delta \chi^{2}$ as a function of each variable: $\sin ^{2}\left(2 \theta_{13}\right), \sin ^{2}\left(\theta_{23}\right), \Delta m_{31}^{2}$, and $\delta_{C P}$ for the assumption of True $\mathrm{MH}=\mathrm{NH}$ and True $\mathrm{MH}=\mathrm{IH}$ respectively. The $\Delta \chi^{2}$ value is computed using the method outlined in Chapter 5 . The true values of the oscillation parameters are set to the Capozzi et al. global fit values while the fit value of the oscillation parameter being scanned is set to the $\mathrm{x}$-axis value. All of the other oscillation parameter fit values are profiled in the minimization of the $\Delta \chi^{2}$ and are constrained by the global fit $1 \sigma$ ranges. The $\Delta \chi^{2}$ is summed for all four samples: $\nu_{e}$ appearance, $\bar{\nu}_{e}$ appearance, $\nu_{\mu}$ disappearance, and $\bar{\nu}_{\mu}$ disappearance. Normalization systematics on the signal and background are included at the nominal levels. The mass hierarchy is also profiled by computing a $\Delta \chi^{2}$ using both the $\mathrm{NH}$ and $\mathrm{IH}$ assumptions for the fit spectra and taking the minimum between the two $\Delta \chi^{2}$ values.

Figures $6.17 \mathrm{~A}$ and $6.18 \mathrm{~A}$ show the $\Delta \chi^{2}$ versus the fit value of $\sin ^{2}\left(2 \theta_{13}\right)$ used in the $\Delta \chi^{2}$ computation. The $\Delta \chi^{2}$ grows as the scanned value of $\sin ^{2}\left(2 \theta_{13}\right)$ increasingly differs from the true value of $\sin ^{2}\left(2 \theta_{13}\right)$. There are kinks in the $\mathrm{NO} \nu \mathrm{A}$ and $\mathrm{T} 2 \mathrm{~K}+\mathrm{NO} \nu \mathrm{A}$ curves that come from a change in the $\delta_{C P}$ fit value that minimizes the $\Delta \chi^{2}$. For reference, a value of $\Delta \chi^{2}=1(9)$ corresponds to $1 \sigma(3 \sigma)$. Figure 6.18 A shows a sudden drop in the LBNE and T2HK curves at a $\sin ^{2}\left(2 \theta_{13}\right)$ value below the true value of $\sin ^{2}\left(2 \theta_{13}\right)$. This is a degenerate

region where these experiments cannot distinguish between a value of $\sin ^{2}\left(2 \theta_{13}\right)=0.092$ and 
$\sin ^{2}\left(2 \theta_{13}\right)=0.077$ because they have similar oscillation probabilities and the event spectra are similar enough when the normalization systematics and oscillation parameters systematics are taken into account. Other measurements can be used to break this degeneracy and these will be added in Section 6.2.3

Figures $6.17 \mathrm{~B}$ and $6.18 \mathrm{~B}$ show the $\Delta \chi^{2}$ versus the fit value of $\sin ^{2}\left(\theta_{23}\right)$. The global best fit value is in the first octant at $\sin ^{2}\left(\theta_{23}\right)=0.44(0.46)$ in the $\mathrm{NH}(\mathrm{IH})$. There is a degeneracy in the values of $\sin ^{2}\left(\theta_{23}\right)$ for the $\mathrm{T} 2 \mathrm{~K}, \mathrm{NO} \nu \mathrm{A}$, and $\mathrm{T} 2 \mathrm{~K}+\mathrm{NO} \nu \mathrm{A}$ curves. This means that their $\nu_{e}$ appearance, $\bar{\nu}_{e}$ appearance, $\nu_{\mu}$ disappearance, and $\bar{\nu}_{\mu}$ disappearance spectra predictions for a value of $\sin ^{2}\left(2 \theta_{13}\right)=0.44$ and a value of $\sin ^{2}\left(2 \theta_{13}\right)=0.59$ cannot be distinguished at the $3 \sigma$ level though they can all distinguish them at the $1 \sigma$ level. The lower statistics of $\mathrm{T} 2 \mathrm{~K}$ and $\mathrm{NO} \nu \mathrm{A}$, even in combination, are not enough to distinguish between the two octants at the $3 \sigma$ level. A high statistics sample in $\nu_{e}$ appearance and $\bar{\nu}_{e}$ appearance helps to distinguish between the two octants because there is a strong dependence on the octant in the oscillation probabilities for these samples. The first octant assumption predicts a lower $\nu_{e}$ appearance and $\bar{\nu}_{e}$ appearance probability than the second octant assumption. LBNE and $\mathrm{T} 2 \mathrm{HK}$ have no such degeneracy in the True $\mathrm{MH}=\mathrm{NH}$ case at the $3 \sigma$ level $\left(\Delta \chi^{2}=9\right)$ which is the vertical range covered by this figure, while LBNE sees a small degenerate region in the True $\mathrm{MH}=\mathrm{IH}$ case.

Figure $6.17 \mathrm{~B}$ also shows that the value of $\sin ^{2}\left(\theta_{23}\right)=0.44$ can be distinguished from maximal mixing, or $\sin ^{2}\left(\theta_{23}\right)=0.5$, in $\mathrm{T} 2 \mathrm{~K}$ at the $3 \sigma$ level. It cannot be determined at such a high level in $\mathrm{NO} \nu \mathrm{A}$. In the True $\mathrm{MH}=\mathrm{IH}$ case, Figure $6.18 \mathrm{~B}$ shows that even the combination can only make the distinction at about the $90 \%$ level $\left(\Delta \chi^{2}=2.7\right)$. LBNE just barely reaches the $3 \sigma$ level. The global fit value of $\sin ^{2}\left(\theta_{23}\right)$ for True $\mathrm{MH}=\mathrm{IH}$ is higher than in True $\mathrm{MH}=\mathrm{NH}$ and this makes it more difficult to distinguish from $\sin ^{2}\left(\theta_{23}\right)=0.5$. 
Figures $6.17 \mathrm{C}$ and $6.18 \mathrm{C}$ show the $\Delta \chi^{2}$ versus the fit value of $\Delta m_{31}^{2}$. These measurements are mostly symmetric around the true value $\Delta m_{31}^{2}$. There is some deviation seen in the $\mathrm{NO} \nu \mathrm{A}$ and $\mathrm{T} 2 \mathrm{~K}+\mathrm{NO} \nu \mathrm{A}$ curves. These abrupt changes come from jumps in the values of $\delta_{C P}$ that minimize the $\Delta \chi^{2}$. The value jumps from $\delta_{C P}=0$ to $\delta_{C P}=\pi$ at these points. The difference in the event spectra that come about from changes in $\Delta m_{31}^{2}$ and changing $\delta_{C P}$ from 0 to $\pi$ are similar in that they both result in a shift of the spectra with respect to energy.

Figures $6.17 \mathrm{D}-\mathrm{F}$ and $6.18 \mathrm{D}-\mathrm{F}$ show the $\Delta \chi^{2}$ versus the fit value of $\delta_{C P}$ for $\delta_{C P}^{\text {true }}=0$, $\delta_{C P}^{\text {true }}=\pi / 2$, and $\delta_{C P}^{\text {true }}=-\pi / 2$ respectively for the True $\mathrm{MH}=\mathrm{NH}$ and True $\mathrm{MH}=\mathrm{IH}$ assumptions. In the $\delta_{C P}^{\text {true }}=0$ case there are multiple degeneracies that come about from the similar event spectra predicted for fit assumptions of $\mathrm{NH}$ and $\mathrm{IH}$ and values of $\delta_{C P}$. These degeneracies can be better understood by looking at the predicted number of signal events for the $\mathrm{NH}$ and $\mathrm{IH}$ assumptions as a function of $\delta_{C P}$, as in Figure 6.19. It depicts an integrated number of events in $\nu_{e}$ appearance and does not indicate what $\Delta \chi^{2}$ contributions might come from considering $\bar{\nu}_{e}$ appearance, $\nu_{\mu}$ disappearance, $\bar{\nu}_{\mu}$ disappearance, and shape information. Shape information is particularly important in LBNE to distinguish $\delta_{C P}=0$ and $\delta_{C P}=\pi$. For $6.19 \mathrm{~A}$, depicting T2K $\nu_{e}$ appearance signal events, there are degeneracies where the expected number of signal events at the value of $\delta_{C P}$ on the $\mathrm{x}$-axis is equal to the number expected at $\delta_{C P}=0$ and $\delta_{C P}=\pi$ in the $\mathrm{NH}$ and $\mathrm{IH}$. To compare with the degeneracies seen in the T2K curve in Figure 6.17D, the top most horizontal line in Figure 6.19A, representing $\mathrm{NH}$ and $\delta_{C P}=0$, overlaps with the IH (dashed black) curve at two points just above and below the $\mathrm{x}$-axis value of $\delta_{C P}=-\pi / 2$. These same points are seen to show degeneracies, coming from profiling the $\mathrm{MH}$, in Figure 6.19A, though the T2K curve in this case includes all four samples so is not as degenerate as it would be with a $\nu_{e}$ appearance only fit. A similar degeneracy is seen in the $\mathrm{NO} \nu \mathrm{A}$ curves though it is less pronounced due 
to the longer baseline in $\mathrm{NO} \nu \mathrm{A}$. The degeneracy is non-existent in LBNE because of its long baseline and ability to separate the effects of the $\mathrm{MH}$ and $\delta_{C P}$. T2HK sees a degeneracy between $\delta_{C P}=-\pi$ and $\delta_{C P}=-\pi / 2$ that comes from profiling the $\mathrm{MH}$.

Similar degeneracies appear in Figure $6.17 \mathrm{~F}$ but are at different points in $\delta_{C P}$. T2K sees less of an effect from the MH degeneracy in this case, when compared with $\mathrm{NO} \nu \mathrm{A}$, because it has such a small MH effect, as can be seen by comparing the solid and dashed black lines in Figure 6.19A. NO $\nu \mathrm{A}$ has a similar number of signal events predicted between $\mathrm{NH}, \delta_{C P}=\pi / 2$ and IH, $\delta_{C P}=-\pi / 2$ so is unable to significantly distinguish between them. LBNE again sees no degeneracies. T2HK sees a small degenerate region near $\pi$, again coming from profiling the $\mathrm{MH}$.

Figure $6.17 \mathrm{~F}$ shows no degeneracies and this is where the signal events have no or little overlap between values of $\delta_{C P}$ and the $\mathrm{MH}$. All of these trends are mirrored, with respect to $\delta_{C P}=0$, in the IH case as seen in Figure 6.18. 

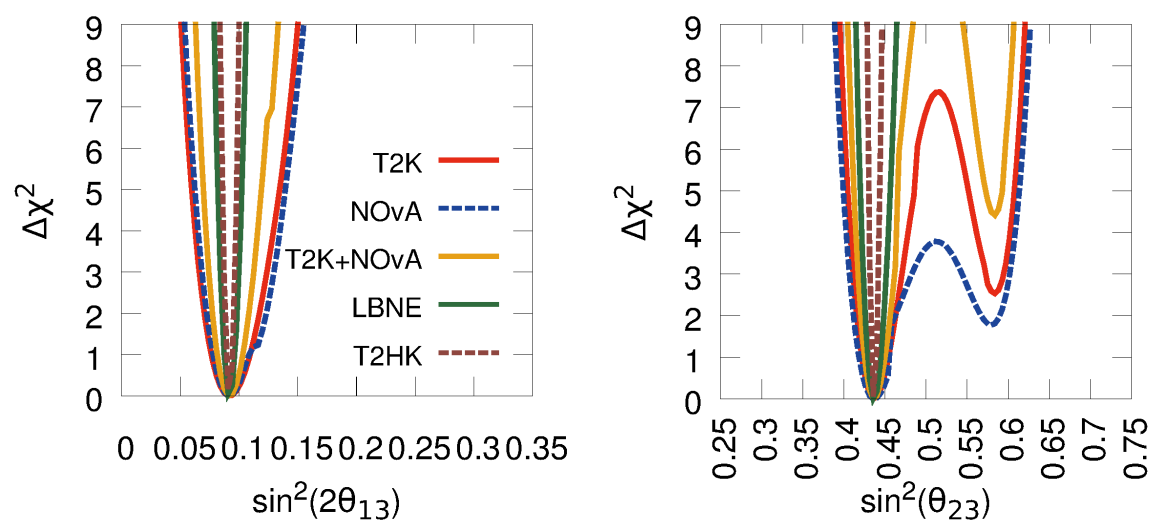

(A) $\delta_{C P}^{\text {true }}=0$, True $\mathrm{MH}=\mathrm{NH}$

(B) $\delta_{C P}^{\text {true }}=0$, True $\mathrm{MH}=\mathrm{NH}$
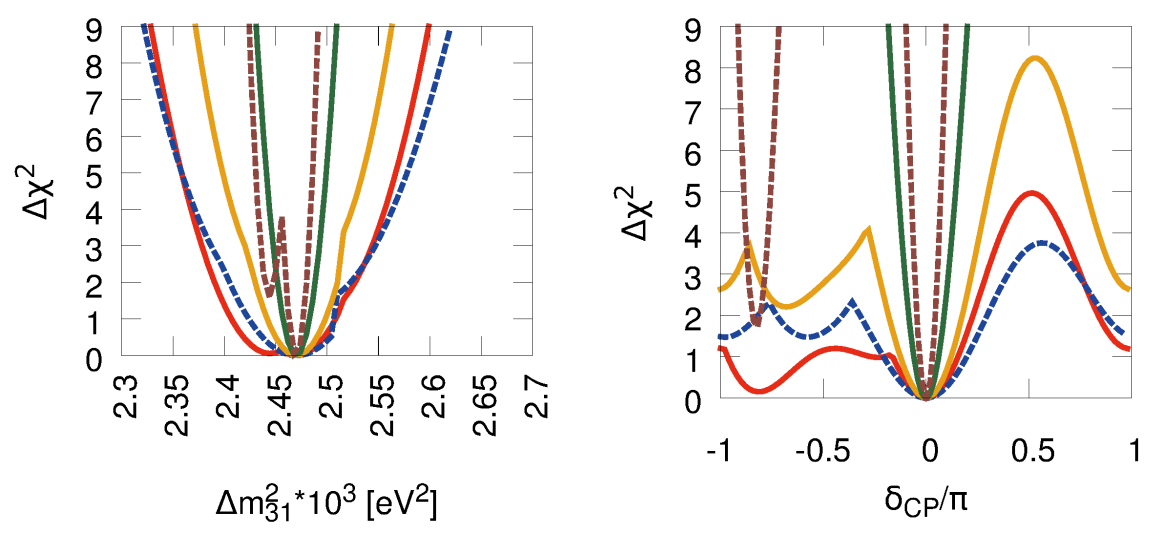

(c) $\delta_{C P}^{\text {true }}=0$, True $\mathrm{MH}=\mathrm{NH}$

(D) $\delta_{C P}^{\text {true }}=0$, True $\mathrm{MH}=\mathrm{NH}$
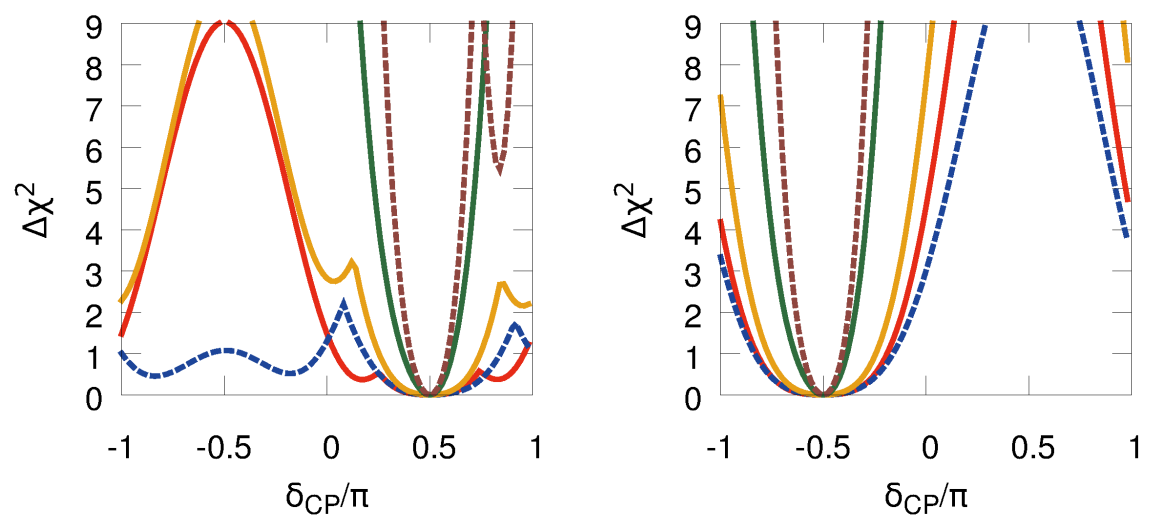

(E) $\delta_{C P}^{\text {true }}=\pi / 2$, True $\mathrm{MH}=\mathrm{NH}$

(F) $\delta_{C P}^{\text {true }}=-\pi / 2$, True $\mathrm{MH}=\mathrm{NH}$

FiguRE 6.17. Behavior of the $\Delta \chi^{2}$ as a function of each oscillation parameter. The Capozzi et al. best-fit values are assumed for the true values, except in the case of $\delta_{C P}$ which is given for $\delta_{C P}=-\pi / 2,0, \pi / 2$. All four samples are fit in each experiment. The nominal assumptions are used for systematics and $\nu: \bar{\nu}$ ratios. 

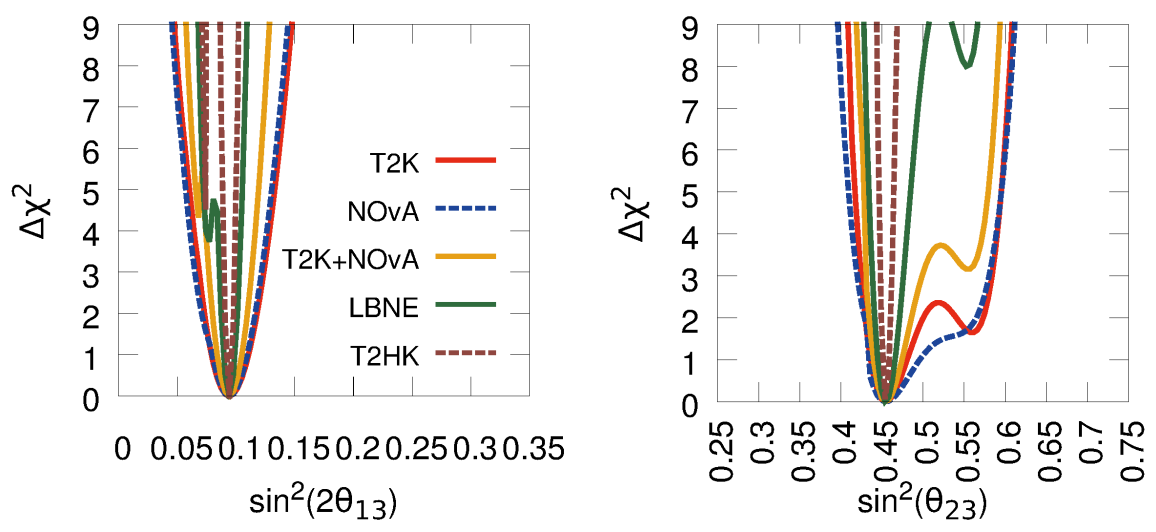

(A) $\delta_{C P}^{\text {true }}=0$, True $\mathrm{MH}=\mathrm{IH}$

(B) $\delta_{C P}^{\text {true }}=0$, True $\mathrm{MH}=\mathrm{IH}$
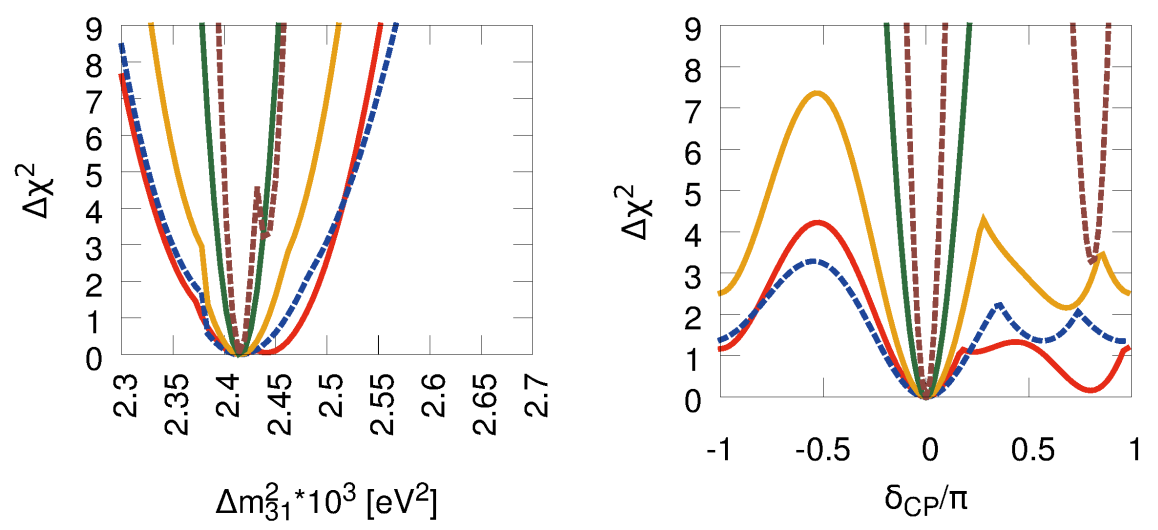

(c) $\delta_{C P}^{\text {true }}=0$, True $\mathrm{MH}=\mathrm{IH}$

(D) $\delta_{C P}^{\text {true }}=0$, True $\mathrm{MH}=\mathrm{IH}$
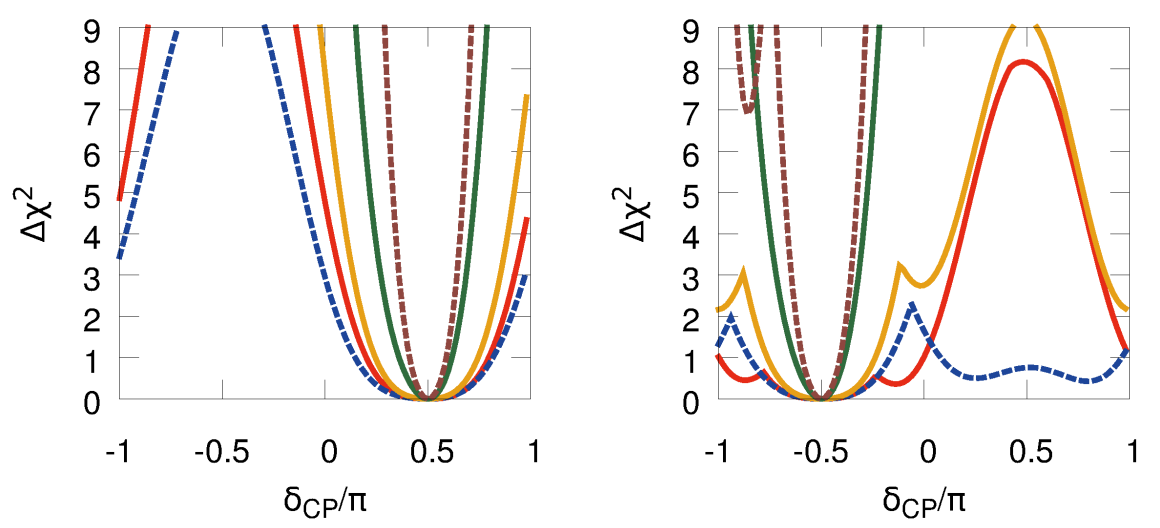

(E) $\delta_{C P}^{\text {true }}=\pi / 2$, True $\mathrm{MH}=\mathrm{IH}$

(F) $\delta_{C P}^{\text {true }}=-\pi / 2$, True $\mathrm{MH}=\mathrm{IH}$

Figure 6.18. As in Figure 6.17 but for True $\mathrm{MH}=\mathrm{IH}$. 


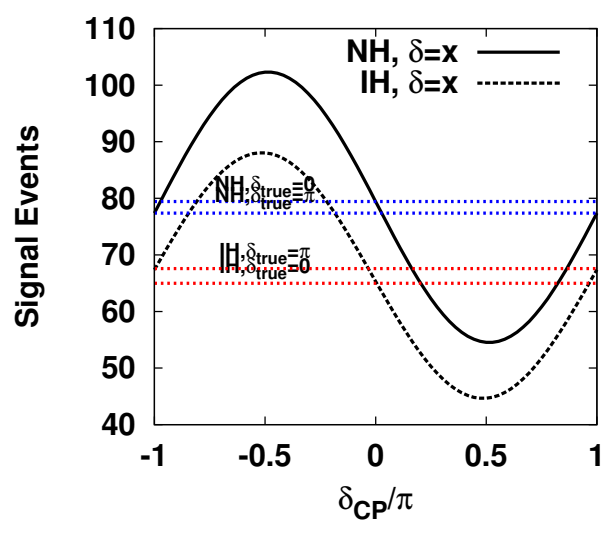

(A) $\mathrm{T} 2 \mathrm{~K} \nu_{e}$ appearance

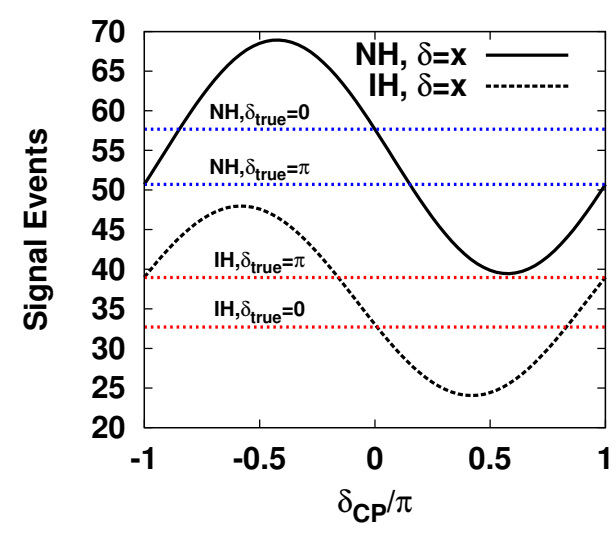

(B) $\mathrm{NO} \nu \mathrm{A} \nu_{e}$ appearance

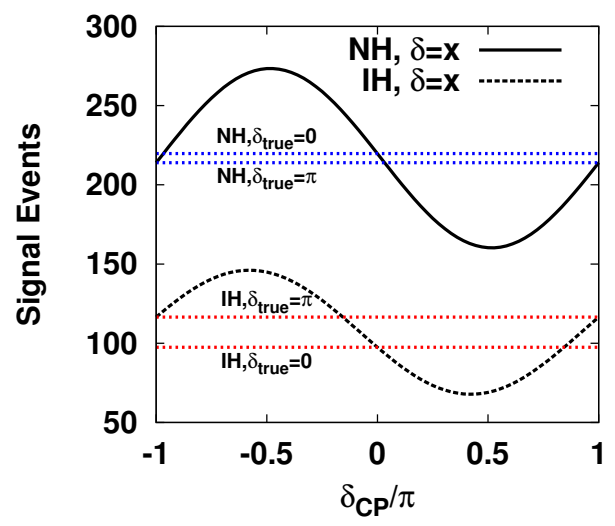

(C) LBNE $\nu_{e}$ appearance

FIGURE 6.19. Integrated number of $\nu_{e}$ appearance signal events predicted in $\mathrm{T} 2 \mathrm{~K}, \mathrm{NO} \nu \mathrm{A}$, and LBNE as a function of the true value of $\delta_{C P}$. The solid black curve is for the $\mathrm{NH}$ assumption and the dashed black curve is for the $\mathrm{IH}$ assumption. The horizontal blue(red) lines are for the $\mathrm{NH}(\mathrm{IH})$ assumption at $\delta_{C P}=0$ and $\delta_{C P}=\pi$ as indicated. The y-axis ranges are different for each figure due to the different number of signal events in each experiment. 
6.2.2. Resolutions. The resolution on a particular oscillation parameter is a useful metric with which to compare the performance of different experiments. Figure 6.20 shows the expected ranges and $1 \sigma$ resolutions, as a percentage, on $\sin ^{2}\left(2 \theta_{13}\right), \sin ^{2}\left(\theta_{23}\right)$, and $\Delta m_{31}^{2}$ along with the Capozzi et al. $20141 \sigma$ regions. The other oscillation parameters are profiled in the $\Delta \chi^{2}$ minimization so that, for example, the $\sin ^{2}\left(2 \theta_{13}\right)$ range includes limitations in the resolution that come from uncertainties in $\sin ^{2}\left(\theta_{23}\right), \Delta m_{31}^{2}$, and $\delta_{C P}$. The $1 \sigma$ resolution is defined as: Resolution $=\frac{\text { Upper-Lower }}{2 \cdot \text { Central }}$. The figure shows how well the oscillation parameters can be constrained if the true value of the parameter is that of the Capozzi et al. best fit value. The Capozzi et al. global fit includes more than just LBL experiment inputs, as described in Chapter 3, such as atmospheric, solar, and reactor measurements.

The figure shows that $\sin ^{2}\left(2 \theta_{13}\right)$ is least constrained in $\mathrm{T} 2 \mathrm{~K}$ and most constrained in T2HK. NO $\nu$ A does slightly better than $\mathrm{T} 2 \mathrm{~K}$ because it has some constraint on the $\mathrm{MH}$ and the combination does even better. Both LBNE and T2HK expected constraints are better than the current constraint on $\sin ^{2}\left(2 \theta_{13}\right)$.

Two values of $\sin ^{2}\left(\theta_{23}\right)$ are fit to show the ability to constrain $\sin ^{2}\left(\theta_{23}\right)$ if $\sin ^{2}\left(\theta_{23}\right)=0.44$ or if $\sin ^{2}\left(\theta_{23}\right)=0.5$. The resolutions are worse for $\sin ^{2}\left(\theta_{23}\right)=0.5$ because the oscillation probability changes more slowly around $\theta_{23}=\pi / 4$ in $\nu_{\mu}$ disappearance and $\bar{\nu}_{\mu}$ disappearance due to the dependence on $\sin ^{2}\left(2 \theta_{23}\right)$. The constraints for the $\sin ^{2}\left(\theta_{23}\right)=0.44$ value are improved by all of the measurements.

The expected resolutions on $\Delta m_{31}^{2}$ are generally an improvement on the current constraints. The $\Delta m_{31}^{2}$ resolution is partially limited by the granularity of the binning around the peak $\nu_{\mu}$ disappearance and $\bar{\nu}_{\mu}$ disappearance probability. The binning structure in T2K and T2HK has been optimized for this, with $50 \mathrm{MeV}$ bins in the region of $E_{\text {reco. }}$. The $\mathrm{NO} \nu \mathrm{A}$ 
and LBNE $E_{\text {reco }}$ binning is not optimized for this measurement with $250 \mathrm{MeV}$ and $125 \mathrm{MeV}$ bins respectively.

These ranges and resolutions can be read off the $\Delta \chi^{2}$ figures in Figure 6.17. Quantifying the experimental performance this way overlooks many subtle effects of degeneracies in the measurements. These effects are better addressed with a more detailed look at two dimensional plots of the $\Delta \chi^{2}$ in the next section.

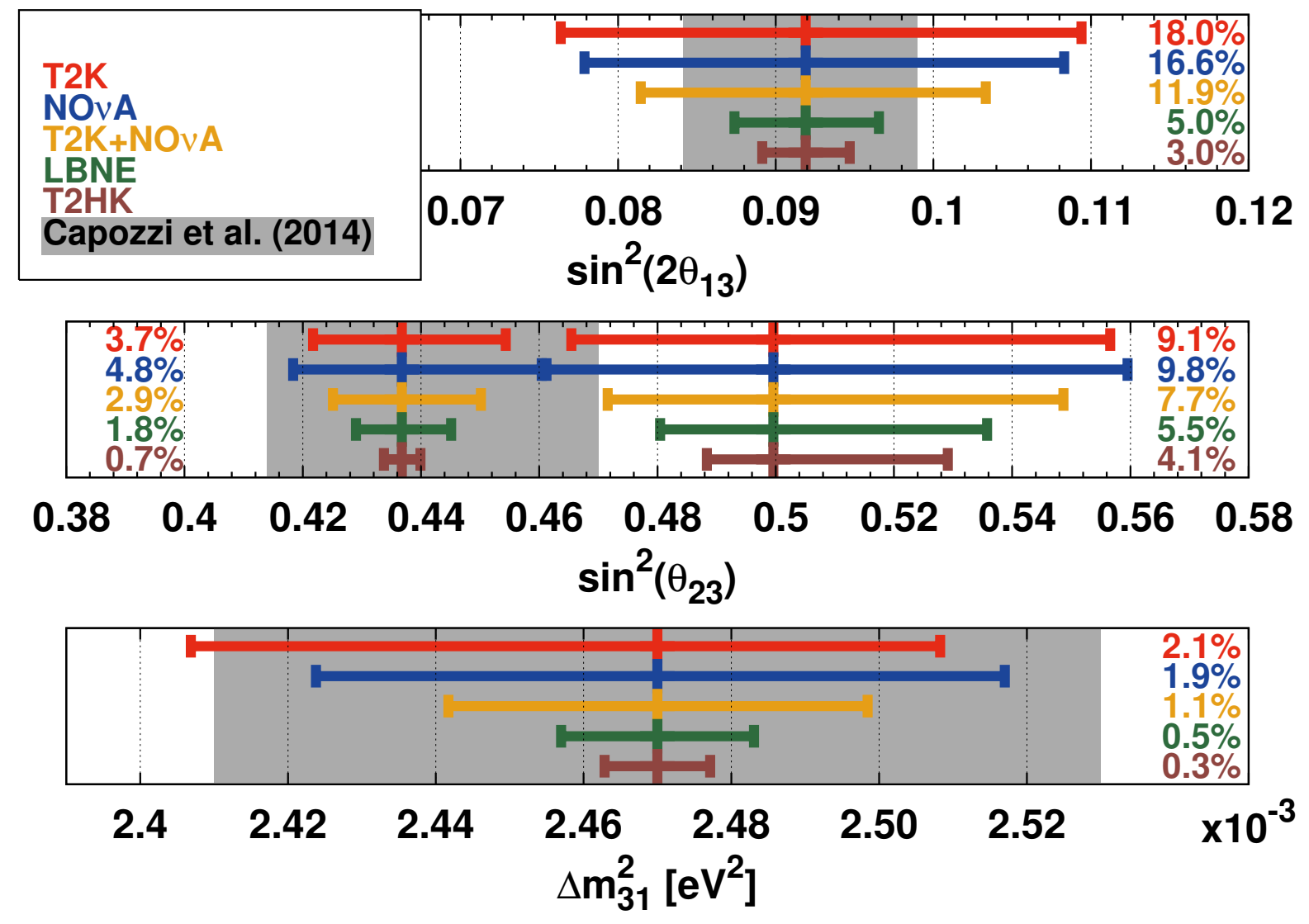

FigurE 6.20. The expected $1 \sigma$ resolutions on $\sin ^{2}\left(2 \theta_{13}\right), \sin ^{2}\left(\theta_{23}\right)$, and $\Delta m_{31}^{2}$ for the four experiments and a $\mathrm{T} 2 \mathrm{~K}+\mathrm{NO} \nu \mathrm{A}$ combined fit. The resolutions are also given as a percentage. The Capozzi et al. best-fit values are given in the gray background regions. All four samples are fit in each experiment: $\nu_{e}$ appearance, $\bar{\nu}_{e}$ appearance, $\nu_{\mu}$ disappearance, and $\bar{\nu}_{\mu}$ disappearance. The nominal assumptions are used for systematics and $\nu: \bar{\nu}$ ratios. The other oscillation parameters and the $\mathrm{MH}$ are profiled in the fit. The resolution on $\sin ^{2}\left(\theta_{23}\right)$ is given for both $\sin ^{2}\left(\theta_{23}\right)=0.437$ and $\sin ^{2}\left(\theta_{23}\right)=0.5$. 
6.2.3. Allowed Regions. Two dimensional allowed regions are contour plots in two of the oscillation parameters and show an experiment's ability to simultaneously constrain two of the parameters. They are generated in a similar way as the $\Delta \chi^{2}$ figures of the previous sections. The true values of the oscillation parameters are set to the Capozzi et al. global fit values while the fit value of the oscillation parameters being plotted are set to the $\mathrm{x}$-axis and $\mathrm{y}$-axis values. All of the other oscillation parameter fit values are profiled in the minimization of the $\Delta \chi^{2}$ and are constrained by the global fit $1 \sigma$ ranges. The $\Delta \chi^{2}$ is summed for all four samples: $\nu_{e}$ appearance, $\bar{\nu}_{e}$ appearance, $\nu_{\mu}$ disappearance, and $\bar{\nu}_{\mu}$ disappearance. Normalization systematics on the signal and background are included at the nominal levels. The mass hierarchy is also profiled by computing a $\Delta \chi^{2}$ using both the NH and IH assumptions for the fit spectra and taking the minimum between the two $\Delta \chi^{2}$ values. The $90 \%$ CL contours are draw assuming two DOF at $\Delta \chi^{2}=4.7$.

Figure 6.21 shows the $90 \%$ CL allowed regions in $\delta_{C P}$ vs $\sin ^{2}\left(2 \theta_{13}\right)$ for the four experiments and for the combination of $\mathrm{T} 2 \mathrm{~K}+\mathrm{NO} \nu \mathrm{A}$. Six figures are given for $\delta_{C P}^{\text {true }}=0, \delta_{C P}^{\text {true }}=\pi / 2$, and $\delta_{C P}^{\text {true }}=-\pi / 2$ with the True $\mathrm{MH}=\mathrm{NH}$ and True $\mathrm{MH}=\mathrm{IH}$ assumptions. The true values of $\delta_{C P}$ and $\sin ^{2}\left(2 \theta_{13}\right)$ are indicated by the cross. Figure 6.21.A shows the regions allowed in a fit for the $\delta_{C P}^{\text {true }}=0$ and True $\mathrm{MH}=\mathrm{NH}$ assumptions. $\mathrm{T} 2 \mathrm{~K}$ and $\mathrm{NO} \nu \mathrm{A}$ are not able to constrain $\delta_{C P}$ at the $90 \%$ level for these values while $\mathrm{T} 2 \mathrm{~K}+\mathrm{NO} \nu \mathrm{A}$ begins to exclude values of $\delta_{C P}$ around $\pi / 2$. LBNE constrains $\delta_{C P}$ and $\sin ^{2}\left(2 \theta_{13}\right)$ simultaneously with no degeneracies. T2HK constrains them as well but has a degenerate region near $\delta_{C P}=-\pi$ due to degeneracies between $\delta_{C P}$ and the $\mathrm{MH}$, as discussed in the previous sections.

Figure $6.21 \mathrm{~B}$ shows the regions allowed in a fit for the $\delta_{C P}^{\text {true }}=0$ and True $\mathrm{MH}=\mathrm{IH}$ assumptions. The T2K and NO $\nu$ A curves do not differ much from the True $\mathrm{MH}=\mathrm{NH}$ versions but the excluded region for the $\mathrm{T} 2 \mathrm{~K}+\mathrm{NO} \nu \mathrm{A}$ combination moves to $\delta_{C P}=-\pi / 2$. For both 
LBNE and T2HK there is a degenerate region in $\sin ^{2}\left(2 \theta_{13}\right)$, near $\sin ^{2}\left(2 \theta_{13}\right)=0.08$ and away from the true value, that comes about from uncertainties in $\sin ^{2}\left(\theta_{23}\right)$ allowing for a value of $\sin ^{2}\left(\theta_{23}\right)$ in the second octant and a lower value of $\sin ^{2}\left(2 \theta_{13}\right)$ in the fit. T2HK also sees the degenerate region from the uncertain $\mathrm{MH}$ move to near $\delta_{C P}=\pi$.

For non-zero values of $\delta_{C P}^{\text {true }}$, as shown in Figures $6.21 \mathrm{C}-\mathrm{F}, \mathrm{T} 2 \mathrm{~K}$ is able to exclude between $\pi / 2$ and $\pi$ radians surrounding the wrong value of $\delta_{C P}$, depending on the true $\mathrm{MH}$. NO $\nu \mathrm{A}$ excludes a similar region, but only in the cases where the $\mathrm{MH}$ and $\delta_{C P}$ are not degenerate. In cases where $\delta_{C P}$ and $\mathrm{MH}$ are degenerate, $\mathrm{NO} \nu \mathrm{A}$ is unable to exclude any of $\delta_{C P}$ at the $90 \%$ level. Both LBNE and T2HK are able to constrain $\delta_{C P}$ and $\sin ^{2}\left(2 \theta_{13}\right)$ without degeneracies except in the case of True $\mathrm{MH}=\mathrm{IH}$ as above.

There already exists constraints on $\sin ^{2}\left(2 \theta_{13}\right)$ that can be included to improve these expected allowed regions. As discussed in Section 5.4.2.4, the global fit constraints on $\sin ^{2}\left(2 \theta_{13}\right)$ can be added to the $\Delta \chi^{2}$ values and the contours regenerated. This is done in Figure 6.22 With this constraint in place, the degeneracies in $\sin ^{2}\left(2 \theta_{13}\right)$ in the True $\mathrm{MH}=\mathrm{IH}$ case are no longer present and the regions are slightly smaller in the $\delta_{C P}$ dimension as well. It is useful to find out what each experiment can measure without external input as well as with external input. There could be tensions, for example, in a future global fit between reactor SBL and accelerator LBL measurements of $\sin ^{2}\left(2 \theta_{13}\right)$. In that case a measurement using only the experimental LBL data may be necessary to quantify the differences between the measurements. 

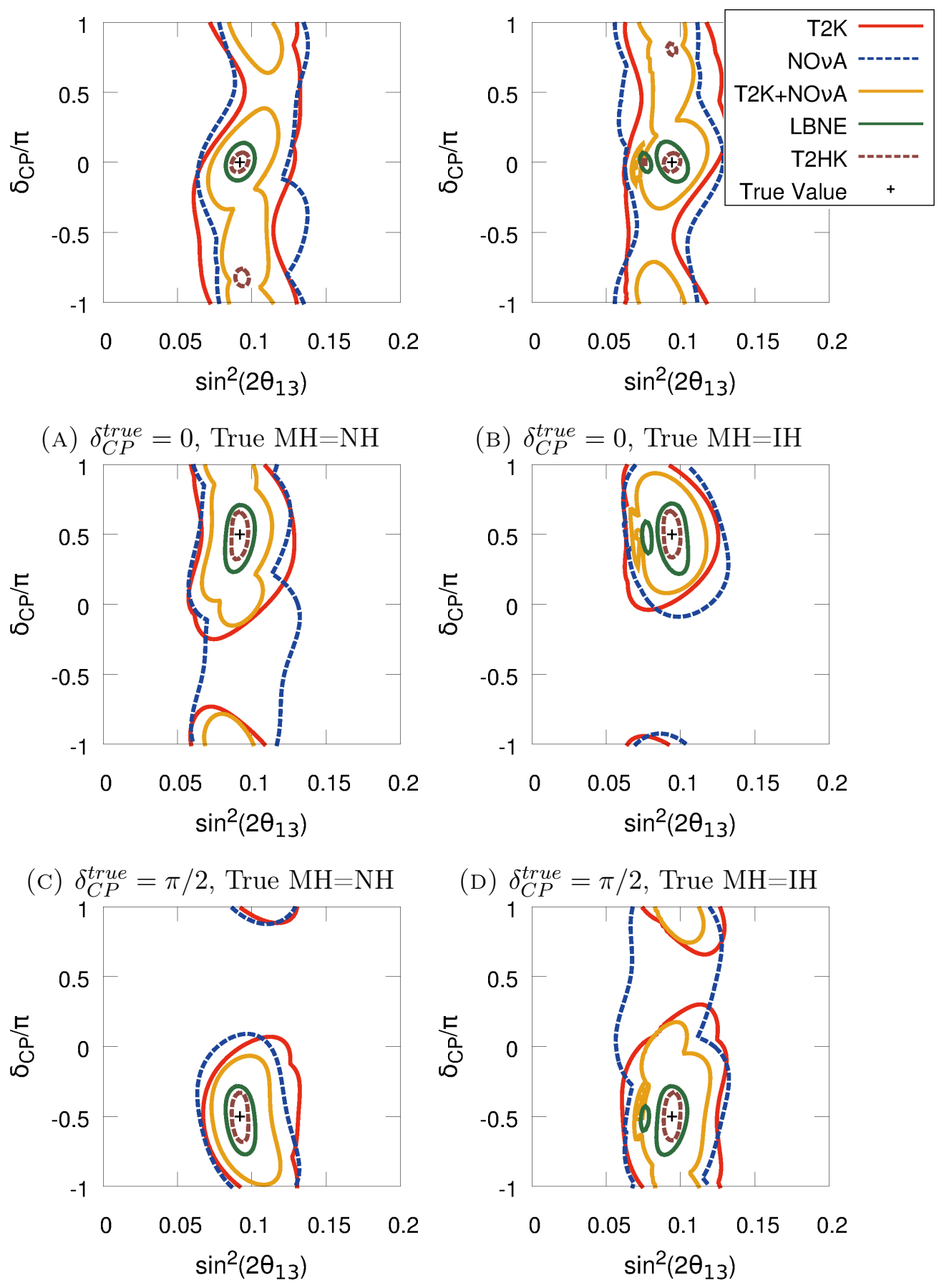

(E) $\delta_{C P}^{\text {true }}=-\pi / 2$, True $\mathrm{MH}=\mathrm{NH}$

(F) $\delta_{C P}^{\text {true }}=-\pi / 2$, True $\mathrm{MH}=\mathrm{IH}$

Figure 6.21. The $90 \% \mathrm{CL}$ allowed regions in $\delta_{C P}$ vs $\sin ^{2}\left(2 \theta_{13}\right)$ for the indicated true values. The Capozzi et al. best-fit values are assumed for the true values, except in the case of $\delta_{C P}$ which is given for $\delta_{C P}=-\pi / 2,0, \pi / 2$. All four samples are fit in each experiment. The nominal assumptions are used for systematics and $\nu: \bar{\nu}$ ratios. The other oscillation parameters and the $\mathrm{MH}$ are profiled. 

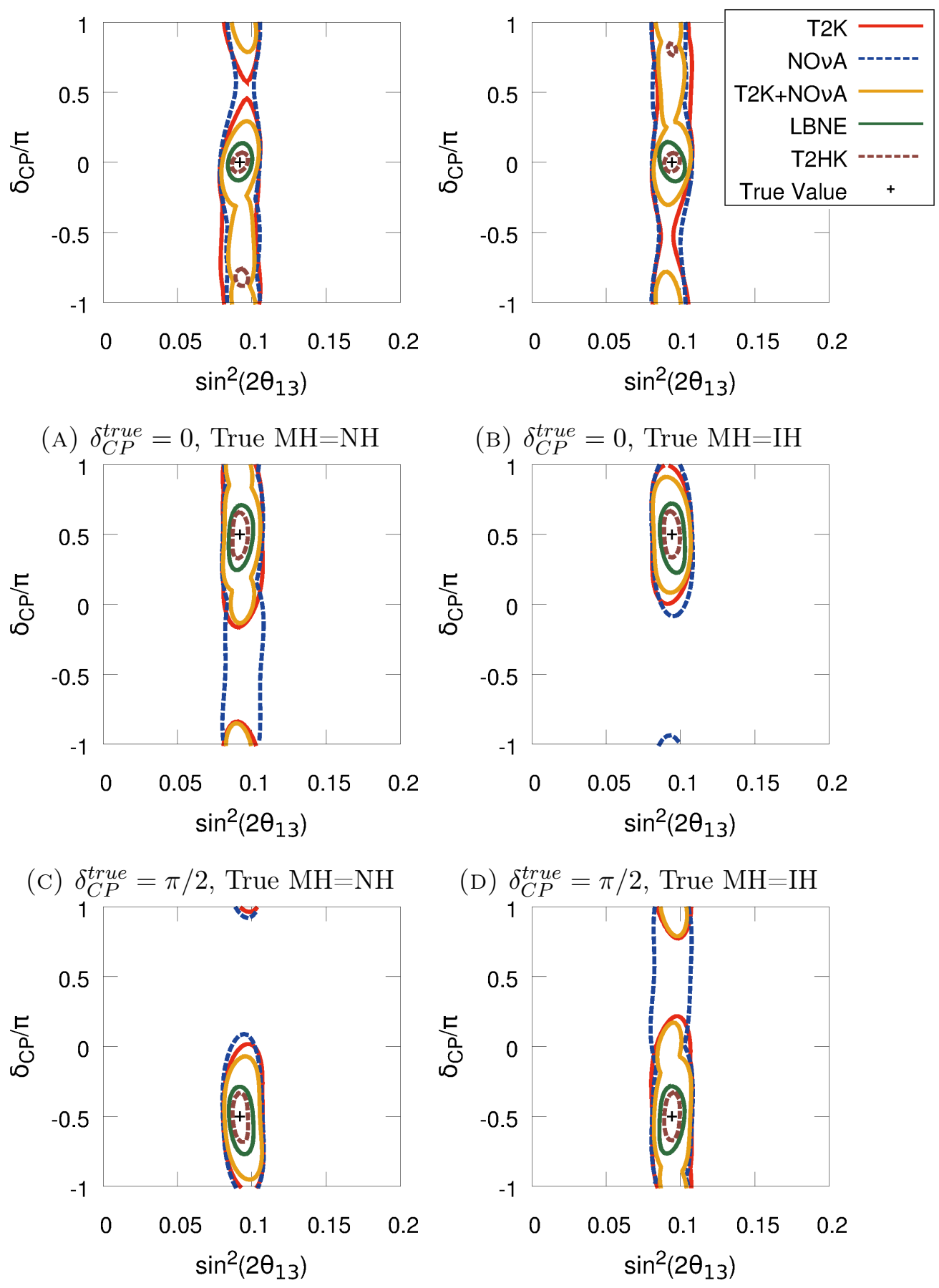

(E) $\delta_{C P}^{\text {true }}=-\pi / 2$, True $\mathrm{MH}=\mathrm{NH}$

(F) $\delta_{C P}^{\text {true }}=-\pi / 2$, True $\mathrm{MH}=\mathrm{IH}$

Figure 6.22. The $90 \% \mathrm{CL}$ allowed regions in $\delta_{C P}$ vs $\sin ^{2}\left(2 \theta_{13}\right)$ for the indicated true values. The Capozzi et al. best-fit values are assumed for the true values, except in the case of $\delta_{C P}$ which is given for $\delta_{C P}=-\pi / 2,0, \pi / 2$. All four samples are fit in each experiment. The nominal assumptions are used for systematics and $\nu: \bar{\nu}$ ratios. The measurement on $\sin ^{2}\left(2 \theta_{13}\right)$ from reactor experiments has been added to the $\Delta \chi^{2}$. The other oscillation parameters and the $\mathrm{MH}$ are profiled. 
Figure 6.23 shows a similar set of $90 \% \mathrm{CL}$ allowed regions in $\delta_{C P}$ vs $\sin ^{2}\left(\theta_{23}\right)$. The true values of $\delta_{C P}$ and $\sin ^{2}\left(\theta_{23}\right)$ are indicated by the cross. Figure $6.23 \mathrm{~A}$ shows the regions allowed in a fit for the $\delta_{C P}^{\text {true }}=0$ and True $\mathrm{MH}=\mathrm{NH}$ assumptions. Ignoring the second octant for a moment, the excluded regions of $\delta_{C P}$ for $\mathrm{T} 2 \mathrm{~K}, \mathrm{NO} \nu \mathrm{A}$, and $\mathrm{T} 2 \mathrm{~K}+\mathrm{NO} \nu \mathrm{A}$ are similar to those in the $\delta_{C P}$ vs $\sin ^{2}\left(2 \theta_{13}\right)$ regions. But there are degenerate regions in the second octant that make the excluded regions different in this case. These regions in $\delta_{C P}$ do not appear in the $\delta_{C P}$ vs $\sin ^{2}\left(2 \theta_{13}\right)$ regions of Figures 6.21 and 6.22 because in that fit the value of $\sin ^{2}\left(\theta_{23}\right)$ is constrained by the Capozzi et al. $1 \sigma$ global fit constraints. Here it is constrained only by the experiment itself. This means, for example, that in a $\mathrm{T} 2 \mathrm{~K}$ measurement a value of $\delta_{C P}^{\text {true }}=0$ in the first octant looks similar to a value of $\delta_{C P}^{\text {true }}=\pi / 2$ in the second octant. External measurements on $\sin ^{2}\left(\theta_{23}\right)$ will need to included in order to remove these degenerate regions for $\mathrm{T} 2 \mathrm{~K}, \mathrm{NO} \nu \mathrm{A}$, and $\mathrm{T} 2 \mathrm{~K}+\mathrm{NO} \nu \mathrm{A}$.

If the $\mathrm{MH}$ is assumed to be known, then the degenerate regions decrease dramatically. Figure 6.24 shows the effects of assuming the $\mathrm{MH}$ to be known on the $\delta_{C P}$ vs $\sin ^{2}\left(\theta_{23}\right)$ allowed regions. $\mathrm{NO} \nu \mathrm{A}$ especially benefits because the degeneracies between $\delta_{C P}$ and the $\mathrm{MH}$ are resolved. This also removes the degenerate region in $\delta_{C P}$ for T2HK. LBNE is completely unaffected because LBNE is able to resolve the MH on its own.

Maximal mixing, or $\sin ^{2}\left(\theta_{23}\right)=0.5$ also affects the contours in $\delta_{C P}$ vs $\sin ^{2}\left(\theta_{23}\right)$. Figure 6.25 shows the allowed regions again but with $\sin ^{2}\left(\theta_{23}\right)=0.5$ and with the MH profiled. The contours grow wider in $\sin ^{2}\left(\theta_{23}\right)$ as compared with Figure 6.23. They also grow wider in $\delta_{C P}$ for $\mathrm{T} 2 \mathrm{~K}, \mathrm{NO} \nu \mathrm{A}$, and $\mathrm{T} 2 \mathrm{~K}+\mathrm{NO} \nu \mathrm{A}$. 

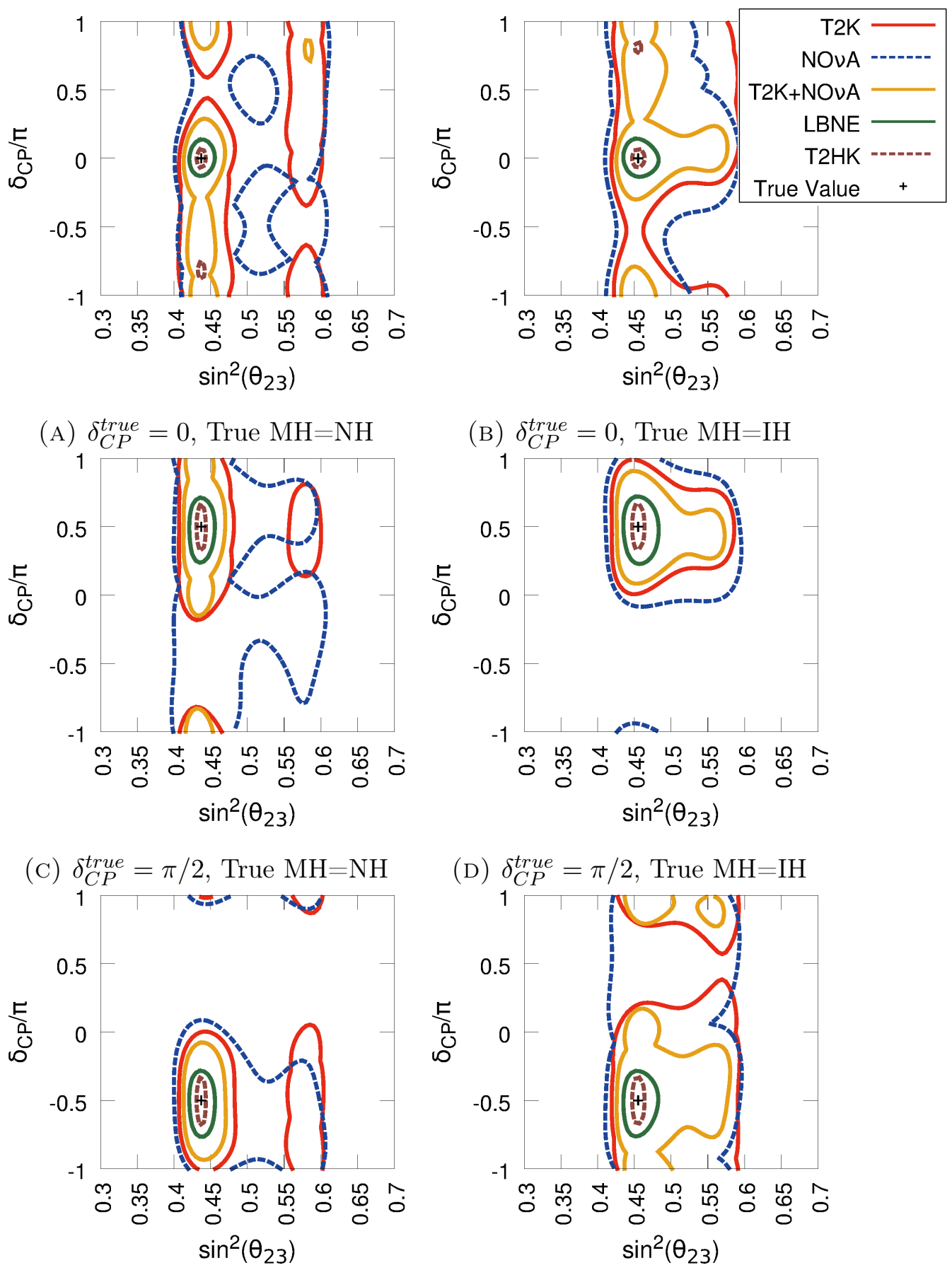

(E) $\delta_{C P}^{\text {true }}=-\pi / 2$, True $\mathrm{MH}=\mathrm{NH}$

(F) $\delta_{C P}^{\text {true }}=-\pi / 2$, True $\mathrm{MH}=\mathrm{IH}$

FiguRE 6.23. The 90\% CL allowed regions in $\delta_{C P}$ vs $\sin ^{2}\left(\theta_{23}\right)$ for the indicated true values. The Capozzi et al. best-fit values are assumed for the true values, except in the case of $\delta_{C P}$ which is given for $\delta_{C P}=-\pi / 2,0, \pi / 2$. All four samples are fit in each experiment. The nominal assumptions are used for systematics and $\nu: \bar{\nu}$ ratios. The other oscillation parameters and the $\mathrm{MH}$ are profiled. 

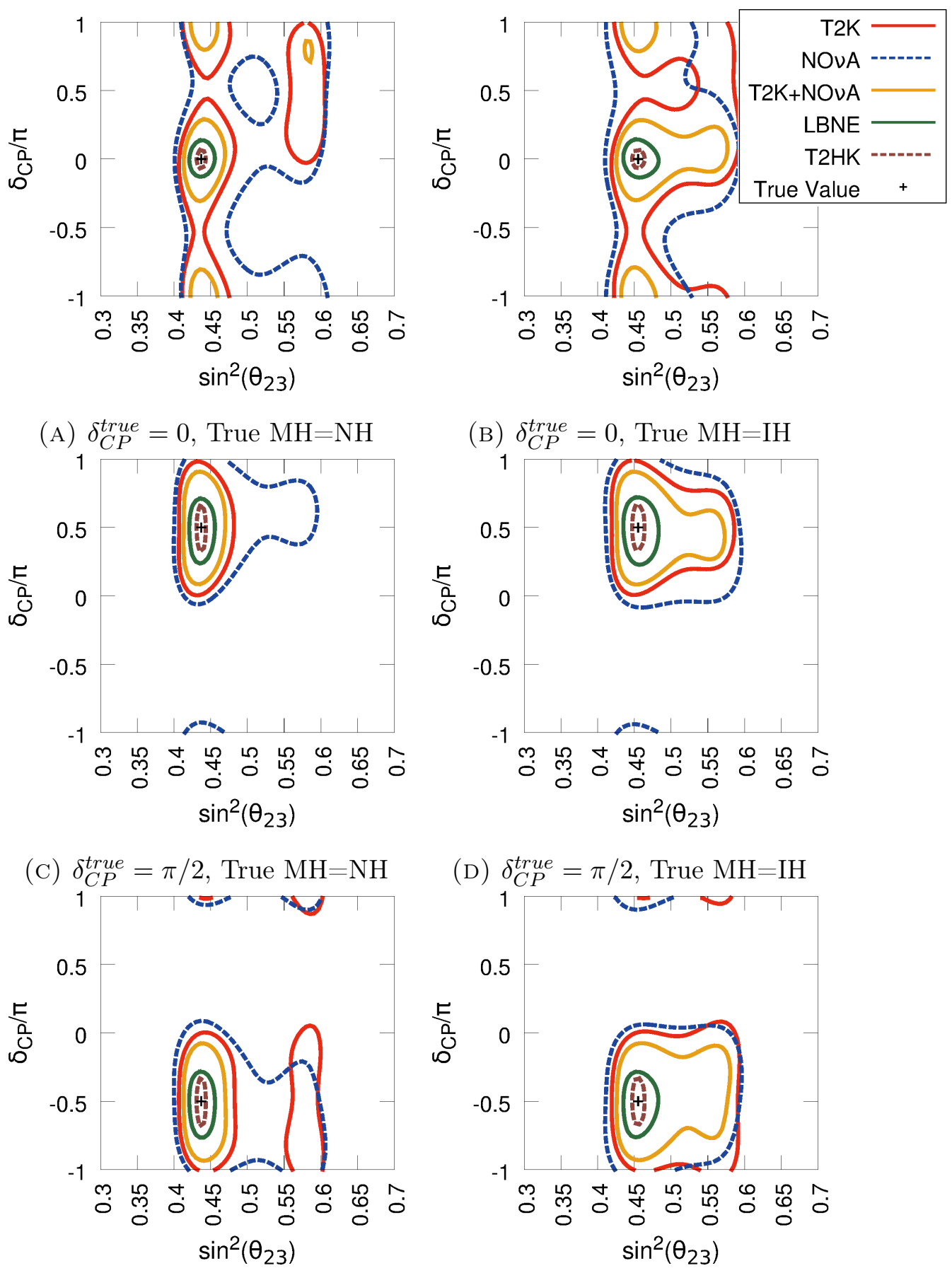

(E) $\delta_{C P}^{\text {true }}=-\pi / 2$, True $\mathrm{MH}=\mathrm{NH}$

(F) $\delta_{C P}^{\text {true }}=-\pi / 2$, True $\mathrm{MH}=\mathrm{IH}$

Figure 6.24. The $90 \%$ CL allowed regions in $\delta_{C P}$ vs $\sin ^{2}\left(\theta_{23}\right)$ for the indicated true values. The Capozzi et al. best-fit values are assumed for the true values, except in the case of $\delta_{C P}$ which is given for $\delta_{C P}=-\pi / 2,0, \pi / 2$. All four samples are fit in each experiment. The nominal assumptions are used for systematics and $\nu: \bar{\nu}$ ratios. The other oscillation parameters are profiled and the $\mathrm{MH}$ is assumed to be known. 

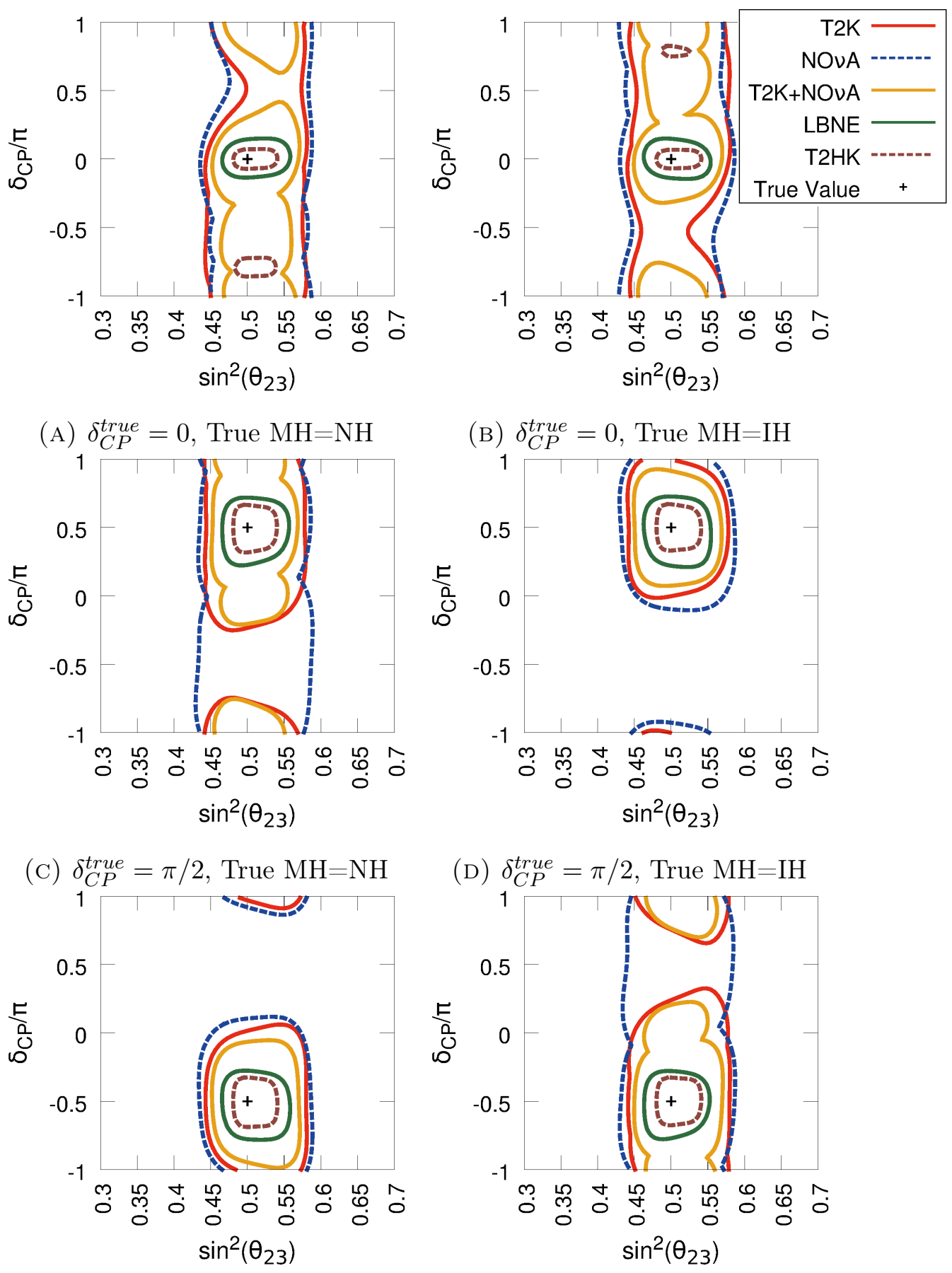

(E) $\delta_{C P}^{\text {true }}=-\pi / 2$, True $\mathrm{MH}=\mathrm{NH}$

(F) $\delta_{C P}^{\text {true }}=-\pi / 2$, True $\mathrm{MH}=\mathrm{IH}$

Figure 6.25. The 90\% CL allowed regions in $\delta_{C P}$ vs $\sin ^{2}\left(\theta_{23}\right)$ for the indicated true values and maximal mixing. The Capozzi et al. best-fit values are assumed for the true values, except in the case of $\delta_{C P}$ which is given for $\delta_{C P}=-\pi / 2,0, \pi / 2$ and for $\sin ^{2}\left(\theta_{23}\right)$ which is assumed to be $\sin ^{2}\left(\theta_{23}\right)=0.5$. All four samples are fit in each experiment. The nominal assumptions are used for systematics and $\nu: \bar{\nu}$ ratios. The other oscillation parameters and the $\mathrm{MH}$ are profiled. 
Figure 6.26 shows $90 \%$ CL allowed regions in $\sin ^{2}\left(2 \theta_{13}\right)$ vs $\sin ^{2}\left(\theta_{23}\right)$ for the True $\mathrm{MH}=\mathrm{NH}$ and True $\mathrm{MH}=\mathrm{IH}$ assumptions. No external measurements on $\sin ^{2}\left(2 \theta_{13}\right)$ and $\sin ^{2}\left(\theta_{23}\right)$ are applied here so that the experiments ability to constrain $\sin ^{2}\left(2 \theta_{13}\right)$ and $\sin ^{2}\left(\theta_{23}\right)$ on their own is indicated by the contours. For both the True $\mathrm{MH}=\mathrm{NH}$ and True $\mathrm{MH}=\mathrm{IH}$ and assumptions there is a degenerate region where the amplitude for $\nu_{e}$ appearance and $\bar{\nu}_{e}$ appearance, $\sin ^{2}\left(\theta_{23}\right) \sin ^{2}\left(2 \theta_{13}\right)$, is the same. The $\nu_{\mu}$ disappearance and $\bar{\nu}_{\mu}$ disappearance contributions are unable to constrain this degeneracy because they are predominantly dependent on $\sin ^{2}\left(2 \theta_{23}\right)$ so the octant is not resolved by this measurement. All four experiments are affected by this degeneracy and will need an external measurement to break the degeneracy.

Figure 6.27 applies the global fit constraints on $\sin ^{2}\left(2 \theta_{13}\right)$, discussed in Section 5.4.2.4. This breaks the degeneracy in $\mathrm{T} 2 \mathrm{~K}+\mathrm{NO} \nu \mathrm{A}$ for True $\mathrm{MH}=\mathrm{NH}$ and in $\mathrm{T} 2 \mathrm{HK}$ and LBNE for True $\mathrm{MH}=\mathrm{IH}$ by constraining $\sin ^{2}\left(2 \theta_{13}\right)$ to be within a small range that excludes the second octant degenerate region.

Figure 6.28 shows the contours under the assumption of maximal mixing, $\sin ^{2}\left(\theta_{23}\right)=0.5$, and without the global fit constraints on $\sin ^{2}\left(2 \theta_{13}\right)$. The correlations between $\sin ^{2}\left(2 \theta_{13}\right)$ and $\sin ^{2}\left(\theta_{23}\right)$ are more obvious in this Figure; as $\sin ^{2}\left(2 \theta_{13}\right)$ increases, $\sin ^{2}\left(\theta_{23}\right)$ must decrease to produce the same, degenerate event rate. 


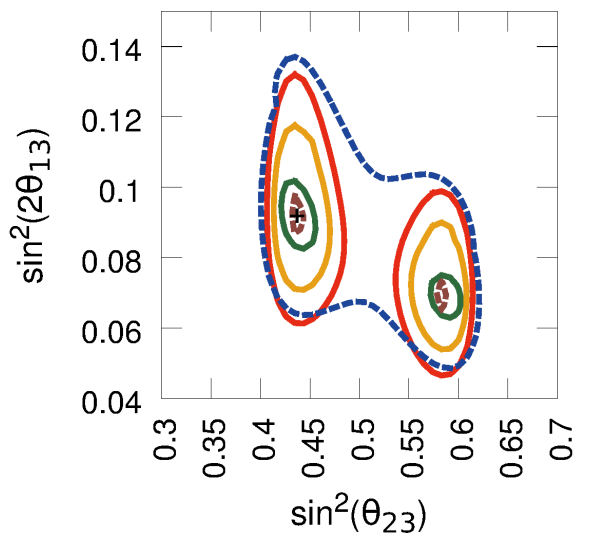

(A) $\delta_{C P}^{\text {true }}=0$, True $\mathrm{MH}=\mathrm{NH}$

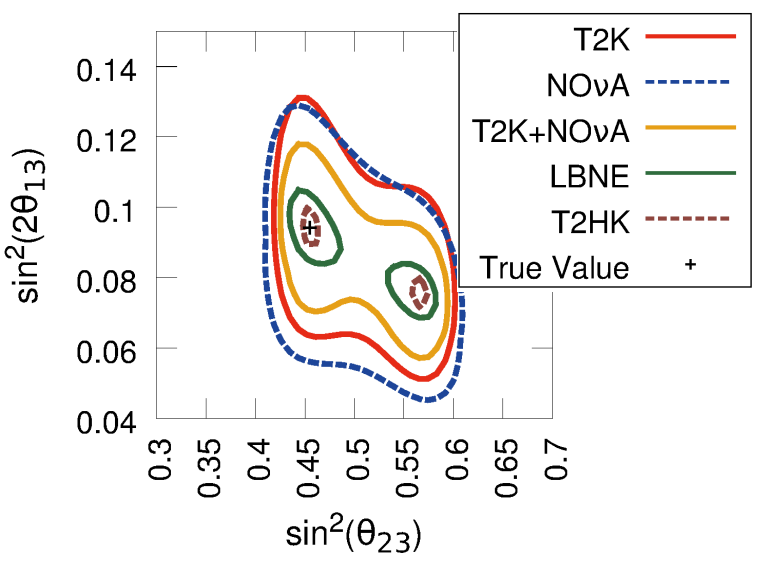

(B) $\delta_{C P}^{\text {true }}=0$, True $\mathrm{MH}=\mathrm{IH}$

Figure 6.26. The $90 \% \mathrm{CL}$ allowed regions in $\sin ^{2}\left(2 \theta_{13}\right)$ vs $\sin ^{2}\left(\theta_{23}\right)$ for the indicated true values. The Capozzi et al. best-fit values are assumed for the true values. All four samples are fit in each experiment. The nominal assumptions are used for systematics and $\nu: \bar{\nu}$ ratios. The other oscillation parameters and the $\mathrm{MH}$ are profiled.

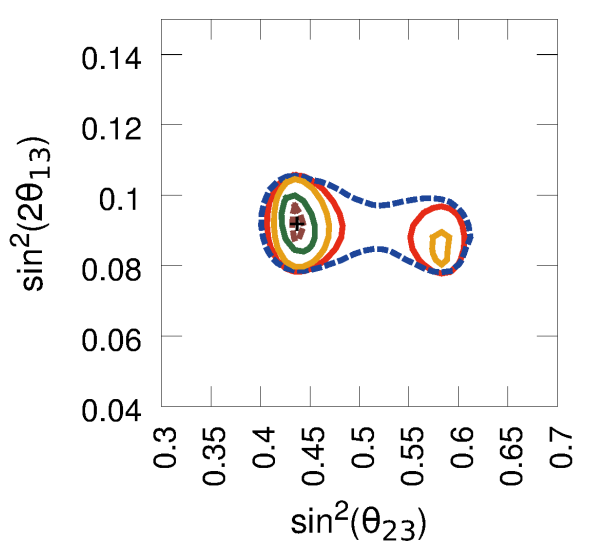

(A) $\delta_{C P}^{\text {true }}=0$, True $\mathrm{MH}=\mathrm{NH}$

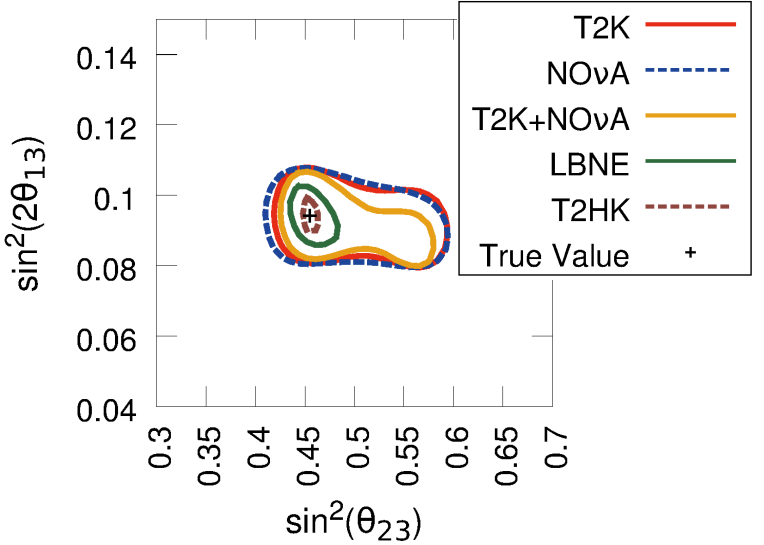

(в) $\delta_{C P}^{\text {true }}=0$, True $\mathrm{MH}=\mathrm{IH}$

Figure 6.27. The $90 \% \mathrm{CL}$ allowed regions in $\sin ^{2}\left(2 \theta_{13}\right)$ vs $\sin ^{2}\left(\theta_{23}\right)$ for the indicated true values. The Capozzi et al. best-fit values are assumed for the true values. All four samples are fit in each experiment. The nominal assumptions are used for systematics and $\nu: \bar{\nu}$ ratios. The measurement on $\sin ^{2}\left(2 \theta_{13}\right)$ from reactor experiments has been added to the $\Delta \chi^{2}$. The other oscillation parameters and the $\mathrm{MH}$ are profiled. 


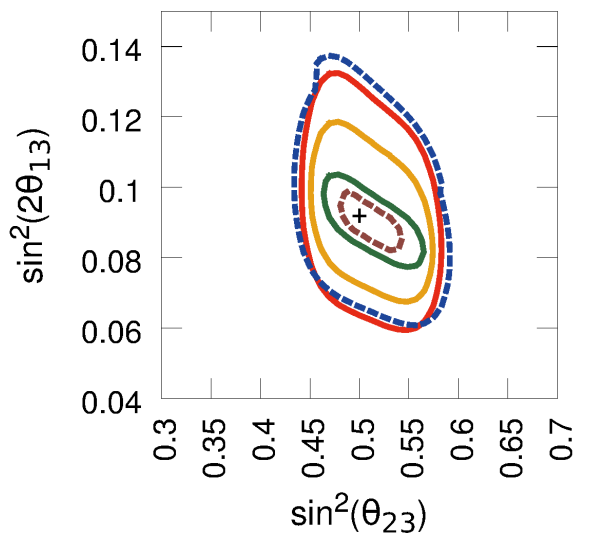

(A) $\delta_{C P}^{\text {true }}=0$, True $\mathrm{MH}=\mathrm{NH}$

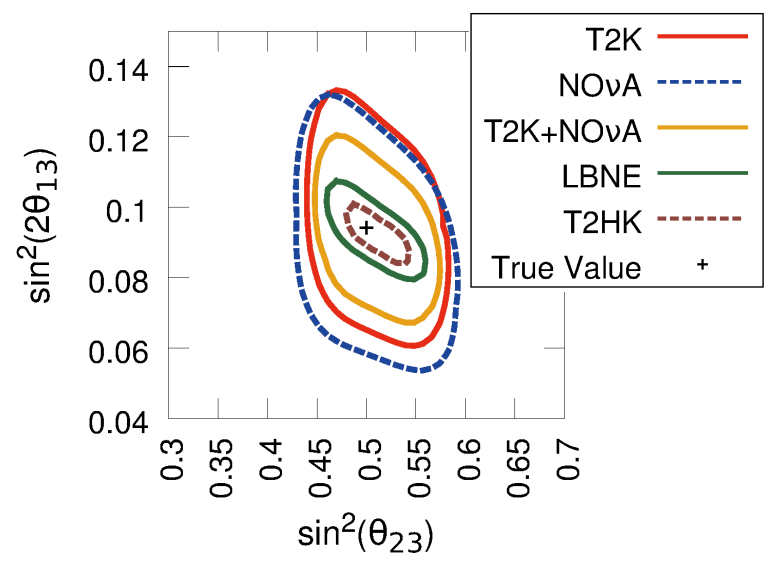

(B) $\delta_{C P}^{\text {true }}=0$, True $\mathrm{MH}=\mathrm{IH}$

Figure 6.28. The $90 \% \mathrm{CL}$ allowed regions in $\sin ^{2}\left(2 \theta_{13}\right)$ vs $\sin ^{2}\left(\theta_{23}\right)$ for the indicated true values and maximal mixing. The Capozzi et al. best-fit values are assumed for the true values except for $\sin ^{2}\left(\theta_{23}\right)=0.5$. All four samples are fit in each experiment. The nominal assumptions are used for systematics and $\nu: \bar{\nu}$ ratios. The other oscillation parameters and the $\mathrm{MH}$ are profiled. 
Concluding the set of $90 \%$ CL allowed regions, Figures 6.29 through 6.31 give the allowed regions in $\Delta m_{31}^{2}$ vs $\sin ^{2}\left(\theta_{23}\right)$ at the global best fit $\sin ^{2}\left(\theta_{23}\right)$ both unknown and known MH cases and with maximal mixing, $\sin ^{2}\left(\theta_{23}\right)=0.5$, and unknown MH. In Figure 6.29 the degeneracies in the octant are still present for $\mathrm{T} 2 \mathrm{~K}$ and $\mathrm{NO} \nu \mathrm{A}$, but $\mathrm{T} 2 \mathrm{~K}+\mathrm{NO} \nu \mathrm{A}$ is able to distinguish between the two octants in the NH, but not the IH. LBNE and T2HK are able to resolve the octant degeneracy on their own in this case, in contrast to the $\sin ^{2}\left(2 \theta_{13}\right)$ vs $\sin ^{2}\left(\theta_{23}\right)$ regions, because the constraint on $\sin ^{2}\left(2 \theta_{13}\right)$ is included when it is profiled during the $\Delta \chi^{2}$ minimization. With the maximal mixing in Figure 6.31, the degeneracies are replaced by wider regions in $\sin ^{2}\left(\theta_{23}\right)$. There is an interesting effect coming from profiling the MH in this Figure that is especially pronounced for T2HK. It is obvious that the T2HK allowed region is a merger of two allowed regions that are separated in $\Delta m_{31}^{2}$. These two regions come from the different values of $\Delta m_{31}^{2}$ in each mass hierarchy. Figure 6.30 shows the effect of assuming the MH to be known. In this case the width of the region in $\Delta m_{31}^{2}$ is reduced.

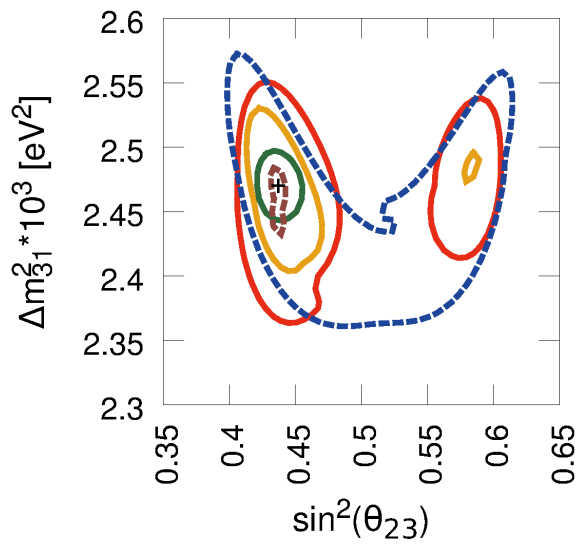

(A) $\delta_{C P}^{\text {true }}=0$, True $\mathrm{MH}=\mathrm{NH}$

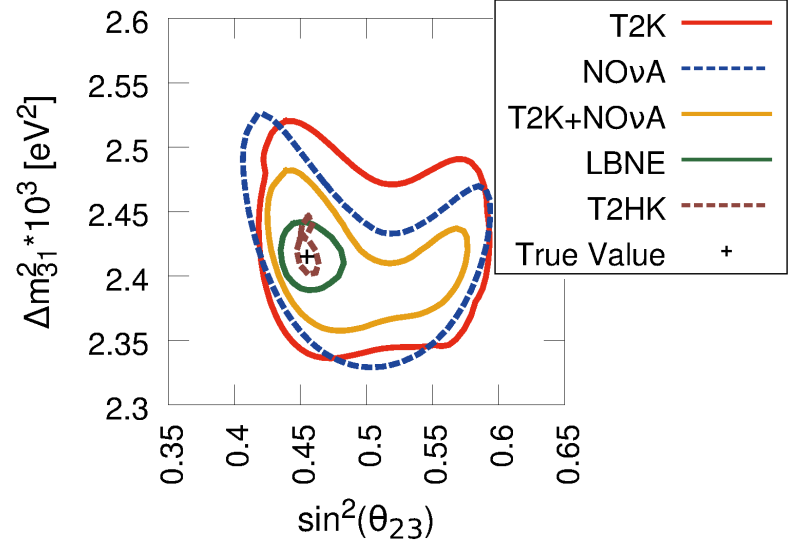

(B) $\delta_{C P}^{\text {true }}=0$, True $\mathrm{MH}=\mathrm{IH}$

Figure 6.29. The $90 \% \mathrm{CL}$ allowed regions in $\Delta m_{31}^{2}$ vs $\sin ^{2}\left(\theta_{23}\right)$ for the indicated true values. The Capozzi et al. best-fit values are assumed for the true values. All four samples are fit in each experiment. The nominal assumptions are used for systematics and $\nu: \bar{\nu}$ ratios. The other oscillation parameters and the $\mathrm{MH}$ are profiled. 


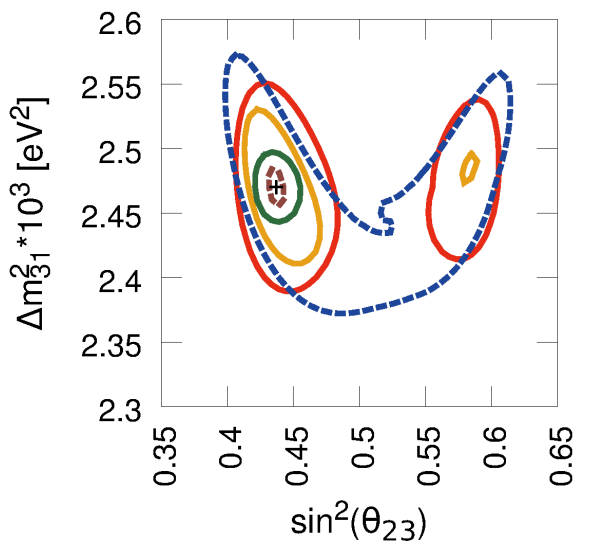

(A) $\delta_{C P}^{\text {true }}=0$, True $\mathrm{MH}=\mathrm{NH}(\mathrm{MH}$ known)

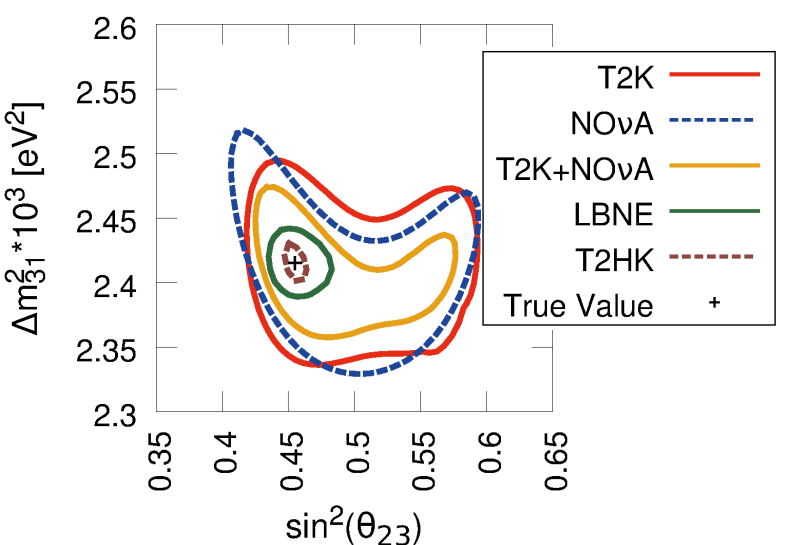

(B) $\delta_{C P}^{\text {true }}=0$, True $\mathrm{MH}=\mathrm{IH}(\mathrm{MH}$ known)

Figure 6.30. The $90 \% \mathrm{CL}$ allowed regions in $\Delta m_{31}^{2}$ vs $\sin ^{2}\left(\theta_{23}\right)$ for the indicated true values. The Capozzi et al. best-fit values are assumed for the true values. All four samples are fit in each experiment. The nominal assumptions are used for systematics and $\nu: \bar{\nu}$ ratios. The other oscillation parameters are profiled but the $\mathrm{MH}$ is assumed to be known.

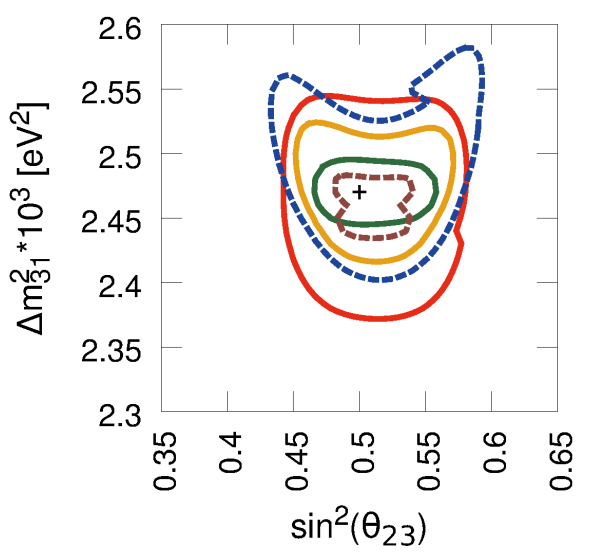

(A) $\delta_{C P}^{\text {true }}=0$, True $\mathrm{MH}=\mathrm{NH}$

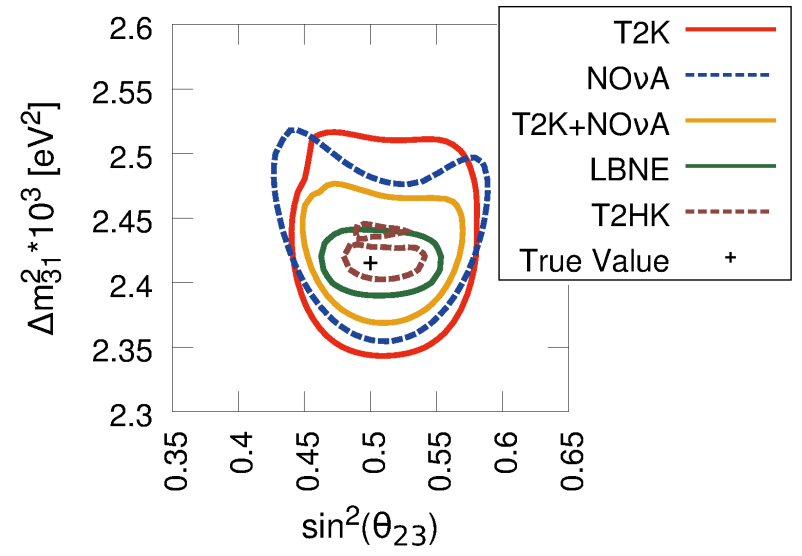

(B) $\delta_{C P}^{\text {true }}=0$, True $\mathrm{MH}=\mathrm{IH}$

Figure 6.31. The $90 \% \mathrm{CL}$ allowed regions in $\Delta m_{31}^{2} \mathrm{vs} \sin ^{2}\left(\theta_{23}\right)$ for the indicated true values. The Capozzi et al. best-fit values are assumed for the true values except for $\sin ^{2}\left(\theta_{23}\right)=0.5$. All four samples are fit in each experiment. The nominal assumptions are used for systematics and $\nu: \bar{\nu}$ ratios. The other oscillation parameters and the $\mathrm{MH}$ are profiled. 


\subsection{Sensitivity to CP Violation, Mass Hierarchy, and $\theta_{\mathbf{2} 3}$ Octant}

The $\Delta \chi^{2}$ method described in Chapter 5 can also be used to compute the ability of each experiment, or combination of experiments, to reject a null hypothesis. The $\Delta \chi^{2}$ will be used in this Section to compute the sensitivity to detecting $\mathrm{CP}$ violation, determining the $\mathrm{MH}$, and determining the $\theta_{23}$ octant.

The $\Delta \chi^{2}$, as defined in Equation 28, is used with the true set of oscillation parameters set to the global fit values but with one or more of the parameters set to other values to be tested in a raster scan. The test values used depend on the type of sensitivity regions being computed:

- CP violation $\left(\sin \delta_{C P} \neq 0\right)$ : The test value for $\delta_{C P}$ is constrained to either 0 and $\pi$. The test octant and test $\mathrm{MH}$ are left unconstrained.

- Mass Hierarchy: The test value for MH is constrained to the MH opposite the true MH. The test octant and test $\delta_{C P}$ are left unconstrained.

- $\theta_{23}$ octant: The test value for $\sin ^{2}\left(\theta_{23}\right)$ is constrained to the octant opposite that of the true value. The test $\mathrm{MH}$ and test $\delta_{C P}$ are left unconstrained.

All of the other test oscillation parameters are profiled. 
6.3.1. Pre-fit And Post-fit Spectra. Figures 6.32 to 6.47 depict the process of fitting the four samples in each experiment by showing the true event spectrum overlaid with a test spectrum before the systematic parameters are fit (pre-fit) and after the fit (post-fit). The post-fit spectrum includes the effects of minimizing the $\Delta \chi^{2}$ with respect to the oscillation parameters and with respect to normalization uncertainties on the signal and the background. The pre-fit and post-fit background spectra are shown in the dashed lines. The histograms are stacked so that the true, pre-fit, and post-fit spectra are for signal plus background. The values of the total $\Delta \chi^{2}$ comparing the true to the pre-fit and post-fit spectra are indicated and include the contributions from $\nu_{e}$ appearance, $\bar{\nu}_{e}$ appearance, $\nu_{\mu}$ disappearance, and $\bar{\nu}_{\mu}$ disappearance. All of the figures assume True $\mathrm{MH}=\mathrm{NH}$. The right y-axis of the figures represents the $\Delta \chi^{2}$ value, computed using Equation 28, in the yellow histogram. This value is computed using the true and post-fit spectrum so that it includes the effects of systematics uncertainties but does not include the penalty terms that constrain the systematics nuisance parameters that are in line two of Equation 28. These spectral comparisons answer a few questions about the sensitivities:

- How are the sensitivities affected by systematic uncertainties?

- Where does the sensitivity to the oscillation hypothesis come from in terms of $E_{\text {reco }}$ ?

- How do the $\nu_{e}$ appearance, $\bar{\nu}_{e}$ appearance, $\nu_{\mu}$ disappearance, and $\bar{\nu}_{\mu}$ disappearance samples contribute to the total $\Delta \chi^{2}$ ?

If the cross-sections and flux were perfectly known, or flat with respect to energy, then these questions could be addressed in a very straight forward way because they would depend only on the values and uncertainties of the oscillation parameters. In the case of realistic flux and detector responses and systematics it is necessary to answer these questions by evaluating the event spectra for each sample in each experiment. These questions will be addressed in the 
following sections for $\delta_{C P}$ variations, $\mathrm{MH}$ variations, and $\sin ^{2}\left(\theta_{23}\right)$ variations for distinguish the octant and maximal mixing.

6.3.1.1. Spectra: $\delta_{C P}$ Variations. Figures 6.32 to 6.35 show the spectra for all four samples in each of the experiments for variations in $\delta_{C P}$. The true event spectrum is for $\delta_{C P}=0$ and the pre-fit and post-fit spectra are for $\delta_{C P}=-\pi / 2$. This comparison does not represent the full sensitivity to $\mathrm{CP}$ violation, but it gives an idea of where the best-case sensitivity to $\mathrm{CP}$ violation comes from in terms of the event spectra and how the systematic uncertainties diminish the sensitivity.

Figure 6.32 shows these spectra for T2K. The sensitivity to these variations in $\delta_{C P}$ comes from the difference between the expected event spectrum at $\delta_{C P}=-\pi / 2$ (red and blue lines) and $\delta_{C P}=0$ (gray areas). The difference between the blue line and the gray histogram visually depicts the sensitivity to detecting the effects of these different $\delta_{C P}$ values after systematic uncertainties have been included. Most of the $\Delta \chi^{2}$ contribution comes from $6.32 \mathrm{~A}$ for $\nu_{e}$ appearance. There is very little contribution to this sensitivity from $\nu_{\mu}$ disappearance and $\bar{\nu}_{\mu}$ disappearance. The total $\Delta \chi^{2}$ before the fit is 6 and 4 after the fit, indicating a $2 \sigma$ difference between the spectra for these particular $\delta_{C P}$ values. With much larger POT and detector mass, as in T2HK in Figure 6.35, these scale to much larger values, reaching greater than $5 \sigma$ significance.

For $\mathrm{NO} \nu \mathrm{A}$, in Figure 6.33 , these variations in $\delta_{C P}$ cause the largest $\Delta \chi^{2}$ contribution in the $\bar{\nu}_{e}$ appearance sample. This sample has the largest contribution because the total number of events at $\delta_{C P}=-\pi / 2$ is more similar to $\delta_{C P}=0$ for $\nu_{e}$ appearance than for $\bar{\nu}_{e}$ appearance. There is very little contribution to the $\Delta \chi^{2}$ from $\nu_{\mu}$ disappearance and $\bar{\nu}_{\mu}$ disappearance for $\mathrm{NO} \nu \mathrm{A} .{ }^{1}$

\footnotetext{
${ }^{1}$ Although there is often little contribution to the $\Delta \chi^{2}$ from $\nu_{\mu}$ disappearance and $\bar{\nu}_{\mu}$ disappearance, including these samples helps to constrain the other oscillation parameters in the $\Delta \chi^{2}$ minimization.
} 
For LBNE, in Figure 6.34, the largest $\Delta \chi^{2}$ contribution is in the $\nu_{e}$ appearance sample. There is an obvious shape dependence visible in these spectra. The first oscillation maximum is dominant with the $\Delta \chi^{2}$ contribution coming from $E_{\text {reco }}$ values that are shifted from the oscillation peak due to the shift in energy of the fit spectrum at $\delta_{C P}=-\pi / 2$ with respect to the true spectrum at $\delta_{C P}=0$. There is also a contribution from the second oscillation maximum below $1 \mathrm{GeV}$, though it is significantly smaller than the $\Delta \chi^{2}$ contribution from the first oscillation maximum. 


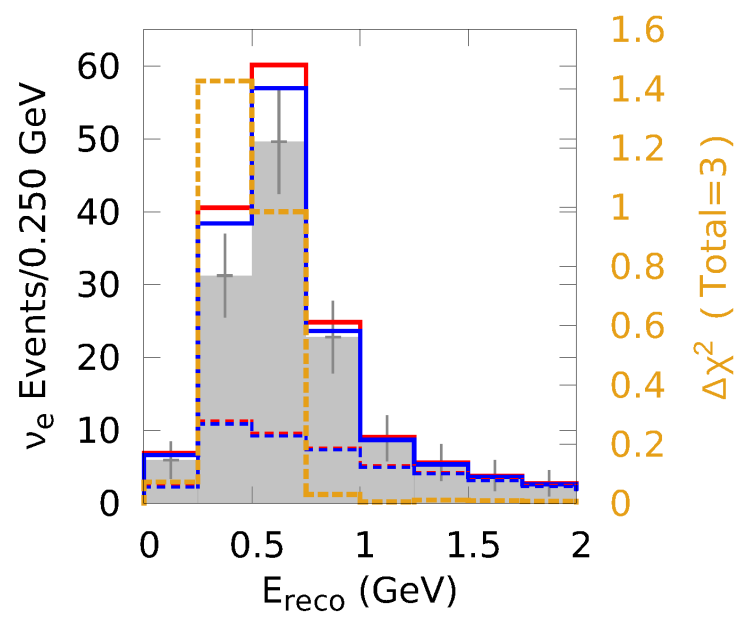

(A) $\nu_{e}$ appearance

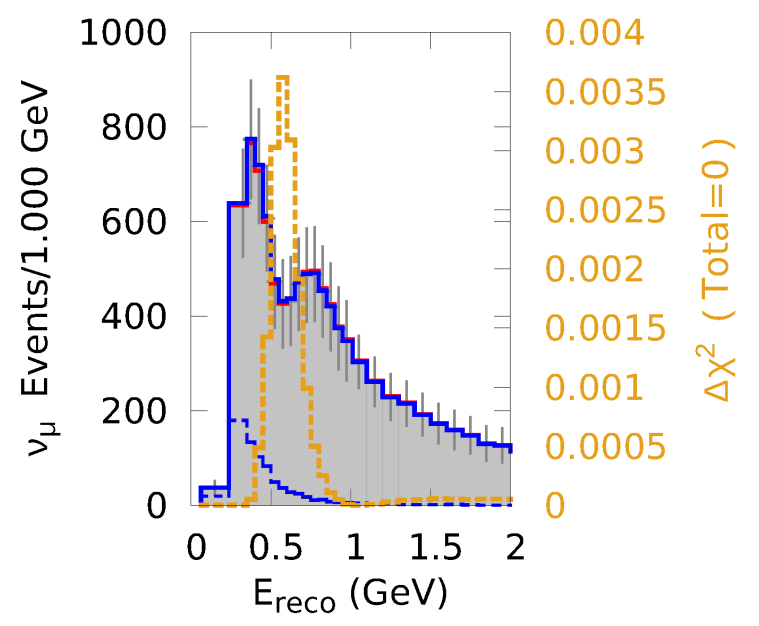

(C) $\nu_{\mu}$ disappearance

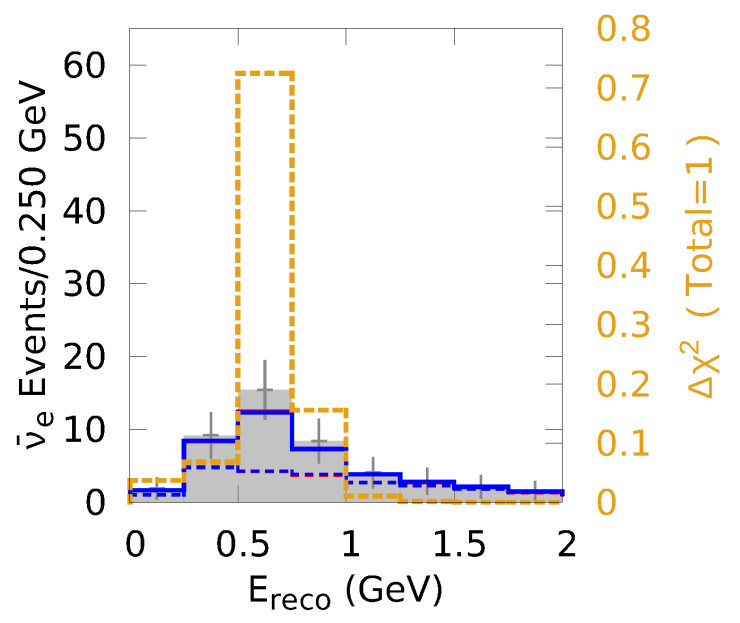

(B) $\bar{\nu}_{e}$ appearance

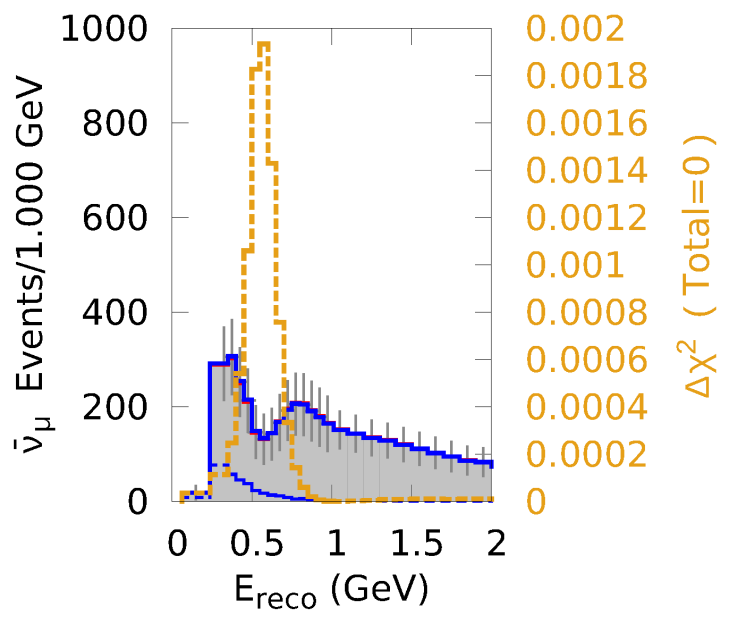

(D) $\bar{\nu}_{\mu}$ disappearance

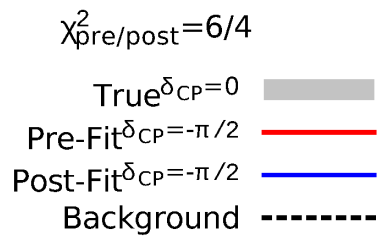

Figure 6.32. T2K pre-fit (red) and post-fit (blue) spectra, each with $\delta_{C P}=$ $-\pi / 2$, compared to the true spectrum for $\delta_{C P}^{\text {true }}=0$. The background spectra are given by the dashed lines and the signal+background spectra are given by the solid lines and gray region. The error bars on the gray region indicate statistical uncertainties. The total $\Delta \chi^{2}$ before and after the fit are indicated and include $\nu_{e}$ appearance, $\bar{\nu}_{e}$ appearance, $\nu_{\mu}$ disappearance, and $\bar{\nu}_{\mu}$ disappearance. The nominal assumptions are used for systematics and $\nu: \bar{\nu}$ ratios. The $\Delta \chi^{2}$ as a function of $E_{\text {reco }}$ is given by the yellow histogram. 


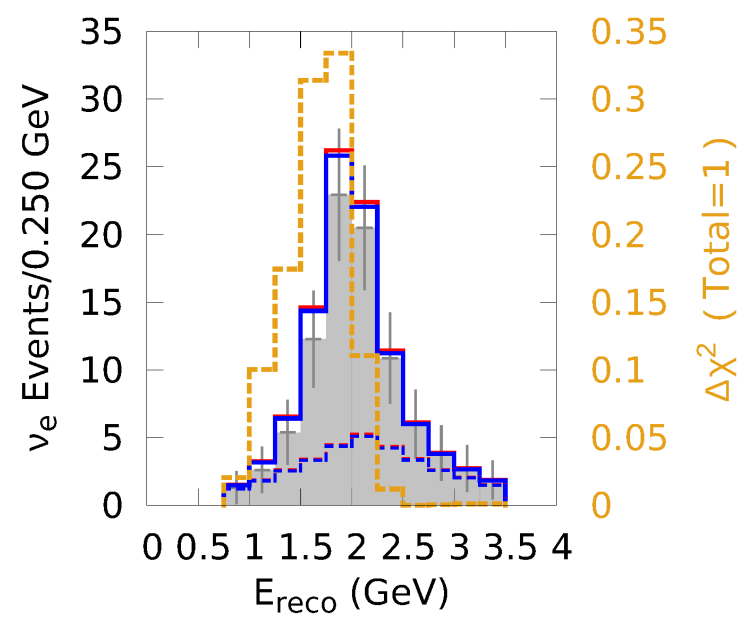

(A) $\nu_{e}$ appearance

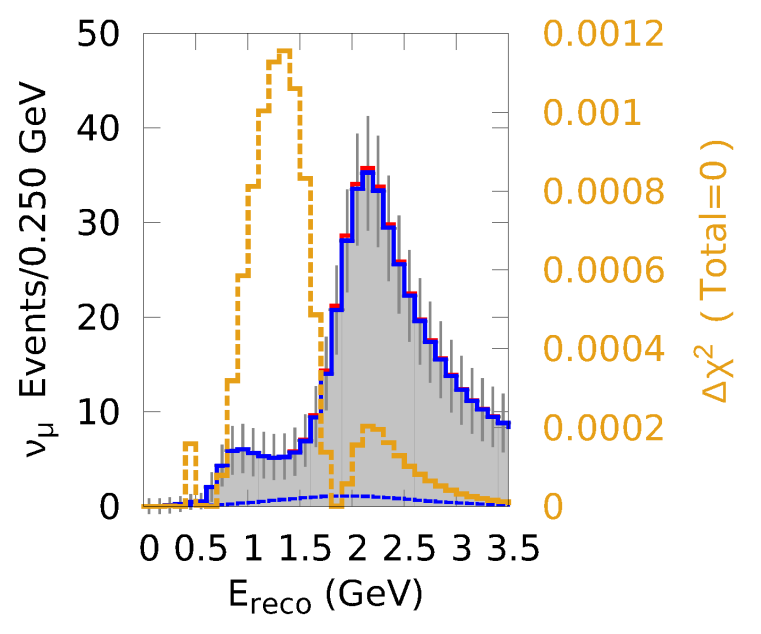

(C) $\nu_{\mu}$ disappearance

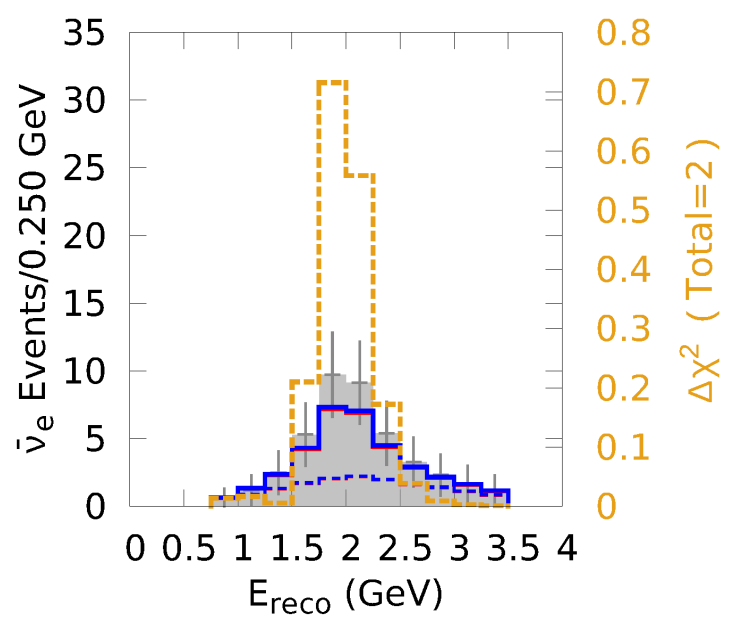

(B) $\bar{\nu}_{e}$ appearance

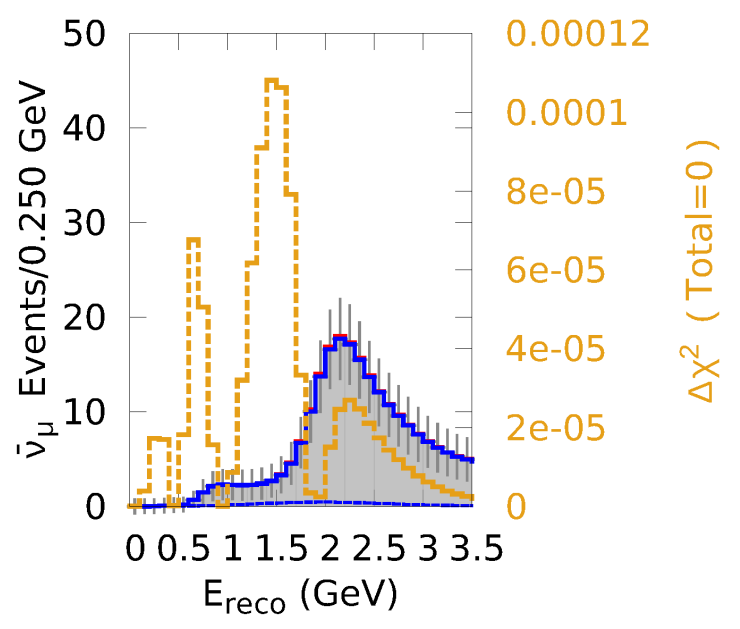

(D) $\bar{\nu}_{\mu}$ disappearance

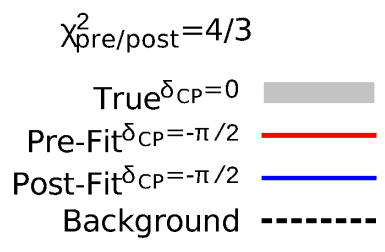

Figure 6.33. As in Figure 6.36 but for $\mathrm{NO} \nu \mathrm{A}$. 


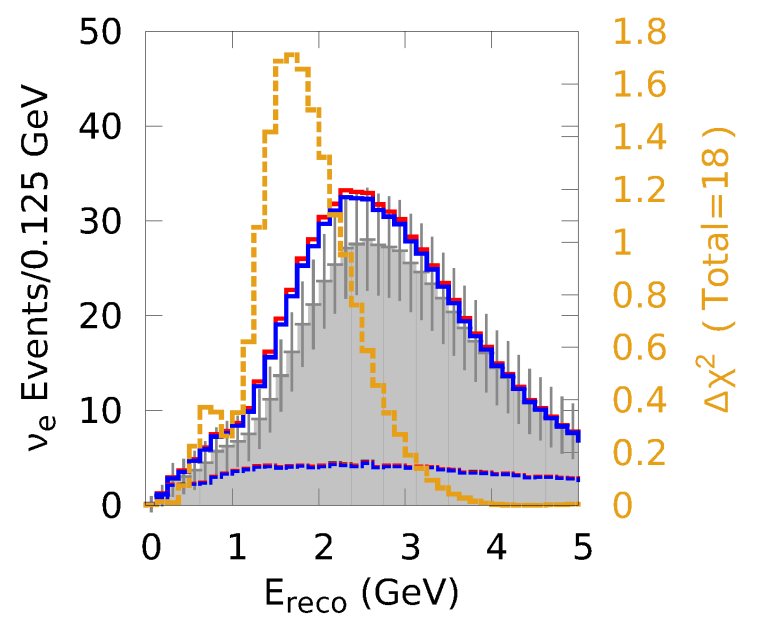

(A) $\nu_{e}$ appearance

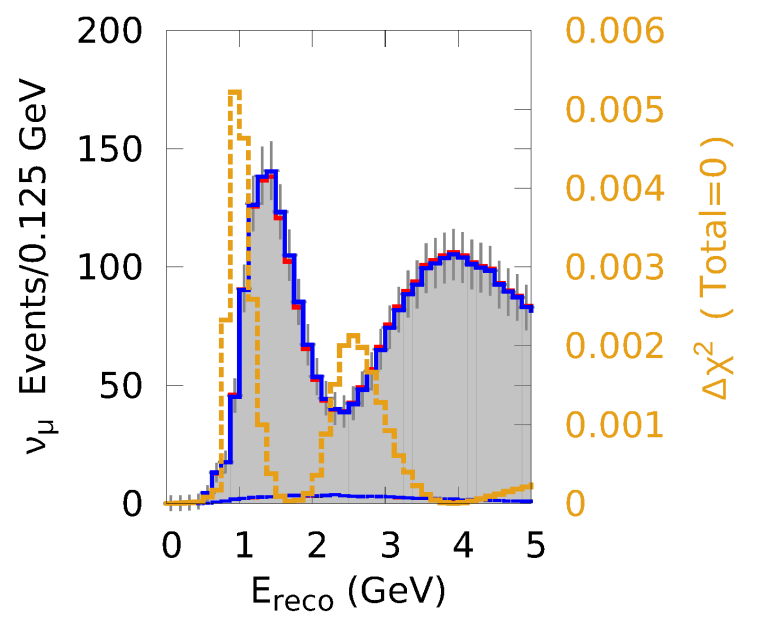

(C) $\nu_{\mu}$ disappearance

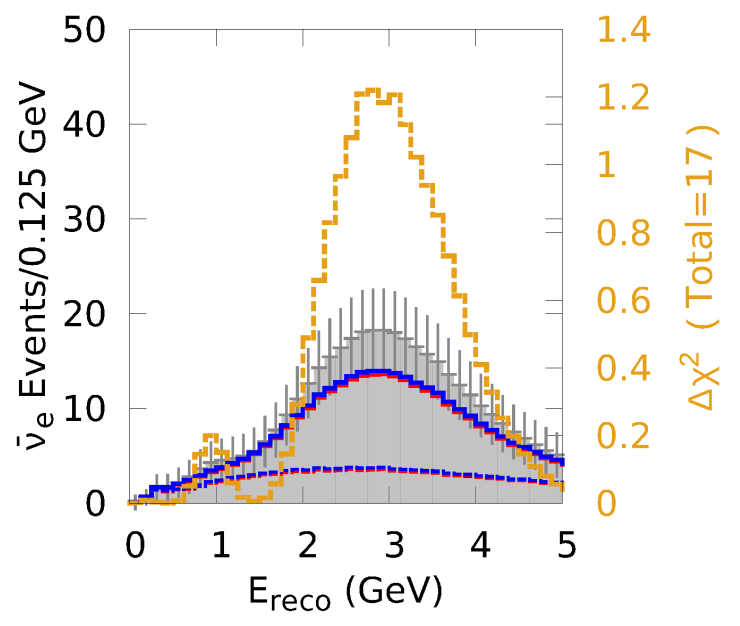

(в) $\bar{\nu}_{e}$ appearance

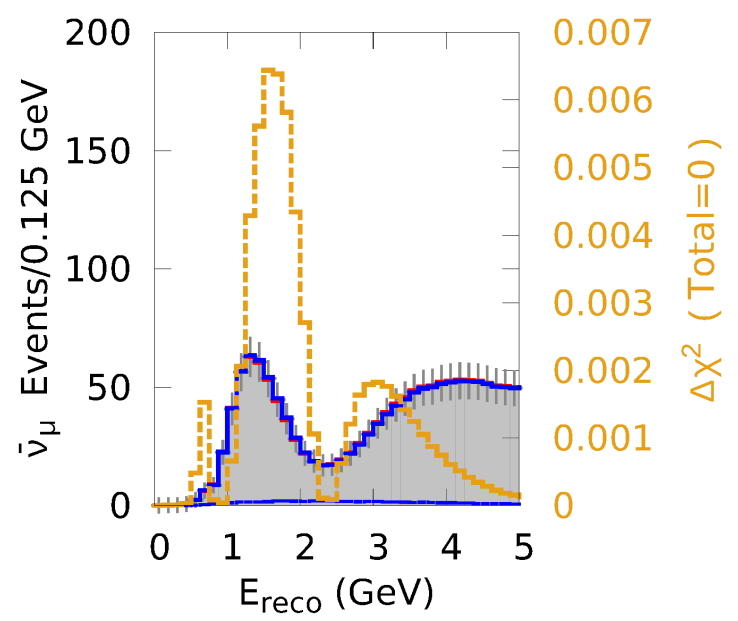

(D) $\bar{\nu}_{\mu}$ disappearance

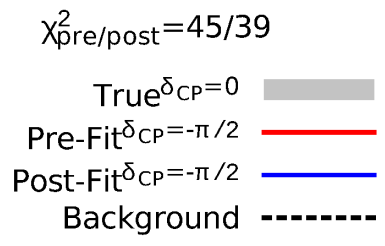

Figure 6.34. As in Figure 6.36 but for LBNE. 


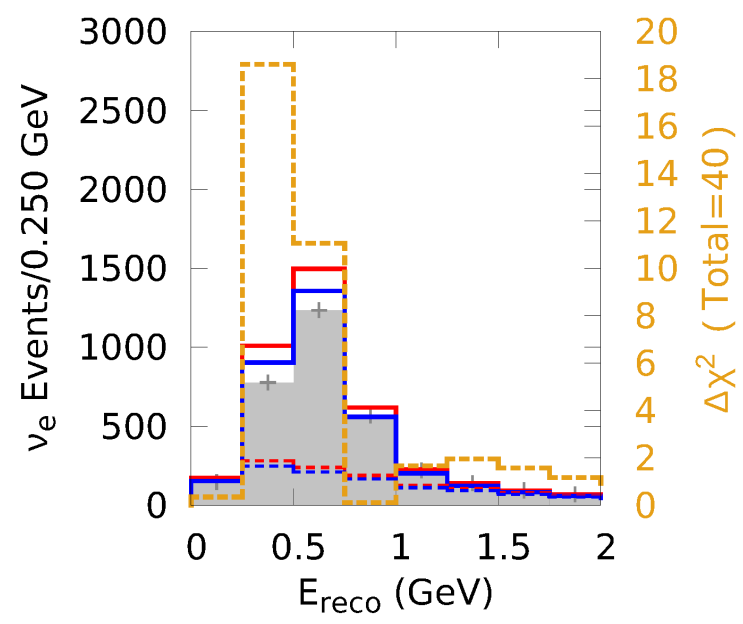

(A) $\nu_{e}$ appearance

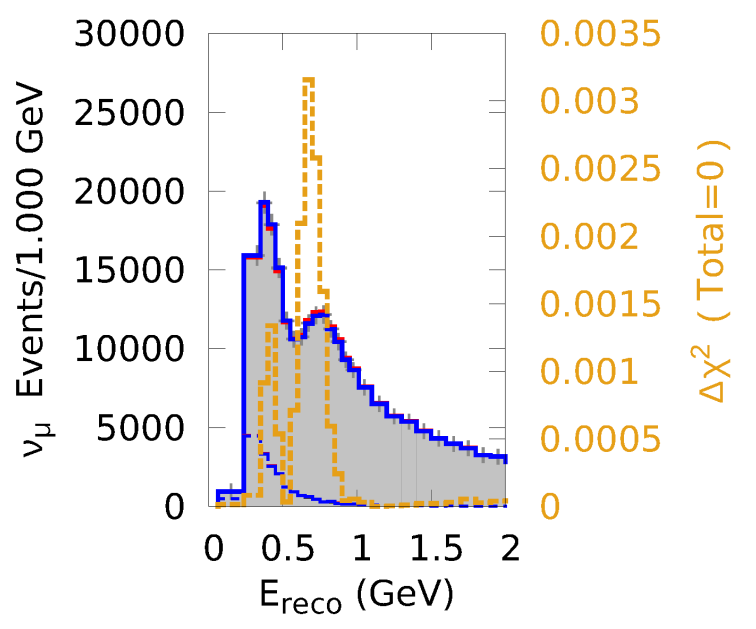

(C) $\nu_{\mu}$ disappearance

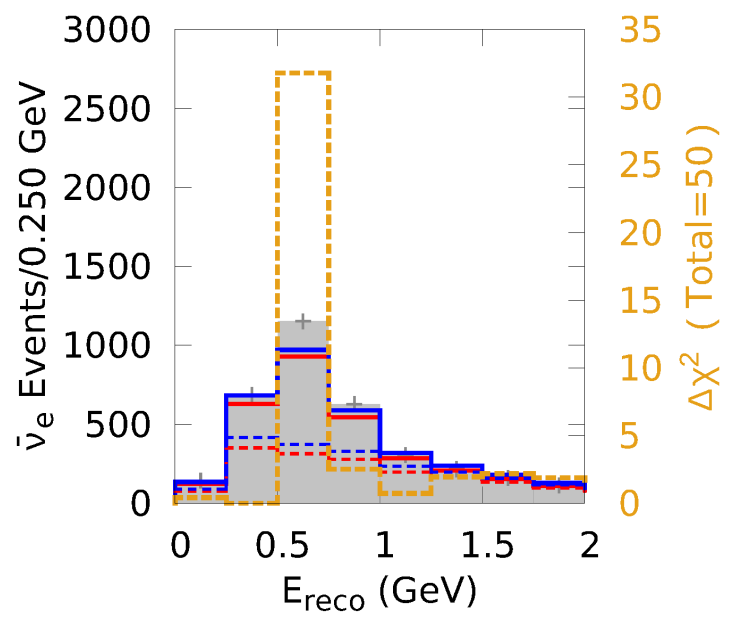

(в) $\bar{\nu}_{e}$ appearance

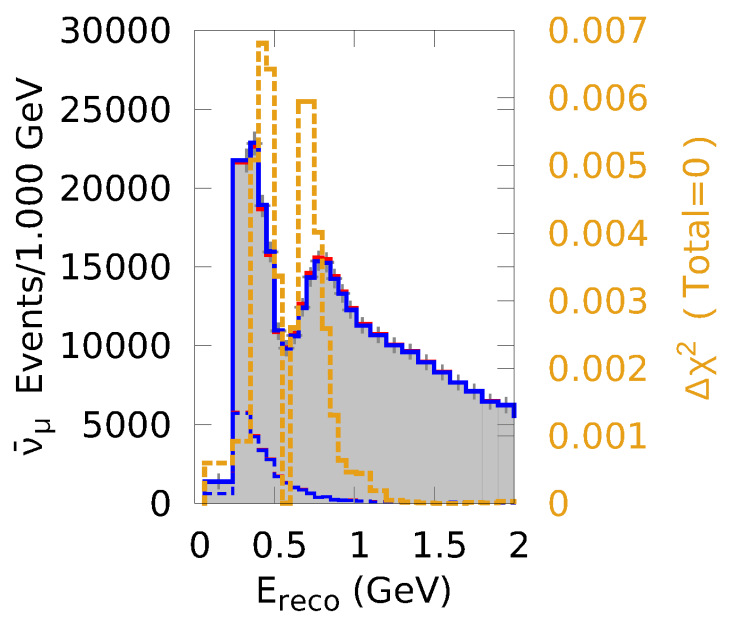

(D) $\bar{\nu}_{\mu}$ disappearance

$$
\begin{gathered}
X_{\text {pre/post }}^{2}=189 / 102 \\
\text { True }^{\delta_{C P}=0} \\
\text { Pre-Fit }{ }_{C P}=-\pi / 2 \\
\text { Post-Fit } \delta_{C P}=-\pi / 2 \\
\text { Background ---.--- }
\end{gathered}
$$

Figure 6.35. As in Figure 6.36 but for T2HK. 
6.3.1.2. Spectra: $M H$ Variations. Figures 6.36 to 6.39 show the spectra for all four samples in each of the experiments for variations in the $\mathrm{MH}$. The true event spectrum is for $\mathrm{NH}$ and the pre-fit and post-fit spectra are for IH. The true values of the oscillation parameters are those of the Capozzi et al. best-fit values and with $\delta_{C P}^{\text {true }}=0$. All of the other oscillation parameters are profiled in the fit.

In Figure 6.36 T2K has very little sensitivity to spectrum variations caused by changing the mass hierarchy. It is interesting to note that there is more sensitivity in the $\nu_{\mu}$ disappearance and $\bar{\nu}_{\mu}$ disappearance samples than in the $\nu_{e}$ appearance and $\bar{\nu}_{e}$ appearance samples. This trend continues in T2HK, in Figure 6.39, but because the statistics are so large in T2HK the significance increases to a value of $\Delta \chi^{2}=2$. This significance varies heavily depending on the assumed true values of the other parameters and this will be explored further in Section 6.3.2.

The sensitivity to the $\mathrm{MH}$ in $\mathrm{NO} \nu \mathrm{A}$, as see in Figure 6.37, comes primarily from the $\nu_{e}$ appearance sample. Comparing the red (pre-fit) and blue (post-fit) histograms in Figure 6.37A, the systematics have a large effect and diminish the total $\Delta \chi^{2}$ from $\Delta \chi^{2}=11$ before the fit to $\Delta \chi^{2}=1$ after the fit. Again, this $\Delta \chi^{2}$ is highly dependent on the assumed true value of $\delta_{C P}$ as will become more evident in later sections.

In LBNE, the MH variations have a very large effect on the event spectra, as shown in Figure 6.38. The scale of the contributions from $\nu_{e}$ appearance and $\bar{\nu}_{e}$ appearance are the same. As in the $\delta_{C P}$ variations spectra, the shape of the spectrum contributes significantly to the fit. The second oscillation maximum, below $1 \mathrm{GeV}$, again is contributing to the $\Delta \chi^{2}$. The total $\Delta \chi^{2}$ is 80 after the fit. 


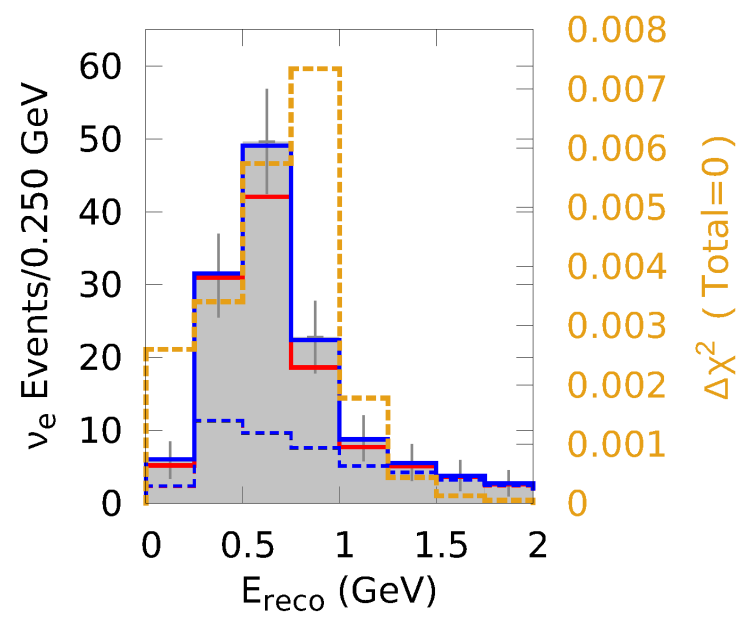

(A) $\nu_{e}$ appearance

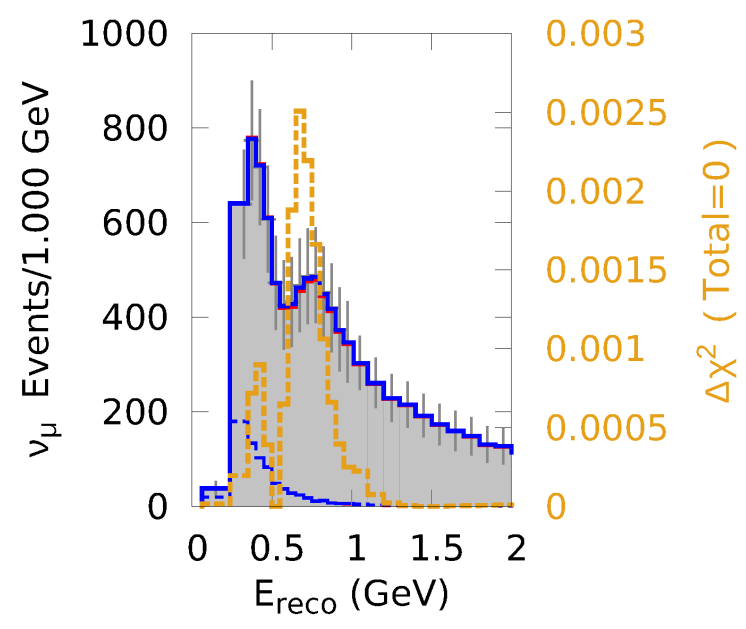

(C) $\nu_{\mu}$ disappearance

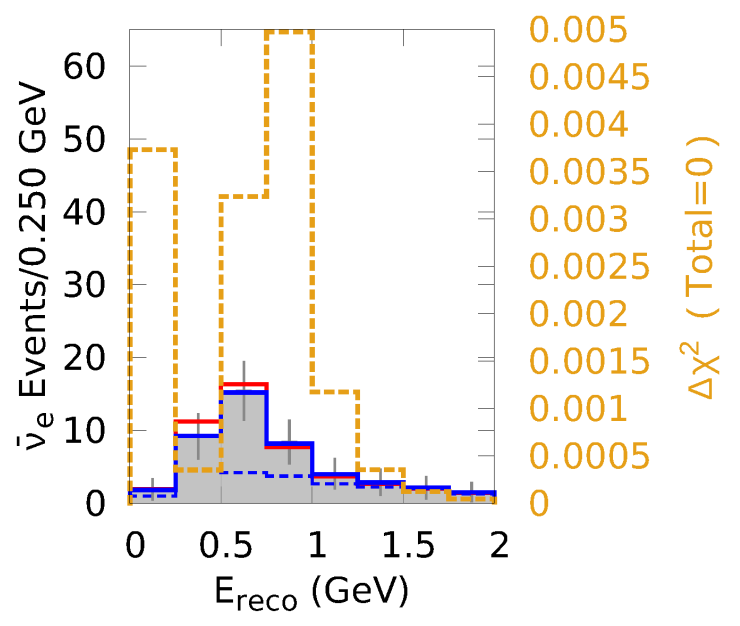

(B) $\bar{\nu}_{e}$ appearance

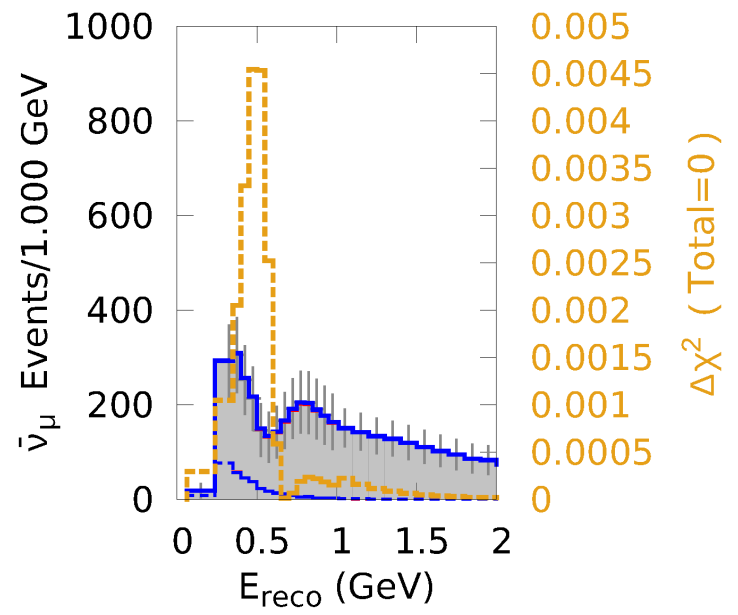

(D) $\bar{\nu}_{\mu}$ disappearance

\footnotetext{
$\chi_{\text {pre }}^{2}$ post $=3 / 0$

$\operatorname{True}(\mathrm{MH}=\mathrm{NH})$

Pre-Fit $(\mathrm{MH}=\mathrm{IH})$

Post-Fit(MH=IH)

Background
}

Figure 6.36. T2K pre-fit (red) and post-fit (blue) spectra, each with $\mathrm{MH}=\mathrm{IH}$, compared to the true spectrum for True $\mathrm{MH}=\mathrm{NH}\left(\delta_{C P}^{\text {true }}=0\right)$. The background spectra are given by the dashed lines and the signal + background spectra are given by the solid lines and gray region. The error bars on the gray region indicate statistical uncertainties. The total $\Delta \chi^{2}$ before and after the fit are indicated and include $\nu_{e}$ appearance, $\bar{\nu}_{e}$ appearance, $\nu_{\mu}$ disappearance, and $\bar{\nu}_{\mu}$ disappearance. The nominal assumptions are used for systematics and $\nu: \bar{\nu}$ ratios. The $\Delta \chi^{2}$ as a function of $E_{\text {reco }}$ is given by the yellow histogram. 


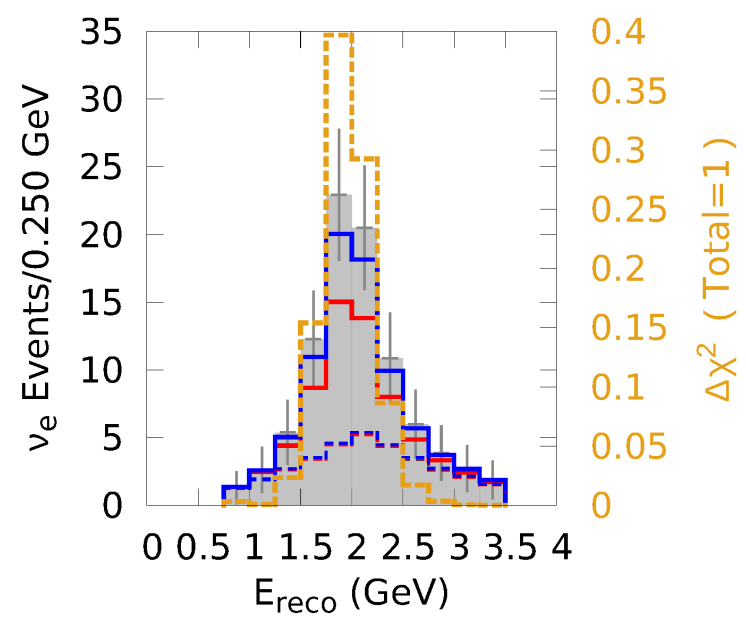

(A) $\nu_{e}$ appearance

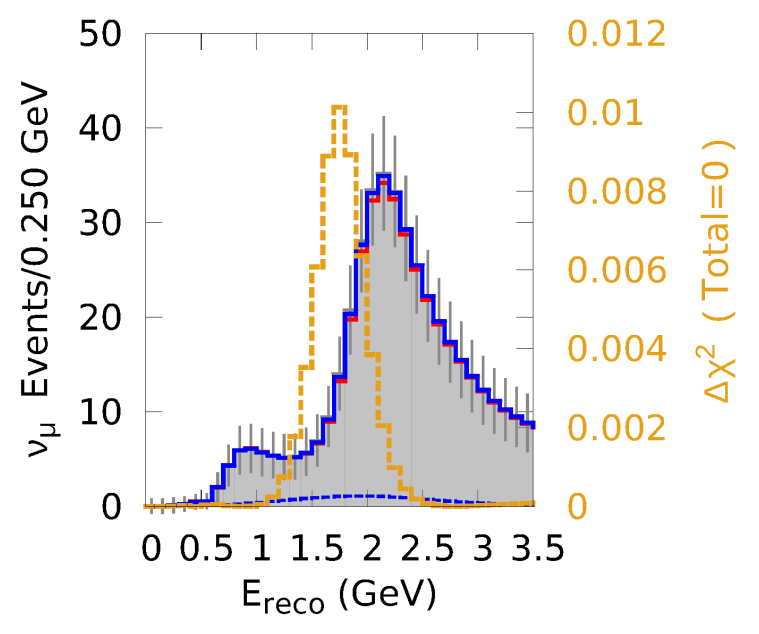

(C) $\nu_{\mu}$ disappearance

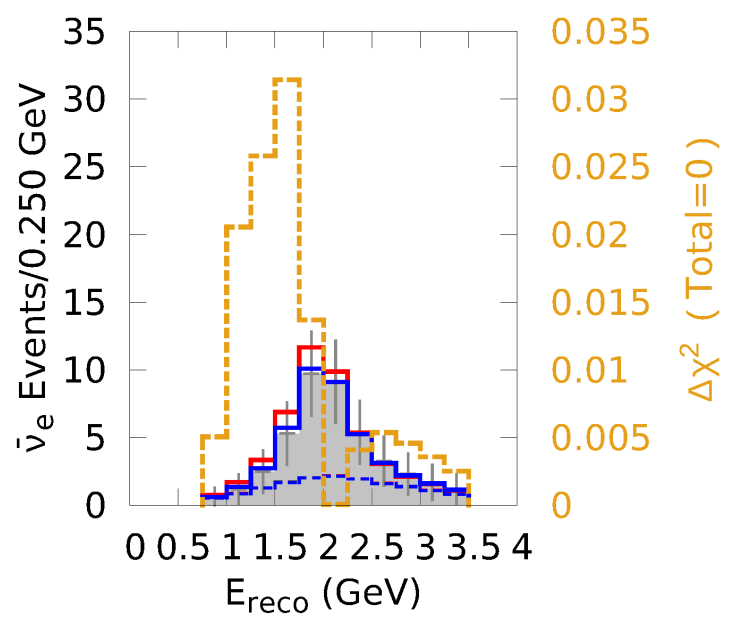

(в) $\bar{\nu}_{e}$ appearance

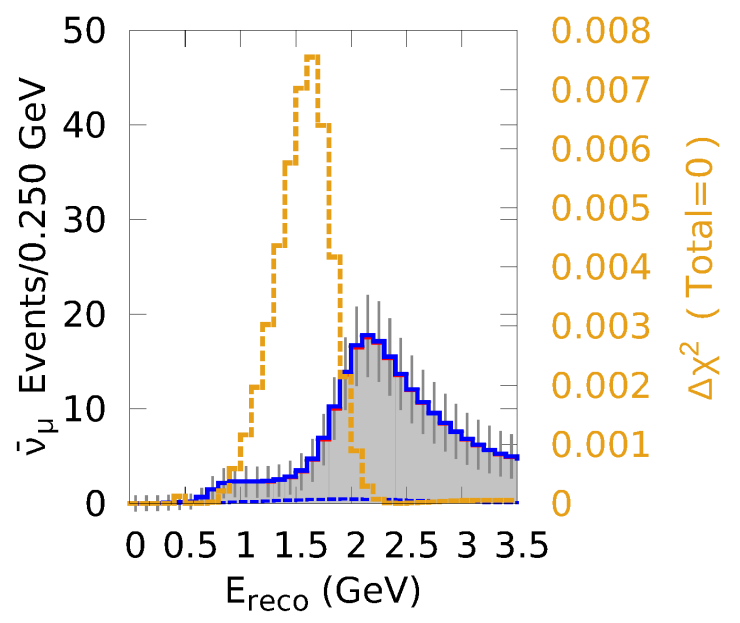

(D) $\bar{\nu}_{\mu}$ disappearance

$$
\begin{array}{r}
\chi_{\text {pre/post }}^{2}=11 / 1 \\
\text { True }(\mathrm{MH}=\mathrm{NH}) \\
\text { Pre-Fit }(\mathrm{MH}=\mathrm{IH}) \\
\text { Post-Fit }(\mathrm{MH}=\mathrm{IH}) \\
\text { Background }
\end{array}
$$

Figure 6.37. As in Figure 6.36 but for $\mathrm{NO} \nu \mathrm{A}$. 


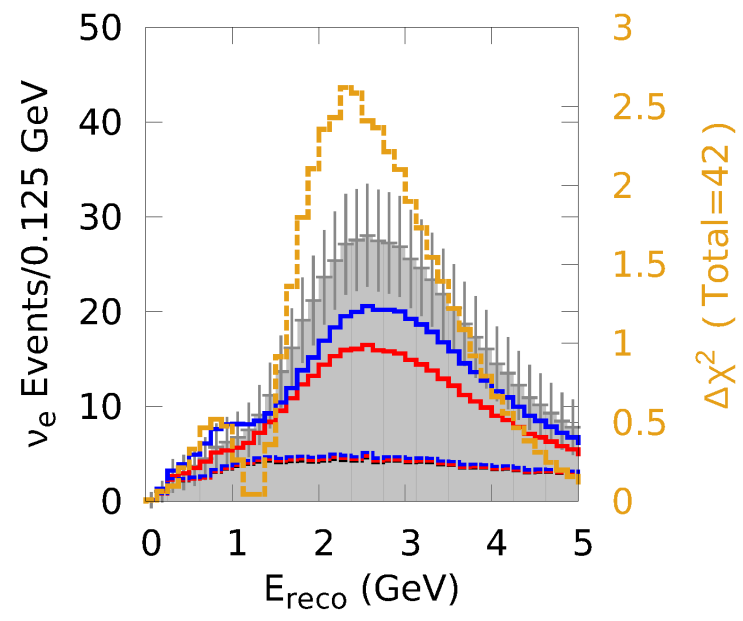

(A) $\nu_{e}$ appearance

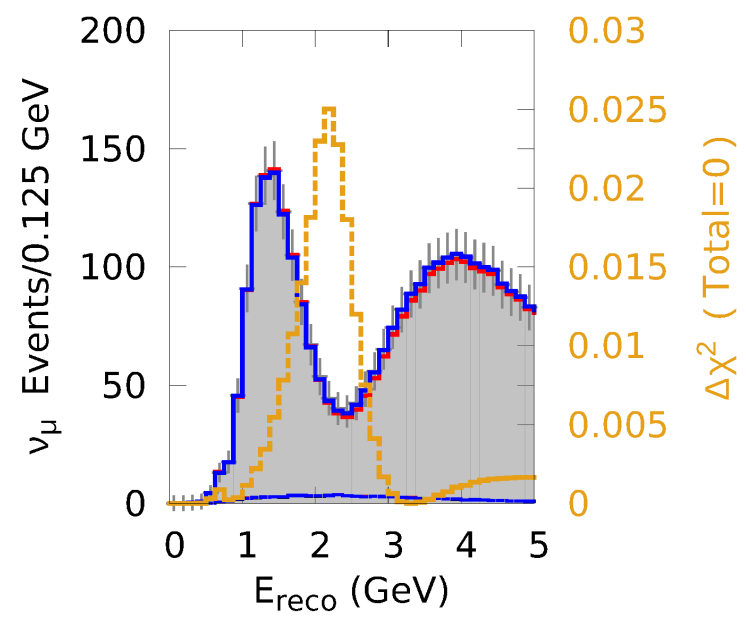

(C) $\nu_{\mu}$ disappearance

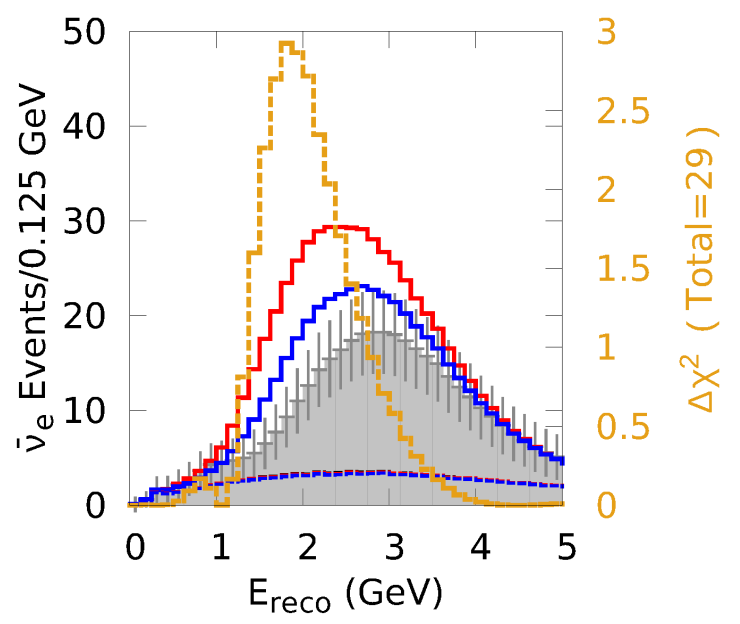

(B) $\bar{\nu}_{e}$ appearance

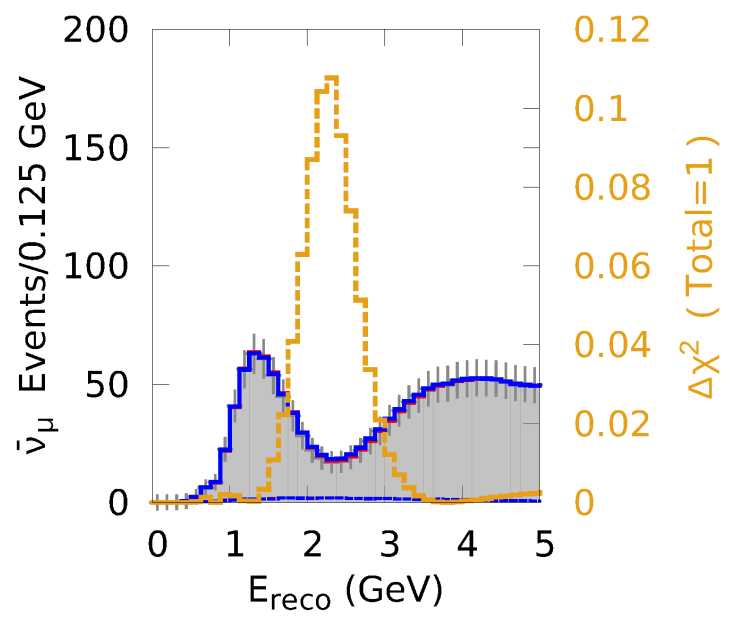

(D) $\bar{\nu}_{\mu}$ disappearance

$$
\begin{aligned}
& X_{\text {pre/post }}^{2}=240 / 80 \\
& \text { True }(\mathrm{MH}=\mathrm{NH}) \\
& \text { Pre-Fit }(\mathrm{MH}=\mathrm{IH}) \\
& \text { Post-Fit }(\mathrm{MH}=\mathrm{IH}) \\
& \text { Background }
\end{aligned}
$$

Figure 6.38. As in Figure 6.36 but for LBNE. 


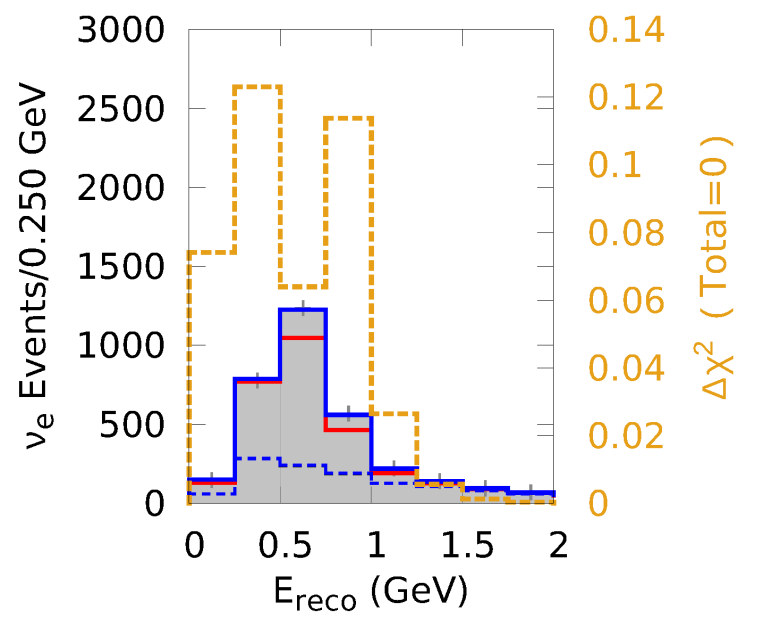

(A) $\nu_{e}$ appearance

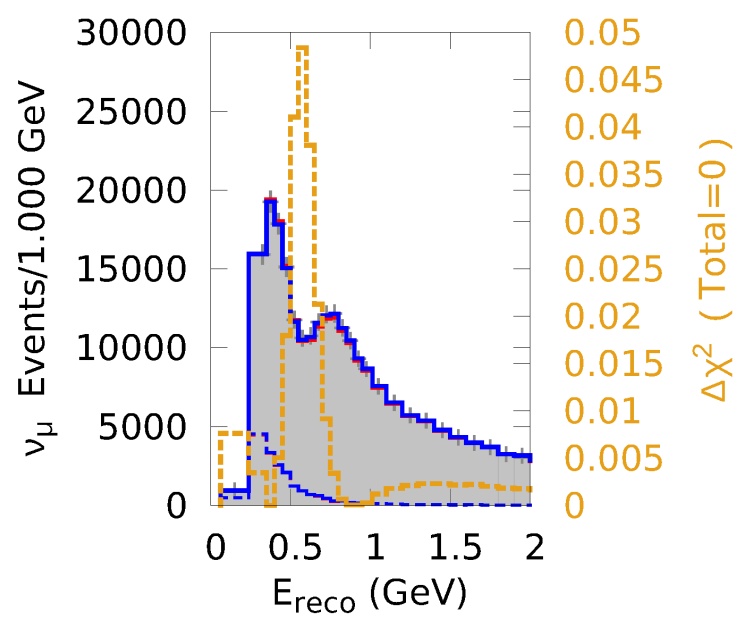

(C) $\nu_{\mu}$ disappearance

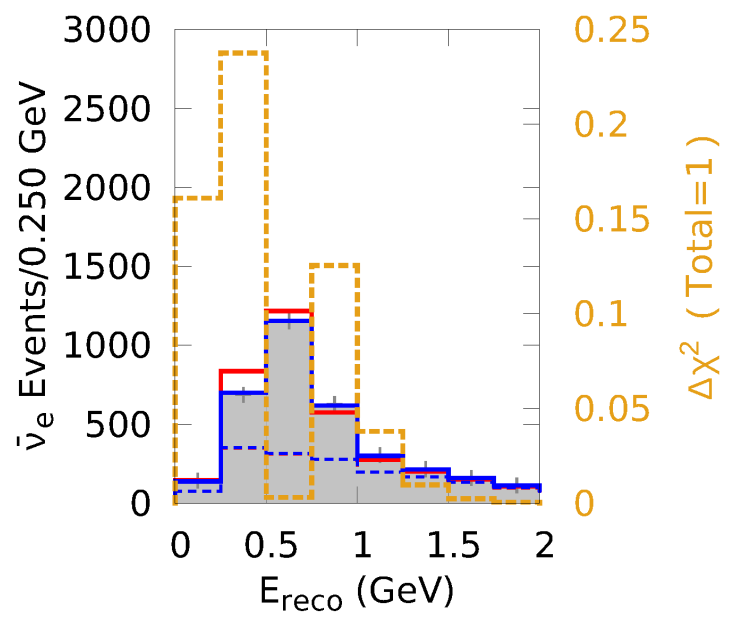

(B) $\bar{\nu}_{e}$ appearance

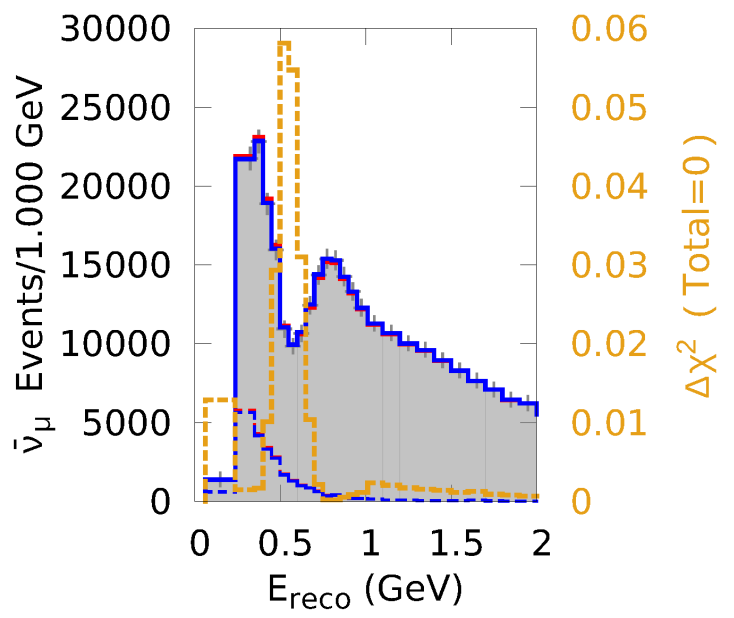

(D) $\bar{\nu}_{\mu}$ disappearance

\footnotetext{
$\chi_{\text {pre } / \text { post }}^{2}=104 / 2$

True $(\mathrm{MH}=\mathrm{NH})$

Pre-Fit $(\mathrm{MH}=\mathrm{IH})$

Post-Fit $(\mathrm{MH}=\mathrm{IH})$

Background --------
}

Figure 6.39. As in Figure 6.36 but for T2HK. 
6.3.1.3. Spectra: $\sin ^{2}\left(\theta_{23}\right)$ Variations: Maximal Mixing. Figures 6.40 to 6.43 show the spectra for all four samples in each of the experiments for variations in $\sin ^{2}\left(\theta_{23}\right)$. The true event spectrum is for $\sin ^{2}\left(\theta_{23}\right)=0.5$ and the pre-fit and post-fit spectra are for $\sin ^{2}\left(\theta_{23}\right)=$ 0.43. This comparison evaluates the ability of the experiment to detect non-maximal mixing. The true values of the other oscillation parameters are those of the Capozzi et al. best-fit values and with $\delta_{C P}^{\text {true }}=0$. All of the other oscillation parameters are profiled in the fit.

The dominant contribution to detecting non-maximal $\sin ^{2}\left(\theta_{23}\right)$ in T2K comes from $\nu_{\mu}$ disappearance, as shown in Figure 6.40. The $\Delta \chi^{2}$ values are peaked around the oscillation maximum in the $\nu_{\mu}$ disappearance and $\bar{\nu}_{\mu}$ disappearance samples because the $\theta_{23}$ oscillation parameter controls the amplitude of the disappearance. Scaling to T2HK exposures, as in Figure 6.43, leads to a much higher $\Delta \chi^{2}$ value. The $\Delta \chi^{2}$ contributions are more balanced between $\nu_{\mu}$ disappearance and $\bar{\nu}_{\mu}$ disappearance in the T2HK case because of the 1:3 $\nu: \bar{\nu}$ running ratio.

The sensitivity to this effect in $\mathrm{NO} \nu \mathrm{A}$ and LBNE also comes predominantly from $\nu_{\mu}$ disappearance with the largest contributions centered around the oscillation maximum. The $\Delta \chi^{2}$ value is higher before the fit in $\mathrm{NO} \nu \mathrm{A}$ then in $\mathrm{T} 2 \mathrm{~K}$ yet it ends up lower after the fit so the systematics play a larger role in $\mathrm{NO} \nu \mathrm{A}$ for non-maximal $\sin ^{2}\left(\theta_{23}\right)$ sensitivity. 


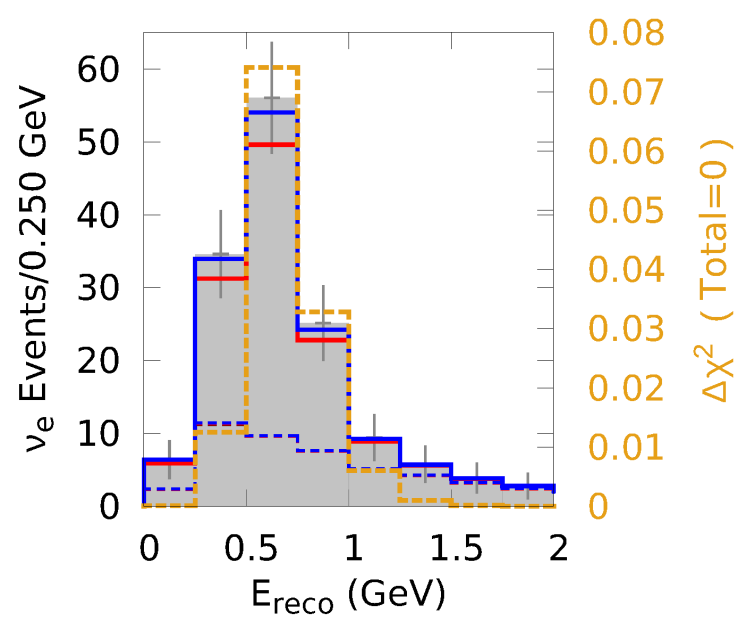

(A) $\nu_{e}$ appearance

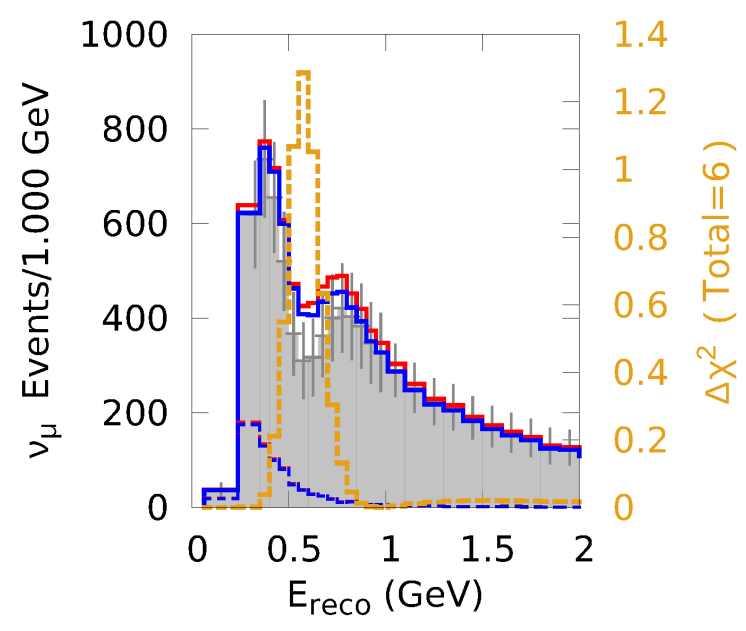

(C) $\nu_{\mu}$ disappearance

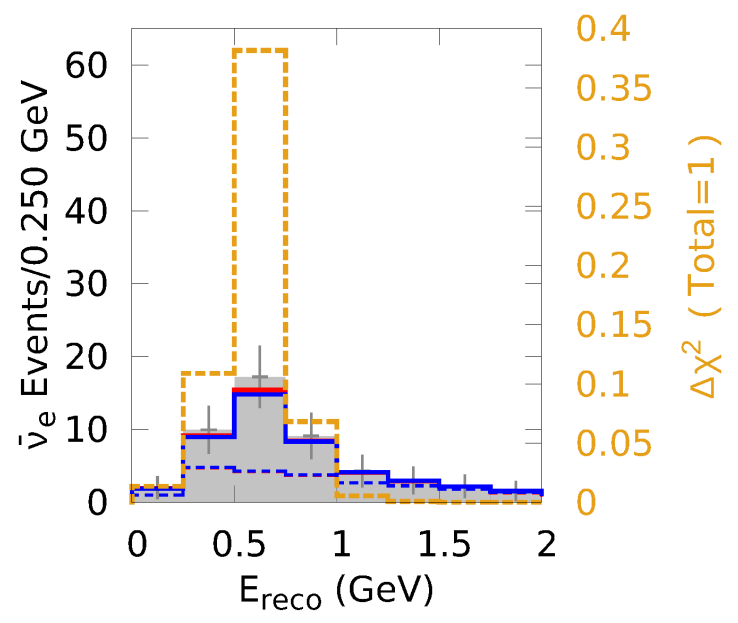

(B) $\bar{\nu}_{e}$ appearance

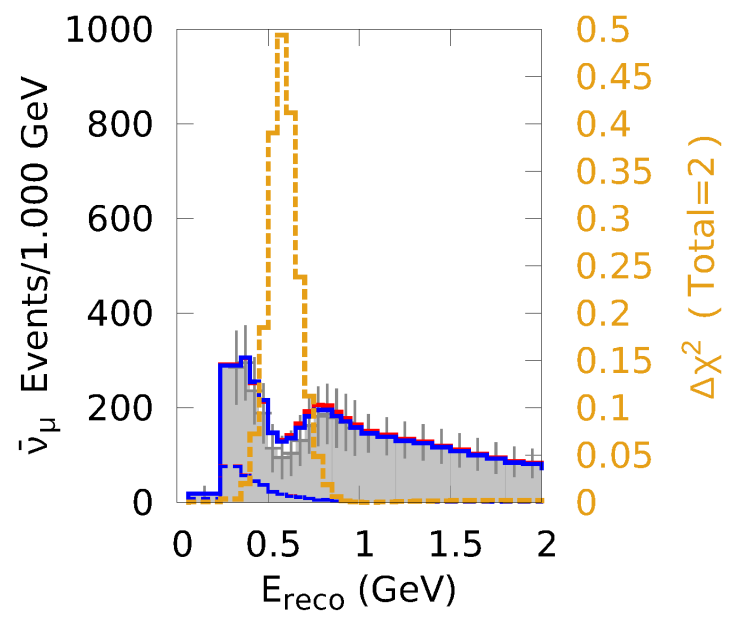

(D) $\bar{\nu}_{\mu}$ disappearance

$$
\begin{array}{r}
X_{\text {pre/post }}^{2}=14 / 10 \\
\text { True }^{\sin (\theta)_{23}}=0.5 \\
\text { Pre-Fit }{ }^{\sin (\theta)_{23}=0.43} \\
\text { Post-Fit } \sin (\theta)_{23}=0.43 \\
\text { Background ------- }
\end{array}
$$

Figure 6.40. T2K pre-fit (red) and post-fit (blue) spectra, each with $\sin ^{2}\left(\theta_{23}\right)=0.43$, compared to the true spectrum for $\sin ^{2}\left(\theta_{23}\right)=0.5$. The background spectra are given by the dashed lines and the signal+background spectra are given by the solid lines and gray region. The error bars on the gray region indicate statistical uncertainties. The total $\Delta \chi^{2}$ before and after the fit are indicated and include $\nu_{e}$ appearance, $\bar{\nu}_{e}$ appearance, $\nu_{\mu}$ disappearance, and $\bar{\nu}_{\mu}$ disappearance. The nominal assumptions are used for systematics and $\nu: \bar{\nu}$ ratios. The $\Delta \chi^{2}$ as a function of $E_{\text {reco }}$ is given by the yellow histogram. 


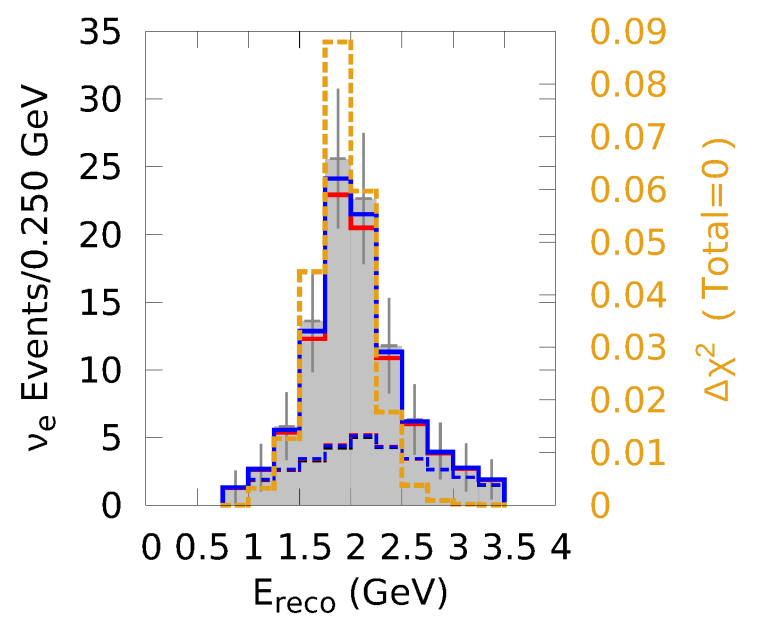

(A) $\nu_{e}$ appearance

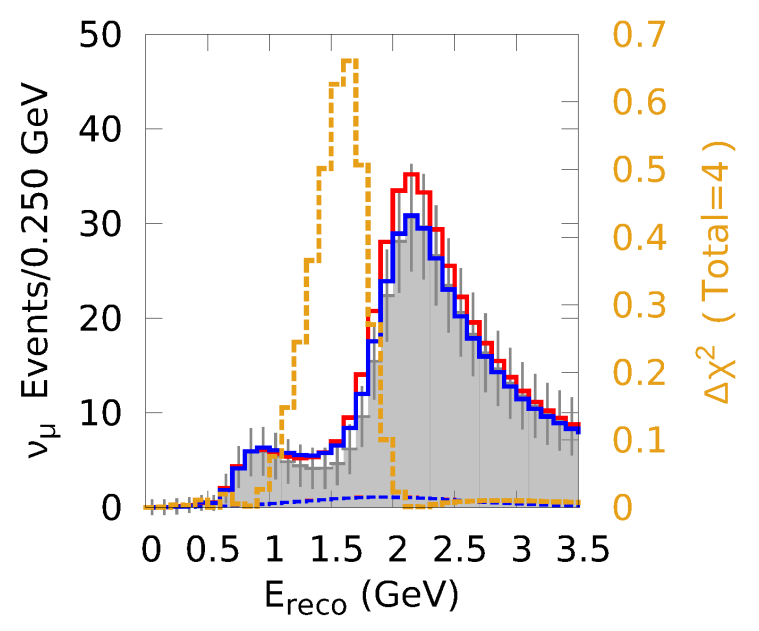

(C) $\nu_{\mu}$ disappearance

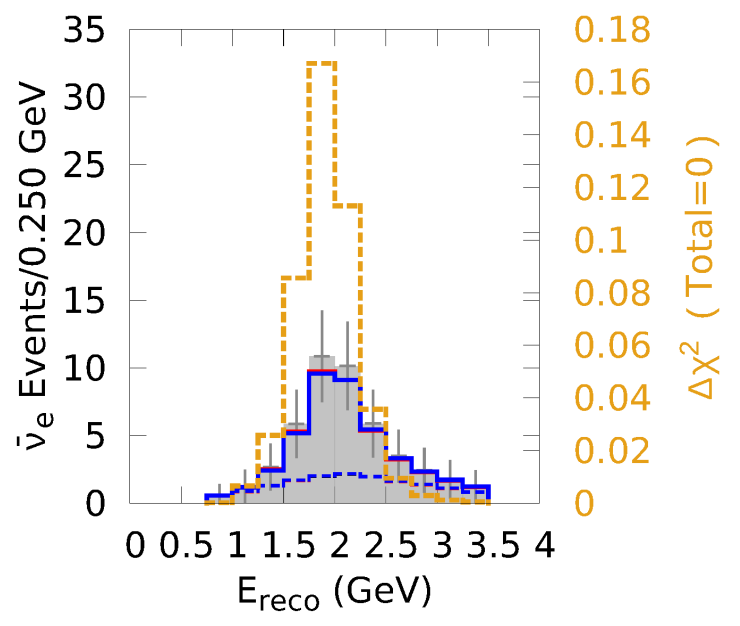

(B) $\bar{\nu}_{e}$ appearance

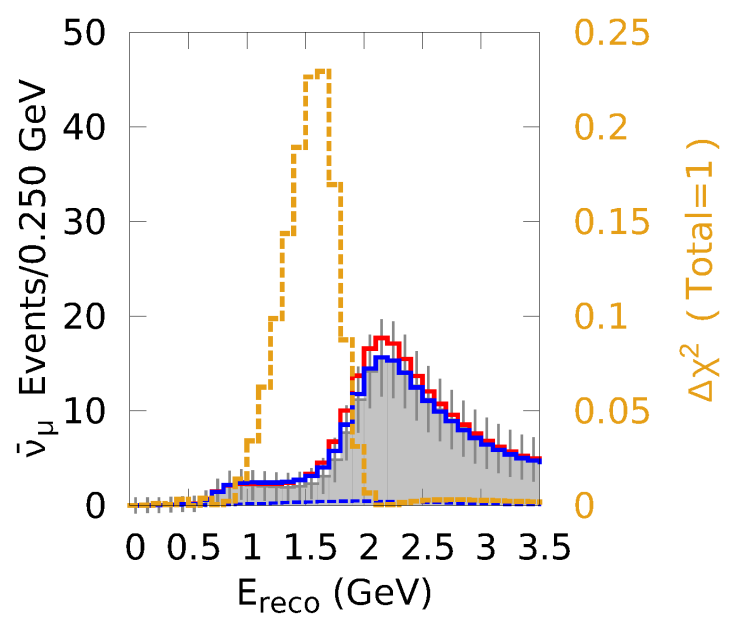

(D) $\bar{\nu}_{\mu}$ disappearance

\footnotetext{
$\chi_{\text {pre } / \text { post }}^{2}=16 / 7$

True $^{\sin (\theta)_{23}=0.5}$

Pre-Fit $\sin (\theta)_{23}=0.43$

Post-Fit $\sin (\theta)_{23}=0.43$

Background ---------
}

Figure 6.41. As in Figure 6.40 but for $\mathrm{NO} \nu \mathrm{A}$. 


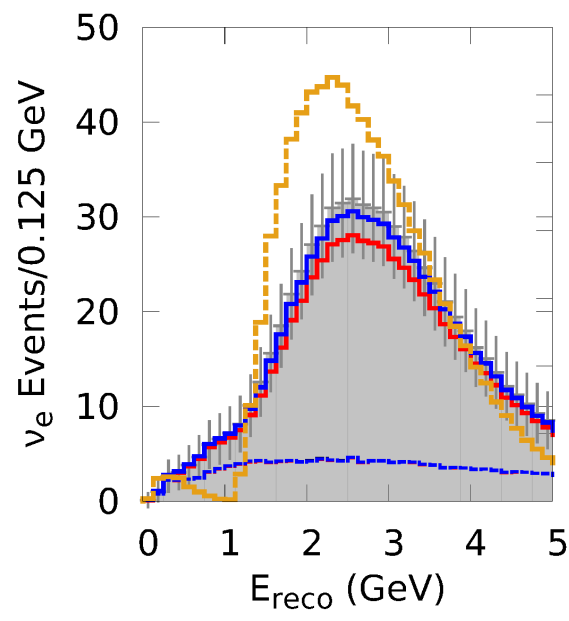

(A) $\nu_{e}$ appearance

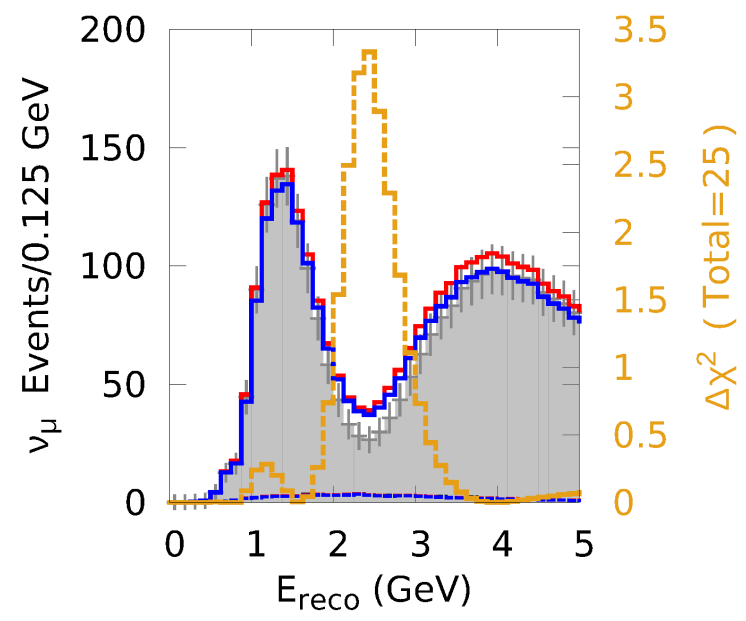

(C) $\nu_{\mu}$ disappearance

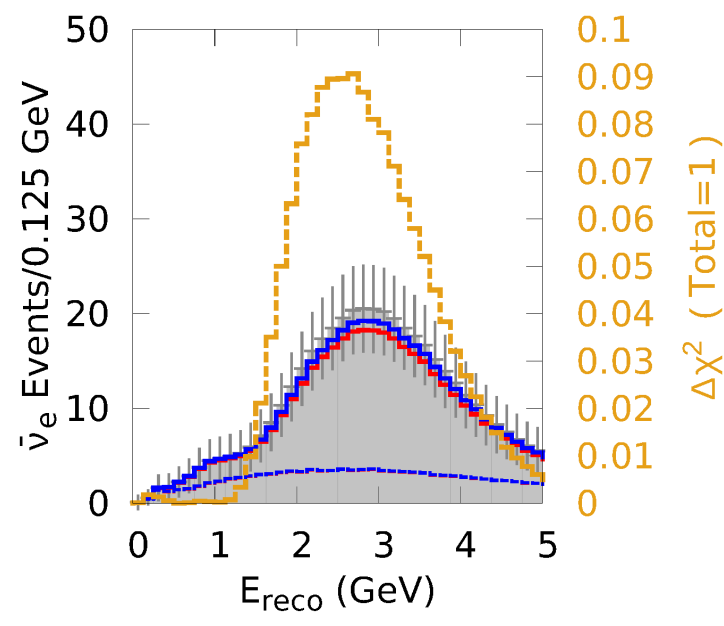

(B) $\bar{\nu}_{e}$ appearance

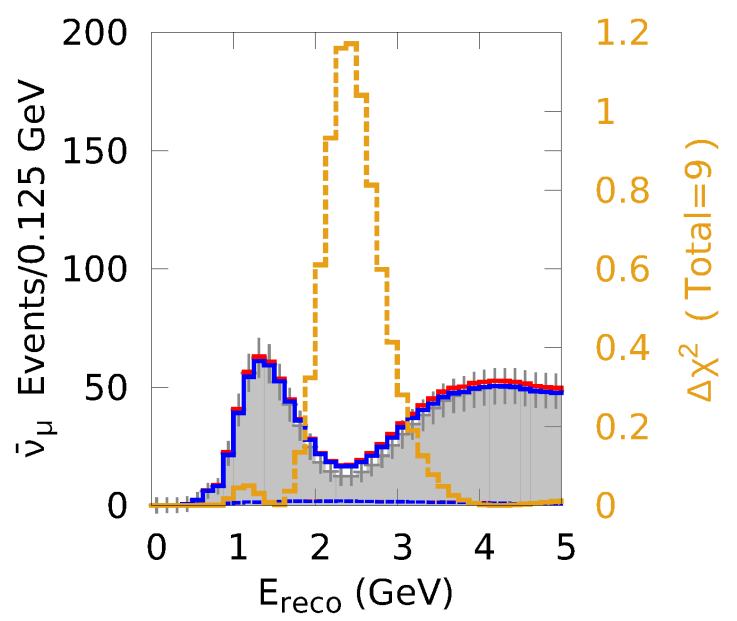

(D) $\bar{\nu}_{\mu}$ disappearance

$$
\begin{array}{r}
X_{\text {pre/post }}^{2}=68 / 41 \\
\text { True }^{\sin (\theta)_{23}}=0.5 \\
\text { Pre-Fit }{\sin (\theta)_{23}=0.43} \\
\text { Post-Fit } \sin (\theta)_{23}=0.43 \\
\text { Background ---.--- }
\end{array}
$$

Figure 6.42. As in Figure 6.40 but for LBNE. 


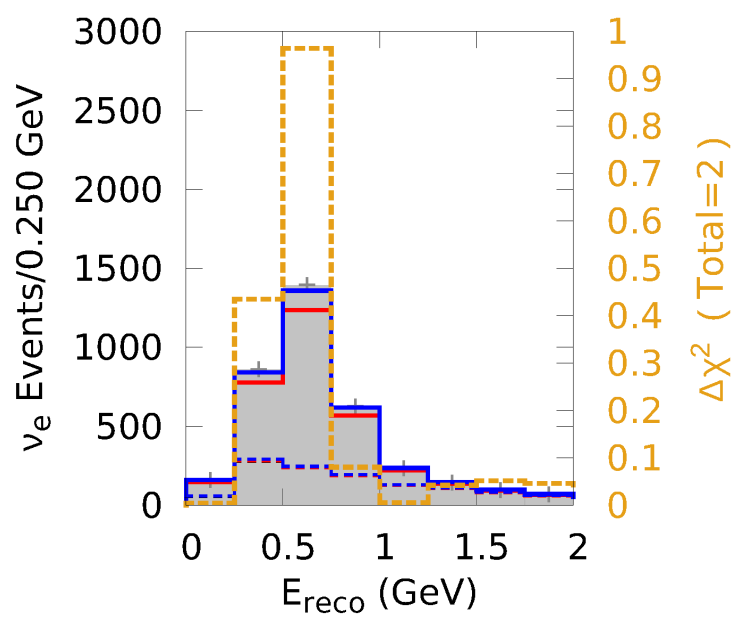

(A) $\nu_{e}$ appearance

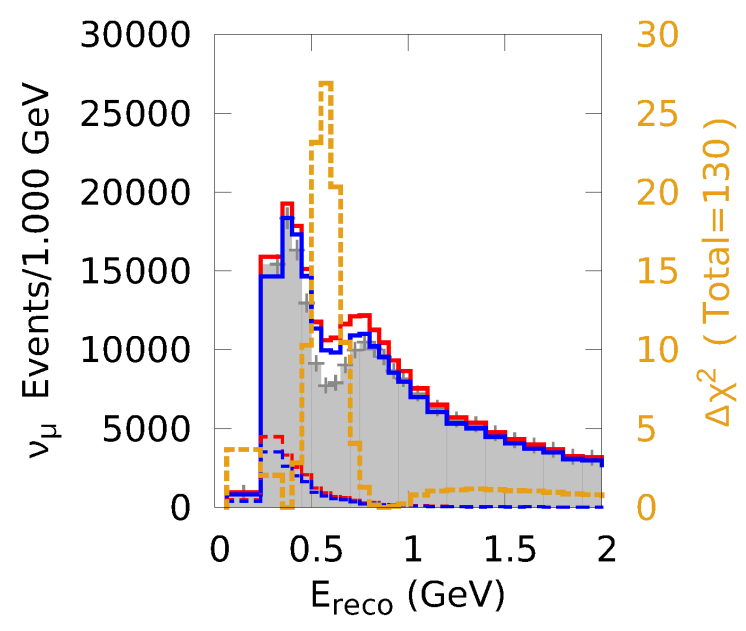

(C) $\nu_{\mu}$ disappearance

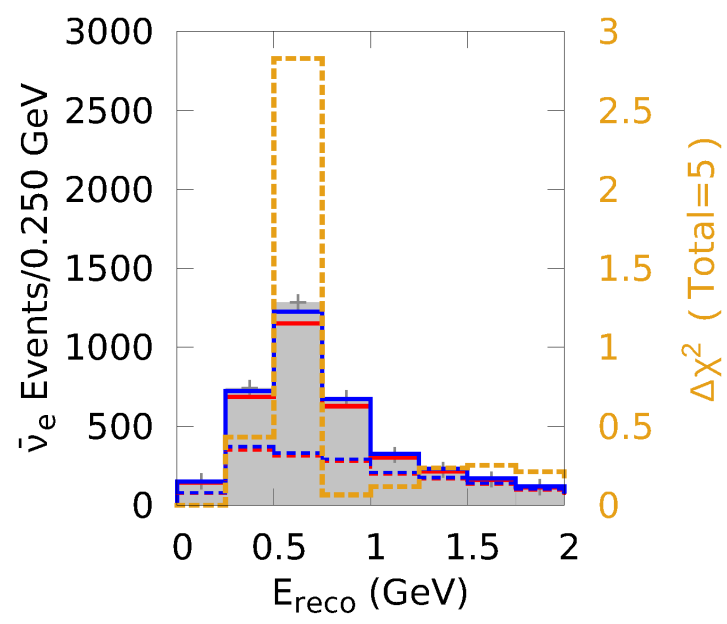

(B) $\bar{\nu}_{e}$ appearance

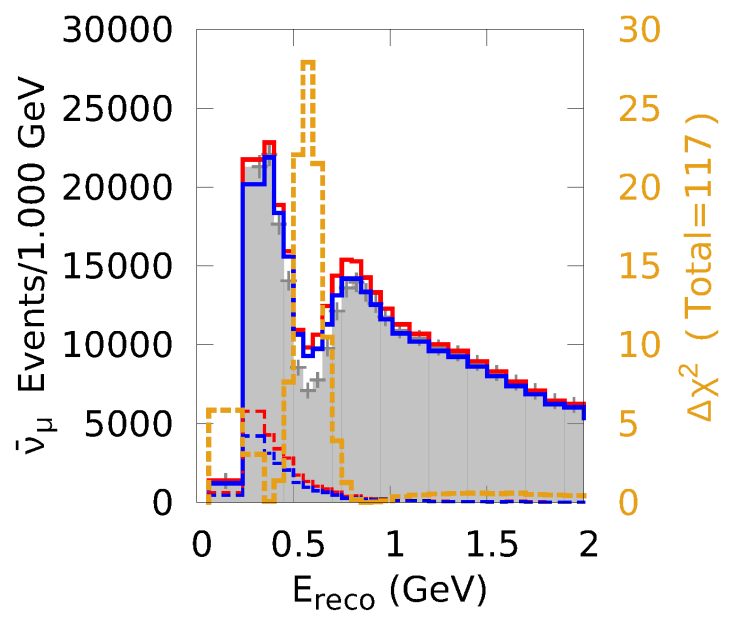

(D) $\bar{\nu}_{\mu}$ disappearance

$$
\begin{aligned}
& X_{\text {pre } / \text { post }}^{2}=490 / 270 \\
& \text { True }^{\sin (\theta)_{23}=0.5} \\
& \text { Pre-Fit } \sin (\theta)_{23}=0.43 \\
& \text { Post-Fit } \sin (\theta)_{23}=0.43 \\
& \text { Background ----.---- }
\end{aligned}
$$

Figure 6.43. As in Figure 6.40 but for T2HK. 
6.3.1.4. Spectra: $\sin ^{2}\left(\theta_{23}\right)$ Variations: Octant. Figures 6.44 to 6.47 show similar spectra for a different set of variations in $\sin ^{2}\left(\theta_{23}\right)$. The true event spectrum is for $\sin ^{2}\left(\theta_{23}\right)=0.43$ and the pre-fit and post-fit spectra are for $\sin ^{2}\left(\theta_{23}\right)=0.57$. This comparison evaluates the ability of the experiment to distinguish between the first and second octants. In this case, the disappearance spectra are less able to distinguish between the two spectra. The dependence of the disappearance amplitude on $\sin ^{2}\left(2 \theta_{23}\right)$ means that the spectra in the first and second octant are similar. The appearance amplitude depends on $\sin ^{2}\left(\theta_{23}\right)$ so it is sensitive to the octant and for this reason the $\Delta \chi^{2}$ contributions are greater for these spectra than those in the previous section. 


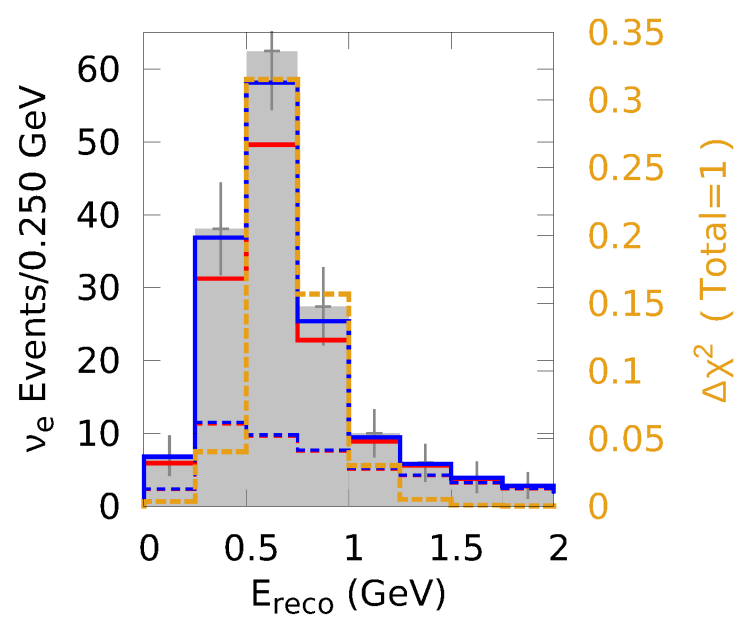

(A) $\nu_{e}$ appearance

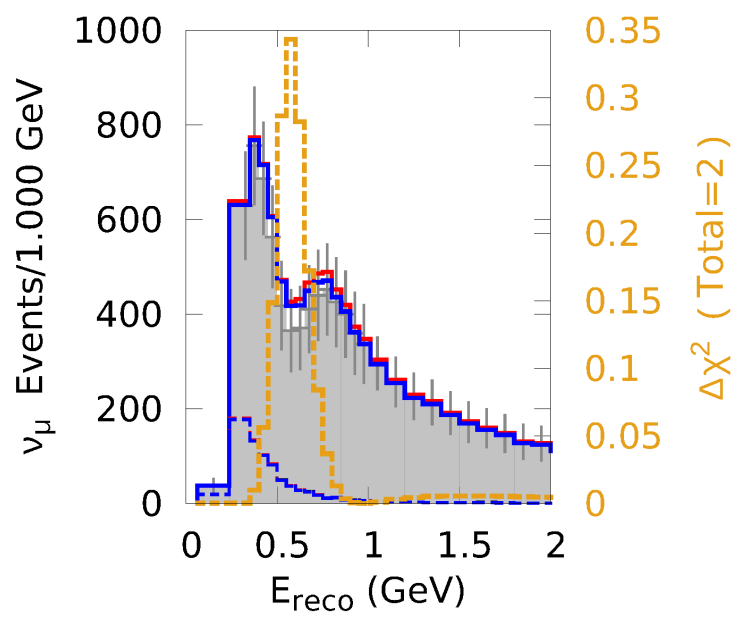

(C) $\nu_{\mu}$ disappearance

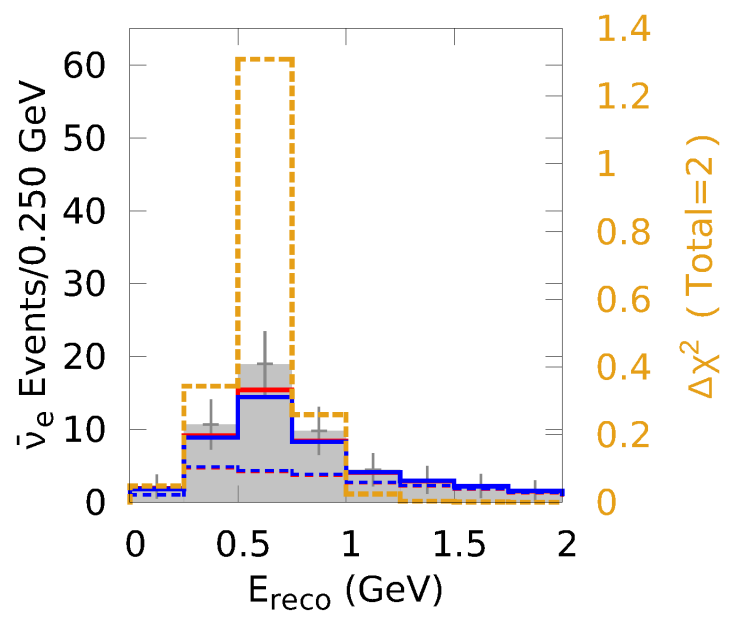

(в) $\bar{\nu}_{e}$ appearance

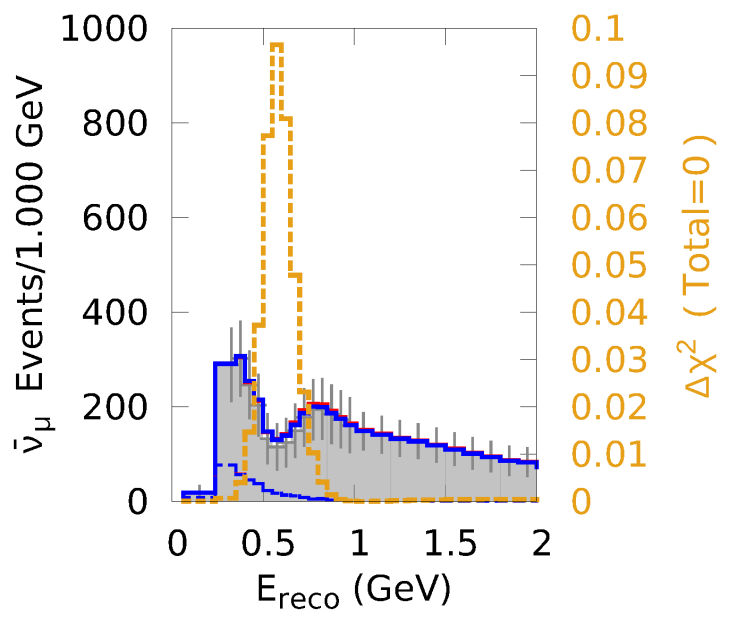

(D) $\bar{\nu}_{\mu}$ disappearance

\footnotetext{
$\chi_{\text {pre/post }}^{2}=10 / 5$

True $^{\sin (\theta)_{23}=0.43}$

Pre-Fit $\sin (\theta)_{23}=0.57$

Post-Fit $\sin (\theta)_{23}=0.57$

Background --------
}

FiguRE 6.44. T2K pre-fit (red) and post-fit (blue) spectra, each with $\sin ^{2}\left(\theta_{23}\right)=0.43$, compared to the true spectrum for $\sin ^{2}\left(\theta_{23}\right)=0.57$ in the second octant. The background spectra are given by the dashed lines and the signal + background spectra are given by the solid lines and gray region. The error bars on the gray region indicate statistical uncertainties. The total $\Delta \chi^{2}$ before and after the fit are indicated and include $\nu_{e}$ appearance, $\bar{\nu}_{e}$ appearance, $\nu_{\mu}$ disappearance, and $\bar{\nu}_{\mu}$ disappearance. The nominal assumptions are used for systematics and $\nu: \bar{\nu}$ ratios. The $\Delta \chi^{2}$ as a function of $E_{\text {reco }}$ is given by the yellow histogram. 


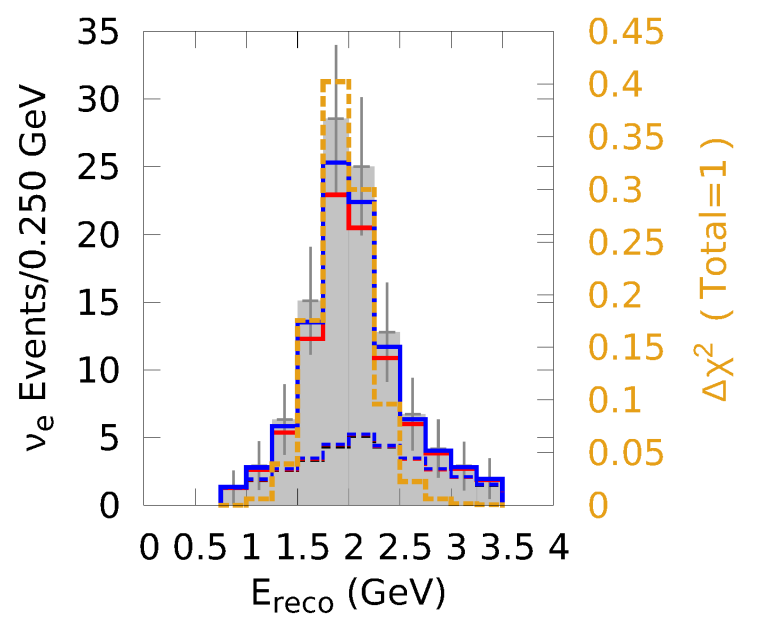

(A) $\nu_{e}$ appearance

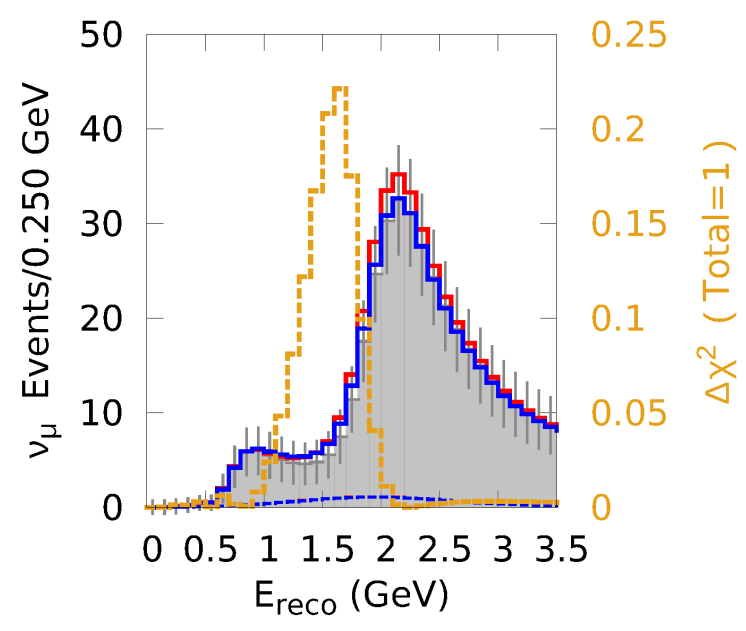

(C) $\nu_{\mu}$ disappearance

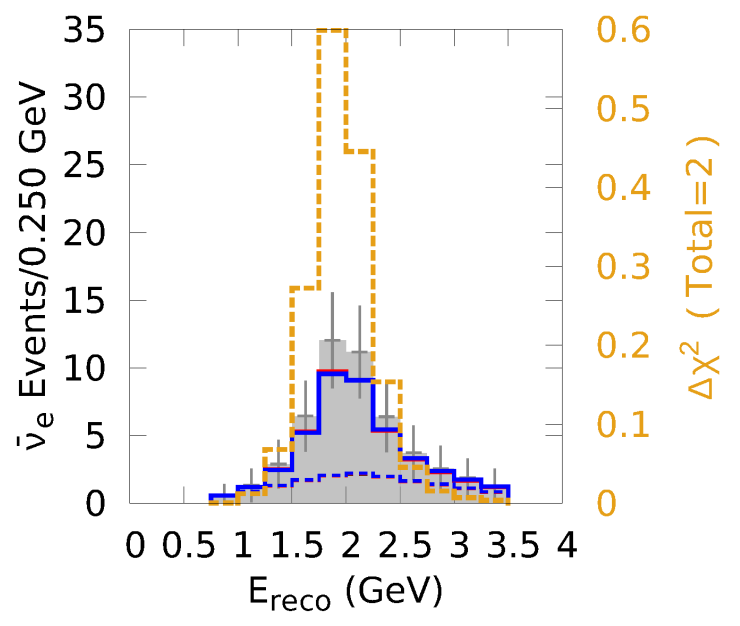

(B) $\bar{\nu}_{e}$ appearance

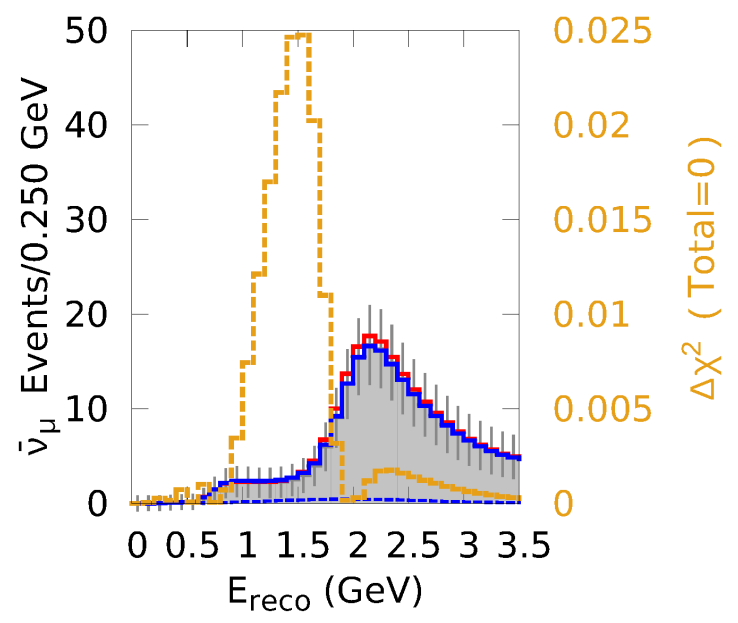

(D) $\bar{\nu}_{\mu}$ disappearance

\footnotetext{
$\chi_{\text {pre } / \text { post }}^{2}=9 / 5$

True $\mathrm{sin}^{\sin (\theta)_{23}=0.43}$

Pre-Fit $\sin (\theta)_{23}=0.57$

Post-Fit $\sin (\theta)_{23}=0.57$

Background --------
}

Figure 6.45. As in Figure 6.44 but for $\mathrm{NO} \nu \mathrm{A}$. 


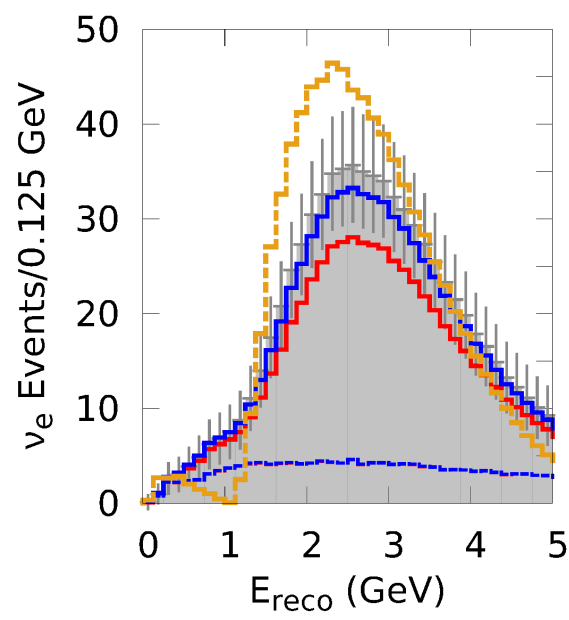

(A) $\nu_{e}$ appearance

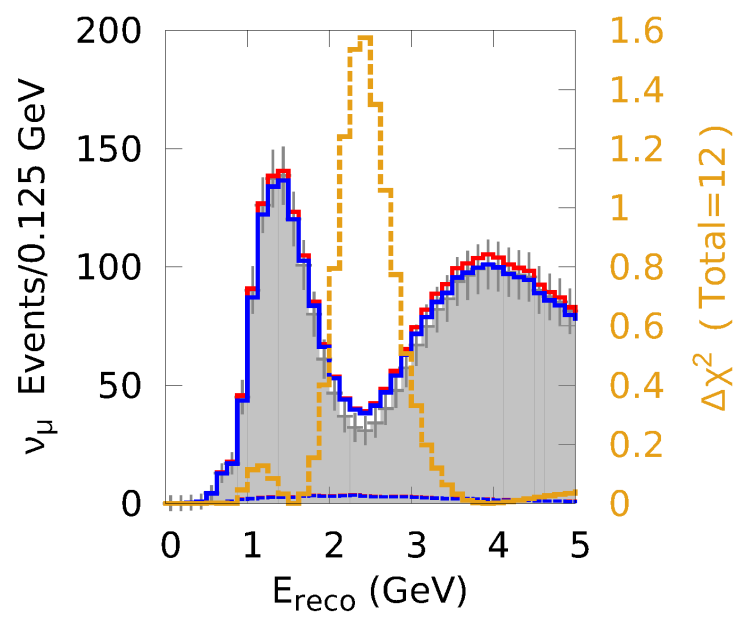

(C) $\nu_{\mu}$ disappearance

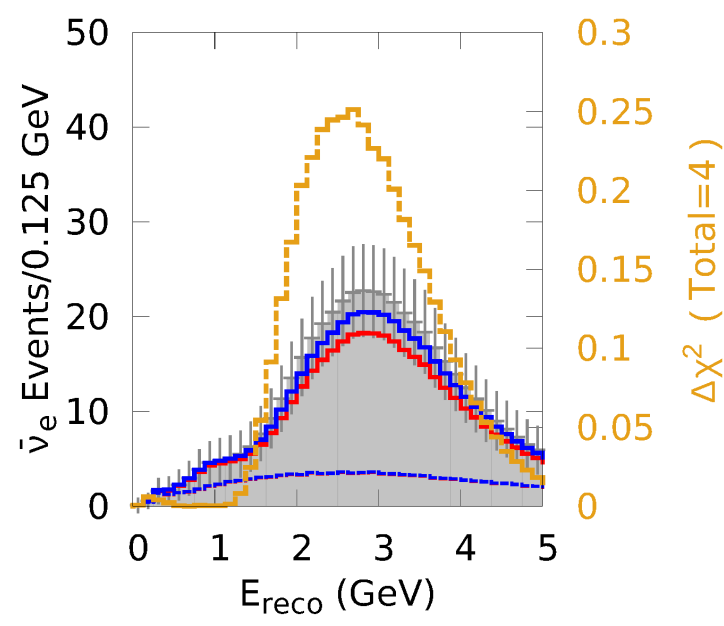

(в) $\bar{\nu}_{e}$ appearance

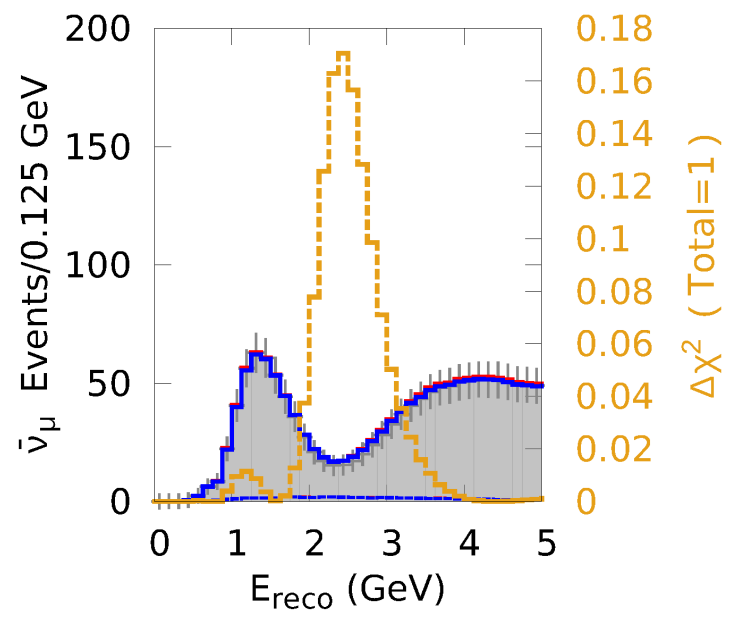

(D) $\bar{\nu}_{\mu}$ disappearance

$$
\begin{array}{r}
X_{\text {pre/post }}^{2}=74 / 32 \\
\text { True } \mathrm{S}^{\sin (\theta)_{23}}=0.43 \\
\text { Pre-Fit }{ }^{\sin (\theta)_{23}}=0.57 \\
\text { Post-Fit } \sin (\theta)_{23}=0.57 \\
\text { Background --.---. }
\end{array}
$$

Figure 6.46. As in Figure 6.44 but for LBNE. 


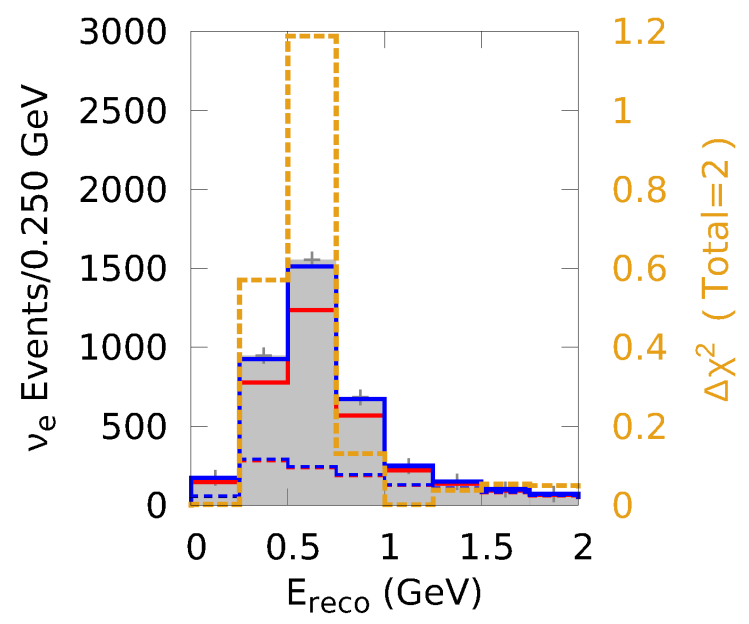

(A) $\nu_{e}$ appearance

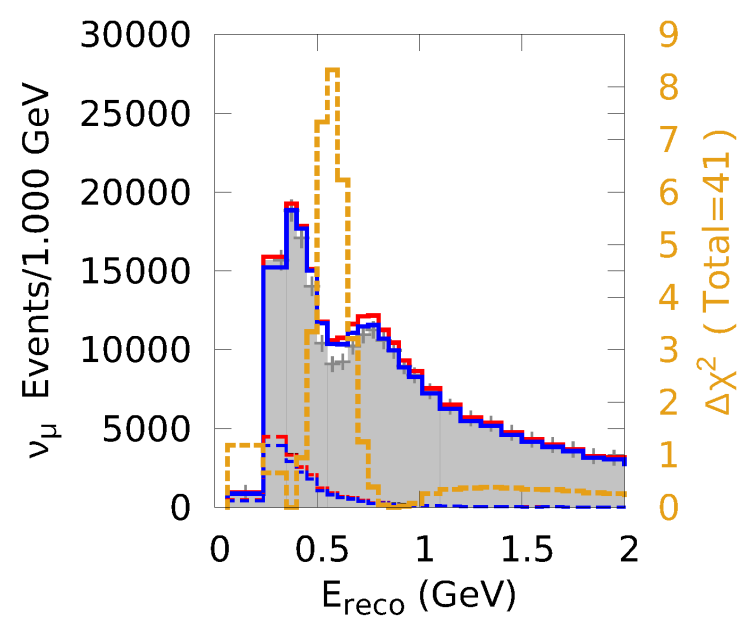

(C) $\nu_{\mu}$ disappearance

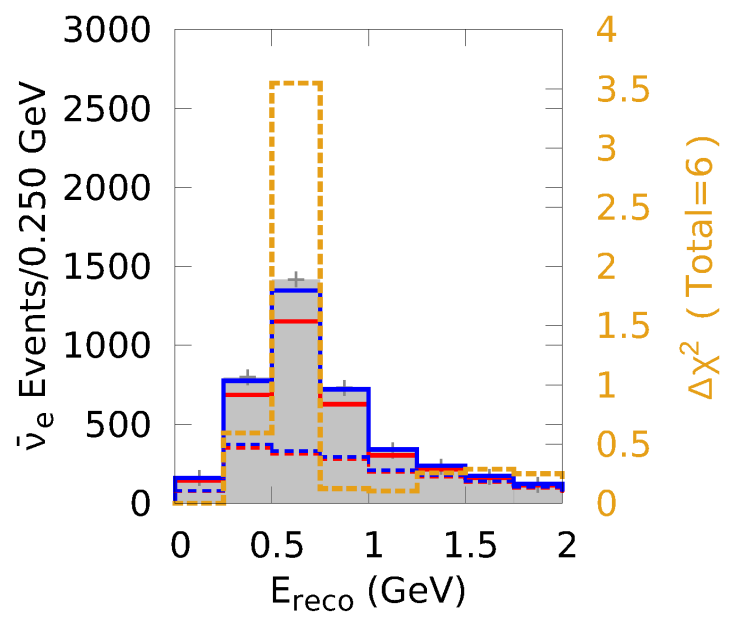

(B) $\bar{\nu}_{e}$ appearance

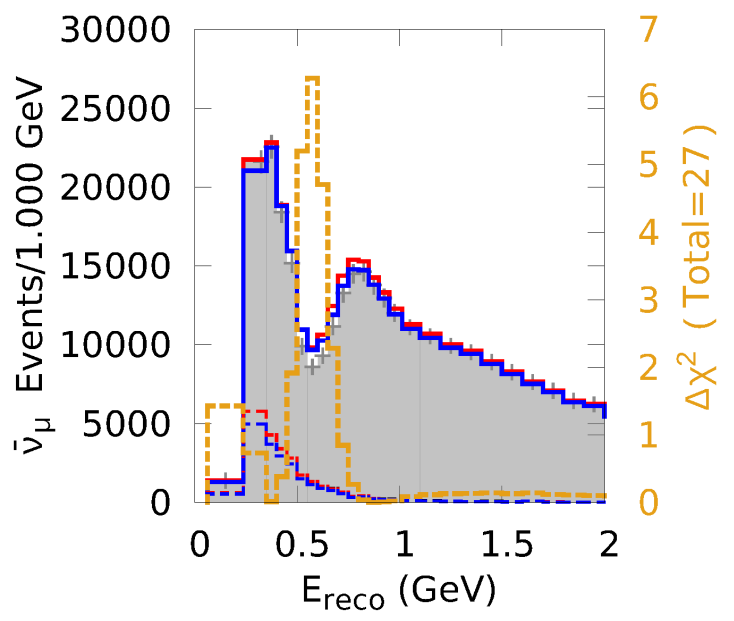

(D) $\bar{\nu}_{\mu}$ disappearance

$$
\begin{array}{r}
\text { Xpre/post }^{2}=338 / 97 \\
\text { True } \mathrm{S}^{\sin (\theta)_{23}}=0.43 \\
\text { Pre-Fit }{ }^{\sin (\theta)_{23}}=0.57 \\
\text { Post-Fit } \sin (\theta)_{23}=0.57 \\
\text { Background }
\end{array}
$$

Figure 6.47. As in Figure 6.44 but for T2HK. 
6.3.2. Sensitivity Regions in $\sin ^{2}\left(\theta_{23}\right)$ vs $\delta_{C P}$. All of the oscillation parameters are well constrained in the global fit except for $\sin ^{2}\left(\theta_{23}\right)$ and $\delta_{C P}$. For this reason, the ability of each experiment to reject hypotheses as a function of the true value of $\sin ^{2}\left(\theta_{23}\right)$ and $\delta_{C P}$ will be explored here. These plots are qualitative in nature and make three effects very obvious:

- Decreasing CP asymmetry with increasing $\sin ^{2}\left(\theta_{23}\right)$

- Increasing matter asymmetry with increasing $\sin ^{2}\left(\theta_{23}\right)$

- Increasing CP asymmetry with increasing $\sin \delta_{C P}$

Figures 6.48 through 6.62 show sensitivity regions for the indicated confidence levels at which each experiment can reject each of the hypotheses. These sensitivity regions $\operatorname{in}^{\sin ^{2}}\left(\theta_{23}\right)$ vs $\delta_{C P}$ are $2 \mathrm{D} \Delta \chi^{2}$ contours assuming the $\Delta \chi^{2}$ has one degree of freedom. In contrast to the plots in Section 6.2.3 that have two degrees of freedom, the plots here have one degree of freedom because all of the parameters are profiled in the fit except for one: the hypothesis being tested $\left(\right.$ e.g. $\left.\sin \delta_{C P} \neq 0\right)$. The $\Delta \chi^{2}$ values are computed at points in the $\sin ^{2}\left(\theta_{23}\right)$ vs $\delta_{C P}$ plane sampled by an evenly spaced $40 \times 40$ grid. The true values of $\sin ^{2}\left(\theta_{23}\right)$ and $\delta_{C P}$ are set to the values of the grid point. The $\mathrm{T} 2 \mathrm{~K}, \mathrm{NO} \nu \mathrm{A}$, and $\mathrm{T} 2 \mathrm{~K}+\mathrm{NO} \nu \mathrm{A}$ sensitivity regions are limited to the $90 \%$ and $3 \sigma$ significance levels, while the LBNE and T2HK show the $3 \sigma$ and $5 \sigma$ levels. The Capozzi et al. global fit value, $1 \sigma$ region, and $3 \sigma$ region for $\sin ^{2}\left(\theta_{23}\right)$ are indicated by the horizontal black lines and differ for the True $\mathrm{MH}=\mathrm{NH}$ and True $\mathrm{MH}=\mathrm{IH}$ assumptions. All of the figures profile all of the oscillation parameters, the octant, and the $\mathrm{MH}$ in the fit. The nominal assumptions for $\nu: \bar{\nu}$ running and normalization systematics are assumed here.

6.3.2.1. CP Violation. The first set of sensitivity regions in Figures 6.48.6.52 are for $\mathrm{CP}$ violation. At a particular point in the plane, which indicates a true value of $\sin ^{2}\left(\theta_{23}\right)$ 
and a true value of $\delta_{C P}$, the null hypothesis, $\sin \delta_{C P}=0$, can be rejected at the indicated significance level.

For T2K, in Figure 6.48, CP violation can be detected at the $90 \%$ level in a region around $\delta_{C P}^{\text {true }}=-\pi / 2$ for the True $\mathrm{MH}=\mathrm{NH}$ and $\delta_{C P}^{\text {true }}=\pi / 2$ for True $\mathrm{MH}=\mathrm{IH}$. In the True $\mathrm{MH}=\mathrm{NH}$ case the significance falls below $90 \%$ for some values of $\sin ^{2}\left(\theta_{23}\right)$ because of the degeneracy between $\delta_{C P}$ and $\sin ^{2}\left(\theta_{23}\right)$ A similar drop-off is seen for True $\mathrm{MH}=\mathrm{IH}$ but for the opposite sign of $\delta_{C P}$ and in the second octant. It is a general feature of these sensitivities that the sensitivity decreases as the true value of $\sin ^{2}\left(\theta_{23}\right)$ increases. This is because of the dependence of the CP asymmetry on $\cot \left(\theta_{13}\right)$ as in Equation 19 . T2K can make a $90 \% \mathrm{CL}$ determination of $\mathrm{CP}$ violation if $\delta_{C P}$ is near $-(+) \pi / 2$ in the $\mathrm{NH}(\mathrm{IH})$ and assuming $\sin ^{2}\left(\theta_{23}\right)$ is at the current global fit value. The sensitivity falls off slightly for the second octant.

$\mathrm{NO} \nu \mathrm{A}$, in Figure 6.49, sees similar sensitivity to $\mathrm{T} 2 \mathrm{~K}$ around the global best-fit value of $\sin ^{2}\left(\theta_{23}\right)$, though it has $90 \%$ CL sensitivity over a narrower region in $\delta_{C P}$. The T2K and $\mathrm{NO} \nu \mathrm{A}$ regions complement each other very well in some regions of the plane; in areas where $\mathrm{T} 2 \mathrm{~K}$ sees lower $\Delta \chi^{2}$ values, $\mathrm{NO} \nu \mathrm{A}$ sees higher $\Delta \chi^{2}$ values, and vice versa. However, even the combination of $\mathrm{T} 2 \mathrm{~K}+\mathrm{NO} \nu \mathrm{A}$, in Figure 6.49, does not see much 90\% CL sensitivity in the first octant. This is due to the degeneracy between the NH and the IH. This degeneracy decreases in the second octant because the matter effects become larger in the second octant and $\mathrm{NO} \nu \mathrm{A}$ starts to distinguish between the $\mathrm{NH}$ and $\mathrm{IH}$. The $\mathrm{T} 2 \mathrm{~K}+\mathrm{NO} \nu \mathrm{A}$ combination can make a $90 \%$ CL determination of $\mathrm{CP}$ violation for $\delta_{C P}$ near $-(+) \pi / 2$ in the $\mathrm{NH}(\mathrm{IH})$ and for all $\sin ^{2}\left(\theta_{23}\right)$ values, but falls short of $90 \%$ CL in the first octant for $\delta_{C P}$ near $+(-) \pi / 2$ for the True $\mathrm{MH}=\mathrm{NH}$ (True $\mathrm{MH}=\mathrm{IH}$ ) assumption due to $\mathrm{MH}$ degeneracies.

Figure 6.51 shows the equivalent sensitivity in LBNE. There are no degeneracies between the octant and $\mathrm{MH}$ here so that the regions are relatively uniform in $\delta_{C P}$ and exhibit the 
expected falloff in the $\mathrm{CP}$ asymmetry with increasing $\sin ^{2}\left(\theta_{23}\right)$. LBNE can make a $5 \sigma$ determination of $\mathrm{CP}$ violation for values of $\sin ^{2}\left(\theta_{23}\right)$ near the global best-fit and $\delta_{C P}$ near $\pm \pi / 2$. A $3 \sigma$ determination can be made for all values of $\sin ^{2}\left(\theta_{23}\right)$ for $\delta_{C P}$ values near $\pm \pi / 2$.

The widths of these $\delta_{C P}$ regions will be further quantified in Section 6.4.

T2HK, in Figure 6.51, sees greater than $5 \sigma$ sensitivity for $\delta_{C P}$ values near $-(+) \pi / 2$ for the True $\mathrm{MH}=\mathrm{NH}$ (True $\mathrm{MH}=\mathrm{IH}$ ) assumptions and the coverage of this region, in terms of $\delta_{C P}$, falls of with increasing $\sin ^{2}\left(\theta_{23}\right)$. There is a large effect coming from the MH degeneracy for $\delta_{C P}$ near $+(-) \pi / 2$ for the True $\mathrm{MH}=\mathrm{NH}$ (True $\mathrm{MH}=\mathrm{IH}$ ) assumptions. As the matter effect increases with higher $\sin ^{2}\left(\theta_{23}\right)$ the T2HK sensitivity is increasingly affected by the degeneracy between $\delta_{C P}$ and the $\mathrm{MH}$.

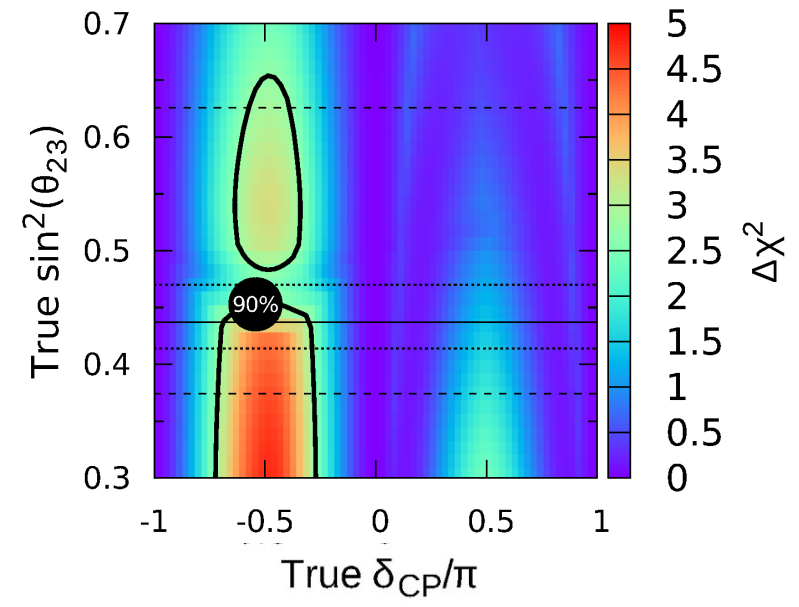

(A) True $\mathrm{MH}=\mathrm{NH}$

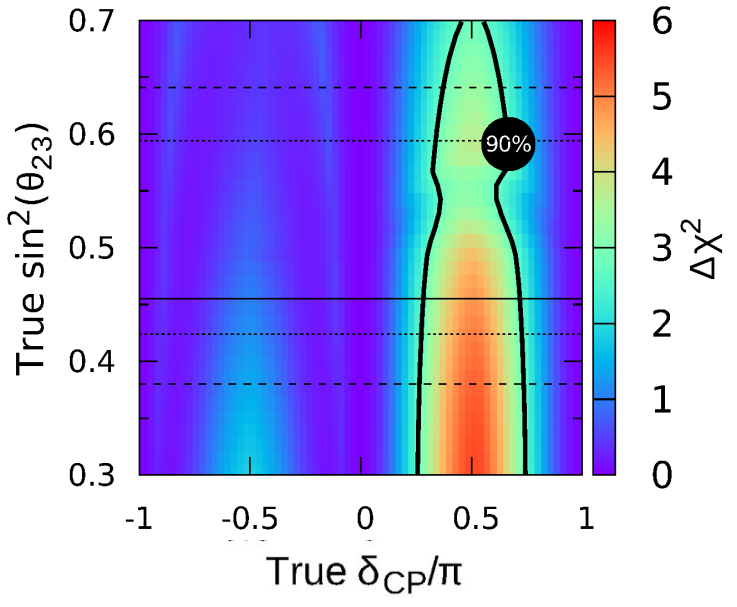

(B) True $\mathrm{MH}=\mathrm{IH}$

Figure 6.48. T2K 90\% CL CP violation sensitivity regions in True $\sin ^{2}\left(\theta_{23}\right)$ vs True $\delta_{C P}$. The color scale represents the $\Delta \chi^{2}$ value. The Capozzi et al. global fit best fit, $1 \sigma$, and $3 \sigma$ regions are indicated by the horizontal black lines. The Capozzi et al. best-fit values are assumed for the true values of the other oscillation parameters. All four samples are fit: $\nu_{e}$ appearance, $\bar{\nu}_{e}$ appearance, $\nu_{\mu}$ disappearance, and $\bar{\nu}_{\mu}$ disappearance. The nominal assumptions are used for systematics and $\nu: \bar{\nu}$ ratios. The other oscillation parameters, $\mathrm{MH}$, and octant are profiled. 


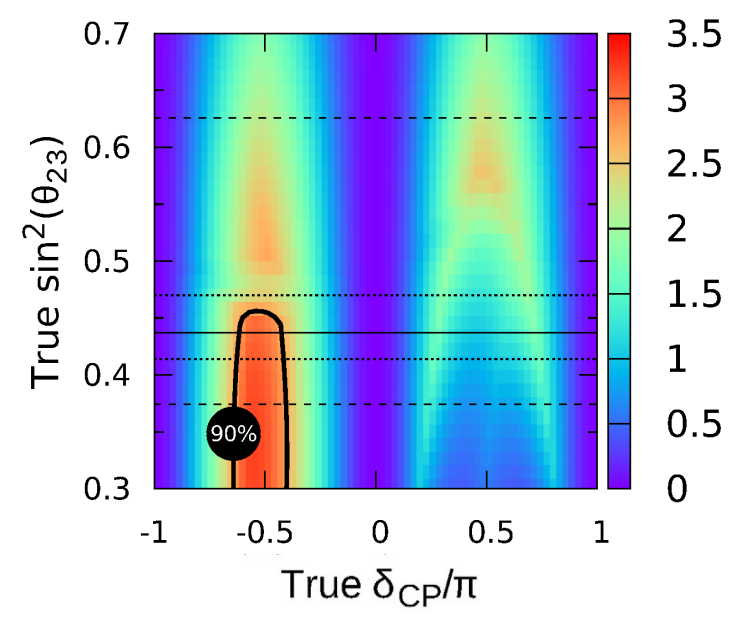

(A) True $\mathrm{MH}=\mathrm{NH}$

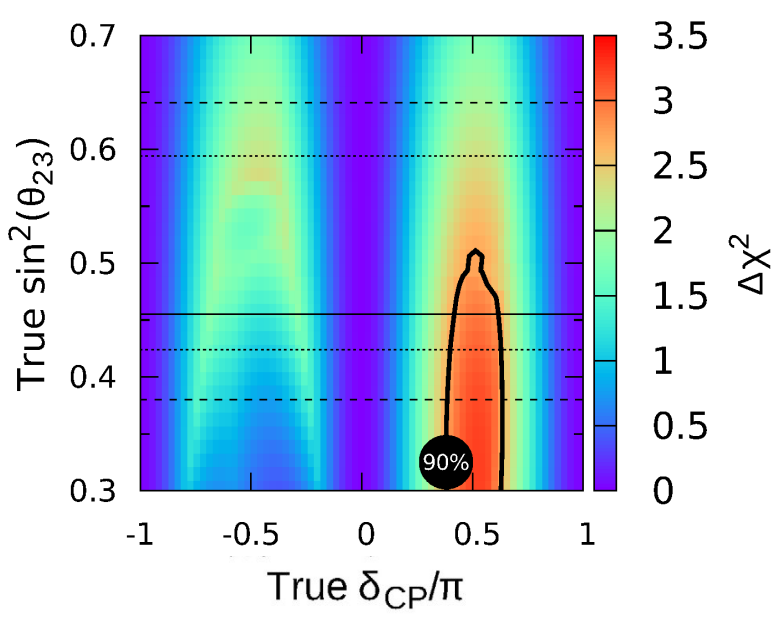

(B) True $\mathrm{MH}=\mathrm{IH}$

Figure 6.49. As in Figure 6.48 but for $\mathrm{NO} \nu \mathrm{A}$.

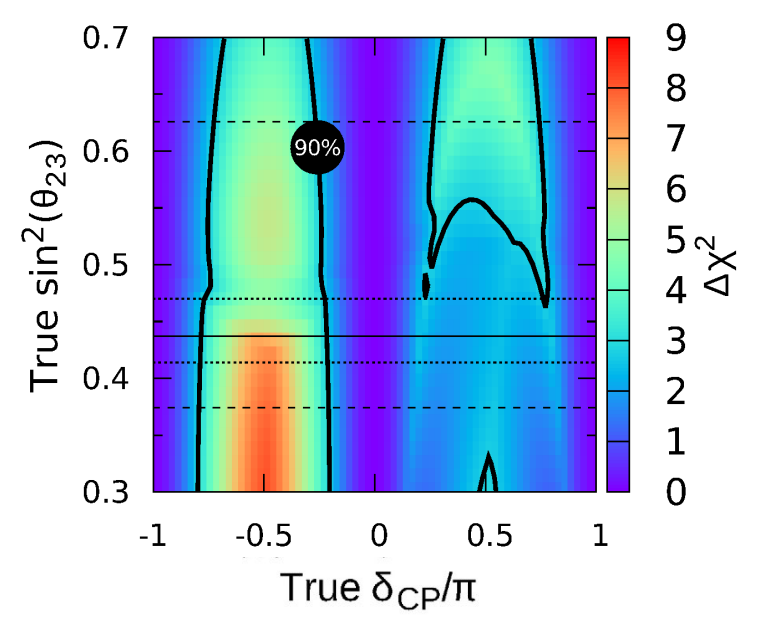

(A) True $\mathrm{MH}=\mathrm{NH}$

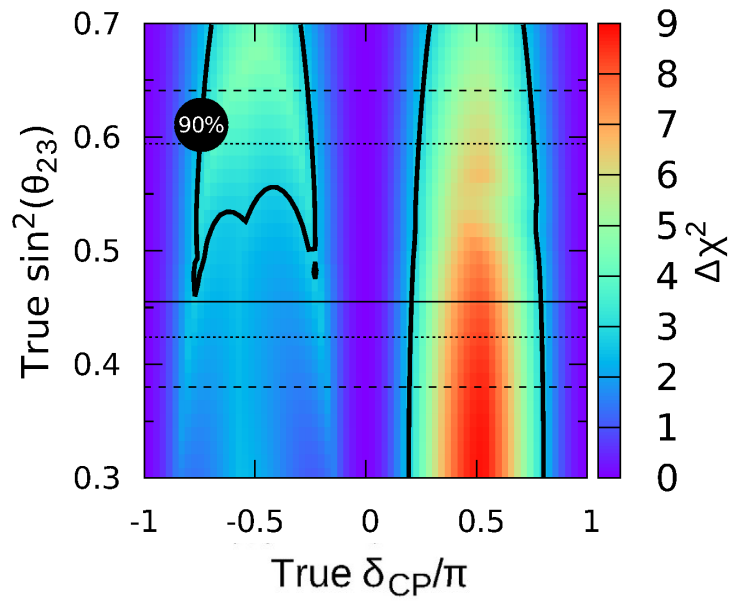

(B) True $\mathrm{MH}=\mathrm{IH}$

Figure 6.50. As in Figure 6.48 but for $\mathrm{T} 2 \mathrm{~K}+\mathrm{NO} \nu \mathrm{A}$. 


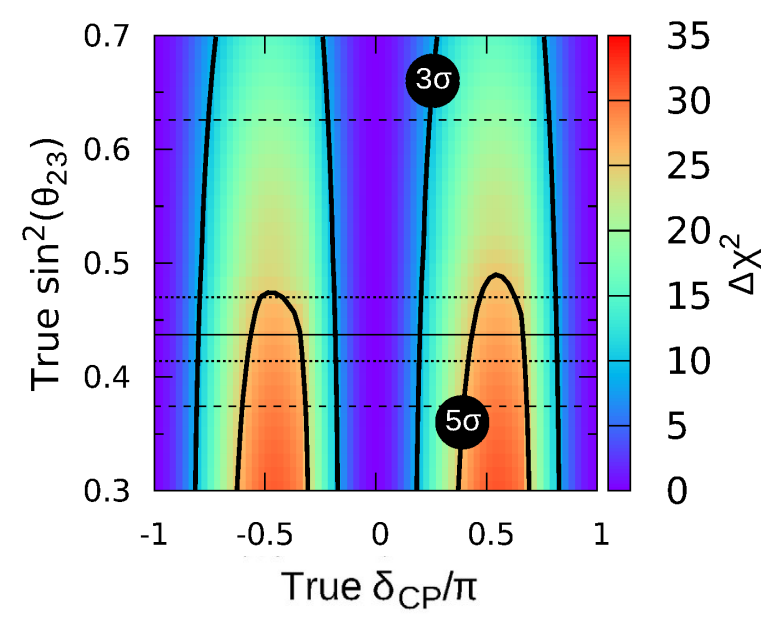

(A) True $\mathrm{MH}=\mathrm{NH}$

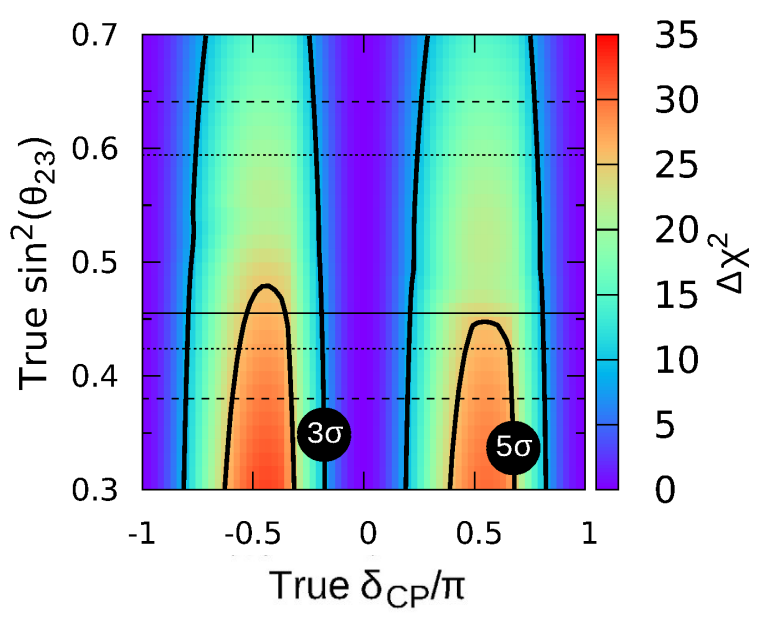

(B) True $\mathrm{MH}=\mathrm{IH}$

Figure 6.51. As in Figure 6.48 but for LBNE $3 \sigma$ and $5 \sigma$.

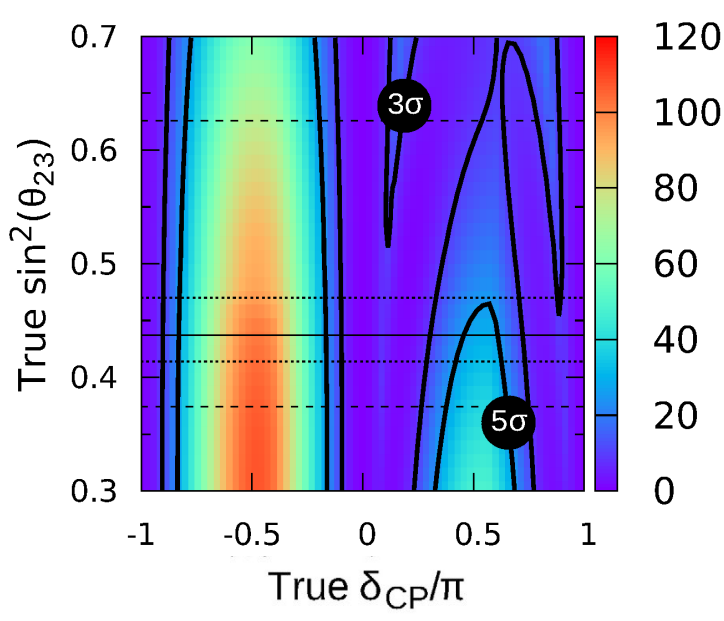

(A) True $\mathrm{MH}=\mathrm{NH}$

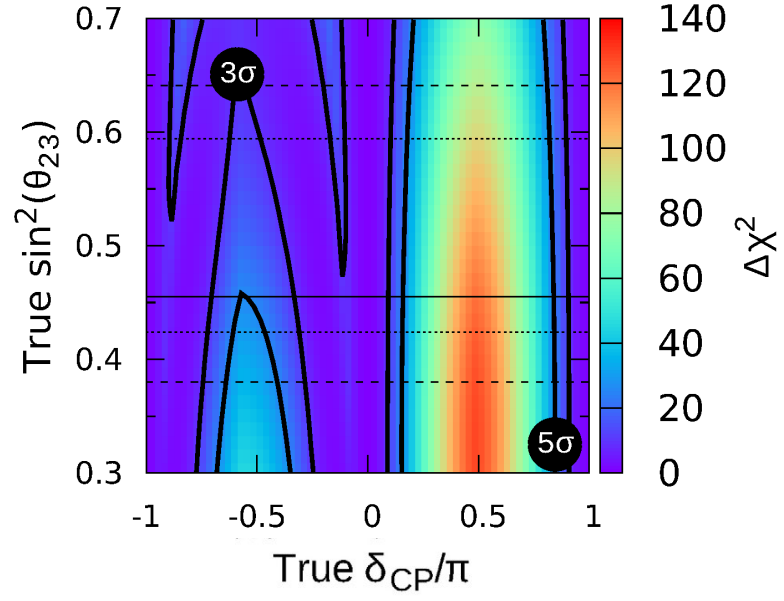

(B) True $\mathrm{MH}=\mathrm{IH}$

Figure 6.52. As in Figure 6.48 but for T2HK $3 \sigma$ and $5 \sigma$. 
6.3.2.2. Mass Hierarchy. The next set of sensitivity regions in Figures 6.53-6.57 are for MH determination. At a particular point in the plane, which indicates the true value of $\sin ^{2}\left(\theta_{23}\right)$ and the true value of $\delta_{C P}$ used in the $\Delta \chi^{2}$, the null hypothesis of the opposite MH can be rejected at the indicated significance level. For the True $\mathrm{MH}=\mathrm{NH}$ (True $\mathrm{MH}=\mathrm{IH}$ ) assumption the fit spectrum is for the $\mathrm{IH}(\mathrm{MH})$ and this fit spectrum is allowed to vary with respect to the oscillation parameters and normalization systematics. This means that for the true $\mathrm{MH}$ assumption, the opposite $\mathrm{MH}$ can be rejected at the indicated significance level. The distribution of this $\Delta \chi^{2}$ does not actually follow a typical $\chi^{2}$ distribution, especially for low values of $\Delta \chi^{2}$, but this feature will be revisited in Chapter 8 and for now the significance will be interpreted as $\sigma=\sqrt{\Delta \chi^{2}}$.

T2K has very little sensitivity to the $\mathrm{MH}$ due to the short baseline, but there is a small effect coming from the matter effect shown in Figure 6.53. But this significance only reaches the $1 \sigma$ level for a small region in $\delta_{C P}$ around the global best-fit value of $\sin ^{2}\left(\theta_{23}\right)$. Any significance around $\delta_{C P}=+(-) \pi / 2$ is lost by the degeneracy between $\delta_{C P}$ and the MH for the case of the True $\mathrm{MH}=\mathrm{NH}$ (True $\mathrm{MH}=\mathrm{IH}$ ) assumption. There is an interesting effect that occurs around $\delta_{C P}=-(+) \pi / 2$ for True $\mathrm{MH}=\mathrm{NH}$ (True $\mathrm{MH}=\mathrm{IH}$ ). The fit value of $\delta_{C P}$ that minimizes the $\Delta \chi^{2}$ jumps from 0 to $\pi$ producing a ridge of slightly more significant $\Delta \chi^{2}$ values. A more extreme example of this effect is given by the $\mathrm{MH}$ sensitivity regions for T2HK in Figure 6.57 where there are regions of $3 \sigma$ and $5 \sigma$ sensitivity to the MH. A significant portion of the total $\Delta \chi^{2}$ comes from the $\nu_{\mu}$ disappearance spectral distortions at these particular values of $\delta_{C P}$ and $\sin ^{2}\left(\theta_{23}\right)$.

By itself $\mathrm{NO} \nu \mathrm{A}$ see significant regions, in Figure 6.54, of $\delta_{C P}$ covered at the $90 \% \mathrm{CL}$ around the global best-fit value of $\sin ^{2}\left(\theta_{23}\right)$ around $\delta_{C P}^{\text {true }}=-\pi / 2\left(\delta_{C P}^{\text {true }}=\pi / 2\right)$ for the case of True $\mathrm{MH}=\mathrm{NH}($ True $\mathrm{MH}=\mathrm{IH})$. The degeneracy between $\delta_{C P}$ and $\mathrm{MH}$ makes a significant 
measurement difficult for the opposite sign of true $\delta_{C P}$. Combining $\mathrm{T} 2 \mathrm{~K}+\mathrm{NO} \nu \mathrm{A}$, as in Figure 6.55, breaks the degeneracy for higher values of $\sin ^{2}\left(\theta_{23}\right)$ and this extends the $90 \%$ regions in the degenerate region for both True $\mathrm{MH}=\mathrm{NH}$ and True $\mathrm{MH}=\mathrm{IH}$.

A combination of high resolution on $\delta_{C P}$ and sensitivity to spectral shape differences between $\mathrm{NH}$ and $\mathrm{IH}$ is critical to the ability to make a significant measurement for all values of $\delta_{C P}$ and $\sin ^{2}\left(\theta_{23}\right)$. LBNE is able to break these degeneracies and make a greater than $5 \sigma$ determination of the $\mathrm{MH}$ for most values of $\sin ^{2}\left(\theta_{23}\right)$ and $\delta_{C P}$. There is a small region where the degeneracies are still present when the matter effect is lower at lower values of $\sin ^{2}\left(\theta_{23}\right)$ so that $\delta_{C P}$ and $\mathrm{MH}$ effects are degenerate. Covering the second oscillation maximum, as discussed in Chapter 4, is critical to breaking the degeneracy in this region and will be discussed further in Chapter 9.

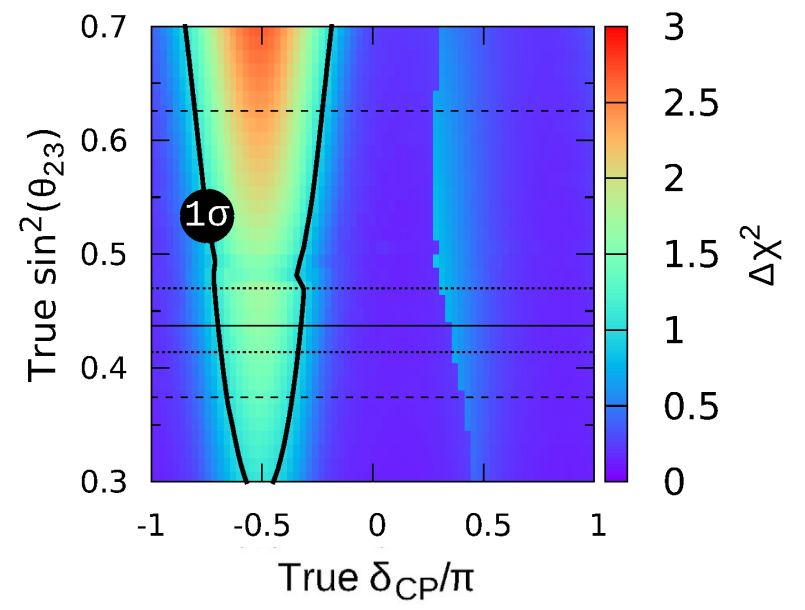

(A) True $\mathrm{MH}=\mathrm{NH}$

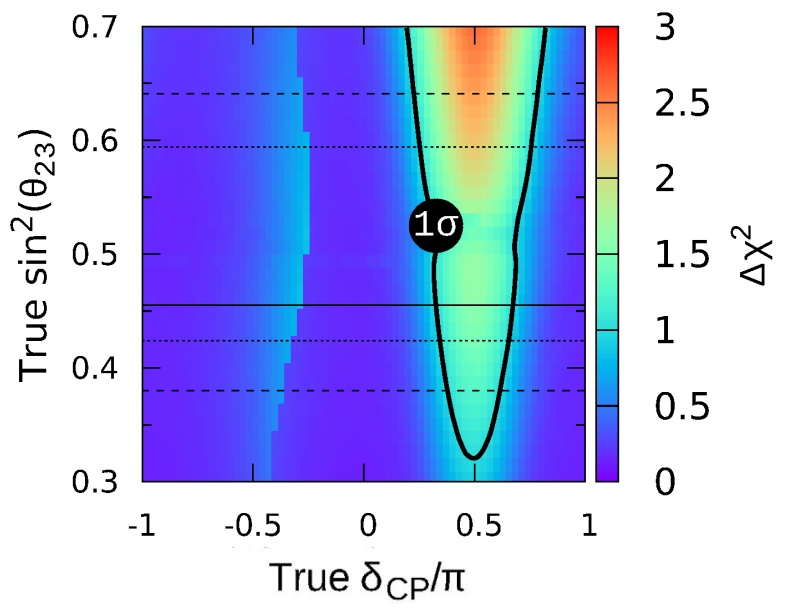

(B) True $\mathrm{MH}=\mathrm{IH}$

Figure 6.53. T2K $1 \sigma$ CL MH sensitivity regions in True $\sin ^{2}\left(\theta_{23}\right)$ vs True $\delta_{C P}$. The color scale represents the $\Delta \chi^{2}$ value. The Capozzi et al. global fit best fit, $1 \sigma$, and $3 \sigma$ regions are indicated by the horizontal black lines. The Capozzi et al. best-fit values are assumed for the true values of the other oscillation parameters. All four samples are fit: $\nu_{e}$ appearance, $\bar{\nu}_{e}$ appearance, $\nu_{\mu}$ disappearance, and $\bar{\nu}_{\mu}$ disappearance. The nominal assumptions are used for systematics and $\nu: \bar{\nu}$ ratios. The other oscillation parameters and the octant are profiled. 


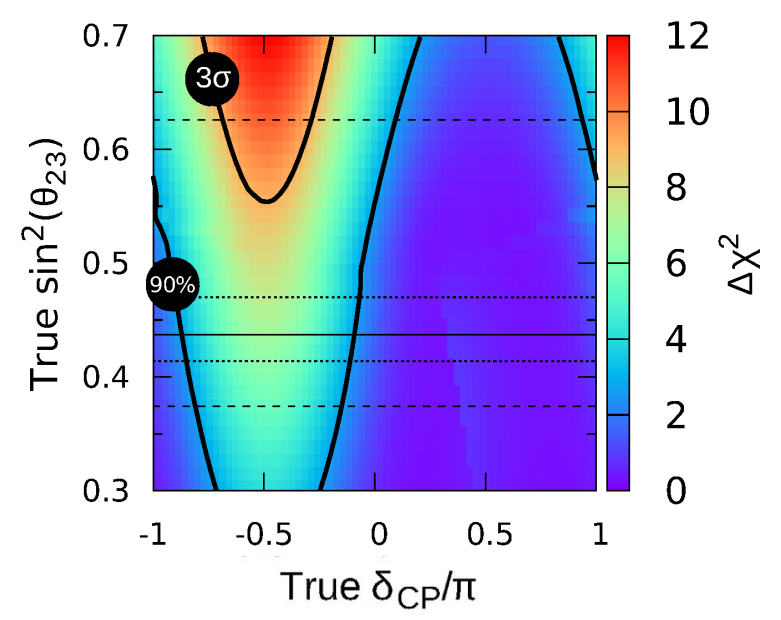

(A) True $\mathrm{MH}=\mathrm{NH}$

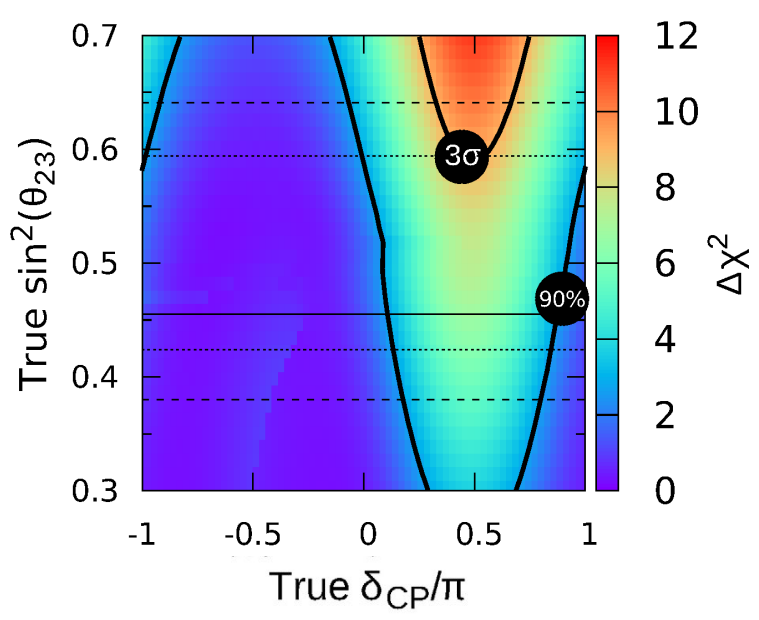

(B) True $\mathrm{MH}=\mathrm{IH}$

Figure 6.54. As in Figure 6.53 but for $\mathrm{NO} \nu \mathrm{A}$.

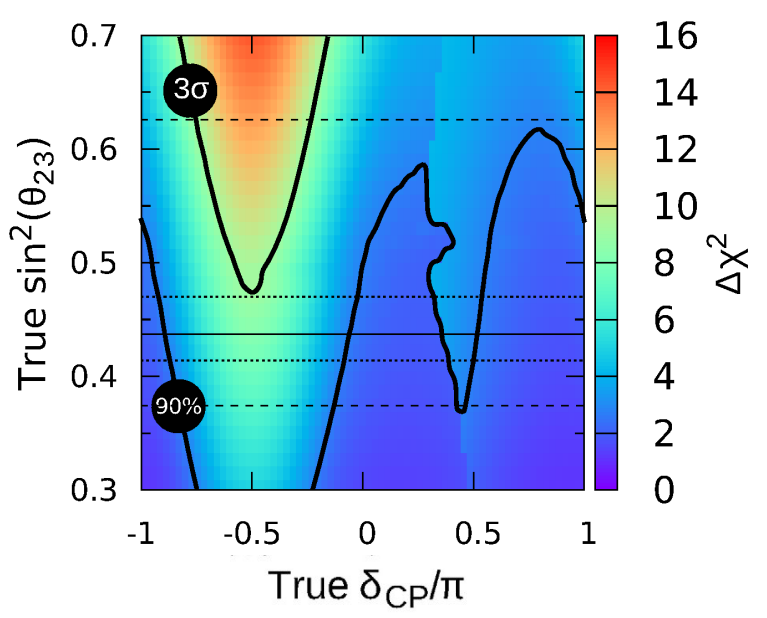

(A) True $\mathrm{MH}=\mathrm{NH}$

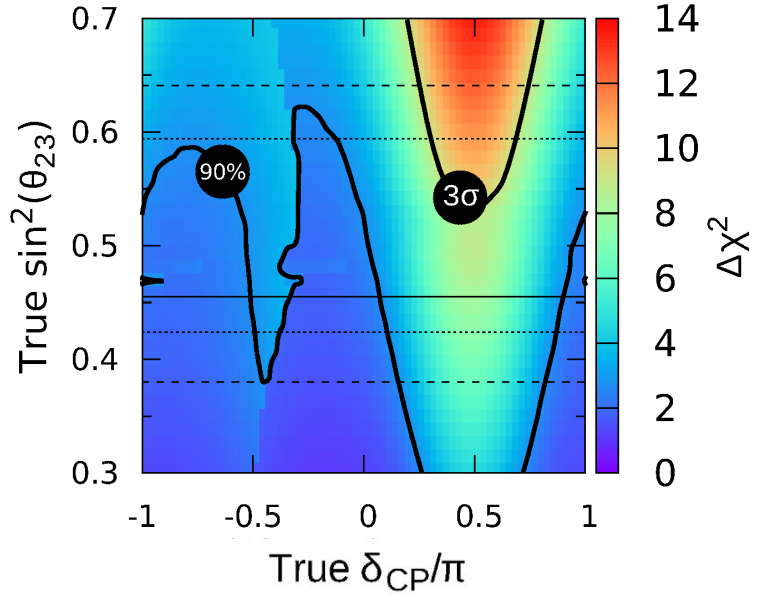

(B) True $\mathrm{MH}=\mathrm{IH}$

Figure 6.55. As in Figure 6.53 but for $\mathrm{T} 2 \mathrm{~K}+\mathrm{NO} \nu \mathrm{A}$. 


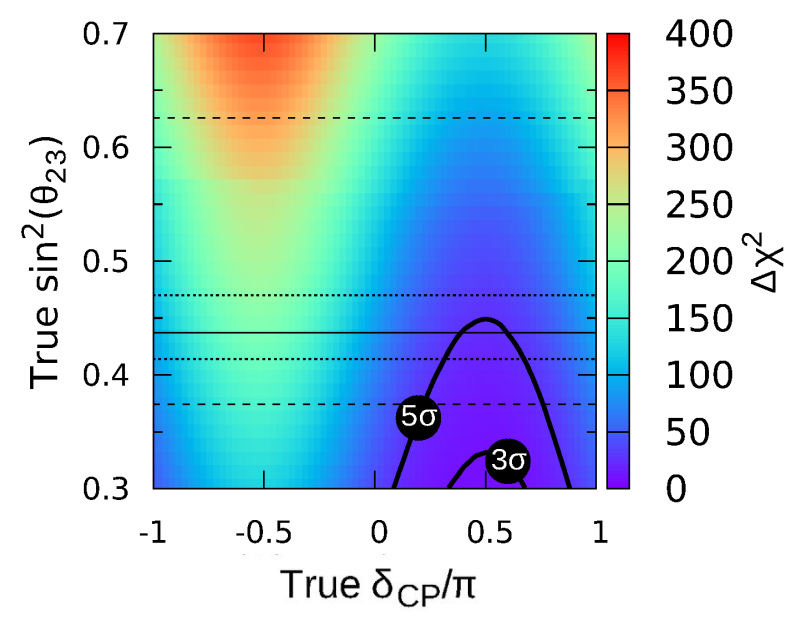

(A) True $\mathrm{MH}=\mathrm{NH}$

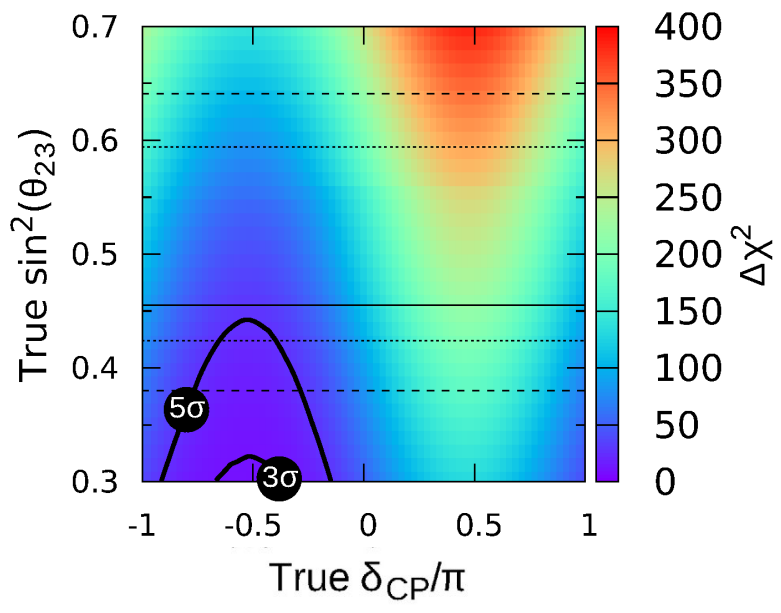

(B) True $\mathrm{MH}=\mathrm{IH}$

Figure 6.56. As in Figure 6.53 but for LBNE $3 \sigma$ and $5 \sigma$.

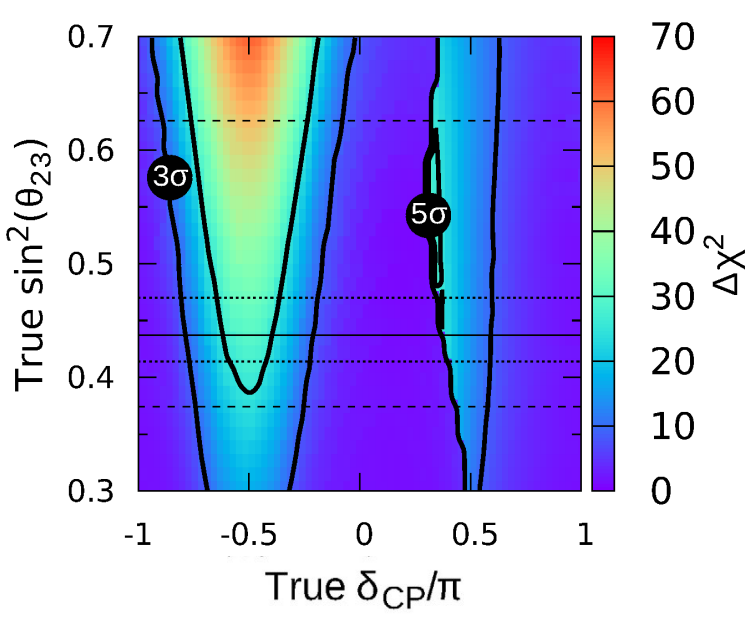

(A) True $\mathrm{MH}=\mathrm{NH}$

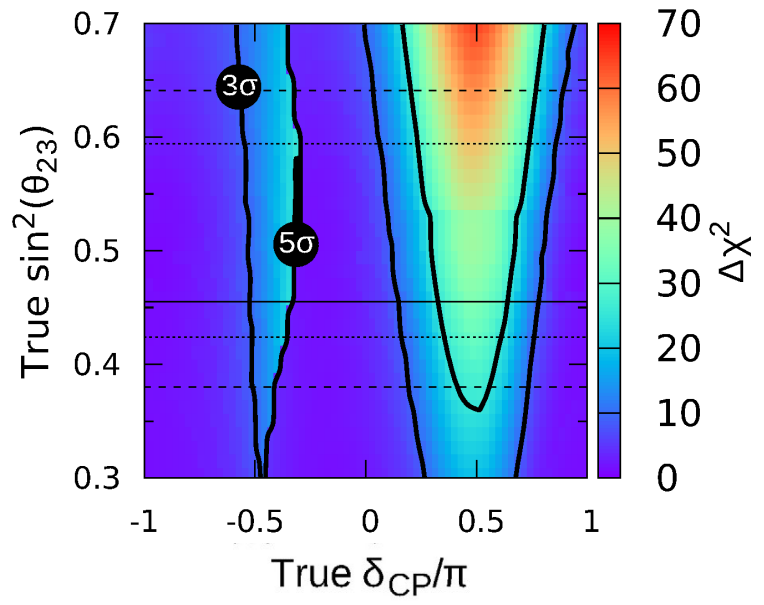

(B) True $\mathrm{MH}=\mathrm{IH}$

Figure 6.57. As in Figure 6.53 but for T2HK $3 \sigma$ and $5 \sigma$. 
6.3.2.3. $\theta_{23}$ Octant. The final set of sensitivity regions in Figures 6.58.6.62 are for $\theta_{23}$ octant determination. At a particular point in the plane, which again indicates the true value of $\sin ^{2}\left(\theta_{23}\right)$ and the true value of $\delta_{C P}$ used in the $\Delta \chi^{2}$, the null hypothesis of the opposite octant can be rejected at the indicated significance level. The true octant is given by the $\sin ^{2}\left(\theta_{23}\right)$ value in the plane and the $\sin ^{2}\left(\theta_{23}\right)$ value of the fit spectra is forced to be in the opposite octant but the fit $\sin ^{2}\left(\theta_{23}\right)$ value is still profiled in the $\Delta \chi^{2}$ minimization.

T2K and $\mathrm{NO} \nu \mathrm{A}$ have comparable $90 \%$ and $3 \sigma$ octant sensitivity regions, shown in Figures 6.58 and 6.59 , although there is variation in each of them with respect to $\delta_{C P}$ that comes from their ability to constrain $\delta_{C P}$ in the fit. Their combination sees a relatively flat response in $\delta_{C P}$ and can determine the octant to $90 \%$ CL from $\sin ^{2}\left(\theta_{23}\right) \sim 0.46$ to $\sin ^{2}\left(\theta_{23}\right) \sim 0.56$. LBNE and T2HK, in Figures 6.61 and 6.62 , both see $3 \sigma$ octant sensitivity for $\sin ^{2}\left(\theta_{23}\right)$ values between 0.46 and 0.57 . 


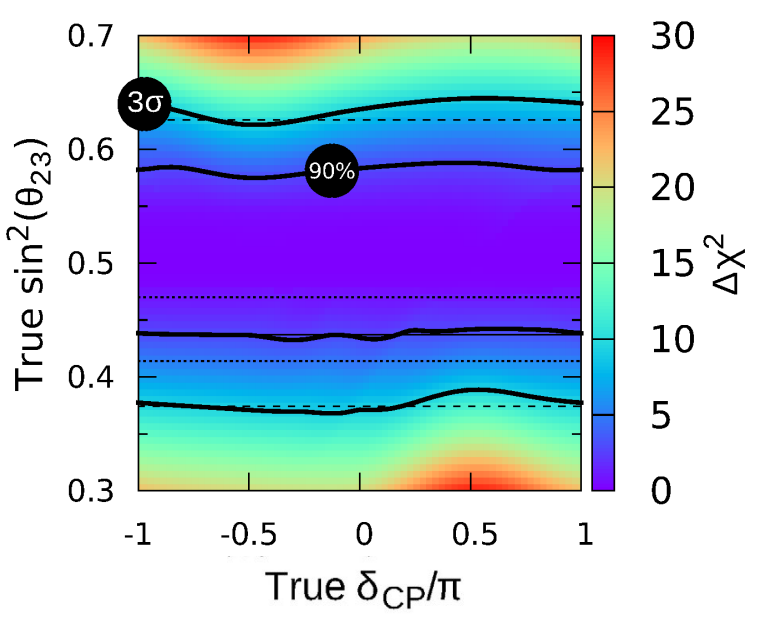

(A) True $\mathrm{MH}=\mathrm{NH}$

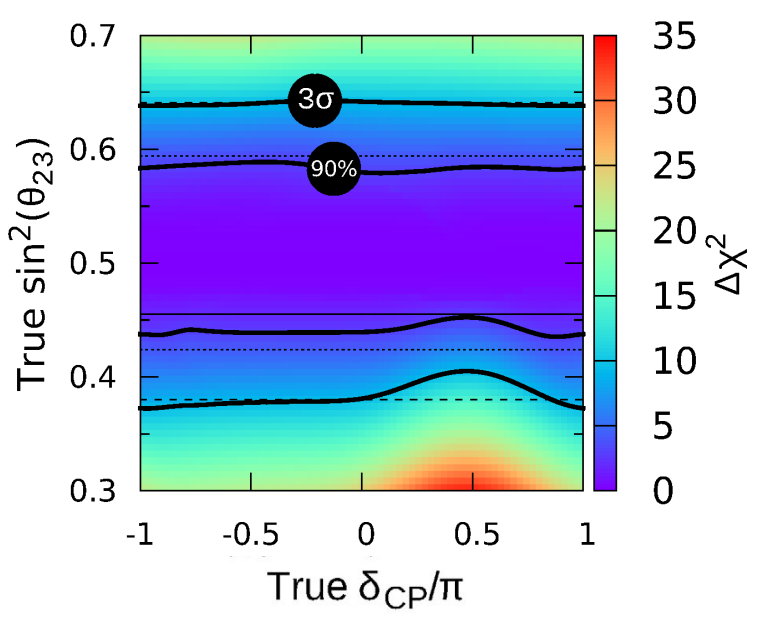

(B) True $\mathrm{MH}=\mathrm{IH}$

Figure 6.58. T2K $90 \%$ and $3 \sigma$ CL octant sensitivity regions in True $\sin ^{2}\left(\theta_{23}\right)$ vs True $\delta_{C P}$. The color scale represents the $\Delta \chi^{2}$ value. The Capozzi et al. global fit best fit, $1 \sigma$, and $3 \sigma$ regions are indicated by the horizontal black lines. The Capozzi et al. best-fit values are assumed for the true values of the other oscillation parameters. All four samples are fit: $\nu_{e}$ appearance, $\bar{\nu}_{e}$ appearance, $\nu_{\mu}$ disappearance, and $\bar{\nu}_{\mu}$ disappearance. The nominal assumptions are used for systematics and $\nu: \bar{\nu}$ ratios. The other oscillation parameters and the $\mathrm{MH}$ are profiled.

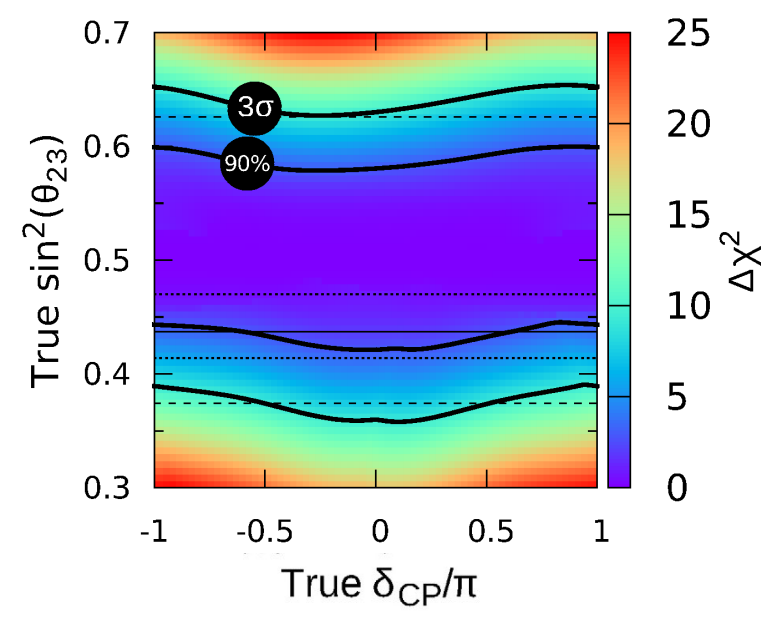

(A) True $\mathrm{MH}=\mathrm{NH}$

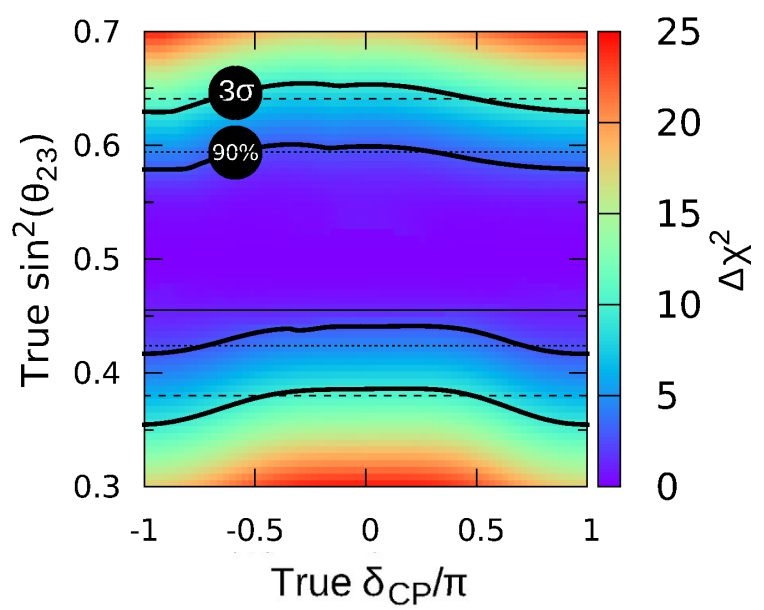

(B) True $\mathrm{MH}=\mathrm{IH}$

Figure 6.59. As in Figure 6.58 but for $\mathrm{NO} \nu \mathrm{A}$. 


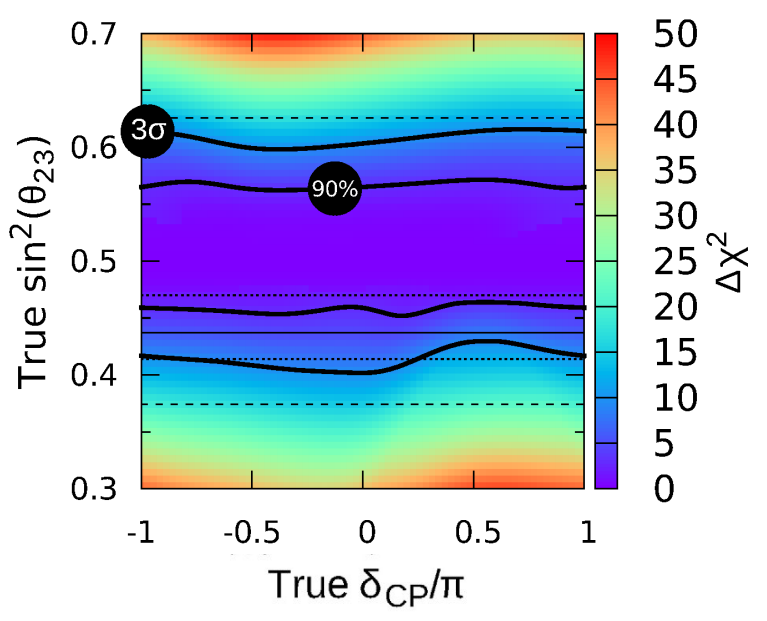

(A) True $\mathrm{MH}=\mathrm{NH}$

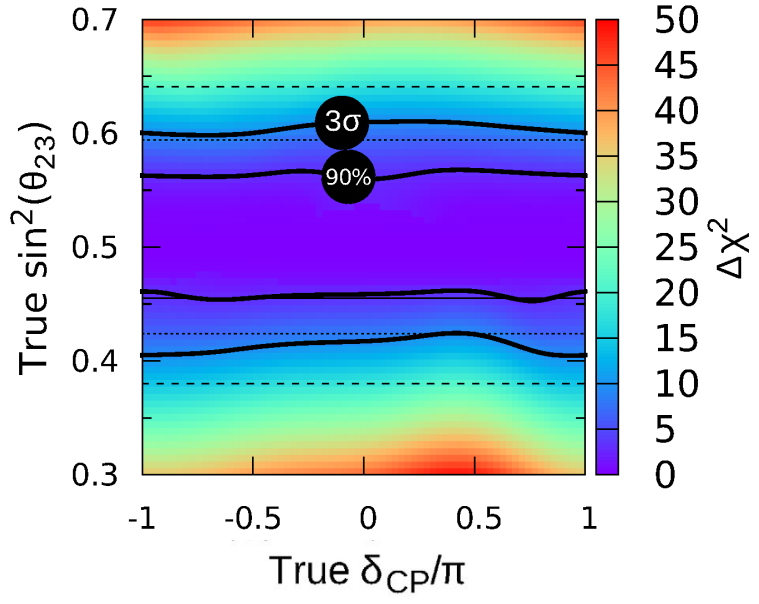

(B) True $\mathrm{MH}=\mathrm{IH}$

Figure 6.60. As in Figure 6.58 but for $\mathrm{T} 2 \mathrm{~K}+\mathrm{NO} \nu \mathrm{A}$.

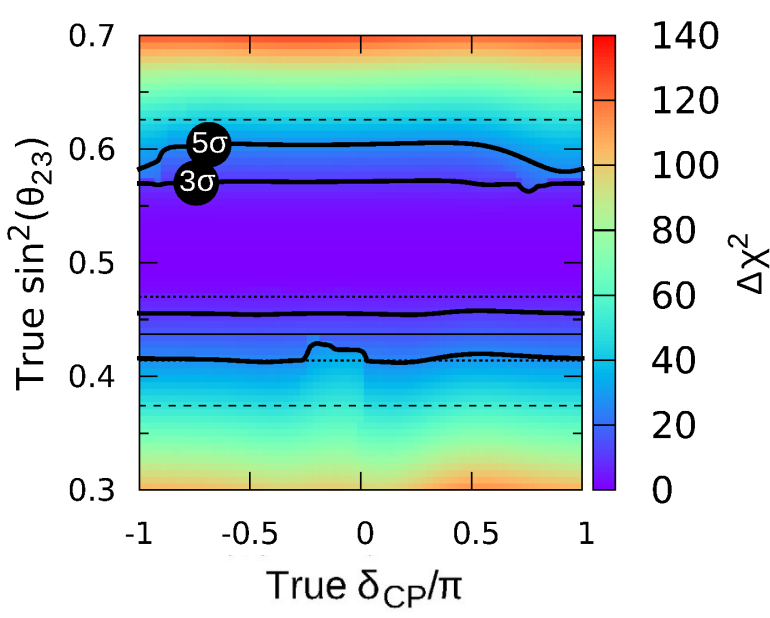

(A) True $\mathrm{MH}=\mathrm{NH}$

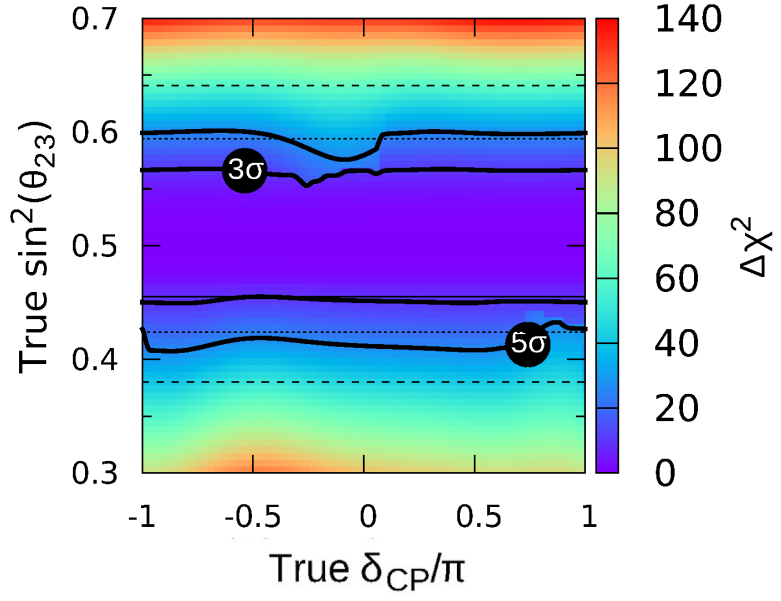

(B) True $\mathrm{MH}=\mathrm{IH}$

Figure 6.61. As in Figure 6.58 but for LBNE $3 \sigma$ and $5 \sigma$. 


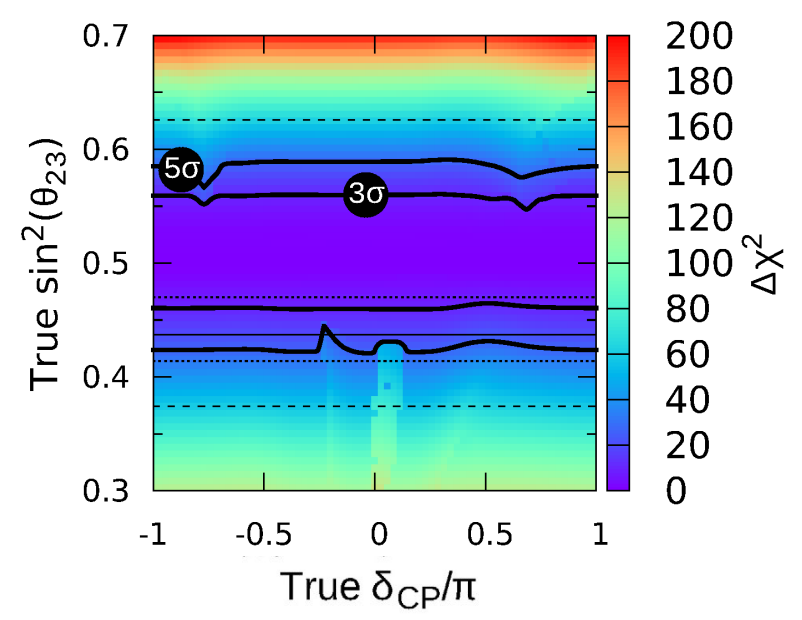

(A) True $\mathrm{MH}=\mathrm{NH}$

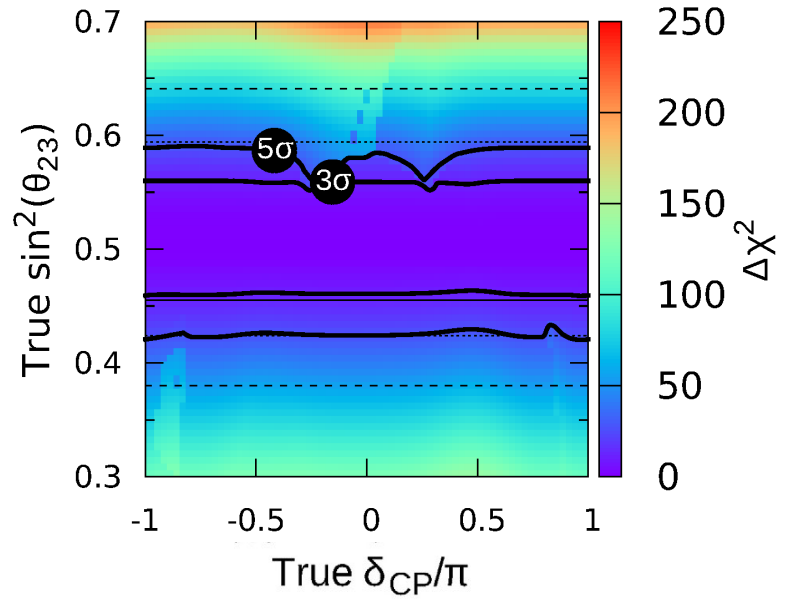

(B) True $\mathrm{MH}=\mathrm{IH}$

Figure 6.62. As in Figure 6.58 but for T2HK $3 \sigma$ and $5 \sigma$. 
6.3.3. Sensitivity vs $\delta_{C P}$. It is also possible to simplify the sensitivity regions produced in the previous section to a two dimensional plot of significance (or $\Delta \chi^{2}$ ) vs the true value of $\delta_{C P}$. The true value of $\sin ^{2}\left(\theta_{23}\right)$ is assumed to be that of the Capozzi et al. global best-fit in this case. This format allows the sensitivities of each of the experiments to be directly compared.

Figures 6.63 and 6.64 give the sensitivity, in terms of $\sqrt{\Delta \chi^{2}}$, vs the true value of $\delta_{C P}$ for detecting $\mathrm{CP}$ violation or determining the $\mathrm{MH}$ respectively. The colored regions represent the maximum and minimum sensitivity that come from considering all the combinations of $1 \sigma$ uncertainties on $\sin ^{2}\left(2 \theta_{13}\right), \sin ^{2}\left(\theta_{23}\right)$, and $\Delta m_{31}^{2}$ in the indicated $\mathrm{MH}$ assumption. The colored regions are larger for the True $\mathrm{MH}=\mathrm{IH}$ because the Capozzi et al. $1 \sigma$ constraints on $\sin ^{2}\left(\theta_{23}\right)$ are much larger, reaching into the second octant in the IH case.

For CP violation sensitivity in Figure 6.63 the curves for all of the experiments in the True $\mathrm{MH}=\mathrm{NH}$ case are free of degeneracies for $\delta_{C P}<0$ but there are degeneracies with the $\mathrm{MH}$ for $\delta_{C P}>0$. The reverse is true for True $\mathrm{MH}=\mathrm{IH} . \mathrm{T} 2 \mathrm{~K}$ and $\mathrm{NO} \nu \mathrm{A}$ have similar sensitivity but $\mathrm{T} 2 \mathrm{~K}$ has greater sensitivity when not affected by the $\mathrm{MH}$ degeneracy and $\mathrm{NO} \nu \mathrm{A}$ has greater sensitivity when the $\mathrm{MH}$ is degenerate with $\delta_{C P}$ for true $\delta_{C P}$ values greater(less) than zero for the True $\mathrm{MH}=\mathrm{NH}$ (True $\mathrm{MH}=\mathrm{IH}$ ) case. The $\mathrm{T} 2 \mathrm{~K}+\mathrm{NO} \nu \mathrm{A}$ combination reaches a maximum of about $2.5 \sigma$ in the favorable case and the combination makes the sensitivity nearly uniform in the degenerate case. LBNE is the only experiment not affected by $\mathrm{MH}$ degeneracies here and is able to make a $3 \sigma$ measurement for a significant fraction of $\delta_{C P}$ values. In the $\delta_{C P}<0$ region T2HK has the greatest sensitivity, reaching a maximum of nearly $7 \sigma$, but suffers from the $\mathrm{MH}$ degeneracy in the $\delta_{C P}>0$ case. If the mass hierarchy is assumed to be known, then the $\mathrm{T} 2 \mathrm{~K}, \mathrm{NO} \nu \mathrm{A}, \mathrm{T} 2 \mathrm{~K}+\mathrm{NO} \nu \mathrm{A}$, and $\mathrm{T} 2 \mathrm{HK}$ curves will be nearly symmetric between the $\delta_{C P}<0$ and $\delta_{C P}>0$ regions. 
For MH sensitivity in Figure 6.63 similar features are seen as in the sensitivity regions of the previous section. The figure shows full and scaled versions to make all of the features of the curves visible. T2K, as expected, shows relatively little sensitivity to the $\mathrm{MH}$, but with the much larger statistics of $\mathrm{T} 2 \mathrm{HK}$ is able to make measurements on par with $\mathrm{T} 2 \mathrm{~K}+\mathrm{NO} \nu \mathrm{A}$ combined and even exceeding it for some values of $\delta_{C P}$. There is an interesting feature just below $\delta_{C P}^{\text {true }}=-\pi / 2\left(\delta_{C P}^{\text {true }}=\pi / 2\right)$ for the True $\mathrm{MH}=\mathrm{NH}$ (True $\left.\mathrm{MH}=\mathrm{IH}\right)$ case where the $\Delta \chi^{2}$ value spikes for $\mathrm{T} 2 \mathrm{~K}, \mathrm{NO} \nu \mathrm{A}, \mathrm{T} 2 \mathrm{~K}+\mathrm{NO} \nu \mathrm{A}$, and $\mathrm{T} 2 \mathrm{HK}$. This feature comes about because there is a small region where the degeneracy between the $\mathrm{MH}$ and $\delta_{C P}$ does not exist because the number of signal events differs from any number of events possible in the opposite mass hierarchy for any value of $\delta_{C P}$. LBNE is able make a greater than $5 \sigma \mathrm{MH}$ determination for most values of $\delta_{C P}$.

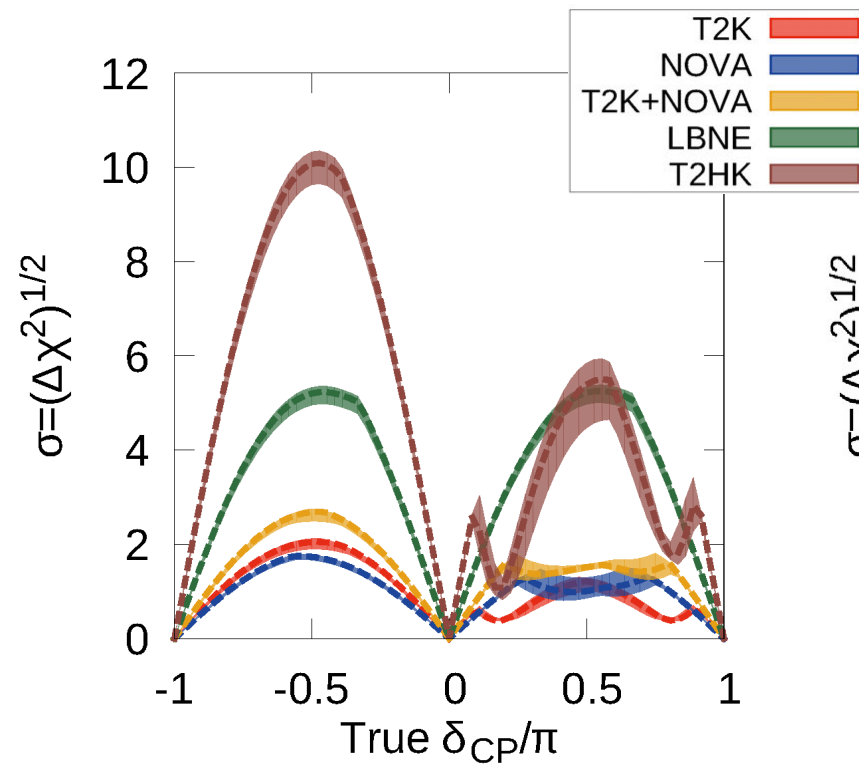

(A) True $\mathrm{MH}=\mathrm{NH}$

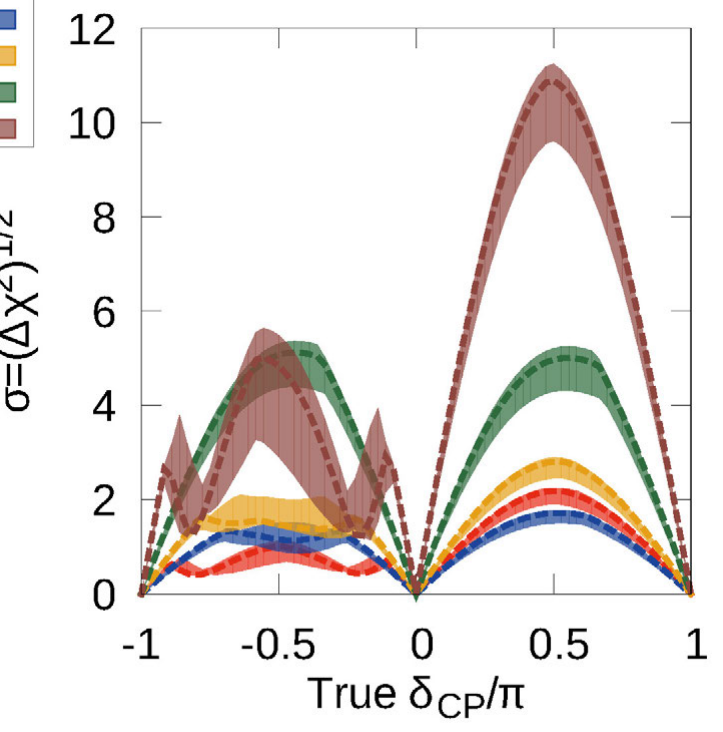

(B) True $\mathrm{MH}=\mathrm{IH}$

Figure 6.63. CP violation sensitivity vs True $\delta_{C P}$. The Capozzi et al. bestfit values are assumed for the true values of the other oscillation parameters. All four samples are fit: $\nu_{e}$ appearance, $\bar{\nu}_{e}$ appearance, $\nu_{\mu}$ disappearance, and $\bar{\nu}_{\mu}$ disappearance. The nominal assumptions are used for systematics and $\nu: \bar{\nu}$ ratios. The other oscillation parameters and $\mathrm{MH}$ are profiled. 


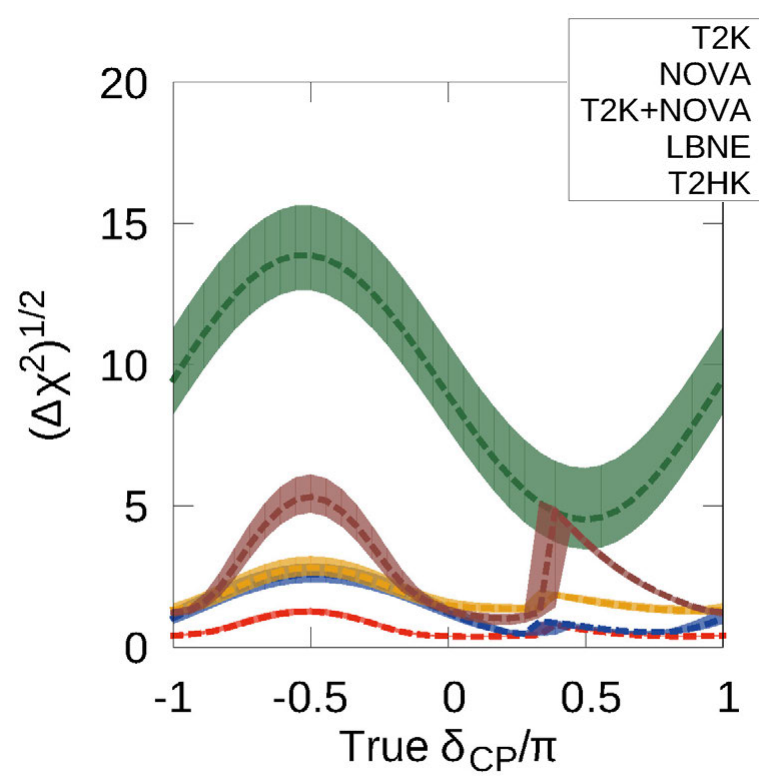

(A) True $\mathrm{MH}=\mathrm{NH}$

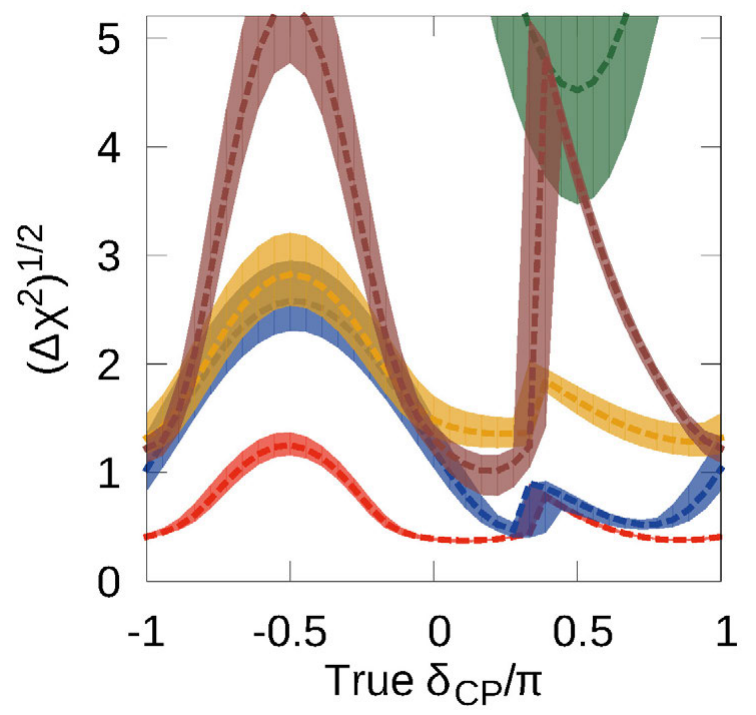

(c) True $\mathrm{MH}=\mathrm{NH}$ (zoomed)

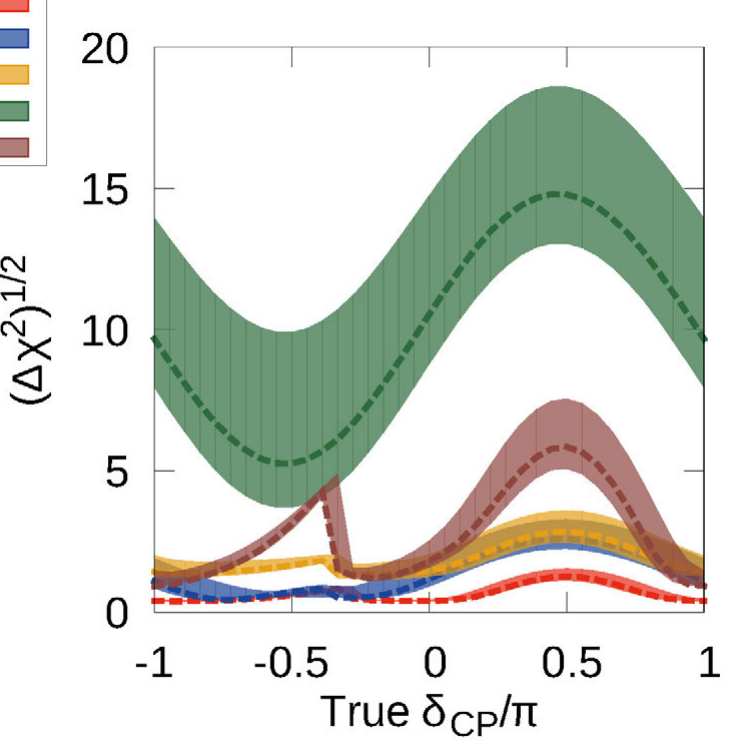

(B) True $\mathrm{MH}=\mathrm{IH}$

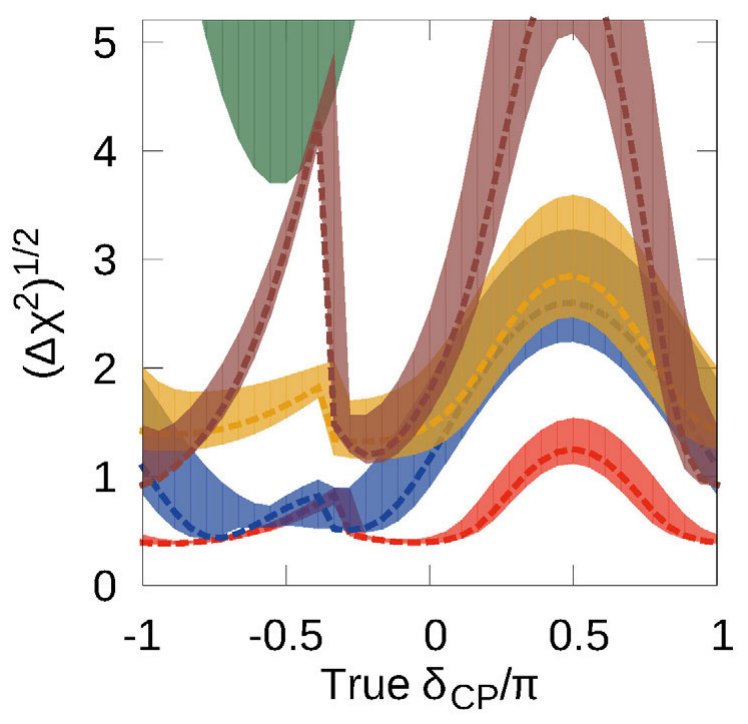

(D) True $\mathrm{MH}=\mathrm{IH}$ (zoomed)

Figure 6.64. MH sensitivity vs True $\delta_{C P}$. The Capozzi et al. best-fit values are assumed for the true values of the other oscillation parameters. All four samples are fit: $\nu_{e}$ appearance, $\bar{\nu}_{e}$ appearance, $\nu_{\mu}$ disappearance, and $\bar{\nu}_{\mu}$ disappearance. The nominal assumptions are used for systematics and $\nu: \bar{\nu}$ ratios. The other oscillation parameters are profiled. The bottom two figures have the y-axis scaled to a smaller value to show features in the lower sensitivities. 


\subsection{Sensitivity vs Exposure}

All of the figures up to this point have assumed the nominal exposure planned by each experiment. The experiments are each planning to run over many years, between 6 and 10 generally, so many measurements of the oscillation parameters and constraints on $\mathrm{CP}$ violation, $\mathrm{MH}$, and the octant could be made before the end of the experiment. This section will evaluate the sensitivity as a function of the number of POT the experiment has received.

Figures 6.65 through 6.72 show resolutions on oscillation parameters and expected sensitivities for MH, CP violation, and the octant. The method is similar to the $\Delta \chi^{2}$ computation defined before, but it is repeated at each POT value to compute a resolution or a sensitivity. The vertical lines indicate the nominal planned exposure for $\mathrm{NO} \nu \mathrm{A}$, LBNE, and $\mathrm{T} 2 \mathrm{~K}$ with the color matching that of the curve for the experiment. T2HK has a nominal running POT of $15.6 \times 10^{21}$ POT so it is beyond the bounds of these plots. The bands represent the best and worst case resolutions that result from $1 \sigma$ variations in the true values of $\sin ^{2}\left(2 \theta_{13}\right)$, $\sin ^{2}\left(\theta_{23}\right)$, and $\Delta m_{31}^{2}$. The dashed lines within the bands represent the best-fit values. So each curve is giving a range of resolutions, at each POT value, on sensitivities that might be achieved taking into account the current $1 \sigma$ uncertainties in these oscillation parameters. All of the other oscillation parameters are assumed to be at the best-fit values and are profiled in the $\Delta \chi^{2}$ minimization. This curves are computed at 30 steps in POT. The horizontal dashed black lines in Figures 6.65 through 6.67 represent the current constraints on the plotted resolution from the Capozzi et al. global fit.

Figure 6.65 shows the $1 \sigma \sin ^{2}\left(2 \theta_{13}\right)$ resolutions vs $\mathrm{POT}$ in $\mathrm{T} 2 \mathrm{~K}, \mathrm{NO} \nu \mathrm{A}, \mathrm{LBNE}$, and

$\mathrm{T} 2 \mathrm{HK}$. The resolution is defined as Resolution $=\frac{\sin ^{2}\left(2 \theta_{13}^{\text {upper }}\right)-\sin ^{2}\left(2 \theta_{13}^{\text {lower }}\right)}{2}$ where the upper and lower values are those $1 \sigma(1 \mathrm{DOF})$ values to which $\sin ^{2}\left(2 \theta_{13}\right)$ can be measured if the true value of $\sin ^{2}\left(2 \theta_{13}\right)=0.092\left(\sin ^{2}\left(2 \theta_{13}\right)=0.094\right)$ in the $\mathrm{NH}(\mathrm{IH})$ is assumed. Two versions are given 
for each true $\mathrm{MH}$ value that show a zoomed y-axis to make details in the LBNE and T2HK curves more visible. $\mathrm{NO} \nu \mathrm{A}$ sees a tighter constraint on $\sin ^{2}\left(2 \theta_{13}\right)$ than $\mathrm{T} 2 \mathrm{~K}$ at the same POT due to higher event rates, but T2K will produce a similar resolution with a higher final POT. T2HK sees the best constraint on $\sin ^{2}\left(2 \theta_{13}\right)$ for all POT values. The current constraint on $\sin ^{2}\left(2 \theta_{13}\right)$ from the 2014 Capozzi et al. global fit, indicated by the horizontal dashed black line, is primarily dominated by results from reactor experiments. Only LBNE and T2HK have high enough statistics to compete with the reactor measurements at their current levels. However, by the time LBNE or T2HK could reach a competitive level, experiments like RENO and Daya Bay will have produced even tighter constraints on $\sin ^{2}\left(2 \theta_{13}\right)$ and the global fit constraints will continue to be dominated by reactor results.

Figure 6.66 shows curves for the $1 \sigma \sin ^{2}\left(\theta_{23}\right)$ resolutions. Here the resolution is defined as Resolution $=\frac{\sin ^{2}\left(\theta_{23}^{\text {upper }}\right)-\sin ^{2}\left(\theta_{23}^{\text {lower }}\right)}{2}$ and the true value of $\sin ^{2}\left(\theta_{23}\right)$ is $\sin ^{2}\left(2 \theta_{13}\right)=0.092$ $\left(\sin ^{2}\left(2 \theta_{13}\right)=0.094\right)$ in the $\mathrm{NH}(\mathrm{IH})$ case. No bands are included in this figure as the $\sin ^{2}\left(\theta_{23}\right)$ constraint variations have a very large effect and make the figure indecipherable. T2K and $\mathrm{NO} \nu \mathrm{A}$ in the True $\mathrm{MH}=\mathrm{NH}$ case show a large slope at early running as the second octant is quickly excluded due to the slightly lower value of $\mathrm{IH} \sin ^{2}\left(\theta_{23}\right)$. The $\mathrm{T} 2 \mathrm{~K}$ and $\mathrm{NO} \nu \mathrm{A}$ curves in the True $\mathrm{MH}=\mathrm{IH}$ case have a relatively low slope at early POT as the first and second octant are still included in the $1 \sigma$ region for $\sin ^{2}\left(\theta_{23}\right)$. But as the POT increases further a large jump is seen as the second octant is finally excluded. Both T2K and $\mathrm{NO} \nu \mathrm{A}$ quickly surpass the current constraints on $\sin ^{2}\left(\theta_{23}\right)$ even with only $50 \%$ of the total POT in the $\mathrm{NO} \nu \mathrm{A}$ case and $\sim 25 \%$ of the total POT in the T2K case. LBNE will reach a resolution of 0.0064, or 1.5\%, under the nominal assumptions and T2HK will exceed this resolution due to the very high exposure and large event rates in $\nu_{\mu}$ disappearance and $\bar{\nu}_{\mu}$ disappearance. 


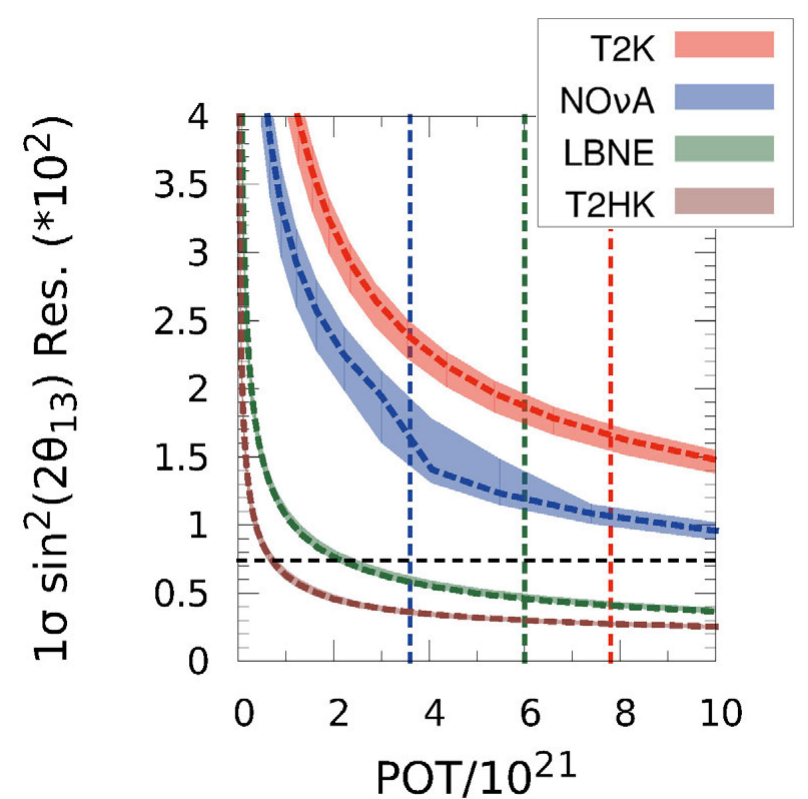

(A) True $\mathrm{MH}=\mathrm{NH}$

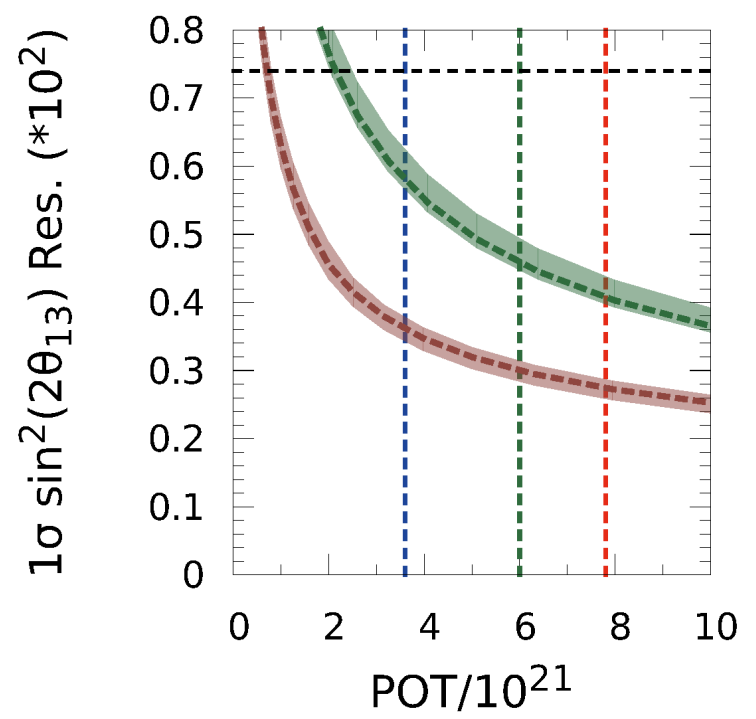

(c) True $\mathrm{MH}=\mathrm{NH}$ (zoomed)

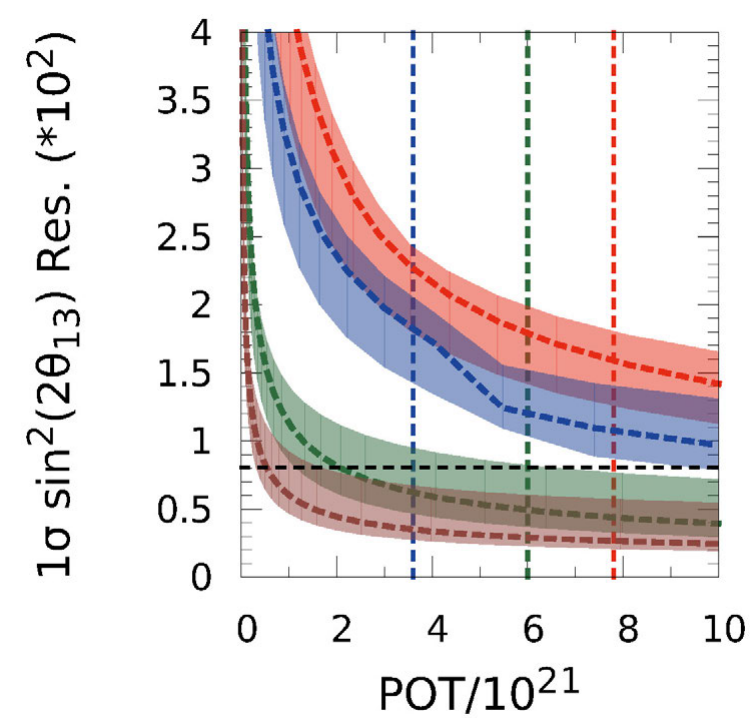

(B) True $\mathrm{MH}=\mathrm{IH}$

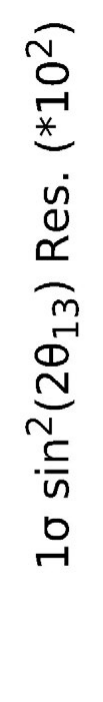

(D) True $\mathrm{MH}=\mathrm{IH}$ (zoomed)

Figure 6.65. $1 \sigma \sin ^{2}\left(2 \theta_{13}\right)$ resolutions vs $\mathrm{POT}$ in $\mathrm{T} 2 \mathrm{~K}, \mathrm{NO} \nu \mathrm{A}, \mathrm{LBNE}$, and T2HK. The Capozzi et al. best-fit values are assumed for the true values of the oscillation parameters and the true value of $\sin ^{2}\left(2 \theta_{13}\right)=0.092$ $\left(\sin ^{2}\left(2 \theta_{13}\right)=0.094\right)$ in the $\mathrm{NH}(\mathrm{IH})$ is used. All four samples are fit: $\nu_{e}$ appearance, $\bar{\nu}_{e}$ appearance, $\nu_{\mu}$ disappearance, and $\bar{\nu}_{\mu}$ disappearance. The nominal assumptions are used for systematics and $\nu: \bar{\nu}$ ratios. The bands represent the best and worst case resolutions that result from $1 \sigma$ variations in the true values of $\sin ^{2}\left(2 \theta_{13}\right), \sin ^{2}\left(\theta_{23}\right)$, and $\Delta m_{31}^{2}$. The other oscillation parameters and $\mathrm{MH}$ are profiled. The vertical dashed lines represent the nominal exposures for $\mathrm{NO} \nu \mathrm{A}, \mathrm{T} 2 \mathrm{~K}$, and LBNE respectively. 


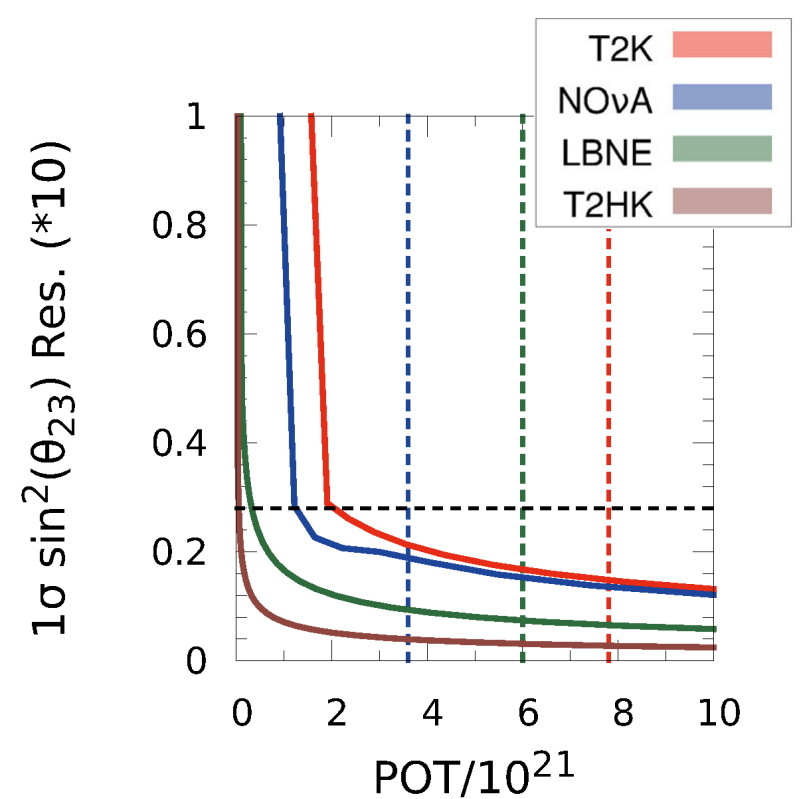

(A) True $\mathrm{MH}=\mathrm{NH}$

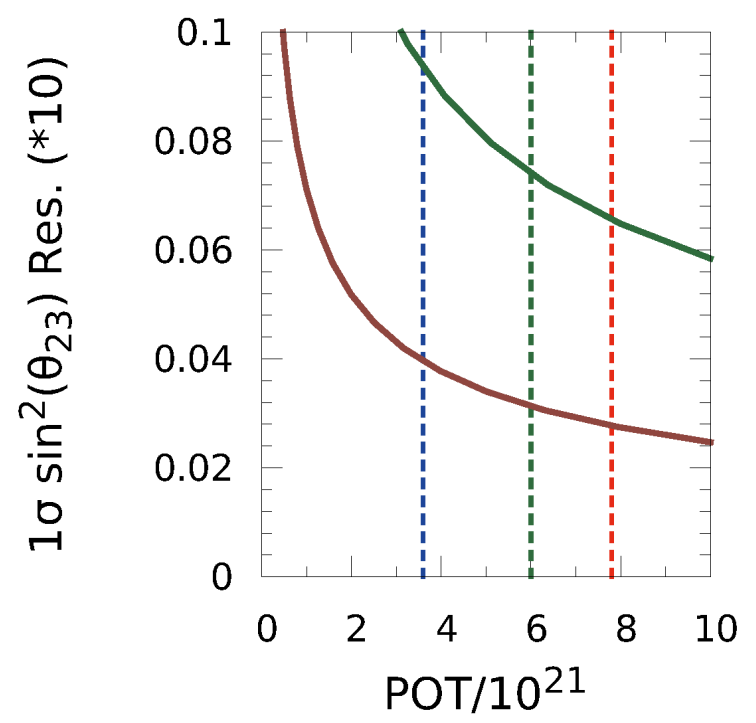

(c) True $\mathrm{MH}=\mathrm{NH}$ (zoomed)

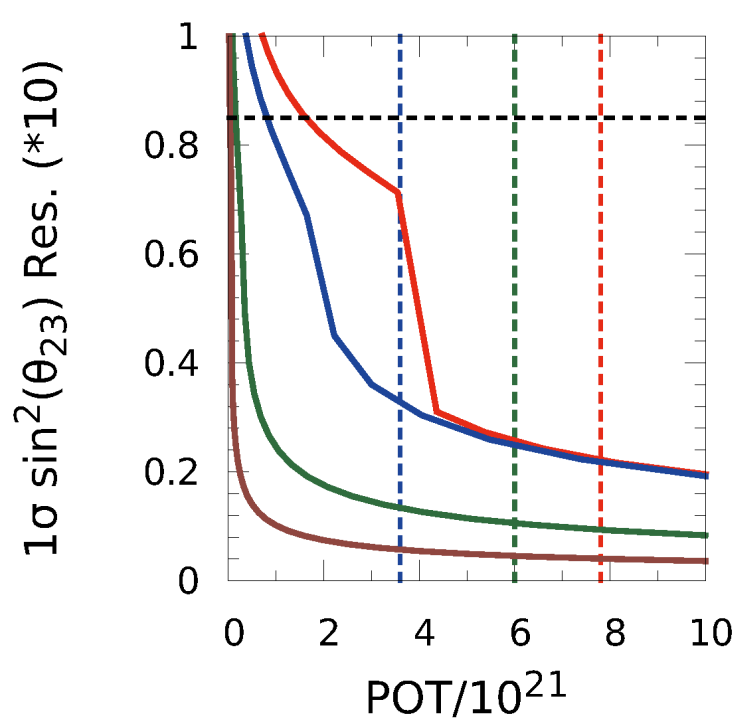

(B) True $\mathrm{MH}=\mathrm{IH}$

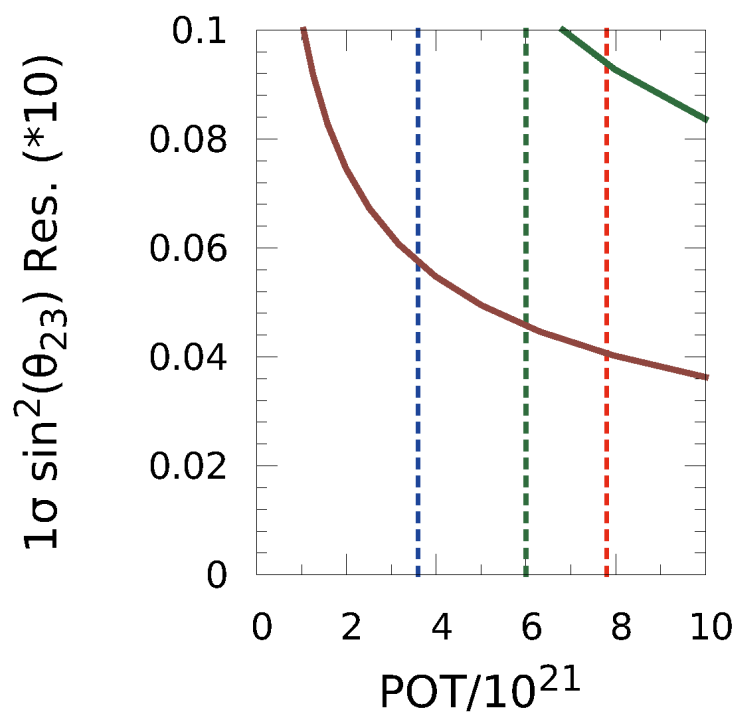

(D) True $\mathrm{MH}=\mathrm{IH}$ (zoomed)

Figure 6.66. $1 \sigma \sin ^{2}\left(\theta_{23}\right)$ resolutions vs POT in $\mathrm{T} 2 \mathrm{~K}, \mathrm{NO} \nu \mathrm{A}, \mathrm{LBNE}$, and T2HK. The Capozzi et al. best-fit values are assumed for the true values of the oscillation parameters and the true value of $\sin ^{2}\left(\theta_{23}\right)=0.44\left(\sin ^{2}\left(\theta_{23}\right)=0.46\right)$ in the $\mathrm{NH}(\mathrm{IH})$ is used. All four samples are fit: $\nu_{e}$ appearance, $\bar{\nu}_{e}$ appearance, $\nu_{\mu}$ disappearance, and $\bar{\nu}_{\mu}$ disappearance. The nominal assumptions are used for systematics and $\nu: \bar{\nu}$ ratios. The other oscillation parameters and $\mathrm{MH}$ are profiled. The vertical dashed lines represent the nominal exposures for $\mathrm{NO} \nu \mathrm{A}$, $\mathrm{T} 2 \mathrm{~K}$, and LBNE respectively. 
Figure 6.67 shows curves for the $1 \sigma \Delta m_{31}^{2}$ resolutions. No True $\mathrm{MH}=\mathrm{IH}$ version is included here as the resolutions for either $\mathrm{MH}$ are very similar. Here the resolution is defined as Resolution $=\frac{\Delta m_{31}^{2} \text { (upper) }-\Delta m_{31}^{2} \text { (lower) }}{2}$ and is around the true value $\Delta m_{31}^{2}=2.47 \times 10^{-3} \mathrm{eV}^{2}$. All of the experiments will make improvements over the current global-fit resolution, indicated by the horizontal black line, at their final POT level. NO $\nu$ A will constrain $\Delta m_{31}^{2}$ more than $\mathrm{T} 2 \mathrm{~K}$ for all $\mathrm{POT}$ values since $\mathrm{NO} \nu \mathrm{A}$ has sensitivity to the $\mathrm{MH}$ and is able to better distinguish between the two solutions in the $\Delta m_{31}^{2}$ value in each mass hierarchy. The resolution is computed by scanning out from the central (best-fit) value until the $1 \sigma \Delta \chi^{2}$ is reached. This will exclude disconnected regions like those in the T2HK allowed regions of Figure 6.29 leading to the smaller widths corresponding to the $\Delta m_{32}^{2}$ widths in 6.30 where the $\mathrm{MH}$ is known. The jump in the T2HK resolution near $5 \times 10^{20} \mathrm{POT}$ is where the region due to the IH solution separates from the region due to the (correct) NH solution. LBNE sees no such jump as it is able to distinguish between the NH and IH spectra. LBNE would also, in a sense, be blind to the incorrect solution because of the coarse $125 \mathrm{MeV}$ binning in $E_{\text {reco }}$ in disappearance mode, future studies for LBNE sensitivities should include optimization studies of the binning in disappearance mode, particularly around the maximum disappearance dip, to see if this resolution might be improved.

Figures 6.68 and 6.69 show curves for the $1 \sigma \delta_{C P}$ resolutions for $\delta_{C P}^{\text {true }}=0$ and $\delta_{C P}^{\text {true }}=\pi / 2$ respectively. Here the resolution is defined as Resolution $=\frac{\delta_{C P}^{\text {upper }}-\delta_{C P}^{\text {lower }}}{2}$ and is around the indicated true value of $\delta_{C P}$. The resolution is given in degrees. The $1 \sigma$ global-fit constraints on $\delta_{C P}$ are relatively weak so they are not indicated in these Figures nor are the central best-fit values used as the true central values. Values of $\delta_{C P}^{\text {true }}=0$ and $\delta_{C P}^{\text {true }}=\pi / 2$ are used to encompass the range of expected resolutions on $\delta_{C P}$. 


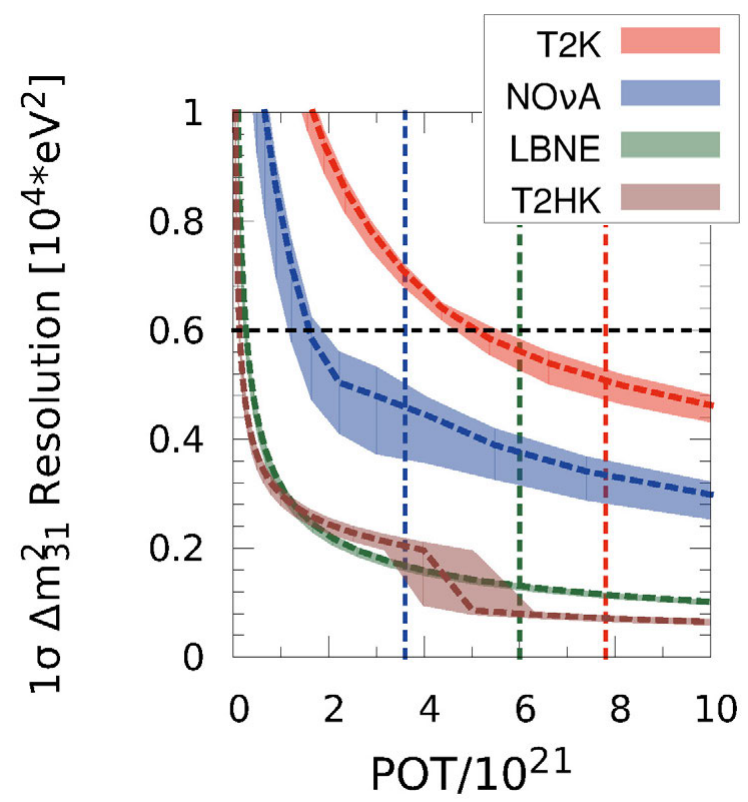

(A) True $\mathrm{MH}=\mathrm{NH}$

Figure 6.67. $1 \sigma \Delta m_{31}^{2}$ resolutions vs POT in $\mathrm{T} 2 \mathrm{~K}, \mathrm{NO} \nu \mathrm{A}, \mathrm{LBNE}$, and T2HK. The Capozzi et al. best-fit values are assumed for the true values of the oscillation parameters and the true value of $\Delta m_{31}^{2}=2.47 \times 10^{-3} \mathrm{eV}^{2}$ in the NH is used. Similar resolutions are expected in the IH case. All four samples are fit: $\nu_{e}$ appearance, $\bar{\nu}_{e}$ appearance, $\nu_{\mu}$ disappearance, and $\bar{\nu}_{\mu}$ disappearance. The nominal assumptions are used for systematics and $\nu: \bar{\nu}$ ratios. The bands represent the best and worst case resolutions that result from $1 \sigma$ variations in the true values of $\sin ^{2}\left(2 \theta_{13}\right), \sin ^{2}\left(\theta_{23}\right)$, and $\Delta m_{31}^{2}$. The other oscillation parameters and $\mathrm{MH}$ are profiled. The vertical dashed lines represent the nominal exposures for $\mathrm{NO} \nu \mathrm{A}, \mathrm{T} 2 \mathrm{~K}$, and LBNE respectively.

In Figure 6.68, T2K sees a strong effect from the degeneracy between the $\mathrm{MH}, \delta_{C P}$, and the $\theta_{23}$ octant making the bands very wide starting at around $6 \times 10^{21}$ POT for both the True $\mathrm{MH}=\mathrm{NH}$ and True $\mathrm{MH}=\mathrm{IH}$ cases. In the lower edge of this band, the $\sin ^{2}\left(\theta_{23}\right)$ true value is at the lower edge of the $1 \sigma$ range, at $\sin ^{2}\left(\theta_{23}\right)=0.414(0.424)$ so the octant is able to be resolved and thus the opposite octant solutions that widen the $\delta_{C P}$ allowed regions are removed. The upper edge corresponds to the $\sin ^{2}\left(\theta_{23}\right)$ true value that is at the upper edge of the $1 \sigma$ range, $\sin ^{2}\left(\theta_{23}\right)=0.470(0.594)$ for $\mathrm{NH}(\mathrm{IH})$, and the octant is not resolved so the resolution does not decrease. 


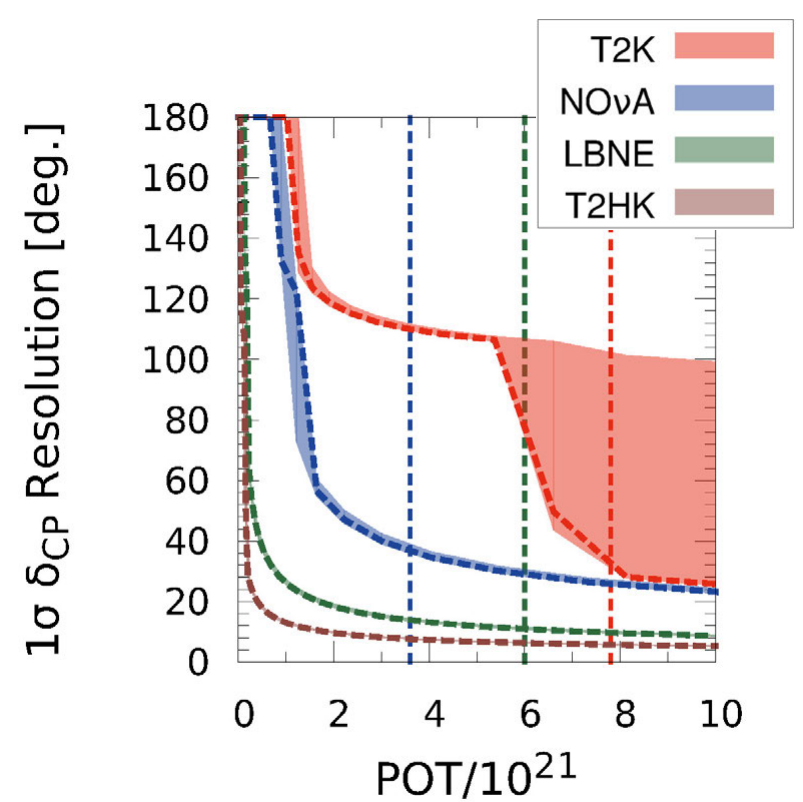

(A) True $\mathrm{MH}=\mathrm{NH}, \delta_{C P}^{\text {true }}=0$

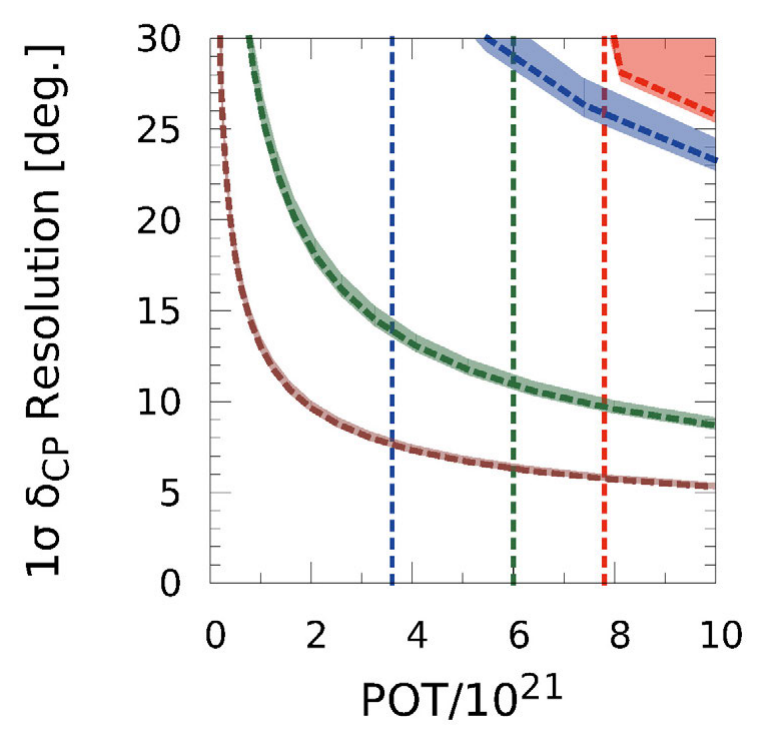

(c) True $\mathrm{MH}=\mathrm{NH}, \delta_{C P}^{\text {true }}=0$ (zoomed)

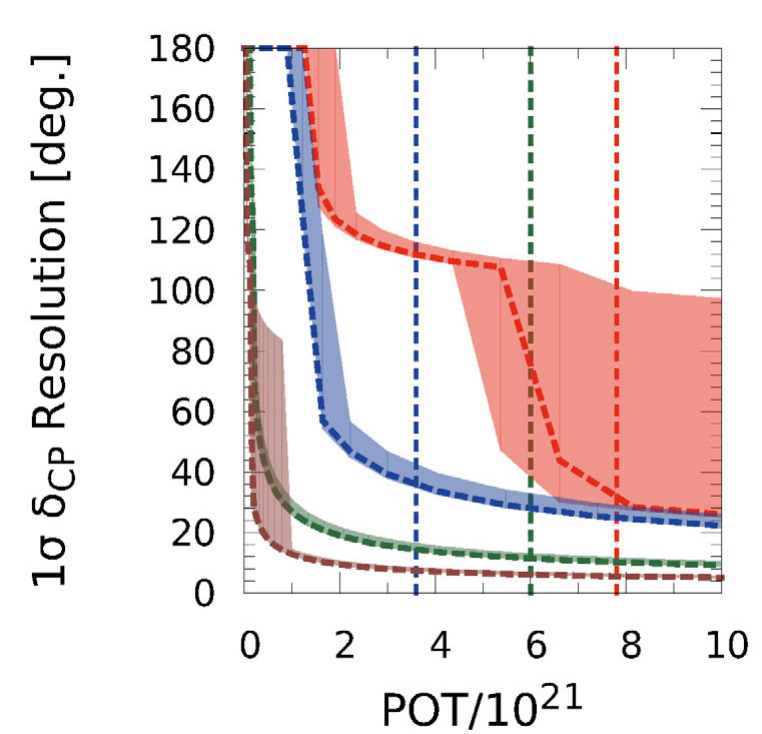

(B) True $\mathrm{MH}=\mathrm{IH}, \delta_{C P}^{\text {true }}=0$

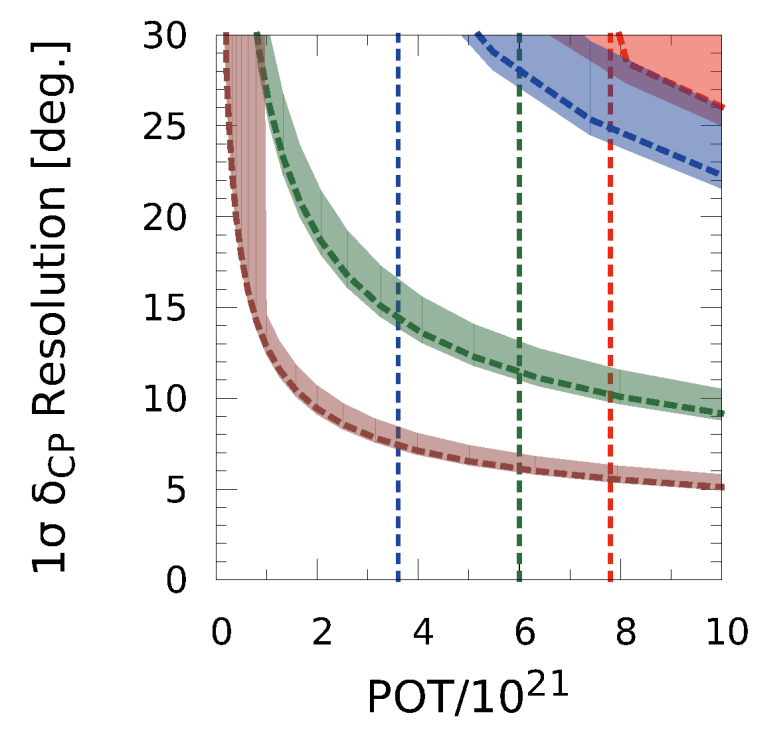

(D) True $\mathrm{MH}=\mathrm{IH}, \delta_{C P}^{\text {true }}=0$ (zoomed)

Figure 6.68. $1 \sigma \delta_{C P}$ resolutions vs POT in T2K, NO $\nu \mathrm{A}, \mathrm{LBNE}$, and T2HK for $\delta_{C P}^{\text {true }}=0$. The Capozzi et al. best-fit values are assumed for the true values of the oscillation parameters. All four samples are fit: $\nu_{e}$ appearance, $\bar{\nu}_{e}$ appearance, $\nu_{\mu}$ disappearance, and $\bar{\nu}_{\mu}$ disappearance. The nominal assumptions are used for systematics and $\nu: \bar{\nu}$ ratios. The bands represent the best and worst case resolutions that result from $1 \sigma$ variations in the true values of $\sin ^{2}\left(2 \theta_{13}\right), \sin ^{2}\left(\theta_{23}\right)$, and $\Delta m_{31}^{2}$. The other oscillation parameters and $\mathrm{MH}$ are profiled. The vertical dashed lines represent the nominal exposures for $\mathrm{NO} \nu \mathrm{A}$, $\mathrm{T} 2 \mathrm{~K}$, and LBNE respectively. 


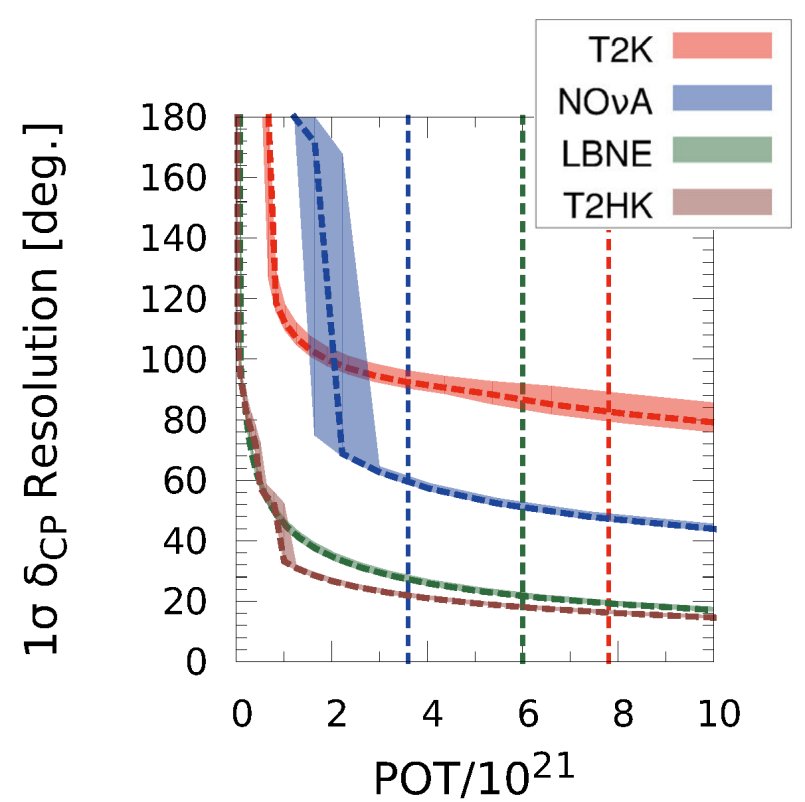

(A) True $\mathrm{MH}=\mathrm{NH}, \delta_{C P}^{\text {true }}=\pi / 2$

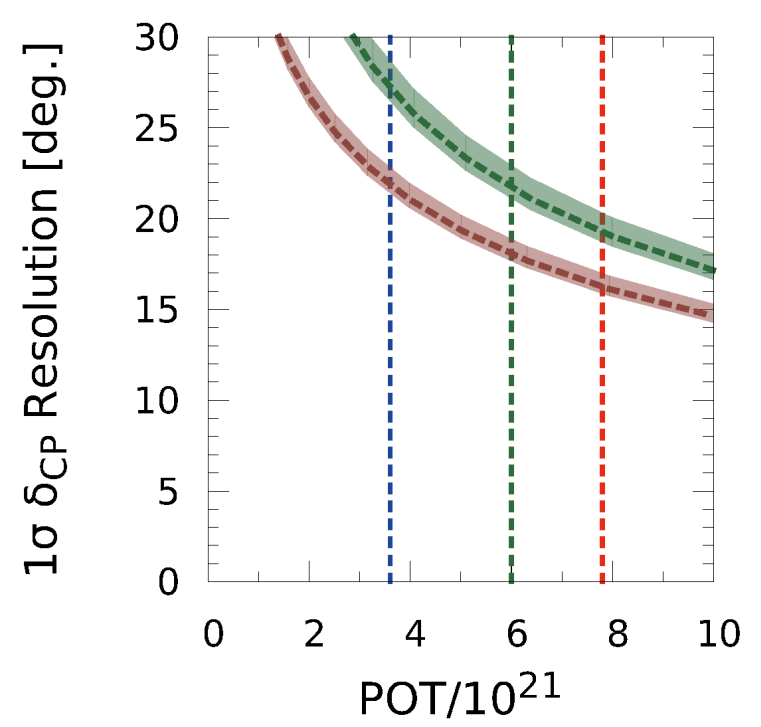

(c) True $\mathrm{MH}=\mathrm{NH}, \delta_{C P}^{\text {true }}=\pi / 2$ (zoomed)

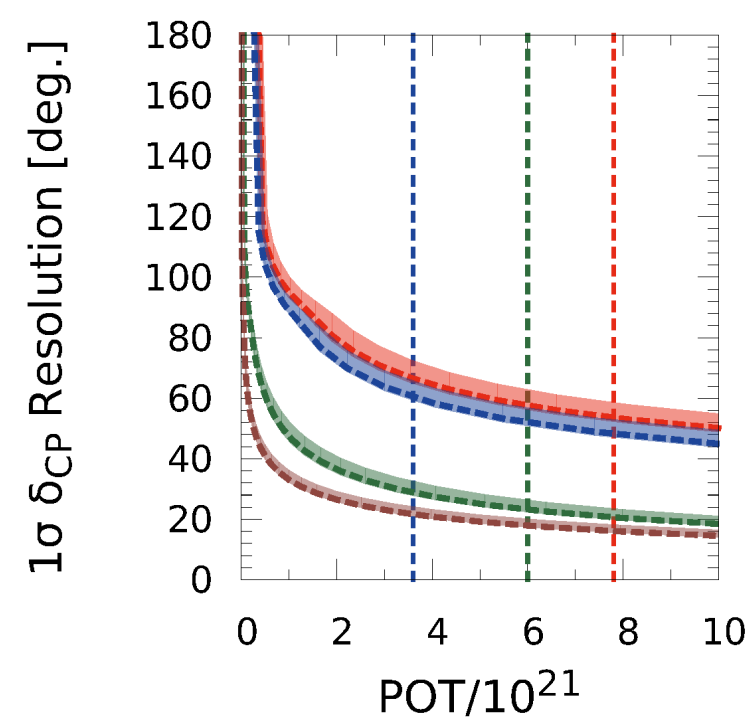

(B) True $\mathrm{MH}=\mathrm{IH}, \delta_{C P}^{\text {true }}=\pi / 2$

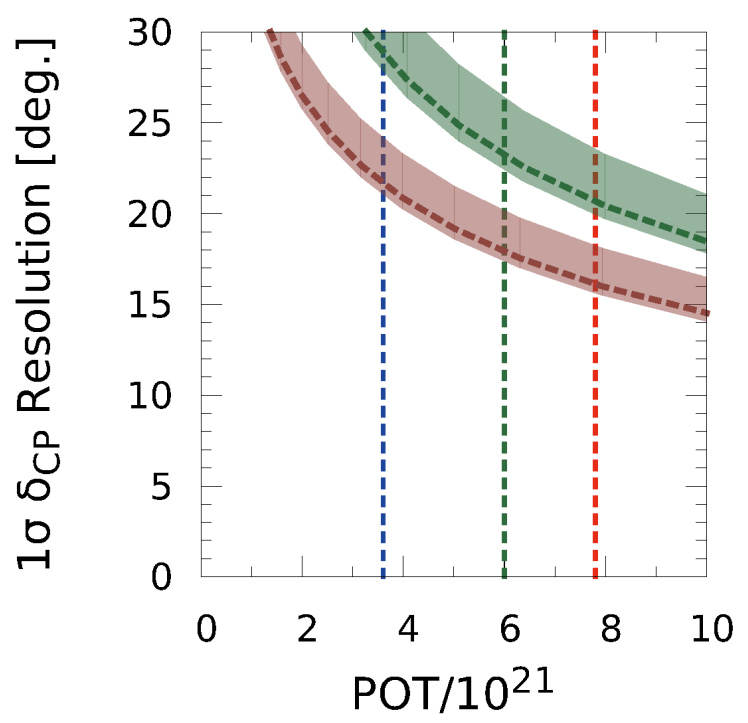

(D) True $\mathrm{MH}=\mathrm{IH}, \delta_{C P}^{\text {true }}=\pi / 2$ (zoomed)

Figure 6.69. $1 \sigma \delta_{C P}$ resolutions vs POT in T2K, NO $\nu \mathrm{A}, \mathrm{LBNE}$, and T2HK for $\delta_{C P}^{\text {true }}=\pi / 2$. The Capozzi et al. best-fit values are assumed for the true values of the oscillation parameters. All four samples are fit: $\nu_{e}$ appearance, $\bar{\nu}_{e}$ appearance, $\nu_{\mu}$ disappearance, and $\bar{\nu}_{\mu}$ disappearance. The nominal assumptions are used for systematics and $\nu: \bar{\nu}$ ratios. The bands represent the best and worst case resolutions that result from $1 \sigma$ variations in the true values of $\sin ^{2}\left(2 \theta_{13}\right), \sin ^{2}\left(\theta_{23}\right)$, and $\Delta m_{31}^{2}$. The other oscillation parameters and $\mathrm{MH}$ are profiled. The vertical dashed lines represent the nominal exposures for $\mathrm{NO} \nu \mathrm{A}$, T2K, and LBNE respectively. 
Figure 6.70 shows the sensitivity to $\mathrm{CP}$ violation as a function of POT. The $\mathrm{y}$-axis represents the median significance of the rejection of the $\sin \delta_{C P}=0$ hypothesis, in units of $\sigma$. This median means that, in terms of significance, $50 \%$ of $\delta_{C P}$ values produce a significance above and $50 \%$ below the $\mathrm{y}$-axis value. This is also referred to as $\delta_{C P}$ coverage. No experiment will ever reach $100 \% \delta_{C P}$ coverage for CP violation because there are values of $\delta_{C P}, 0$ and $\pi$, for which $\mathrm{CP} \sin \delta_{C P}=0$. A value of $50 \%$ was chosen to represent the distribution. The bands are primarily influenced by the value of $\sin ^{2}\left(\theta_{23}\right)$, as the value of $\sin ^{2}\left(\theta_{23}\right)$ increases the $\mathrm{CP}$ asymmetry decreases so the upper edge of the band corresponds to the lower $\sin ^{2}\left(\theta_{23}\right)$ $1 \sigma$ global-fit value. LBNE has the highest median significance for all but the earliest POT values and reaches $3.8 \sigma$ for the nominal LBNE POT. T2HK suffers from the MH degeneracy, but if the $\mathrm{MH}$ is resolved then this plot would show T2HK to have a much higher median significance. $\mathrm{T} 2 \mathrm{~K}$ and $\mathrm{NO} \nu \mathrm{A}$ are both below $1 \sigma$ due to the $\mathrm{MH}$ degeneracy not being resolved. Their combination will allow for a greater median significance to be reached.

Figure 6.71 shows the sensitivity to determining the MH vs POT. The value plotted on the $\mathrm{y}$-axis is the worst case significance, in terms of $\delta_{C P}$, so it is the significance for which $100 \%$ of $\delta_{C P}$ values are covered. The significance for $\mathrm{MH}$ determination does not have the same meaning here as the null hypothesis is not distributed as a $\chi^{2}$ distribution and this feature will be studied in Chapter 8 . The bands again are primarily influenced by the value of $\sin ^{2}\left(\theta_{23}\right)$, but in this case as the value of $\sin ^{2}\left(\theta_{23}\right)$ increases the matter effect increases so that the upper edge of the band corresponds to the higher $\sin ^{2}\left(\theta_{23}\right) 1 \sigma$ global-fit value. LBNE has the highest significance again because it is the only experiment able to simultaneously resolve the $\delta_{C P}, \mathrm{MH}$, and octant degeneracies.

Figure 6.72 shows the values for which the octant can be determined at $3 \sigma$ as a function of POT. For example, in the case of True $\mathrm{MH}=\mathrm{NH}$, T2K at full POT can resolve the octant 


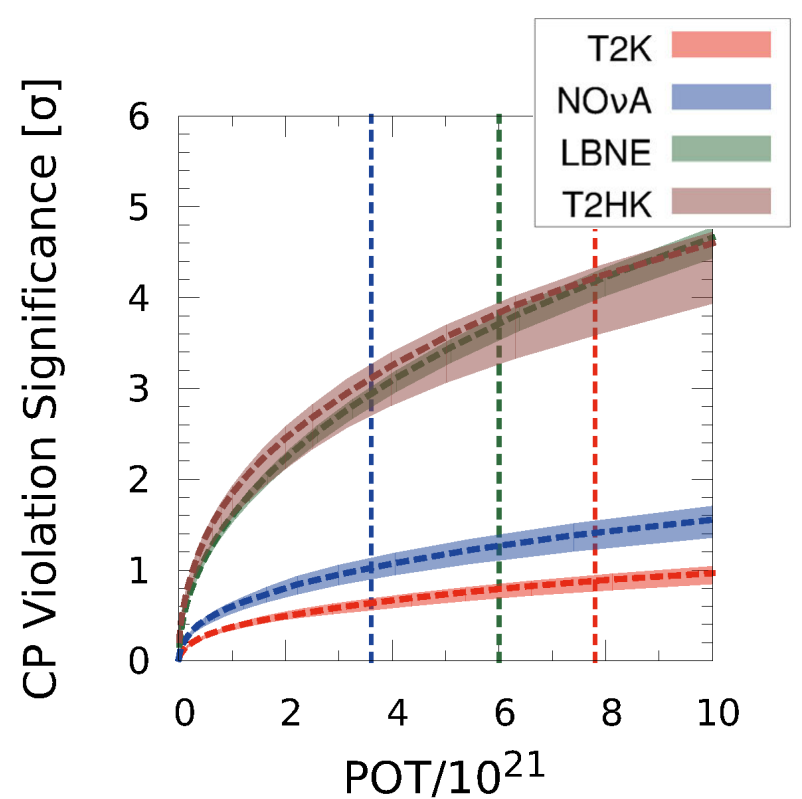

(A) True $\mathrm{MH}=\mathrm{NH}$

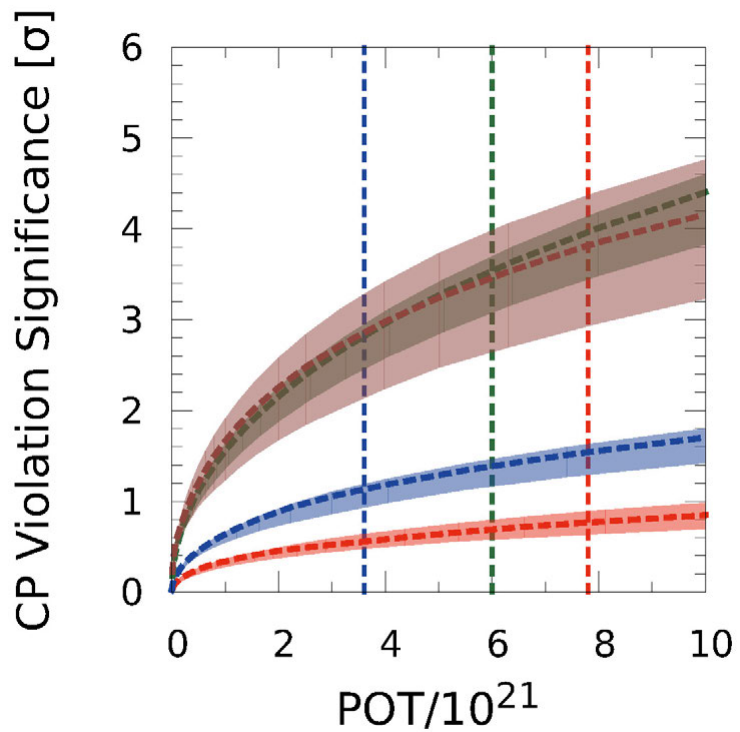

(B) True $\mathrm{MH}=\mathrm{IH}$

Figure 6.70. CP violation median sensitivity vs POT in $\mathrm{T} 2 \mathrm{~K}, \mathrm{NO} \nu \mathrm{A}, \mathrm{LBNE}$, and T2HK. The Capozzi et al. best-fit values are assumed for the true values of the oscillation parameters. All four samples are fit: $\nu_{e}$ appearance, $\bar{\nu}_{e}$ appearance, $\nu_{\mu}$ disappearance, and $\bar{\nu}_{\mu}$ disappearance. The nominal assumptions are used for systematics and $\nu: \bar{\nu}$ ratios. The bands represent the best and worst case resolutions that result from $1 \sigma$ variations in the true values of $\sin ^{2}\left(2 \theta_{13}\right), \sin ^{2}\left(\theta_{23}\right)$, and $\Delta m_{31}^{2}$. The other oscillation parameters and $\mathrm{MH}$ are profiled. The vertical dashed lines represent the nominal exposures for $\mathrm{NO} \nu \mathrm{A}$, T2K, and LBNE respectively.

at the $3 \sigma$ level if the true value of $\sin ^{2}\left(\theta_{23}\right)$ is greater than 0.64 or less than 0.37 . T2HK and LBNE quickly reach a limit on their ability to resolve the octant at this level and can do so if the true value of $\sin ^{2}\left(\theta_{23}\right)$ is greater than 0.57 or less than 0.45 


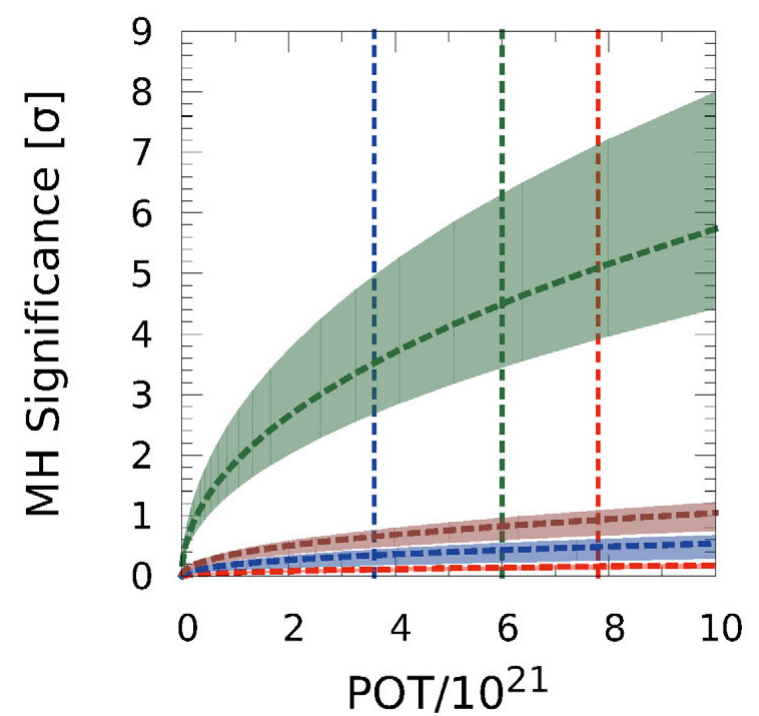

(A) True $\mathrm{MH}=\mathrm{NH}$

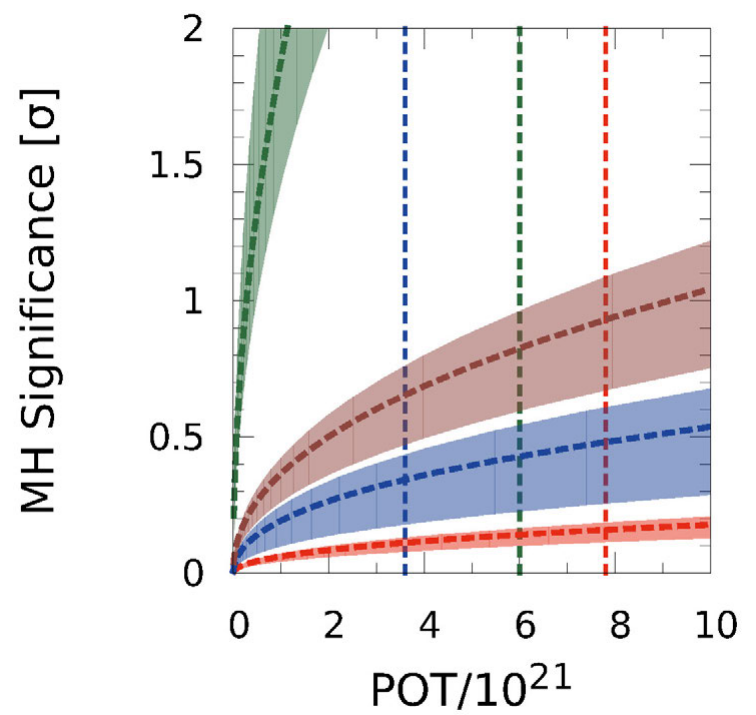

(c) True $\mathrm{MH}=\mathrm{NH}$ (zoomed)

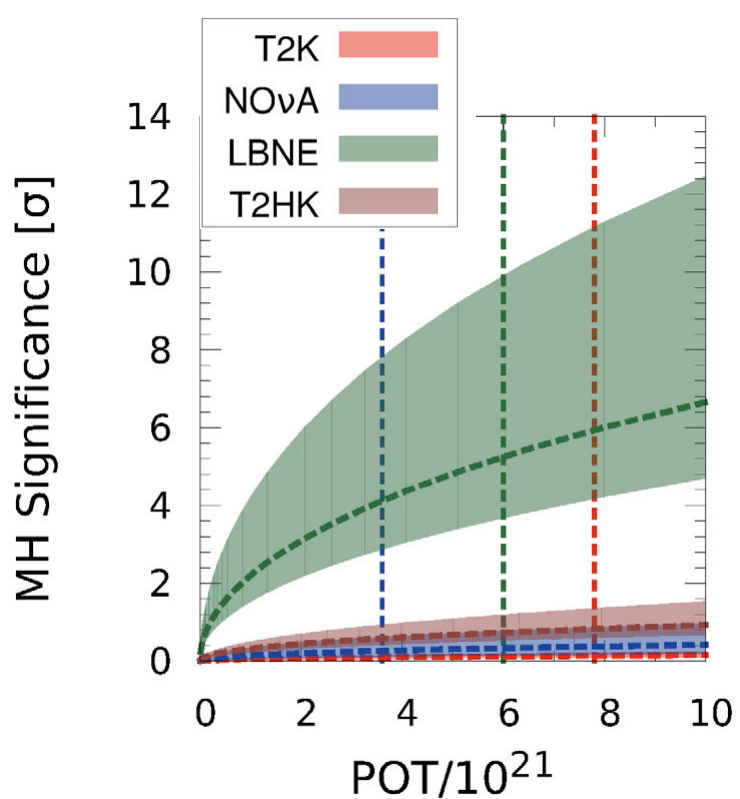

(B) True $\mathrm{MH}=\mathrm{IH}$

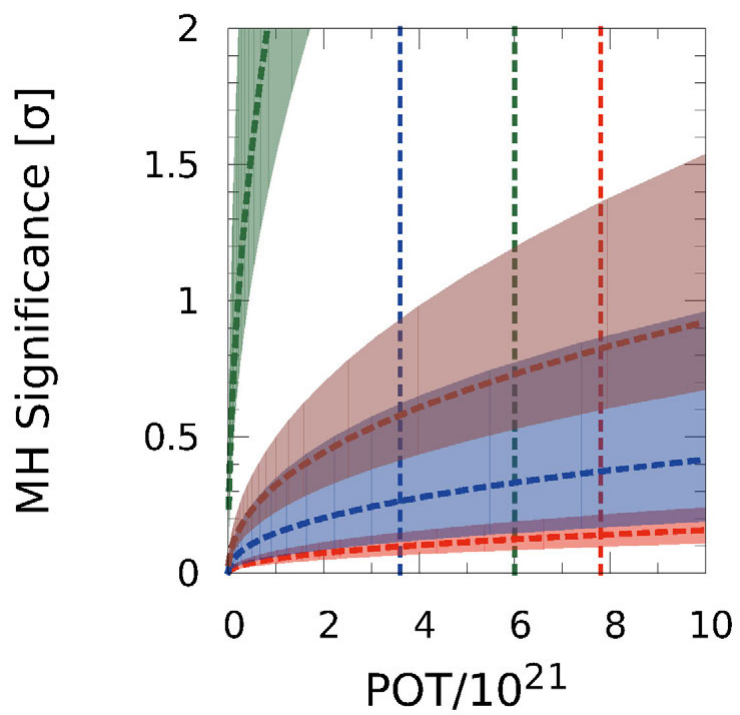

(D) True $\mathrm{MH}=\mathrm{IH}$ (zoomed)

Figure 6.71. MH worst case sensitivity vs POT in T2K, NO $\nu$ A, LBNE, and T2HK. The Capozzi et al. best-fit values are assumed for the true values of the oscillation parameters. All four samples are fit: $\nu_{e}$ appearance, $\bar{\nu}_{e}$ appearance, $\nu_{\mu}$ disappearance, and $\bar{\nu}_{\mu}$ disappearance. The nominal assumptions are used for systematics and $\nu: \bar{\nu}$ ratios. The bands represent the best and worst case resolutions that result from $1 \sigma$ variations in the true values of $\sin ^{2}\left(2 \theta_{13}\right)$, $\sin ^{2}\left(\theta_{23}\right)$, and $\Delta m_{31}^{2}$. The other oscillation parameters and $\mathrm{MH}$ are profiled. The vertical dashed lines represent the nominal exposures for $\mathrm{NO} \nu \mathrm{A}, \mathrm{T} 2 \mathrm{~K}$, and LBNE respectively. 


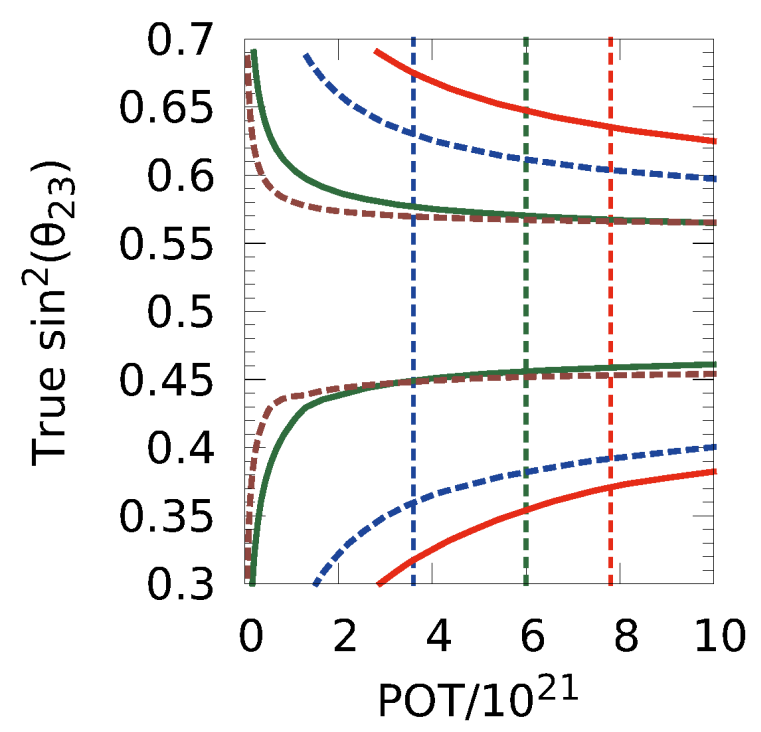

(A) True $\mathrm{MH}=\mathrm{NH}$

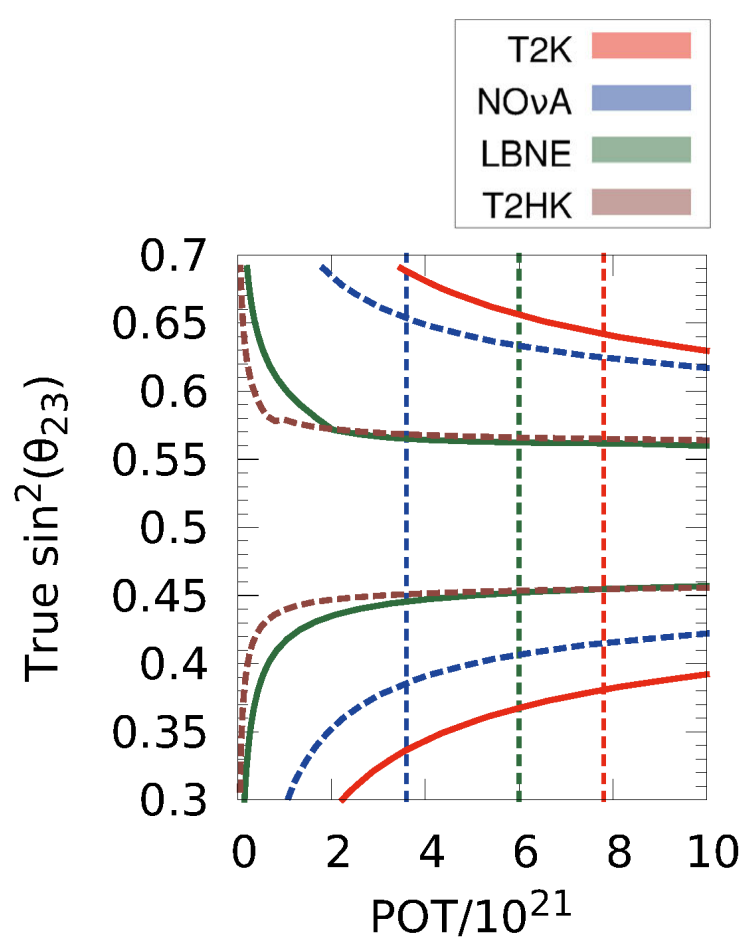

(B) True $\mathrm{MH}=\mathrm{IH}$

Figure 6.72. $3 \sigma$ Octant sensitivity vs POT in T2K, NO $\nu \mathrm{A}, \mathrm{LBNE}$, and T2HK. The Capozzi et al. best-fit values are assumed for the true values of the oscillation parameters. All four samples are fit: $\nu_{e}$ appearance, $\bar{\nu}_{e}$ appearance, $\nu_{\mu}$ disappearance, and $\bar{\nu}_{\mu}$ disappearance. The nominal assumptions are used for systematics and $\nu: \bar{\nu}$ ratios. The bands represent the best and worst case resolutions that result from $1 \sigma$ variations in the true values of $\sin ^{2}\left(2 \theta_{13}\right)$, $\sin ^{2}\left(\theta_{23}\right)$, and $\Delta m_{31}^{2}$. The other oscillation parameters and $\mathrm{MH}$ are profiled. The vertical dashed lines represent the nominal exposures for $\mathrm{NO} \nu \mathrm{A}, \mathrm{T} 2 \mathrm{~K}$, and LBNE respectively. 


\subsection{Concluding Remarks}

A full suite of sensitivities to the neutrino oscillation parameters in a three flavor analysis has been presented in this chapter. The set of assumptions that went into these sensitivities was outlined in Table 6.1. In particular, the assumption of normalization systematics on the signal and background and the Capozzi et al. global fit values and $1 \sigma$ ranges were used to model uncertainties in the measured event spectra.

The constraints on $\delta_{C P}$ that are indicated in the Capozzi et al. global fit and plotted in Figure 3.9 were not used in generating these sensitivities. The fit values of $\delta_{C P}$ were assumed to be unconstrained in the fits and the true values were either computed at each value of

$\delta_{C P}$ or assumed to be $\delta_{C P}^{\text {true }}=0, \delta_{C P}^{\text {true }}=-\pi / 2$, or $\delta_{C P}^{\text {true }}=\pi / 2$. Figures 6.63 and 6.64 give an idea of the sensitivity in the case of the best-fit $\delta_{C P}$ value that is nearly $-\pi / 2$. In the True $\mathrm{MH}=\mathrm{NH}$ case the $\mathrm{CP}$ violation detection and $\mathrm{MH}$ determination potential is highest at this value. In the True $\mathrm{MH}=\mathrm{IH}$ case the degeneracy between the $\mathrm{MH}$ and $\delta_{C P}$ will make measurements difficult until LBNE (or another experiment) can conclusively resolve the MH.

The next three chapters will focus on expanding the statistical interpretation of these sensitivities, incorporating more sophisticated systematic uncertainties, and varying the running assumptions to optimize sensitivity. 


\section{CHAPTER 7}

\section{SystematiC UnCERTAINTIES}

Systematic uncertainties are a critical piece to estimating the sensitivity of oscillations in current and future LBL experiments. It is important to evaluate their effects on the sensitivity in order to plan for their mitigation. In the $\Delta \chi^{2}$ computation, these systematic uncertainties take into account how well the predicted event spectra are expected to be constrained by measurements in the near and far detectors as well as by external data. This chapter will discuss the normalization systematics introduced in Chapter 5 in more detail and describe multiple studies that were done with the goal of more accurately representing the expected systematic uncertainties.

The systematic uncertainties assumed to generate all of the sensitivities of Chapter 6 assumed normalization uncertainties on the signal and background at between 1-5\% for the total signal and $5-10 \%$ for the total background. These uncertainties were incorporated into the $\Delta \chi^{2}$ formulation using nuisance parameters. The nuisance parameters for the signal and background normalizations modify the total number of events for $\nu_{e}$ appearance $\left(N_{\nu_{e}}\right), \bar{\nu}_{e}$

appearance $\left(N_{\bar{\nu}_{e}}\right), \nu_{\mu}$ disappearance $\left(N_{\nu_{\mu}}\right)$, and $\bar{\nu}_{\mu}$ disappearance $\left(N_{\bar{\nu}_{\mu}}\right)$ using the following definitions:

$$
\begin{aligned}
& N_{\nu_{e}}=\left(1+f_{\nu_{e}}^{\text {signal }}\right)\left(n_{\nu_{e}-\text { osc }}+n_{\bar{\nu}_{e}-\text { osc }}\right) \\
& \quad+\left(1+f_{\nu_{e}}^{\text {background }}\right)\left(n_{\nu_{e}-\text { beam }}+n_{\bar{\nu}_{e}-\text { beam }}+n_{\nu_{\tau}}+n_{\bar{\nu}_{\tau}}+n_{N C}+n_{\nu_{\mu}}+n_{\bar{\nu}_{\mu}}\right) \\
& N_{\bar{\nu}_{e}}=\left(1+f_{\bar{\nu}_{e}}^{\text {signal }}\right)\left(n_{\nu_{e}-\text { osc }}+n_{\bar{\nu}_{e}-\text { osc }}\right) \\
& \quad+\left(1+f_{\bar{\nu}_{e}}^{\text {background }}\right)\left(n_{\nu_{e}-\text { beam }}+n_{\bar{\nu}_{e}-\text { beam }}+n_{\nu_{\tau}}+n_{\bar{\nu}_{\tau}}+n_{N C}+n_{\nu_{\mu}}+n_{\bar{\nu}_{\mu}}\right)
\end{aligned}
$$




$$
\begin{aligned}
N_{\nu_{\mu}} & =\left(1+f_{\nu_{\mu}}^{\text {signal }}\right)\left(n_{\nu_{\mu}}+n_{\bar{\nu}_{\mu}}\right) \\
& +\left(1+f_{\nu_{\mu}}^{\text {background }}\right)\left(n_{\nu_{e}-\text { beam }}+n_{\bar{\nu}_{e}-\text { beam }}+n_{\nu_{\tau}}+n_{\bar{\nu}_{\tau}}+n_{N C}\right) \\
N_{\bar{\nu}_{\mu}} & =\left(1+f_{\bar{\nu}_{\mu}}^{\text {signal }}\right)\left(n_{\nu_{\mu}}+n_{\bar{\nu}_{\mu}}\right) \\
& +\left(1+f_{\bar{\nu}_{\mu}}^{\text {background }}\right)\left(n_{\nu_{e}-\text { beam }}+n_{\bar{\nu}_{e}-\text { beam }}+n_{\nu_{\tau}}+n_{\bar{\nu}_{\tau}}+n_{N C}\right)
\end{aligned}
$$

The index to indicate the bin in $E_{\text {reco }}$ has been left off to simplify these equations. As discussed in Chapter 5 the nuisance parameters are profiled and that means that they are varied to minimize the $\Delta \chi^{2}$. In this formulation there are eight nuisance parameters; two each for $\nu_{e}$ appearance, $\bar{\nu}_{e}$ appearance, $\nu_{\mu}$ disappearance, and $\bar{\nu}_{\mu}$ disappearance. The nuisance parameters have the effect of allowing the signal or the backgrounds that make up the fit spectra to shift upwards or downwards to match the true spectra and reducing the ability to differentiate between two oscillation hypotheses. The nuisance parameters are not correlated in any way in this formulation so that they float independently of one another.

These systematic uncertainties are put in place to incorporate the uncertainties from multiple effects. The major categories of uncertainties are:

- flux uncertainties that arise primarily from uncertainties in the modeling of the production of hadrons in the target

- $\nu$ interaction cross section uncertainties

- nuclear model uncertainties that affect, for example, the number of nucleons ejected in an interaction

- far detector reconstruction and fiducial volume uncertainties 
It is beyond the scope of this thesis to give a detailed accounting of each of these sources. This chapter will instead focus on two approaches to modeling the uncertainties in a more advanced way.

The dominant uncertainty for an LBL neutrino oscillation measurement is the flux uncertainty. In the simplest formulation the flux error introduces an uncertainty on the initial flux of $\nu_{\mu}$ in the beam. The flux uncertainties will be constrained by measurements in a near detector and measurements in the other samples at the far detector. For example, the $\nu_{\mu}$ disappearance measurement will be used to constrain the $\nu_{e}$ appearance measurement. The justification behind the use of normalization uncertainties for signal and background is that this is the uncorrelated piece of the uncertainties assuming the other correlated pieces will cancel. There could be a $5 \%$ flux uncertainty, for example, in the $\nu_{\mu}$ flux but this will cancel by simultaneously fitting multiples samples in the near and far detector, leaving only the uncertainties that do not cancel. An analysis below will treat the correlations more directly by allowing them to cancel in the minimization of the $\Delta \chi^{2}$.

A normalization uncertainty only affects the overall rate by multiplying every bin in the signal or background spectrum by a normalization factor. It does not affect the shape of the fit spectra because each bin increases or decreases by the same multiplicative factor and so the relative number of events in one bin to the next bin remains the same. Each of the methods discussed in this chapter moves progressively towards implementing more shape uncertainty. First, a comparison of the relative contributions of rate and shape to the sensitivity in each experiment will be made. 


\subsection{Shape and Rate Contributions to Sensitivity}

The rate contribution to the sensitivity is computed by counting the total number of events irrespective of $E_{\text {reco. }}$ This gives the sensitivity if the experiment were a single bin counting experiment with no ability to distinguish between the energy of one event or another.

The shape contribution is determined by using the binning structure as before, but allowing the entire fit spectrum (signal and background) to float without constraint. The shape contribution to the $\Delta \chi^{2}$ is determined by the binning structure and binning granularity of the experiment and the energy range covered by the experiment.

Figures 7.1 through 7.5 show the contributions of shape and rate to the sensitivity to CP violation, $\mathrm{MH}$, and $\delta_{C P}$ resolution in each experiment. The contributions are determined using the criteria outlined above. These figures include no non-oscillation parameter systematics but do profile the oscillation parameters to minimize the $\Delta \chi^{2}$. The contributions of rate and shape do not necessarily add directly to give the shape+rate curve because of constraints that they provide on the other oscillation parameters when they are profiled.

For $\mathrm{T} 2 \mathrm{~K}$ and $\mathrm{NO} \nu \mathrm{A}$ it is not critical to incorporate a shape uncertainty as there is little contribution to the sensitivity from the shape of the fit spectra. However for LBNE the lack of uncertainty on the shape of the fit spectra is an overly optimistic assumption. The rest of this chapter will focus on implementing shape uncertainties using multiple methods to vary the shape of the fit spectra. The remaining studies for this chapter will be for LBNE only since it is affected significantly by shape uncertainties. 

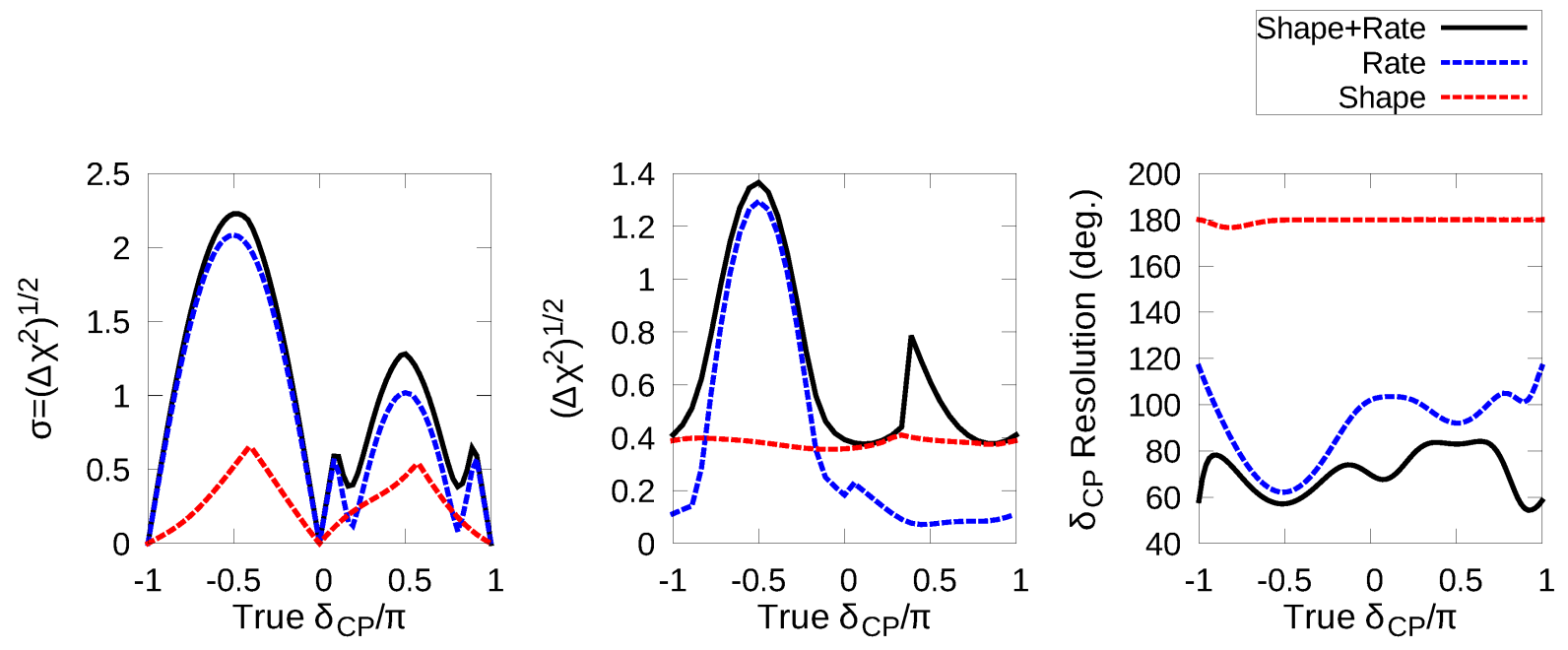

FiguRE 7.1. The contributions of rate and shape to the sensitivity to CP violation, $\mathrm{MH}$, and $\delta_{C P}$ resolution in $\mathrm{T} 2 \mathrm{~K}$. The Capozzi et al. best-fit values are assumed for the true values and $\delta_{C P}^{\text {true }}=0$. The nominal running assumptions and $\nu: \bar{\nu}$ ratios, but no systematics are used. The other oscillation parameters and the $\mathrm{MH}$ are profiled.
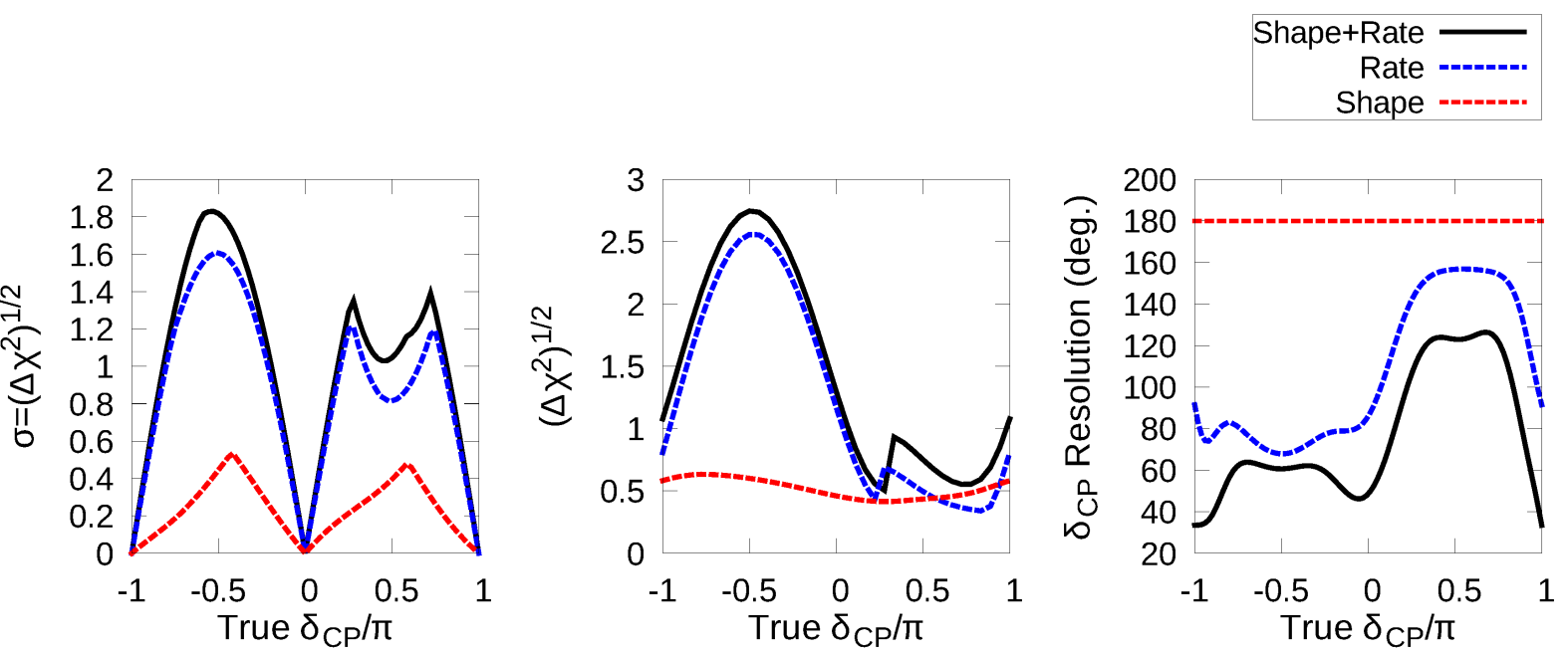

FIGURE 7.2. The contributions of rate and shape to the sensitivity to CP violation, $\mathrm{MH}$, and $\delta_{C P}$ resolution in $\mathrm{NO} \nu \mathrm{A}$. The Capozzi et al. best-fit values are assumed for the true values and $\delta_{C P}^{\text {true }}=0$. The nominal running assumptions and $\nu: \bar{\nu}$ ratios, but no systematics are used. The other oscillation parameters and the $\mathrm{MH}$ are profiled. 

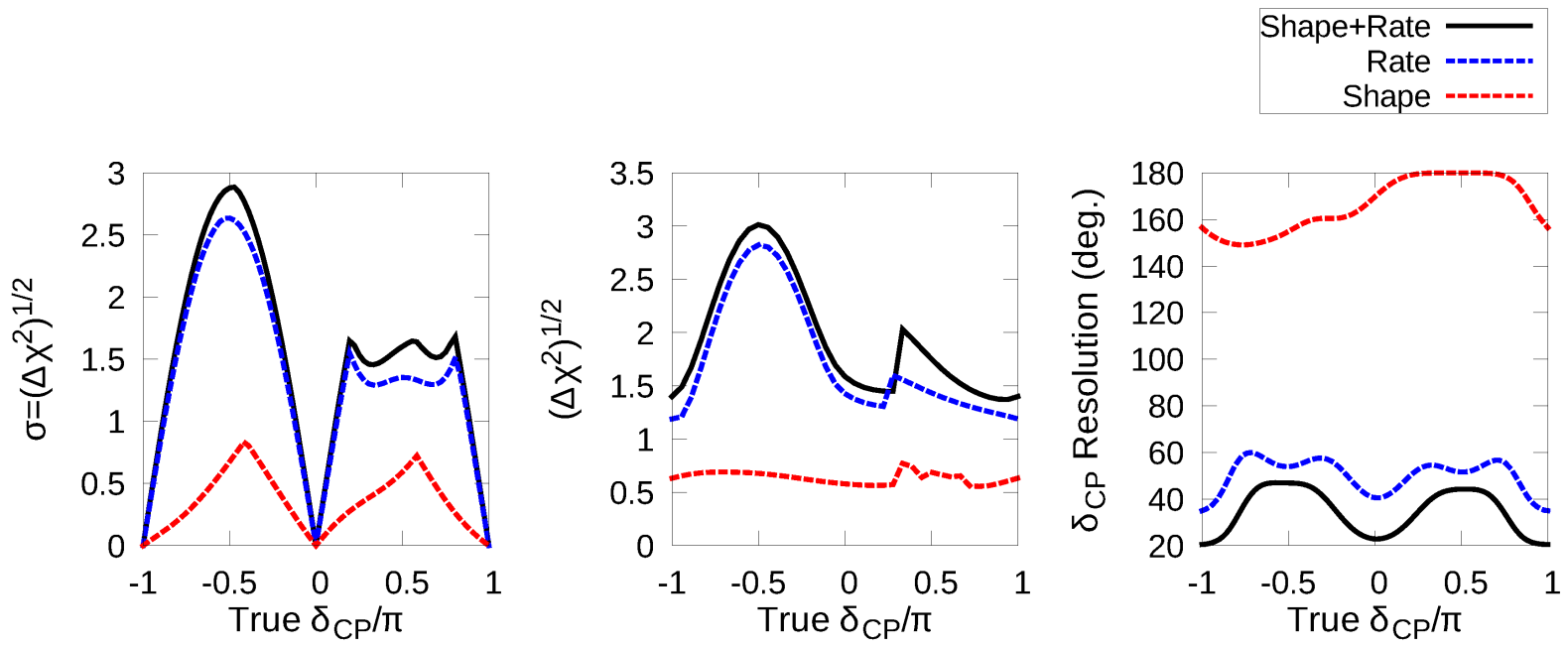

Figure 7.3. The contributions of rate and shape to the sensitivity to CP violation, $\mathrm{MH}$, and $\delta_{C P}$ resolution in a $\mathrm{T} 2 \mathrm{~K}+\mathrm{NO} \nu \mathrm{A}$ combined fit. The Capozzi et al. best-fit values are assumed for the true values and $\delta_{C P}^{t r u e}=0$. The nominal running assumptions and $\nu: \bar{\nu}$ ratios, but no systematics are used. The other oscillation parameters and the $\mathrm{MH}$ are profiled.

Shape+Rate

Rate

Shape
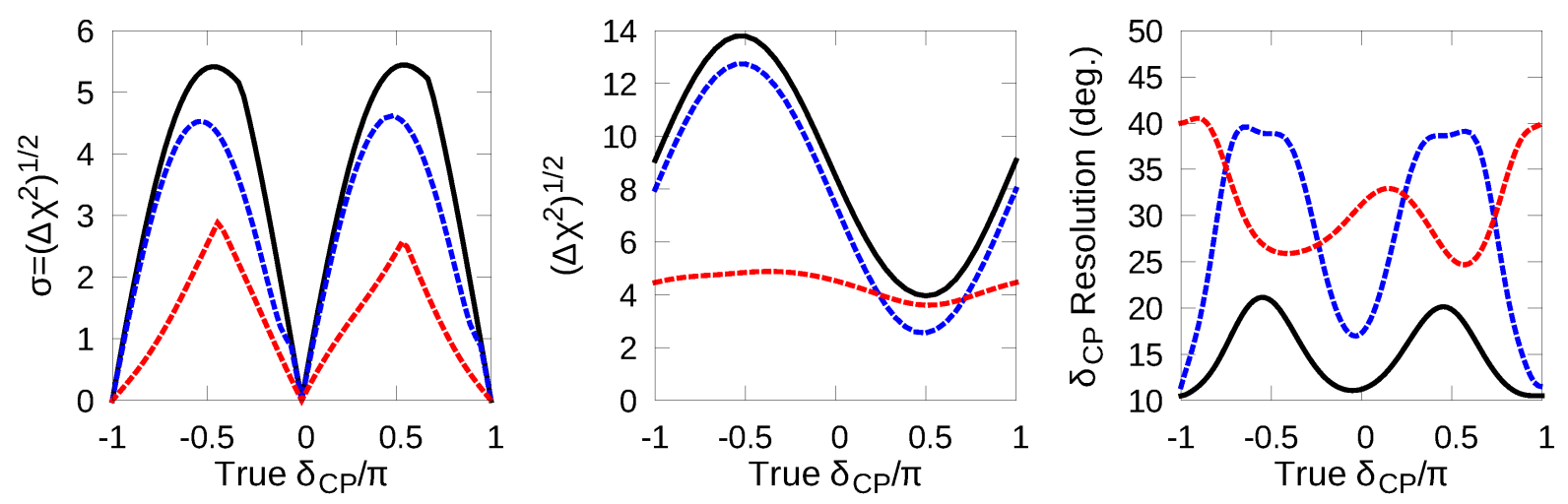

Figure 7.4. The contributions of rate and shape to the sensitivity to CP violation, $\mathrm{MH}$, and $\delta_{C P}$ resolution in LBNE. The Capozzi et al. best-fit values are assumed for the true values and $\delta_{C P}^{\text {true }}=0$. The nominal running assumptions and $\nu: \bar{\nu}$ ratios, but no systematics are used. The other oscillation parameters and the $\mathrm{MH}$ are profiled. 

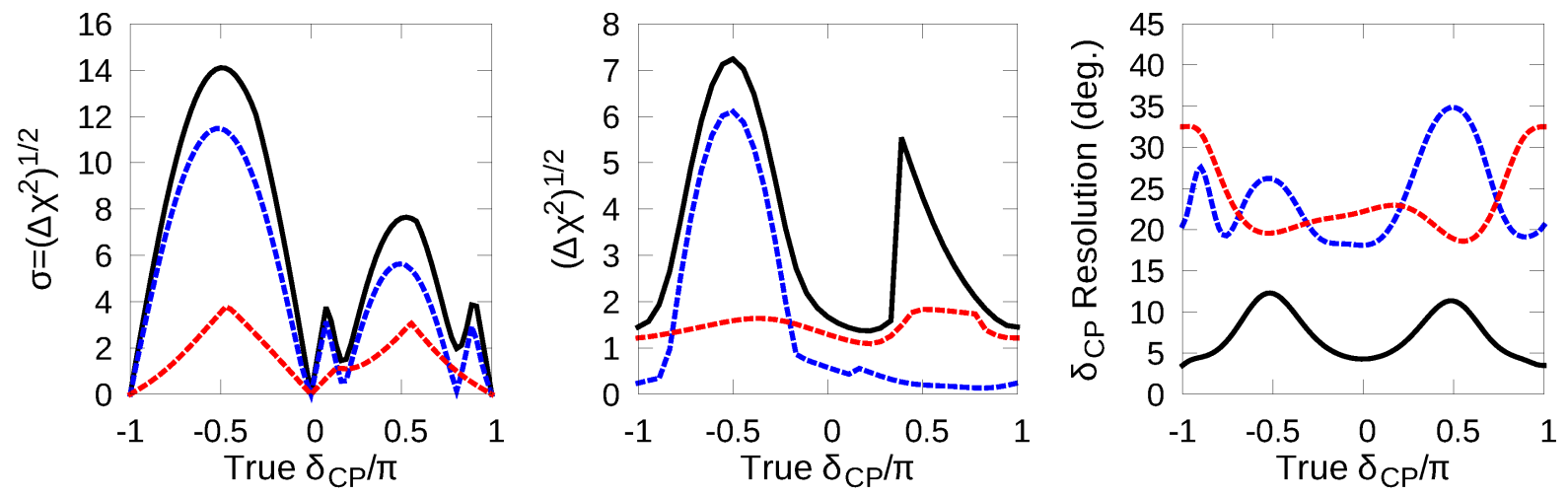

FiguRE 7.5. The contributions of rate and shape to the sensitivity to CP violation, $\mathrm{MH}$, and $\delta_{C P}$ resolution in T2HK. The Capozzi et al. best-fit values are assumed for the true values and $\delta_{C P}^{\text {true }}=0$. The nominal running assumptions and $\nu: \bar{\nu}$ ratios, but no systematics are used. The other oscillation parameters and the $\mathrm{MH}$ are profiled. 


\subsection{Implementing Shape Uncertainties}

The author of this thesis has extended the GLoBES library described in Chapter 5 to implement multiple methods of including uncertainties in the shape of the fit spectrum. This involved developing implementations of the $\Delta \chi^{2}$ to modify the response of the fit spectrum with respect to nuisance parameters that represent uncertainties in the systematic parameters of a particular model of the uncertainty. One such implementation will be described here although many others were also developed.

There are multiple effects that were added to study more detailed systematic uncertainties. First is the inclusion of correlations among the samples. As described above, the normalization systematic uncertainties were completely uncorrelated which means that the nuisance parameters were varied irrespective of one another. If the flux of $\nu_{\mu}$ is measured then this also serves as a constraint on the oscillated $\nu_{e}$ flux since it oscillates from the $\nu_{\mu}$ flux. So it is sensible to make the nuisance parameters correlated, that is, make them the same for the four samples.

The second effect is to separate the uncertainties on the backgrounds by allowing them to vary independently. It is important that they are allowed to vary in a way that accurately models their actual uncertainties. Summing the backgrounds into one spectrum and allowing them to float together, as was done in the previous chapter, overly constrains the backgrounds. The formulation being produced here allows the backgrounds to float separately and, when these fluctuations are taken together, introduces a shape uncertainty in the total background spectrum. 
The equations used to compute the total number of events in each sample in LBNE with the new nuisance parameters included are

$$
\begin{aligned}
& N_{\nu_{e}}=\left(1+f_{\text {signal }}\right)\left(n_{\nu_{e}-o s c}+n_{\bar{\nu}_{e}-o s c}\right)+\left(1+f_{\nu_{e}}\right)\left(n_{\nu_{e}-\text { beam }}+n_{\bar{\nu}_{e}-\text { beam }}\right) \\
& +\left(1+f_{\nu_{\tau}}\right)\left(n_{\nu_{\tau}}+n_{\bar{\nu}_{\tau}}\right)+\left(1+f_{N C}\right)\left(n_{N C}+n_{\nu_{\mu}}+n_{\nu_{\mu}}\right) \\
& N_{\bar{\nu}_{e}}=\left(1+f_{\text {signal }}\right)\left(n_{\nu_{e}-o s c}+n_{\bar{\nu}_{e}-o s c}\right)+\left(1+f_{\nu_{e}}\right)\left(n_{\nu_{e}-\text { beam }}+n_{\bar{\nu}_{e}-\text { beam }}\right) \\
& +\left(1+f_{\nu_{\tau}}\right)\left(n_{\nu_{\tau}}+n_{\bar{\nu}_{\tau}}\right)+\left(1+f_{N C}\right)\left(n_{N C}+n_{\nu_{\mu}}+n_{\nu_{\mu}}\right) \\
& N_{\nu_{\mu}}=\left(1+f_{\text {signal }}\right)\left(n_{\nu_{\mu}}+n_{\bar{\nu}_{\mu}}\right)+\left(1+f_{\nu_{e}}\right)\left(n_{\nu_{e}-\text { beam }}+n_{\bar{\nu}_{e}-\text { beam }}\right) \\
& +\left(1+f_{\nu_{\tau}}\right)\left(n_{\nu_{\tau}}+n_{\bar{\nu}_{\tau}}\right)+\left(1+f_{N C}\right)\left(n_{N C}\right) \\
& N_{\bar{\nu}_{\mu}}=\left(1+f_{\text {signal }}\right)\left(n_{\nu_{\mu}}+n_{\bar{\nu}_{\mu}}\right)+\left(1+f_{\nu_{e}}\right)\left(n_{\nu_{e}-\text { beam }}+n_{\bar{\nu}_{e}-\text { beam }}\right) \\
& +\left(1+f_{\nu_{\tau}}\right)\left(n_{\nu_{\tau}}+n_{\bar{\nu}_{\tau}}\right)+\left(1+f_{N C}\right)\left(n_{N C}\right)
\end{aligned}
$$

where it is implied that each of the channel variables $\left(n_{*}\right)$ depend on the oscillation parameters $(\boldsymbol{\theta})$. This formulation will be referred to as correlated sample, multiple background (CSMB) uncertainties while the previous will be referred to as uncorrelated sample, single background (USSB) uncertainties. The constraints on these nuisance parameters are implemented using a pull term as discussed in Chapter 5. The uncertainties on the four parameters are: $\sigma_{\text {signal }}=0.05, \sigma_{\nu_{e}}=0.05, \sigma_{N C}=0.1, \sigma_{\nu_{\tau}}=0.2$. These uncertainties were chose to 
correspond with studies done in other experiments to estimate the independent background contributions to the fit|46].

To contrast with the USSB approach the pre-fit and post-fit spectra for both systematic uncertainty formulations will be presented here. Figure 7.6 shows the spectra for USSB systematic uncertainties and 7.7 for CSMB uncertainties. These figures have the oscillation parameters fixed in the fit in order to show the effects of only the USSB and CSMB approaches.

The total $\Delta \chi^{2}$ for the USSB approach, shown in Figure 7.6, is reduced by including the systematics, from 47 to 44 . In the $\nu_{e}$ appearance event spectra, Figure 7.6. A, the normalization on the signal shifts the fit spectrum downward to minimize the $\Delta \chi^{2}$. The opposite is true for $\bar{\nu}_{e}$ appearance in Figure 7.6B, where the fit spectrum is shifted upwards. The $\nu_{\mu}$ disappearance and $\bar{\nu}_{\mu}$ disappearance spectra play very little role in this sensitivity with both of the $\Delta \chi^{2}$ values nearly equal to zero.

The total $\Delta \chi^{2}$ for the CSMB approach, shown in Figure 7.7, remains basically the same (the total value is rounded to the nearest integer). In order to reduce the $\Delta \chi^{2}$ value computed from the $\nu_{e}$ appearance sample, in Figure 7.7A, the $f_{\text {signal }}$ will have to have a negative value, but in the $\bar{\nu}_{e}$ appearance sample, Figure $7.7 \mathrm{~B}$, it would require a positive value to decrease the $\Delta \chi^{2}$. The balance between these two effects is found during the minimization and leaves the $f_{\text {signal }}$ nearly at zero. It is this constraint that comes from measuring spectra in both neutrino and antineutrino modes that leads to no reduction in sensitivity for $\mathrm{CP}$ violation when using the CSMB approach.

The $\nu$ samples will not be perfectly constrained by the $\bar{\nu}$ samples in a full analysis, however. For example, reduced statistics from the reduced cross sections for $\bar{\nu}$ mode may lead to a weaker constraint on the $\bar{\nu}$ flux overall so that the ratio of expected events in each 
mode will not be known perfectly. Section 7.3 will have new nuisance parameters introduced to account for these differences.

Figure 7.8 shows the $\mathrm{CP}$ violation and MH sensitivity $\left(\sqrt{\Delta \chi^{2}}\right)$ vs $\delta_{C P}$ for the no systematics, USSB, and CSMB systematics cases. The reduction that comes in either formulation is small, but the CP violation sensitivity is effectively not reduced at all in the CSMB case. The MH sensitivity is reduced near $\delta_{C P}=-\pi / 2$ where there is a strong dependence on the rate and the overall normalization uncertainty on the signal is able to fit the differences between the NH spectra and IH spectra.

In addition to these studies presented in this section, other studies were done that evaluated the effects of various models of the uncertainties for the shape of the signal and background event spectra. In every case the systematics were found to have a small effect if they were able to constrain the shape of the signal to a high degree. It will take a more advanced model of the fluctuations induced in the $E_{\text {reco }}$ fit spectra to accurately depict the expected reductions in sensitivity. 


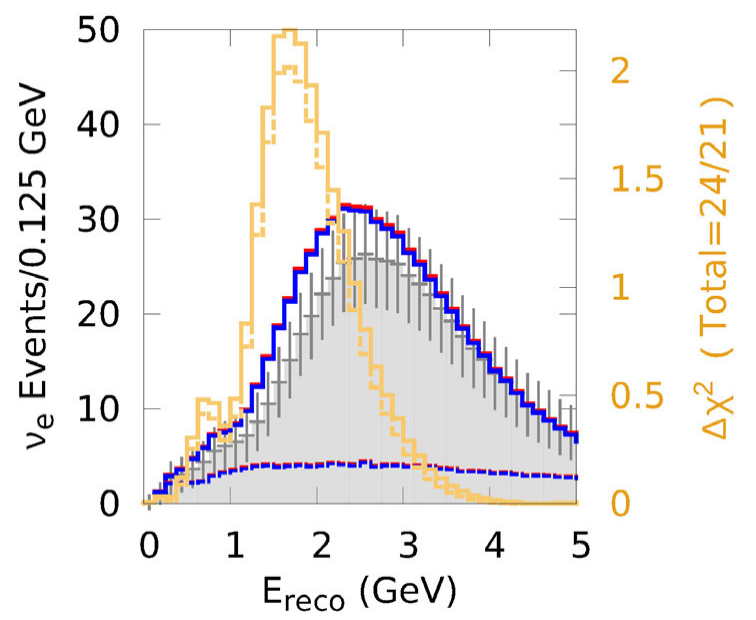

(A) $\nu_{e}$ appearance

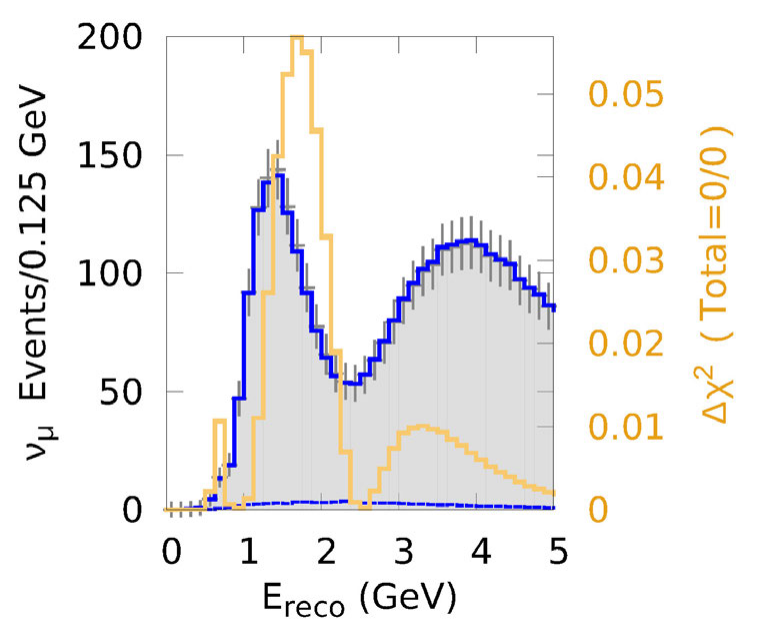

(C) $\nu_{\mu}$ disappearance

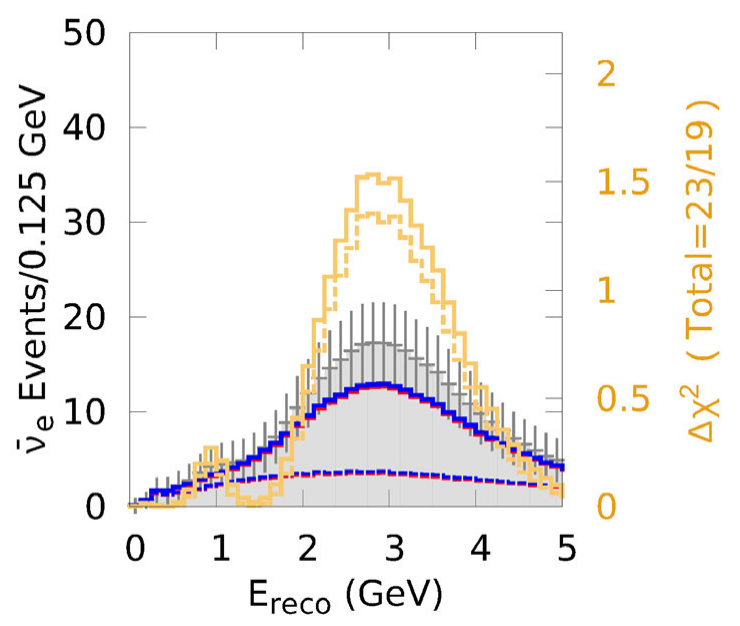

(B) $\bar{\nu}_{e}$ appearance

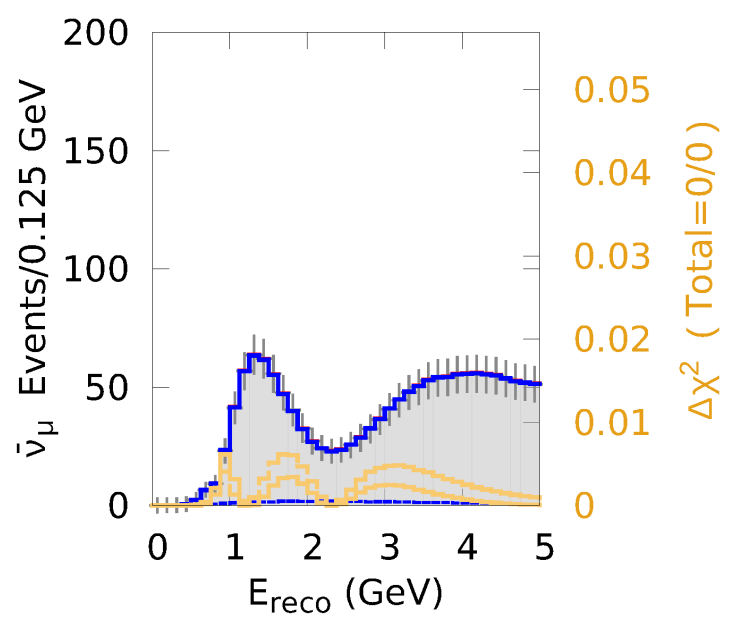

(D) $\bar{\nu}_{\mu}$ disappearance

$$
\begin{array}{r}
\chi_{\text {pre/post }}^{2}=47 / 44 \\
\operatorname{True}^{\delta_{C P}=0} \\
\text { Pre-Fit } \delta_{C P}=-\pi / 2 \\
\text { Post-Fit } \delta_{C P}=-\pi / 2 \\
\text { Background ------- }
\end{array}
$$

Figure 7.6. LBNE pre-fit (red) and post-fit (blue) spectra, each with $\delta_{C P}=$ $-\pi / 2$, compared to the true spectrum for $\delta_{C P}^{\text {true }}=0$. The background spectra are given by the dashed lines and the signal+background spectra are given by the solid lines and gray region. The error bars on the gray region indicate statistical uncertainties. The total $\Delta \chi^{2}$ before and after the fit are indicated and include $\nu_{e}$ appearance, $\bar{\nu}_{e}$ appearance, $\nu_{\mu}$ disappearance, and $\bar{\nu}_{\mu}$ disappearance. The nominal assumptions are used for systematic (uncorrelated sample, single background systematics) uncertainties and $\nu: \bar{\nu}$ ratios. The $\Delta \chi^{2}$ as a function of $E_{\text {reco }}$ is given by the yellow histogram. 


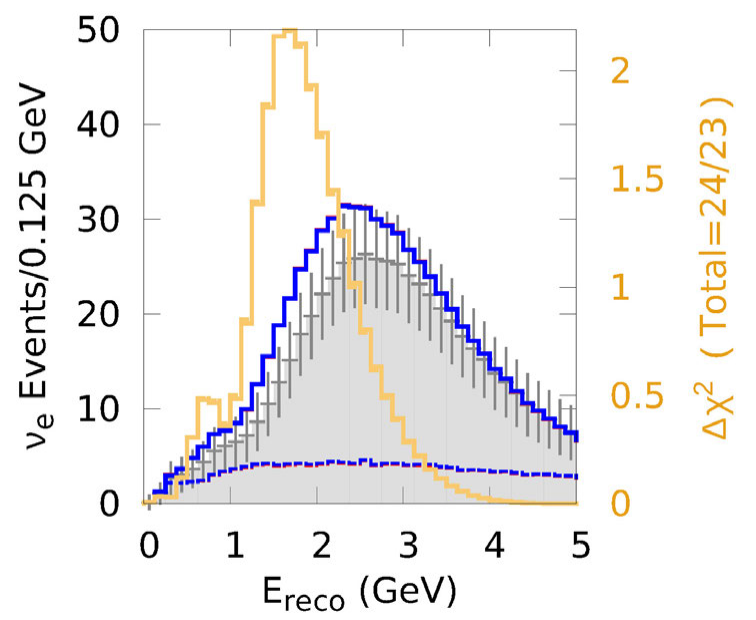

(A) $\nu_{e}$ appearance

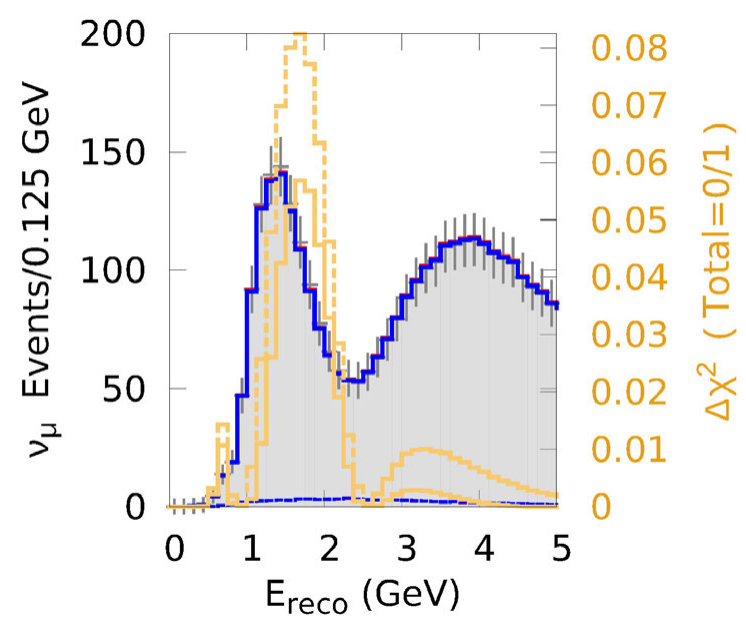

(C) $\nu_{\mu}$ disappearance

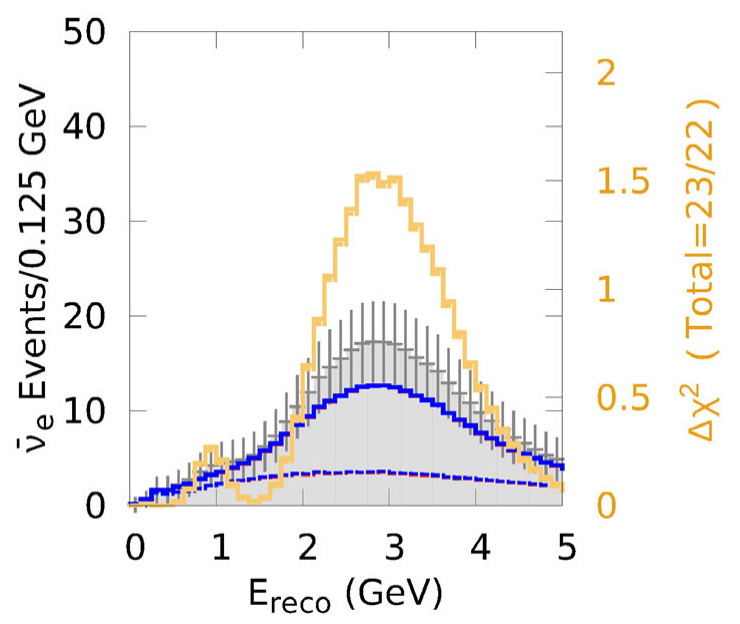

(B) $\bar{\nu}_{e}$ appearance

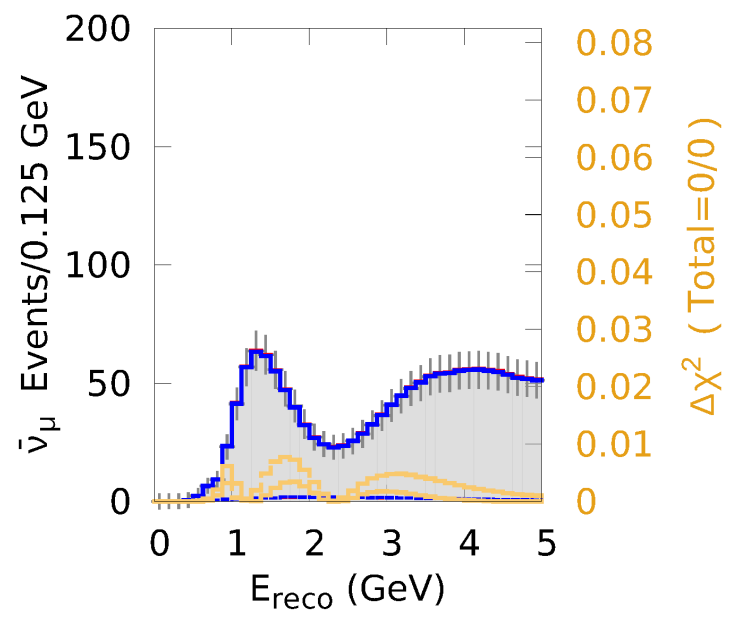

(D) $\bar{\nu}_{\mu}$ disappearance

$$
\begin{array}{r}
X_{\text {pre/post }}^{2}=47 / 47 \\
\operatorname{True}^{\delta_{C P}=0} \\
\text { Pre-Fit } \delta_{C P}=-\pi / 2 \\
\text { Post-Fit } \delta_{C P}=-\pi / 2 \\
\text { Background -------- }
\end{array}
$$

Figure 7.7. LBNE pre-fit (red) and post-fit (blue) spectra, each with $\delta_{C P}=$ $-\pi / 2$, compared to the true spectrum for $\delta_{C P}^{\text {true }}=0$. The background spectra are given by the dashed lines and the signal+background spectra are given by the solid lines and gray region. The error bars on the gray region indicate statistical uncertainties. The total $\Delta \chi^{2}$ before and after the fit are indicated and include $\nu_{e}$ appearance, $\bar{\nu}_{e}$ appearance, $\nu_{\mu}$ disappearance, and $\bar{\nu}_{\mu}$ disappearance. The nominal assumptions are used for systematic (correlated sample, multiple background systematics) uncertainties and $\nu: \bar{\nu}$ ratios. The $\Delta \chi^{2}$ as a function of $E_{\text {reco }}$ is given by the yellow histogram. 


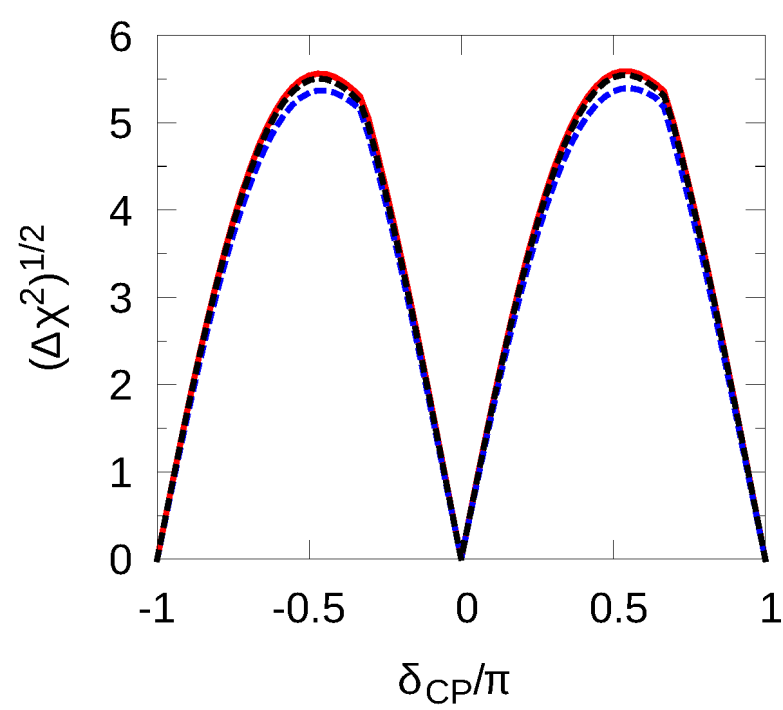

(A) CP Violation Sensitivity vs $\delta_{C P}$ True $\mathrm{MH}=\mathrm{NH}$

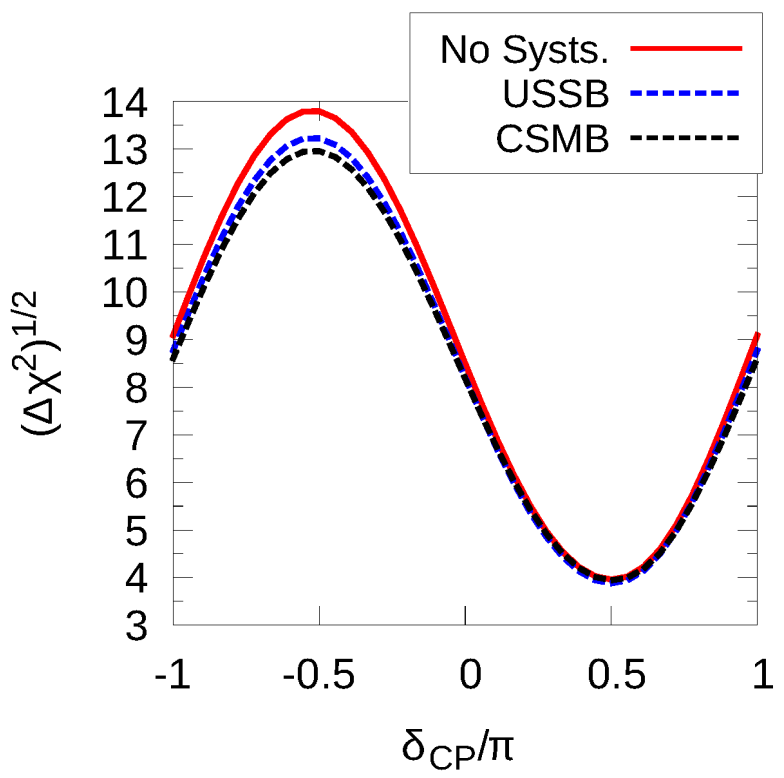

(B) $\mathrm{MH}$ Sensitivity vs $\delta_{C P}$ True $\mathrm{MH}=\mathrm{NH}$

FIgURE 7.8. LBNE CP Violation \& MH Sensitivity sensitivity vs True $\delta_{C P}$ for no systematics and the two different systematics formulations. The Capozzi et al. best-fit values are assumed for the true values of the other oscillation parameters. All four samples are fit: $\nu_{e}$ appearance, $\bar{\nu}_{e}$ appearance, $\nu_{\mu}$ disappearance, and $\bar{\nu}_{\mu}$ disappearance. The nominal assumptions are used for the $\nu: \bar{\nu}$ ratios. The other oscillation parameters and $\mathrm{MH}$ are profiled. 


\subsection{LBNE Systematic Response Functions}

The LBNE Fast Monte Carlo (Fast MC) simulation is being developed to simulate the neutrino production, propagation, and interactions in both near and far detectors for LBNE. As described in Chapter 4, the inputs to produce all of the figures in previous sections were made using the outputs of this Fast MC simulation. Not used in previous sections were variations in the fit event spectra that come from considering variations in the systematic uncertainty parameters. As of this writing, systematic uncertainties in the flux and crosssections have been implemented and propagated to the far detector analysis samples in order to estimate their effects on the sensitivity to the neutrino oscillation parameters. The work in this section was done within the LBNE collaboration and includes the inputs from other members of the collaboration. Work or figures that were not done by the author of this thesis will be labeled as such.

Propagating the systematic uncertainties through the Fast MC allows the effects of each uncertainty to be seen on each sample at the FD and this has been incorporated, by the author of this thesis, into sensitivity analyses using the GLoBES library in a similar way to the method that was outlined in Chapter 5. This adds realistic systematic uncertainties to the $\nu_{e}$ appearance, $\bar{\nu}_{e}$ appearance, $\nu_{\mu}$ disappearance, and $\bar{\nu}_{\mu}$ disappearance FD oscillation analysis samples that might be used in a full data analysis. The variations in a channel are collected into a response function that takes a true neutrino energy and a nuisance parameter encoding the number of standard deviations the systematic parameter should vary and returns a weight that multiplies the number of events for that channel and $E_{\text {true }}$. 
The number of events in $E_{\text {reco }}$ bin $i$ and channel $c$ is given by:

$$
n_{i, c}(\boldsymbol{\theta}, f)=\sum_{j}^{N_{E_{\text {true }}}}\left(R_{j}^{c}(f) \cdot P_{j}^{c}(\boldsymbol{\theta}) \cdot n_{j}^{c} \cdot S_{i j}^{c}\right)
$$

where $n_{j}^{c}$ is the Fast $\mathrm{MC}$ prediction for the event rate at the $\mathrm{FD}$ in $E_{\text {true }}$ bin $j, P^{c}(\boldsymbol{\theta})$ is the oscillation probability for the set of oscillation parameters $\boldsymbol{\theta}, S_{i j}^{c}$ is the Fast MC smearing matrix that maps $E_{\text {true }} \rightarrow E_{\text {reco }}$, and $R_{j}^{c}(f)$ is the Fast MC systematic response function at nuisance parameter value $f$ and in energy bin $j$. The response functions are generated at discrete values of $f$ by the Fast MC and splines are used to form a continuous response function when used in the $\Delta \chi^{2}$.

Event rates are computed separately for each channel and combined to form a signal and background where a number of nuisance parameters are varied in order to apply theoretical and statistical uncertainties to the samples. Four nuisance parameters that represent theoretical uncertainties between channels and samples are incorporated:

- $f_{\nu_{\mu}}$ varies the channels depending on $\nu_{\mu}$

- $f_{\bar{\nu} / \nu}$ varies the channels depending on $\bar{\nu}_{\mu}, \bar{\nu}_{e}$, and $\bar{\nu}_{\tau}$ relative to the $\nu_{\mu}, \nu_{e}$, and $\nu_{\tau}$ channels respectively and incorporates uncertainty in the relative cross sections between $\nu$ and $\bar{\nu}$

- $f_{\nu_{e} / \nu_{\mu}}$ varies the channels depending on $\nu_{e}$ relative to the $\nu_{\mu}$ channel

- $f_{\nu_{\tau} / \nu_{\mu}}$ varies the channels depending on $\nu_{\tau}$ relative to the $\nu_{\mu}$ channel

Eight nuisance parameters represent statistical uncertainty from statistical limitations in the measurement of $\nu_{\mu}$ flux and incorporate statistical uncertainties between samples within the Asimov data set framework: $f_{\bar{\nu}_{\mu} / \nu_{\mu}}^{\text {stat }}, f_{\nu_{e} / \nu_{\mu}}^{s t a t}, f_{\bar{\nu}_{e} / \nu_{\mu}}^{s t a t}, f_{N C / \nu_{\mu}}^{s t a t}, f_{\nu_{e}^{o s c} / \nu_{\mu}}^{s t a t}, f_{\bar{\nu}_{e}^{o s c} / \nu_{\mu}}^{s t a t}, f_{\nu_{\tau} / \nu_{\mu}}^{s t a t}$, and $f_{\bar{\nu}_{\tau} / \nu_{\mu}}^{s t a t}$. 
The number of events in a particular bin then is computed using the raw event rates for each channel (lower case $\mathrm{n}$ ) and this set of nuisance parameters. In $\nu_{e}$ appearance and $\bar{\nu}_{e}$ appearance the number of events is

$$
\begin{aligned}
N_{\nu_{e}, \bar{\nu}_{e}} & =R^{\nu_{e}-o s c}\left(f_{\nu_{\mu}}+f_{\nu_{e} / \nu_{\mu}}+f_{\nu_{e}^{o s c} / \nu_{\mu}}^{s t a t}\right) n_{\nu_{e}-o s c} \\
& +R^{\bar{\nu}_{e}-o s c}\left(f_{\nu_{\mu}}+f_{\bar{\nu} / \nu}+f_{\nu_{e} / \nu_{\mu}}+f_{\bar{\nu}_{e} \text { sc } / \nu_{\mu}}^{s t a t}\right) n_{\bar{\nu}_{e}-o s c} \\
& +R^{\nu_{\mu}}\left(f_{\nu_{\mu}}\right) n_{\nu_{\mu}} \\
& +R^{\bar{\nu}_{\mu}}\left(f_{\nu_{\mu}}+f_{\bar{\nu} / \nu}+f_{\bar{\nu}_{\mu} / \nu_{\mu}}^{s t a t}\right) n_{\bar{\nu}_{\mu}} \\
& +R^{N C}\left(f_{\nu_{\mu}}+f_{N C / \nu_{\mu}}^{s t a t}\right) n_{N C} \\
& +R^{\nu_{e}-b e a m}\left(f_{\nu_{\mu}}+f_{\nu_{e} / \nu_{\mu}}+f_{\nu_{e} / \nu_{\mu}}^{s t a t}\right) n_{\nu_{e}-\text { beam }} \\
& +R^{\bar{\nu}_{e}-b e a m}\left(f_{\nu_{\mu}}+f_{\bar{\nu} / \nu}+f_{\nu_{e} / \nu_{\mu}}+f_{\bar{\nu}_{e} / \nu_{\mu}}^{s t a t}\right) n_{\bar{\nu}_{e}-\text { beam }} \\
& +R^{\nu_{\tau}}\left(f_{\nu_{\mu}}+f_{\nu_{\tau} / \nu_{\mu}}+f_{\nu_{\tau} / \nu_{\mu}}^{s t a t}\right) n_{\nu_{\tau}} \\
& +R^{\bar{\nu}_{\tau}}\left(f_{\nu_{\mu}}+f_{\bar{\nu} / \nu}+f_{\nu_{\tau} / \nu_{\mu}}+f_{\bar{\nu}_{\tau} / \nu_{\mu}}^{s t a t}\right) n_{\bar{\nu}_{\tau}}
\end{aligned}
$$


where the first two rows are the signal channels and the last seven are the background channels. In $\nu_{\mu}$ disappearance and $\bar{\nu}_{\mu}$ disappearance the number of events is

$$
\begin{aligned}
& N_{\nu_{\mu}, \bar{\nu}_{\mu}}=R^{\nu_{\mu}}\left(f_{\nu_{\mu}}\right) n_{\nu_{\mu}} \\
& +R^{\bar{\nu}_{\mu}}\left(f_{\nu_{\mu}}+f_{\bar{\nu} / \nu}+f_{\bar{\nu}_{\mu} / \nu_{\mu}}^{s t a t}\right) n_{\bar{\nu}_{\mu}} \\
& +R^{\nu_{e}-o s c}\left(f_{\nu_{\mu}}+f_{\nu_{e} / \nu_{\mu}}+f_{\nu_{e}^{s o c} / \nu_{\mu}}^{s t a t}\right) n_{\nu_{e}-o s c} \\
& +R^{\bar{\nu}_{e}-o s c}\left(f_{\nu_{\mu}}+f_{\bar{\nu} / \nu}+f_{\nu_{e} / \nu_{\mu}}+f_{\bar{\nu}_{e}^{o s c} / \nu_{\mu}}^{s t a t}\right) n_{\bar{\nu}_{e}-o s c} \\
& +R^{N C}\left(f_{\nu_{\mu}}+f_{N C / \nu_{\mu}}^{s t a t}\right) n_{N C} \\
& +R^{\nu_{e}-\text { beam }}\left(f_{\nu_{\mu}}+f_{\nu_{e} / \nu_{\mu}}+f_{\nu_{e} / \nu_{\mu}}^{\text {stat }}\right) n_{\nu_{e}-\text { beam }} \\
& +R^{\bar{\nu}_{e}-\text { beam }}\left(f_{\nu_{\mu}}+f_{\bar{\nu} / \nu}+f_{\nu_{e} / \nu_{\mu}}+f_{\bar{\nu}_{e} / \nu_{\mu}}^{s t a t}\right) n_{\bar{\nu}_{e}-\text { beam }} \\
& +R^{\nu_{\tau}}\left(f_{\nu_{\mu}}+f_{\nu_{\tau} / \nu_{\mu}}+f_{\nu_{\tau} / \nu_{\mu}}^{s t a t}\right) n_{\nu_{\tau}} \\
& +R^{\bar{\nu}_{\tau}}\left(f_{\nu_{\mu}}+f_{\bar{\nu} / \nu}+f_{\nu_{\tau} / \nu_{\mu}}+f_{\bar{\nu}_{\tau} / \nu_{\mu}}^{s t a t}\right) n_{\bar{\nu}_{\tau}}
\end{aligned}
$$

and again the first two rows are the signal channels and the last seven are the background channels. The response functions are different for each sample so that, for example, the $R^{\nu_{\mu}}$ in $\nu_{e}$ appearance differs from the $R^{\nu_{\mu}}$ in $\nu_{\mu}$ disappearance. It is important to note that the 12 nuisance parameters here are shared between the four samples so that the sensitivity benefits from the large samples in $\nu_{\mu}$ acting to constrain the predicted $\nu_{e}$ flux in the $\nu_{e}$ appearance and $\bar{\nu}_{e}$ appearance samples. Without the $f^{\text {stat }}$ parameters and the theoretical uncertainties then these constraints would be perfect and the $\nu_{\mu}$ and $\nu_{e}$ samples would be completely correlated, as would the $\nu_{e}$ and $\bar{\nu}_{e}$ samples. With these $f^{\text {stat }}$ nuisance parameters in place the constraints on the systematic uncertainties are limited by the statistics of the experiment and the constraints provided by other experiments on the model parameters. 
The nuisance parameters are not completely free but constrained by penalty terms with Gaussian priors on each parameter. The theoretical nuisance parameters are constrained with prior $1 \sigma$ widths of $\sigma_{\bar{\nu} / \nu}=10 \%, \sigma_{\nu_{e} / \nu_{\mu}}=2.5 \%, \sigma_{\nu_{\tau} / \nu_{\mu}}=10 \%$. These constraints are based on external measurements and are estimates that will need to be justified and expanded in future work.

The statistical nuisance parameters represent statistical uncertainties through constraints of the nuisance parameters so these constraints are computed from the available simulated data samples. The uncertainties in these nuisance parameters are the constraints that will come from measurements in the far detector. In particular the constraints on the statistical nuisance parameters depend on the sample statistics and get better with increased POT. The constraints are computed by evaluating the $\Delta \chi^{2}$ as a function of $f_{\nu_{\mu}}$ and determining the constraint provided on $f_{\nu_{\mu}}$ by the simulated data. Figure 7.9 shows an example of a response of the $\Delta \chi^{2}$ to changes in the $f_{\nu_{\mu}}$ parameter for the CC $M_{A}^{R E S}$ systematic that will be described in more detail below. The $1 \sigma$ response for this systematic is at $\chi^{2}(0.15)=1$. For this systematic, then, the $\sigma_{\text {stat }}$ prior is $15 \%$, i.e., there is a $15 \%$ constraint on this systematic parameter that comes from the $\nu_{\mu}$ sample. The improvement over the prior constraint is visible by comparing the gray curve to the red curve. This does not represent a measurement of the systematic parameter, but is a characterization of how much the parameter affects the analysis samples in the oscillation analyses. All eight $f^{\text {stat }}$ parameters use this same constraint and so incorporate uncertainty in the $\nu_{\mu}$ channel measurement into the constraints on the other channels.

Figure 7.9 shows the response, or fractional change, of each channel making up the $\nu_{e}$ appearance sample to variations in $\mathrm{CC} M_{A}^{R E S}$. The color scale represents the response function, $R_{j}^{c}(f)$, value. The x-axis represents the true energy bin $(\mathrm{j})$, and the $\mathrm{y}$-axis the value 


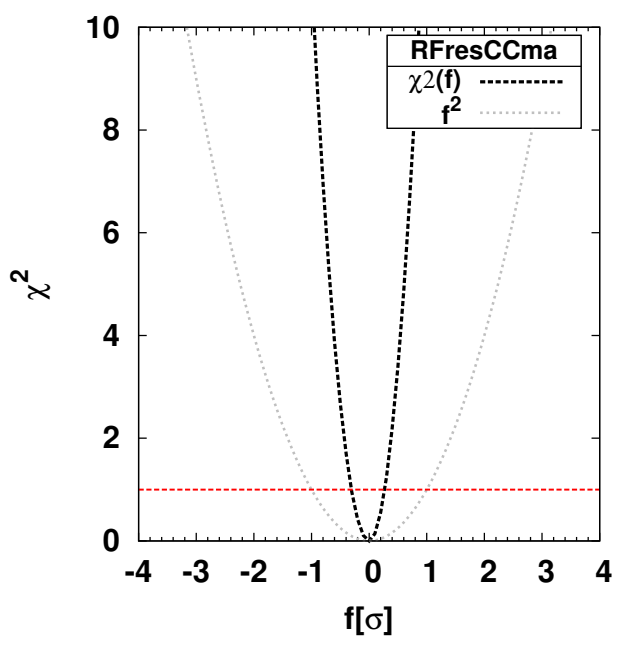

Figure 7.9. Response function $\Delta \chi^{2}$ vs f for CC $M_{A}^{R E S}$. The $\Delta \chi^{2}$ curve shows the effect of varying the $f_{\nu_{\mu}}$ nuisance parameter by the x-axis value on the $\Delta \chi^{2}$. The gray line shows the prior constraint on the $f_{\nu_{\mu}}$ parameter $\left(\sigma_{\nu_{\mu}}=1\right)$ where a $+1 \sigma$ variation corresponds to a $+20 \%$ change in $\mathrm{CC} M_{A}^{R E S}$. The $1 \sigma$ sigma response is extracted and used to constrain the $f^{\text {stat }}$ nuisance parameters. The $1 \sigma$ level is indicated by the horizontal red line.

of the nuisance parameter (f). For example a $1 \sigma$ change in CC $M_{A}^{R E S}$ change $(+20 \%$ change in parameter) produces a $+10 \%$ change in number of signal $\nu_{e}$ events at $2 \mathrm{GeV}$. In this way the nuisance parameter fluctuations are propagated to all of the channels using Equation 40.

After propagating the response function effects to the channels they can be combined into the $\nu_{e}$ appearance, $\bar{\nu}_{e}$ appearance, $\nu_{\mu}$ disappearance, and $\bar{\nu}_{\mu}$ disappearance samples as in Figures 7.11 and 7.12. These figures show the effect of a $+1 \sigma$ fluctuation in the CC $M_{A}^{R E S}$ parameter. The $+1 \sigma$ variation corresponds to a $+20 \%$ change in the value of $\mathrm{CC} M_{A}^{R E S}$. To the extent that the fluctuations in the systematic parameters allow the spectrum to mimic oscillation effects, these response function systematic parameters will reduce the sensitivity to the oscillation parameters. These reductions will be evaluated for a preliminary set of the systematic parameters in the next section.

There are 13 cross section systematics that are currently considered in the analysis of the LBNE sensitivity. These represent variations in the models that affect the cross section 

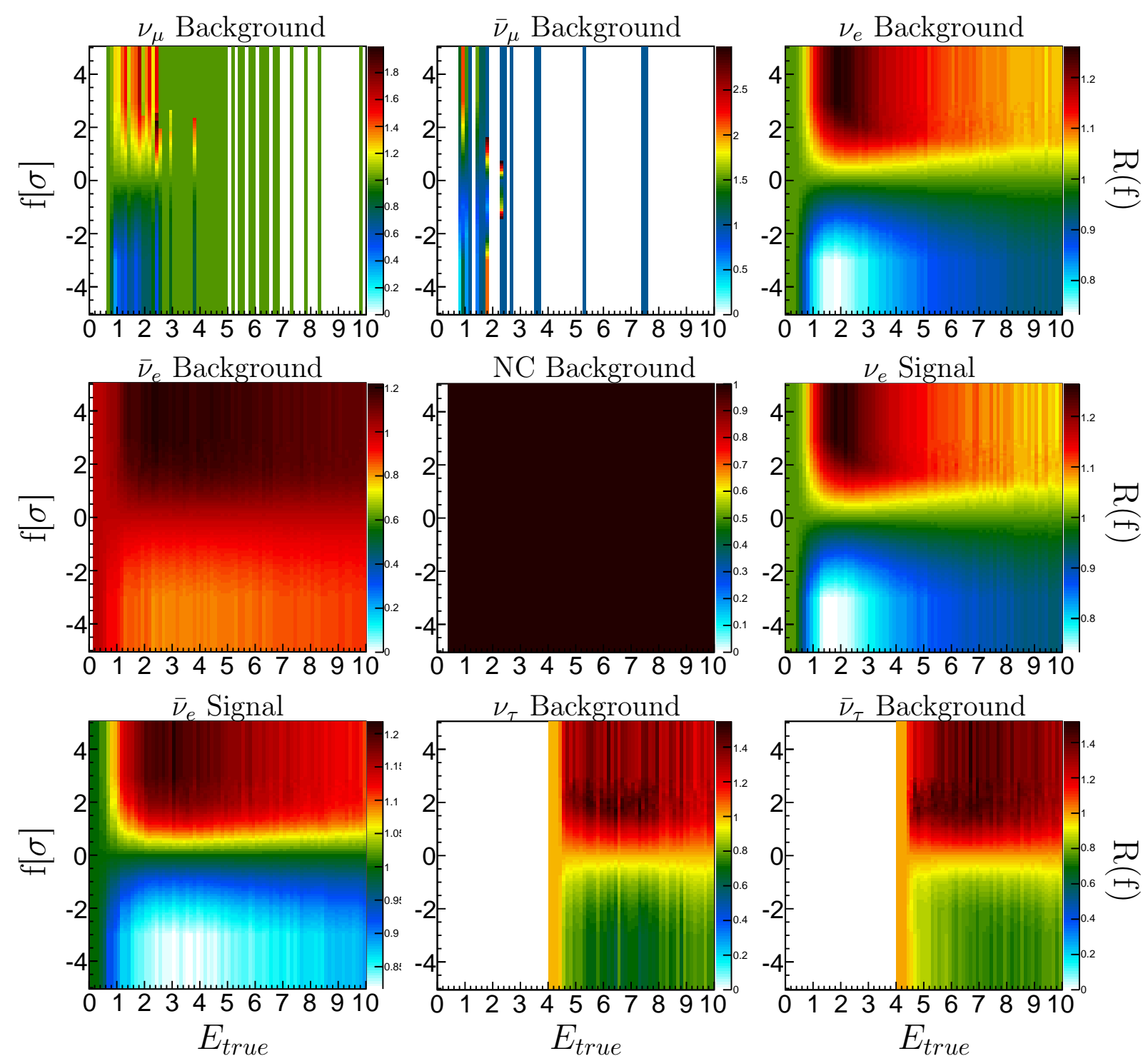

FiguRE 7.10. LBNE Fast MC response functions in $\nu_{e}$ appearance for the $\mathrm{CC} M_{A}^{R E S}$ systematic parameter. Each channel has a response function which varies the spectrum as the nuisance parameter is varied. The empty (white) bins are bins where no events occur. This figure is from the LBNE Fast MC. The NC channel sees no effect from this particular systematic parameter so it is constant at one.

of the various interactions measured in an oscillation analysis and they affect the shape and rate of the predicted spectrum. In addition, there are 10 flux systematic parameters that represent uncertainties in the configuration of the beam that must be constrained by near detector measurements. No near detector is currently implemented in these studies to 
constrain these parameters so the resulting sensitivities are preliminary studies to inform the design constraints on a near detector. Constraints coming from the near detector will greatly reduce the uncertainties in the flux at the far detector.
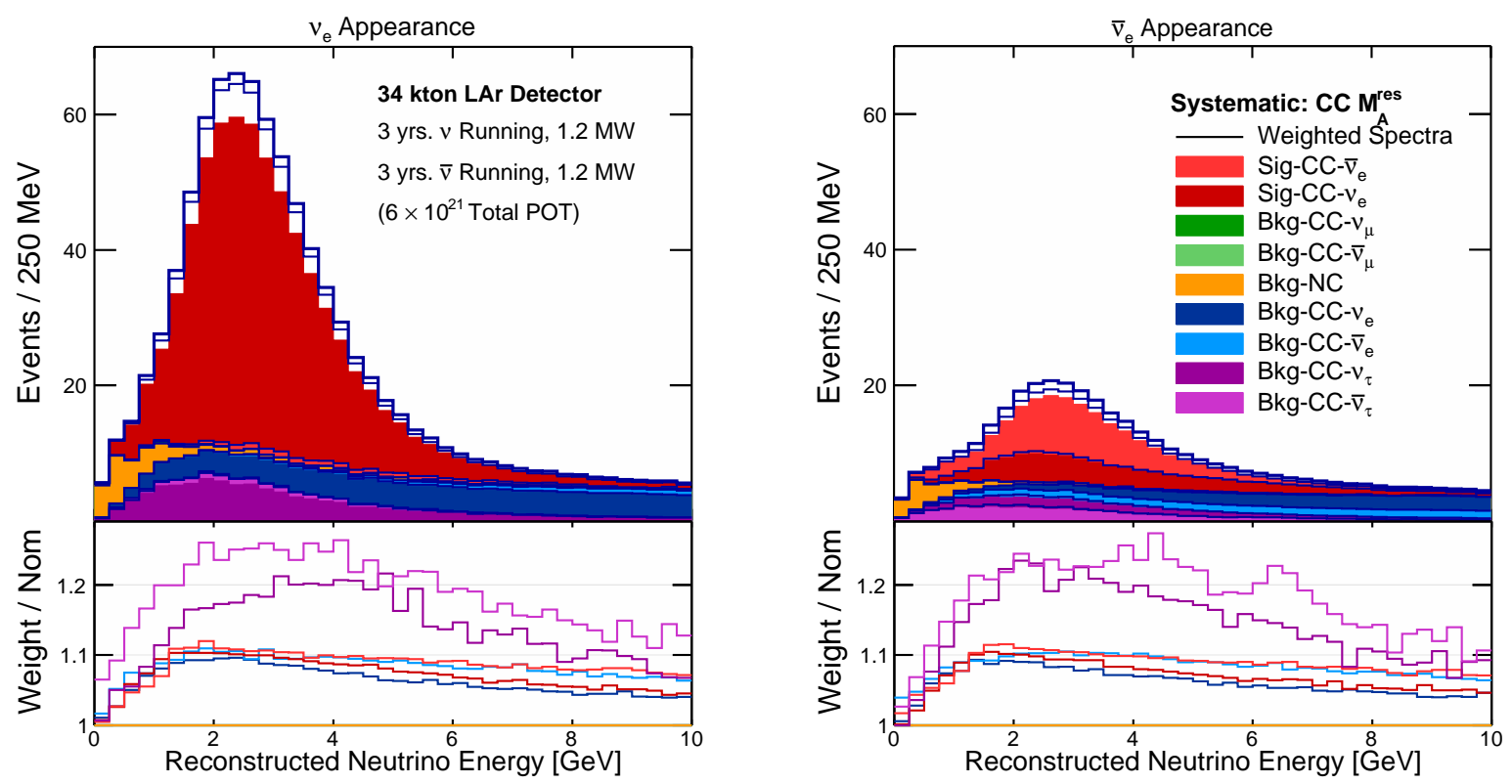

FigURE 7.11. LBNE Fast MC event spectra for $\nu_{e}$ appearance (left) and $\bar{\nu}_{e}$ appearance (right). The nominal spectrum for each channel is indicated by the filled histograms and the variation produced by a $+1 \sigma$ shift in $\mathrm{CC} M_{A}^{R E S}$ is indicated by the lines above each filled histogram. The bottom portion of the plot shows the ratio of the weighted spectrum to the nominal, unweighted spectrum for each channel. Figures reproduced from [39]. 

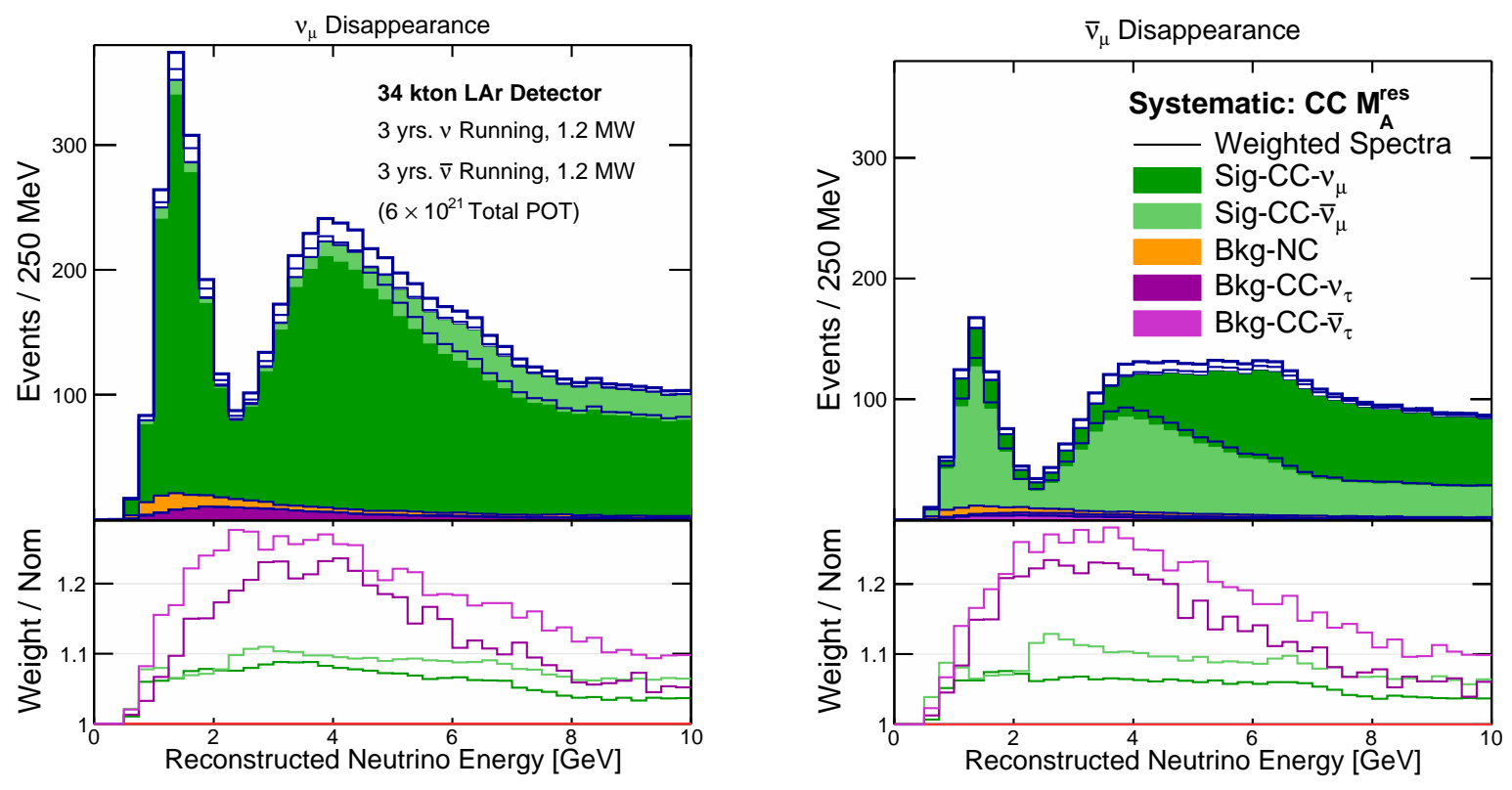

FigURE 7.12. LBNE Fast MC event spectra for $\nu_{\mu}$ disappearance (left) and $\bar{\nu}_{\mu}$ disappearance (right). The nominal spectrum for each channel is indicated by the filled histograms and the variation produced by a $+1 \sigma$ shift in $\mathrm{CC} M_{A}^{R E S}$ is indicated by the lines above each filled histogram. The bottom portion of the plot shows the ratio of the weighted spectrum to the nominal, unweighted spectrum for each channel. Figures reproduced from [39]. 
7.3.1. Pre-Fit \& Post-Fit Spectra. To illustrate the effects of incorporating the response function systematics the pre-fit and post-fit spectra for $\delta_{C P}$ variations are shown in Figures 7.13 through 7.18. These figures are for the $\mathrm{CC} M_{A}^{Q E}$ and $\mathrm{CC} M_{A}^{R E S}$ systematics individually and then in combination, and then for combinations of the flux and/or cross section parameters. The $\mathrm{CC} M_{A}^{Q E}$ parameter affects the region around the QE peak near 0.6 $\mathrm{GeV}$, while the resonant (RES) region dominates between 1-3 GeV as illustrated in Figure 4.6. Applying both of these systematic effects together gives the fit spectra freedom to move in a wide range of $E_{\text {reco. }}$ The $\Delta \chi^{2}$ for the combination of the systematics has 12 nuisance parameters per systematic for a total of 24 nuisance parameters.

Figure 7.13 shows the pre-fit and post-fit spectra for $\delta_{C P}$ with the variations allowed by the CC $M_{A}^{Q E}$ response functions allowed to shift the spectrum. There is a small reduction in the $\Delta \chi^{2}$ from the $\nu_{e}$ appearance sample, from 24 to 21 , while the other samples, particularly $\bar{\nu}_{e}$ appearance and $\nu_{\mu}$ disappearance, are acting to constrain the $\mathrm{CC} M_{A}^{Q E}$ parameter and have their $\Delta \chi^{2}$ increased in order to reduce the $\Delta \chi^{2}$ from the $\nu_{e}$ appearance spectrum. The overall $\Delta \chi^{2}$ is reduced by allowing the pre-fit $\nu_{e}$ appearance spectrum to float downward slightly which also slightly decreases the $\bar{\nu}_{e}$ appearance event spectrum and the $\nu_{\mu}$ disappearance event spectrum. Overall this systematic has a small effect on the ability of LBNE to differentiate between $\delta_{C P}=0$ and $\delta_{C P}=-\pi / 2$ and reduces the total $\Delta \chi^{2}$ from 47 to 46 . A very similar effect is seen in Figure 7.14 for the CC $M_{A}^{R E S}$ parameter.

The combination of these two systematic parameters, in Figure 7.15, allows more freedom in the fit spectrum variations and is able to reduce the total $\Delta \chi^{2}$ from 47 to 45 .

The combined effect on the sensitivity to variations in $\delta_{C P}$ of all 13 of the cross section parameters can be seen in Figure 7.16. The total $\Delta \chi^{2}$ is reduced much more significantly, from 47 to 36 , by the extra freedom in the fit allowed by the additional systematic parameters. 
For the 10 flux systematic parameters an even more significant reduction is seen in Figure 7.17 decreasing the $\Delta \chi^{2}$ fro 47 to 30 . Finally, when combining all of the systematic parameters currently simulated in the Fast MC simulation, in Figure 7.18, the $\Delta \chi^{2}$ is reduced from 47 to 25 .

The $\Delta \chi^{2}$ in the case of 13,10 , and 23 systematic parameters has 156,120 , or 286 nuisance parameters, respectively. This large number of systematic parameters makes the minimization take much longer and required special handling to parallelize the processing of adjacent points in $\delta_{C P}$. 


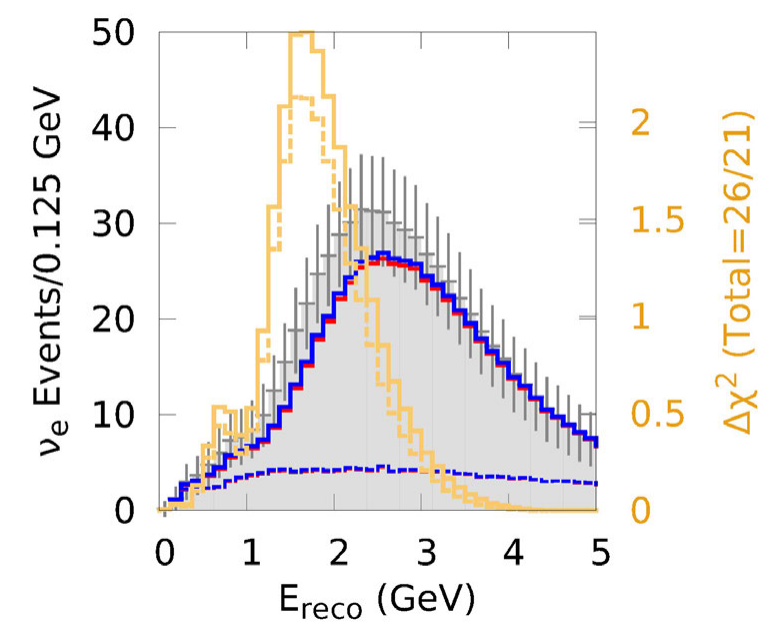

(A) $\nu_{e}$ appearance

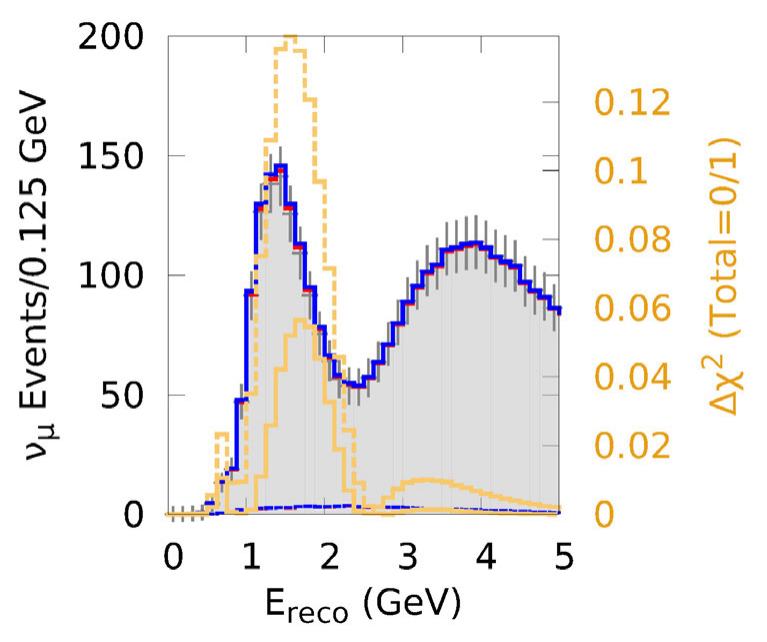

(C) $\nu_{\mu}$ disappearance

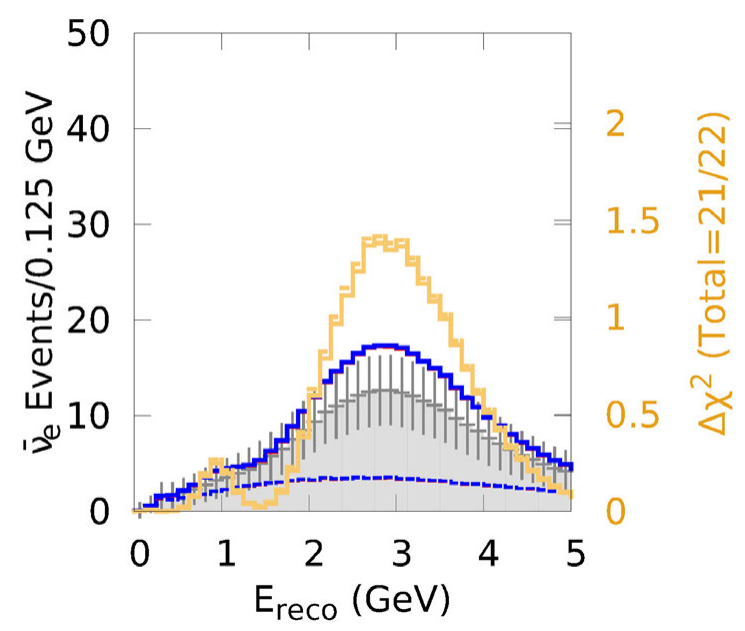

(B) $\bar{\nu}_{e}$ appearance

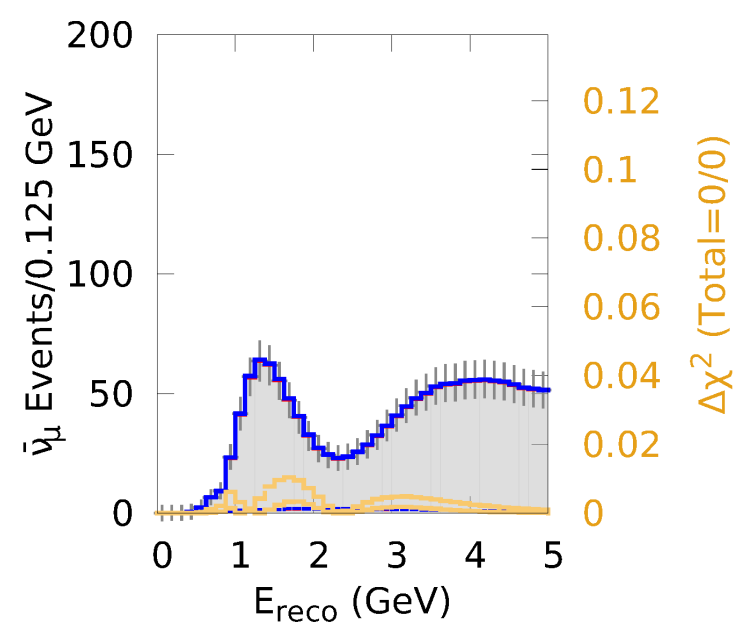

(D) $\bar{\nu}_{\mu}$ disappearance

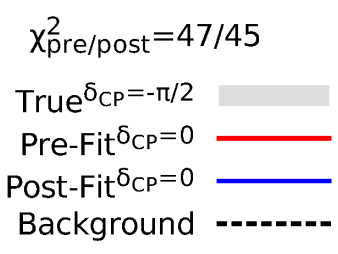

FiguRE 7.13. LBNE Fast MC pre-fit (red) and post-fit (blue) spectra, each with $\delta_{C P}=-\pi / 2$, compared to the true spectrum for $\delta_{C P}^{\text {true }}=0$. The CC $M_{A}^{Q E}$ systematic response functions were used in the fit. The background spectra are given by the dashed lines and the signal+background spectra are given by the solid lines and gray region. The error bars on the gray region indicate statistical uncertainties. The total $\Delta \chi^{2}$ before and after the fit are indicated in the legend and include $\nu_{e}$ appearance, $\bar{\nu}_{e}$ appearance, $\nu_{\mu}$ disappearance, and $\bar{\nu}_{\mu}$ disappearance. The nominal assumptions are used the $\nu: \bar{\nu}$ ratio. The $\Delta \chi^{2}$ as a function of $E_{\text {reco }}$ is given by the yellow histogram before (solid) and after (dashed) the fit. The other oscillation parameters are fixed. 


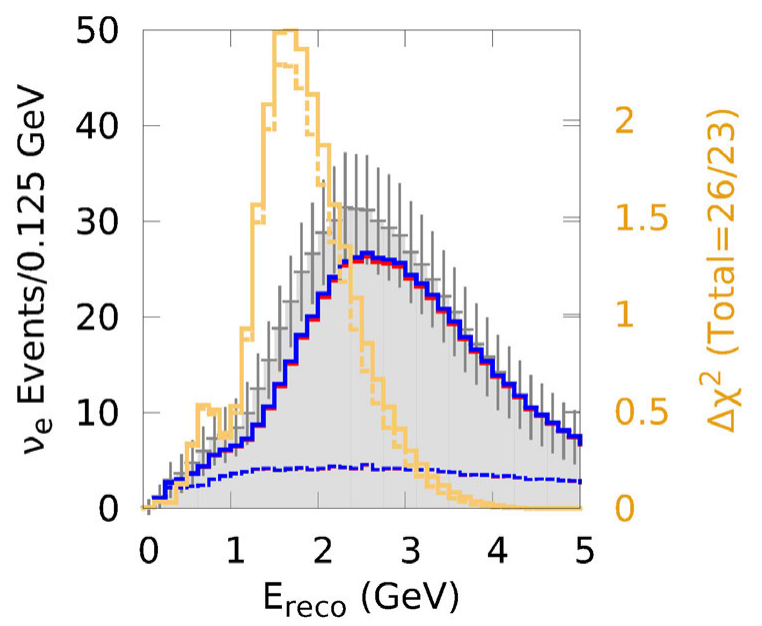

(A) $\nu_{e}$ appearance

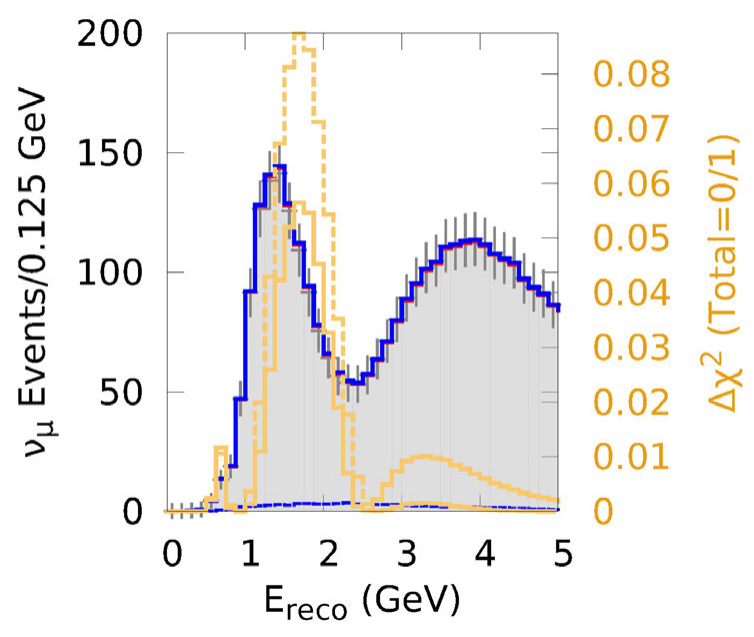

(C) $\nu_{\mu}$ disappearance

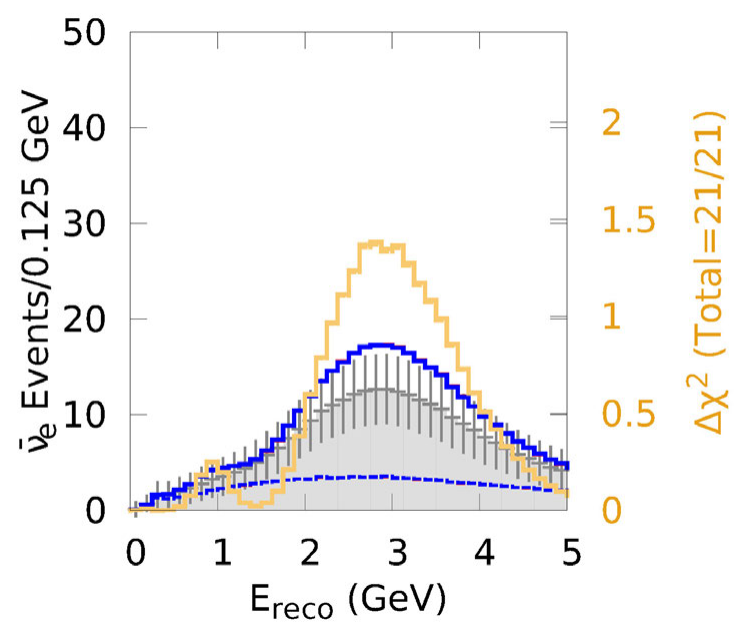

(B) $\bar{\nu}_{e}$ appearance

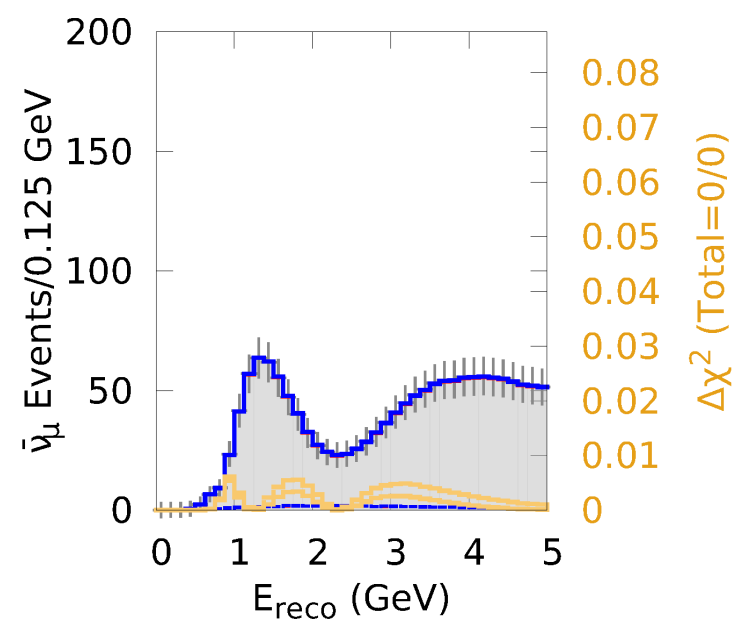

(D) $\bar{\nu}_{\mu}$ disappearance

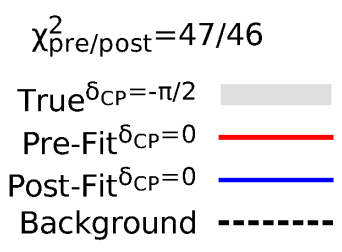

Figure 7.14. As in Figure 7.13 but for the $\mathrm{CC} M_{A}^{R E S}$ systematic parameter. 


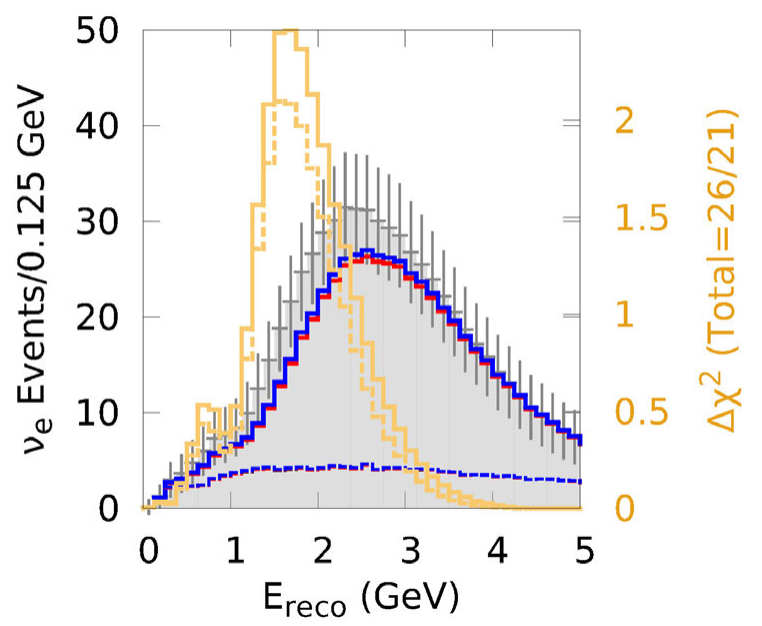

(A) $\nu_{e}$ appearance

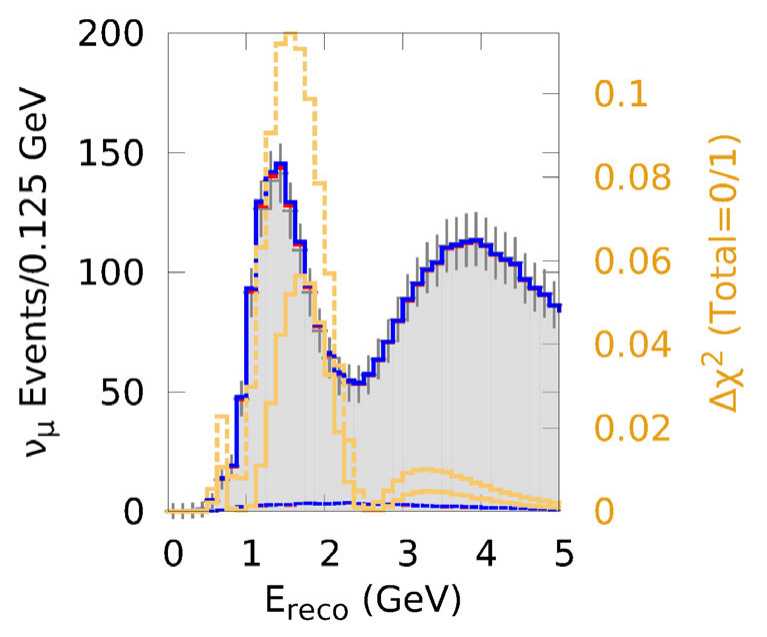

(C) $\nu_{\mu}$ disappearance

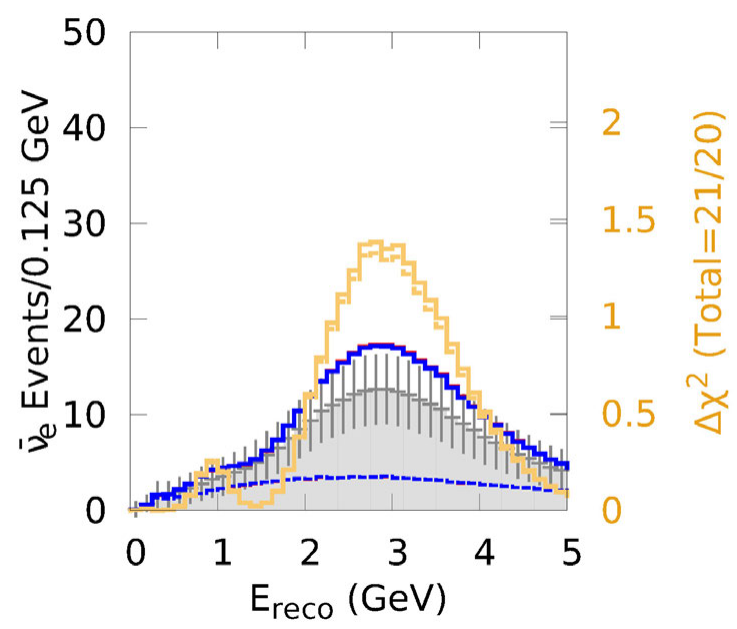

(B) $\bar{\nu}_{e}$ appearance

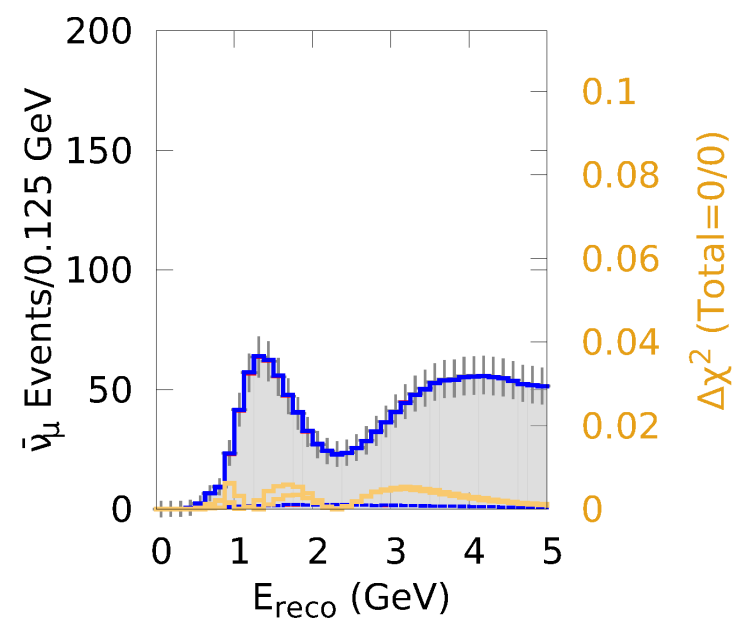

(D) $\bar{\nu}_{\mu}$ disappearance

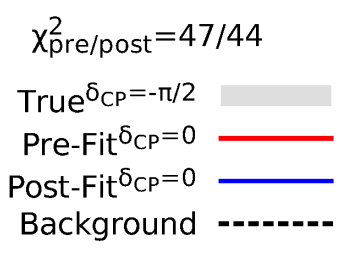

FiguRE 7.15. As in Figure 7.13 but for both the CC $M_{A}^{Q E}$ and $M_{A}^{R E S}$ systematic parameters. 


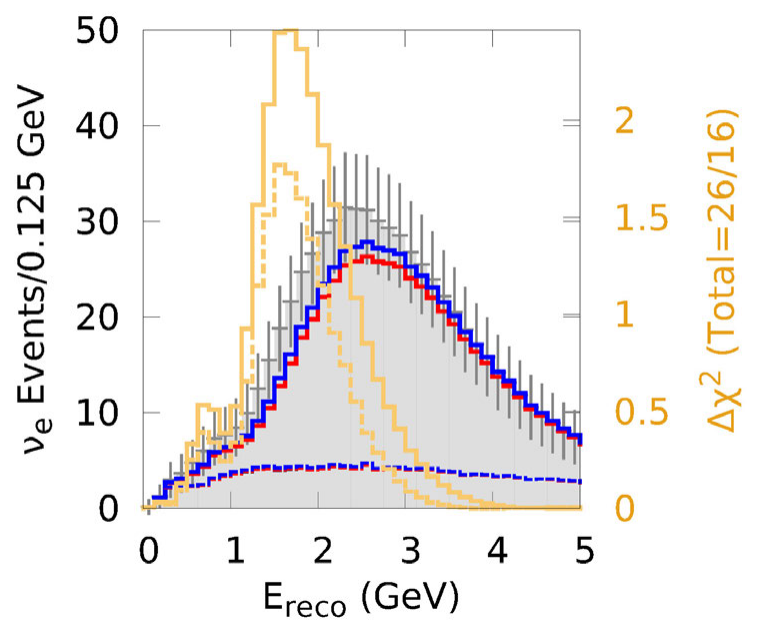

(A) $\nu_{e}$ appearance

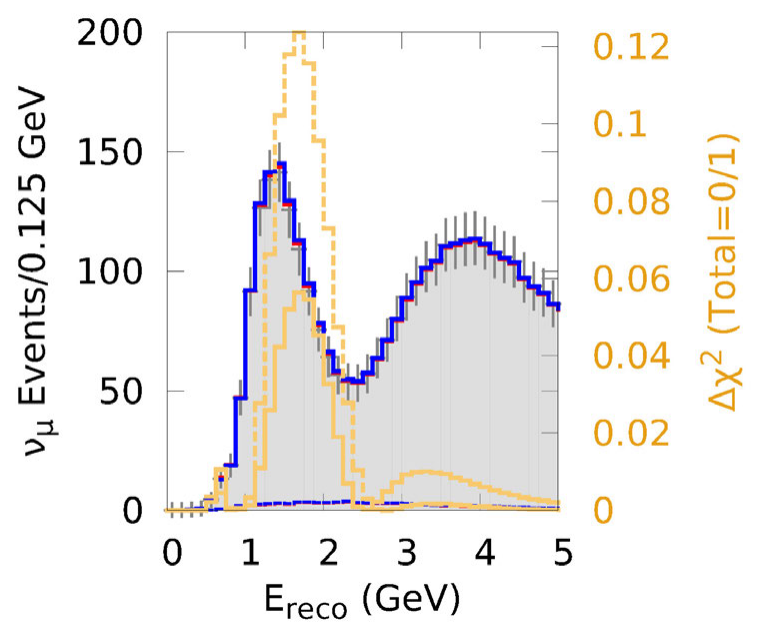

(C) $\nu_{\mu}$ disappearance

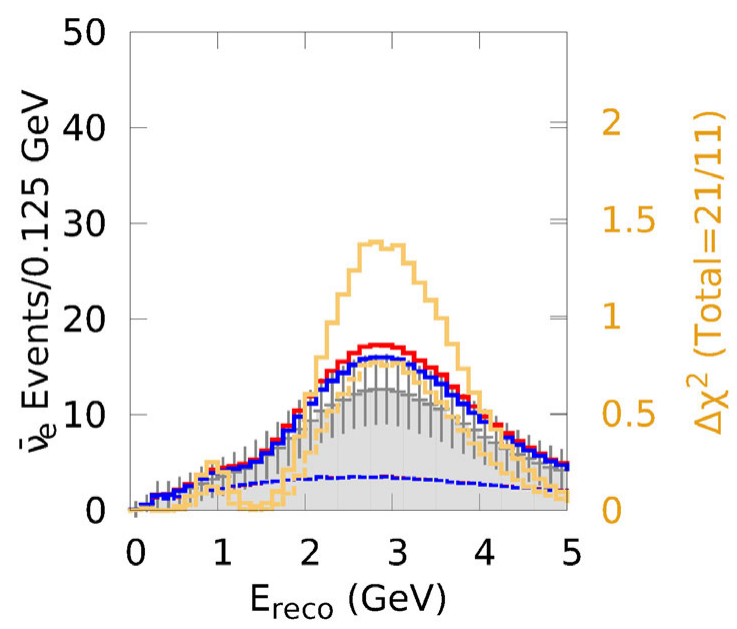

(B) $\bar{\nu}_{e}$ appearance

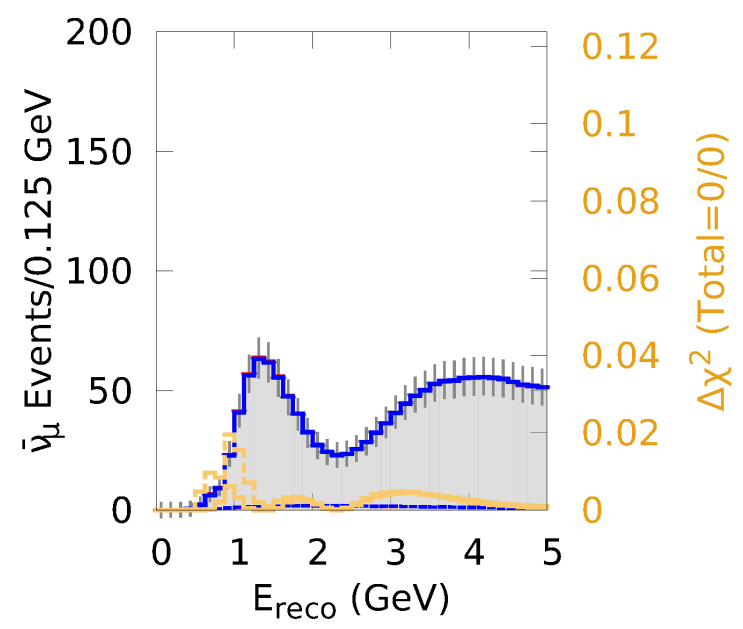

(D) $\bar{\nu}_{\mu}$ disappearance

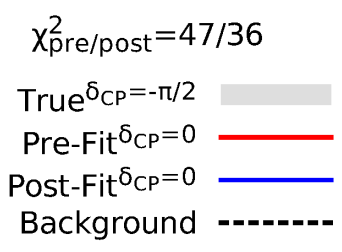

Figure 7.16. As in Figure 7.13 but for all 13 of the neutrino cross section systematic parameters. 


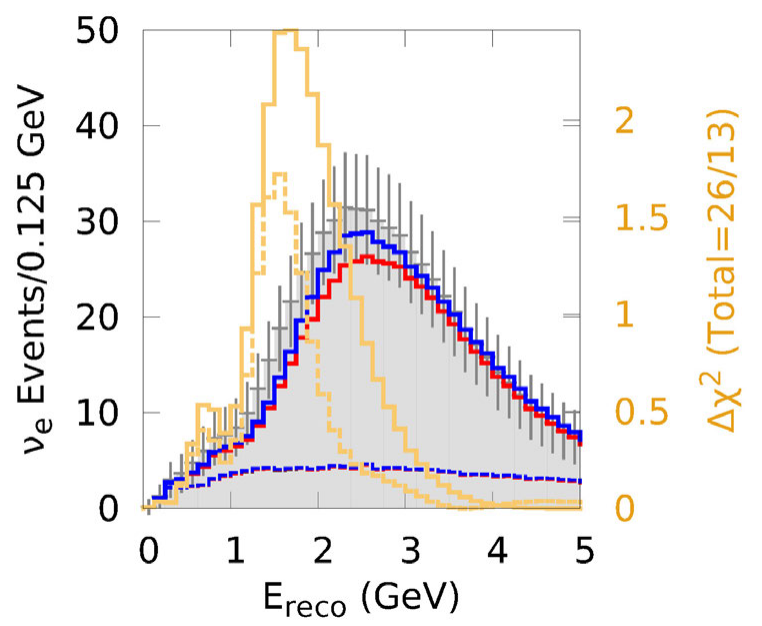

(A) $\nu_{e}$ appearance

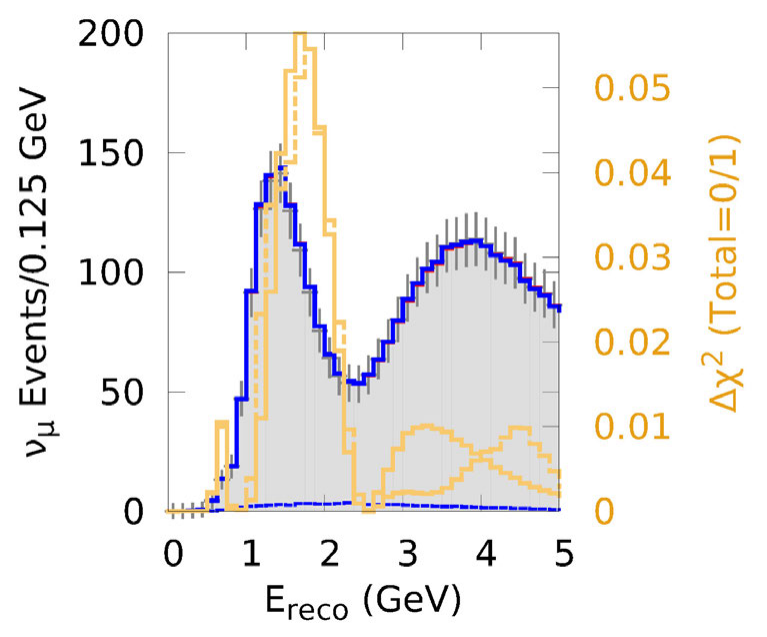

(C) $\nu_{\mu}$ disappearance

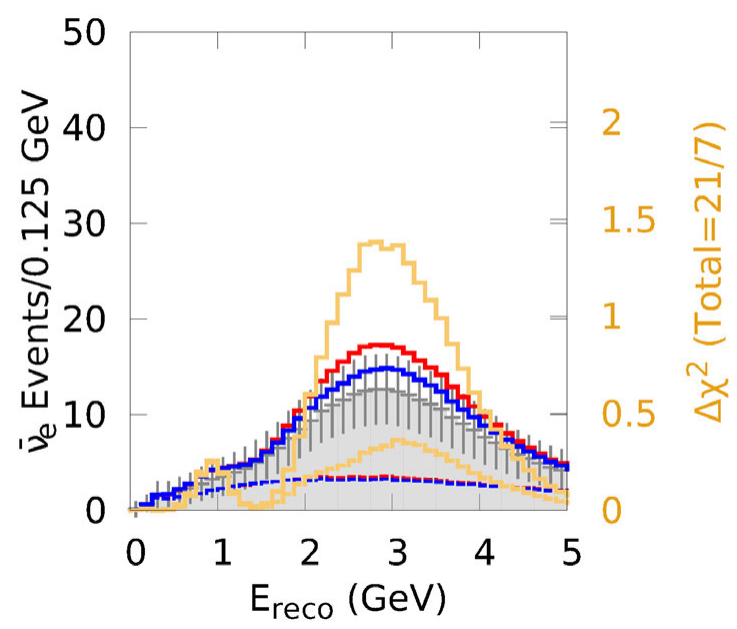

(B) $\bar{\nu}_{e}$ appearance

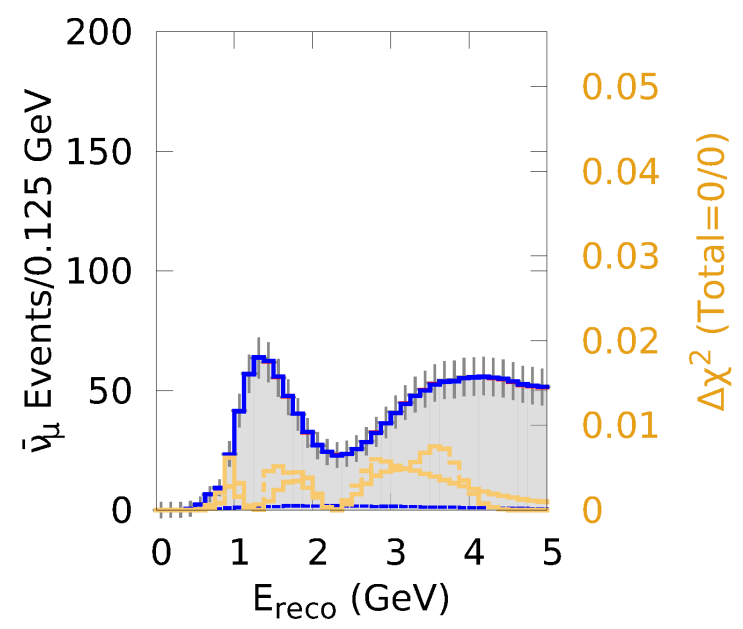

(D) $\bar{\nu}_{\mu}$ disappearance

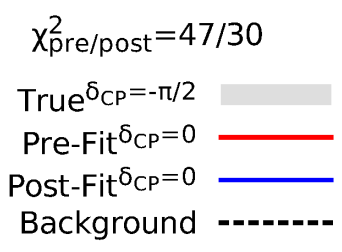

Figure 7.17. As in Figure 7.13 but for all 10 of the flux systematic parameters. 


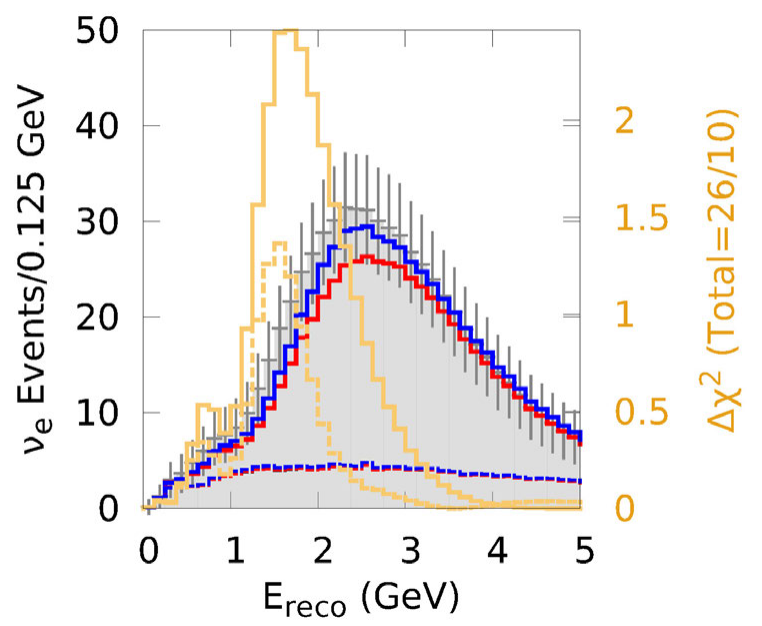

(A) $\nu_{e}$ appearance

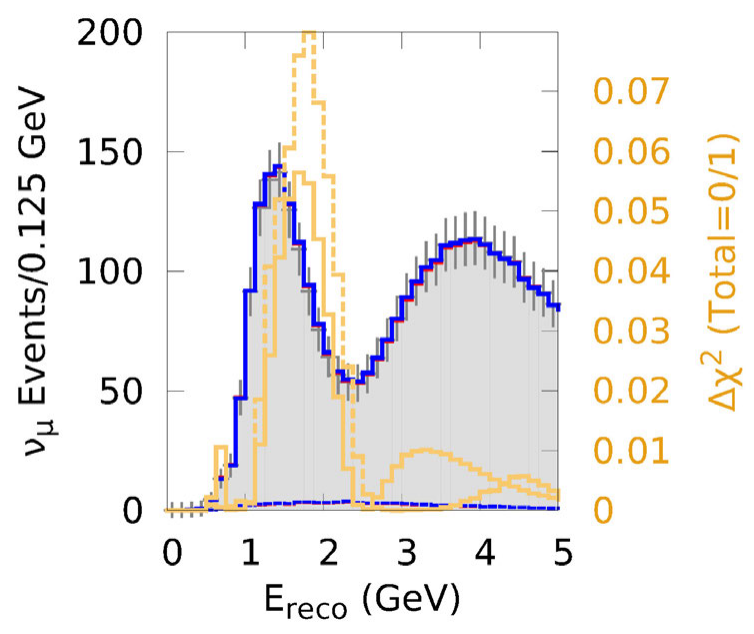

(C) $\nu_{\mu}$ disappearance

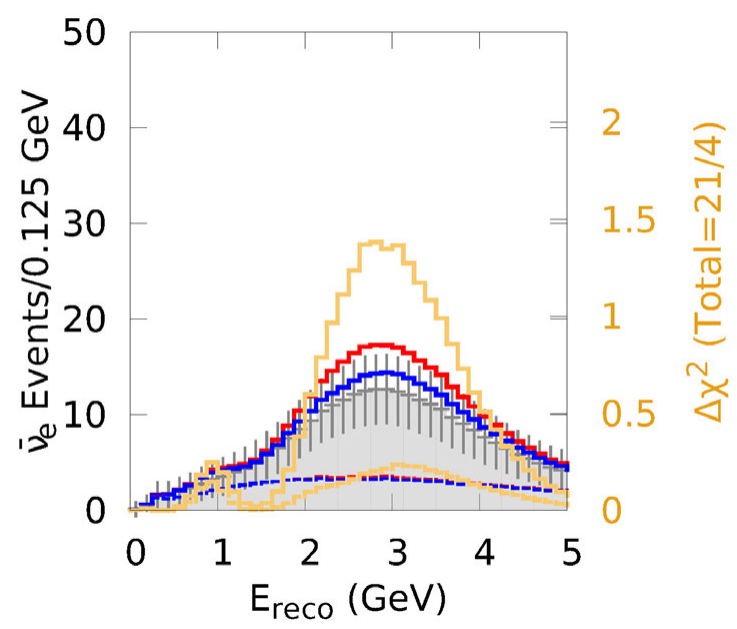

(B) $\bar{\nu}_{e}$ appearance

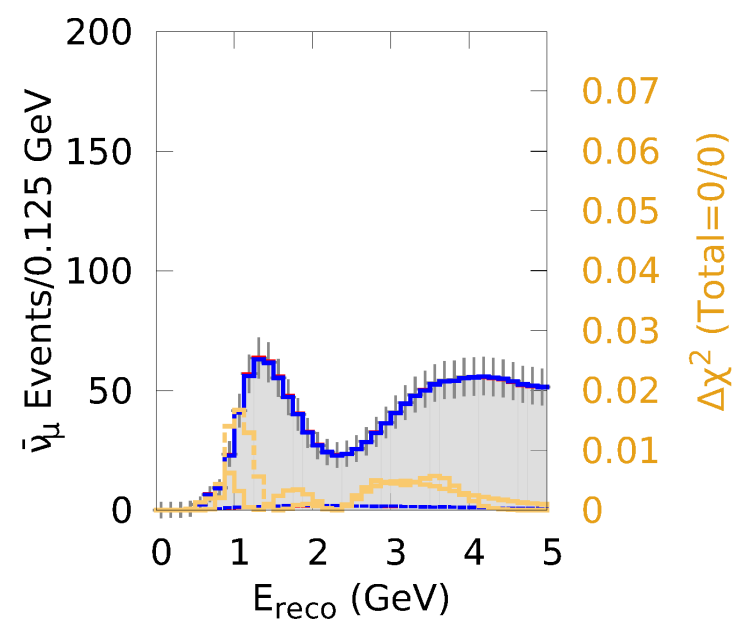

(D) $\bar{\nu}_{\mu}$ disappearance

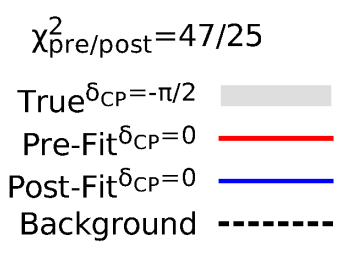

Figure 7.18. As in Figure 7.13 but for all 23 of the systematic parameters. 
The systematic variations caused by a systematic parameter will, in general, cause variations in all four of the samples. It is informative to look at the fit for only one sample so that the effects of the systematic parameter, without constraints from the other samples, can be seen. The effect of including only one sample in the analysis, $\nu_{e}$ appearance, is shown in Figure 7.19. Without the other samples present in the fit the fit spectra for $\nu_{e}$ appearance are allowed to float without constraints from the other samples and with only the constraints from the penalty terms present. The total $\Delta \chi^{2}$ is reduced from 26 to 6 in this case because the systematic parameters allow the fit spectra to float in a way that mimics the effects of $\delta_{C P}$ variations. This significant reduction in sensitivity emphasizes the need for constraining the systematic parameters via either other FD samples or by using ND samples.
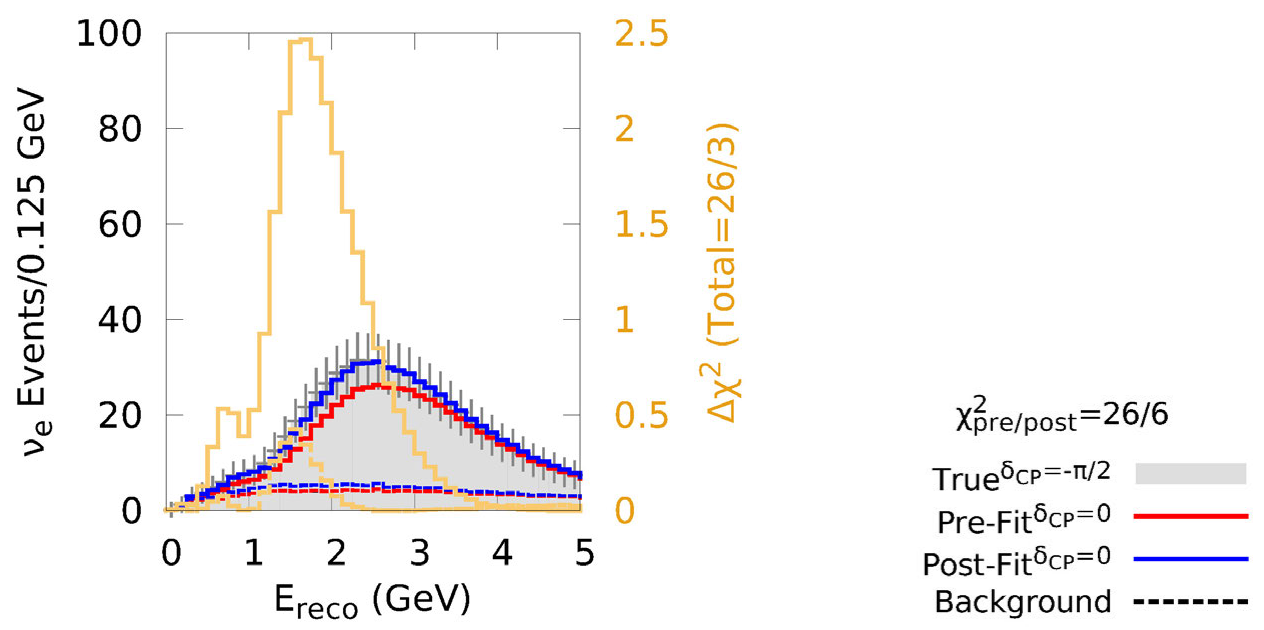

FiguRE 7.19. As in Figure 7.13 but for all 23 of the systematic parameters and including only the $\nu_{e}$ appearance sample. 
7.3.2. Sensitivities. Figures 7.20 through 7.25 evaluate the effects of systematic uncertainties on the $\mathrm{CP}$ violation and $\mathrm{MH}$ sensitivities as a function of the true value of $\delta_{C P}$ using the Fast MC systematic parameter response functions. The red curves include all four samples: $\nu_{e}$ appearance, $\bar{\nu}_{e}$ appearance, $\nu_{\mu}$ disappearance, and $\bar{\nu}_{\mu}$ disappearance. The blue curves include only the $\nu_{e}$ appearance sample with $50 \%$ neutrino running in order to show the effect of constraints coming from fitting multiple samples. The solid lines include no systematics while the dashed curves include the indicated systematic response function or set of response functions. The top set of figures include no oscillation parameter systematics while the bottom set profile the other oscillation parameters in order to minimize the $\Delta \chi^{2}$.

Figure 7.20 shows these sensitivities vs $\delta_{C P}$ with the $\mathrm{CC} M_{A}^{Q E}$ systematic parameter included. For the $\nu_{e}$ appearance only case (blue) there is a large reduction of the sensitivity when only the effects of the systematics are applied in Figures 7.20A-B. When all four samples are included (red) the effects of the systematic are constrained and there is very little reduction. In Figures 7.20C-D the other oscillation parameters are profiled so that the effects of the systematic parameter relative to the oscillation parameter systematics can be seen by comparison. For most of the $\delta_{C P}$ parameter space the CC $M_{A}^{Q E}$ systematic is only able to decrease the sensitivity further by a small amount. There is still a large reduction in the $\nu_{e}$ appearance only MH sensitivity near $\delta_{C P}=-\pi / 2$.

There is a larger reduction of each of the sensitivities for the CC $M_{A}^{R E S}$ systematic parameter seen in Figure 7.21. The CC $M_{A}^{R E S}$ parameter has a bigger effect at the energy of the first oscillation maximum in LBNE whereas the CC $M_{A}^{Q E}$ parameter varies the fit spectra closer to the second oscillation maximum. The dominant contribution to the sensitivity is from the first oscillation maximum and this explains the larger effect of this systematic parameter on the sensitivities. 
The combination of the $\mathrm{CC} M_{A}^{Q E}$ and $\mathrm{CC} M_{A}^{R E S}$ systematic parameters, in Figure 7.22, degrades the sensitivity even further in the $\nu_{e}$ appearance only (blue) case but the combined sample only decreases slightly.

When the effects of multiple systematics are included, as in Figures 7.23 through 7.25, the increased uncertainty in the fit spectrum degrades the sensitivity even further. For all 23 systematics and with the oscillation parameters profiled, in Figure 7.25 the best case sensitivity for CP violation is reduced from $5.5 \sigma$ to $4 \sigma$. This study does not yet include constraints on the systematic parameters that will come from measurements in a near detector and the studies will be used to help establish design criteria for a near detector.

The studies here regarding the response function systematics are preliminary and a more detailed study is underway. This work is being used in a comprehensive study by the LBNE collaboration of the effects of systematics on the sensitivities in LBNE. Efforts are underway to either validate or update the $1 \% / 5 \%$ signal/background uncertainties for $\nu_{e}$ appearance (5\%/10\% for $\nu_{\mu}$ disappearance) that were assumed throughout this thesis using these detailed systematic studies. 


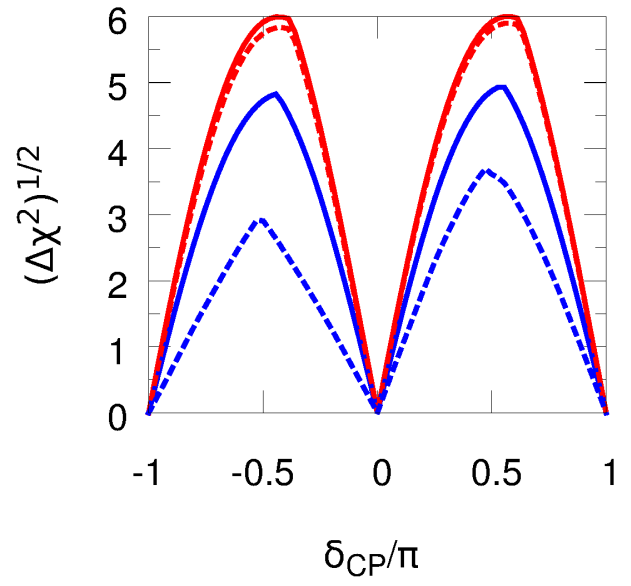

(A) CP Violation Sensitivity vs $\delta_{C P}$ True $\mathrm{MH}=\mathrm{NH}$

Osc. Systs. Fixed

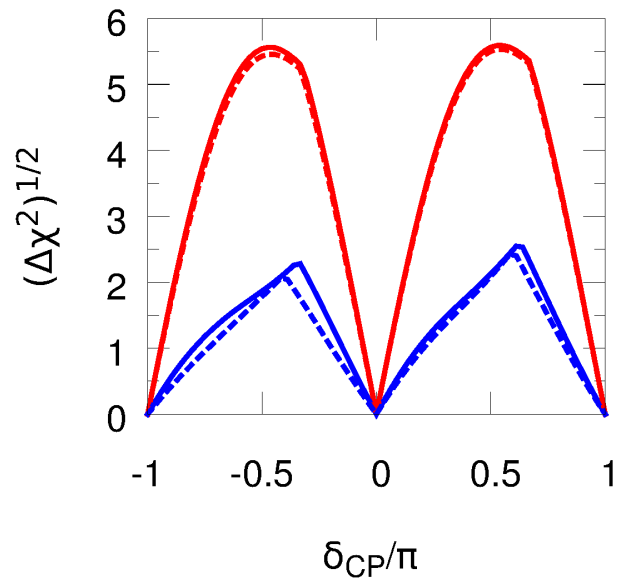

(C) CP Violation Sensitivity vs $\delta_{C P}$ True $\mathrm{MH}=\mathrm{NH}$

Osc. Systs. Profiled

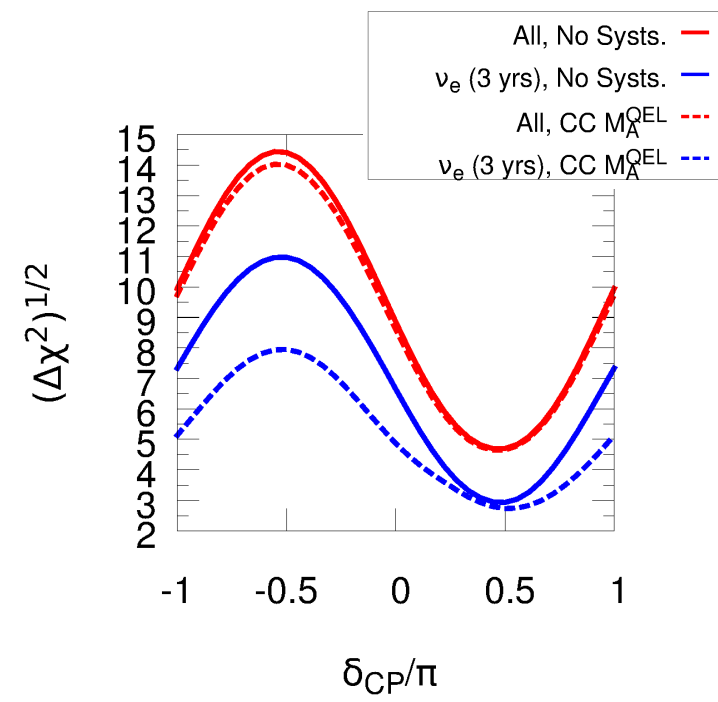

(B) $\mathrm{MH}$ Sensitivity vs $\delta_{C P}$ True $\mathrm{MH}=\mathrm{NH}$

Osc. Systs. Fixed

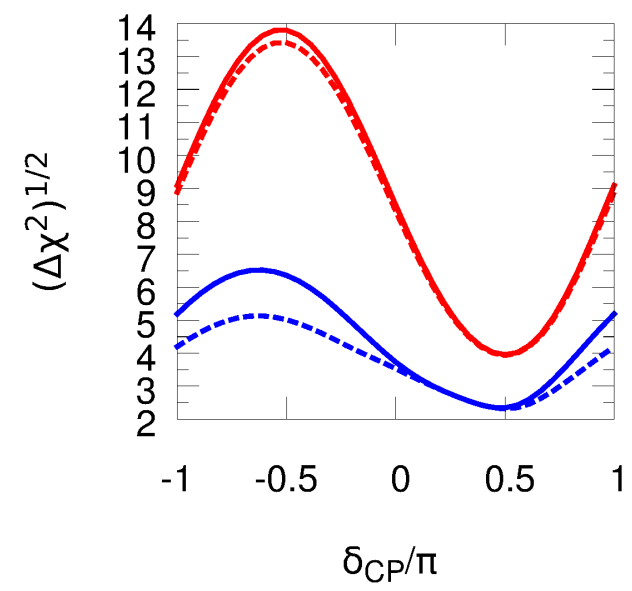

(D) $\mathrm{MH}$ Sensitivity vs $\delta_{C P}$ True $\mathrm{MH}=\mathrm{NH}$ Osc. Systs. Profiled

FiguRE 7.20. LBNE CP Violation and MH Sensitivity sensitivity vs $\delta_{C P}$ for no systematics (solid) with the CC $M_{A}^{Q E}$ response function systematic (dashed) in place. All four samples are fit in the "all" cases (red) while only the $\nu_{e}$ appearance sample is fit for the $\nu_{e}$ cases (blue). The Capozzi et al. best-fit values are assumed for the true values of the other oscillation parameters. The fit oscillation parameters are fixed in the top figures and are profiled in the bottom figures. 


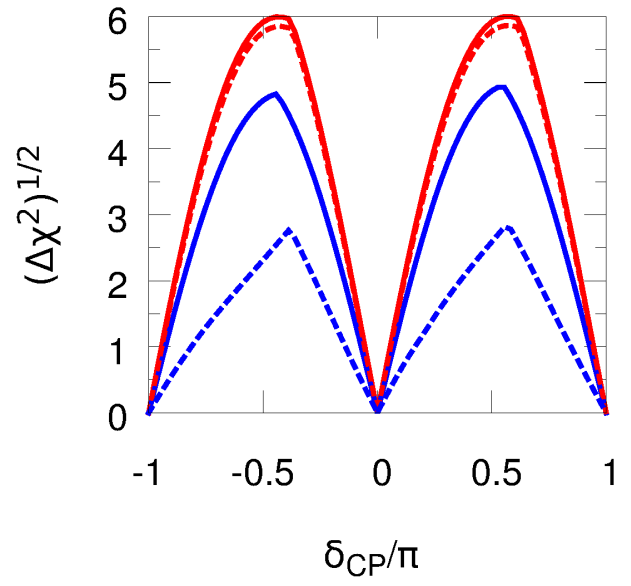

(A) CP Violation Sensitivity vs $\delta_{C P}$ True $\mathrm{MH}=\mathrm{NH}$

Osc. Systs. Fixed

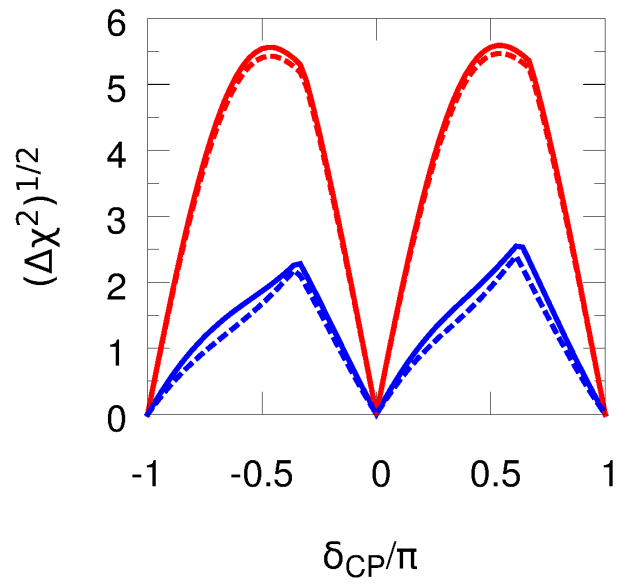

(C) CP Violation Sensitivity vs $\delta_{C P}$ True $\mathrm{MH}=\mathrm{NH}$

Osc. Systs. Profiled

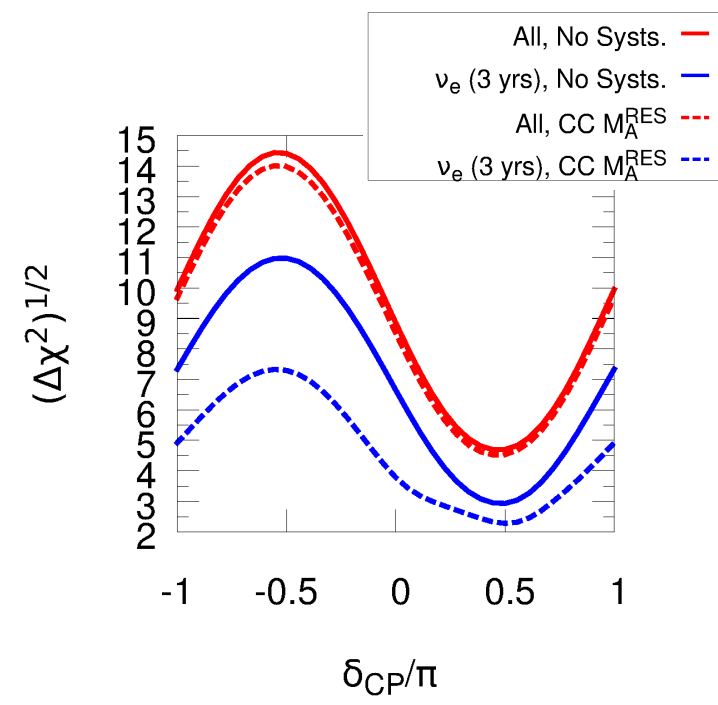

(B) MH Sensitivity vs $\delta_{C P}$ True $\mathrm{MH}=\mathrm{NH}$ Osc. Systs. Fixed

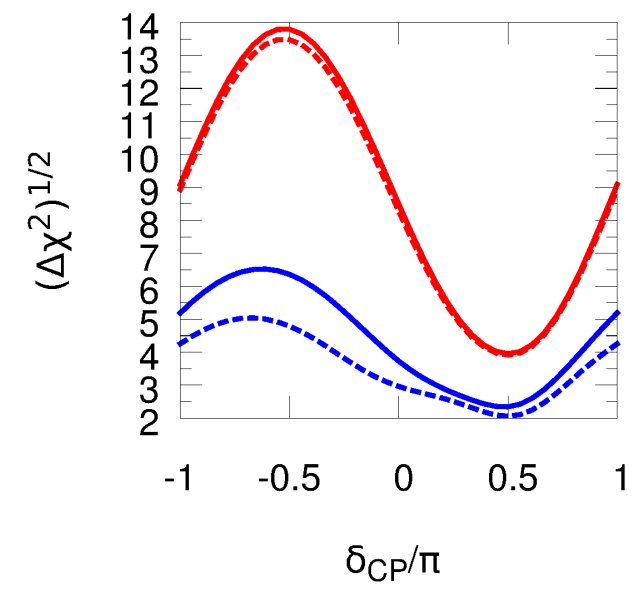

(D) $\mathrm{MH}$ Sensitivity vs $\delta_{C P}$ True $\mathrm{MH}=\mathrm{NH}$ Osc. Systs. Profiled

Figure 7.21. As in Figure 7.20 but for CC $M_{A}^{R E S}$. 


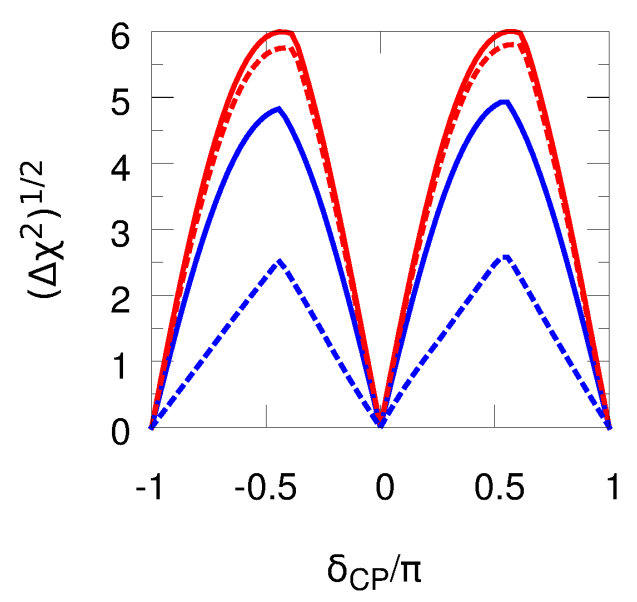

(A) CP Violation Sensitivity vs $\delta_{C P}$ True $\mathrm{MH}=\mathrm{NH}$

Osc. Systs. Fixed

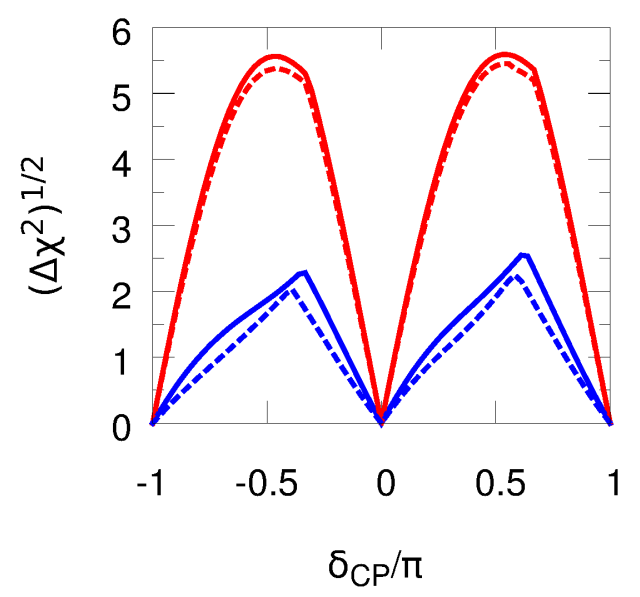

(C) CP Violation Sensitivity vs $\delta_{C P}$ True $\mathrm{MH}=\mathrm{NH}$ Osc. Systs. Profiled

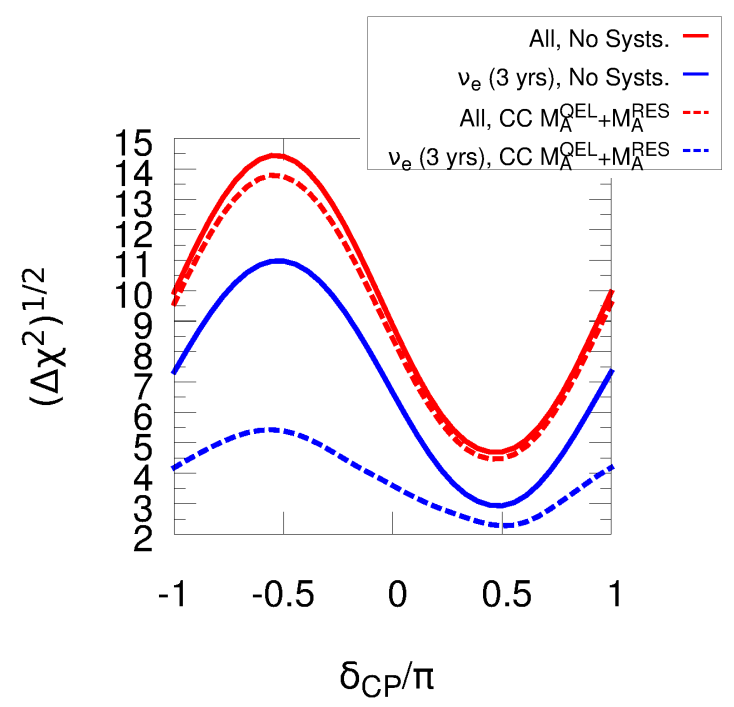

(B) MH Sensitivity vs $\delta_{C P}$ True $\mathrm{MH}=\mathrm{NH}$ Osc. Systs. Fixed

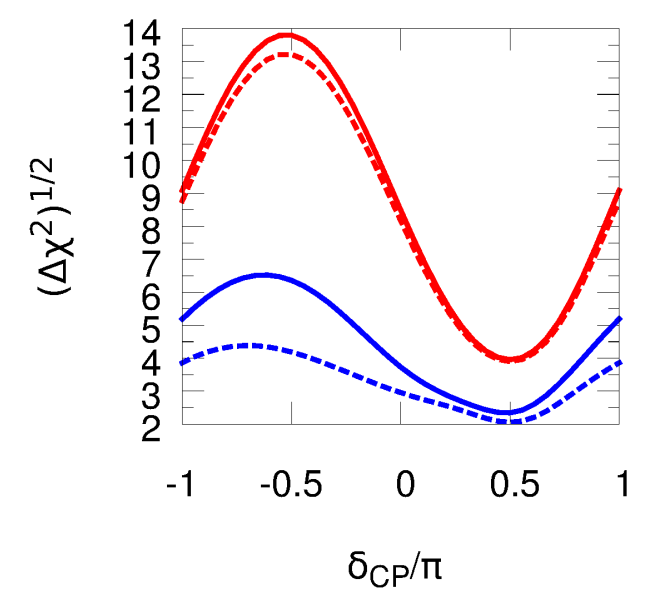

(D) MH Sensitivity vs $\delta_{C P}$ True $\mathrm{MH}=\mathrm{NH}$ Osc. Systs. Profiled

Figure 7.22. As in Figure 7.20 but for both CC $M_{A}^{R E S}$ and CC $M_{A}^{Q E L}$. 


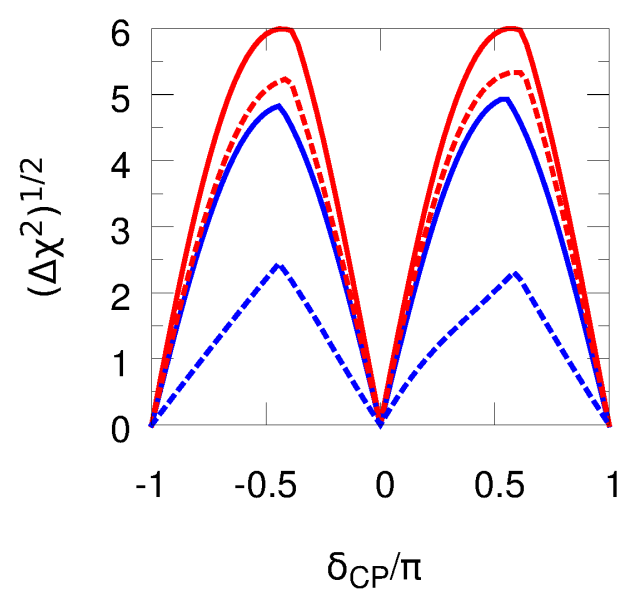

(A) CP Violation Sensitivity vs $\delta_{C P}$ True $\mathrm{MH}=\mathrm{NH}$

Osc. Systs. Fixed

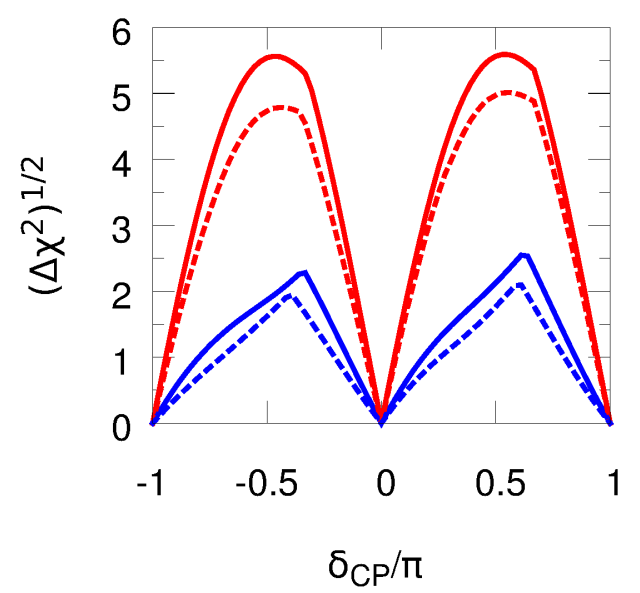

(C) CP Violation Sensitivity vs $\delta_{C P}$ True $\mathrm{MH}=\mathrm{NH}$

Osc. Systs. Profiled

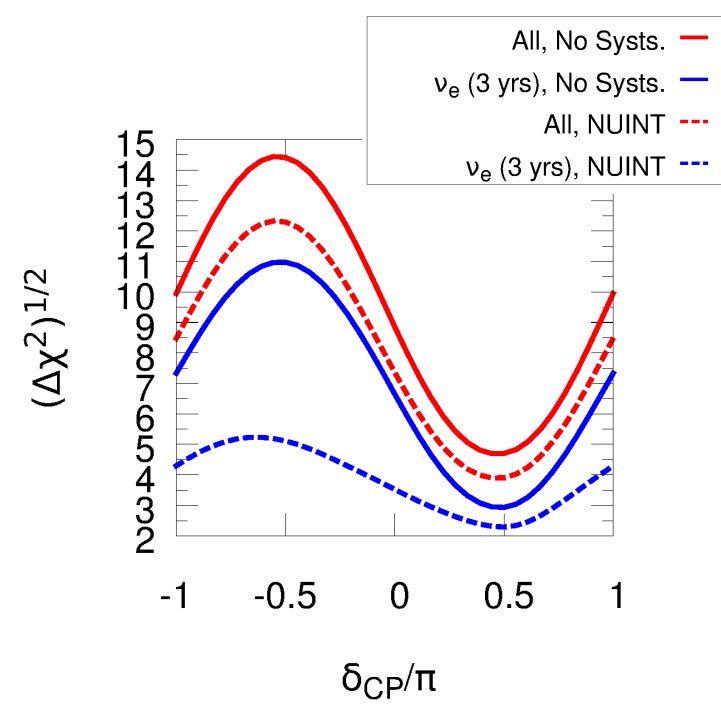

(B) MH Sensitivity vs $\delta_{C P}$ True $\mathrm{MH}=\mathrm{NH}$ Osc. Systs. Fixed

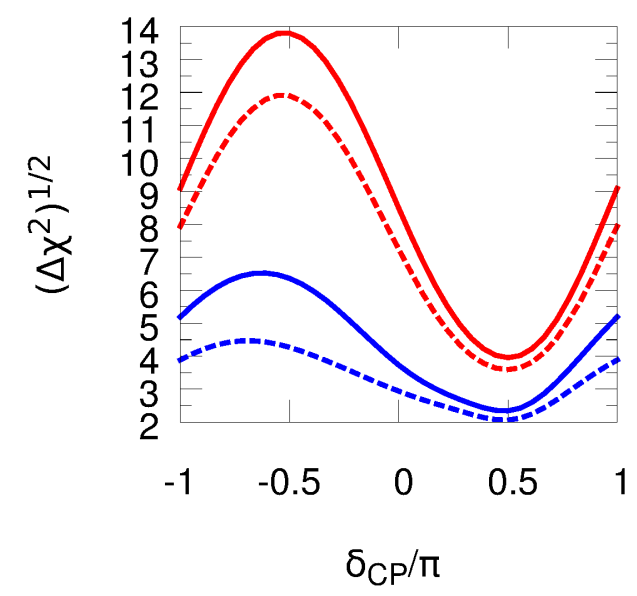

(D) MH Sensitivity vs $\delta_{C P}$ True $\mathrm{MH}=\mathrm{NH}$ Osc. Systs. Profiled

FiguRE 7.23. As in Figure 7.20 but for all 13 cross section systematic parameters. 


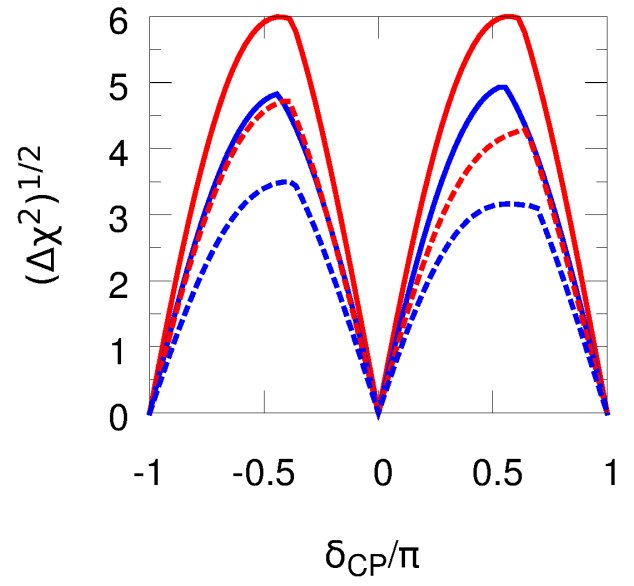

(A) CP Violation Sensitivity vs $\delta_{C P}$ True $\mathrm{MH}=\mathrm{NH}$

Osc. Systs. Fixed

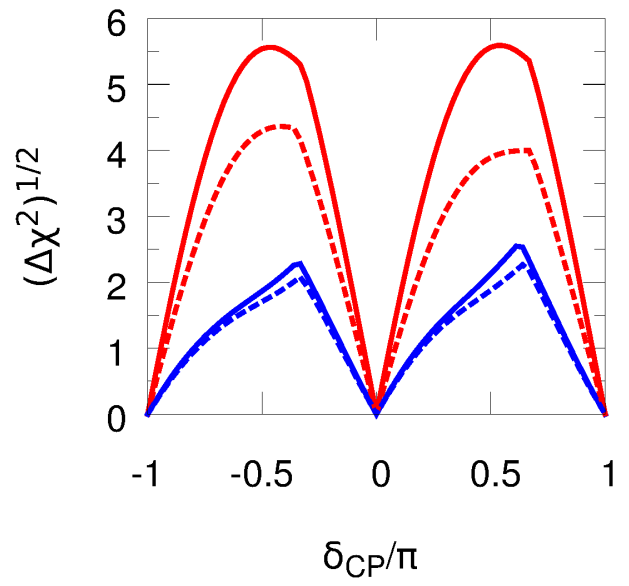

(C) CP Violation Sensitivity vs $\delta_{C P}$ True $\mathrm{MH}=\mathrm{NH}$ Osc. Systs. Profiled

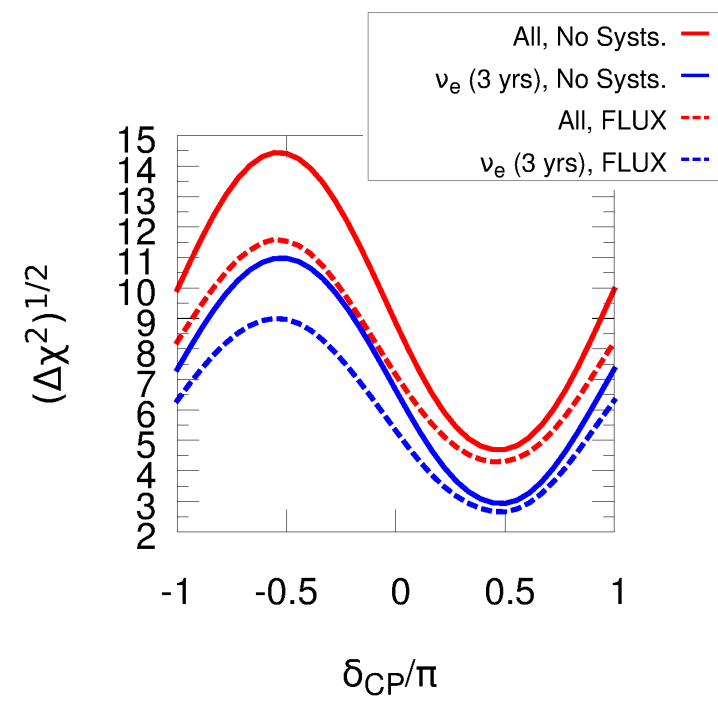

(B) $\mathrm{MH}$ Sensitivity vs $\delta_{C P}$ True $\mathrm{MH}=\mathrm{NH}$ Osc. Systs. Fixed

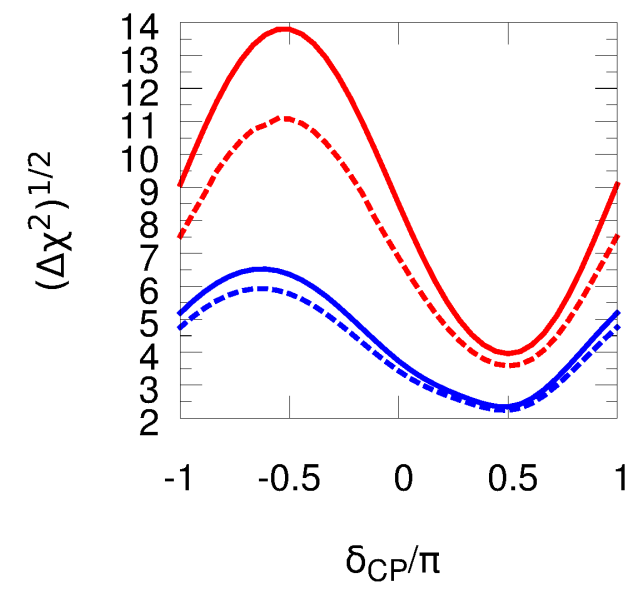

(D) MH Sensitivity vs $\delta_{C P}$ True $\mathrm{MH}=\mathrm{NH}$ Osc. Systs. Profiled

FIGURE 7.24. As in Figure 7.20 but for all 10 flux systematic parameters. 


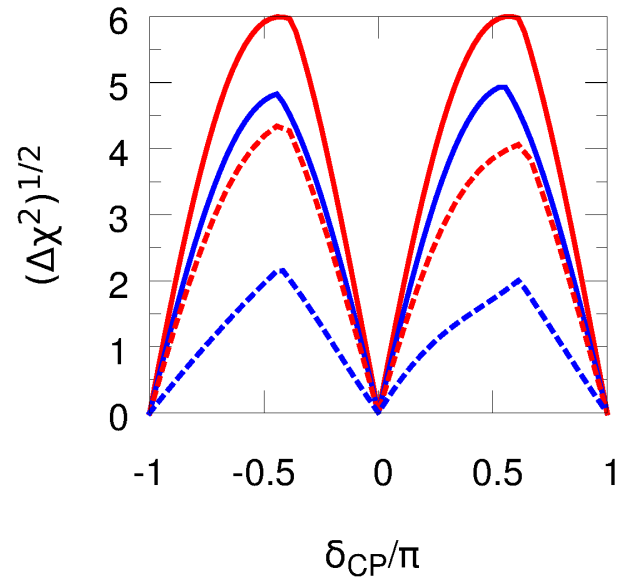

(A) CP Violation Sensitivity vs $\delta_{C P}$ True $\mathrm{MH}=\mathrm{NH}$

Osc. Systs. Fixed

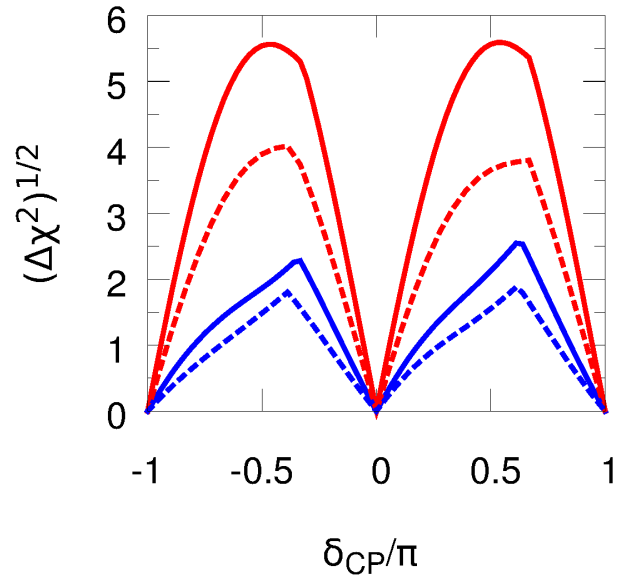

(C) CP Violation Sensitivity vs $\delta_{C P}$ True $\mathrm{MH}=\mathrm{NH}$ Osc. Systs. Profiled

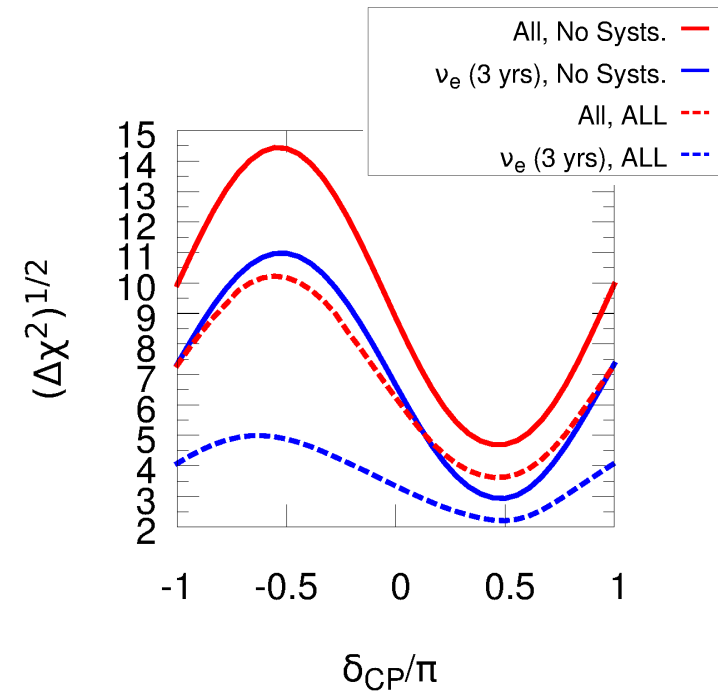

(B) MH Sensitivity vs $\delta_{C P}$ True $\mathrm{MH}=\mathrm{NH}$ Osc. Systs. Fixed

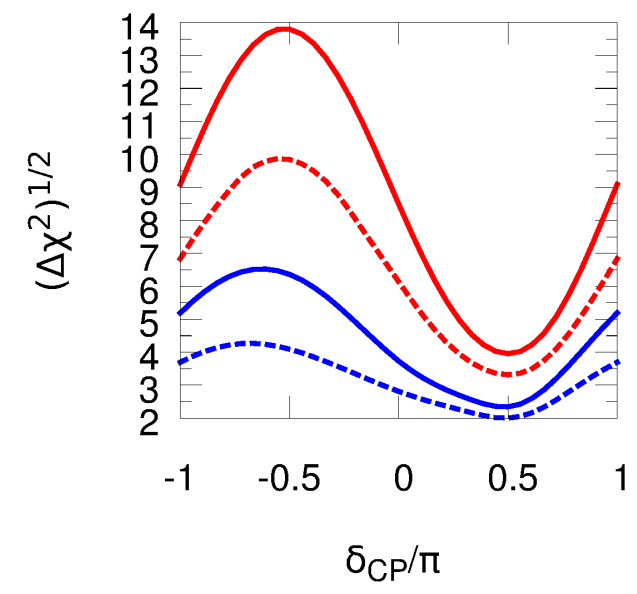

(D) $\mathrm{MH}$ Sensitivity vs $\delta_{C P}$ True $\mathrm{MH}=\mathrm{NH}$ Osc. Systs. Profiled

FIGURE 7.25. As in Figure 7.20 but for all 23 systematic parameters. 


\subsection{Conclusions \& Future Work}

This chapter has presented studies that show the shape and rate contributions to the sensitivities to the neutrino oscillation parameters. The dominant contribution in $\mathrm{T} 2 \mathrm{~K}$, $\mathrm{NO} \nu \mathrm{A}$, and $\mathrm{T} 2 \mathrm{HK}$ is from the rate while LBNE depends heavily on both the rate and the shape to achieve high sensitivity to the $\mathrm{MH}$ and to $\mathrm{CP}$ violation. Methods were developed to explore the effects of alternative systematics treatments in LBNE, in particular methods that include realistic degradation of the shape contributions to the sensitivity were explored.

These studies are part of an ongoing effort in the LBNE collaboration to understand the effects of systematic uncertainties on the projected oscillation sensitivities. The author of this thesis has developed the software tools used by the collaboration to study these effects. Future work in the LBNE Fast MC simulations will enable a complete study of the systematics that are expected to affect the oscillation sensitivities. The work will inform the design of the near and far detectors for the experiment as well as prioritize the systematic effects that must be constrained by the experiment in order to meet the stated experimental goals.

The most up to date work to include these systematic parameters from the LBNE Fast $\mathrm{MC}$ into the sensitivity estimates for $\mathrm{CP}$ violation and $\mathrm{MH}$ determination were shown in this chapter. For example, including all 23 of the parameters that are currently implemented reduces the $\mathrm{CP}$ violation sensitivity at the best case point $\left(\delta_{C P}=-\pi / 2\right.$ in the $\left.\mathrm{NH}\right)$ from $5.5 \sigma$ to $4 \sigma$. These dramatic reductions in the sensitivity do not necessarily reflect what is

expected for a complete LBNE simulation. These results represent the current state of the simulation considering the far detector only. Future work will see more accurate estimates of future sensitivity in LBNE by including near detector simulations and a more complete set of systematic parameters. 


\section{CHAPTER 8}

\section{Statistical Fluctuations of Event SpeCtra}

The method used in this thesis for computing a $\Delta \chi^{2}$ comparing a null hypothesis, H0, to a test hypothesis, H1, was outlined in Chapter 5. This chapter will study the implications of

looking beyond the Asimov data set where the median $\Delta \chi^{2}\left(\overline{\Delta \chi^{2}}\right)$ value is computed from the most likely values of the sample spectra and no statistical fluctuations are included. The Asimov data set approach to computing $\overline{\Delta \chi^{2}}$ is suitable for evaluating the power of the average experiment to reject hypotheses but leaves out the range of possible experiments that could be expected.

To study the effects of statistical fluctuations on the distribution of $\Delta \chi^{2}$ values the GLoBES library was modified by the author of this thesis to allow for repeatedly computing the $\Delta \chi^{2}$ with statistical fluctuations included. Before the $\Delta \chi^{2}$ is computed, the true event spectrum is fluctuated, bin-by-bin, by pulling from the Poisson distribution with median value equal to that of the median value from the bin. This fluctuation is done on each of the four samples, $\nu_{e}$ appearance, $\bar{\nu}_{e}$ appearance, $\nu_{\mu}$ disappearance, and $\bar{\nu}_{\mu}$ disappearance, and then the $\Delta \chi^{2}$ value is computed as before using the newly fluctuated true event spectrum, which serves as a mock data spectrum. This process is repeated thousands of times to generate a distribution of $\Delta \chi^{2}$ values.

This statistical approach, referred to as a toy Monte Carlo (MC), will be used to study the $\Delta \chi^{2}$ distributions for determining the $\mathrm{MH}$ and detecting $\mathrm{CP}$ violation.

\subsection{Mass Hierarchy \& Statistical Fluctuations}

The test for MH sensitivity is different from the tests for the other sensitivities in that it compares two discrete possibilities: the normal and inverted mass hierarchies. A test 
statistic $\mathrm{T}$ is defined as:

$$
T \equiv \Delta \chi^{2}=\chi_{I H}^{2}-\chi_{N H}^{2}
$$

Where the definition $T \equiv \Delta \chi^{2}$ is used to emphasize the fact that the null hypothesis for this test statistic does not necessarily follow a $\chi^{2}$ distribution. Note that in the case of the Asimov data set and for the True $\mathrm{MH}=\mathrm{NH}$ (True $\mathrm{MH}=\mathrm{IH})$ then $\chi_{N H}^{2}=0\left(\chi_{I H}^{2}=0\right)$ as pointed out in Chapter 5. Figure 8.1 shows the distributions of $T$ for $\mathrm{T} 2 \mathrm{~K}+\mathrm{NO} \nu \mathrm{A}$. The red histograms of Figures 8.1A, C, E represent the distribution of T values assuming True $\mathrm{MH}=\mathrm{NH}$ and $\delta_{C P}$ at the indicated value, while the blue histogram assumes True $\mathrm{MH}=\mathrm{IH}$ but integrates over $\delta_{C P}$. The blue histograms of Figures 8.1B, D, F represent the distribution of $\mathrm{T}$ values assuming True $\mathrm{MH}=\mathrm{IH}$ and $\delta_{C P}$ at the indicated value, while the blue histogram assumes True $\mathrm{MH}=\mathrm{IH}$ but integrates over $\delta_{C P}$. A value of $T$ less than zero indicates that the IH would be favored by the measurement and a $T$ value greater than zero favors the NH. There are significant portions of the distributions that fall below(above) zero in the True $\mathrm{MH}=\mathrm{NH}$ (True $\mathrm{MH}=\mathrm{IH})$ case. These figures also indicate the value of $T$ computed from the Asimov data set $\left(T_{\overline{\Delta \chi^{2}}}\right)$ and from the median of the toy MC $\left(T_{M C}\right)$ as vertical lines. These vertical lines are close together or overlapping in almost every case which validates the assumption that the Asimov data set accurately reproduces the median of the toy MC distribution of $T$ values with some deviations where the degeneracies between $\delta_{C P}$ and the MH are significant.

The results of these toy $\mathrm{MC} \mathrm{T}$ distributions are used to compute the probability distribution function (PDF) for the null hypothesis and the test hypothesis. These two PDFs are then used to compute the p-value for which the null hypothesis is correctly rejected for all toy MC experiments. The probability to reject $\mathrm{H} 0$ when it is false, or Type II error|45], is 


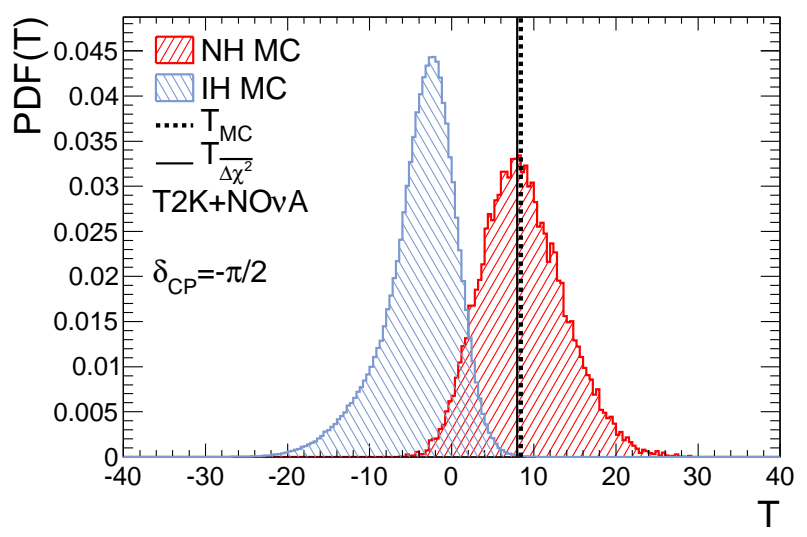

(A) $\delta_{C P}=-\pi / 2$, True $\mathrm{MH}=\mathrm{NH}$

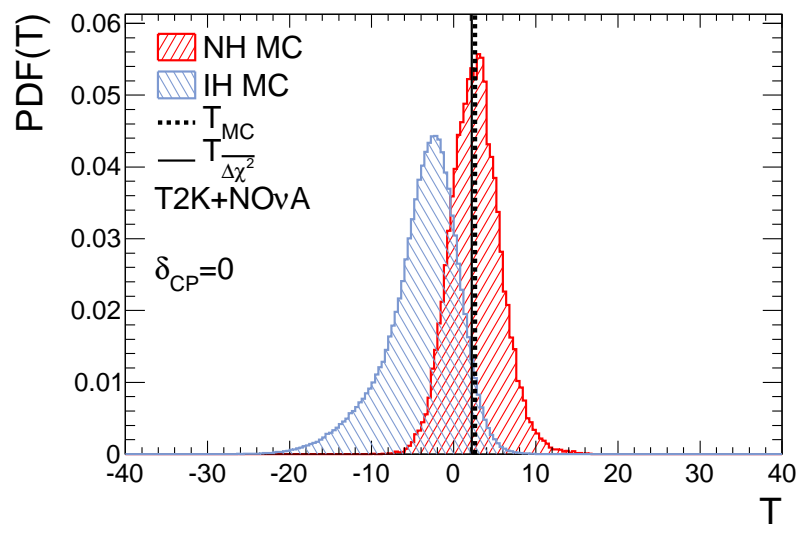

(c) $\delta_{C P}=0$, True $\mathrm{MH}=\mathrm{NH}$

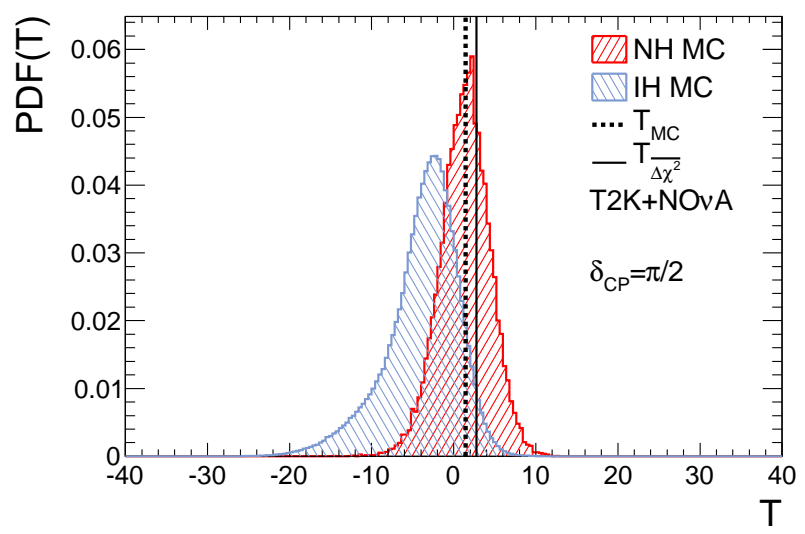

(E) $\delta_{C P}=\pi / 2$, True $\mathrm{MH}=\mathrm{NH}$

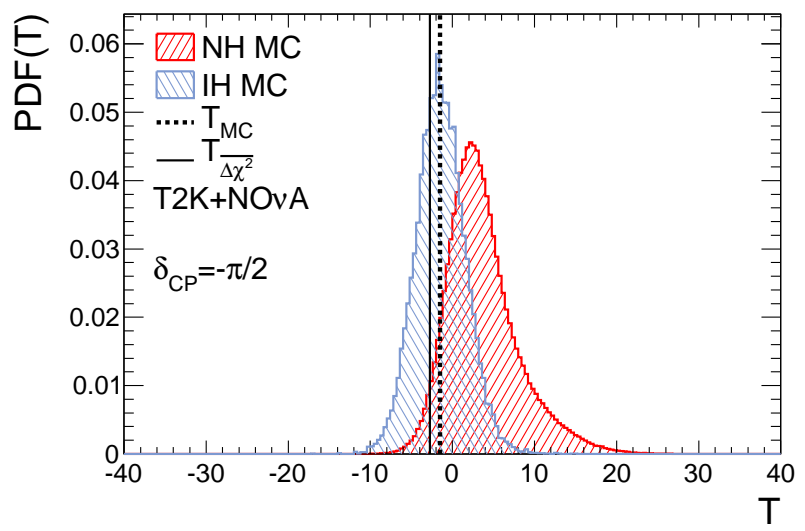

(B) $\delta_{C P}=-\pi / 2^{\circ}$, True $\mathrm{MH}=\mathrm{IH}$

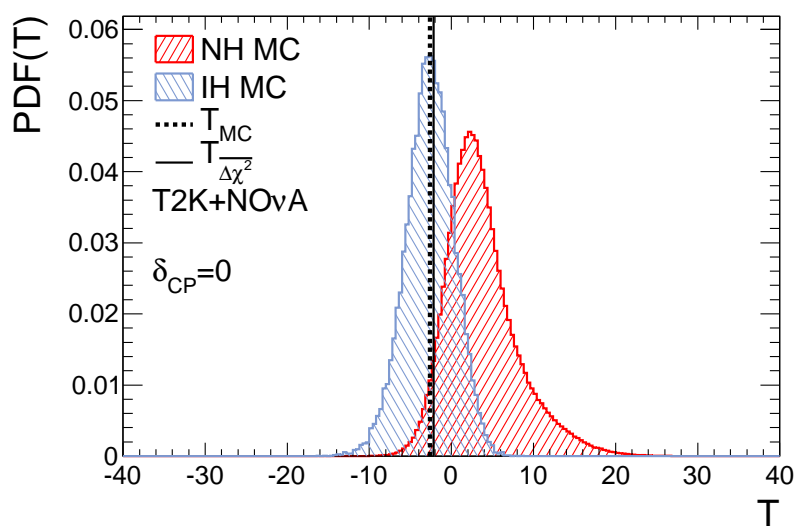

(D) $\delta_{C P}=0$, True $\mathrm{MH}=\mathrm{IH}$

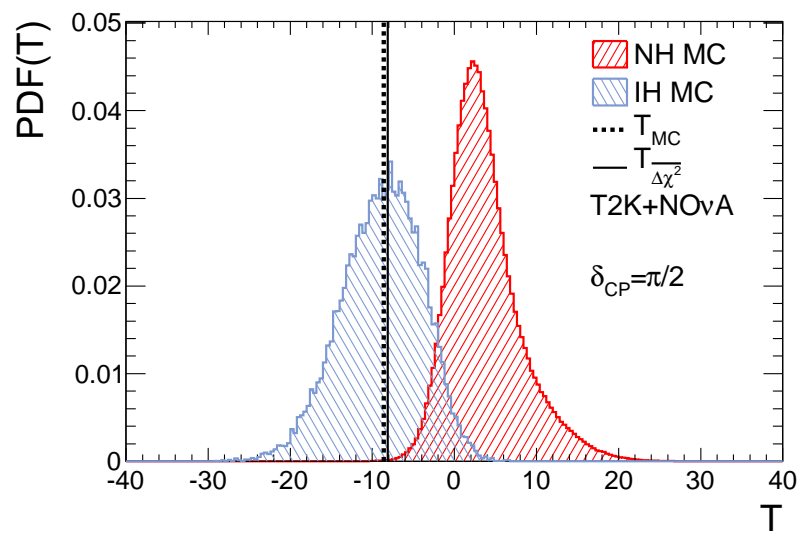

(F) $\delta_{C P}=\pi / 2$, True $\mathrm{MH}=\mathrm{IH}$

FIgURE 8.1. Distributions of the test statistic, $T$, for a combined $\mathrm{T} 2 \mathrm{~K}+\mathrm{NO} \nu \mathrm{A}$ fit for $\delta_{C P}=-\pi / 2,0$, and $\pi / 2$ for both the True $\mathrm{MH}=\mathrm{NH}$ and True $\mathrm{MH}=\mathrm{IH}$ cases. The red(blue) histogram is for the $\mathrm{NH}(\mathrm{IH})$ distribution. The vertical lines represent the median of the toy MC $T$ distribution (dashed) and the $T$ value computed from the Asimov data set (solid). The nominal assumptions are assumed for systematics and $\nu: \bar{\nu}$ ratios. The fit oscillation parameters are profiled. 
referred to as $\beta$ and is directly related to the $\mathrm{p}$-value. The $\mathrm{p}$-value, or $1-\beta$, is computed using

$$
p=1-\beta=\int_{-\infty}^{T_{\text {median }}} \operatorname{PDF}(T \mid I H) d T
$$

for the True $\mathrm{MH}=\mathrm{NH}$ cases and

$$
p=1-\beta=\int_{T_{\text {median }}}^{\infty} \operatorname{PDF}(T \mid N H) d T
$$

for the True $\mathrm{MH}=\mathrm{IH}$ cases.

Table 8.1 lists the $T$-values and $p$-values for the True $\mathrm{MH}=\mathrm{NH}$ and True $\mathrm{MH}=\mathrm{IH}$ cases using $T_{\text {median }}=T_{M C}$ or $T_{\text {median }}=T_{\overline{\Delta \chi^{2}}}$ for three values of $\delta_{C P}$ in $\mathrm{T} 2 \mathrm{~K}+\mathrm{NO} \nu \mathrm{A}$. Two values are given for the p-value. The first is computed from the distribution of $T$ values for H0 using the integrals in Equations 44.45 using either the $T_{\overline{\Delta \chi^{2}}}$ or $T_{M C}$ value for the appropriate limit of the integration. The second is computed by integrating the $\chi^{2}$ distribution for $1 \mathrm{DOF}$ above the same limit. Comparing these two approaches to computing the p-value quantifies how the assumption that the H0 hypothesis is $\chi^{2}$ distributed and that Wilk's theorem applies skews the interpretation of the metric.

The $T_{M C}$ values are similar to the $T_{\overline{\Delta \chi^{2}}}$ values, but there are differences particularly for the cases where the degeneracies between $\delta_{C P}$ and the MH are significant at $\delta_{C P}=+(-) \pi / 2$ in the $\mathrm{NH}(\mathrm{IH})$. In these degenerate cases the statistical fluctuations tend to smooth out any abrupt transitions in the fit values of the oscillation parameters that may cause the $\overline{\Delta \chi^{2}}$ to over/under estimate the central value of the $\Delta \chi^{2}$ distribution. Another example of this effect is discussed in the next section. Because of this smoothing effect the test statistic computed using the Asimov data set, $T_{\overline{\Delta \chi^{2}}}$, overestimates the median of the $\Delta \chi^{2}$ distribution in the 
degenerate cases, i.e. it predicts a more significant measurement than is likely. In the other cases the Asimov data set is slightly conservative.

The p-values in columns 3, 6, and 9 of Table 8.1 give the fraction of experiments for which the IH will be measured when it is false if the criteria for accepting the hierarchy measurement is that the $\Delta \chi^{2}$ is greater(lesser) than the median expected value for the True $\mathrm{MH}=\mathrm{NH}$ (True $\mathrm{MH}=\mathrm{IH}$ ) case. If $\delta_{C P}=-\pi / 2$ and the $\mathrm{MH}$ is normal then there is a $0.03 \%$ probability that the wrong mass hierarchy determination will be made. This increases to $7 \%$ in the $\delta_{C P}=+\pi / 2$ case. If the $\chi^{2}$ distribution is used to interpret the test statistic then a more conservative estimate of the p-value will be made.

TABLE 8.1. Median MC and $\overline{\Delta \chi^{2}}$ test statistic values and $\mathrm{p}$-values for $\delta_{C P}=$ $-\pi / 2,0$, and $\pi / 2$ corresponding to the distributions shown in Figure 8.1 for a $\mathrm{T} 2 \mathrm{~K}+\mathrm{NO} \nu \mathrm{A}$ combined fit. The p-values are computed with the $\mathrm{H} 0$ and $\mathrm{H} 1$ distributions computed with 20,000 toy MC experiments. The values are given for both True $\mathrm{MH}=\mathrm{NH}$ and True $\mathrm{MH}=\mathrm{IH}$ cases.

\begin{tabular}{l|ccc|ccc|ccc} 
& \multicolumn{3}{|c|}{$\delta_{C P}=-\pi / 2$} & \multicolumn{3}{c|}{$\delta_{C P}=0$} & \multicolumn{3}{c}{$\delta_{C P}=\pi / 2$} \\
& \multicolumn{3}{c|}{$\mathrm{p}(\mathrm{T})$} & $\mathrm{p}(\mathrm{T})$ & \multicolumn{3}{c}{$\mathrm{p}(\mathrm{T})$} & $\mathrm{p}(\mathrm{T})$ & \multicolumn{3}{c}{$\mathrm{p}(\mathrm{T})$} & $\mathrm{p}(\mathrm{T})$ \\
& $\mathrm{T}$ & Toy MC & $\chi^{2}$ & $\mathrm{~T}$ & Toy MC & $\chi^{2}$ & $\mathrm{~T}$ & Toy MC & $\chi^{2}$ \\
\hline NH $T_{M C}$ & 8.39 & 0.000348 & 0.00377 & 2.60 & 0.0330 & 0.107 & 1.44 & 0.0731 & 0.229 \\
NH $T_{\overline{\Delta \chi^{2}}}$ & 7.97 & 0.000485 & 0.00475 & 2.20 & 0.0436 & 0.138 & 2.75 & 0.0330 & 0.0973 \\
IH $T_{M C}$ & -1.46 & 0.0741 & 0.13 & -3.79 & 0.0334 & 0.107 & -8.94 & 0.000201 & 0.00332 \\
IH $T_{\overline{\Delta \chi^{2}}}$ & -2.74 & 0.0334 & 0.068 & -3.19 & 0.0440 & 0.142 & -8.11 & 0.000355 & 0.00441
\end{tabular}

Figure 8.2 shows the distributions as a function of the true $\delta_{C P}$ value. The median of the distribution is given by the solid red line while the $T_{\overline{\Delta \chi^{2}}}$ based on the Asimov data set is given by the dashed black line. The differences between the two methods is very obvious in this figure with the $T_{\text {Asimov }}$ (equivalent to $T_{\overline{\Delta \chi^{2}}}$ ) curve falling below the $T_{\text {Median }}$ (equivalent to $T_{M C}$ ) for all values of $\delta_{C P}$ except around the degeneracies. The $1 \sigma$ and $2 \sigma$ regions both fall below zero indicating that there are significant portions of phase space where the data might indicate an(a) $\mathrm{IH}(\mathrm{NH})$ spectrum when true mass hierarchy is $\mathrm{NH}(\mathrm{IH})$. 


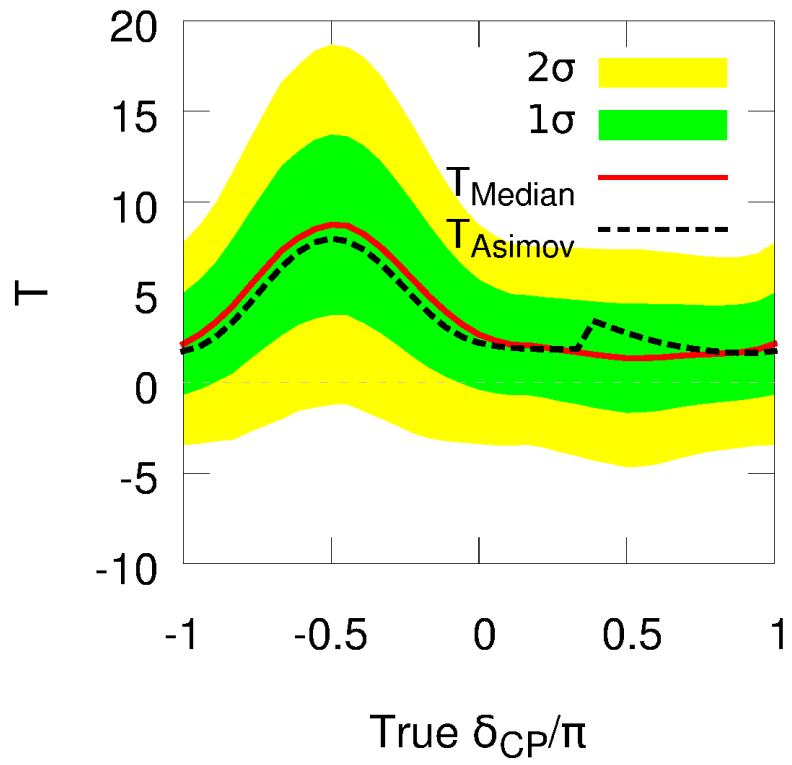

(A) True $\mathrm{MH}=\mathrm{NH}$

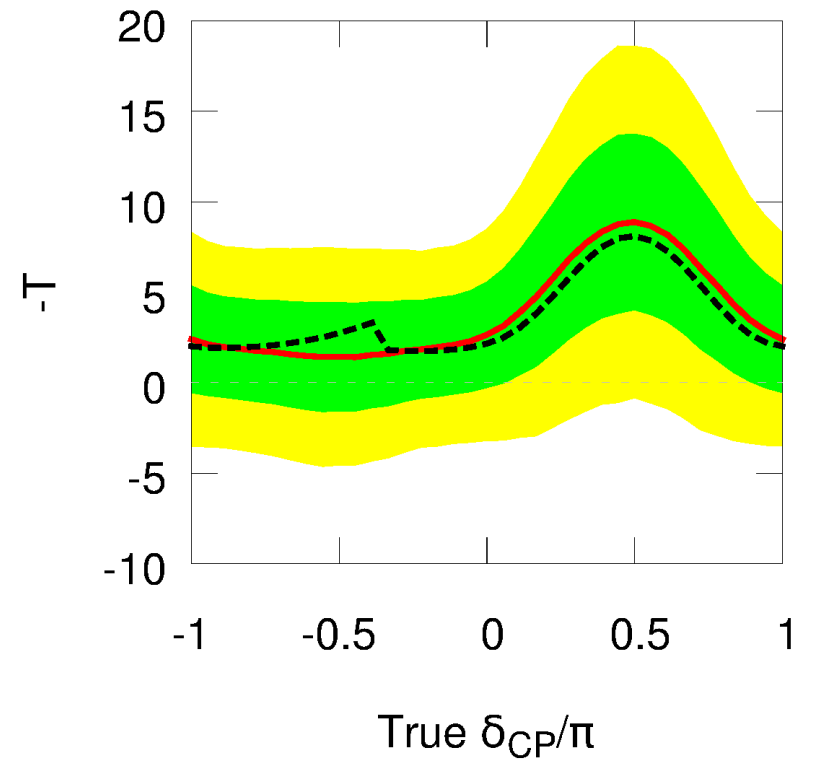

(B) True $\mathrm{MH}=\mathrm{IH}$

Figure 8.2. Distributions of the test statistic $\mathrm{T}$ vs $\delta_{C P}$ for $\mathrm{T} 2 \mathrm{~K}+\mathrm{NO} \nu \mathrm{A}$ in the True $\mathrm{MH}=\mathrm{NH}$ and True $\mathrm{MH}=\mathrm{IH}$ cases. The red line represents the median value computed from the toy $\mathrm{MC}$ while the green and yellow bands represent the widths of the distributions containing $68 \%$ and $95 \%$ of the toy $\mathrm{MC} \mathrm{T}$ values respectively. The $T_{\text {Asimov }}$ (black-dashed) line gives the values computed using the Asimov data set. The nominal assumptions are assumed for systematics and $\nu: \bar{\nu}$ ratios. The fit oscillation parameters are profiled.

8.1.1. MH StATiSTICS IN LBNE. Figure 8.3 shows the corresponding $\Delta \chi^{2}$ distributions for MH determination in LBNE. The H0 distribution has a very different shape, when compared with $\mathrm{T} 2 \mathrm{~K}+\mathrm{NO} \nu \mathrm{A}$, because the values of $\Delta \chi^{2}$ are widely distributed for LBNE, as the true value of $\delta_{C P}$ is varied, because of a high sensitivity to $\delta_{C P}$ and the $\mathrm{MH}$ in LBNE. In 8.3A, for example, the left end of the $\mathrm{H} 0$ distribution (blue) corresponds to $\delta_{C P}=\pi / 2$ while the right end corresponds to $\delta_{C P}=-\pi / 2$. More generally, the $\mathrm{H} 1$ and $\mathrm{H} 0$ distributions are widely separated in every case as compared with $\mathrm{T} 2 \mathrm{~K}+\mathrm{NO} \nu \mathrm{A}$.

In Table 8.2 and in Figure 8.4 the sensitivity test statistic $\mathrm{T}$ is seen to be effectively equivalent between the two approaches. The p-value computed from the Toy MC is below 
TABLE 8.2. Median MC and $\overline{\Delta \chi^{2}}$ test statistic values and $\mathrm{p}$-values for $\delta_{C P}=$ $-\pi / 2,0$, and $\pi / 2$ corresponding to the distributions shown in Figure 8.3 for LBNE. The p-values are computed with the $\mathrm{H} 0$ and $\mathrm{H} 1$ distributions computed with 20,000 toy MC experiments. The values are given for both True $\mathrm{MH}=\mathrm{NH}$ and True $\mathrm{MH}=\mathrm{IH}$ cases.

\begin{tabular}{l|ccc|ccc|ccc} 
& \multicolumn{3}{|c|}{$\delta_{C P}=-\pi / 2$} & \multicolumn{3}{c}{$\delta_{C P}=0$} & \multicolumn{3}{c}{$\delta_{C P}=\pi / 2$} \\
& \multicolumn{3}{c|}{$\mathrm{p}(\mathrm{T})$} & $\mathrm{p}(\mathrm{T})$ & \multicolumn{3}{c}{$\mathrm{p}(\mathrm{T})$} & $\mathrm{p}(\mathrm{T})$ & \multicolumn{3}{c}{$\mathrm{p}(\mathrm{T})$} & $\mathrm{p}(\mathrm{T})$ \\
& $\mathrm{T}$ & Toy MC & $\chi^{2}$ & $\mathrm{~T}$ & Toy MC & $\chi^{2}$ & $\mathrm{~T}$ & Toy MC & $\chi^{2}$ \\
\hline NH $T_{\overline{\Delta \chi^{2}}}$ & 193 & $<0.00005$ & $\sim 0$ & 79.7 & $<0.00005$ & $\sim 0$ & 21.0 & $<0.00005$ & $4.67 \mathrm{e}-06$ \\
NH $T_{M C}$ & 193 & $<0.00005$ & $\sim 0$ & 79.6 & $<0.00005$ & $\sim 0$ & 20.4 & $<0.00005$ & $6.25 \mathrm{e}-06$ \\
IH $T_{\overline{\Delta \chi^{2}}}$ & -28.4 & $<0.00005$ & $1.15 \mathrm{e}-07$ & -112 & $<0.00005$ & $\sim 0$ & -220 & $<0.00005$ & $\sim 0$ \\
IH $T_{M C}$ & -27.7 & $<0.00005$ & $1.43 \mathrm{e}-07$ & -111 & $<0.00005$ & $\sim 0$ & -219 & $<0.00005$ & $\sim 0$
\end{tabular}

the statistical limits of the toy MC. Because the number of toy MC experiments is 20,000 the lowest probability accessible to the MC is $1 / 20000=0.00005$. 


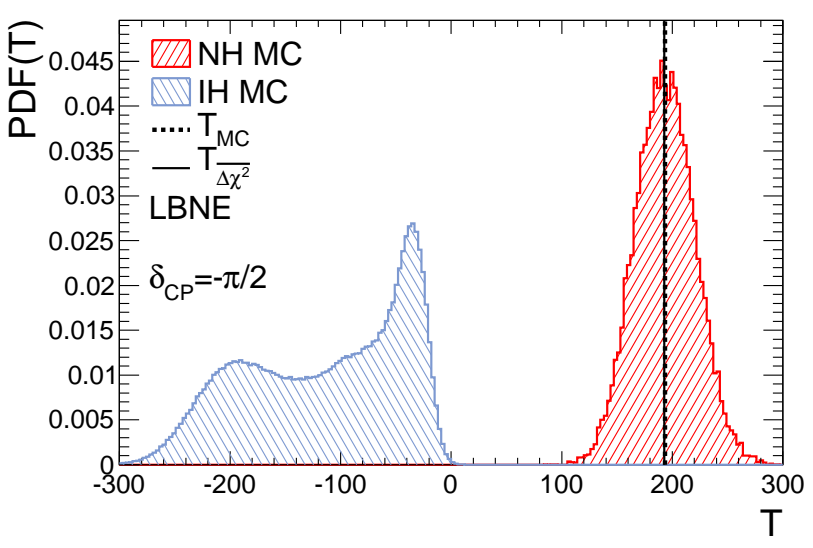

(A) $\delta_{C P}=-\pi / 2$, True $\mathrm{MH}=\mathrm{NH}$

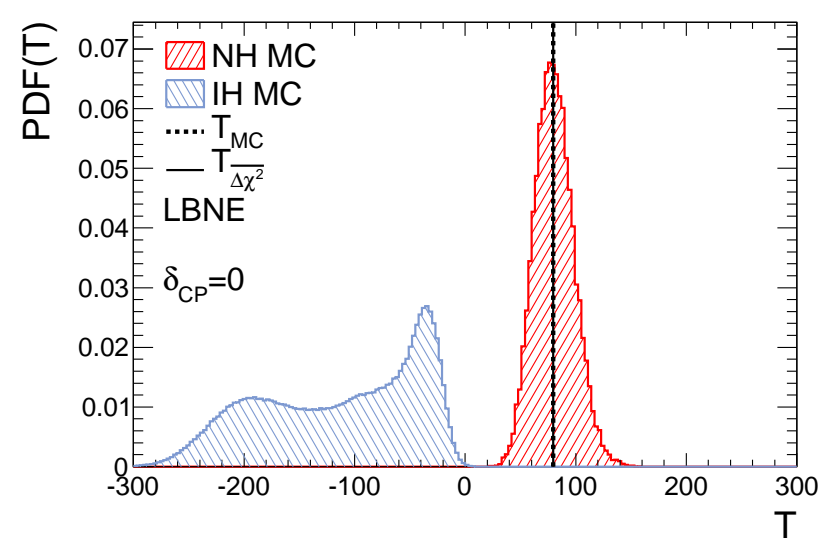

(C) $\delta_{C P}=0$, True $\mathrm{MH}=\mathrm{NH}$

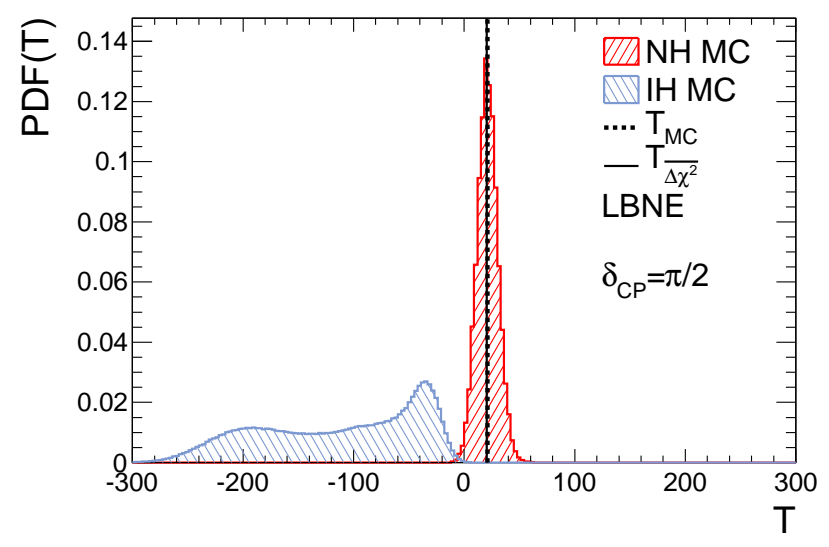

(E) $\delta_{C P}=\pi / 2$, True $\mathrm{MH}=\mathrm{NH}$

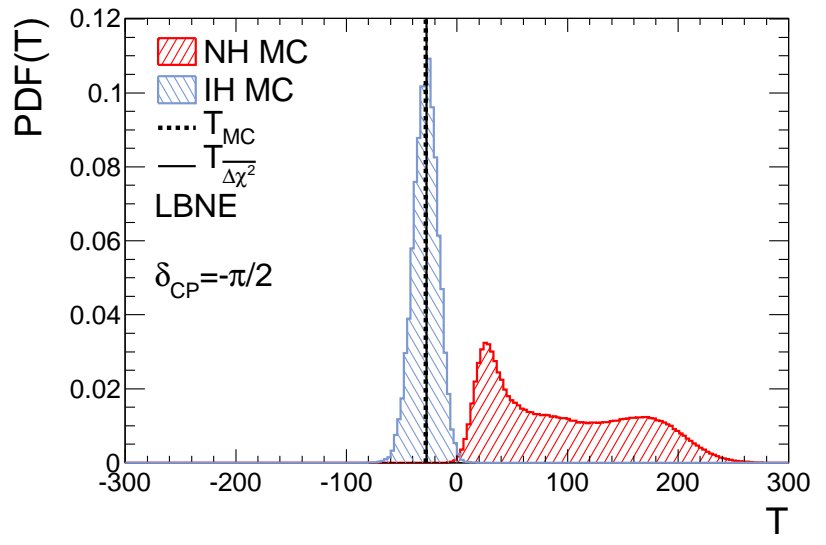

(B) $\delta_{C P}=-\pi / 2^{\circ}$, True $\mathrm{MH}=\mathrm{IH}$

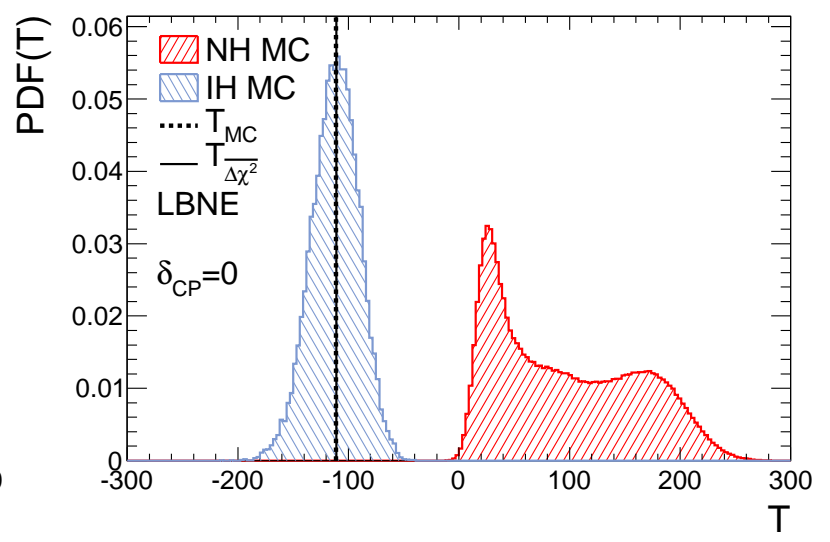

(D) $\delta_{C P}=0$, True $\mathrm{MH}=\mathrm{IH}$

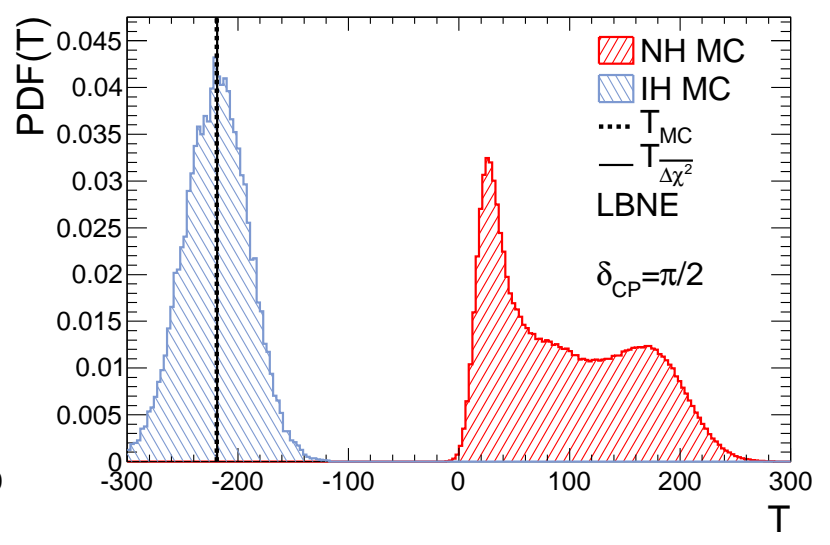

(F) $\delta_{C P}=\pi / 2$, True $\mathrm{MH}=\mathrm{IH}$

Figure 8.3. Distributions of the test statistic, $T$, for LBNE for $\delta_{C P}=-\pi / 2$, 0 , and $\pi / 2$ for both the True $\mathrm{MH}=\mathrm{NH}$ and True $\mathrm{MH}=\mathrm{IH}$ cases. The red(blue) histogram is for the $\mathrm{NH}(\mathrm{IH})$ distribution. The vertical lines represent the median of the toy MC $T$ distribution (dashed) and the $T$ value computed from the Asimov data set (solid). The nominal assumptions are assumed for systematics and $\nu: \bar{\nu}$ ratios. The fit oscillation parameters are profiled. 

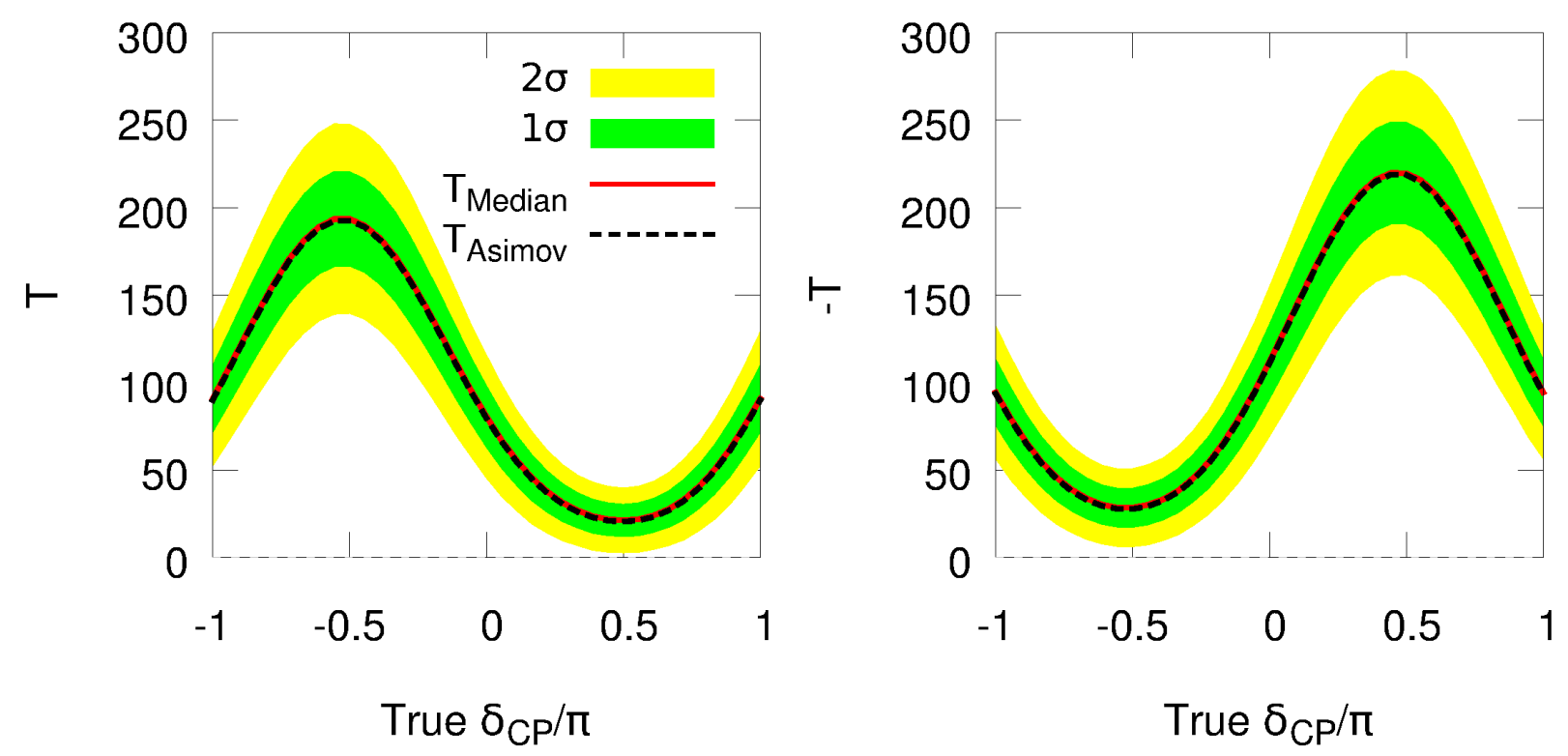

(A) True $\mathrm{MH}=\mathrm{NH}$

(B) True $\mathrm{MH}=\mathrm{IH}$

Figure 8.4. Distributions of the test statistic $\mathrm{T}$ vs $\delta_{C P}$ for LBNE for the True $\mathrm{MH}=\mathrm{NH}$ and True $\mathrm{MH}=\mathrm{IH}$ cases. The red line represents median value computed from the toy $\mathrm{MC}$ while the green and yellow bands represent the widths of the distributions containing $68 \%$ and $95 \%$ of the toy $\mathrm{MC} \mathrm{T}$ values respectively. The $T_{\text {Asimov }}$ (black-dashed) line gives the values computed using the Asimov data set. The nominal assumptions are assumed for systematics and $\nu: \bar{\nu}$ ratios. The fit oscillation parameters are profiled. 
TABLE 8.3. Median MC and $\overline{\Delta \chi^{2}}$ test statistic values and $\mathrm{p}$-values for $\delta_{C P}=$ $-\pi / 2,0$, and $\pi / 2$ corresponding to the distributions shown in Figure 8.5 for $\mathrm{T} 2 \mathrm{HK}$. The p-values are computed with the $\mathrm{H} 0$ and $\mathrm{H} 1$ distributions computed with 20,000 toy MC experiments. The values are given for both True $\mathrm{MH}=\mathrm{NH}$ and True $\mathrm{MH}=\mathrm{IH}$ cases.

\begin{tabular}{l|ccc|ccc|ccc} 
& \multicolumn{3}{|c|}{$\delta_{C P}=-\pi / 2$} & \multicolumn{3}{c|}{$\delta_{C P}=0$} & \multicolumn{3}{c}{$\delta_{C P}=\pi / 2$} \\
& \multicolumn{3}{|c|}{$\mathrm{p}(\mathrm{T})$} & $\mathrm{p}(\mathrm{T})$ & \multicolumn{3}{c}{$\mathrm{p}(\mathrm{T})$} & $\mathrm{p}(\mathrm{T})$ & \multicolumn{3}{c}{$\mathrm{p}(\mathrm{T})$} & $\mathrm{p}(\mathrm{T})$ \\
& $\mathrm{T}$ & Toy MC & $\chi^{2}$ & $\mathrm{~T}$ & Toy MC & $\chi^{2}$ & $\mathrm{~T}$ & Toy MC & $\chi^{2}$ \\
\hline NH $T_{\overline{\Delta \chi^{2}}}$ & 28.3 & $<0.00005$ & $7.66 \mathrm{e}-08$ & 1.71 & 0.0376 & 0.181 & 13.9 & $7.57 \mathrm{e}-05$ & $1.91 \mathrm{e}-04$ \\
NH $T_{M C}$ & 28.9 & $<0.00005$ & $1.06 \mathrm{e}-07$ & 1.79 & 0.0376 & 0.191 & 11.4 & $1.27 \mathrm{e}-04$ & $7.46 \mathrm{e}-04$ \\
IH $T_{\overline{\Delta \chi^{2}}}$ & -97.4 & $1.69 \mathrm{e}-04$ & $1.80 \mathrm{e}-03$ & -3.22 & 0.00947 & 0.0727 & -34.2 & $<0.00005$ & $5.01 \mathrm{e}-09$ \\
IH $T_{M C}$ & -88.6 & $2.99 \mathrm{e}-04$ & $3.73 \mathrm{e}-03$ & -3.37 & 0.00947 & 0.0689 & -35.1 & $<0.00005$ & $3.42 \mathrm{e}-09$
\end{tabular}

8.1.2. MH Statistics In T2HK. Figure 8.5 shows the corresponding $\Delta \chi^{2}$ distributions for $\mathrm{MH}$ determination in T2HK. In the case of $\delta_{C P}=-\pi / 2(\pi / 2)$ in the $\mathrm{NH}(\mathrm{IH})$, T2HK has significant sensitivity to the MH. In these best cases, Table 8.2, gives the $\mathrm{P}$ values for rejection of the correct hypothesis that are less than 0.00005. This sensitivity is not robust for all values of $\delta_{C P}$ however. In the $\delta_{C P}=\pi / 2, \mathrm{NH}$ case of Figure $8.5 \mathrm{E}$ the degeneracy between $\delta_{C P}$ and the $\mathrm{MH}$ is significant and when statistical fluctuations are included the sensitivity is reduced relative to the Asimov data set $\Delta \chi^{2}$. Figure 8.6 shows significant differences between the $T_{\text {Median }}$ and $T_{\text {Asimov }}$ values for the $\delta_{C P}$ values that are degenerate with the MH. 


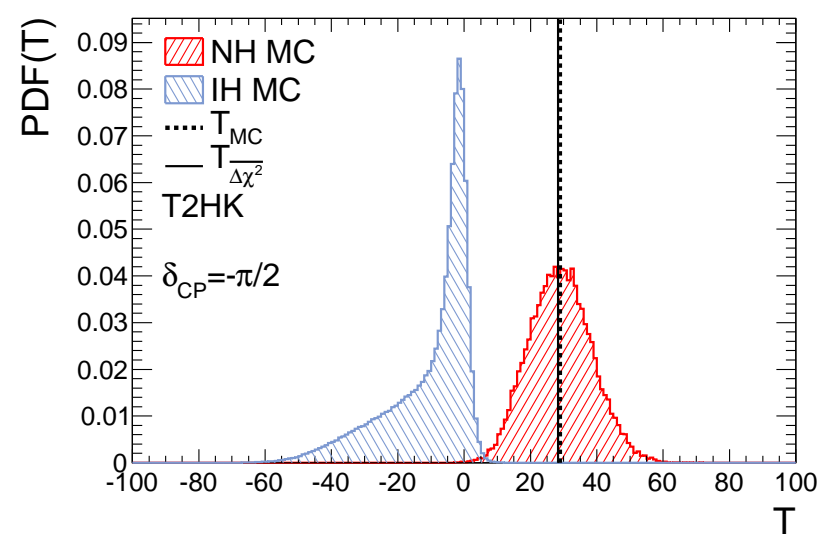

(A) $\delta_{C P}=-\pi / 2$, True $\mathrm{MH}=\mathrm{NH}$

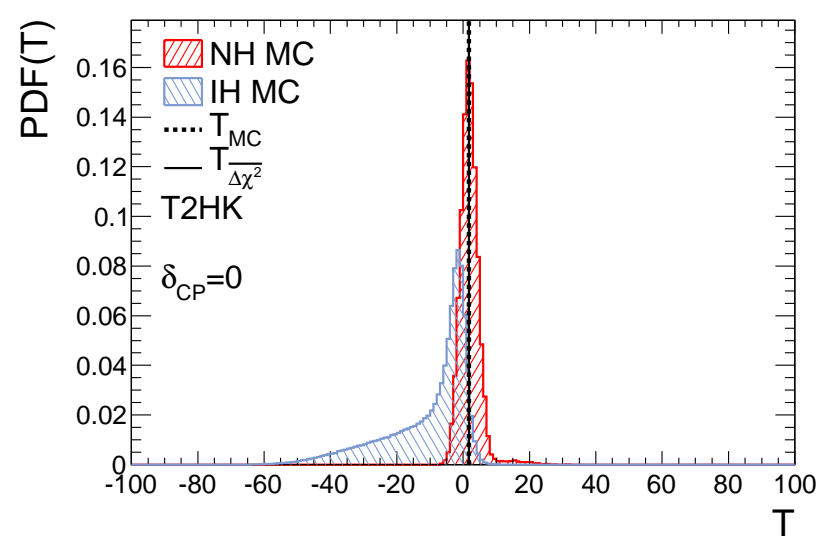

(C) $\delta_{C P}=0$, True $\mathrm{MH}=\mathrm{NH}$

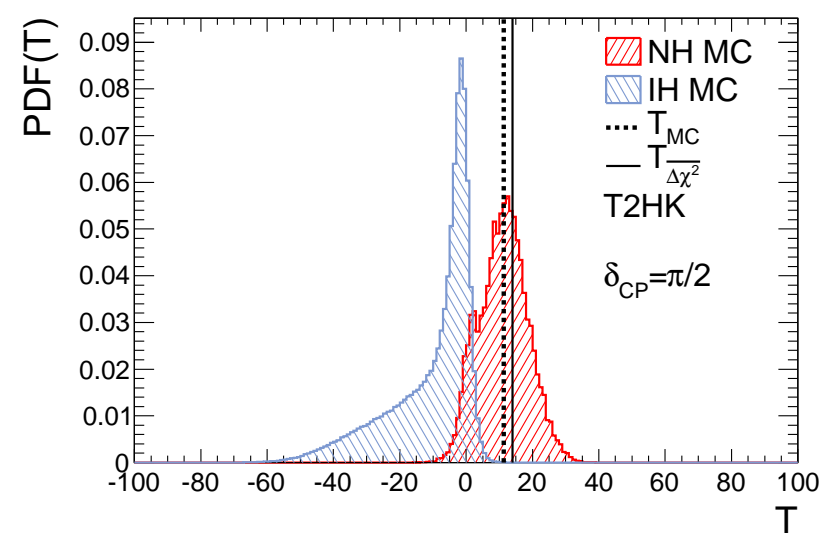

(E) $\delta_{C P}=\pi / 2$, True $\mathrm{MH}=\mathrm{NH}$

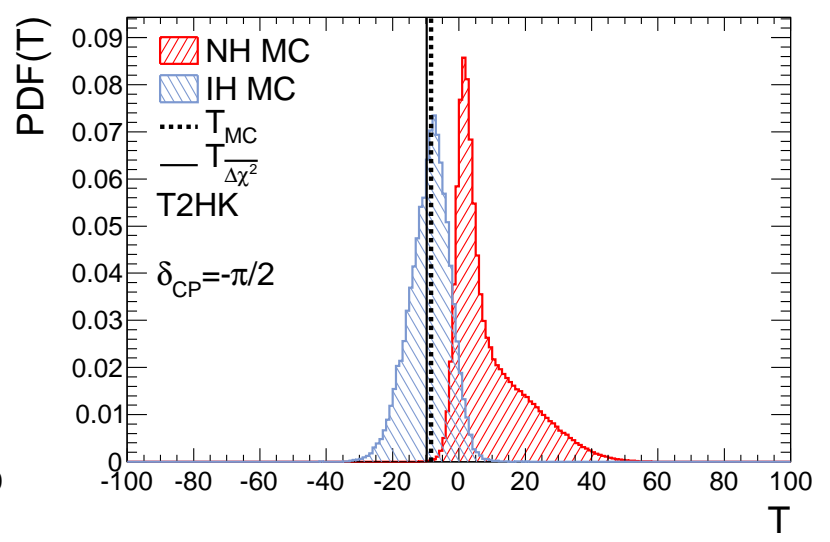

(B) $\delta_{C P}=-\pi / 2^{\circ}$, True $\mathrm{MH}=\mathrm{IH}$

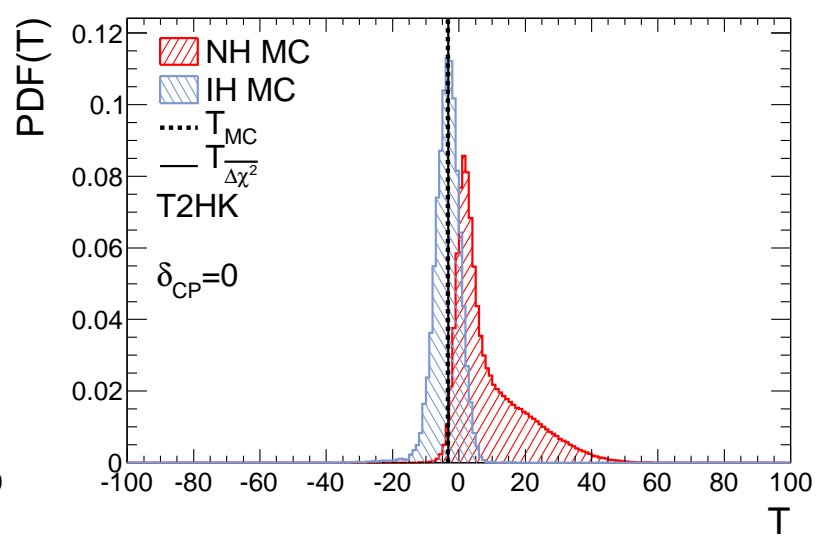

(D) $\delta_{C P}=0$, True $\mathrm{MH}=\mathrm{IH}$

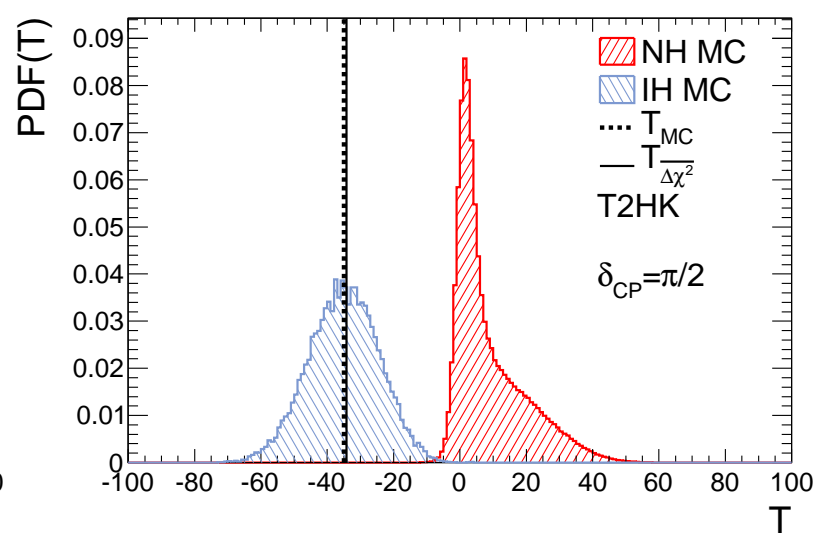

(F) $\delta_{C P}=\pi / 2$, True $\mathrm{MH}=\mathrm{IH}$

Figure 8.5. Distributions of the test statistic, $T$, for T2HK for $\delta_{C P}=-\pi / 2$, 0 , and $\pi / 2$ for both the True $\mathrm{MH}=\mathrm{NH}$ and True $\mathrm{MH}=\mathrm{IH}$ cases. The $\operatorname{red}(\mathrm{blue})$ histogram is for the $\mathrm{NH}(\mathrm{IH})$ distribution. The vertical lines represent the median of the toy MC $T$ distribution (dashed) and the $T$ value computed from the Asimov data set (solid). The nominal assumptions are assumed for systematics and $\nu: \bar{\nu}$ ratios. The fit oscillation parameters are profiled. 


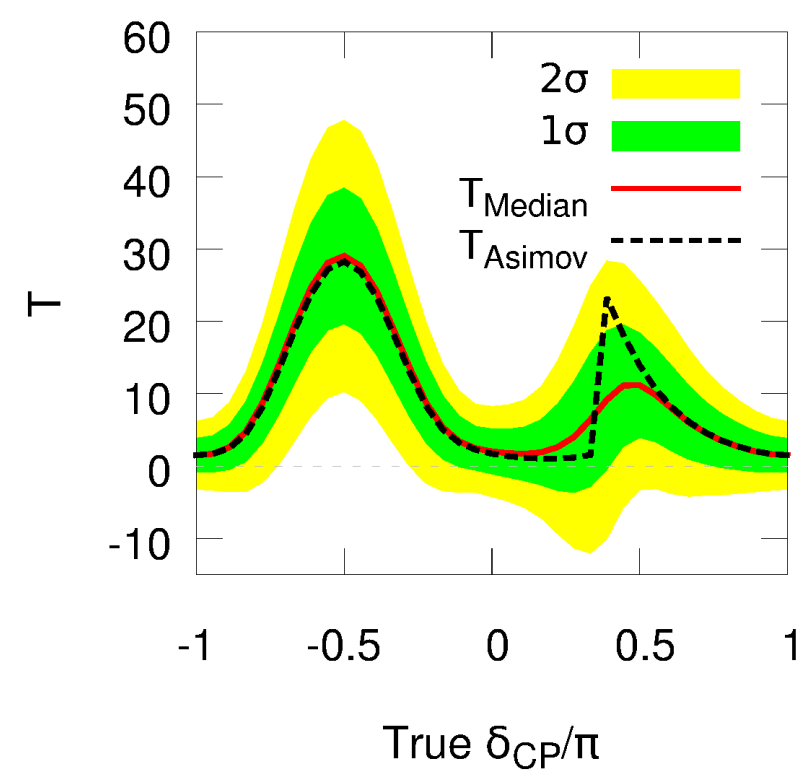

(A) True $\mathrm{MH}=\mathrm{NH}$

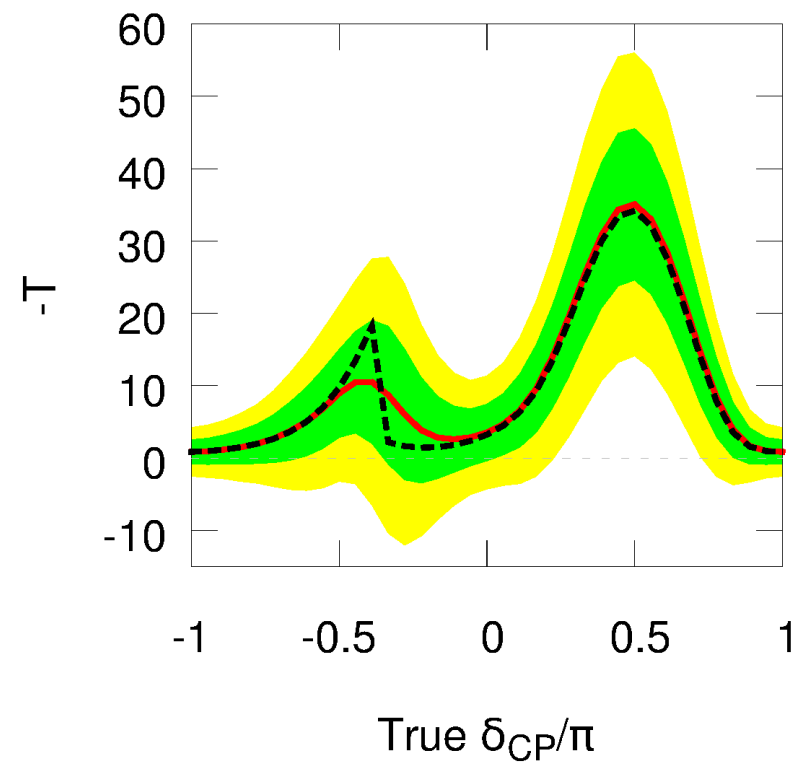

(B) True $\mathrm{MH}=\mathrm{IH}$

Figure 8.6. Distributions of the test statistic $\mathrm{T}$ vs $\delta_{C P}$ for T2HK for the True $\mathrm{MH}=\mathrm{NH}$ and True $\mathrm{MH}=\mathrm{IH}$ cases. The red line represents median value computed from the toy $\mathrm{MC}$ while the green and yellow bands represent the widths of the distributions containing $68 \%$ and $95 \%$ of the toy $\mathrm{MC} \mathrm{T}$ values respectively. The $T_{\text {Asimov }}$ (black-dashed) line gives the values computed using the Asimov data set. The nominal assumptions are assumed for systematics and $\nu: \bar{\nu}$ ratios. The fit oscillation parameters are profiled. 


\subsection{CP Violation \& Statistical Fluctuations}

In the case of sensitivity to $\mathrm{CP}$ violation a test statistic $T_{C P V}$ is defined as

$$
T_{C P V}=\min \left(\chi_{\delta_{C P}=0}^{2}-\chi_{f i t}^{2}, \chi_{\delta_{C P}=\pi}^{2}-\chi_{f i t}^{2}\right)
$$

The $\chi_{\delta_{C P}=0}^{2}$ and $\chi_{\delta_{C P}=\pi}^{2}$ terms compare the $\delta_{C P}=0$ and $\delta_{C P}=\pi$ fit spectra to the Poisson fluctuated true spectrum at a particular value of $\delta_{C P}$ while profiling all of the fit oscillation parameters except for $\delta_{C P}$. The $\chi_{\text {fit }}^{2}$ value is the best fit value of $\chi^{2}$ after all of the oscillation parameters are profiled. For each toy MC throw this value is subtracted from the values of $\chi^{2}$ at $\delta_{C P}=0$ and $\delta_{C P}=\pi$ and the minimum is taken to compute the $T_{C P V}$ for that throw. This is repeated thousands of times to generate a PDF of $T_{C P V}$ values. The studies of $T_{C P V}$ are presented here for LBNE with the nominal assumptions for systematics and $\nu: \bar{\nu}$ ratio and with the fit oscillation parameters profiled.

The null hypothesis, H0, in this case is when $\sin \left(\delta_{C P}\right)=0$ so the minimum is taken between the $T_{C P V}$ distributions at $\delta_{C P}=0$ and $\delta_{C P}=\pi$. The distribution of $T_{C P V}$ for H0 is shown in Figure 8.7. A $\chi^{2}$ distribution for 1 DOF is also plotted to show that the distribution of the PDF for $\mathrm{H} 0$ is $\chi^{2}$ distributed. As discussed in Chapter 5, Wilk's theorem applies when the null hypothesis is distributed as a $\chi^{2}$ and the significance of the determination of CP violation, in units of $\sigma$, is given by $\sigma=\sqrt{T_{C P V}}$.

Figure 8.8 shows the H0 PDF and the test hypothesis, H1, PDF for $\delta_{C P}=-\pi / 2$. The figure also shows the median value $\left(T_{M C}\right)$ computed from the toy $\mathrm{MC}$ and the $T_{\text {Asimov }}$ (which is equivalent to $T_{\overline{\Delta \chi^{2}}}$. These values are nearly equal again showing the equivalence of the approaches. 


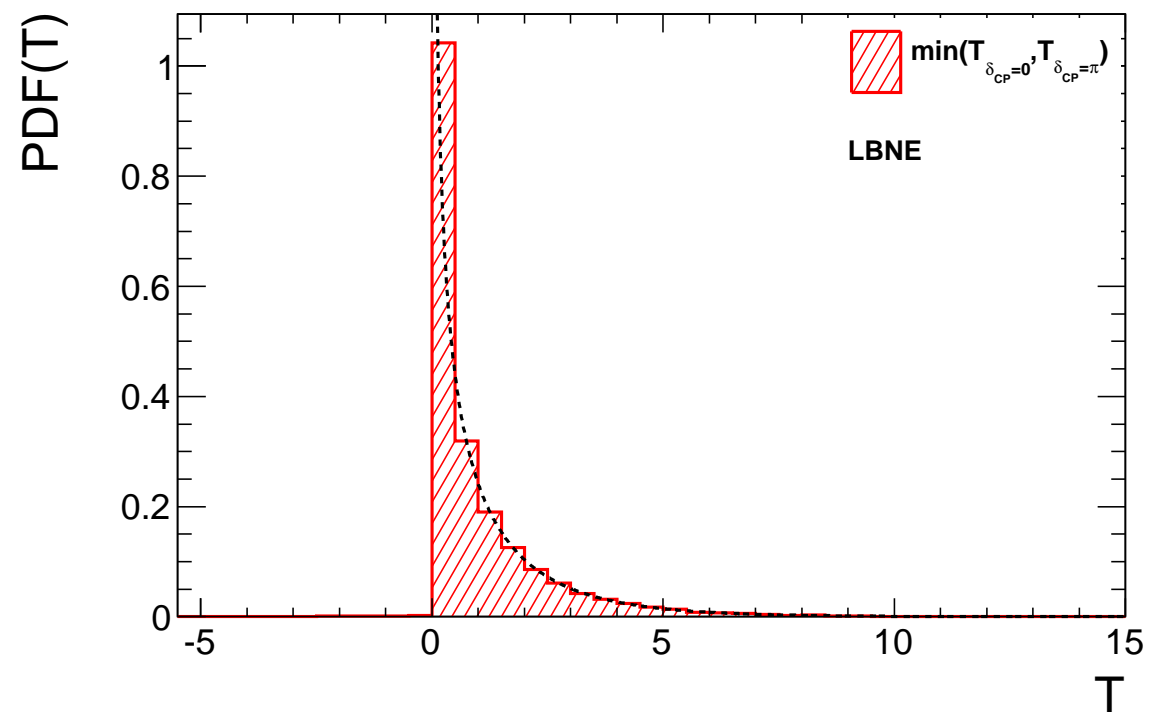

FIGURE 8.7. Distribution of the test statistic, $T_{C P V}$, for LBNE for $\delta_{C P}=0, \pi$. The minimum $T_{C P V}$ is taken between the $\delta_{C P}=0$ and $\delta_{C P}=\pi$ cases to form the PDF. The $\chi^{2}$ distribution for 1 DOF is given by the dashed black line. The nominal assumptions are assumed for systematics and $\nu: \bar{\nu}$ ratios. The fit oscillation parameters are profiled.

Figure 8.9 shows the distributions of $\sqrt{T_{M C}}$ as function of the assumed true $\delta_{C P}$ value. It also overlays the $\sqrt{T_{\text {Asimov }}}$ values in order to compare the approaches. The curves are generally equal except for a notable difference. At $\delta_{C P}=0$ and $\delta_{C P}=\pi$ the $\sqrt{T_{\text {Asimov }}}$ curve goes to zero while the $\sqrt{T_{M C}}$ goes to approximately $1 \sigma$. This happens because the Asimov data set comparison between a test spectrum at $\delta_{C P}=0$ and a fit spectrum at $\delta_{C P}=0$ will always yield a value of $\Delta \chi^{2}=0$. The value of the $\sqrt{T_{M C}}$ in this case is just the median of the $\chi^{2}$ distribution. However, in the case of $\sqrt{T_{M C}}$, the true spectrum is fluctuated so that there is always some difference between the test and fit spectra. This means that if the true value of $\delta_{C P}$ is near 0 or $\pi$ a very large data sample will be required to produce a significant difference. 


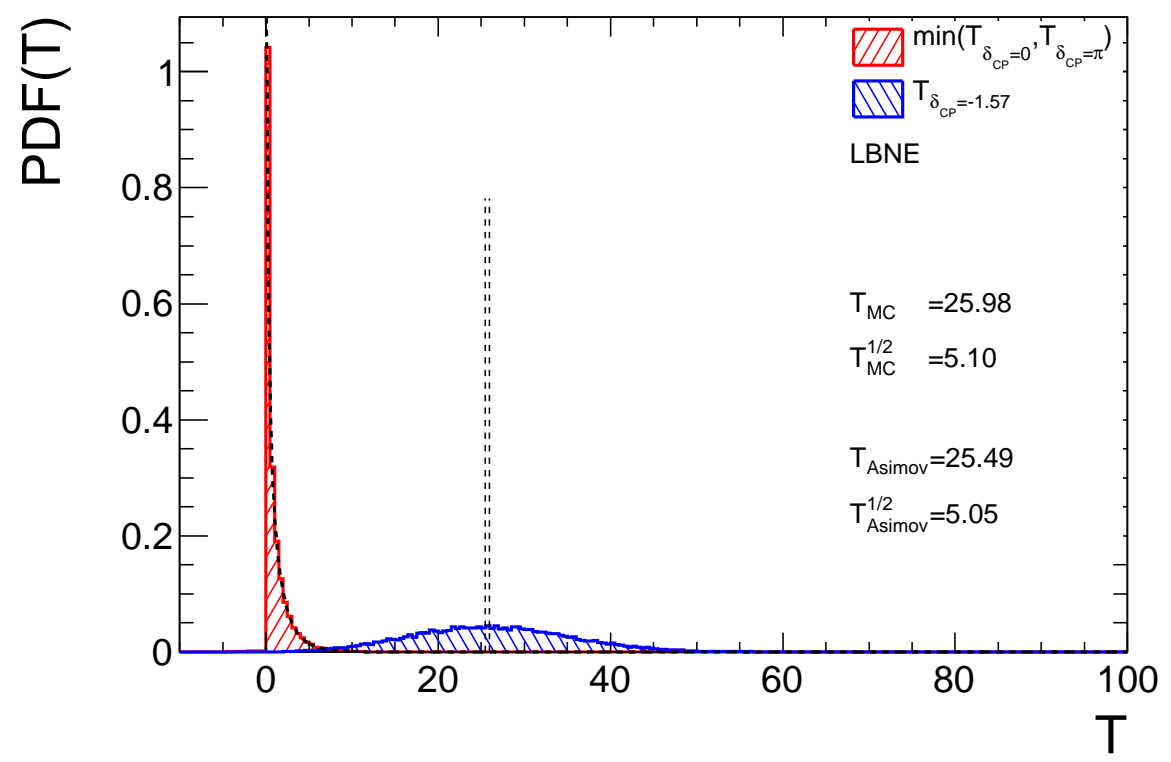

Figure 8.8. Distributions of the test statistic, $T_{C P V}$, for LBNE for $\mathrm{H} 0\left(\min \left(T_{\delta_{C P}=0}, T_{\delta_{C P}=\pi}\right)\right)$ in red and $\mathrm{H} 1$ for the test hypothesis of $\delta_{C P}=-\pi / 2$ in blue. The $\chi^{2}$ distribution for $1 \mathrm{DOF}$ is given by the dashed black curve. The median of the $\mathrm{H} 1$ distribution and the $T_{C P V}$ computed from the Asimov data set are indicated by vertical black lines that are indistinguishable in this case. The nominal assumptions are assumed for systematics and $\nu: \bar{\nu}$ ratios. The fit oscillation parameters are profiled.

\subsection{COnClusions}

This chapter has shown studies done to extend the studies of previous chapters and include the effects of statistical fluctuations on the expected measurement sensitivities to CP violation and the MH. This work was used by the LBNE collaboration to study the effects of statistical fluctuations and was based on the work of Qian et al[47]. Similar studies were also used in the T2K future sensitivity task force to explore the sensitivity for a combined, $\mathrm{T} 2 \mathrm{~K}+\mathrm{NO} \nu \mathrm{A}$ sensitivity to the mass hierarchy. This work also validated the assumption that the Asimov data set, based on the median of the predicted event rate distributions, accurately represents the median sensitivities expected when statistical fluctuations are included. 


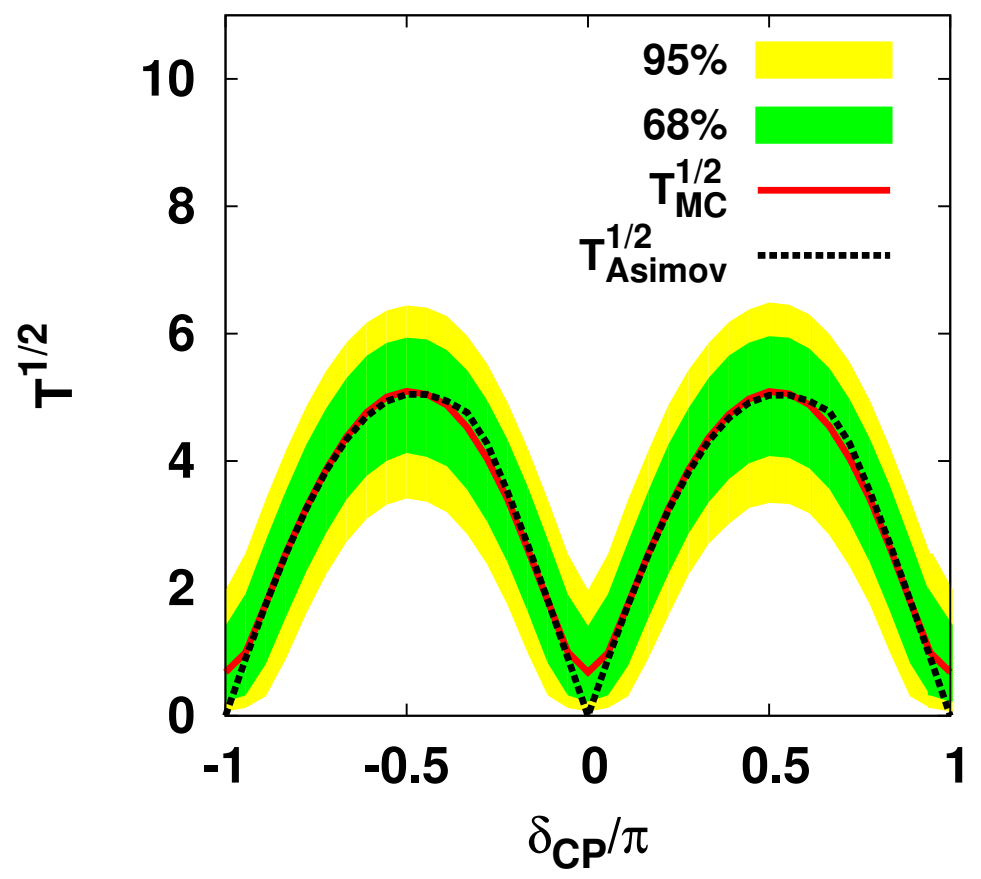

Figure 8.9. Distributions of the test statistic, $T_{C P V}$, for LBNE vs $\delta_{C P}$. The red line represents the median value computed from the toy MC while the green and yellow bands represent the widths of the distributions containing $68 \%$ and $95 \%$ of the toy $\mathrm{MC} T_{C P V}$ values respectively. The $T_{A s i m o v}$ (blackdashed) line gives the values computed using the Asimov data set. The nominal assumptions are assumed for systematics and $\nu: \bar{\nu}$ ratios. The fit oscillation parameters are profiled. 


\section{CHAPTER 9}

\section{Sensitivity Optimizations}

With the machinery in place to compute the sensitivity to the oscillation parameters in each experiment it is possible to evaluate the sensitivity as a function of the run parameters. In this chapter the $\nu: \bar{\nu}$ run ratios will be explored first for a combined $\mathrm{T} 2 \mathrm{~K}+\mathrm{NO} \nu \mathrm{A}$ analysis and then for each experiment individually. Then, for LBNE, the amount of $\nu_{\mu}$ flux at the second oscillation maximum will be varied to optimize $\mathrm{MH}$ sensitivity.

To analyze the effects of different running configurations it is informative to have a set of metrics that characterize the ability of the experiment (or combination of experiments) to constrain variables or reject a hypothesis. The following metrics are used in these studies:

- $\sin ^{2}\left(2 \theta_{13}\right) 1 \sigma$ half-width

- $\sin ^{2}\left(\theta_{23}\right) 1 \sigma$ half-width

- $\Delta m_{31}^{2} 1 \sigma$ half-width

- $\delta_{C P}=[0, \pi / 2, \pi,-\pi / 2]$ half-width

- Median $\Delta \chi^{2}$ for $\sin \delta_{C P} \neq 0$ determination

- Lowest $\Delta \chi^{2}$ for MH determination

These are visualized in Figure 9.1 and were also discussed in Chapter 6. In some cases there are degenerate $1 \sigma$ CI regions in $\delta_{C P}$ that are disconnected from the central value. For example there may be a disconnected degenerate region for a true value of $\delta_{C P}=0$ if event spectra are similar between $\delta_{C P}=0$ and $\delta_{C P}=\pi$. In this case half of the width of the degenerate region is added to this metric.

The first four metrics listed above are measurements of the ability of the experiment to directly constrain the oscillation parameters at a particular set of true parameter values. 
The true values used are from the Capozzi et al. global fit. The fit values of $\mathrm{MH}, \sin ^{2}\left(\theta_{23}\right)$, $\delta_{C P}$, and $\Delta m_{31}^{2}$ are unconstrained in the fit. The fit value of $\sin ^{2}\left(2 \theta_{13}\right)$ is constrained using the Capozzi et al. global fit $1 \sigma$ ranges for $\sin ^{2}\left(2 \theta_{13}\right)$ except in the case of the $\sin ^{2}\left(2 \theta_{13}\right) 1 \sigma$ width where no external constraints on $\sin ^{2}\left(2 \theta_{13}\right)$ are applied.
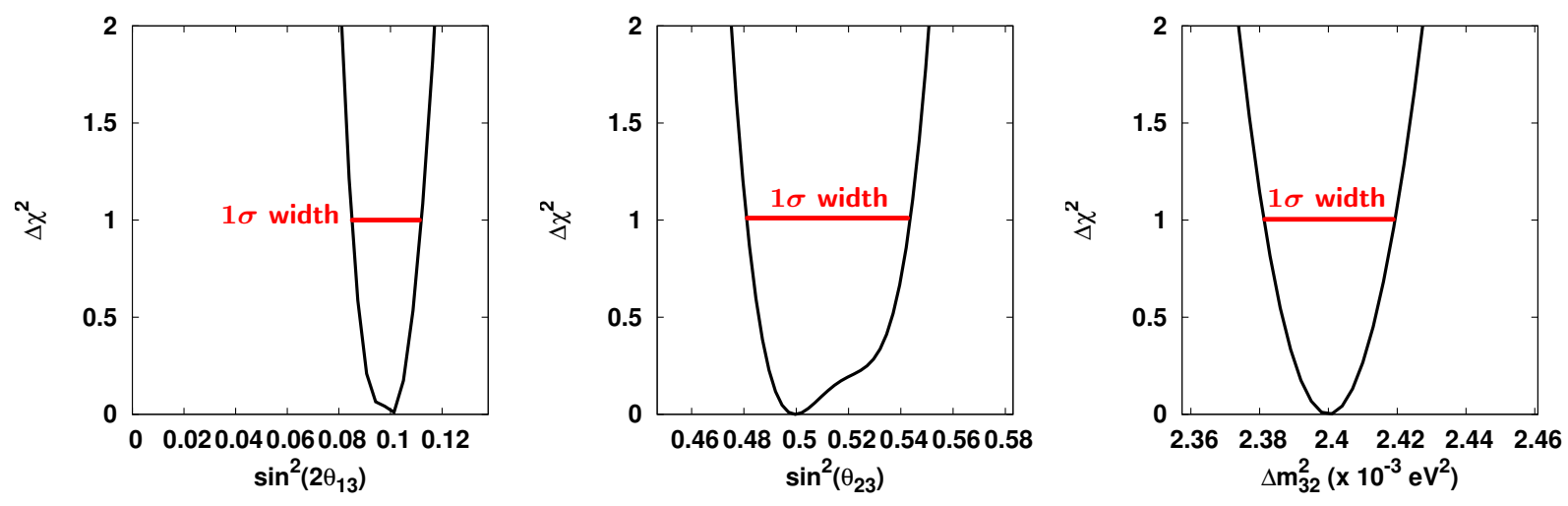

(A) $\sin ^{2}\left(2 \theta_{13}\right) 1 \sigma$ half-width

(B) $\sin ^{2}\left(\theta_{23}\right) 1 \sigma$ half-width

(C) $\Delta m_{31}^{2} 1 \sigma$ half-width
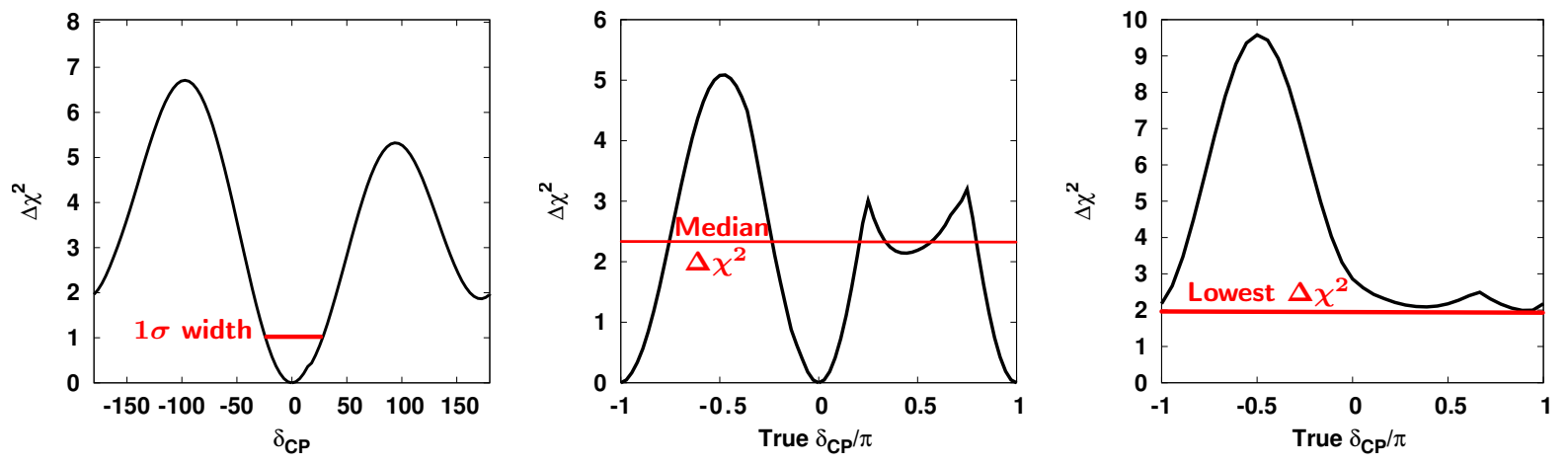

(D) $\delta_{C P}=0^{\circ}$ half-width

(E) Median $\Delta \chi^{2}$ for $\sin \delta_{C P} \neq 0$

(F) Lowest $\Delta \chi^{2}$ for mass hierarchy

Figure 9.1. Metrics of performance for $\nu: \bar{\nu}$ run ratio variations. For Figures 9.1a through $9.1 \mathrm{~d}$ the actual metric is half of the $1 \sigma$ width. These figures are examples of the metrics and do not correspond to a particular experimental configuration used in this thesis. 


\section{1. $\nu: \bar{\nu}$ Run Ratios in EaCh Experiment}

Each experiment will have to decide at which $\nu: \bar{\nu}$ ratio to run in order to optimize sensitivity to particular oscillation effects. This ratio does not have to remain fixed throughout the run and is likely to change depending on other experimental goals and external constraints on the oscillation parameters at the time the experiment runs. This section evaluates the sensitivity variations as a function of $\nu: \bar{\nu}$ from the previous section for $\mathrm{T} 2 \mathrm{~K}, \mathrm{NO} \nu \mathrm{A}, \mathrm{LBNE}$, and T2HK independently.

The sensitivities as a function of $\nu: \bar{\nu}$ ratio produced here will assume the nominal systematics configurations with normalization uncertainties on signal and backgrounds. The systematics implemented in this way will in general affect the sensitivity equally in $\nu$ and $\bar{\nu}$ modes. There could be systematic effects that affect the $\nu$ and $\bar{\nu}$ modes differently. For example a more sophisticated accounting of systematic uncertainties might take into account the fact that the neutrino interaction cross sections are less well known for $\bar{\nu}$ interactions. So it might be desirable to run longer in $\bar{\nu}$ mode to constrain the flux of $\bar{\nu}_{\mu}$ in the beam in that mode. Consequently, the optimal ratios here come with the caveat that further systematics considerations may have to be considered in selecting the final $\nu: \bar{\nu}$ running ratio.

Figure 9.2 shows the expected resolution on $\sin ^{2}\left(2 \theta_{13}\right)$ for the four experiments as a function of the $\nu: \bar{\nu}$ running ratios for both the True $\mathrm{MH}=\mathrm{NH}$ and True $\mathrm{MH}=\mathrm{IH}$ assumptions. The current $\sin ^{2}\left(2 \theta_{13}\right)$ resolution from the Capozzi et al. global fit is indicated by the horizontal dashed black line. All of the experiments show the general feature of needing some mix of $\nu$ and $\bar{\nu}$ running to get the best resolution so that running with 1:0 or 0:1 running ratios leads to the worst resolution on $\sin ^{2}\left(2 \theta_{13}\right)$. This is a result of profiling $\delta_{C P}$ in the fit and running both $\nu$ and $\bar{\nu}$ helps to better constrain the odd terms, from $\sin \delta_{C P}$, in the $\nu_{e}$ appearance and $\bar{\nu}_{e}$ appearance oscillation probabilities, through which $\delta_{C P}$ and $\theta_{13}$ 
are correlated. Running at least some small amount in each mode helps to constrain the $\delta_{C P}$ values and reduce their impact on the $\sin ^{2}\left(2 \theta_{13}\right)$ resolution.

The resolutions on $\sin ^{2}\left(\theta_{23}\right)$ resulting from $\nu: \bar{\nu}$ variations are shown in Figure 9.3. These resolutions generally exclude the second octant solution except in the case of $0: 1 \nu: \bar{\nu}$ running in $\mathrm{NO} \nu \mathrm{A}$ where the resolution jumps to a larger value relative to the other $\nu: \bar{\nu}$ ratios in $\mathrm{NO} \nu \mathrm{A}$. Since $\nu_{\mu}$ disappearance and $\bar{\nu}_{\mu}$ disappearance contribute the most to $\sin ^{2}\left(\theta_{23}\right)$ sensitivity for the true value of $\sin ^{2}\left(\theta_{23}\right)$ used for these figures and those samples do not depend on the $\delta_{C P}$ value, the best $\sin ^{2}\left(\theta_{23}\right)$ resolution comes with mostly $\nu$ running. The overall event rate in $\nu$ mode is higher than in $\bar{\nu}$ so that mode is preferred when statistics dominate the sensitivity.

The resolutions on $\Delta m_{31}^{2}$ show a similar preference for all $\nu$ running in Figure 9.4. Higher statistics enable the dip in the $\nu_{\mu}$ disappearance spectra to be measured with greater precision.

Figures 9.5 through 9.7 show the $1 \sigma$ resolutions on $\delta_{C P}$ for the true values of $\delta_{C P}=0$, $-\pi / 2$, and $\pi / 2$ respectively. $\mathrm{T} 2 \mathrm{~K}$ and $\mathrm{NO} \nu \mathrm{A}$ have a similar preference for all $\nu$ running in the $\delta_{C P}=0$, True $\mathrm{MH}=\mathrm{NH}$ case although $\mathrm{NO} \nu \mathrm{A}$ has better resolution. T2K has a similar preference in the True $\mathrm{MH}=\mathrm{IH}$ case. $\mathrm{NO} \nu \mathrm{A}$ resolutions increase when the $\nu$ ratio is above $80 \%$ because of degeneracies between $\delta_{C P}$ and $\mathrm{MH}$ as can be seen in Figures 6.17 and 6.18. A balance of $\nu: \bar{\nu}$ running in $\mathrm{NO} \nu \mathrm{A}$ better resolves the $\mathrm{MH}$. $\mathrm{NO} \nu \mathrm{A}$ generally has better resolution for $\delta_{C P}=0$, compared with $\mathrm{T} 2 \mathrm{~K}$, because this degeneracy is slightly more resolved in $\mathrm{NO} \nu \mathrm{A}$ with its longer baseline. LBNE and T2HK have similar optimal ratios for $\delta_{C P}=0$ resolution with between $60 \%$ and $70 \% \nu$ running being optimal. T2HK has a jump in the resolution that comes from the degenerate region near $\delta_{C P}=\pi$, visible in Figures 6.17D and 6.18D, not being resolved for all $\nu$ or all $\bar{\nu}$ running. 


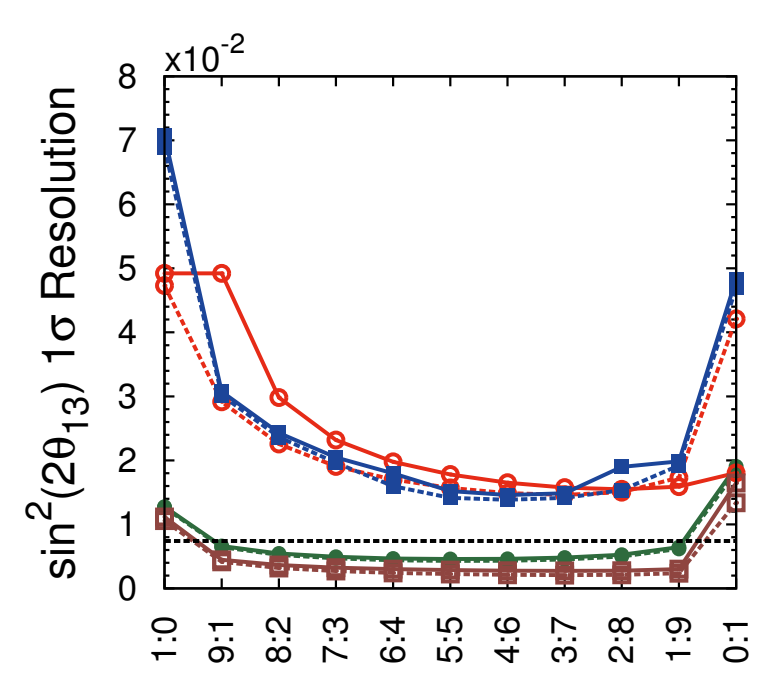

$v: \bar{v}$

(A) True $\mathrm{MH}=\mathrm{NH}$

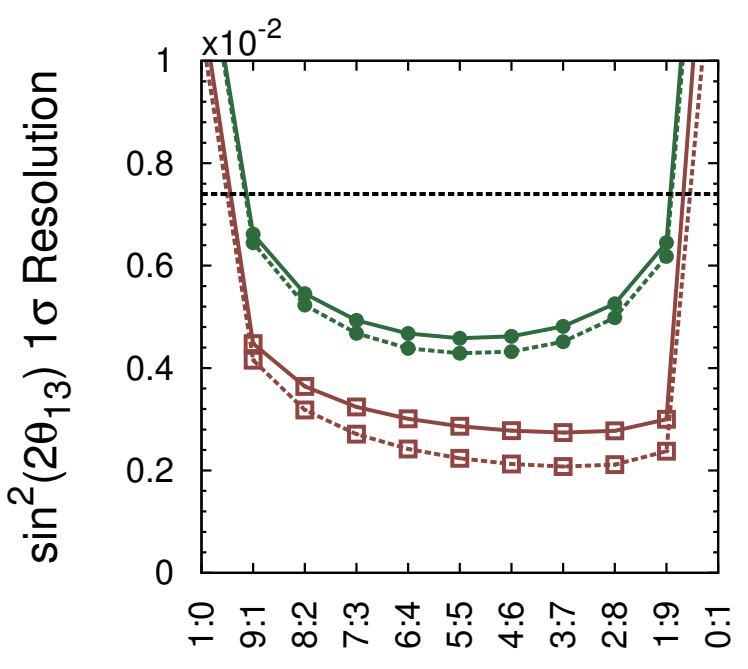

$v: \bar{v}$

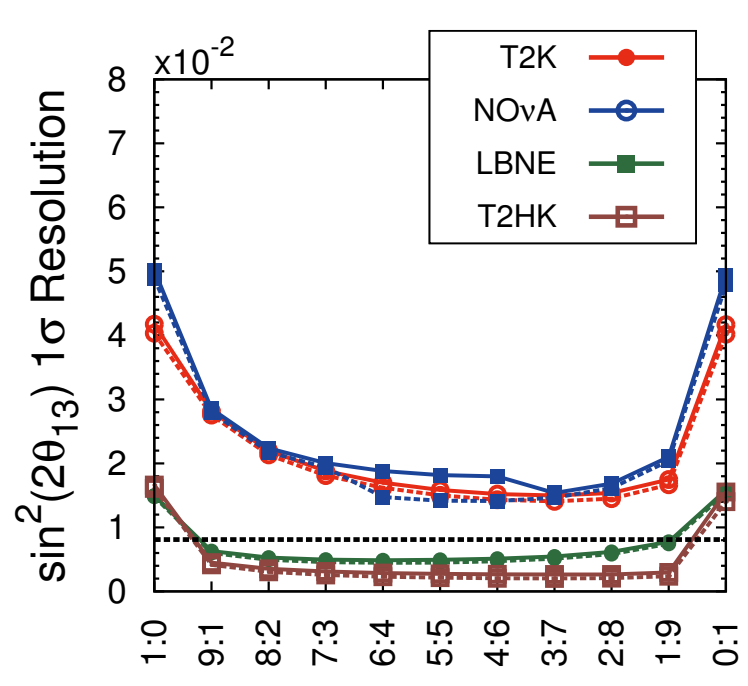

$v: \bar{v}$

(в) True $\mathrm{MH}=\mathrm{IH}$

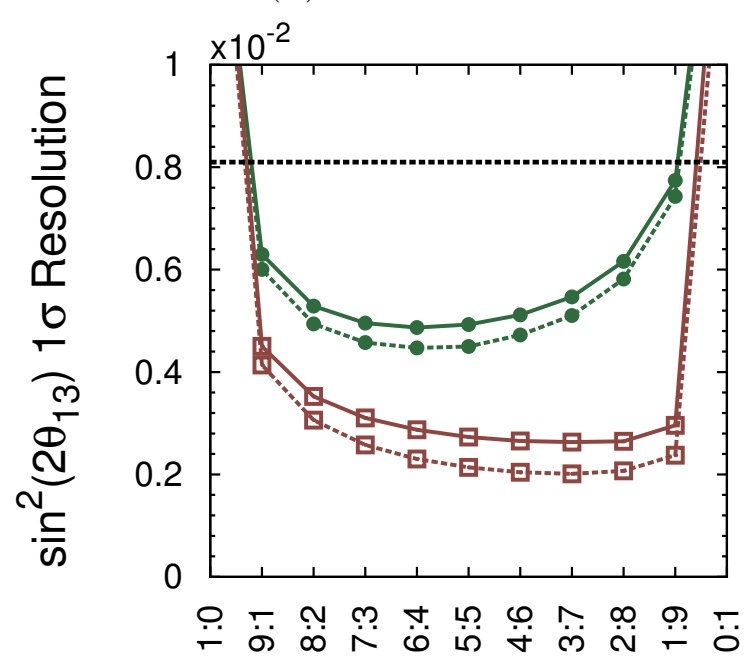

$v: \bar{v}$

(c) True $\mathrm{MH}=\mathrm{NH}$ (zoomed)

(D) True $\mathrm{MH}=\mathrm{IH}$ (zoomed)

Figure 9.2. The $1 \sigma$ resolutions on $\sin ^{2}\left(2 \theta_{13}\right)=0.092\left(\sin ^{2}\left(2 \theta_{13}\right)=0.094\right)$ as a function of $\nu: \bar{\nu}$ running ratio for True $\mathrm{MH}=\mathrm{NH}$ (True $\mathrm{MH}=\mathrm{IH}$ ) for each experiment. Dashed(solid) curves are without(with) normalization systematics at the nominal levels. The horizontal dashed line indicates the current resolution from the Capozzi et al. global fit. The other oscillation parameters and the $\mathrm{MH}$ are profiled. 


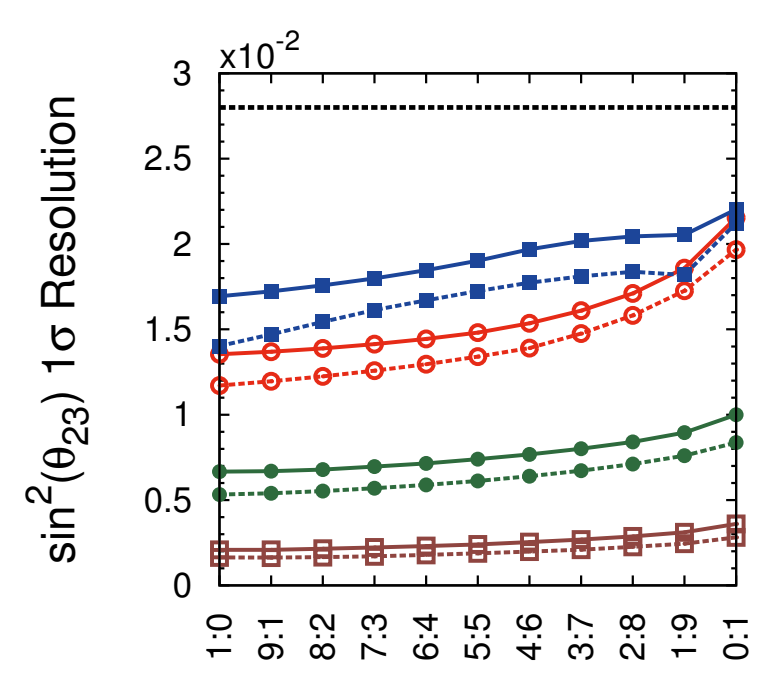

$v: \bar{v}$

(A) True $\mathrm{MH}=\mathrm{NH}$

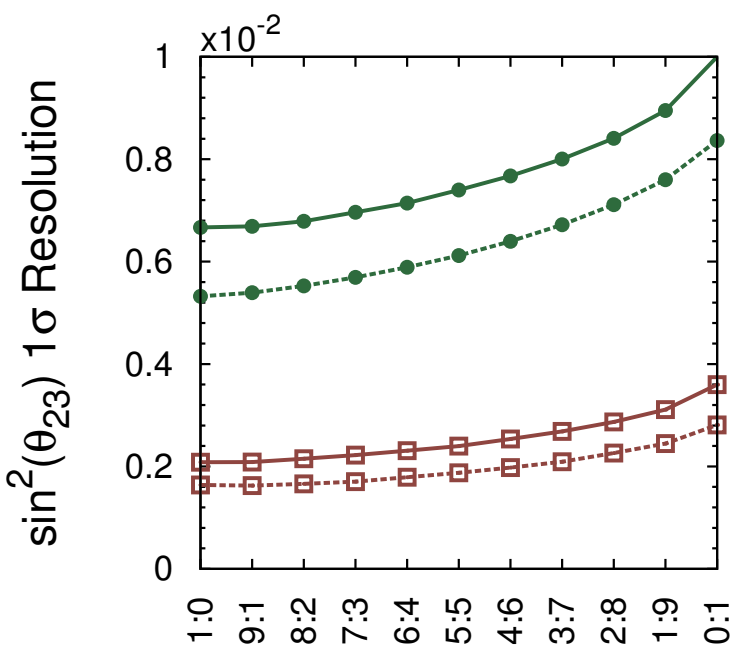

$v: \bar{v}$

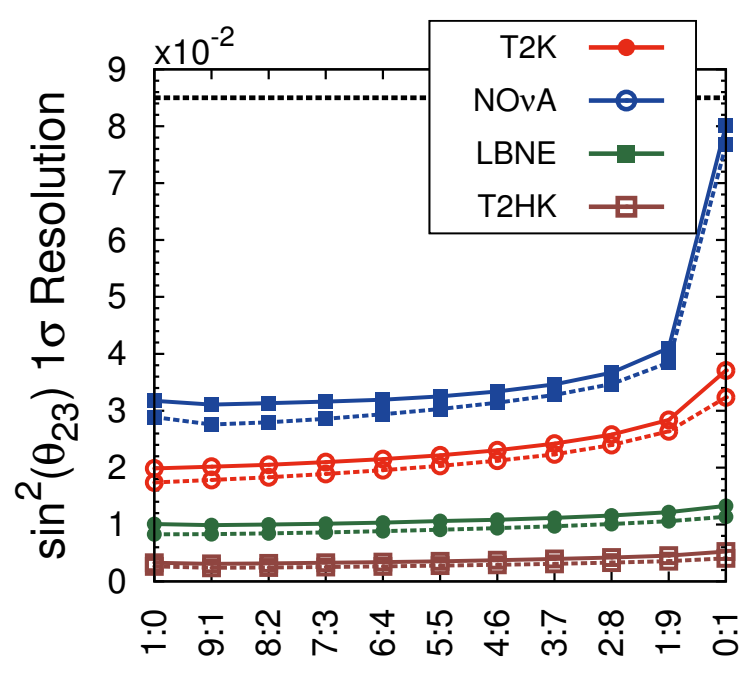

$v: \bar{v}$

(B) True $\mathrm{MH}=\mathrm{IH}$

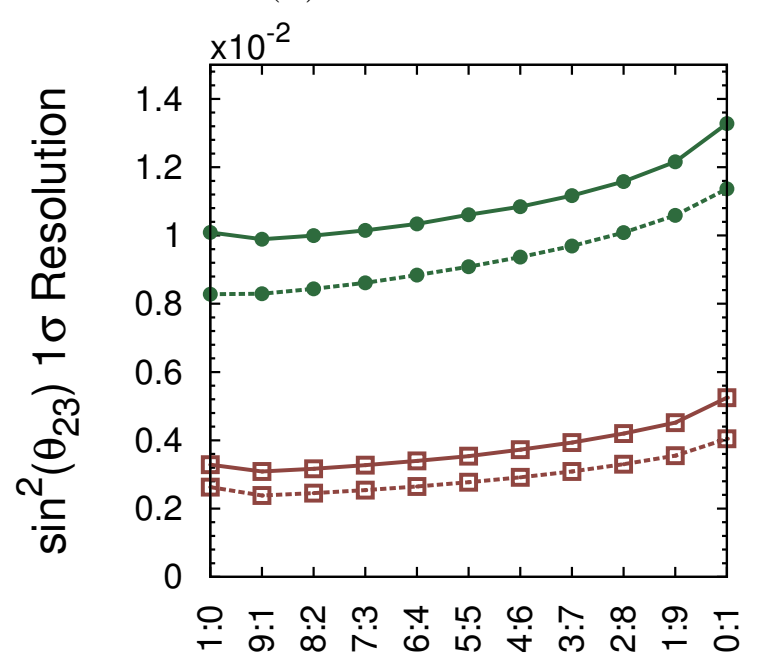

$v: \bar{v}$

(c) True $\mathrm{MH}=\mathrm{NH}$ (zoomed)

(D) True $\mathrm{MH}=\mathrm{IH}$ (zoomed)

FiguRe 9.3. The $1 \sigma$ resolutions on $\sin ^{2}\left(\theta_{23}\right)=0.44\left(\sin ^{2}\left(\theta_{23}\right)=0.46\right)$ as a function of $\nu: \bar{\nu}$ running ratio for True $\mathrm{MH}=\mathrm{NH}$ (True $\mathrm{MH}=\mathrm{IH}$ ) for each experiment. Dashed(solid) curves are without(with) normalization systematics at the nominal levels. The horizontal dashed line indicates the current resolution from the Capozzi et al. global fit. The other oscillation parameters and the $\mathrm{MH}$ are profiled. 


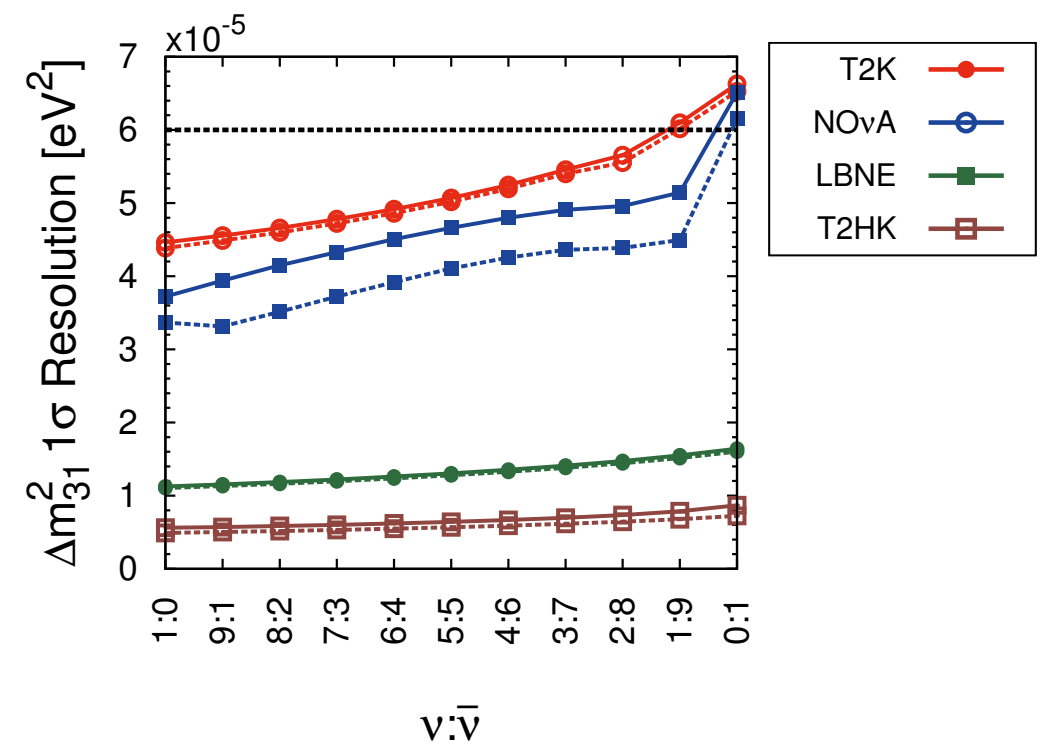

(A) True $\mathrm{MH}=\mathrm{NH}$

Figure 9.4. The $1 \sigma$ resolutions on $\Delta m_{31}^{2}=2.47 \times 10^{-3} \mathrm{eV}^{2}$ as a function of $\nu: \bar{\nu}$ running ratio for True $\mathrm{MH}=\mathrm{NH}$ for each experiment. Dashed(solid) curves are without(with) normalization systematics at the nominal levels. The horizontal dashed line indicates the current resolution from the Capozzi et al. global fit. The other oscillation parameters and the MH are profiled.

In Figures 9.6 and 9.7 the resolutions for $\delta_{C P}=-\pi / 2$ and $\delta_{C P}=\pi / 2$ show responses to $\nu: \bar{\nu}$ variations that are similar to the response for the opposite sign of $\delta_{C P}$ in the opposite MH assumption. For T2K in Figures 9.6A the resolutions are again best for all $\nu$ running while $\mathrm{NO} \nu \mathrm{A}$ requires a balance of $\nu$ and $\bar{\nu}$ running to achieve the best resolution. In the True $\mathrm{MH}=\mathrm{NH}$ case, Figure 9.6C, the LBNE resolutions are relatively flat between $100 \%$ and $50 \% \nu$ running, but in the True $\mathrm{MH}=\mathrm{IH}$ case there is a preference for all $\bar{\nu}$ running. T2HK resolutions for these $\delta_{C P}$ values are relatively flat in response to $\nu: \bar{\nu}$ variations. 


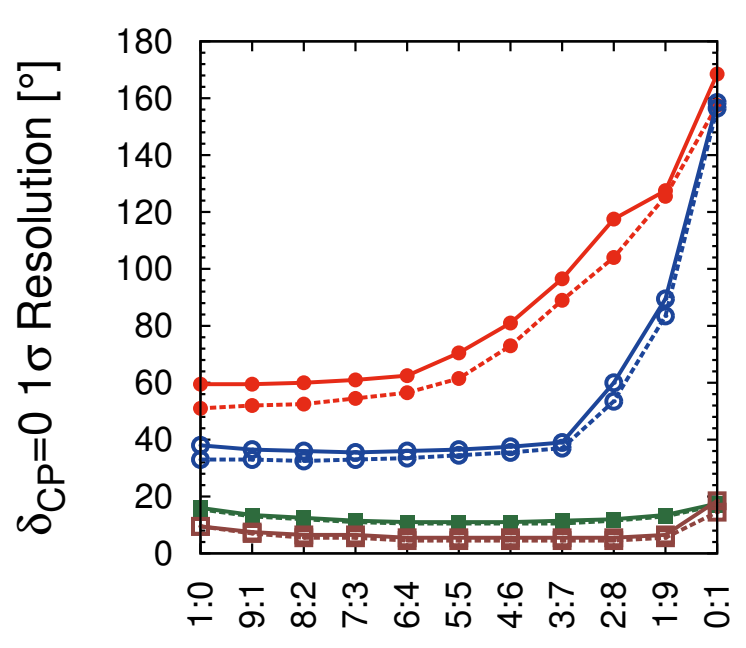

$v: \bar{v}$

(A) True $\mathrm{MH}=\mathrm{NH}$

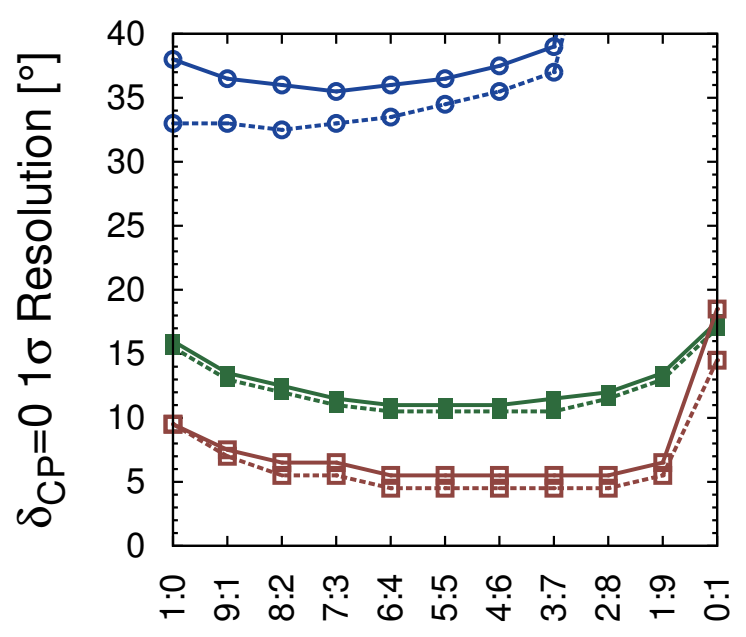

$v: \bar{v}$

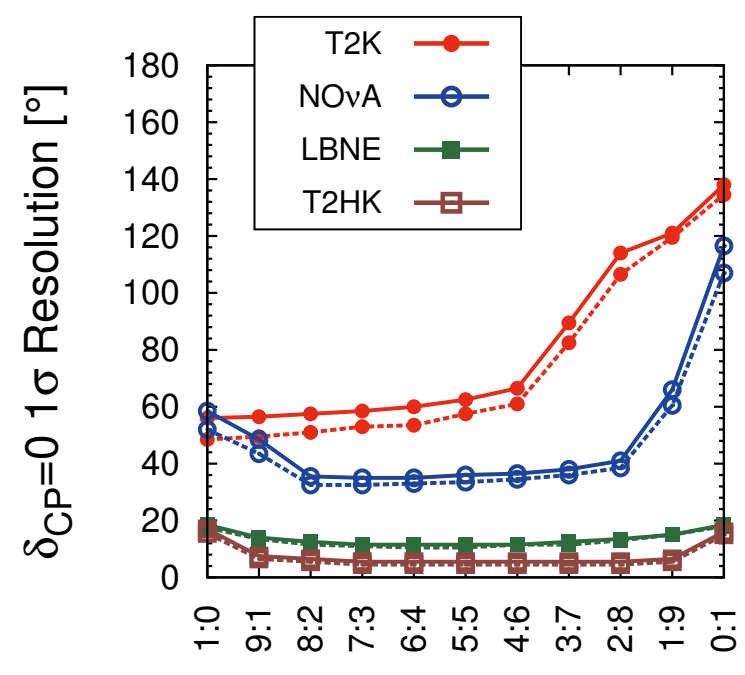

$v: \bar{v}$

(в) True $\mathrm{MH}=\mathrm{IH}$

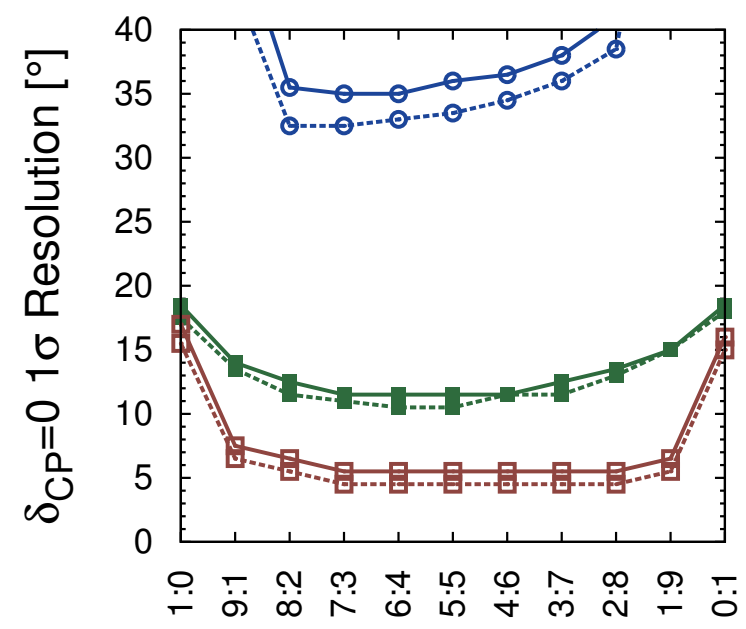

$v: \bar{v}$

(c) True $\mathrm{MH}=\mathrm{NH}$ (zoomed)

(D) True $\mathrm{MH}=\mathrm{IH}$ (zoomed)

FigURE 9.5. The $1 \sigma$ resolutions on $\delta_{C P}=0$ as a function of $\nu: \bar{\nu}$ running ratio for True $\mathrm{MH}=\mathrm{NH}$ (left) and IH (right) for each experiment. Dashed(solid) curves are without(with) normalization systematics at the nominal levels. The other oscillation parameters and the $\mathrm{MH}$ are profiled. 


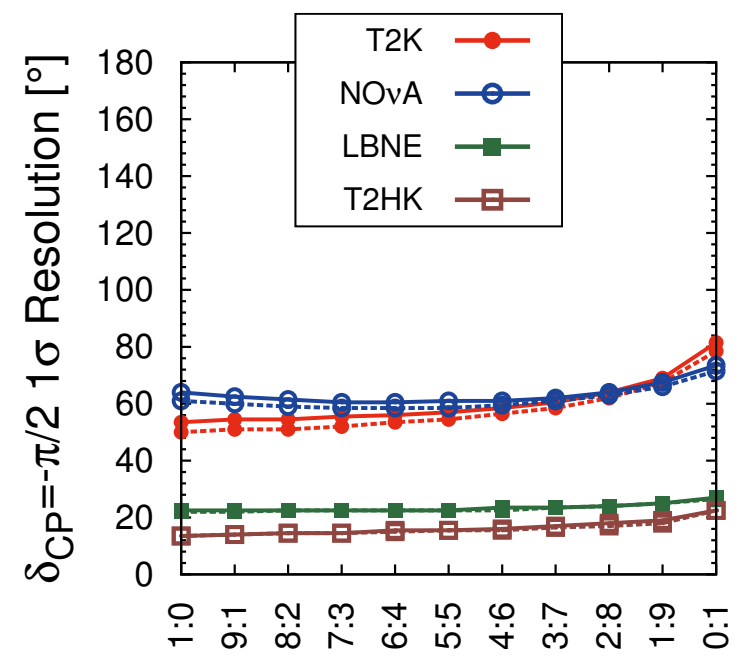

$v: \bar{v}$

(A) True $\mathrm{MH}=\mathrm{NH}$

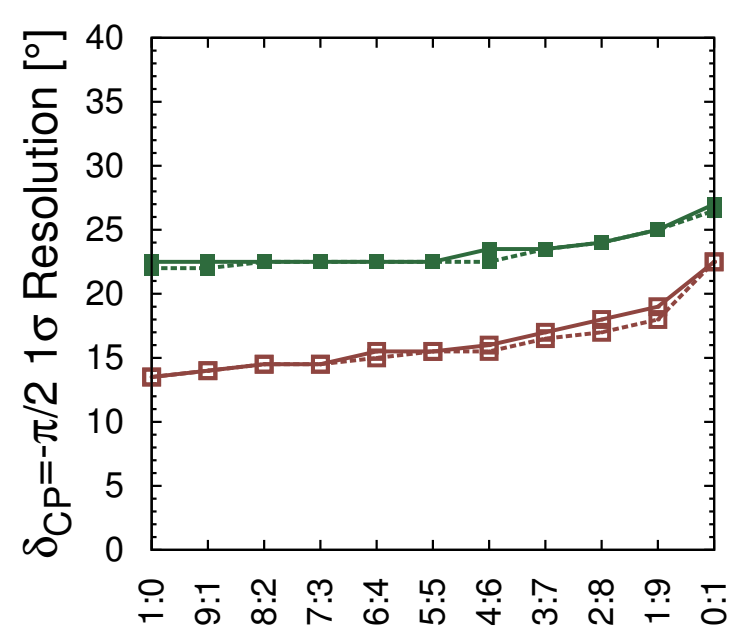

$v: \bar{v}$

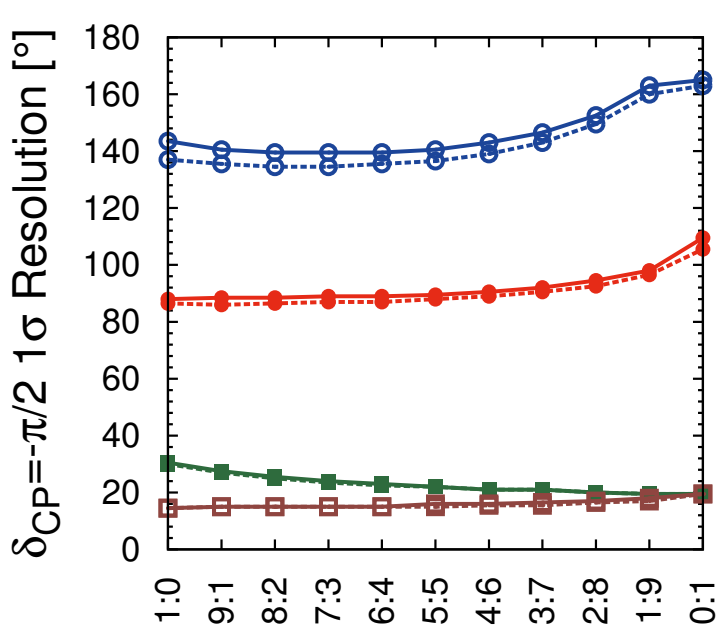

$v: \bar{v}$

(в) True $\mathrm{MH}=\mathrm{IH}$

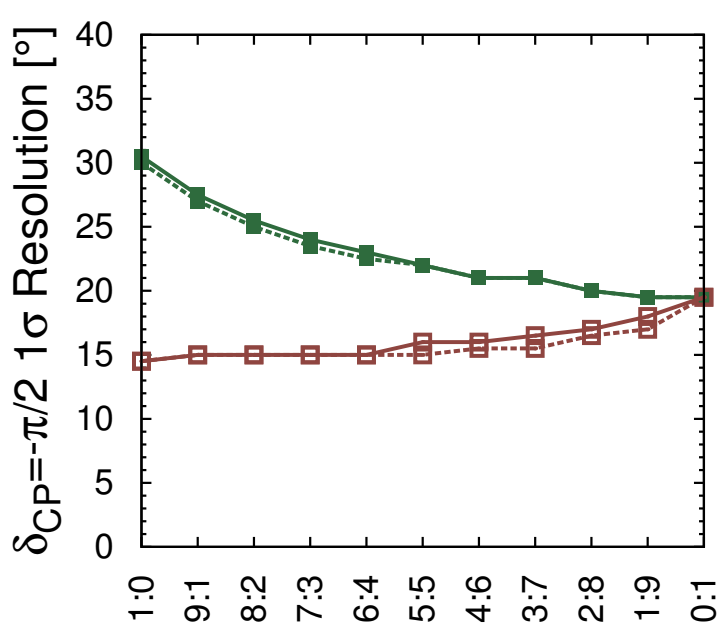

$v: \bar{v}$

(c) True $\mathrm{MH}=\mathrm{NH}$ (zoomed)

(D) True $\mathrm{MH}=\mathrm{IH}$ (zoomed)

Figure 9.6. The $1 \sigma$ resolutions on $\delta_{C P}=-\pi / 2$ as a function of $\nu: \bar{\nu}$ running ratio for True $\mathrm{MH}=\mathrm{NH}$ (left) and $\mathrm{IH}$ (right) for each experiment. Dashed(solid) curves are without(with) normalization systematics at the nominal levels. The other oscillation parameters and the $\mathrm{MH}$ are profiled. 


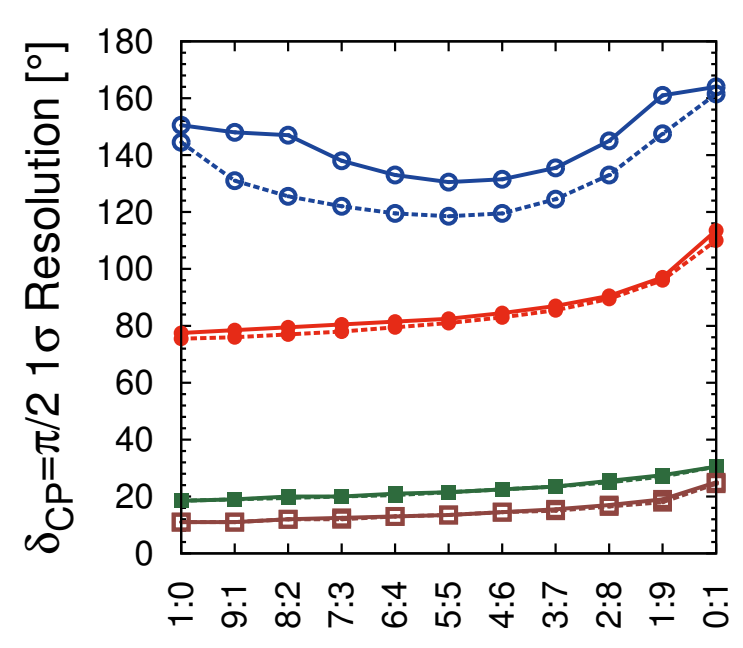

$v: \bar{v}$

(A) True $\mathrm{MH}=\mathrm{NH}$

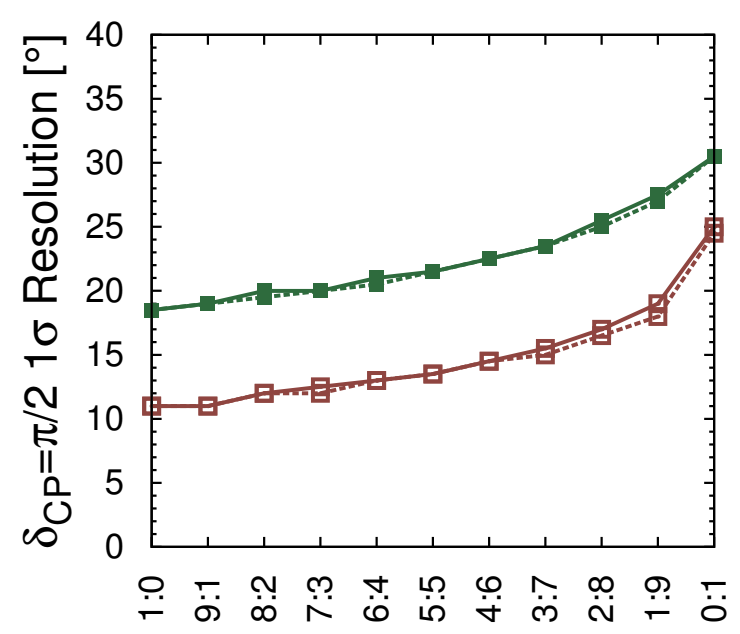

$v: \bar{v}$

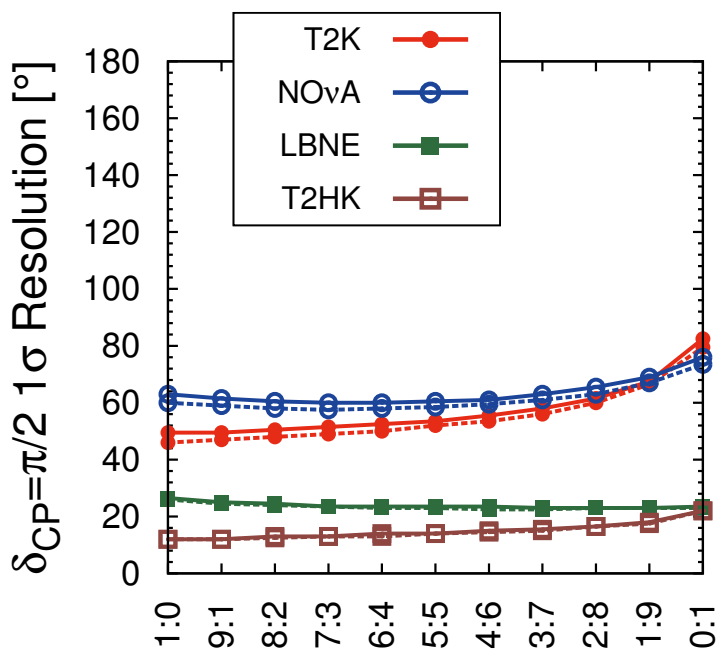

$v: \bar{v}$

(в) True $\mathrm{MH}=\mathrm{IH}$

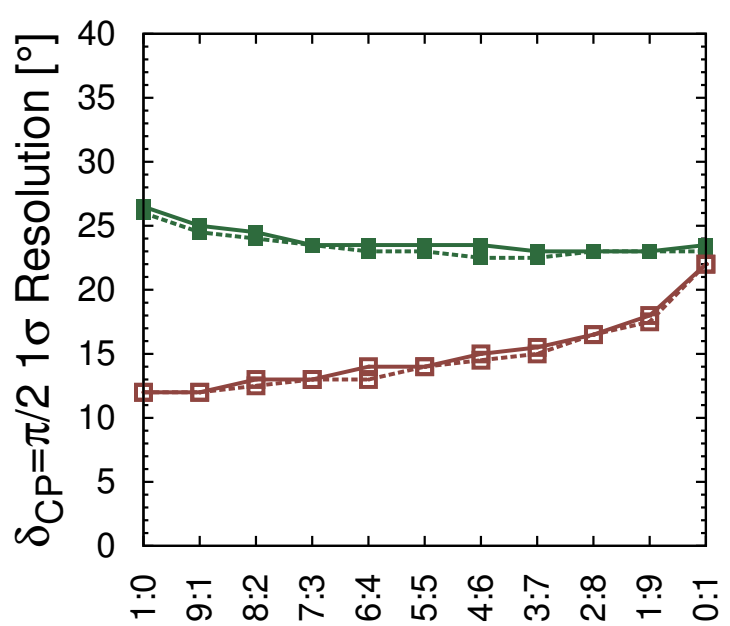

$v: \bar{v}$

(c) True $\mathrm{MH}=\mathrm{NH}$ (zoomed)

(D) True $\mathrm{MH}=\mathrm{IH}$ (zoomed)

Figure 9.7. The $1 \sigma$ resolutions on $\delta_{C P}=\pi / 2$ as a function of $\nu: \bar{\nu}$ running ratio for True $\mathrm{MH}=\mathrm{NH}$ (left) and $\mathrm{IH}$ (right) for each experiment. Dashed(solid) curves are without(with) normalization systematics at the nominal levels. The other oscillation parameters and the $\mathrm{MH}$ are profiled. 
9.1.1. Optimal $\nu: \bar{\nu}$ FOR CP Violation and MH. Each of the experiments is focused on maximizing the possibility of detecting $\mathrm{CP}$ violation and/or determining the $\mathrm{MH}$ so it is reasonable to use one or both of these metrics to choose the $\nu: \bar{\nu}$ run ratio. Figures 9.8 and 9.9 show the median $\Delta \chi^{2}$ value for $\sin \delta_{C P} \neq 0$ and the lowest $\Delta \chi^{2}$ for determining the $\mathrm{MH}$, respectively. These figures characterize the sensitivity for all values of $\delta_{C P}$. However there is a slight indication in the Capozzi et al. global fit for $\delta_{C P}$ near $-\pi / 2$. This value leads to the largest possible $\mathrm{CP}$ asymmetry. This is the best case if the $\mathrm{MH}$ is the $\mathrm{NH}$ in $\mathrm{T} 2 \mathrm{~K}, \mathrm{NO} \nu \mathrm{A}$, and $\mathrm{T} 2 \mathrm{HK}$ and also leads to very high $\mathrm{CP}$ violation sensitivity in LBNE for either MH. Figures 9.10 and 9.11 show the expected $\Delta \chi^{2}$ as a function of the $\nu: \bar{\nu}$ running ratio. Comparing these figures it is possible to plan a run ratio that balances between what is currently known about $\delta_{C P}$ and the possibility that $\delta_{C P}$ is any other value.

For T2K there is very little sensitivity to the $\mathrm{MH}$ so the $\mathrm{CP}$ violation detection sensitivity can guide the selection of the $\nu: \bar{\nu}$ run ratio. For $50 \%$ of $\delta_{C P}$ values T2K can make at least a $\Delta \chi^{2} \sim 1(\sim 1 \sigma)$ measurement with all $\nu$ running in the True $\mathrm{MH}=\mathrm{NH}$ case. If $\delta_{C P}=-\pi / 2$ then the same optimal ratio, 1:0, gives the best $\Delta \chi^{2}$ of $\sim 5$ for True $\mathrm{MH}=\mathrm{NH}$. In the True $\mathrm{MH}=\mathrm{IH}$ case $\mathrm{T} 2 \mathrm{~K}$ suffers from degeneracies between $\delta_{C P}$ and the $\mathrm{MH}$ if $\delta_{C P}-\pi / 2$.

For $\mathrm{CP}$ violation sensitivity in $\mathrm{NO} \nu \mathrm{A}$ the optimal ratio comes about from some mix of $\nu$ and $\bar{\nu}$ running in order to reduce the sensitivity to degeneracies between $\delta_{C P}$ and the MH. The median $\Delta \chi^{2}$ is just above $1 \sigma$ for running ratios near $50 \% \nu$ running. The expected $\Delta \chi^{2}$ for the $\delta_{C P}=-\pi / 2$ case is also highest for ratios near $50 \%$. The lowest $\Delta \chi^{2}$ for $\mathrm{MH}$ determination is a poor metric for $\mathrm{NO} \nu \mathrm{A}$ since the degeneracies between $\delta_{C P}$ and $\mathrm{MH}$ lead to a very small $\Delta \chi^{2}$ in the worst case. In the $\delta_{C P}=-\pi / 2$ case the best $\mathrm{MH}$ sensitivity comes from all $\nu$ running in the True $\mathrm{MH}=\mathrm{NH}$ case simply due to the increased statistics 
in $\nu$ running and the lack of degeneracies for this combination of $\mathrm{MH}$ and $\delta_{C P}$ values. The sensitivity is degraded by degeneracies again in the True $\mathrm{MH}=\mathrm{IH}$ case.

For LBNE the optimal ratio for $\mathrm{CP}$ violation sensitivity is between $30 \%$ and $50 \% \nu$ running considering both $\mathrm{MH}$ cases. The True $\mathrm{MH}=\mathrm{NH}$ case prefers slightly more $\nu$ running than the True $\mathrm{MH}=\mathrm{IH}$ case though the difference in median $\Delta \chi^{2}$ values is very small. The situation is similar in the $\delta_{C P}=-\pi / 2$ case. For $\mathrm{MH}$ determination the optimal ratio is $70 \%$ $\nu$ running for the True $\mathrm{MH}=\mathrm{NH}$ case and $50 \%$ for the True $\mathrm{MH}=\mathrm{IH}$ case and the optimal ratio is again very similar for the $\delta_{C P}=-\pi / 2$ case.

For T2HK the optimal ratio for $\mathrm{CP}$ violation sensitivity is between $30 \%$ and $50 \% \nu$ running considering both $\mathrm{MH}$ cases. For the $\mathrm{MH}$ determination the lowest $\Delta \chi^{2}$ metric shows very little sensitivity but in the case of $\delta_{C P}=-\pi / 2$ the optimal ratio is near $50 \%$ with the metric remaining flat for $\nu$ running between $30 \%$ to $70 \%$. 


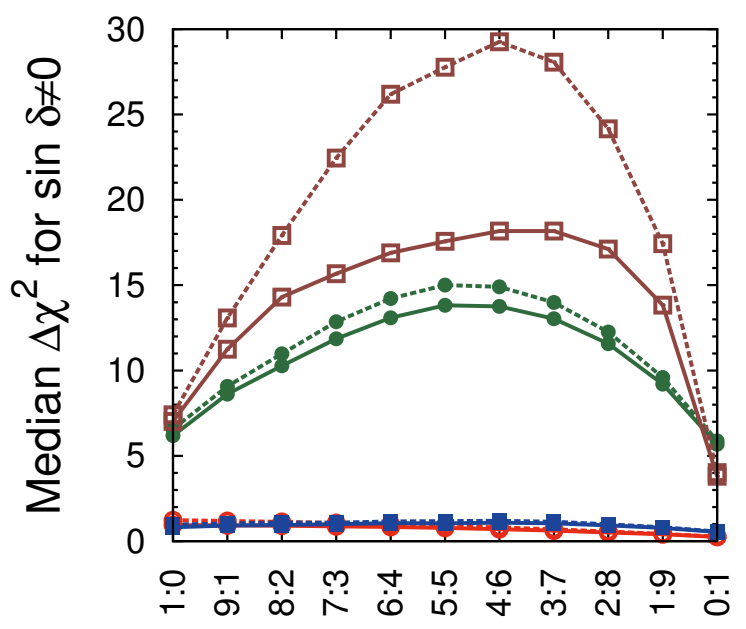

$v: \bar{v}$

(A) True $\mathrm{MH}=\mathrm{NH}$

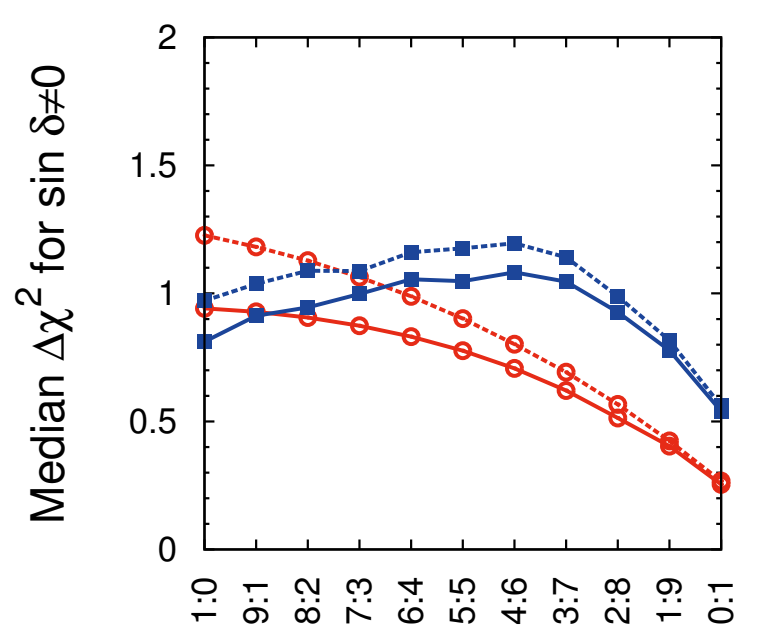

$v: \bar{v}$

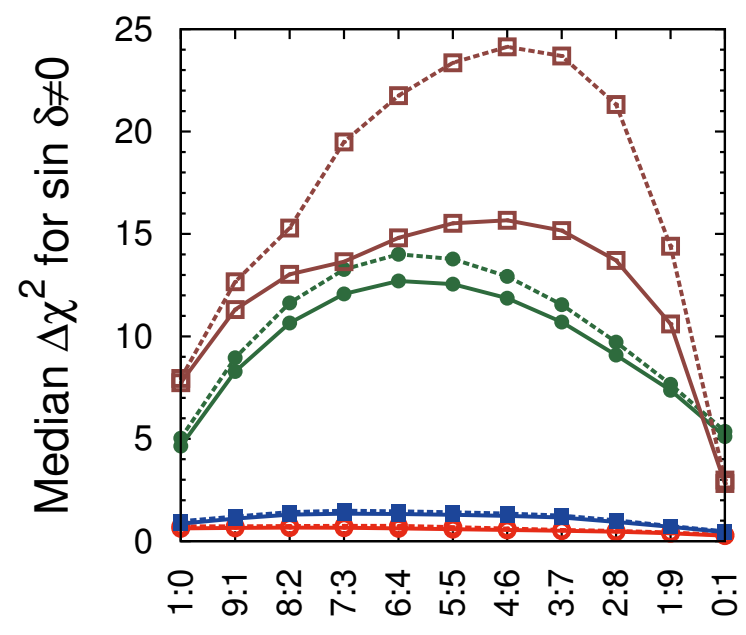

$v: \bar{v}$

(B) True $\mathrm{MH}=\mathrm{IH}$

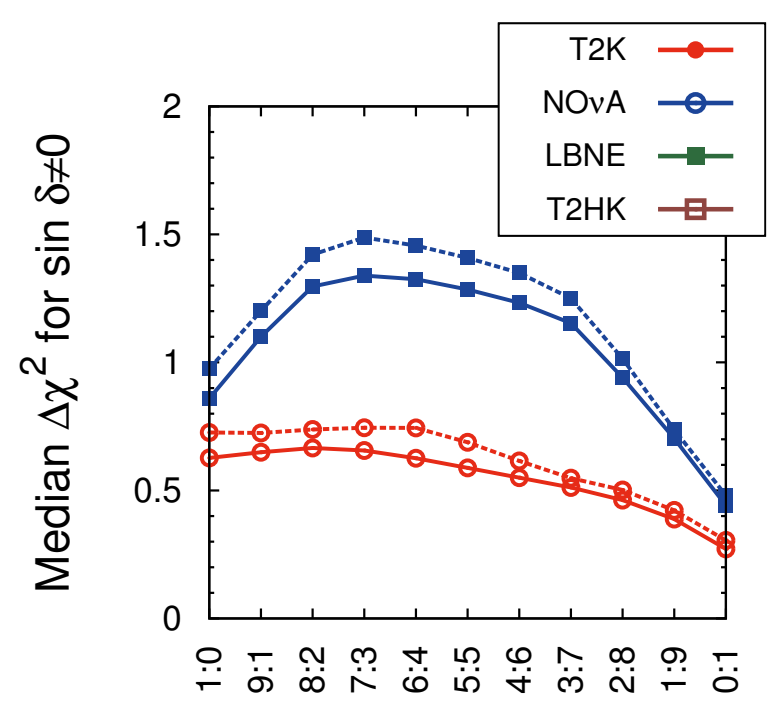

$v: \bar{v}$

(c) True $\mathrm{MH}=\mathrm{NH}$ (zoomed)

(D) True $\mathrm{MH}=\mathrm{IH}$ (zoomed)

Figure 9.8. Median $\Delta \chi^{2}$ for $\mathrm{CP}$ violation as a function of $\nu: \bar{\nu}$ running ratio for True $\mathrm{MH}=\mathrm{NH}$ (left) and $\mathrm{IH}$ (right) for each experiment. Dashed(solid) curves are without(with) normalization systematics at the nominal levels. The other oscillation parameters and the $\mathrm{MH}$ are profiled. 


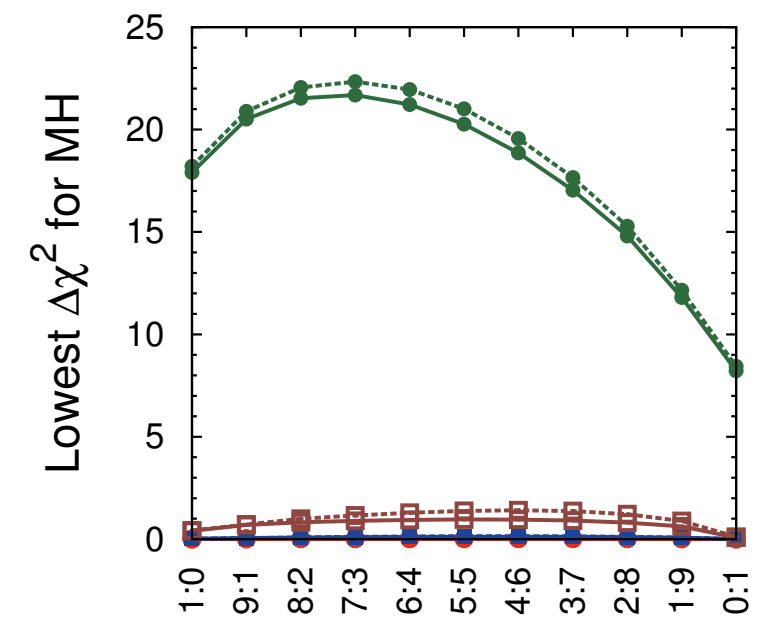

$v: \bar{v}$

(A) True $\mathrm{MH}=\mathrm{NH}$

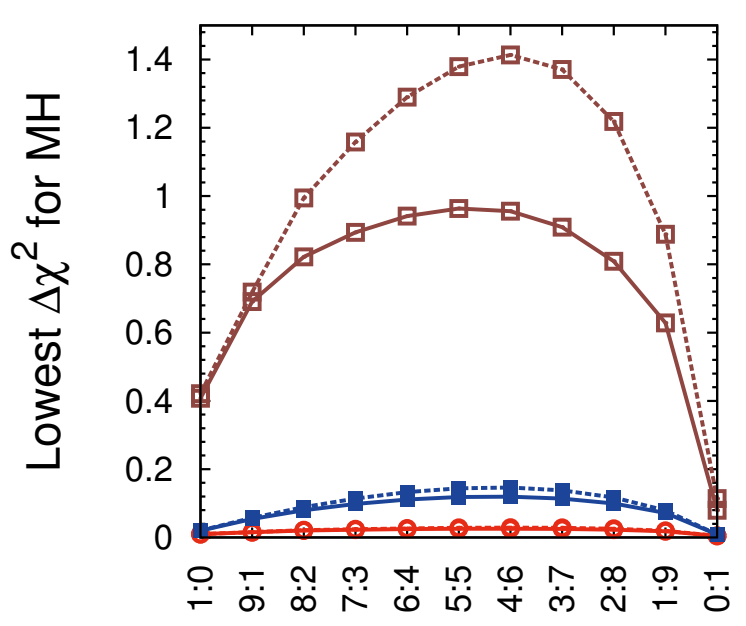

$v: \bar{v}$

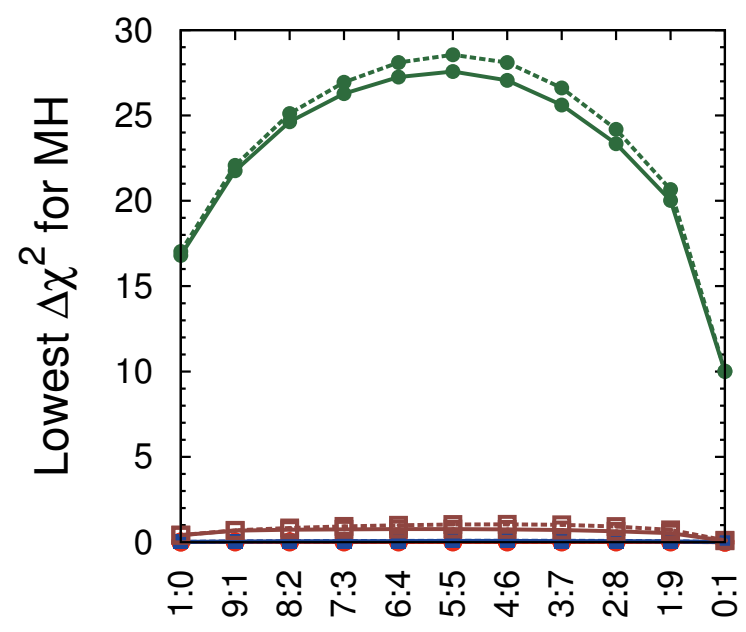

$v: \bar{v}$

(в) True $\mathrm{MH}=\mathrm{IH}$

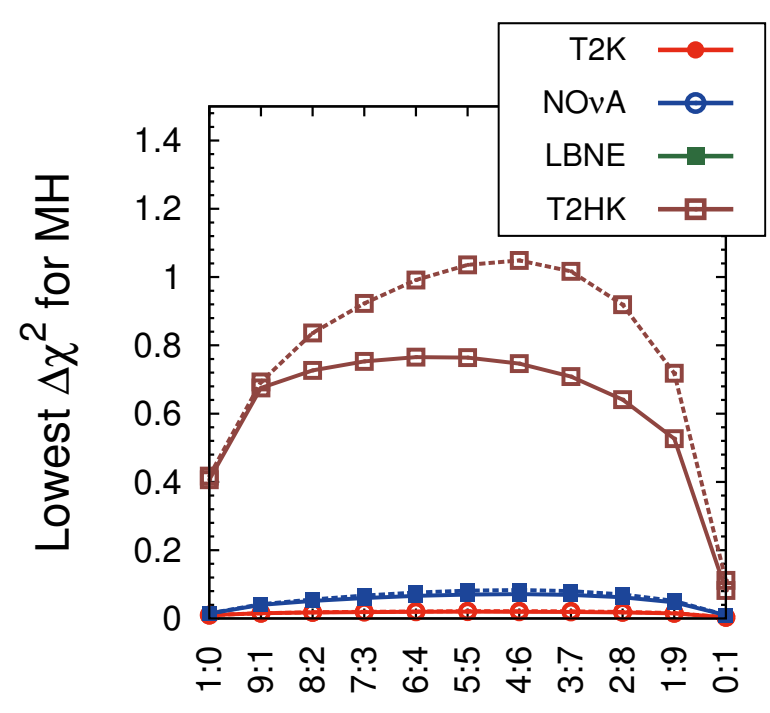

$v: \bar{v}$

(D) True $\mathrm{MH}=\mathrm{IH}$ (zoomed)

(c) True $\mathrm{MH}=\mathrm{NH}$ (zoomed)

FIgURE 9.9. Lowest $\Delta \chi^{2}$ for MH determination as a function of $\nu: \bar{\nu}$ running ratio for True $\mathrm{MH}=\mathrm{NH}$ (left) and $\mathrm{IH}$ (right) for each experiment. Dashed(solid) curves are without(with) normalization systematics at the nominal levels. The other oscillation parameters are profiled. 


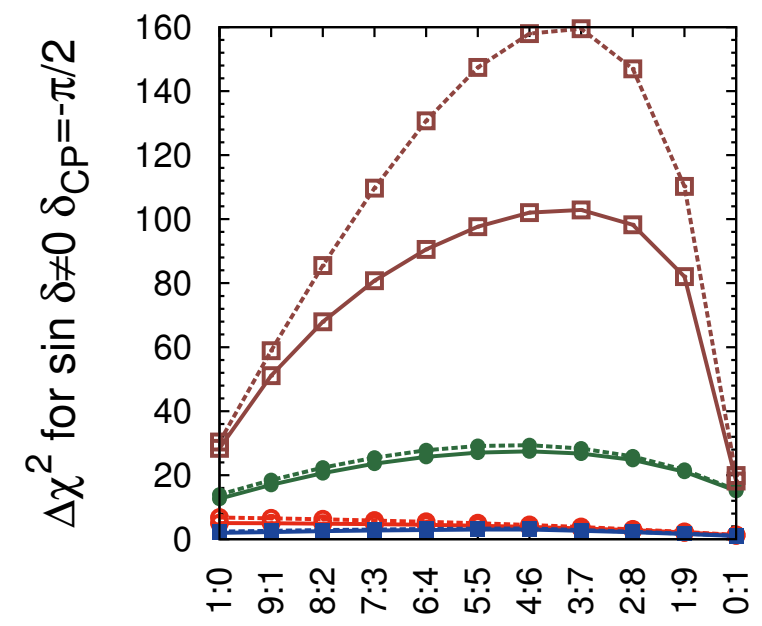

$v: \bar{v}$

(A) True $\mathrm{MH}=\mathrm{NH}$

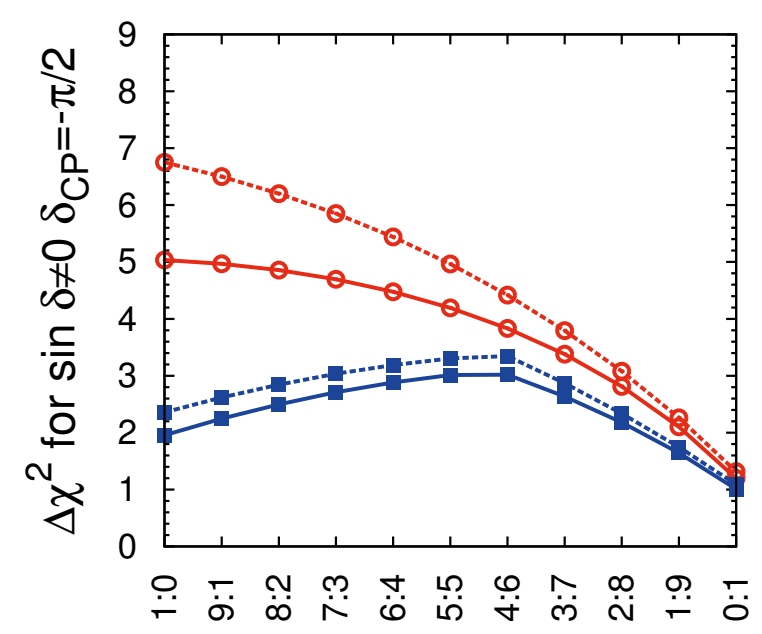

$v: \bar{v}$

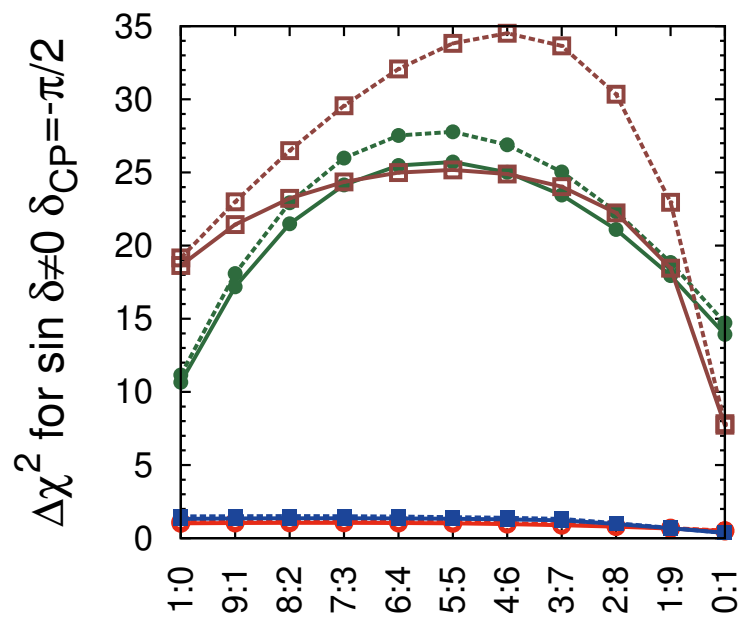

$v: \bar{v}$

(B) True $\mathrm{MH}=\mathrm{IH}$

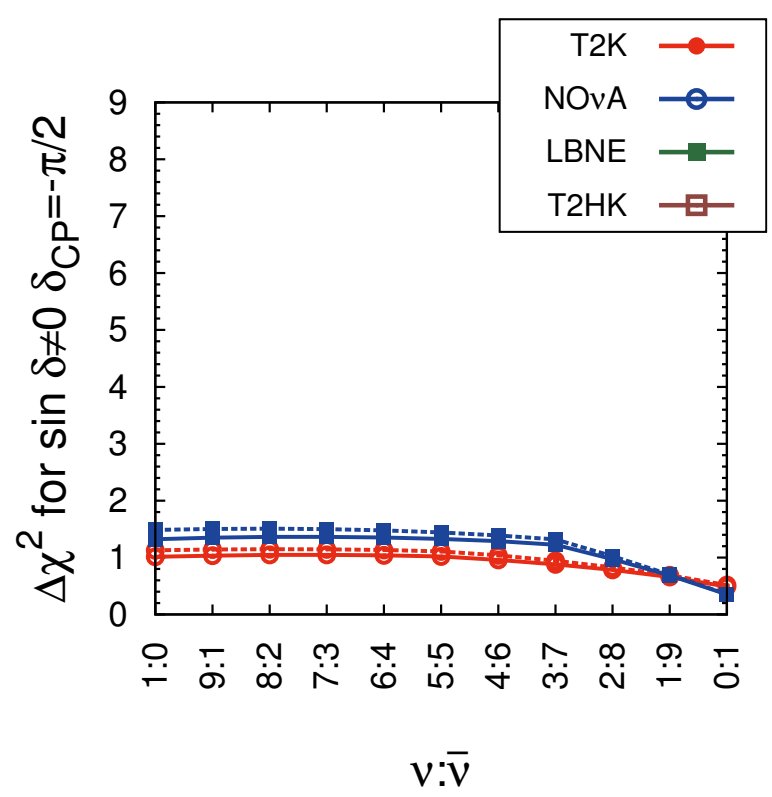

(D) True $\mathrm{MH}=\mathrm{IH}$ (zoomed)

(c) True $\mathrm{MH}=\mathrm{NH}$ (zoomed)

Figure 9.10. $\Delta \chi^{2}$ for $\mathrm{CP}$ violation as a function of $\nu: \bar{\nu}$ running ratio for True $\mathrm{MH}=\mathrm{NH}$ (left) and $\mathrm{IH}$ (right) for each experiment and with the true value of $\delta_{C P}$ at $-\pi / 2$. Dashed(solid) curves are without(with) normalization systematics at the nominal levels. The other oscillation parameters and the MH are profiled. 


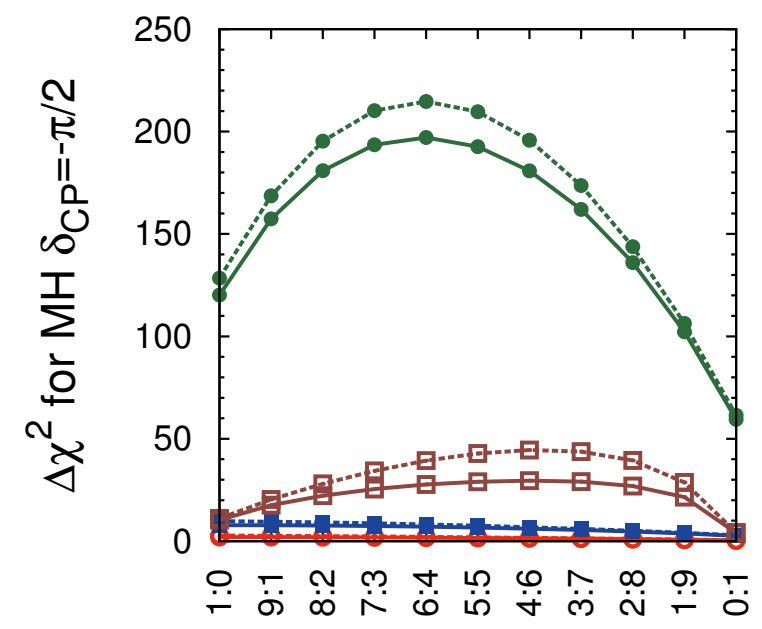

$v: \bar{v}$

(A) True $\mathrm{MH}=\mathrm{NH}$

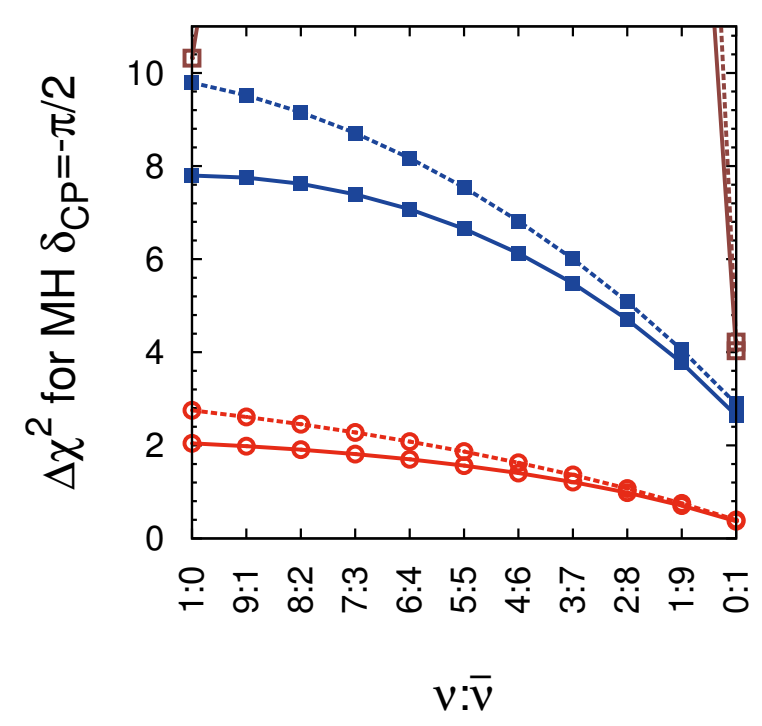

(c) True $\mathrm{MH}=\mathrm{NH}$ (zoomed)

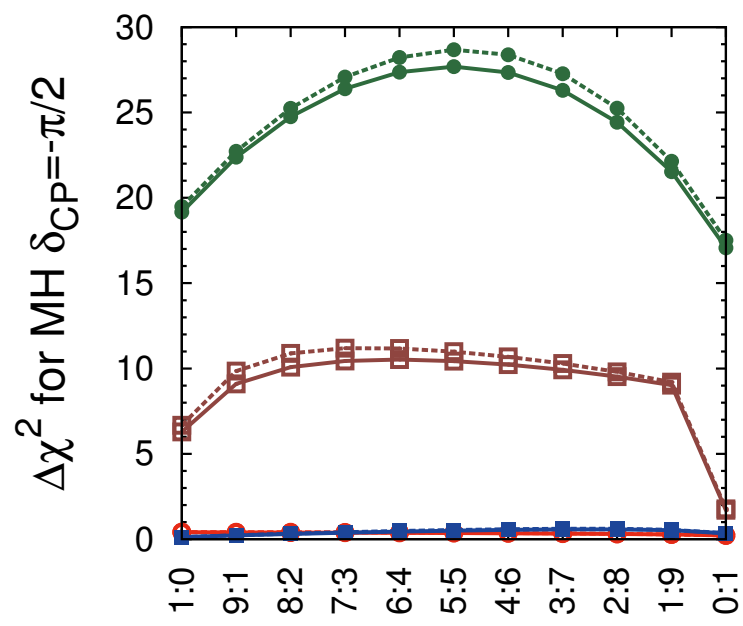

$v: \bar{v}$

(в) True $\mathrm{MH}=\mathrm{IH}$

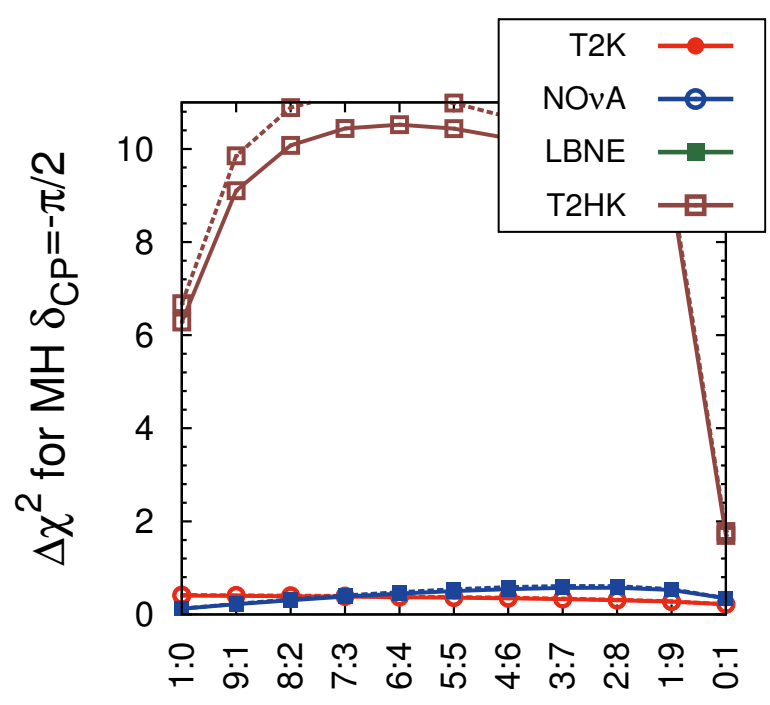

$v: \bar{v}$

(D) True $\mathrm{MH}=\mathrm{IH}$ (zoomed)

FIGURE 9.11. $\Delta \chi^{2}$ for $\mathrm{MH}$ determination as a function of $\nu: \bar{\nu}$ running ratio for True $\mathrm{MH}=\mathrm{NH}$ (left) and $\mathrm{IH}$ (right) for each experiment and with the true value of $\delta_{C P}$ at $-\pi / 2$. Dashed(solid) curves are without(with) normalization systematics at the nominal levels. The other oscillation parameters are profiled. 


\section{2. $\mathrm{T} 2 \mathrm{~K}+\mathrm{NO} \nu \mathrm{A}$ JoInt $\nu: \bar{\nu}$ Ratios}

The metrics introduced in the previous section will be used here to explore the optimal running ratios for a combined $\mathrm{T} 2 \mathrm{~K}+\mathrm{NO} \nu \mathrm{A}$ fit. Each metric is calculated for a $\mathrm{T} 2 \mathrm{~K}+\mathrm{NO} \nu \mathrm{A}$ combined analysis in an $11 \times 11$ grid of $\nu: \bar{\nu}$ run ratios: $0: 1,1: 9,2: 8,3: 7,4: 6,5: 5,6: 4,7: 3,8: 2$, 9:1, 1:0. These plots can indicate the optimal run ratios for both experiments if a combined fit is the ultimate goal.

Figure 9.12 shows the lowest $\Delta \chi^{2}$ values for mass hierarchy determination for $\nu: \bar{\nu}$ variations in a combined $\mathrm{T} 2 \mathrm{~K}+\mathrm{NO} \nu \mathrm{A}$ fit for the True $\mathrm{MH}=\mathrm{NH}$ and True $\mathrm{MH}=\mathrm{IH}$ cases. The best sensitivity for $100 \%$ of $\delta_{C P}$ values comes from $80-90 \% \nu$ running in $\mathrm{NO} \nu \mathrm{A}$ and $100 \% \nu$ running in $\mathrm{T} 2 \mathrm{~K}$ for the True $\mathrm{MH}=\mathrm{NH}$ case. For the True $\mathrm{MH}=\mathrm{IH}$ case it comes from $90 \%$ $\nu$ running in $\mathrm{NO} \nu \mathrm{A}$ and $100 \% \nu$ running in T2K. As seen in the previous section, some $\bar{\nu}$ running is needed in $\mathrm{NO} \nu \mathrm{A}$ to help with the $\delta_{C P}$ and $\mathrm{MH}$ degeneracy but $\mathrm{T} 2 \mathrm{~K}$ has little chance of resolving this degeneracy and contributes most to the combination by running all $\nu$ mode.

Similarly, Figure 9.13 shows the median $\Delta \chi^{2}$ values for $\sin \delta_{C P} \neq 0$ determination for $\nu: \bar{\nu}$ variations in a combined $\mathrm{T} 2 \mathrm{~K}+\mathrm{NO} \nu \mathrm{A}$ fit. The same optimal $\nu: \bar{\nu}$ ratios are seen to give the best sensitivity as in the MH determination case. These ratios concur with the optimal ratios for the experiments in their respective oscillation analyses. 

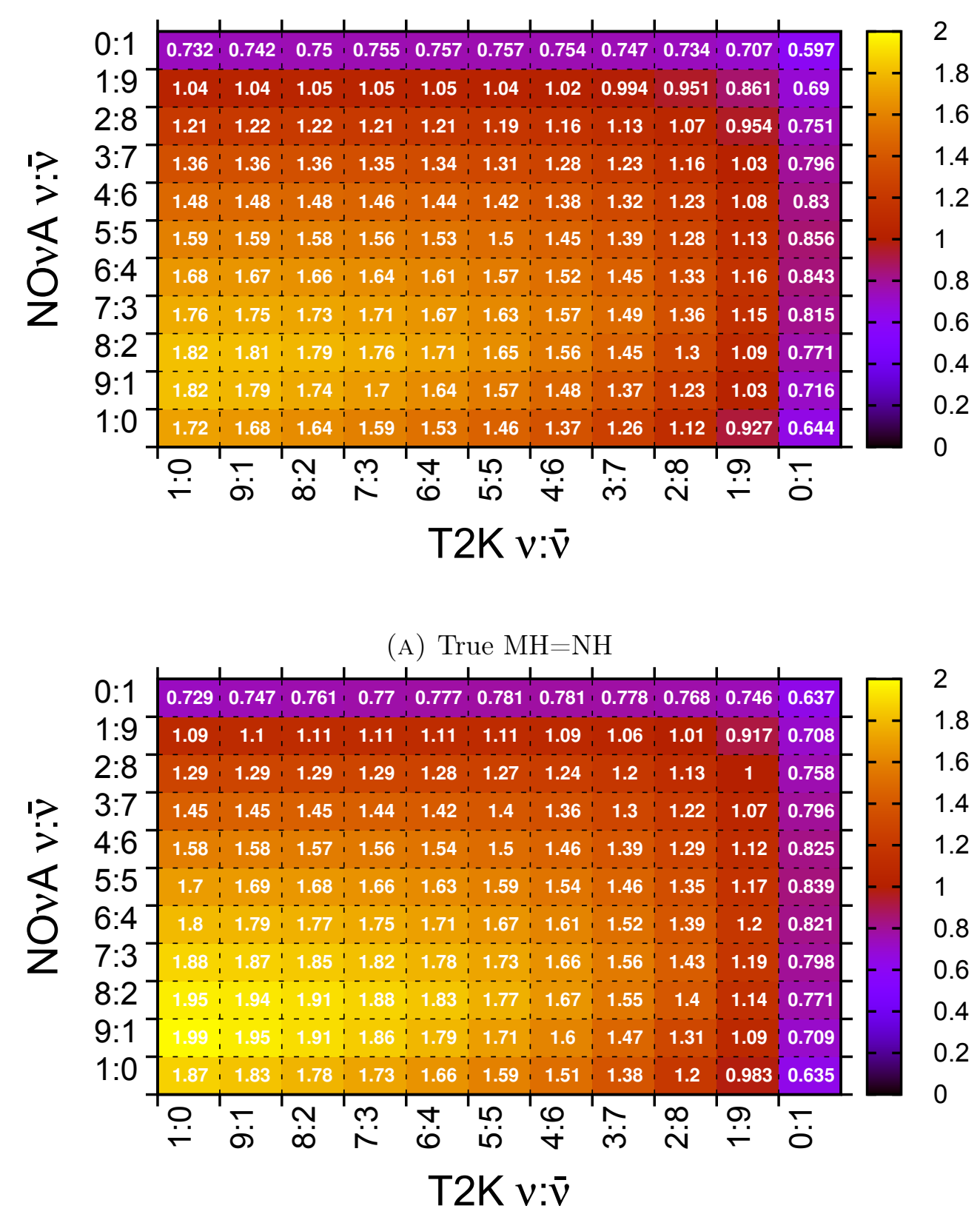

(B) True $\mathrm{MH}=\mathrm{IH}$

Figure 9.12. Lowest $\Delta \chi^{2}$ for a combined $\mathrm{T} 2 \mathrm{~K}+\mathrm{NO} \nu \mathrm{A}$ fit to determine the mass hierarchy as a function of $\nu: \bar{\nu}$ running ratio in $\mathrm{T} 2 \mathrm{~K}$ (x-axis) and $\mathrm{NO} \nu \mathrm{A}$ (y-axis). Each entry represents the $\Delta \chi^{2}$ value which gives $100 \%$ coverage in $\delta_{C P}$. The nominal assumptions for systematic uncertainties are assumed. 

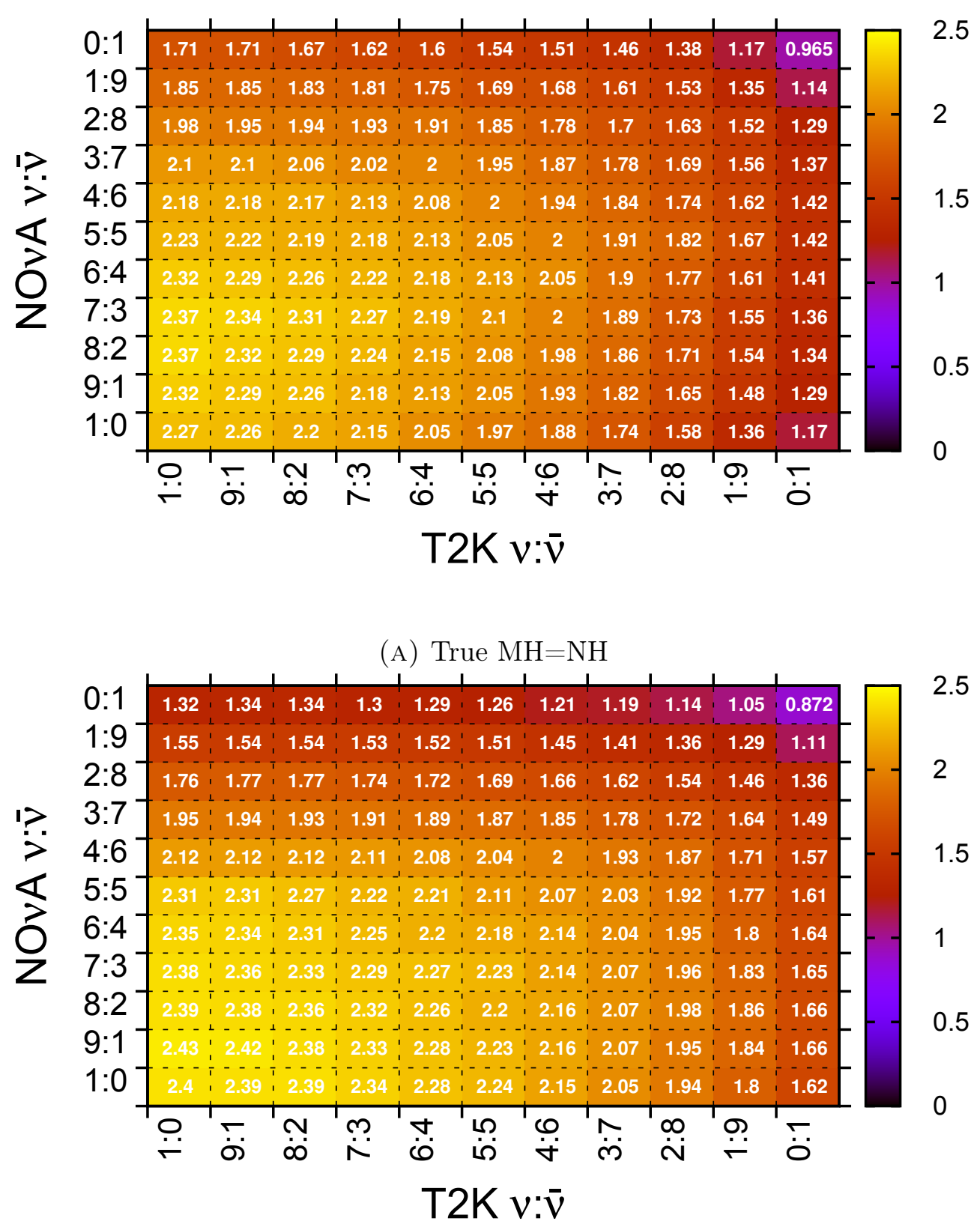

(B) True $\mathrm{MH}=\mathrm{IH}$

Figure 9.13. Median $\Delta \chi^{2}$ for $\mathrm{CP}$ violation in a combined $\mathrm{T} 2 \mathrm{~K}+\mathrm{NO} \nu \mathrm{A}$ fit. Each entry represents the $\Delta \chi^{2}$ value which gives $50 \%$ coverage in $\delta_{C P}$. The nominal assumptions for systematic uncertainties are assumed. 


\subsection{LBNE Second Oscillation Maximum}

In a wide-band neutrino beam such as in LBNE the second oscillation maximum is strongly affected by variations in $\delta_{C P}$ and the MH. This effect was described in Section 4.3.6. In this section the effects of increasing the $\nu_{\mu}$ flux below $1 \mathrm{GeV}$ will be explored. Studying the sensitivities this way motivates the pursuit of more $\nu_{\mu}$ flux at the second oscillation maximum but does not address how this might be put into practice. This study only characterizes the benefits that might be gained from either increasing the flux at the second oscillation maximum by modifying the existing beam or by running the beam in a completely different, perhaps off-axis, configuration.

To test the effects of increasing the initial $\nu_{\mu}$ flux in the neutrino beam near the second oscillation maximum a multiplier was applied to the signal and background channels below $E_{\text {true }}=1 \mathrm{GeV}$. This multiplier is applied to the true $\nu_{\mu}$ energy, before the smearing matrix is applied to map the $E_{\text {true }}$ to $E_{\text {reco }}$ as described in Section 5.4.1. Figure 9.14 shows the effects on the event spectrum, signal and background, for increasing values of this flux multiplier: 1.5, 2, 4, and 10. Table 9.1 lists the integrated numbers of signal, background, and total events for both $\nu_{e}$ appearance and $\bar{\nu}_{e}$ appearance that result from applying these flux multipliers. Increasing the $\nu_{\mu}$ flux in this way increases the signal greatly without significantly increasing the backgrounds.

Figure 9.15 shows the effects of this flux multiplier on the CP violation sensitivity. The median $\Delta \chi^{2}$ increases to $\sim 4 \sigma$ for a $300 \%$ increase in the flux. There is a more significant effect for the $\mathrm{MH}$, in Figure 9.16, especially in the worst case region of true $\delta_{C P}$ values. For even a modest doubling of the flux at the second oscillation maximum the worst case $\mathrm{MH}$ $\Delta \chi^{2}$ exceeds $\Delta \chi^{2}=25$. The addition of flux in this region breaks the degeneracy between 


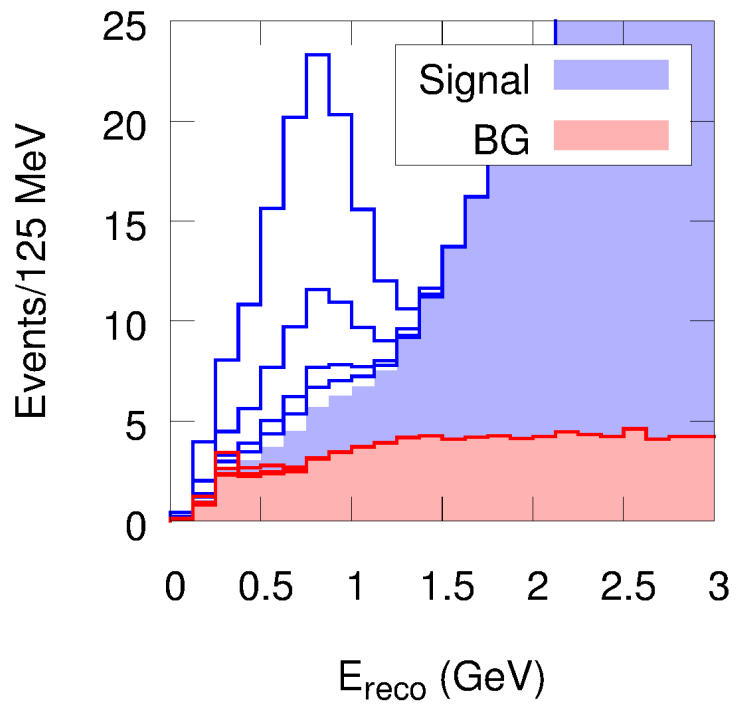

(A) $\nu_{e}$ appearance

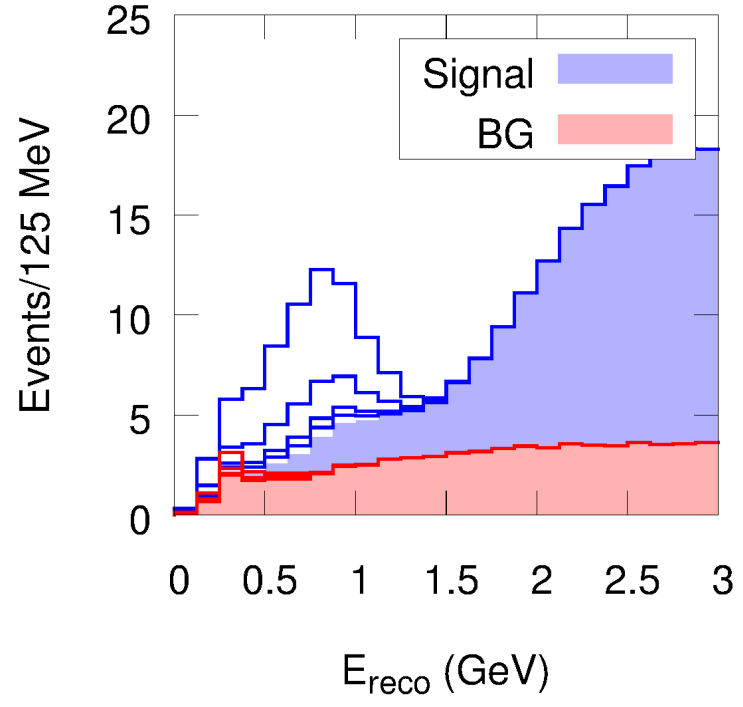

(в) $\bar{\nu}_{e}$ appearance

FIGURE 9.14. Event spectra for $\nu_{e}$ appearance and $\bar{\nu}_{e}$ appearance in LBNE for variations in the flux multiplier to increase the $\nu_{\mu}$ flux below $1 \mathrm{GeV}$. The flux is multiplied by a factor of $1.5,2,4$, and 10. The other nominal running assumptions for LBNE are assumed. The plots are zoomed in to focus on the region, in $E_{\text {reco }}$, of the second oscillation maximum.

TABLE 9.1. Table of event rates expected in LBNE for $6.0 \times 10^{21}$ POT split evenly between neutrino and antineutrino running and with initial $\nu_{\mu}$ flux multiplied by the indicated factor. The Capozzi et al. set of oscillation parameters are assumed.

\begin{tabular}{l|ccc|ccc} 
Multiplier & $\nu_{e}$ Signal & $\nu_{e}$ Bkg & $\nu_{e}$ Total & $\bar{\nu}_{e}$ Signal & $\bar{\nu}_{e}$ Bkg & $\bar{\nu}_{e}$ Total \\
\hline 1 & 501.7 & 194.2 & 695.9 & 303.0 & 147.3 & 450.3 \\
1.5 & 506.5 & 194.5 & 701.0 & 305.5 & 147.5 & 453.0 \\
2 & 511.4 & 194.6 & 706.0 & 307.9 & 147.6 & 455.5 \\
4 & 530.9 & 195.2 & 726.1 & 317.4 & 148.3 & 465.7 \\
10 & 589.6 & 197.2 & 786.8 & 346.2 & 150.2 & 496.4
\end{tabular}

the $\mathrm{MH}$ and $\delta_{C P}$ in this region because the relative oscillation probability between NH vs IH and $\delta_{C P}=\pi / 2$ vs $\delta_{C P}=-\pi / 2$ is different in the first and second oscillation maximum.

The increase in $\nu_{\mu}$ flux below $1 \mathrm{GeV}$ also helps to further distinguish between adjacent $\delta_{C P}$ values because of these opposing changes in the oscillation probability at the first and 


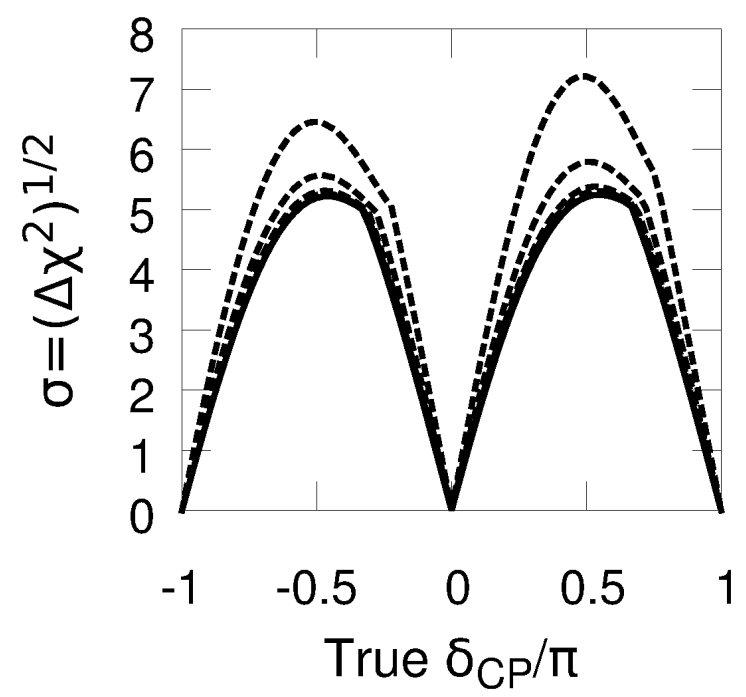

(A) True $\mathrm{MH}=\mathrm{NH}$

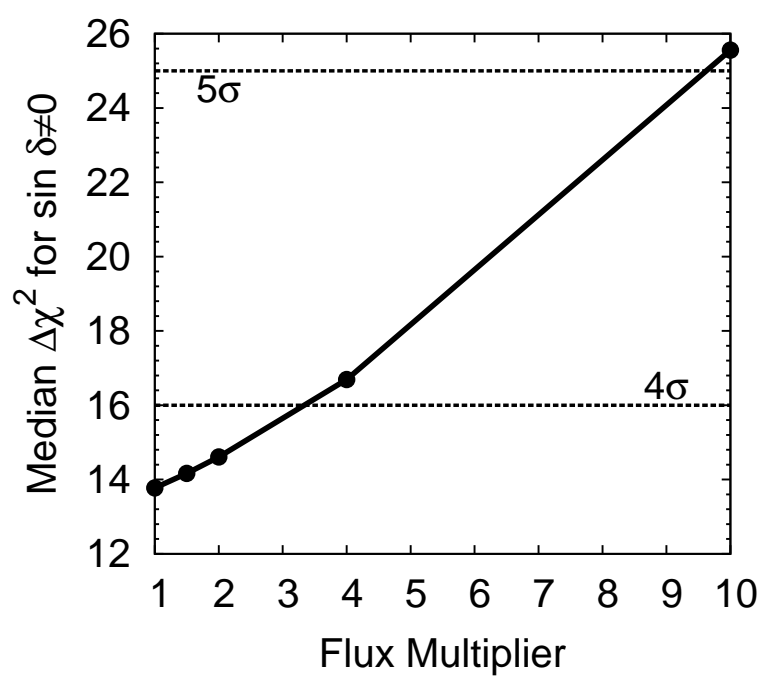

(c) True $\mathrm{MH}=\mathrm{NH}$

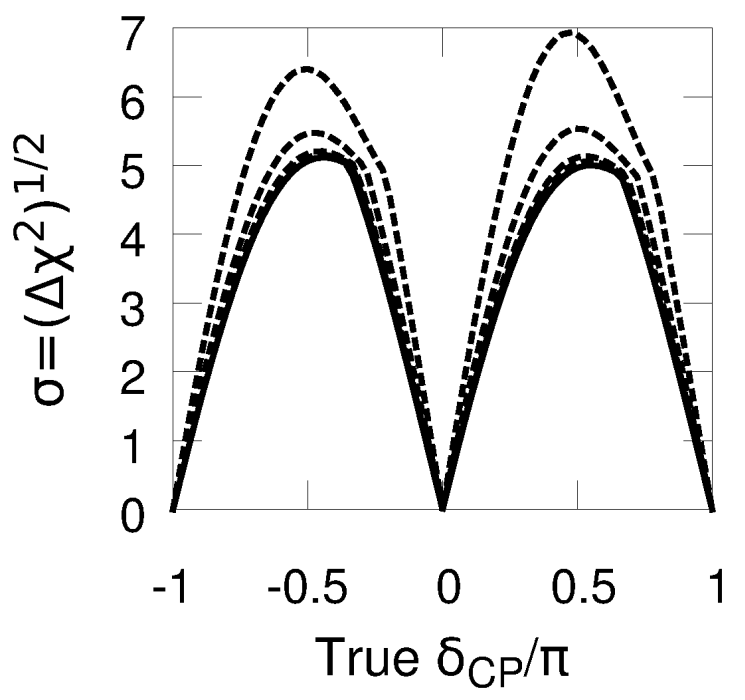

(B) True $\mathrm{MH}=\mathrm{IH}$

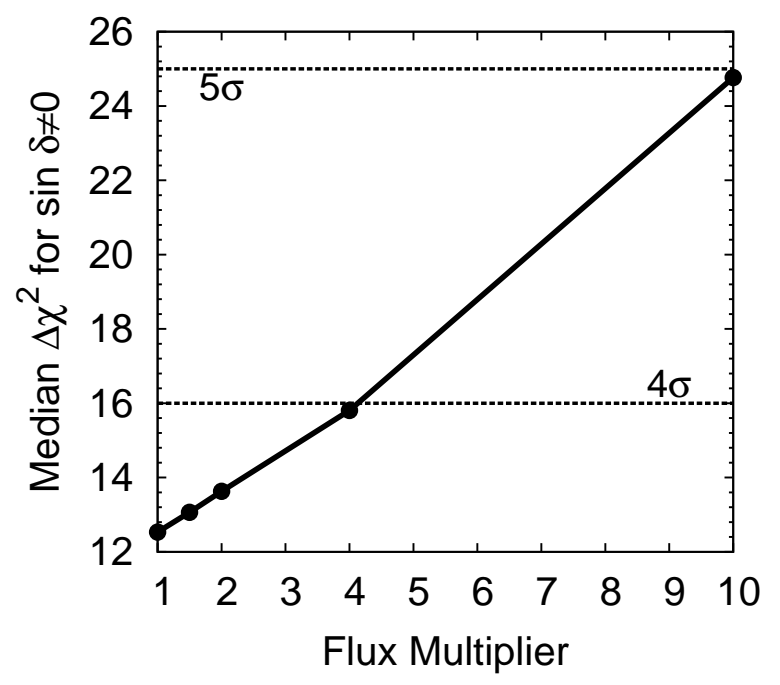

(D) True $\mathrm{MH}=\mathrm{IH}$

Figure 9.15. CP Violation sensitivity for variations in the flux multiplier to increase the $\nu_{\mu}$ flux below $1 \mathrm{GeV}$. The flux is multiplied by a factor of $1.5,2$, 4 , and 10 yielding successively higher $\Delta \chi^{2}$ values. The other nominal running assumptions and normalization systematics for LBNE are assumed. A and B show the sensitivity as a function of true $\delta_{C P}$ while plots $\mathrm{C}$ and $\mathrm{D}$ show the corresponding median $\Delta \chi^{2}$ value as a function of the flux multiplier.

second oscillation maxima. Figure 9.17 shows the resulting increased precision for measuring $\delta_{C P}=0$ and $\delta_{C P}=\pi / 2$ for the True $\mathrm{MH}=\mathrm{NH}$ and True $\mathrm{MH}=\mathrm{IH}$ cases. 


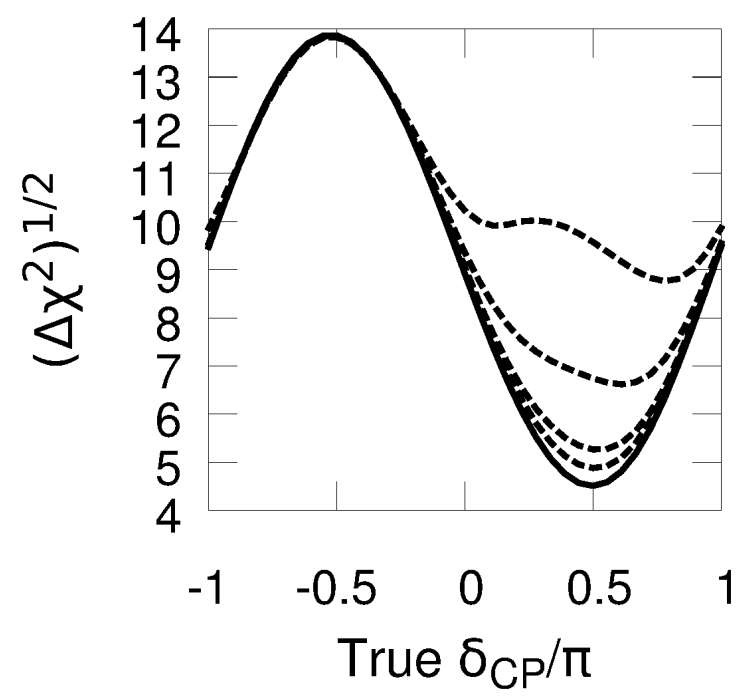

(A) True $\mathrm{MH}=\mathrm{NH}$

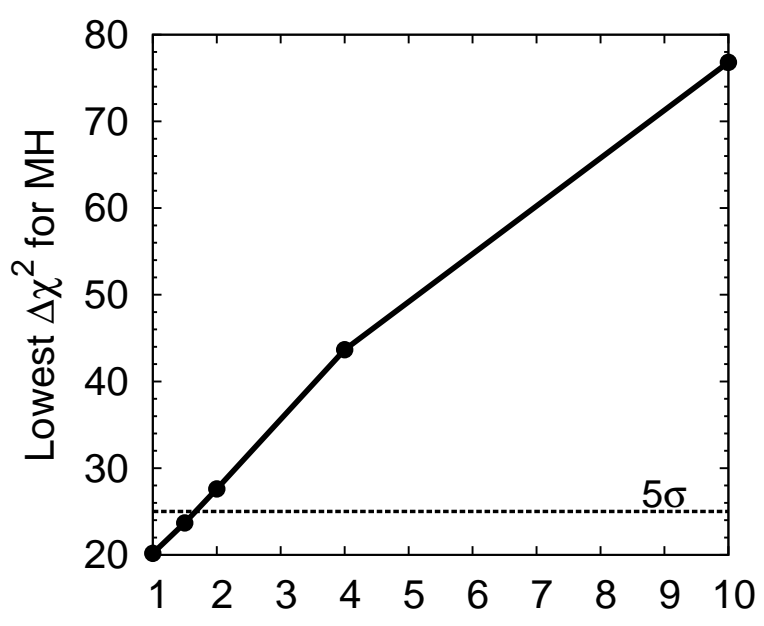

Flux Multiplier

(c) True $\mathrm{MH}=\mathrm{NH}$

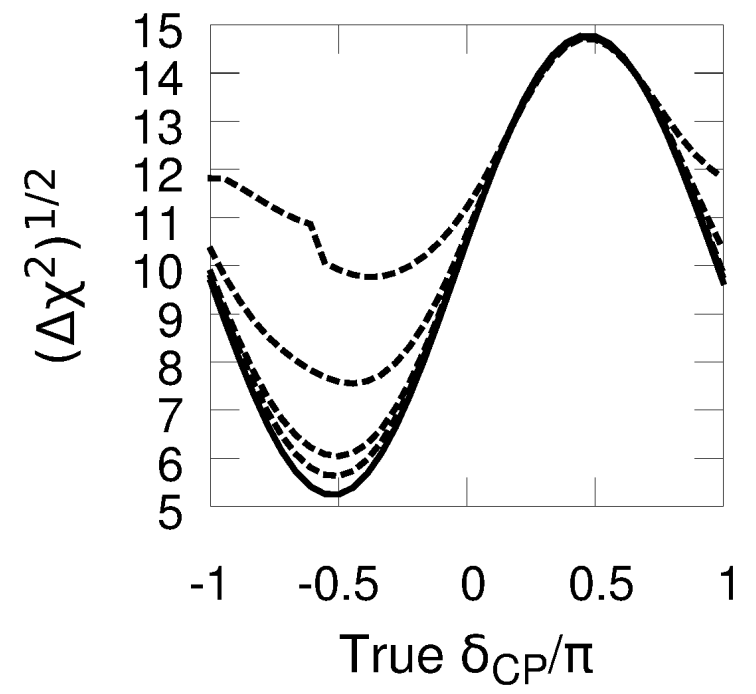

(B) True $\mathrm{MH}=\mathrm{IH}$

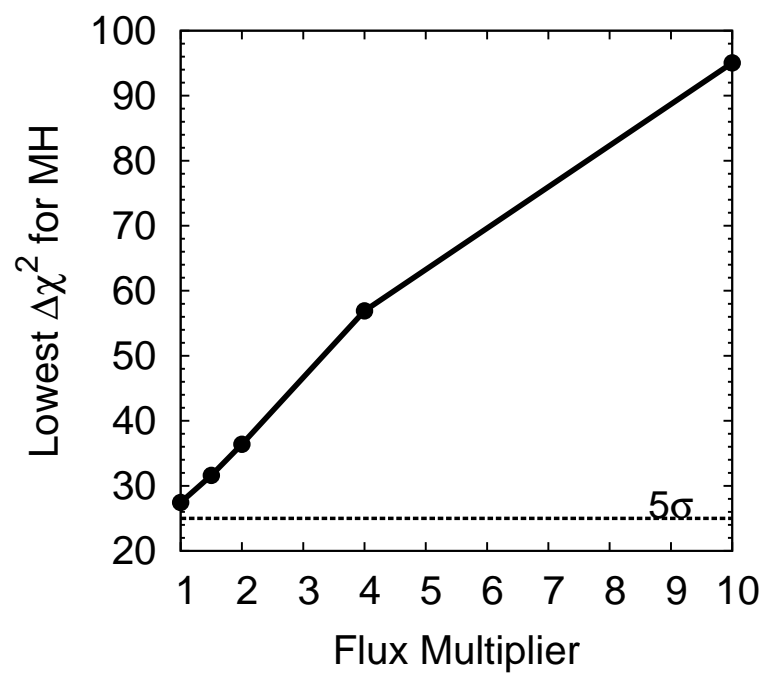

(D) True $\mathrm{MH}=\mathrm{IH}$

FiguRE 9.16. MH sensitivity for variations in the flux multiplier to increase the $\nu_{\mu}$ flux below $1 \mathrm{GeV}$. The flux is multiplied by a factor of $1.5,2,4$, and 10 yielding successively higher $\Delta \chi^{2}$ values. The other nominal running assumptions and normalization systematics for LBNE are assumed. A and B show the sensitivity as a function of true $\delta_{C P}$ while plots $\mathrm{C}$ and $\mathrm{D}$ show the corresponding lowest $\Delta \chi^{2}$ value as a function of the flux multiplier. 

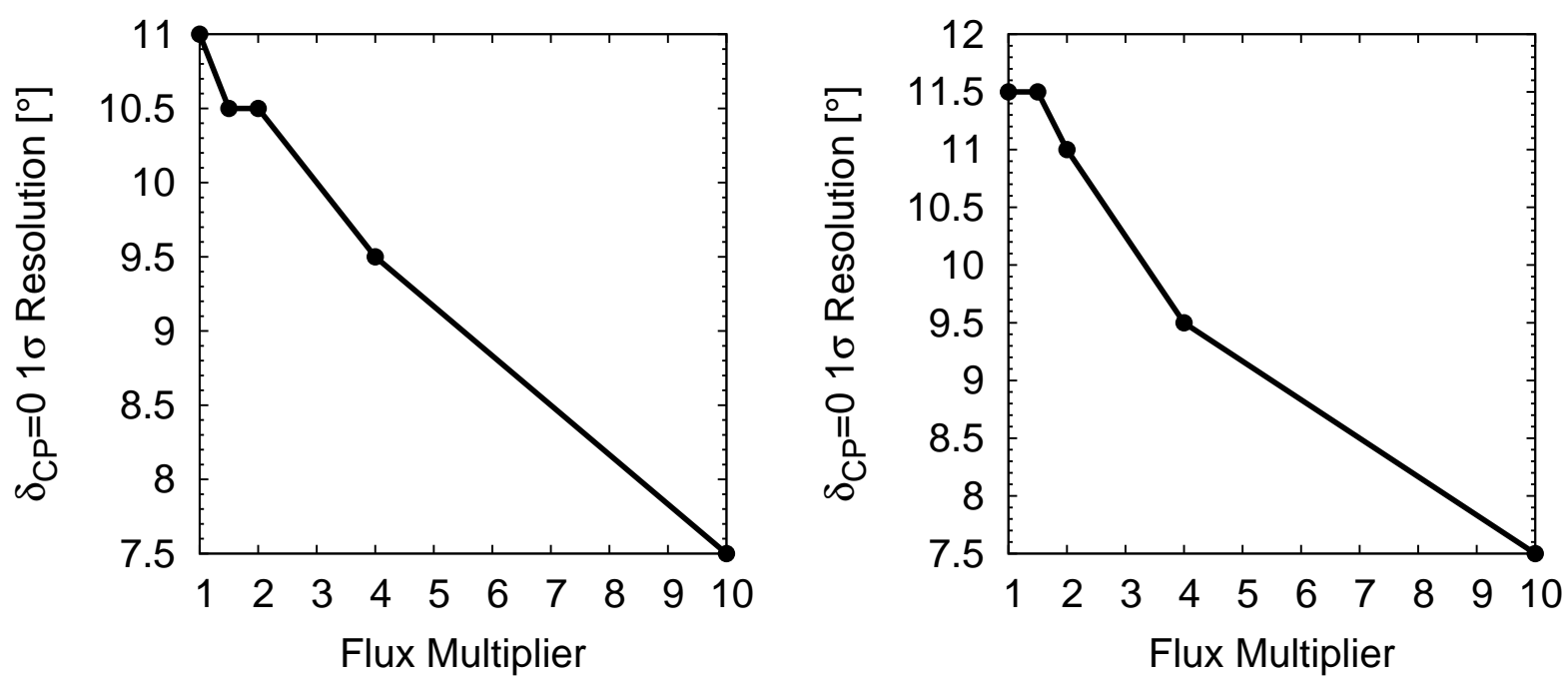

(A) True $\mathrm{MH}=\mathrm{NH}, \delta_{C P}^{\text {true }}=0$

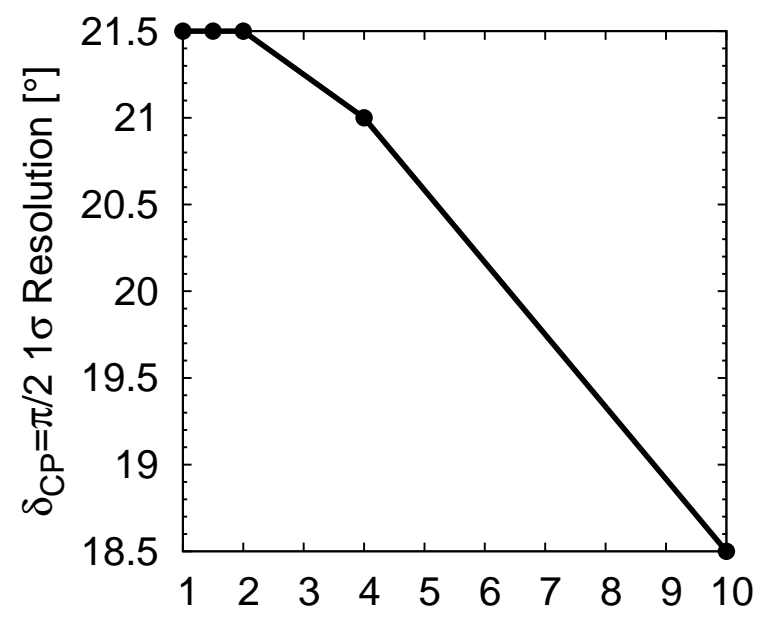

Flux Multiplier

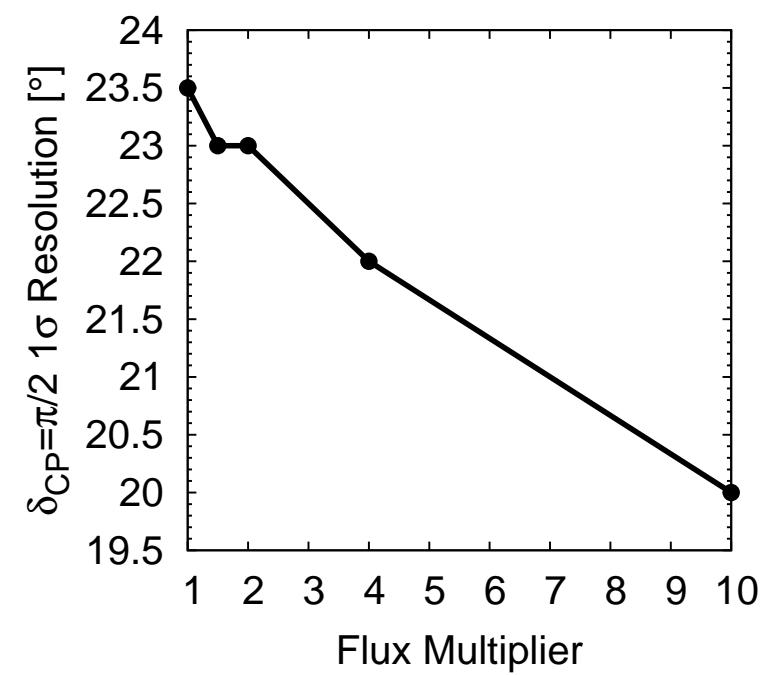

(C) True $\mathrm{MH}=\mathrm{NH}, \delta_{C P}^{\text {true }}=\pi / 2$

(D) True $\mathrm{MH}=\mathrm{IH}, \delta_{C P}^{\text {true }}=\pi / 2$

Figure 9.17. $1 \sigma \delta_{C P}$ resolution for variations in the flux multiplier to increase the $\nu_{\mu}$ flux below $1 \mathrm{GeV}$. The flux is multiplied by a factor of $1.5,2,4$, and 10 yield successively smaller $\delta_{C P}$ resolutions. The other nominal running assumptions and normalization systematics for LBNE are assumed. 


\section{CHAPTER 10}

\section{Summary \& CONCLUSIONS}

The current and next generation of long baseline neutrino oscillation experiments are searching for $\mathrm{CP}$ violation in the lepton sector and attempting to resolve the neutrino mass hierarchy as well as make precision measurements of the oscillation parameters. Their ability to do this depends on experimental design parameters that have been, or are being, selected in order to optimize sensitivity to these parameters. The work presented in this thesis estimates the sensitivity for the T2K, NO $\nu \mathrm{A}, \mathrm{LBNE}$, and T2HK experiments and explores the experimental and theoretical variables that affect this sensitivity.

This thesis has introduced the methods of computing sensitivities to the oscillation parameters in a neutrino oscillation experiment. The neutrino oscillation parameters and the external inputs used to compute event spectra for multiple samples in the far detectors for each experiment were described and the method used to compute a $\Delta \chi^{2}$ in order to quantify the ability of each experiment to distinguish two hypotheses was described. This method was then extended to include the effects of systematic uncertainties and statistical effects relevant to the oscillation physics. Using these methods and inputs, the sensitivity was also optimized with respect to the neutrino and antineutrino running times in each experiment.

Sensitivities were computed for $\mathrm{T} 2 \mathrm{~K}, \mathrm{NO} \nu \mathrm{A}, \mathrm{LBNE}$, and $\mathrm{T} 2 \mathrm{HK}$ and in some cases for a combination of $\mathrm{T} 2 \mathrm{~K}+\mathrm{NO} \nu \mathrm{A}$. Event spectra for each experiment were computed using simulated inputs for the flux, cross sections, energy resolutions, and analysis sample selection efficiencies. These event spectra were considered for $\nu$ and $\bar{\nu}$ modes in both $\nu_{e}$ appearance and $\nu_{\mu}$ disappearance samples in the far detector for each experiment. A $\Delta \chi^{2}$ was computed comparing a true event spectrum with test hypothesis spectra and this $\Delta \chi^{2}$ was minimized 
with respect to oscillation parameter uncertainties, adapted from the Capozzi et al. 2014 global fit, and with various models of the systematic uncertainties taken into account.

The $\mathrm{T} 2 \mathrm{~K}$ and $\mathrm{NO} \nu \mathrm{A}$ experiments will have their full event samples around 2020. With these full samples used in a combined $\mathrm{T} 2 \mathrm{~K}+\mathrm{NO} \nu \mathrm{A}$ fit they can constrain the neutrino oscillation parameters further and determine the octant of $\theta_{23}$ to greater than $90 \%$ CL for the current best-fit value of $\theta_{23}$. They can also begin to see hints of $\mathrm{CP}$ violation but will not be able to make a statistically significant measurement of this effect.

In one possible scenario, the LBNE collaboration projects[39] receiving the full POT used in this thesis by 2035. At that time, LBNE will have the ability to detect CP violation at the $3 \sigma$ confidence level for greater than $60 \%$ of possible $\delta_{C P}$ values, will measure the neutrino mass hierarchy at the $5 \sigma$ level for $84 \%$ of $\delta_{C P}$ values, and determine the octant of $\theta_{23}$ to greater than $3 \sigma$ CL for the current best-fit value of $\theta_{23}$.

T2HK will also have the ability to detect $\mathrm{CP}$ violation with $3 \sigma$ significance for greater than $60 \%$ of possible $\delta_{C P}$ values. T2HK will be limited in ability to resolve the mass hierarchy with long baseline neutrino oscillations due to degeneracies between the oscillation parameters. However, with a very large fiducial volume the T2HK experiment expects sensitivity to the mass hierarchy at better than $3 \sigma$ for $46 \%$ of $\delta_{C P}$ values from atmospheric neutrino samples in the HK detector over 10 years|43|.

These studies of the sensitivities in current and future experiments inform the design and planning of run periods for the experiments and facilitates the communication of the goals and capabilities of the experiments to outside observers and especially decision makers. These sensitivity studies were used by the LBNE collaboration to support the case for constructing LBNE[39||48||41||49||50| and to garner international support and collaborators. Studies on 
the combination of $\mathrm{T} 2 \mathrm{~K}$ and $\mathrm{NO} \nu \mathrm{A}$ in a combined fit were used by the $\mathrm{T} 2 \mathrm{~K}$ collaboration to support requests for future run periods within the T2K experiment|51].

It is an exciting time in neutrino physics with the recent discovery of a large $\theta_{13}$ value opening up the possibility of discovering CP violation in the lepton sector and with two running experiments ( $\mathrm{T} 2 \mathrm{~K}$ and $\mathrm{NO} \nu \mathrm{A}$ ) both collecting data that will contribute to this search. Planning for future experiments is underway and these future experiments are capable of measuring the neutrino mass hierarchy and making a definitive measurement of $\mathrm{CP}$ violation should it exist. These future experiments also lead to an era of precision measurements on the neutrino oscillation parameters that contribute to further establishing the parameters of the three neutrino paradigm. 


\section{BIBLIOGRAPHY}

[1] Particle Data Group Collaboration, J. Beringer et al., "Review of Particle Physics (RPP)", Phys.Rev. D86 (2012) 010001.

[2] M. Freund, "Analytic approximations for three neutrino oscillation parameters and probabilities in matter", Phys.Rev. D64 (2001) 053003, arXiv:hep-ph/0103300.

[3] M. Gonzalez-Garcia and Y. Nir, "Neutrino masses and mixing: Evidence and implications", Rev.Mod.Phys. 75 (2003) 345-402, arXiv:hep-ph/0202058.

[4] P. Huber, M. Lindner, and W. Winter, "Simulation of long-baseline neutrino oscillation experiments with GLoBES: (General Long Baseline Experiment Simulator)", Computer Physics Communications 167 (2005), no. 3, 195 - 202.

[5] P. Huber, J. Kopp, M. Lindner, M. Rolinec, and W. Winter, "New features in the simulation of neutrino oscillation experiments with GLoBES 3.0: (General Long Baseline Experiment Simulator)", Computer Physics Communications 177 (2007), no. $5,432-438$.

[6] W. Marciano and Z. Parsa, "Intense neutrino beams and leptonic CP violation", Nucl.Phys.Proc.Suppl. 221 (2011) 166-172, arXiv:hep-ph/0610258.

[7] A. de Gouvea, J. Jenkins, and B. Kayser, "Neutrino mass hierarchy, vacuum oscillations, and vanishing |U(e3)|", Phys.Rev. D71 (2005) 113009, arXiv:hep-ph/0503079

[8] G. Feldman, J. Hartnell, and T. Kobayashi, "Long-baseline neutrino oscillation experiments", Adv.High Energy Phys. 2013 (2013) 475749, arXiv:1210.1778.

[9] G. Giacomelli, "Atmospheric $\nu$ and Long Baseline $\nu$ experiments II", arXiv:1102.0650 
[10] A. B. McDonald, "Solar neutrinos", New J.Phys. 6 (2004) 121, arXiv:astro-ph/0406253

[11] F. Capozzi, G. Fogli, E. Lisi, A. Marrone, D. Montanino, et al., "Status of three-neutrino oscillation parameters, circa 2013", Phys.Rev. D89 (2014) 093018,

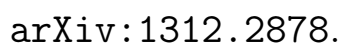

[12] KamLAND Collaboration, A. Gando et al., "Reactor On-Off Antineutrino Measurement with KamLAND", arXiv:1303.4667.

[13] Super-Kamiokande Collaboration, A. Himmel, "Recent Atmospheric Neutrino Results from Super-Kamiokande", arXiv:1310.6677.

[14] Super-Kamiokande Collaboration, R. Wendell et al., "Atmospheric neutrino oscillation analysis with sub-leading effects in Super-Kamiokande I, II, and III", Phys.Rev. D81 (2010) 092004, arXiv:1002.3471.

[15] Daya Bay Collaboration, F. An et al., "Spectral measurement of electron antineutrino oscillation amplitude and frequency at Daya Bay", Phys.Rev.Lett. 112 (2014) 061801, arXiv:1310.6732.

[16] Daya Bay Collaboration, F. An et al., "Improved Measurement of Electron Antineutrino Disappearance at Daya Bay", Chin.Phys. C37 (2013) 011001, $\operatorname{arXiv:1210.6327.}$

[17] H. Minakata, H. Nunokawa, S. J. Parke, and R. Z. Funchal, "Determining neutrino mass hierarchy by precision measurements in electron and muon neutrino disappearance experiments", Phys. Rev. D 74 Sep (2006) 053008.

[18] RENO Collaboration, J. Ahn et al., "Observation of Reactor Electron Antineutrino Disappearance in the RENO Experiment", Phys.Rev.Lett. 108 (2012) 191802, arXiv: 1204.0626 
[19] Double Chooz Collaboration, Y. Abe et al., "Reactor electron antineutrino disappearance in the Double Chooz experiment", Phys.Rev. D86 (2012) 052008, arXiv:1207.6632.

[20] T2K Collaboration, K. Abe et al., "Observation of Electron Neutrino Appearance in a Muon Neutrino Beam", Phys.Rev.Lett. 112 (2014) 061802, arXiv:1311.4750.

[21] T2K Collaboration, K. Abe et al., "Measurement of Neutrino Oscillation Parameters from Muon Neutrino Disappearance with an Off-axis Beam", Phys.Rev.Lett. 111 (2013) 211803, arXiv:1308.0465.

[22] MINOS Collaboration, P. Adamson et al., "Electron neutrino and antineutrino appearance in the full MINOS data sample", Phys.Rev.Lett. 110 (2013), no. 17, 171801, arXiv: 1301.4581.

[23] MINOS Collaboration, P. Adamson et al., "Measurement of Neutrino and Antineutrino Oscillations Using Beam and Atmospheric Data in MINOS", Phys.Rev.Lett. 110 (2013) 251801, arXiv:1304.6335.

[24] MINOS Collaboration, J. Coelho, "Results and prospects from MINOS and MINOS+", 2013. Talk at NuFact 2013, XV International Workshop on Neutrino Factories, Super Beams and Beta Beams (Bejing, China, 2013).

[25] MINOS Collaboration, P. Adamson et al., "Combined analysis of $\nu_{\mu}$ disappearance and $\nu_{\mu} \rightarrow \nu_{e}$ appearance in MINOS using accelerator and atmospheric neutrinos", arXiv:1403.0867.

[26] M. Martini, M. Ericson, and G. Chanfray, "Neutrino energy reconstruction problems and neutrino oscillations", Phys.Rev. D85 (2012) 093012, arXiv: 1202.4745.

[27] S. E. Kopp, "Accelerator-based neutrino beams", Phys.Rept. 439 (2007) 101-159, arXiv: physics/0609129 
[28] G. Danby, J. Gaillard, K. A. Goulianos, L. Lederman, N. B. Mistry, et al., "Observation of High-Energy Neutrino Reactions and the Existence of Two Kinds of Neutrinos", Phys.Rev.Lett. 9 (1962) 36-44.

[29] A. K. Mann and H. Primakoff, "Neutrino oscillations and the number of neutrino types", Phys. Rev. D 15 Feb (1977) 655-665.

[30] T2K Collaboration, "T2K Press Release". http://t2k-experiment.org/wp-content/uploads/NuePressReleaseV2.pdf

[31] T2K Collaboration, K. Abe et al., "The T2K Neutrino Flux Prediction", Phys.Rev. D87 (2013) 012001, arXiv:1211.0469.

[32] NOvA Collaboration, D. Ayres et al., "The NOvA Technical Design Report", 2007. FERMILAB-DESIGN-2007-01.

[33] H. Gallagher, G. Garvey, and G. Zeller, "Neutrino-nucleus interactions", Ann.Rev.Nucl.Part.Sci. 61 (2011) 355-378.

[34] K2K Collaboration, M. Ahn et al., "Measurement of Neutrino Oscillation by the K2K Experiment", Phys.Rev. D74 (2006) 072003, arXiv:hep-ex/0606032.

[35] T2K Collaboration, K. Abe et al., "The T2K Experiment", Nucl.Instrum.Meth. A659 (2011) 106-135, arXiv:1106.1238.

[36] T2K Collaboration, K. Abe et al., "Neutrino Oscillation Physics Potential of the T2K Experiment", arXiv: 1409.7469

[37] NOvA Collaboration, "Talk at LLWI", 2014. http://nova-docdb.fnal .gov/cgi-bin/ShowDocument?docid=10770.

[38] "GLoBES Pre-defined Experiment Files", 2012. http://www.mpi-hd.mpg.de/personalhomes/globes/experiments.html. 
[39] LBNE Collaboration, C. Adams et al., "Scientific Opportunities with the Long-Baseline Neutrino Experiment", arXiv:1307.7335.

[40] Sandbox Studio, Chicago, "Deconstruction: LBNE", 2013. http://www . symmetrymagazine.org/image/lbne-deconstruction

[41] LBNE Collaboration, M. Bass et al., "Baseline optimization for the measurement of $\mathrm{CP}$ violation and mass hierarchy in a long-baseline neutrino oscillation experiment", $\operatorname{arXiv:1311.0212.}$

[42] Hyper-Kamiokande Working Group, "Hyper-Kamiokande Physics Opportunities",

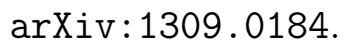

[43] K. Abe, T. Abe, H. Aihara, Y. Fukuda, Y. Hayato, et al., "Letter of Intent: The Hyper-Kamiokande Experiment — Detector Design and Physics Potential", $\operatorname{arXiv:1109.3262.}$

[44] T2HK Collaboration, "A Long Baseline Neutrino Oscillation Experiment Using J-PARC Neutrino Beam and Hyper-Kamiokande", 2014. http://j-parc.jp/researcher/Hadron/en/pac_1405/pdf/P58_2014_2.pdf.

[45] G. Cowan, K. Cranmer, E. Gross, and O. Vitells, "Asymptotic formulae for likelihood-based tests of new physics", European Physical Journal C 71 (2011) 1554, arXiv: 1007.1727

[46] LAGUNA-LBNO Collaboration, S. Agarwalla et al., "The mass-hierarchy and CP-violation discovery reach of the LBNO long-baseline neutrino experiment", JHEP 1405 (2014) 094, arXiv:1312.6520.

[47] X. Qian, A. Tan, W. Wang, J. Ling, R. McKeown, et al., "Statistical Evaluation of Experimental Determinations of Neutrino Mass Hierarchy", Phys.Rev. D86 (2012) 113011, arXiv: 1210.3651 
[48] M. Bass, D. Cherdack, and R. J. Wilson, "Future Neutrino Oscillation Sensitivities for LBNE", arXiv: 1310.6812

[49] A. S. Kronfeld, R. S. Tschirhart, U. Al-Binni, W. Altmannshofer, C. Ankenbrandt, et al., "Project X: Physics Opportunities", arXiv:1306.5009.

[50] J. Hewett, H. Weerts, R. Brock, J. Butler, B. Casey, et al., "Fundamental Physics at the Intensity Frontier", arXiv:1205.2671.

[51] T2K Collaboration, "Physics Potential and Sensitivities of T2K (T2K Report to J-PARC PAC 2013)". http://www.t2k.org/docs/pub/015.

[52] NOvA Collaboration, "Heavy Quarks and Leptons 2012 Conference Talk", 2012. http://nova-docdb.fnal.gov/cgi-bin/ShowDocument?docid=7552

[53] NOvA Collaboration, "NOvA Official Plots and Figures". http://www-nova.fnal.gov/plots_and_figures/plot_and_figures.html. 


\section{APPENDIX A}

\section{NO $\nu$ A Event Rate Reproductions}

The ability to reproduce $\mathrm{NO} \nu \mathrm{A}$ sensitivities is tested by comparisons of numbers of events from official $\mathrm{NO} \nu \mathrm{A}$ documents with those produced using the GLoBES NO$\nu$ A files. Events per channel comparisons are provided in Table A.1. The signal, NC background, $\nu_{\mu} \mathrm{CC}$, and $\nu_{e}$ CC GLoBES efficiencies were tuned to match so that there is no difference between the Official and GLoBES numbers of events. Note that in $\bar{\nu}$ mode the efficiencies were tuned to match assuming that wrong sign contamination was not included in the official totals. The event spectra that result from the GLoBES configuration files are shown in Fig. A.2 for $\nu_{e}$ appearance and $\nu_{\mu}$ disappearance event selections in $\nu$ and $\bar{\nu}$ modes.

TABLE A.1. Comparisons of expected number of $\nu_{e}$ appearance signal and background events between $\mathrm{NO} \nu \mathrm{A}$ official (from [52]) and NO $\nu \mathrm{A}$ GLoBES. The NO $\nu \mathrm{A}$ GLoBES files have been tuned so that these match exactly for $\nu_{e}$ appearance. The mismatch for the Total Bkg field between lines one and two are due to rounding.

\begin{tabular}{lccccc}
\hline Beam & Signal & NC Bkg & $\nu_{\mu} \mathrm{CC}$ & $\nu_{e}$ CC & Total Bkg \\
\hline Official $\nu$ & 72.6 & 20.8 & 5.2 & 8.4 & 34.5 \\
GLoBES $\nu$ & 72.6 & 20.8 & 5.2 & 8.4 & 34.4 \\
Official $\bar{\nu}$ & 33.8 & 10.6 & 0.7 & 5.0 & 16.3 \\
GLoBES $\bar{\nu}$ & 33.8 & 10.6 & 0.7 & 5.0 & 16.3 \\
\hline
\end{tabular}


The accuracy of the GLoBES configuration files for $\mathrm{NO} \nu \mathrm{A}$ is tested by attempting to reproduce the official $\mathrm{NO} \nu$ A sensitivity plots. Each plot (Figs. A.1 through A.3) in this section includes a digitized version of a $\mathrm{NO} \nu \mathrm{A}$ plot from the "official $\mathrm{NO} \nu \mathrm{A}$ plots and figures" website [53]. Unless otherwise specified the reproductions use oscillation parameter values corresponding to the $\mathrm{NO} \nu \mathrm{A}$ plot: $\sin ^{2}\left(2 \theta_{13}\right)=0.095, \sin ^{2}\left(\theta_{23}\right)=0.5$. The NO$\nu$ A GLoBES sensitivities are relatively close to the official $\mathrm{NO} \nu \mathrm{A}$ sensitivities.

In the case of the $1 \sigma$ allowed regions in $2 \sin ^{2}\left(\theta_{23}\right)$ vs $\delta_{C P} /(2 \pi)$ in Fig. A. 1 the $\mathrm{NO} \nu \mathrm{A}$ GLoBES region is slightly shifted upwards. In the case of CP violation sensitivity in Fig. A.2 the NO $\nu$ A GLoBES curves are slightly higher than the official curves. In the case of mass hierarchy sensitivity in Fig. A.3 the NO $\nu$ A GLoBES curves are slightly lower than the official curves. These differences are assumed to be due to differences in signal event predictions in $\nu_{\mu}$ disappearance mode and differences in assumptions for the true oscillation parameters.

In Fig. A.2 the shape of of the significance curves comes from differences in expected event rates between the value of $\delta_{C P}$ indicated on the x-axis and $\delta_{C P}=[0, \pi]$. For $\mathrm{NH}(\mathrm{IH})$ this difference is a maximum at $\delta_{C P}=-\pi / 2(\pi / 2)$. In the $\mathrm{NH}$ there is a degeneracy at $\delta_{C P}=\pi / 2$ where the difference is reduced by using the IH in the fit. This degeneracy causes the dip in the significance seen at $\delta_{C P}=\pi / 2$ for the NH and at $\delta_{C P}=-\pi / 2$ for the IH. The significance falls to zero at $\delta_{C P}=[0, \pi]$ because there is no difference in event rates at those points.

In Fig. A.3 the shape of this significance curve comes from comparing the expected event rates between the $\mathrm{NH}$ and the $\mathrm{IH}$. The maximum sensitivity in the $\mathrm{NH}(\mathrm{IH})$ is at $\delta_{C P}=-\pi / 2(\pi / 2)$ where the largest difference between the event rates occurs. At $\delta_{C P}=$ $\pi / 2(-\pi / 2)$ for the $\mathrm{NH}(\mathrm{IH})$ curve the degeneracy between $\delta_{C P}$ and the mass hierarchy again 
causes the significance to drop. The significance goes to zero where the expected event rate in the $\mathrm{NH}$ is the same as that in the IH at a different value of $\delta_{C P}$.

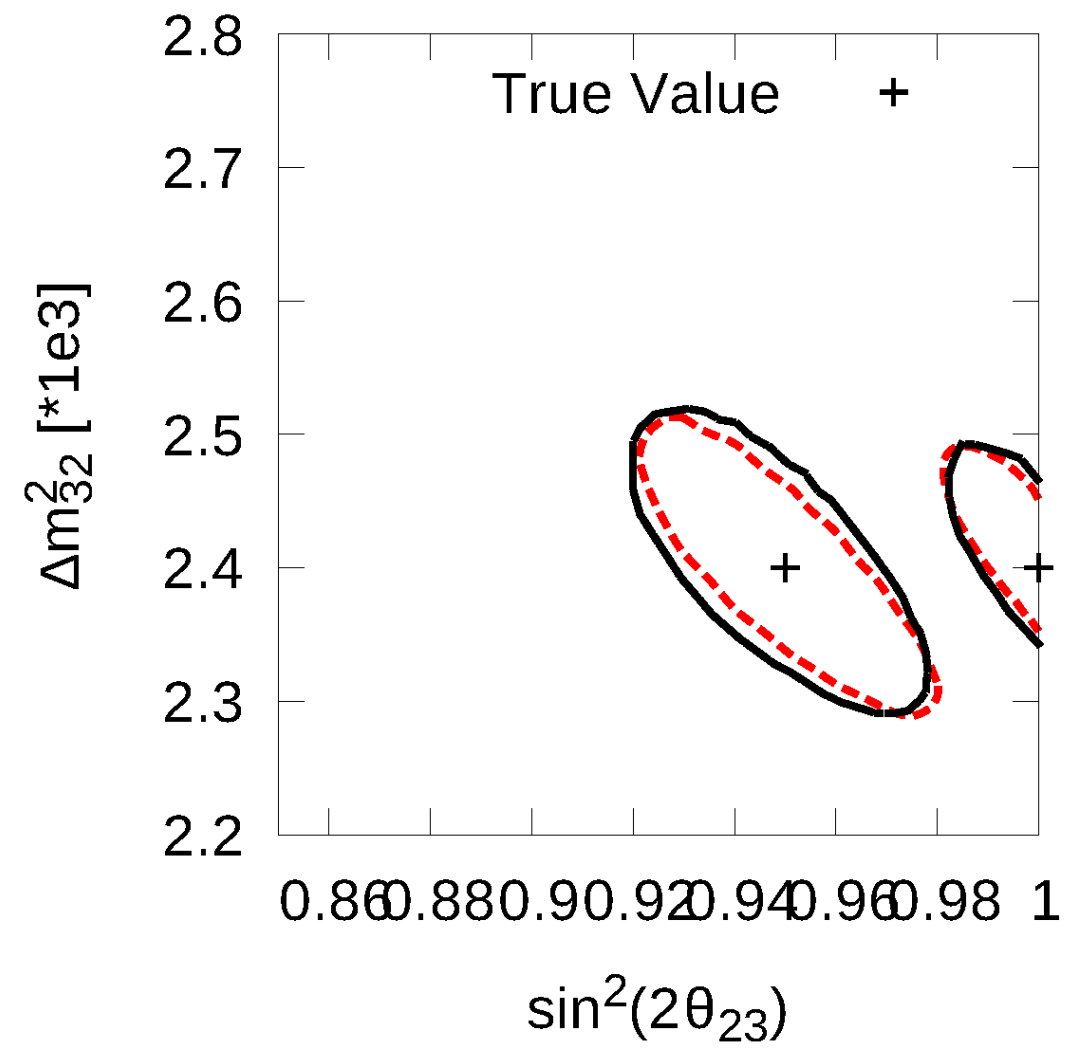

Figure A.1. $1 \sigma$ Allowed regions in $\Delta m_{32}^{2}$ vs $\sin ^{2}\left(2 \theta_{23}\right)$. The GLoBES reproduction(red) makes a slightly. The GLoBES contours are determined assuming $1 \sigma$ with 2 degrees of freedom. 


\section{$\mathrm{CP}$ violation sensitivity}

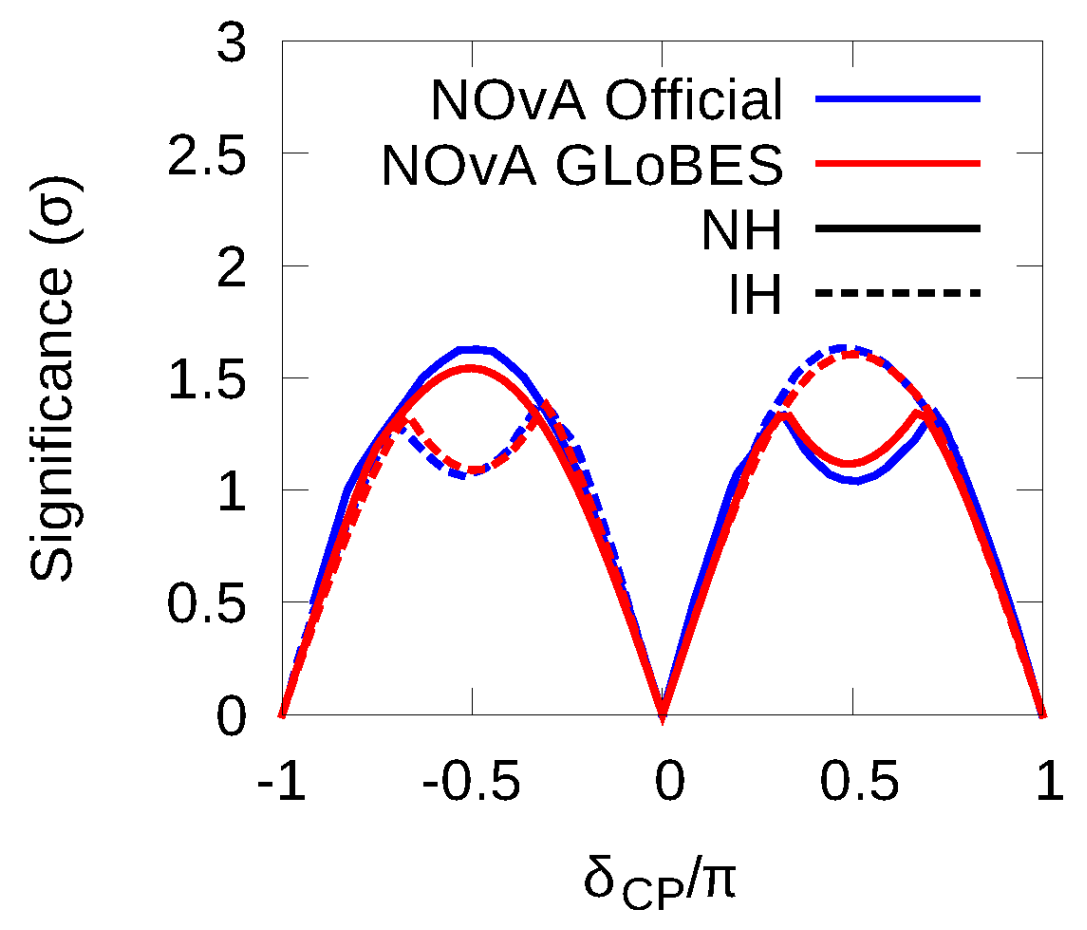

Figure A.2. CP violation sensitivity from official NO $\nu$ A plots (blue) and the GLoBES reproduction (red). The solid/dashed curve is for true normal/inverted hierarchy $(\mathrm{NH} / \mathrm{IH})$. A rate only (single-bin) $\nu_{e}$ appearance analysis is considered for the reproduction. The reproduced curves are all slightly higher than the official curves. 


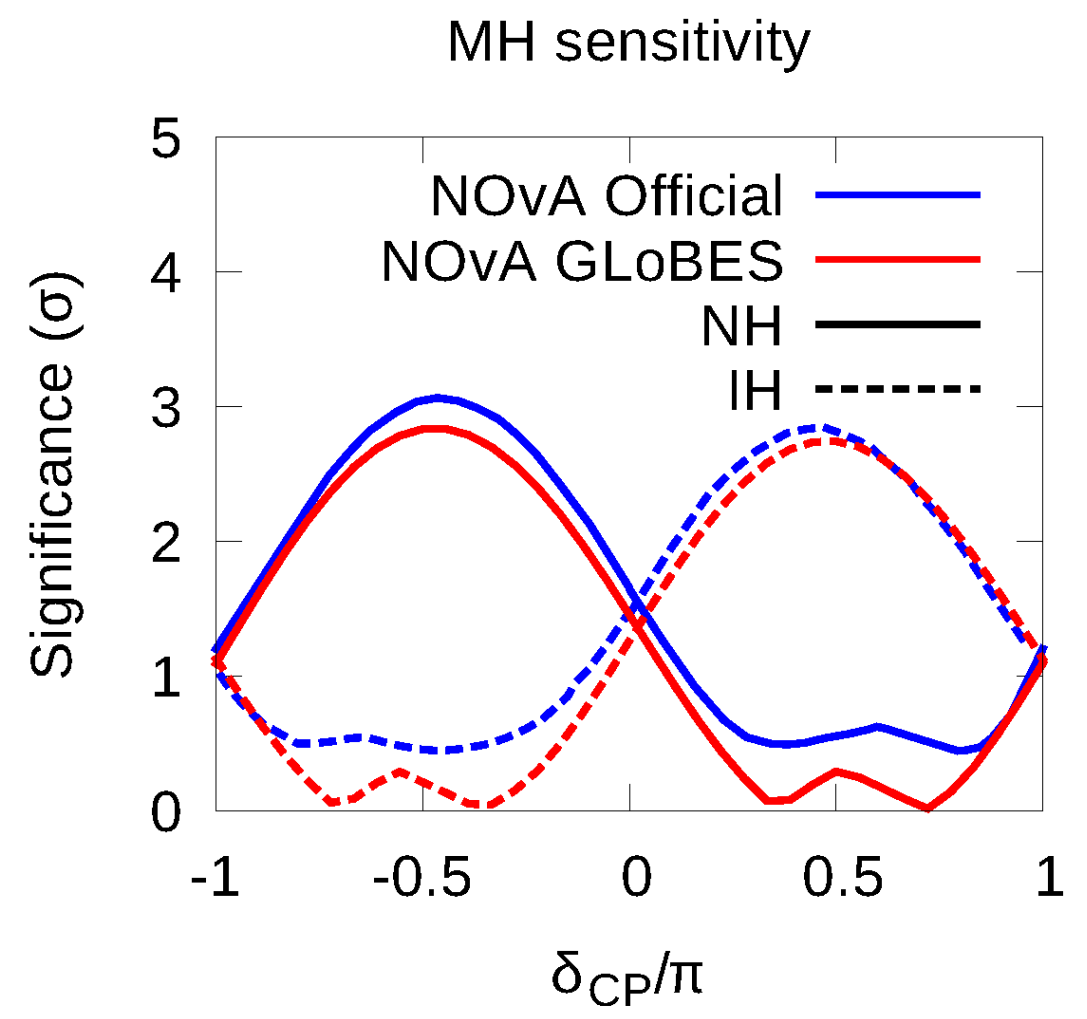

Figure A.3. Mass hierarchy sensitivity from official $\mathrm{NO} \nu \mathrm{A}$ plots (blue) and GLoBES reproduction (red). The solid/dashed curve is for true normal/inverted hierarchy $(\mathrm{NH} / \mathrm{IH})$. A rate only (single-bin) $\nu_{e}$ appearance analysis is considered for the reproduction. The reproduced curves are overall slightly lower than the official curves. 


\section{APPENDIX B}

\section{Event Spectra For Oscillation Parameter}

\section{VARIATIONS IN THE INVERTED HIERARCHY}

This appendix provides IH versions of the oscillation parameter variation spectra presented in Section 6.1. The spectra are given for variations in $\theta_{13}, \theta_{23}, \Delta m_{31}^{2}$, and $\delta_{C P}$.

\section{B.1. $\theta_{13}$ VARIATIONS}

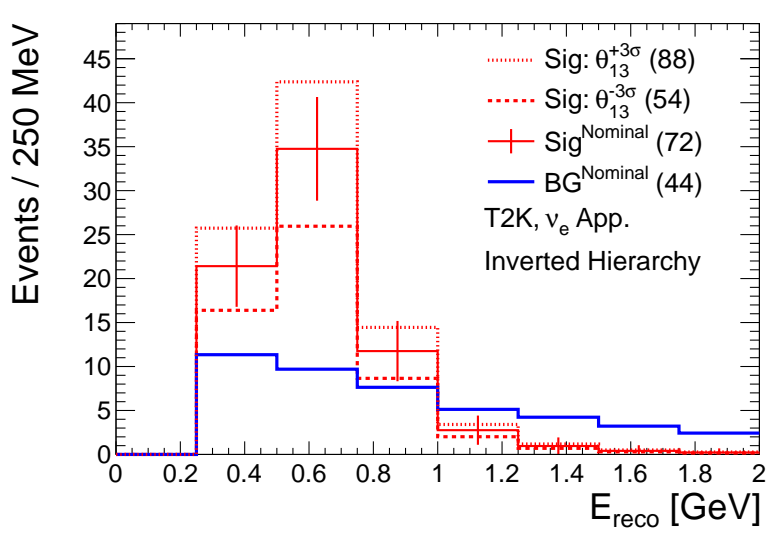

(A) $\nu_{e}$ appearance, True $\mathrm{MH}=\mathrm{NH}$

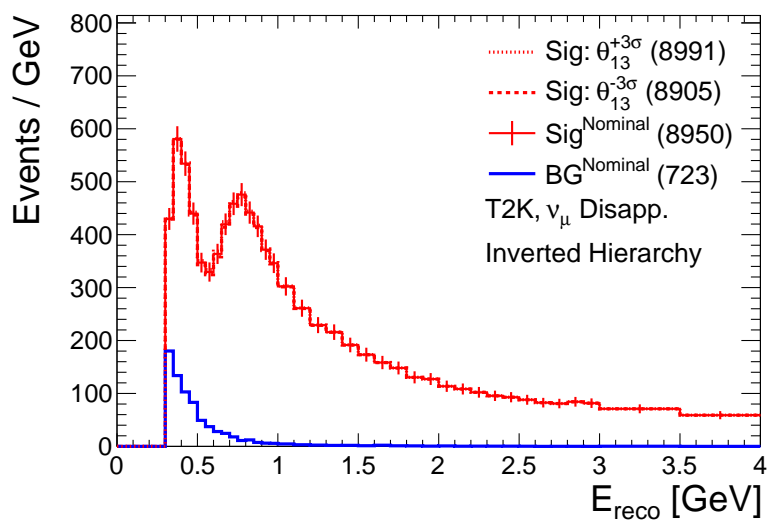

(C) $\nu_{\mu}$ disappearance, True $\mathrm{MH}=\mathrm{NH}$

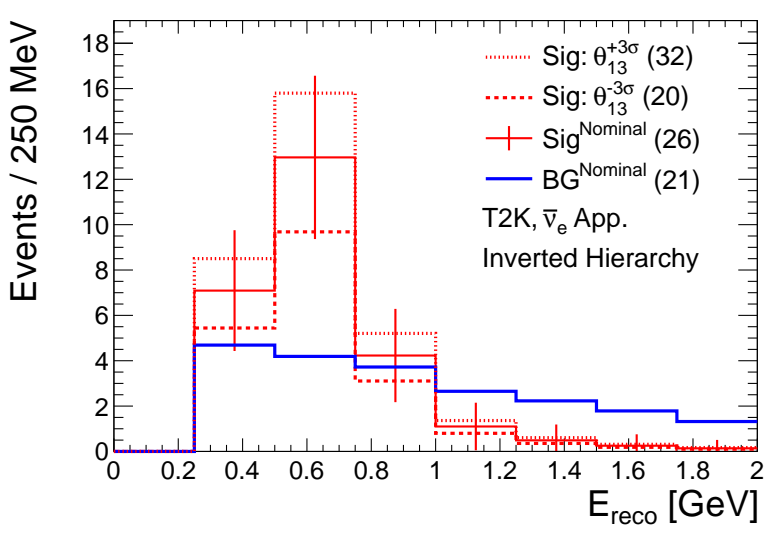

(в) $\bar{\nu}_{e}$ appearance, True $\mathrm{MH}=\mathrm{NH}$

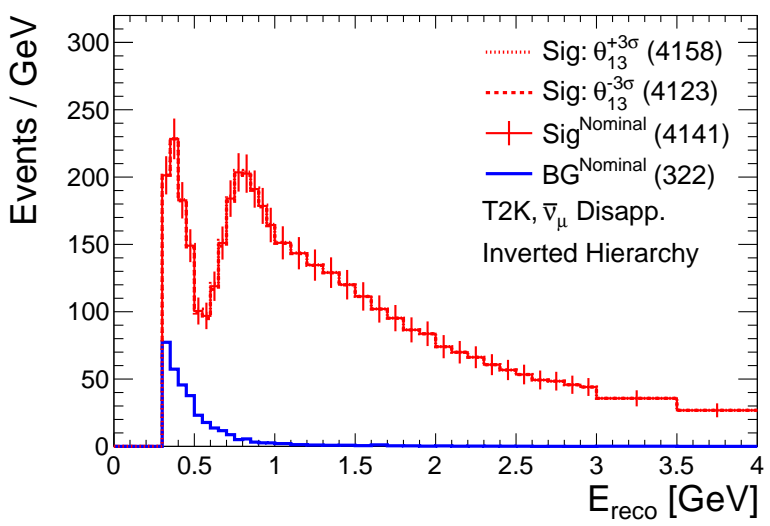

(D) $\bar{\nu}_{\mu}$ disappearance, True $\mathrm{MH}=\mathrm{NH}$

Figure B.1. T2K event spectra for $\pm 3 \sigma$ variations in $\theta_{13}$ for $\nu_{e}$ appearance (top left), $\bar{\nu}_{e}$ appearance (top right), $\nu_{\mu}$ disappearance (bottom left), and $\bar{\nu}_{\mu}$ disappearance (bottom right). The histograms are overlaid and not stacked. The nominal $7.8 \times 10^{21}$ POT with 1:1 $\nu: \bar{\nu}$ ratio is assumed. The integrated numbers of events are given in parentheses in the legend. 


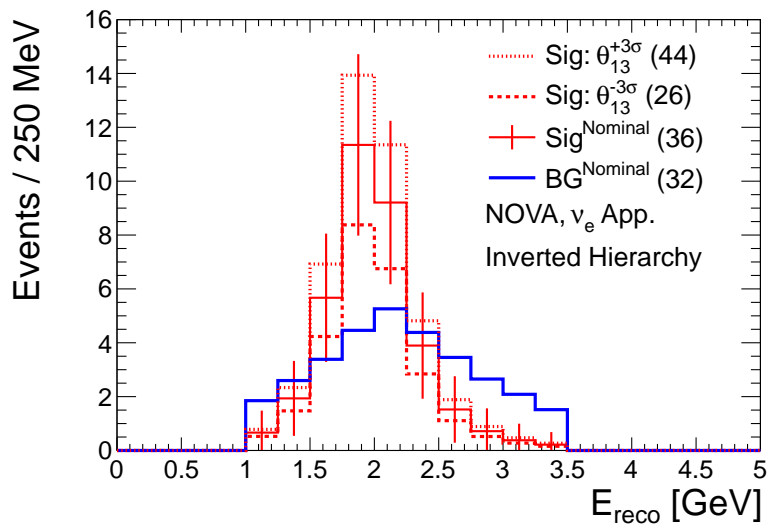

(A) $\nu_{e}$ appearance, True $\mathrm{MH}=\mathrm{NH}$

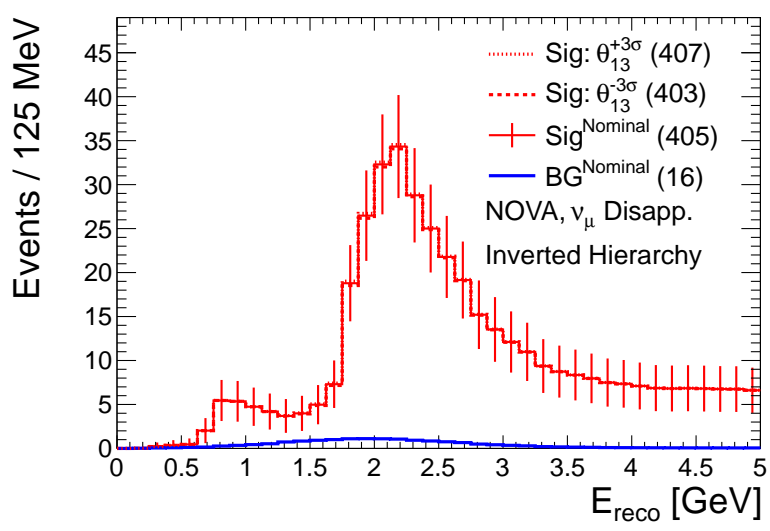

(C) $\nu_{\mu}$ disappearance, True $\mathrm{MH}=\mathrm{NH}$

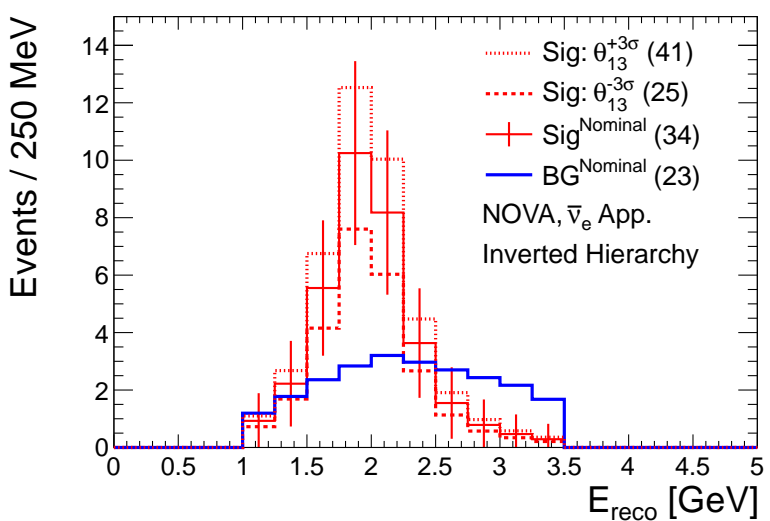

(B) $\bar{\nu}_{e}$ appearance, True $\mathrm{MH}=\mathrm{NH}$

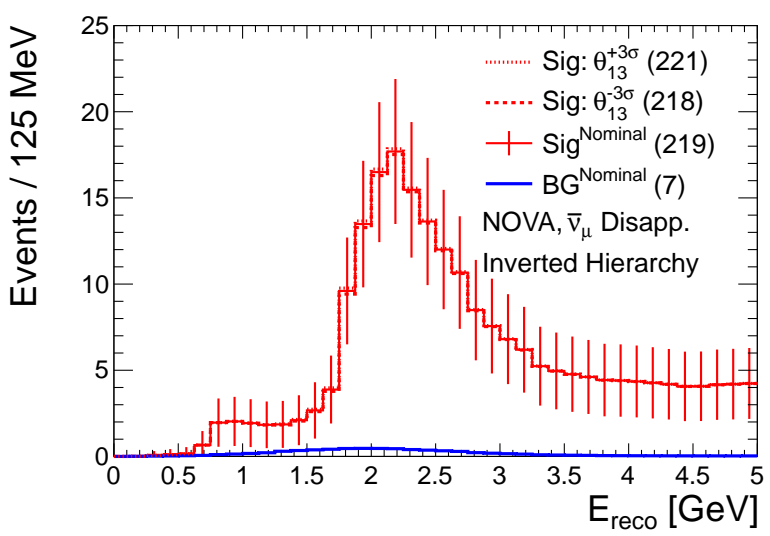

(D) $\bar{\nu}_{\mu}$ disappearance, True $\mathrm{MH}=\mathrm{NH}$

FigurE B.2. $\mathrm{NO} \nu \mathrm{A}$ event spectra for $\pm 3 \sigma$ variations in $\theta_{13}$ for $\nu_{e}$ appearance (top left), $\bar{\nu}_{e}$ appearance (top right), $\nu_{\mu}$ disappearance (bottom left), and $\bar{\nu}_{\mu}$ disappearance (bottom right). The histograms are overlaid and not stacked. The nominal $3.6 \times 10^{21}$ POT with $1: 1 \nu: \bar{\nu}$ ratio is assumed. The integrated numbers of events are given in parentheses in the legend. 


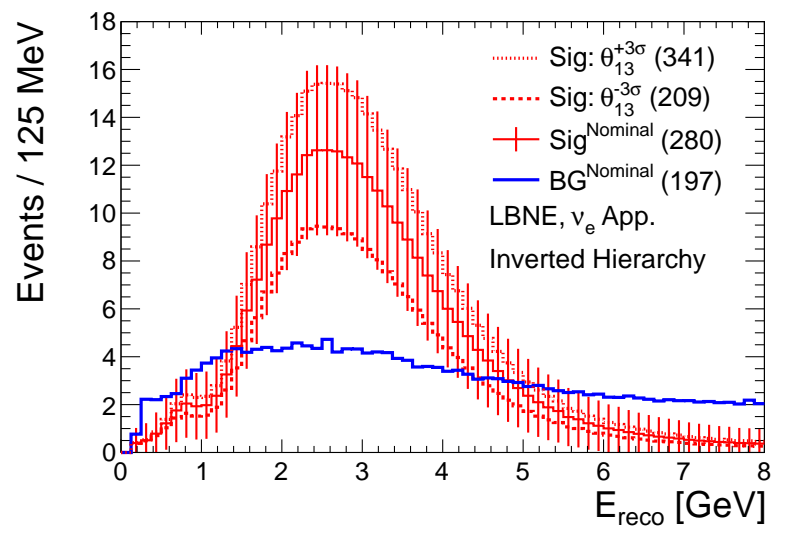

(A) $\nu_{e}$ appearance, True $\mathrm{MH}=\mathrm{NH}$

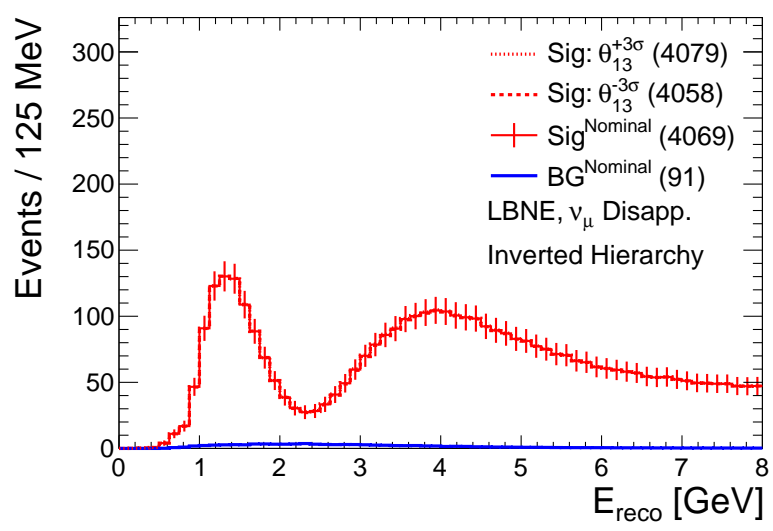

(C) $\nu_{\mu}$ disappearance, True $\mathrm{MH}=\mathrm{NH}$

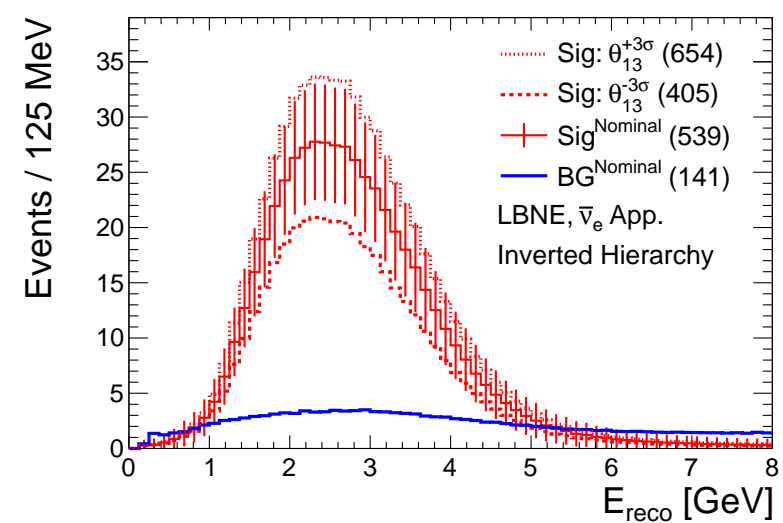

(B) $\bar{\nu}_{e}$ appearance, True $\mathrm{MH}=\mathrm{NH}$

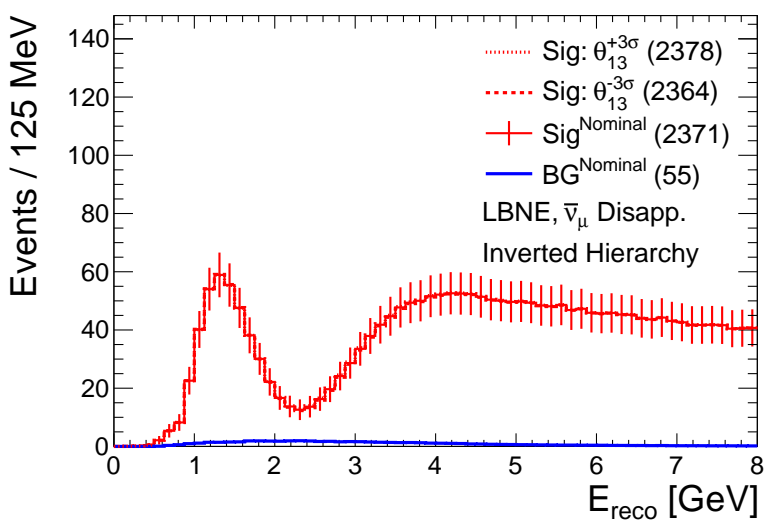

(D) $\bar{\nu}_{\mu}$ disappearance, True $\mathrm{MH}=\mathrm{NH}$

FigurE B.3. LBNE event spectra for $\pm 3 \sigma$ variations in $\theta_{13}$ for $\nu_{e}$ appearance (top left), $\bar{\nu}_{e}$ appearance (top right), $\nu_{\mu}$ disappearance (bottom left), and $\bar{\nu}_{\mu}$ disappearance (bottom right). The histograms are overlaid and not stacked. The nominal $6.0 \times 10^{21}$ POT with $1: 1 \nu: \bar{\nu}$ ratio is assumed. The integrated numbers of events are given in parentheses in the legend. 


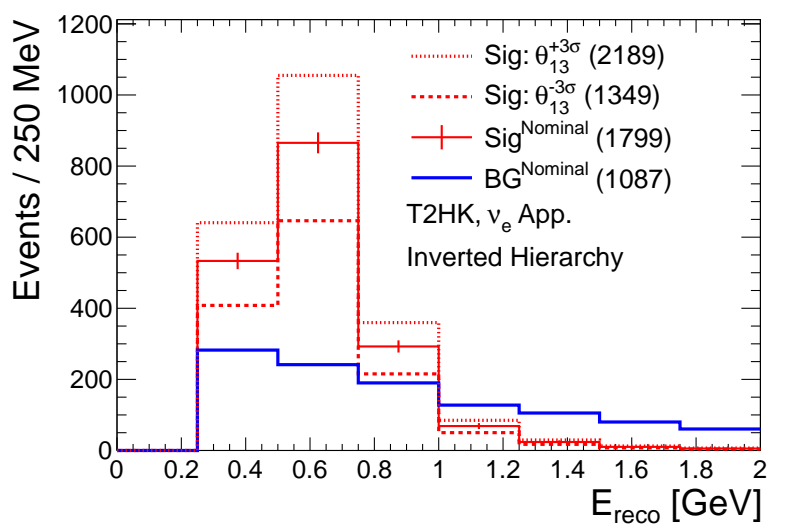

(A) $\nu_{e}$ appearance, True $\mathrm{MH}=\mathrm{NH}$

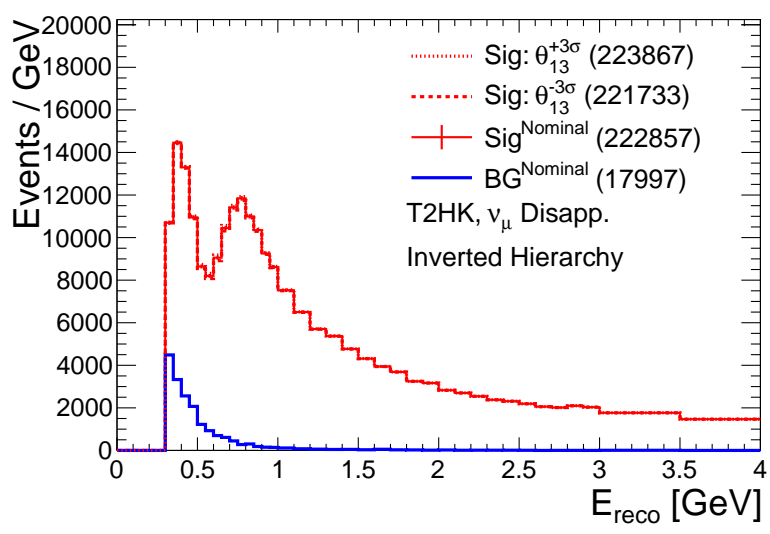

(C) $\nu_{\mu}$ disappearance, True $\mathrm{MH}=\mathrm{NH}$

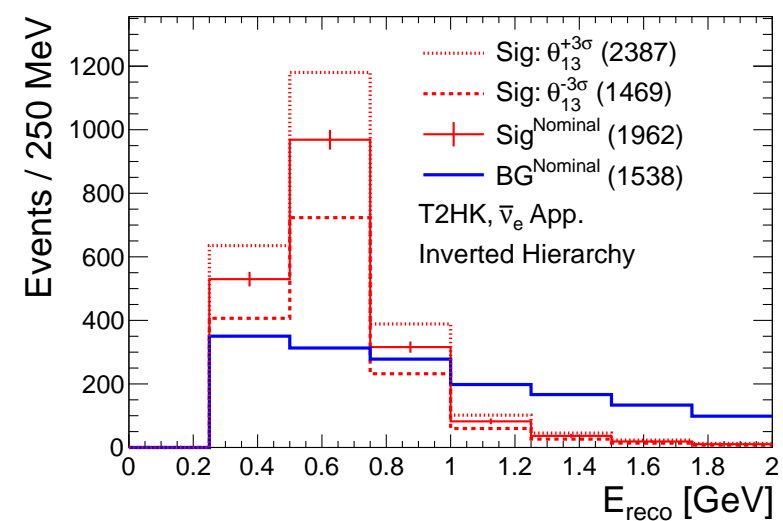

(B) $\bar{\nu}_{e}$ appearance, True $\mathrm{MH}=\mathrm{NH}$

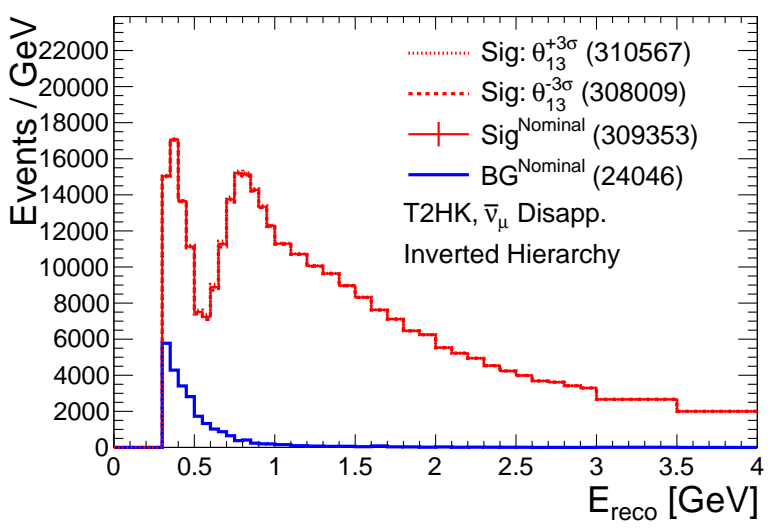

(D) $\bar{\nu}_{\mu}$ disappearance, True $\mathrm{MH}=\mathrm{NH}$

FigurE B.4. T2HK event spectra for $\pm 3 \sigma$ variations in $\theta_{13}$ for $\nu_{e}$ appearance (top left), $\bar{\nu}_{e}$ appearance (top right), $\nu_{\mu}$ disappearance (bottom left), and $\bar{\nu}_{\mu}$ disappearance (bottom right). The histograms are overlaid and not stacked. The nominal $15.6 \times 10^{21}$ POT with $1: 1 \nu: \bar{\nu}$ ratio is assumed. The integrated numbers of events are given in parentheses in the legend. 


\section{B.2. $\theta_{23}$ VARIATIONS}

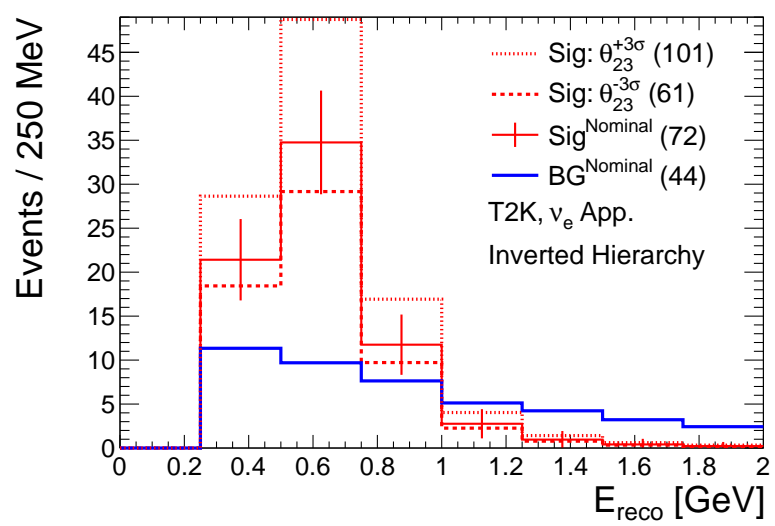

(A) $\nu_{e}$ appearance, True $\mathrm{MH}=\mathrm{NH}$

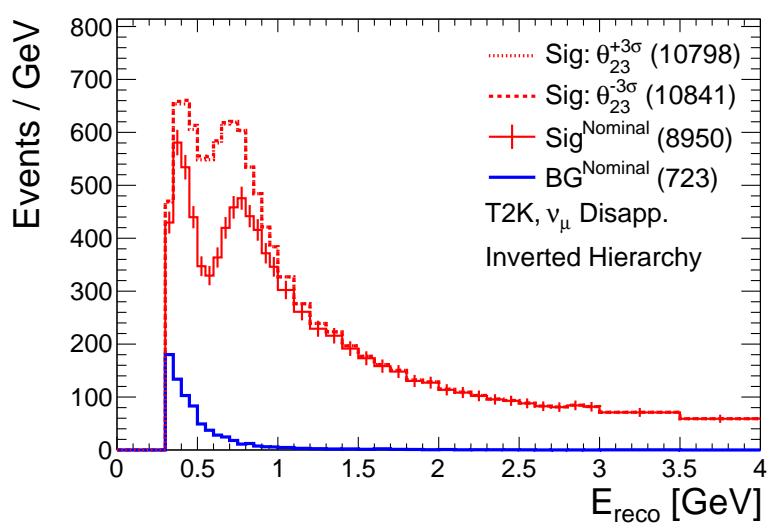

(C) $\nu_{\mu}$ disappearance, True $\mathrm{MH}=\mathrm{NH}$

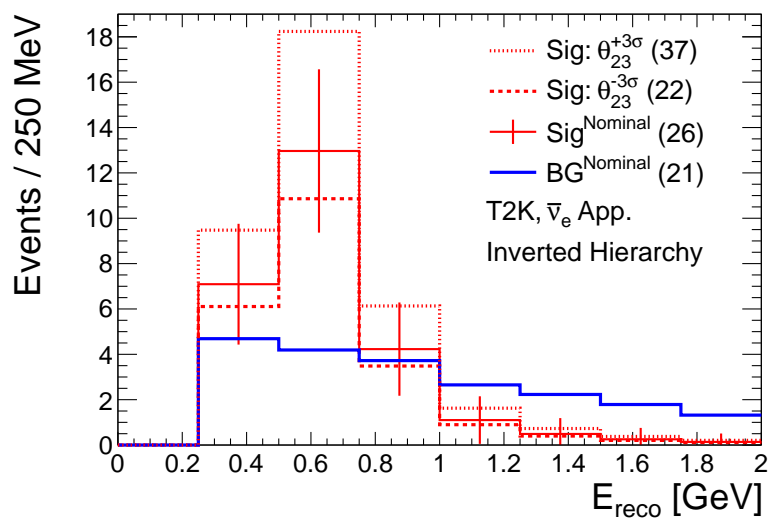

(в) $\bar{\nu}_{e}$ appearance, True $\mathrm{MH}=\mathrm{NH}$

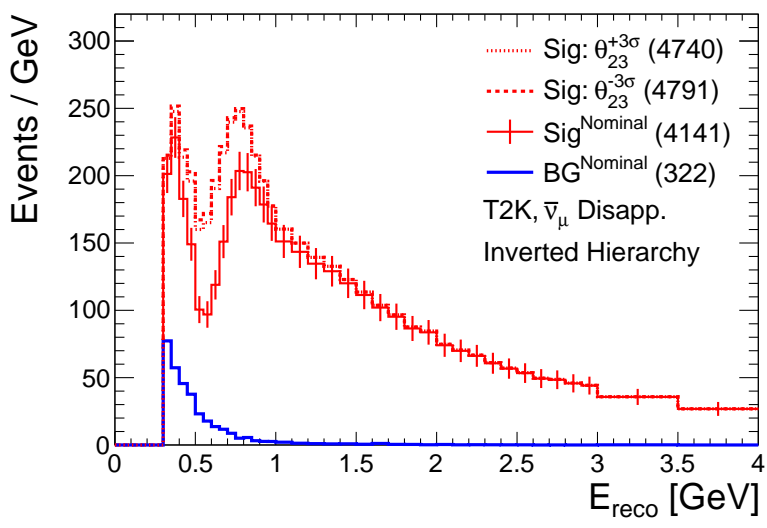

(D) $\bar{\nu}_{\mu}$ disappearance, True $\mathrm{MH}=\mathrm{NH}$

FiguRE B.5. T2K event spectra for $\pm 3 \sigma$ variations in $\theta_{23}$ for $\nu_{e}$ appearance (top left), $\bar{\nu}_{e}$ appearance (top right), $\nu_{\mu}$ disappearance (bottom left), and $\bar{\nu}_{\mu}$ disappearance (bottom right). The histograms are overlaid and not stacked. The nominal $7.8 \times 10^{21}$ POT with $1: 1 \nu: \bar{\nu}$ ratio is assumed. The integrated numbers of events are given in parentheses in the legend. 


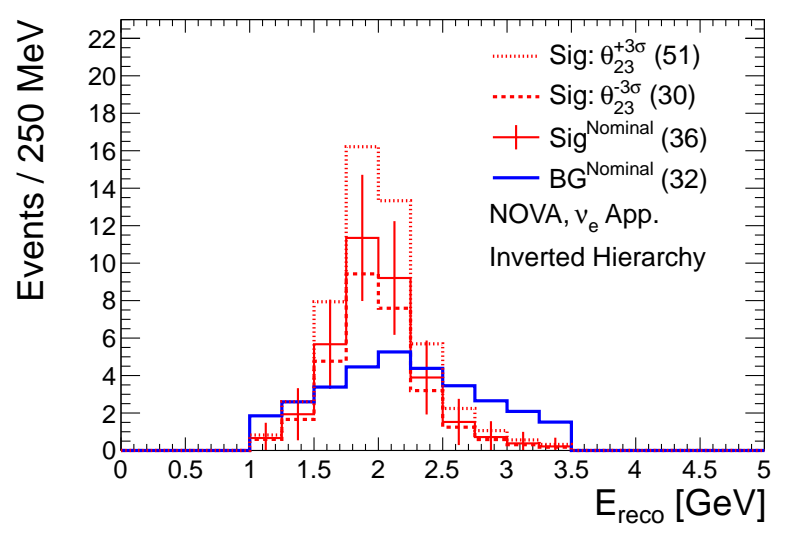

(A) $\nu_{e}$ appearance, True $\mathrm{MH}=\mathrm{NH}$

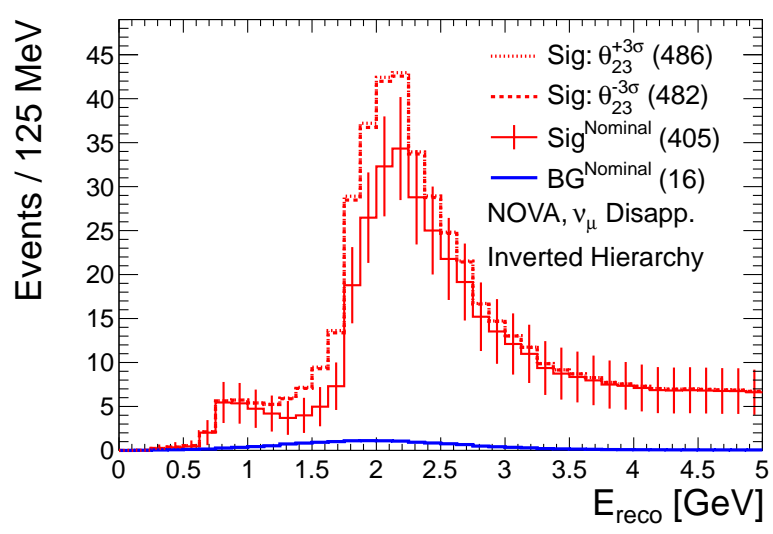

(C) $\nu_{\mu}$ disappearance, True $\mathrm{MH}=\mathrm{NH}$

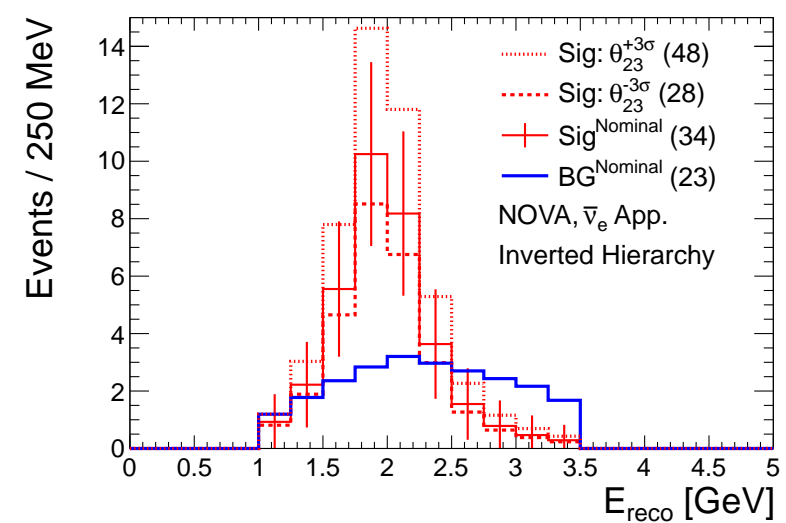

(B) $\bar{\nu}_{e}$ appearance, True $\mathrm{MH}=\mathrm{NH}$

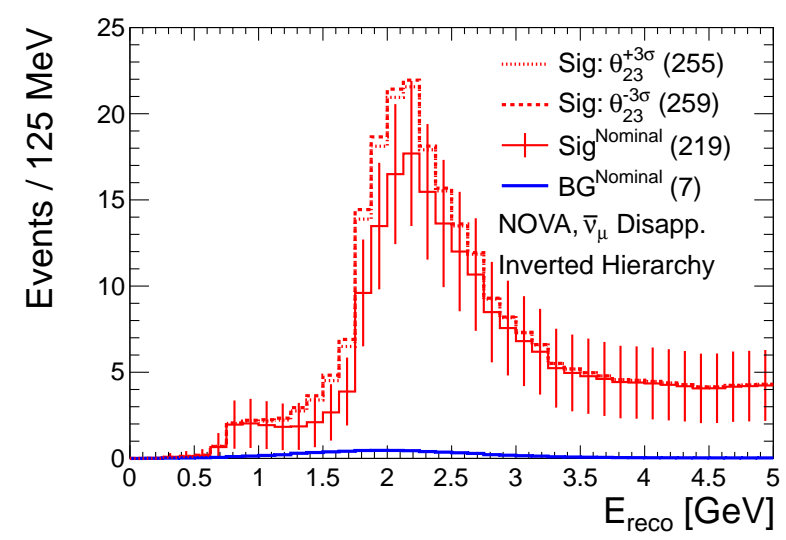

(D) $\bar{\nu}_{\mu}$ disappearance, True $\mathrm{MH}=\mathrm{NH}$

FIGURE B.6. $\mathrm{NO} \nu \mathrm{A}$ event spectra for $\pm 3 \sigma$ variations in $\theta_{23}$ for $\nu_{e}$ appearance (top left), $\bar{\nu}_{e}$ appearance (top right), $\nu_{\mu}$ disappearance (bottom left), and $\bar{\nu}_{\mu}$ disappearance (bottom right). The histograms are overlaid and not stacked. The nominal $3.6 \times 10^{21}$ POT with $1: 1 \nu: \bar{\nu}$ ratio is assumed. The integrated numbers of events are given in parentheses in the legend. 


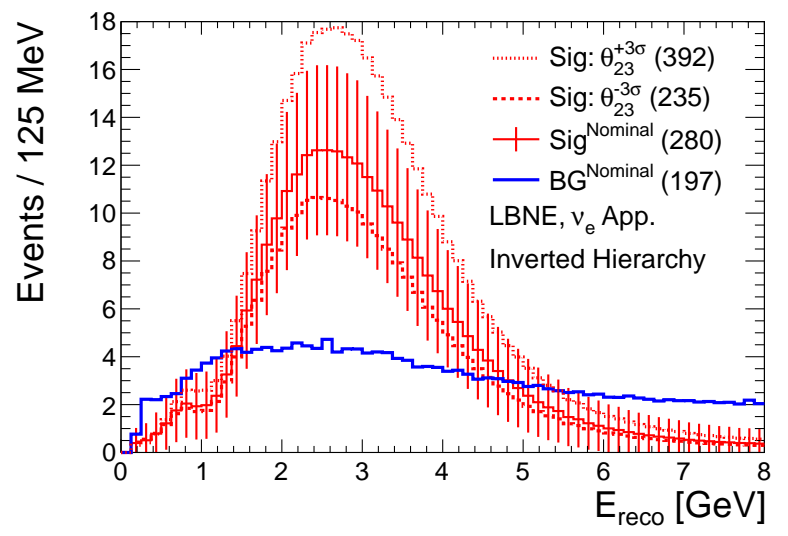

(A) $\nu_{e}$ appearance, True $\mathrm{MH}=\mathrm{NH}$

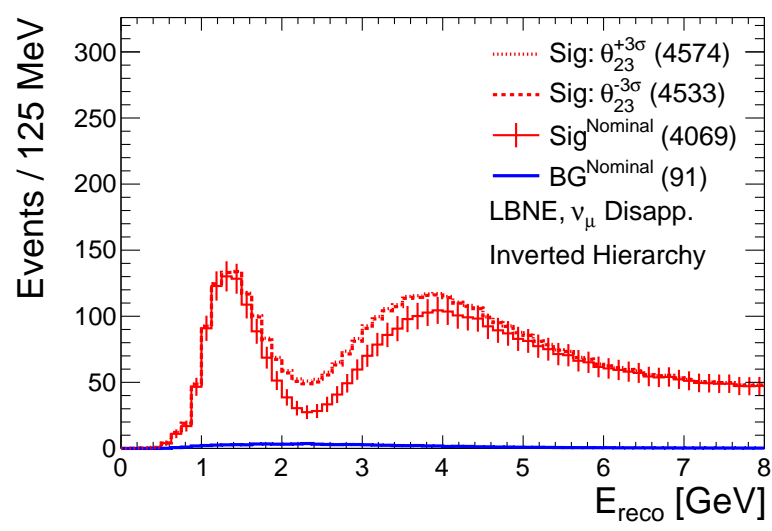

(C) $\nu_{\mu}$ disappearance, True $\mathrm{MH}=\mathrm{NH}$

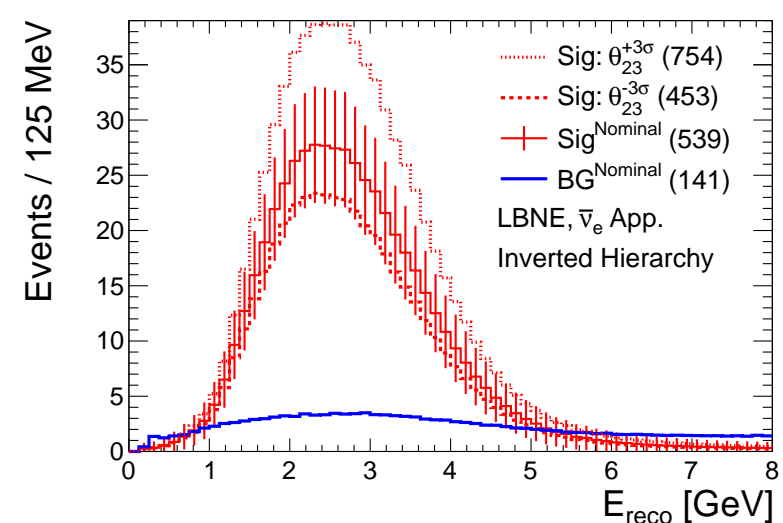

(B) $\bar{\nu}_{e}$ appearance, True $\mathrm{MH}=\mathrm{NH}$

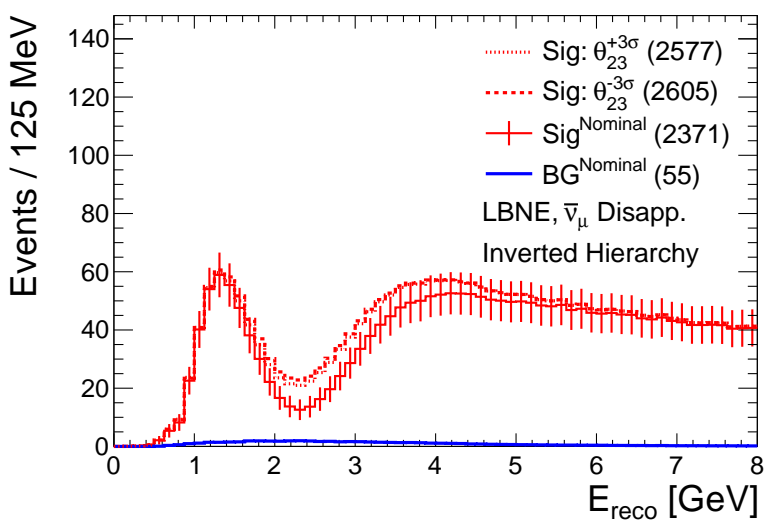

(D) $\bar{\nu}_{\mu}$ disappearance, True $\mathrm{MH}=\mathrm{NH}$

FigurE B.7. LBNE event spectra for $\pm 3 \sigma$ variations in $\theta_{23}$ for $\nu_{e}$ appearance (top left), $\bar{\nu}_{e}$ appearance (top right), $\nu_{\mu}$ disappearance (bottom left), and $\bar{\nu}_{\mu}$ disappearance (bottom right). The histograms are overlaid and not stacked. The nominal $6.0 \times 10^{21}$ POT with $1: 1 \nu: \bar{\nu}$ ratio is assumed. The integrated numbers of events are given in parentheses in the legend. 


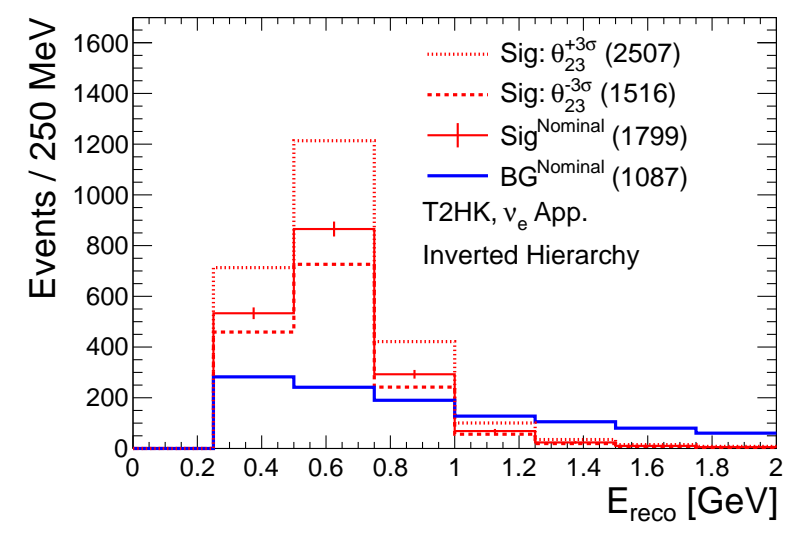

(A) $\nu_{e}$ appearance, True $\mathrm{MH}=\mathrm{NH}$

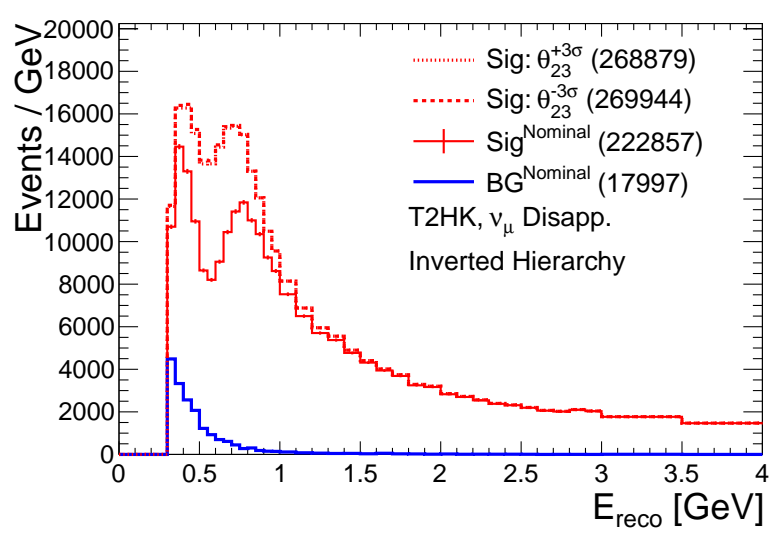

(C) $\nu_{\mu}$ disappearance, True $\mathrm{MH}=\mathrm{NH}$

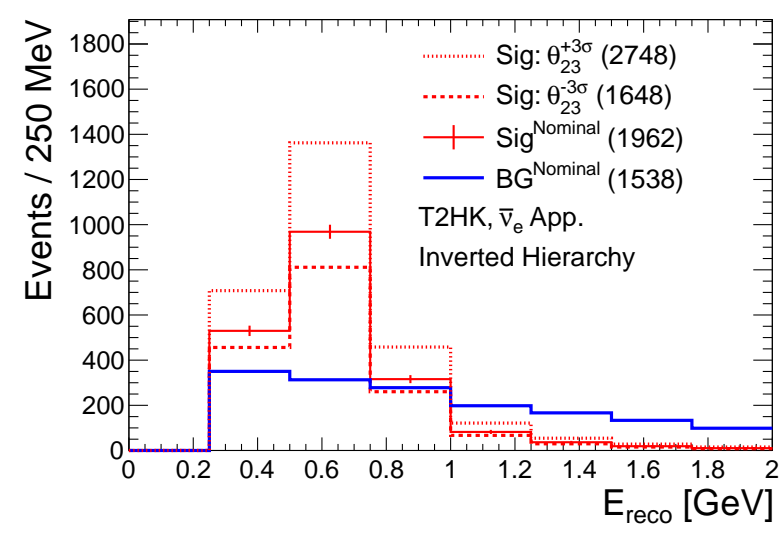

(B) $\bar{\nu}_{e}$ appearance, True $\mathrm{MH}=\mathrm{NH}$

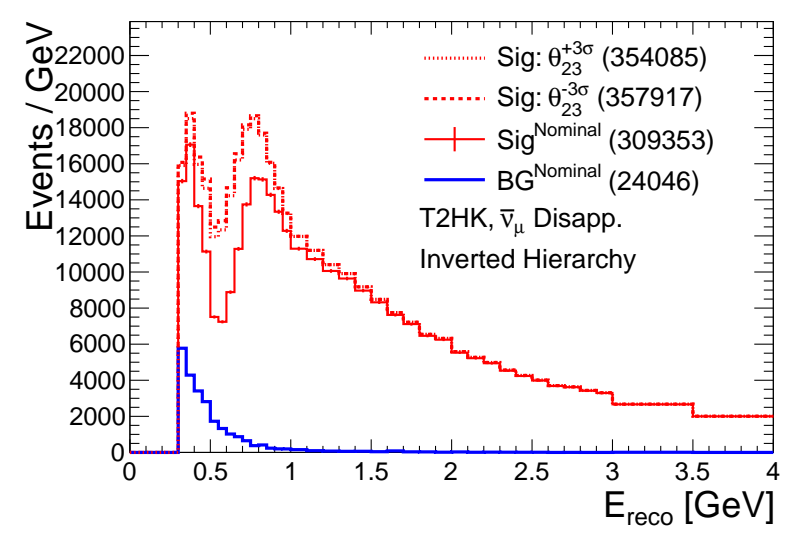

(D) $\bar{\nu}_{\mu}$ disappearance, True $\mathrm{MH}=\mathrm{NH}$

FigurE B.8. T2HK event spectra for $\pm 3 \sigma$ variations in $\theta_{23}$ for $\nu_{e}$ appearance (top left), $\bar{\nu}_{e}$ appearance (top right), $\nu_{\mu}$ disappearance (bottom left), and $\bar{\nu}_{\mu}$ disappearance (bottom right). The histograms are overlaid and not stacked. The nominal $15.6 \times 10^{21} \mathrm{POT}$ with $1: 1 \nu: \bar{\nu}$ ratio is assumed. The integrated numbers of events are given in parentheses in the legend. 


\section{B.3. $\Delta m_{31}^{2}$ VARIATIONS}

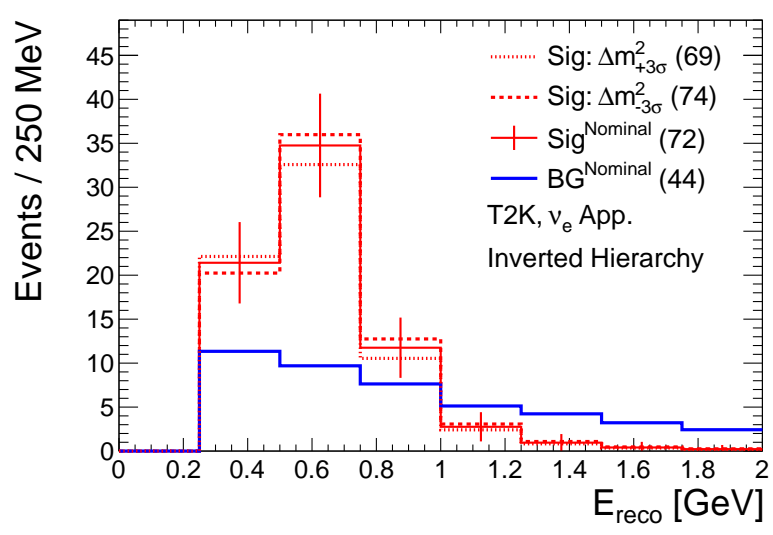

(A) $\nu_{e}$ appearance, True $\mathrm{MH}=\mathrm{NH}$

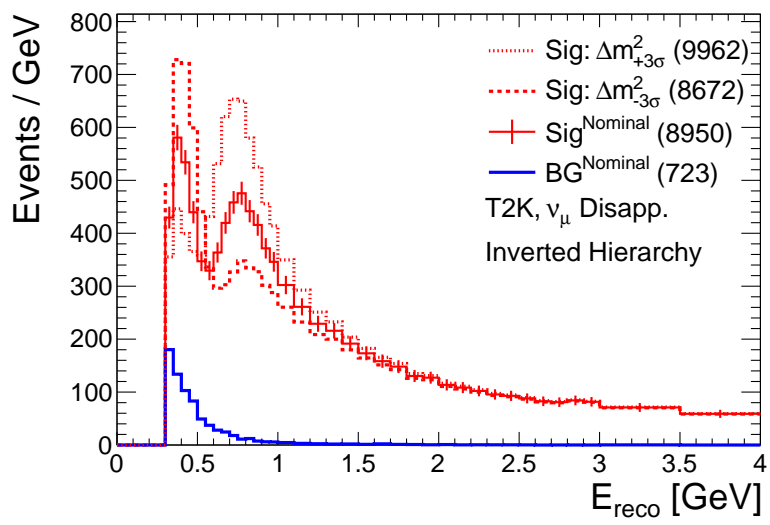

(C) $\nu_{\mu}$ disappearance, True $\mathrm{MH}=\mathrm{NH}$

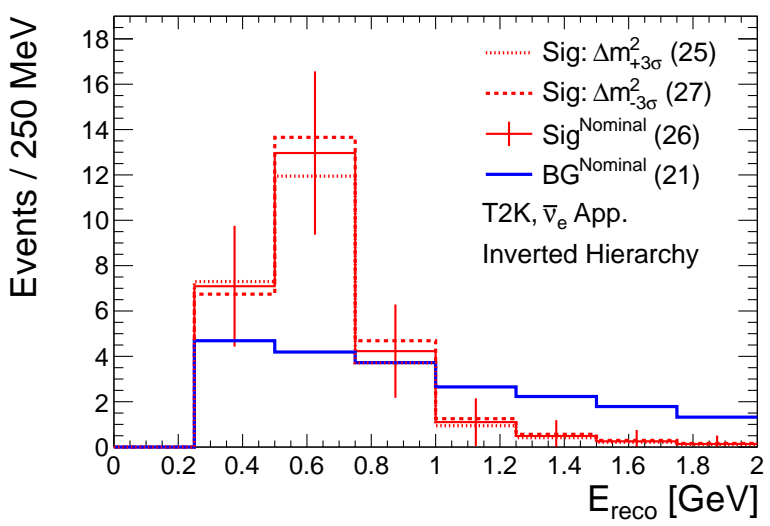

(B) $\bar{\nu}_{e}$ appearance, True $\mathrm{MH}=\mathrm{NH}$

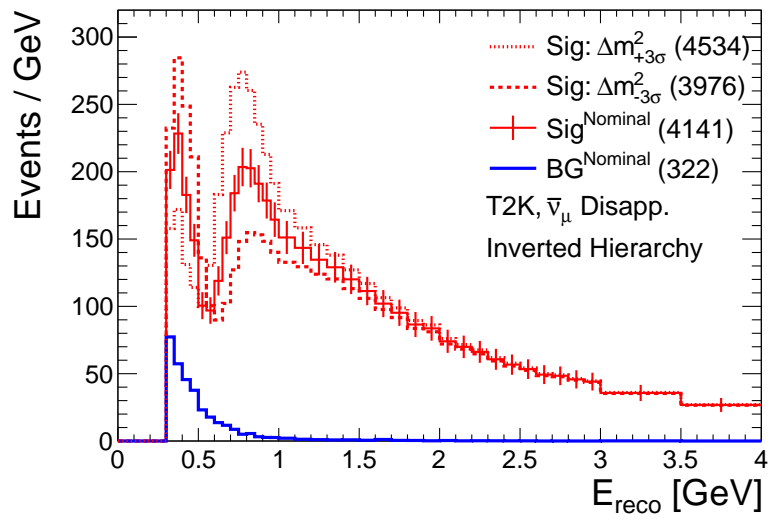

(D) $\bar{\nu}_{\mu}$ disappearance, True $\mathrm{MH}=\mathrm{NH}$

FigurE B.9. T2K event spectra for $\pm 3 \sigma$ variations in $\Delta m_{31}^{2}$ for $\nu_{e}$ appearance (top left), $\bar{\nu}_{e}$ appearance (top right), $\nu_{\mu}$ disappearance (bottom left), and $\bar{\nu}_{\mu}$ disappearance (bottom right). The histograms are overlaid and not stacked. The nominal $7.8 \times 10^{21}$ POT with $1: 1 \nu: \bar{\nu}$ ratio is assumed. The integrated numbers of events are given in parentheses in the legend. 


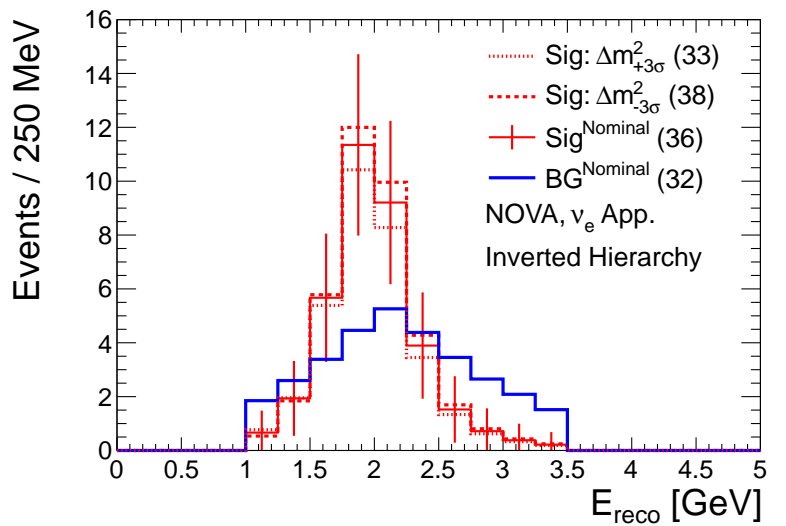

(A) $\nu_{e}$ appearance, True $\mathrm{MH}=\mathrm{NH}$

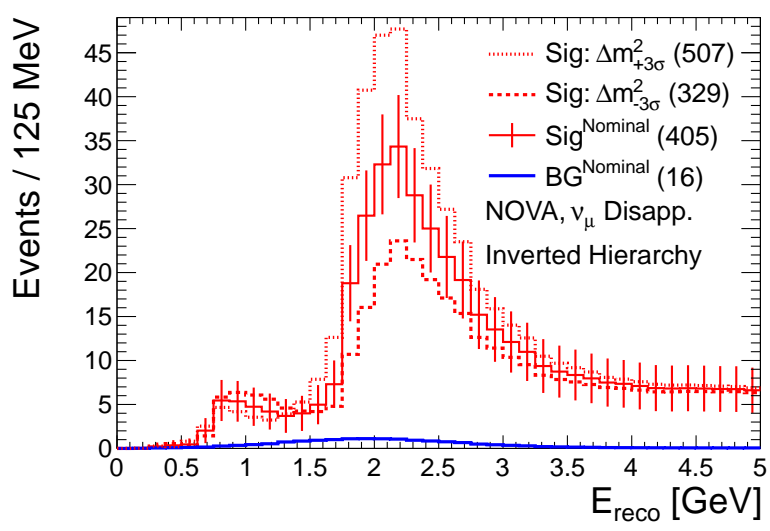

(C) $\nu_{\mu}$ disappearance, True $\mathrm{MH}=\mathrm{NH}$

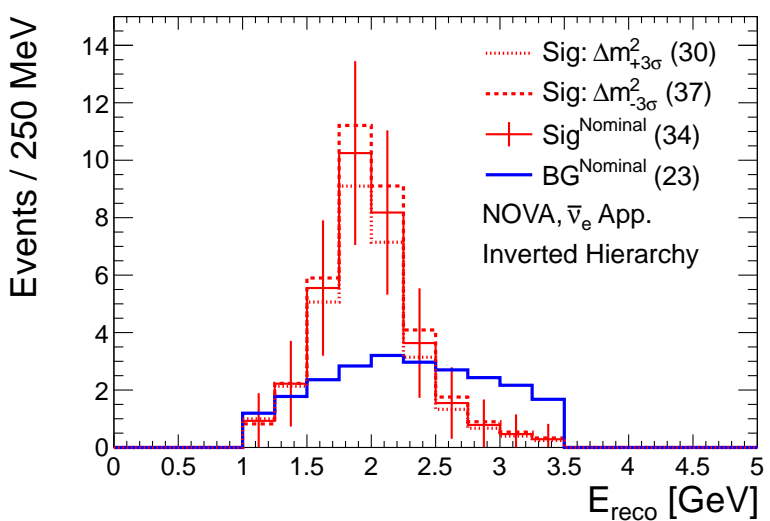

(B) $\bar{\nu}_{e}$ appearance, True $\mathrm{MH}=\mathrm{NH}$

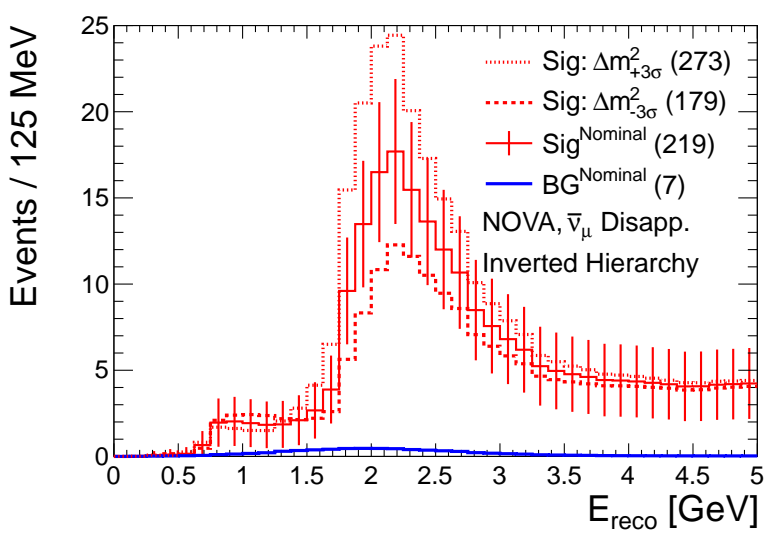

(D) $\bar{\nu}_{\mu}$ disappearance, True $\mathrm{MH}=\mathrm{NH}$

Figure B.10. NO $\nu$ A event spectra for $\pm 3 \sigma$ variations in $\Delta m_{31}^{2}$ for $\nu_{e}$ appearance (top left), $\bar{\nu}_{e}$ appearance (top right), $\nu_{\mu}$ disappearance (bottom left), and $\bar{\nu}_{\mu}$ disappearance (bottom right). The histograms are overlaid and not stacked. The nominal $3.6 \times 10^{21}$ POT with $1: 1 \nu: \bar{\nu}$ ratio is assumed. The integrated numbers of events are given in parentheses in the legend. 


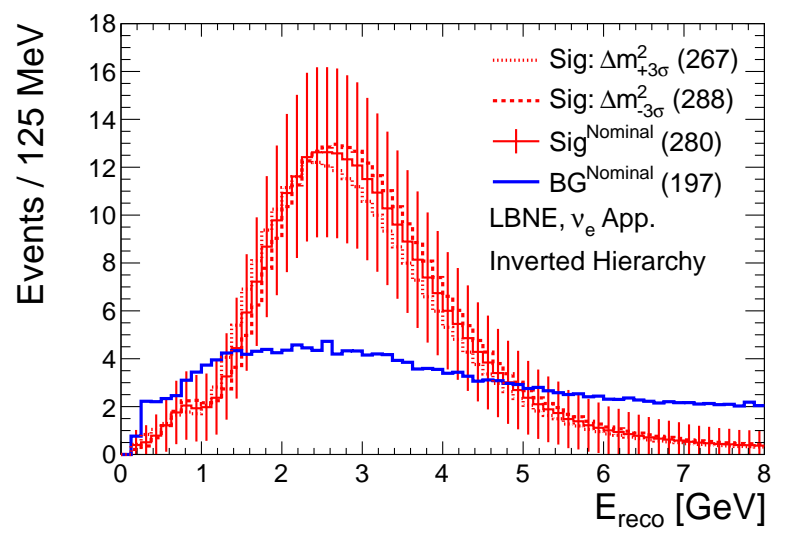

(A) $\nu_{e}$ appearance, True $\mathrm{MH}=\mathrm{NH}$

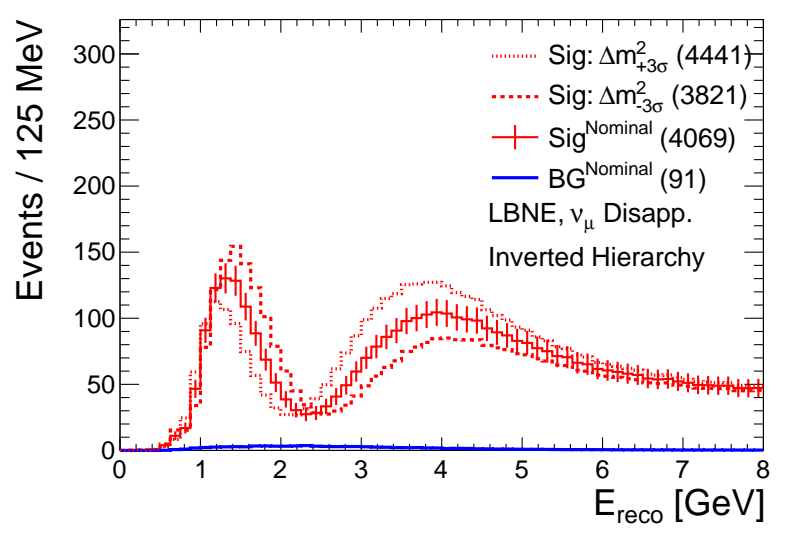

(C) $\nu_{\mu}$ disappearance, True $\mathrm{MH}=\mathrm{NH}$

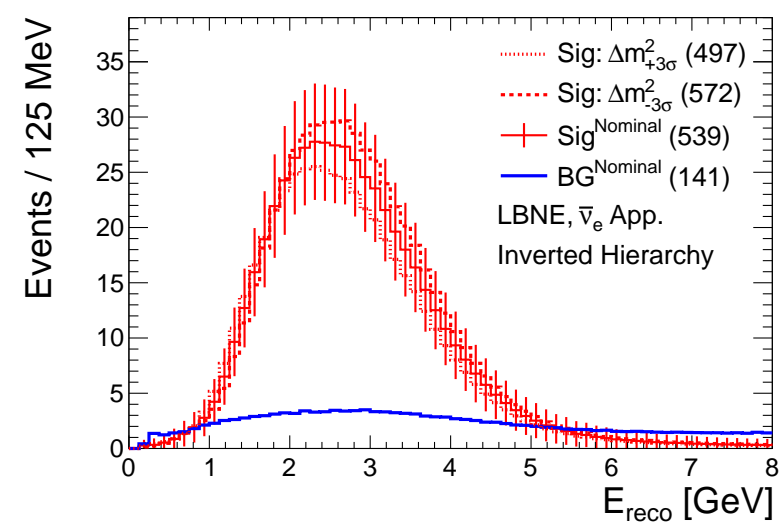

(B) $\bar{\nu}_{e}$ appearance, True $\mathrm{MH}=\mathrm{NH}$

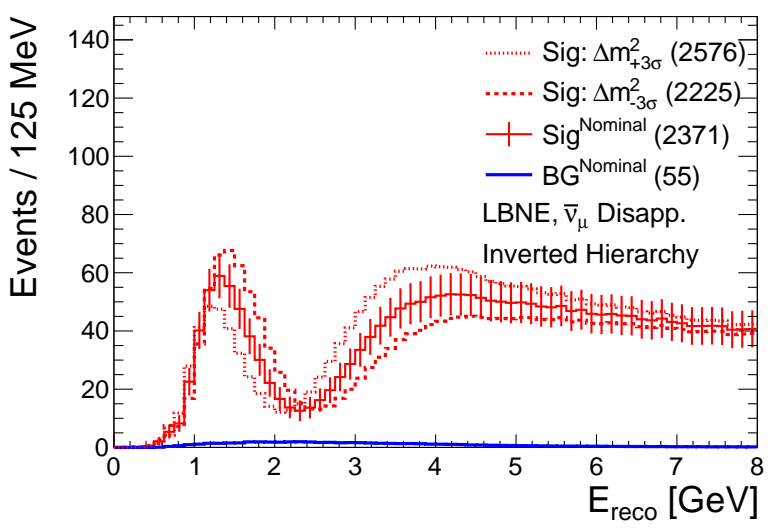

(D) $\bar{\nu}_{\mu}$ disappearance, True $\mathrm{MH}=\mathrm{NH}$

FIGURE B.11. LBNE event spectra for $\pm 3 \sigma$ variations in $\Delta m_{31}^{2}$ for $\nu_{e}$ appearance (top left), $\bar{\nu}_{e}$ appearance (top right), $\nu_{\mu}$ disappearance (bottom left), and $\bar{\nu}_{\mu}$ disappearance (bottom right). The histograms are overlaid and not stacked. The nominal $6.0 \times 10^{21}$ POT with $1: 1 \nu: \bar{\nu}$ ratio is assumed. The integrated numbers of events are given in parentheses in the legend. 


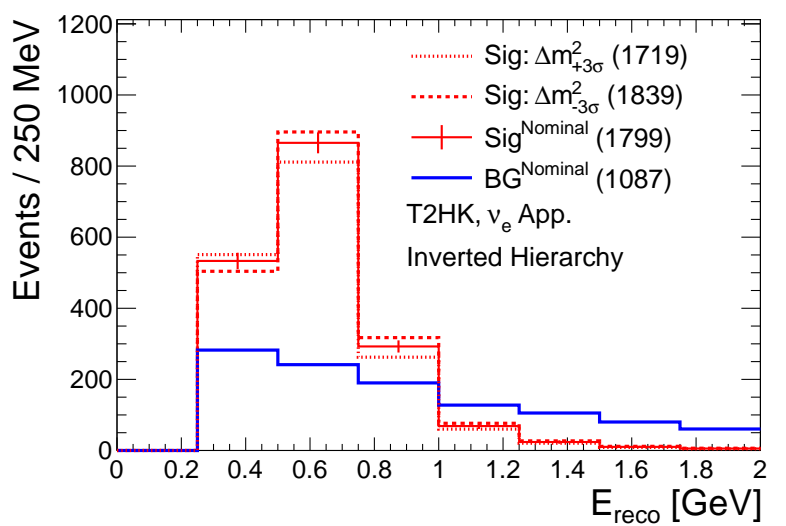

(A) $\nu_{e}$ appearance, True $\mathrm{MH}=\mathrm{NH}$

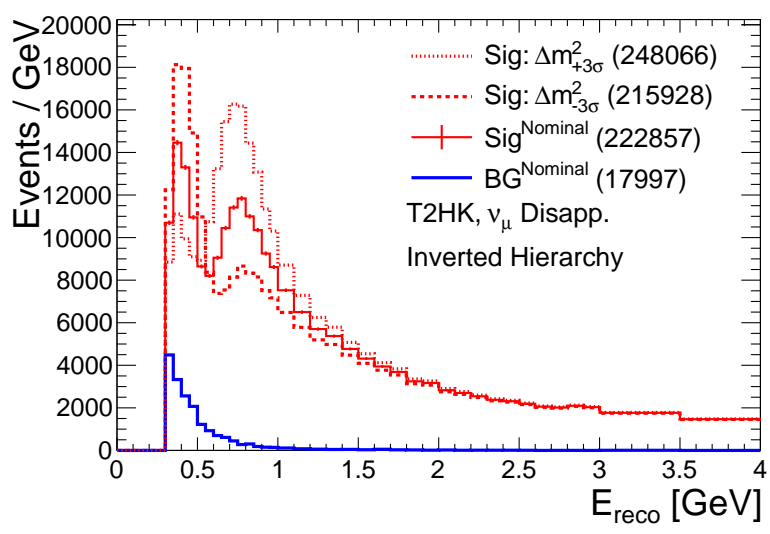

(C) $\nu_{\mu}$ disappearance, True $\mathrm{MH}=\mathrm{NH}$

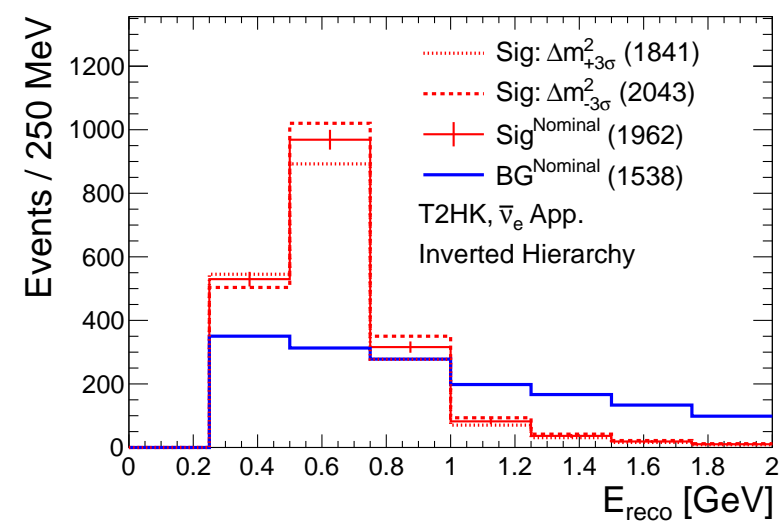

(B) $\bar{\nu}_{e}$ appearance, True $\mathrm{MH}=\mathrm{NH}$

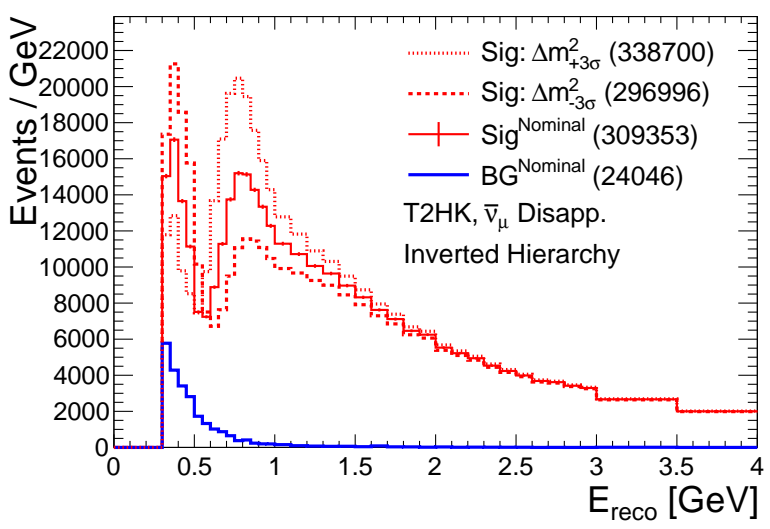

(D) $\bar{\nu}_{\mu}$ disappearance, True $\mathrm{MH}=\mathrm{NH}$

FIGURE B.12. T2HK event spectra for $\pm 3 \sigma$ variations in $\Delta m_{31}^{2}$ for $\nu_{e}$ appearance (top left), $\bar{\nu}_{e}$ appearance (top right), $\nu_{\mu}$ disappearance (bottom left), and $\bar{\nu}_{\mu}$ disappearance (bottom right). The histograms are overlaid and not stacked. The nominal $15.6 \times 10^{21} \mathrm{POT}$ with $1: 1 \nu: \bar{\nu}$ ratio is assumed. The integrated numbers of events are given in parentheses in the legend. 


\section{B.4. $\delta_{C P}$ VARIations}

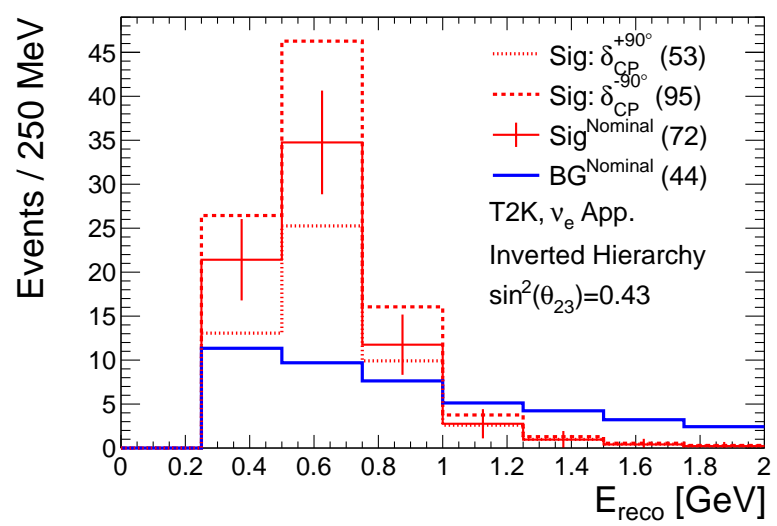

(A) $\nu_{e}$ appearance, True $\mathrm{MH}=\mathrm{NH}$

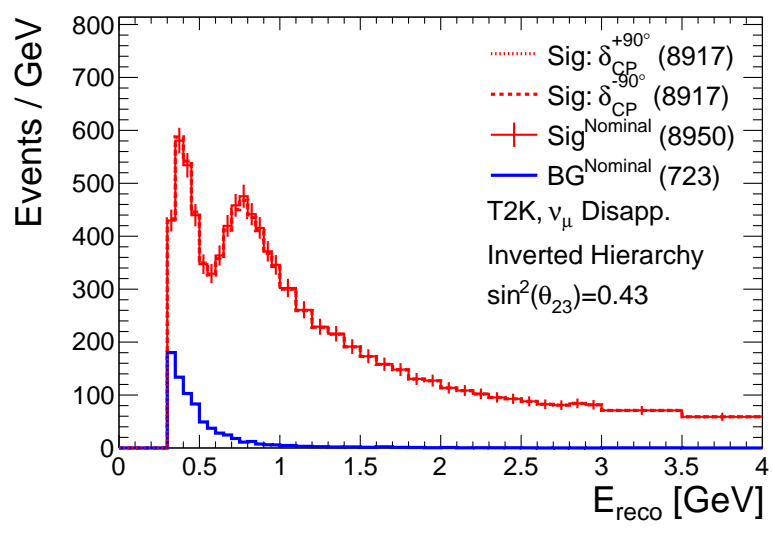

(C) $\nu_{\mu}$ disappearance, True $\mathrm{MH}=\mathrm{NH}$

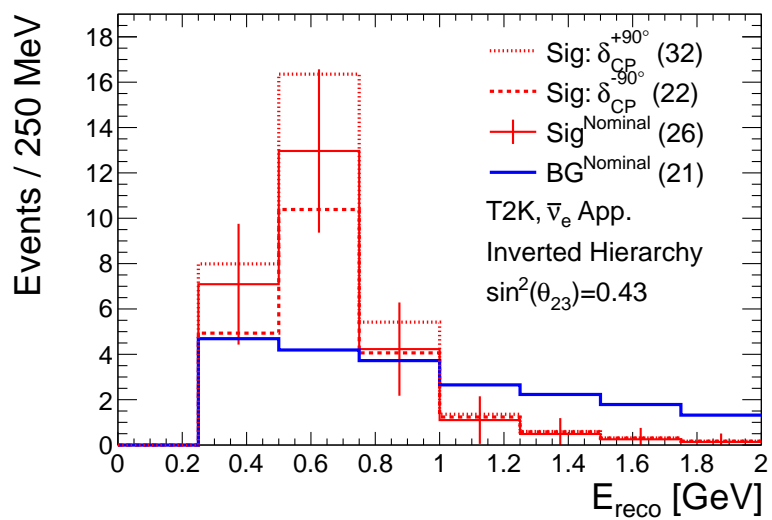

(в) $\bar{\nu}_{e}$ appearance, True $\mathrm{MH}=\mathrm{NH}$

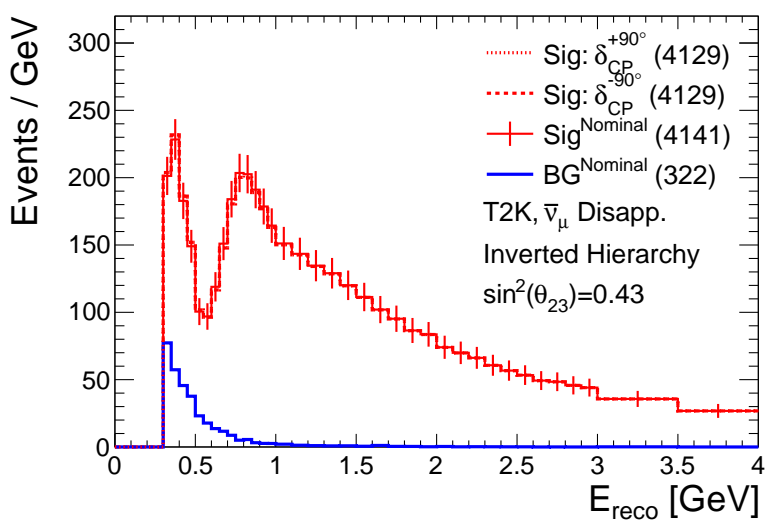

(D) $\bar{\nu}_{\mu}$ disappearance, True $\mathrm{MH}=\mathrm{NH}$

Figure B.13. T2K event spectra for $\pm 3 \sigma$ variations in $\delta_{C P}$ for $\nu_{e}$ appearance (top left), $\bar{\nu}_{e}$ appearance (top right), $\nu_{\mu}$ disappearance (bottom left), and $\bar{\nu}_{\mu}$ disappearance (bottom right). The histograms are overlaid and not stacked. The nominal $7.8 \times 10^{21}$ POT with $1: 1 \nu: \bar{\nu}$ ratio is assumed. The integrated numbers of events are given in parentheses in the legend. 


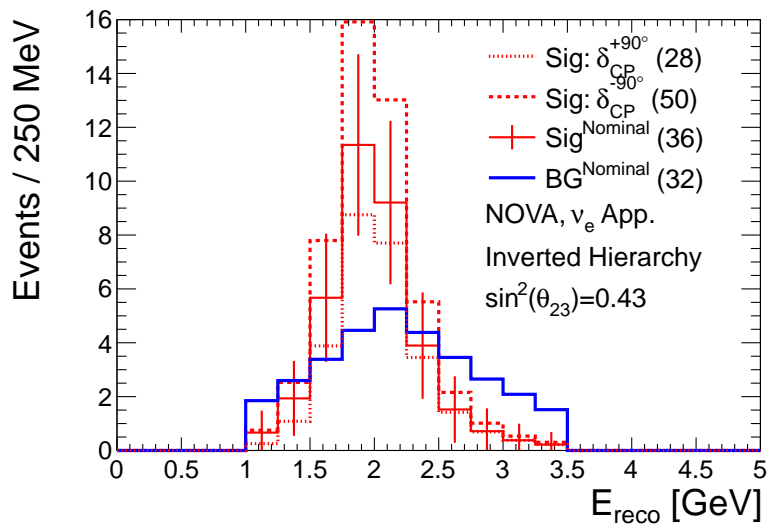

(A) $\nu_{e}$ appearance, True $\mathrm{MH}=\mathrm{NH}$

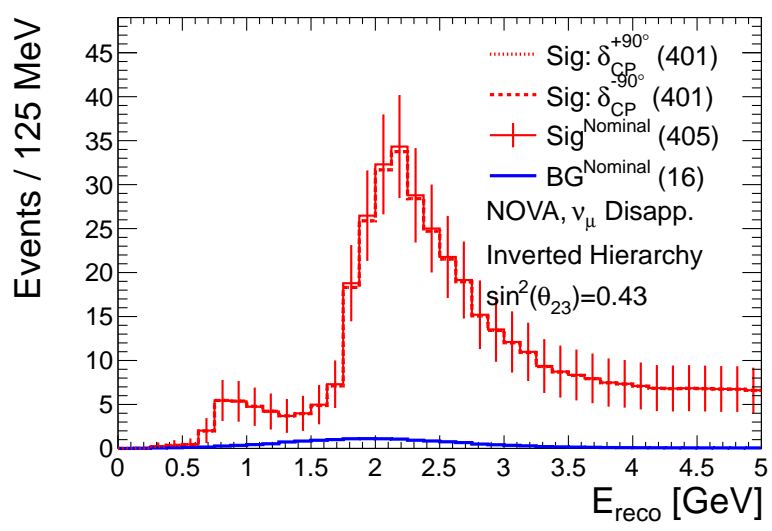

(C) $\nu_{\mu}$ disappearance, True $\mathrm{MH}=\mathrm{NH}$

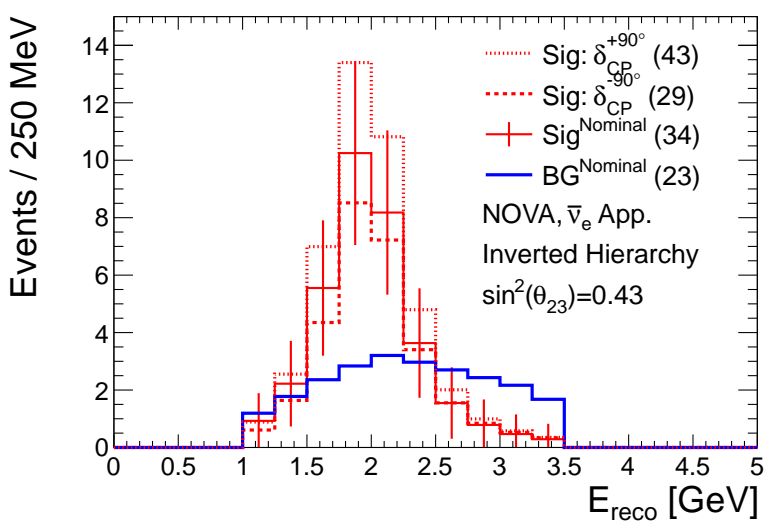

(B) $\bar{\nu}_{e}$ appearance, True $\mathrm{MH}=\mathrm{NH}$

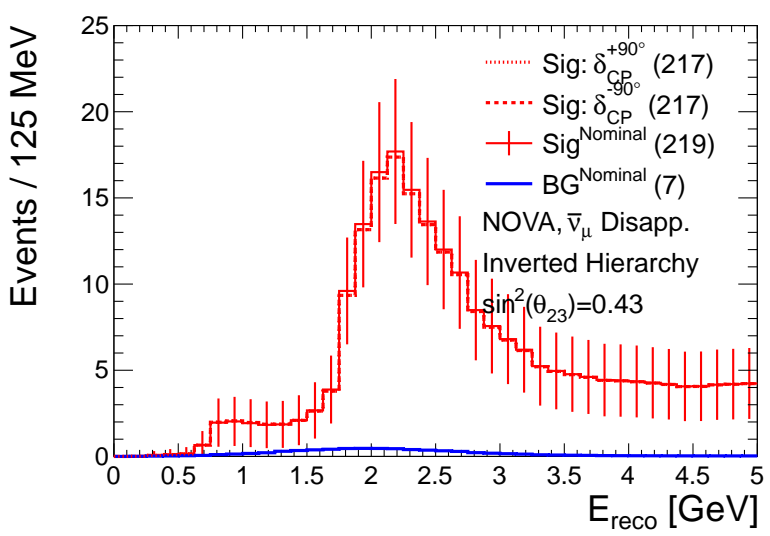

(D) $\bar{\nu}_{\mu}$ disappearance, True $\mathrm{MH}=\mathrm{NH}$

Figure B.14. NO $\nu$ A event spectra for $\pm 3 \sigma$ variations in $\delta_{C P}$ for $\nu_{e}$ appearance (top left), $\bar{\nu}_{e}$ appearance (top right), $\nu_{\mu}$ disappearance (bottom left), and $\bar{\nu}_{\mu}$ disappearance (bottom right). The histograms are overlaid and not stacked. The nominal $3.6 \times 10^{21}$ POT with $1: 1 \nu: \bar{\nu}$ ratio is assumed. The integrated numbers of events are given in parentheses in the legend. 


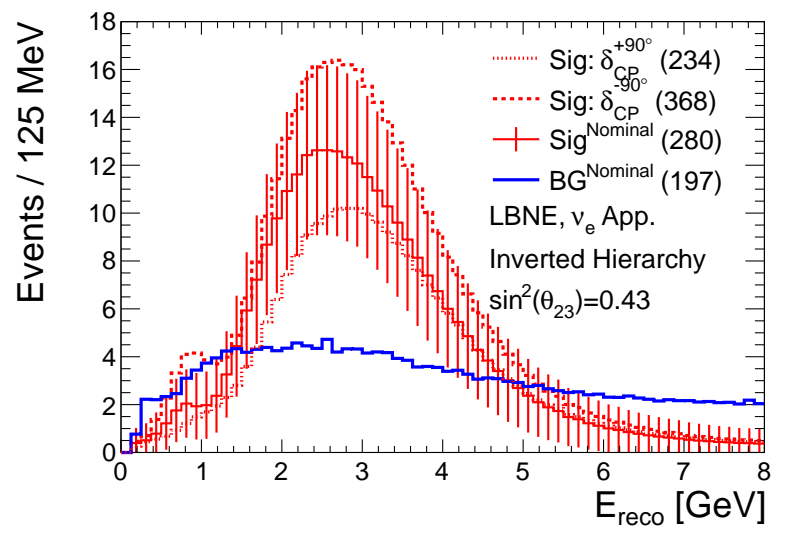

(A) $\nu_{e}$ appearance, True $\mathrm{MH}=\mathrm{NH}$

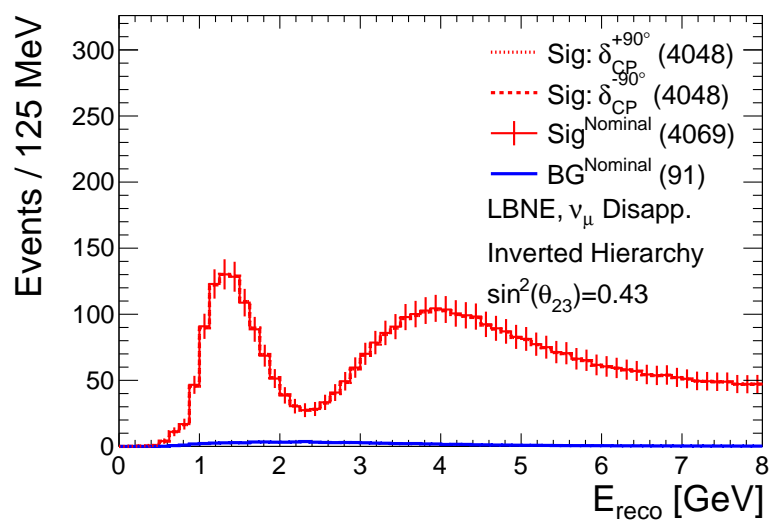

(C) $\nu_{\mu}$ disappearance, True $\mathrm{MH}=\mathrm{NH}$

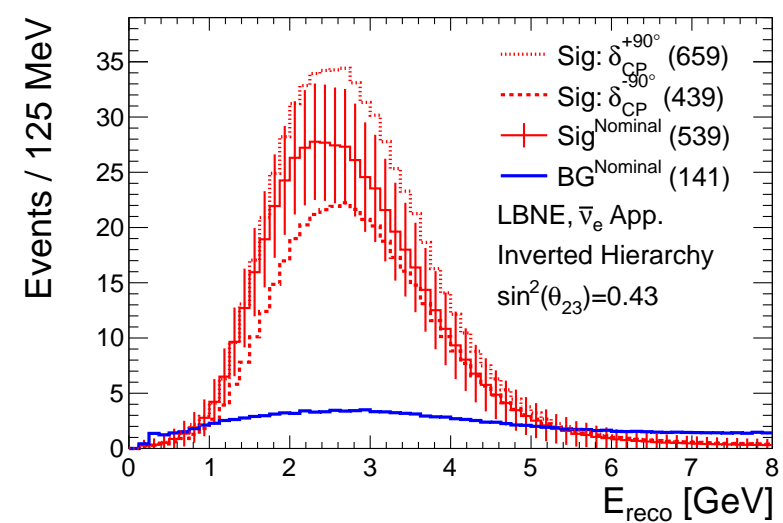

(B) $\bar{\nu}_{e}$ appearance, True $\mathrm{MH}=\mathrm{NH}$

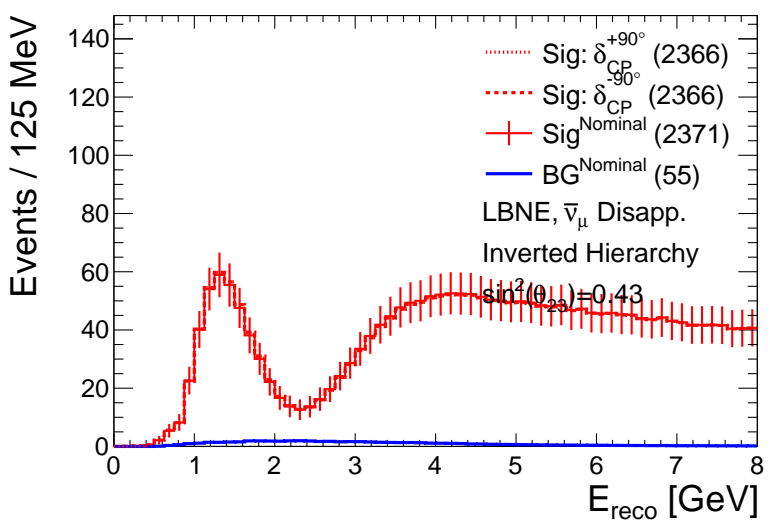

(D) $\bar{\nu}_{\mu}$ disappearance, True $\mathrm{MH}=\mathrm{NH}$

Figure B.15. LBNE event spectra for $\pm 3 \sigma$ variations in $\delta_{C P}$ for $\nu_{e}$ appearance (top left), $\bar{\nu}_{e}$ appearance (top right), $\nu_{\mu}$ disappearance (bottom left), and $\bar{\nu}_{\mu}$ disappearance (bottom right). The histograms are overlaid and not stacked. The nominal $6.0 \times 10^{21}$ POT with $1: 1 \nu: \bar{\nu}$ ratio is assumed. The integrated numbers of events are given in parentheses in the legend. 


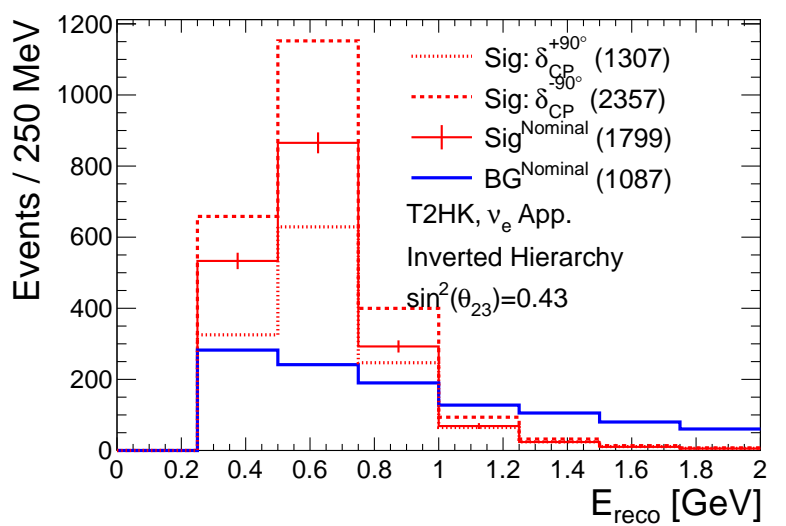

(A) $\nu_{e}$ appearance, True $\mathrm{MH}=\mathrm{NH}$

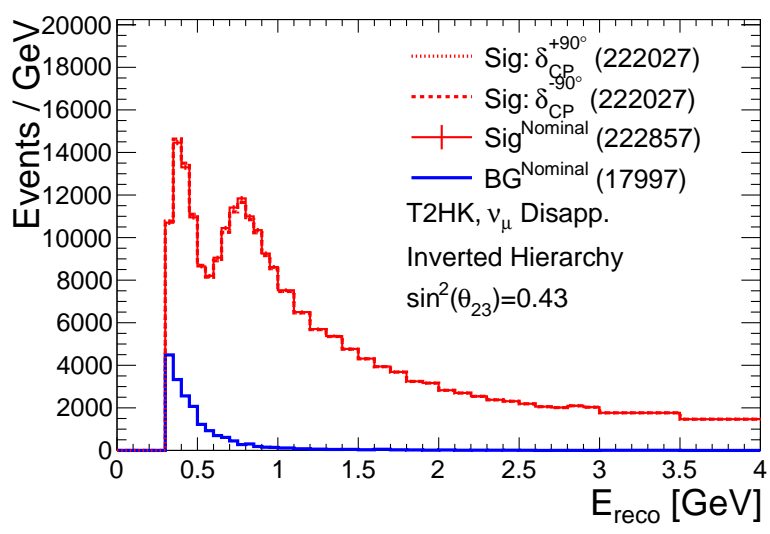

(C) $\nu_{\mu}$ disappearance, True $\mathrm{MH}=\mathrm{NH}$

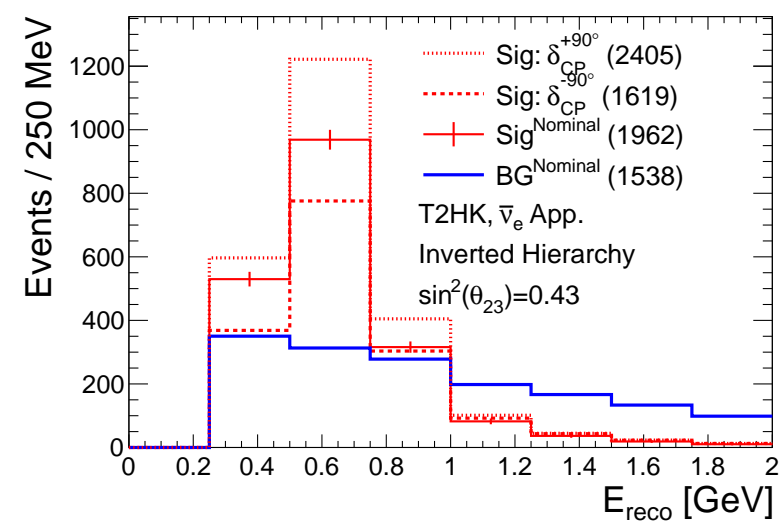

(B) $\bar{\nu}_{e}$ appearance, True $\mathrm{MH}=\mathrm{NH}$

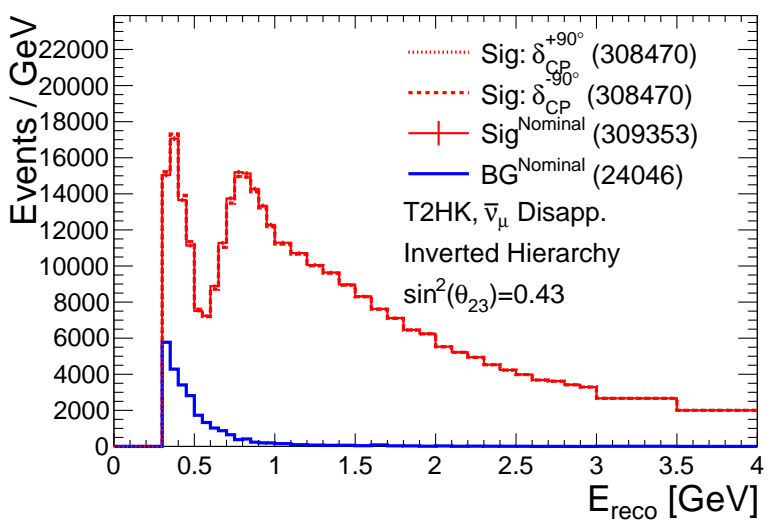

(D) $\bar{\nu}_{\mu}$ disappearance, True $\mathrm{MH}=\mathrm{NH}$

FiguRE B.16. T2HK event spectra for $\pm 3 \sigma$ variations in $\delta_{C P}$ for $\nu_{e}$ appearance (top left), $\bar{\nu}_{e}$ appearance (top right), $\nu_{\mu}$ disappearance (bottom left), and $\bar{\nu}_{\mu}$ disappearance (bottom right). The histograms are overlaid and not stacked. The nominal $15.6 \times 10^{21}$ POT with $1: 1 \nu: \bar{\nu}$ ratio is assumed. The integrated numbers of events are given in parentheses in the legend. 


\section{APPENDIX C}

\section{Resolutions on Oscillation Parameters}

Tables C.1 through C.3 give a complete set of the resolutions and other metrics for CP violation and $\mathrm{MH}$ sensitivity. The resolutions on the parameters were defined in Chapter 9. The $\mathrm{CP}$ violation metric "CPV - $1 \sigma, 3 \sigma$, or $5 \sigma \delta_{C P}$ coverage" gives the percentage of $\delta_{C P}$ values for which the sensitivity is above the indicated level of $1 \sigma, 3 \sigma$, or $5 \sigma$. The CP violation metric "CPV - $\left(\Delta \chi^{2}\right)^{1 / 2}$ for $25 \%, 50 \%$, or $75 \%$ of $\delta_{C P}$ " gives the $\sigma=\sqrt{\Delta \chi^{2}}$ value for which there is $25 \%, 50 \%, 75 \% \delta_{C P}$ coverage.

TABLE C.1. Resolutions on the oscillation parameters for each experiment. Sensitivities are given for both mass hierarchies and for four different systematics scenarios: A) oscillation parameters fixed and no systematics, B) oscillation parameters fixed and with systematics, C) Oscillation parameters profiled and no systematics, D) Oscillation parameters profiled and with systematics. The nominal running assumptions for each experiment are assumed.

\begin{tabular}{|c|c|c|c|c|c|c|c|c|c|c|}
\hline & \multicolumn{2}{|c|}{ T2K } & \multicolumn{2}{|c|}{ NOVA } & \multicolumn{2}{|c|}{ T2K+NOVA } & \multicolumn{2}{|c|}{ LBNE } & \multicolumn{2}{|c|}{ T2HK } \\
\hline & $\mathrm{NH}$ & IH & $\mathrm{NH}$ & $\mathrm{IH}$ & $\mathrm{NH}$ & $\mathrm{IH}$ & $\mathrm{NH}$ & $\mathrm{IH}$ & $\mathrm{NH}$ & $\mathrm{IH}$ \\
\hline $\sin ^{2}\left(2 \theta_{13}\right)$ resolution $\mathrm{A}(\%)$ & 12.4 & 12.6 & 13.9 & 14.5 & 9.3 & 9.5 & 4.4 & 4.2 & 2.1 & 2.1 \\
\hline $\sin ^{2}\left(2 \theta_{13}\right)$ resolution $\mathrm{B}(\%)$ & 13.6 & 13.7 & 14.9 & 15.3 & 10.1 & 10.2 & 4.6 & 4.4 & 2.8 & 2.7 \\
\hline $\sin ^{2}\left(2 \theta_{13}\right)$ resolution $\mathrm{C}(\%)$ & 17.1 & 15.9 & 15.4 & 15.0 & 11.3 & 10.5 & 4.7 & 4.8 & 2.3 & 2.2 \\
\hline $\sin ^{2}\left(2 \theta_{13}\right)$ resolution D $(\%)$ & 18.0 & 16.8 & 16.6 & 19.3 & 11.9 & 11.2 & 5.0 & 5.2 & 3.0 & 2.8 \\
\hline $\sin ^{2}\left(\theta_{23}\right)$ resolution $\mathrm{A}(\%)$ & 2.9 & 4.0 & 2.7 & 3.9 & 2.0 & 2.7 & 1.4 & 1.8 & 0.5 & 0.6 \\
\hline $\sin ^{2}\left(\theta_{23}\right)$ resolution $\mathrm{B}(\%)$ & 3.3 & 4.5 & 3.2 & 4.5 & 2.3 & 3.1 & 1.7 & 2.1 & 0.6 & 0.8 \\
\hline $\sin ^{2}\left(\theta_{23}\right)$ resolution $\mathrm{C}(\%)$ & 3.1 & 4.5 & 4.0 & 6.7 & 2.5 & 3.6 & 1.4 & 2.0 & 0.5 & 0.7 \\
\hline $\sin ^{2}\left(\theta_{23}\right)$ resolution D (\%) & 3.5 & 4.9 & 4.5 & 7.2 & 2.7 & 3.8 & 1.7 & 2.3 & 0.6 & 0.9 \\
\hline$\Delta m_{31}^{2}$ resolution $\mathrm{A}(\%)$ & 1.4 & - & 1.1 & - & 0.9 & - & 0.5 & - & 0.2 & - \\
\hline$\Delta m_{31}^{2}$ resolution $\mathrm{B}(\%)$ & 1.5 & - & 1.3 & - & 1.0 & - & 0.5 & - & 0.3 & - \\
\hline$\Delta m_{31}^{2}$ resolution $\mathrm{C}(\%)$ & 2.0 & - & 1.7 & - & 1.1 & - & 0.5 & - & 0.3 & - \\
\hline$\Delta m_{31}^{2}$ resolution D $(\%)$ & 2.1 & - & 1.9 & - & 1.1 & - & 0.5 & - & 0.3 & - \\
\hline$\delta_{C P}=0$ resolution $\mathrm{A}\left(^{\circ}\right)$ & 46.5 & 42.0 & 33.5 & 33.5 & 19.5 & 19.5 & 10.5 & 10.5 & 4.5 & 4.5 \\
\hline$\delta_{C P}=0$ resolution $\mathrm{B}\left({ }^{\circ}\right)$ & 52.0 & 48.5 & 36.0 & 35.5 & 21.0 & 21.5 & 10.5 & 10.5 & 5.5 & 5.5 \\
\hline$\delta_{C P}=0$ resolution $\mathrm{C}\left({ }^{\circ}\right)$ & 61.5 & 57.5 & 34.5 & 33.5 & 20.5 & 20.5 & 10.5 & 10.5 & 4.5 & 4.5 \\
\hline$\delta_{C P}=0$ resolution $\mathrm{D}\left({ }^{\circ}\right)$ & 70.5 & 62.5 & 36.5 & 36.0 & 22.0 & 22.0 & 11.0 & 11.5 & 5.5 & 5.5 \\
\hline$\delta_{C P}=\pi / 2$ resolution $\mathrm{A}\left({ }^{\circ}\right)$ & 62.5 & 49.5 & 55.0 & 55.5 & 40.5 & 41.0 & 19.5 & 20.5 & 14.0 & 13.5 \\
\hline$\delta_{C P}=\pi / 2$ resolution $\mathrm{B}\left({ }^{\circ}\right)$ & 64.0 & 52.0 & 66.5 & 58.0 & 42.0 & 43.0 & 19.5 & 21.5 & 14.5 & 14.5 \\
\hline$\delta_{C P}=\pi / 2$ resolution $\mathrm{C}\left({ }^{\circ}\right)$ & 81.0 & 52.0 & 118.5 & 58.5 & 43.0 & 43.5 & 21.5 & 23.0 & 15.5 & 15.5 \\
\hline$\delta_{C P}=\pi / 2$ resolution $\mathrm{D}\left({ }^{\circ}\right)$ & 82.5 & 53.5 & 130.5 & 60.5 & 44.5 & 44.5 & 21.5 & 23.5 & 16.0 & 16.5 \\
\hline$\delta_{C P}=-\pi / 2$ resolution $\mathrm{A}\left({ }^{\circ}\right)$ & 52.0 & 68.5 & 56.0 & 56.0 & 42.5 & 43.0 & 20.5 & 19.5 & 14.5 & 13.5 \\
\hline$\delta_{C P}=-\pi / 2$ resolution $\mathrm{B}\left({ }^{\circ}\right)$ & 55.0 & 70.5 & 58.5 & 65.0 & 44.5 & 45.0 & 20.5 & 20.0 & 15.5 & 14.5 \\
\hline$\delta_{C P}=-\pi / 2$ resolution $\mathrm{C}\left({ }^{\circ}\right)$ & 54.5 & 88.0 & 58.5 & 136.5 & 45.5 & 45.5 & 22.5 & 22.0 & 16.5 & 16.0 \\
\hline$\delta_{C P}=-\pi / 2$ resolution $\mathrm{D}\left({ }^{\circ}\right)$ & 57.0 & 89.5 & 61.0 & 140.5 & 47.0 & 47.5 & 22.5 & 22.0 & 17.0 & 16.5 \\
\hline
\end{tabular}


TABle C.2. As in Table C.1 but for CP violation.

\begin{tabular}{|c|c|c|c|c|c|c|c|c|c|c|}
\hline & \multicolumn{2}{|c|}{ T2K } & \multicolumn{2}{|c|}{ NOVA } & \multicolumn{2}{|c|}{$\mathrm{T} 2 \mathrm{~K}+\mathrm{NOVA}$} & \multicolumn{2}{|c|}{ LBNE } & \multicolumn{2}{|c|}{ T2HK } \\
\hline & $\mathrm{NH}$ & $\mathrm{IH}$ & $\mathrm{NH}$ & $\mathrm{IH}$ & $\mathrm{NH}$ & $\mathrm{IH}$ & $\mathrm{NH}$ & $\mathrm{IH}$ & $\mathrm{NH}$ & $\mathrm{IH}$ \\
\hline CPV $-2 \sigma \delta_{C P}$ coverage A $(\%)$ & 18.0 & 19.0 & 0.0 & 0.0 & 43.0 & 43.0 & 78.0 & 78.0 & 90.0 & 90.0 \\
\hline CPV $-2 \sigma \delta_{C P}$ coverage B $(\%)$ & 12.0 & 14.0 & 0.0 & 0.0 & 24.0 & 27.0 & 76.0 & 75.0 & 86.0 & 86.0 \\
\hline CPV - $2 \sigma \delta_{C P}$ coverage $\mathrm{C}(\%)$ & 14.0 & 17.0 & 0.0 & 0.0 & 25.0 & 25.0 & 76.0 & 75.0 & 83.0 & 81.0 \\
\hline CPV $-2 \sigma \delta_{C P}$ coverage D (\%) & 7.0 & 12.0 & 0.0 & 0.0 & 23.0 & 23.0 & 74.0 & 74.0 & 77.0 & 74.0 \\
\hline CPV $-3 \sigma \delta_{C P}$ coverage A $(\%)$ & 0.0 & 0.0 & 0.0 & 0.0 & 6.0 & 7.0 & 64.0 & 64.0 & 86.0 & 86.0 \\
\hline CPV $-3 \sigma \delta_{C P}$ coverage B $(\%)$ & 0.0 & 0.0 & 0.0 & 0.0 & 0.0 & 0.0 & 63.0 & 62.0 & 82.0 & 82.0 \\
\hline CPV $-3 \sigma \delta_{C P}$ coverage C $(\%)$ & 0.0 & 0.0 & 0.0 & 0.0 & 0.0 & 0.0 & 64.0 & 62.0 & 72.0 & 69.0 \\
\hline CPV $-3 \sigma \delta_{C P}$ coverage D $(\%)$ & 0.0 & 0.0 & 0.0 & 0.0 & 0.0 & 0.0 & 62.0 & 60.0 & 64.0 & 61.0 \\
\hline CPV $-5 \sigma \delta_{C P}$ coverage A $(\%)$ & 0.0 & 0.0 & 0.0 & 0.0 & 0.0 & 0.0 & 34.0 & 34.0 & 71.0 & 74.0 \\
\hline CPV $-5 \sigma \delta_{C P}$ coverage B $(\%)$ & 0.0 & 0.0 & 0.0 & 0.0 & 0.0 & 0.0 & 28.0 & 29.0 & 59.0 & 59.0 \\
\hline CPV $-5 \sigma \delta_{C P}$ coverage C $(\%)$ & 0.0 & 0.0 & 0.0 & 0.0 & 0.0 & 0.0 & 30.0 & 23.0 & 53.0 & 49.0 \\
\hline CPV $-5 \sigma \delta_{C P}$ coverage D $(\%)$ & 0.0 & 0.0 & 0.0 & 0.0 & 0.0 & 0.0 & 24.0 & 9.0 & 42.0 & 35.0 \\
\hline$\overline{C P P V-\left(\Delta \chi^{2}\right)^{1 / 2} \text { for } 25 \% \text { of } \delta_{C P} \mathrm{~A}}$ & 1.6 & 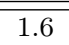 & 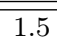 & 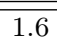 & 2.2 & $\overline{2.2}$ & 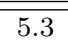 & 5.3 & 9.0 & 9.2 \\
\hline CPV $-\left(\Delta \chi^{2}\right)^{1 / 2}$ for $25 \%$ of $\delta_{C P} \mathrm{~B}$ & 1.5 & 1.5 & 1.4 & 1.5 & 2.0 & 2.0 & 5.1 & 5.1 & 7.1 & 7.3 \\
\hline CPV $-\left(\Delta \chi^{2}\right)^{1 / 2}$ for $25 \%$ of $\delta_{C P} \mathrm{C}$ & 1.5 & 1.6 & 1.3 & 1.4 & 1.9 & 2.0 & 5.1 & 4.9 & 8.5 & 9.1 \\
\hline CPV $-\left(\Delta \chi^{2}\right)^{1 / 2}$ for $25 \%$ of $\delta_{C P} \mathrm{D}$ & 1.4 & 1.4 & 1.3 & 1.3 & 1.8 & 1.8 & 4.9 & 4.7 & 6.8 & 7.1 \\
\hline CPV $-\left(\Delta \chi^{2}\right)^{1 / 2}$ for $50 \%$ of $\delta_{C P} \mathrm{~A}$ & 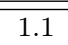 & 1.1 & 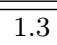 & 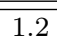 & $\overline{1.9}$ & 2.0 & 4.0 & 4.0 & 6.5 & 6.5 \\
\hline CPV $-\left(\Delta \chi^{2}\right)^{1 / 2}$ for $50 \%$ of $\delta_{C P} \mathrm{~B}$ & 1.1 & 1.0 & 1.2 & 1.2 & 1.8 & 1.8 & 3.9 & 3.8 & 5.5 & 5.4 \\
\hline CPV $-\left(\Delta \chi^{2}\right)^{1 / 2}$ for $50 \%$ of $\delta_{C P} \mathrm{C}$ & 0.9 & 0.8 & 1.1 & 1.2 & 1.5 & 1.5 & 3.9 & 3.7 & 5.1 & 4.8 \\
\hline CPV $-\left(\Delta \chi^{2}\right)^{1 / 2}$ for $50 \%$ of $\delta_{C P} \mathrm{D}$ & 0.9 & 0.8 & 1.0 & 1.1 & 1.4 & 1.5 & 3.7 & 3.5 & 4.2 & 3.8 \\
\hline$\overline{C P P V}-\left(\Delta \chi^{2}\right)^{1 / 2}$ for $75 \%$ of $\delta_{C P} \mathrm{~A}$ & 0.8 & 0.8 & 0.7 & 0.6 & 1.1 & 1.1 & 2.1 & 2.0 & 4.9 & 4.9 \\
\hline CPV $-\left(\Delta \chi^{2}\right)^{1 / 2}$ for $75 \%$ of $\delta_{C P} \mathrm{~B}$ & 0.8 & 0.8 & 0.6 & 0.6 & 1.0 & 1.0 & 2.0 & 1.9 & 3.9 & 3.8 \\
\hline CPV $-\left(\Delta \chi^{2}\right)^{1 / 2}$ for $75 \%$ of $\delta_{C P} \mathrm{C}$ & 0.5 & 0.5 & 0.7 & 0.6 & 1.0 & 1.0 & 2.0 & 1.9 & 2.4 & 2.4 \\
\hline CPV $-\left(\Delta \chi^{2}\right)^{1 / 2}$ for $75 \%$ of $\delta_{C P} \mathrm{D}$ & 0.5 & 0.5 & 0.6 & 0.6 & 1.0 & 1.0 & 1.9 & 1.8 & 2.0 & 1.9 \\
\hline
\end{tabular}

Table C.3. As in Table C.1 but for MH determination.

\begin{tabular}{|c|c|c|c|c|c|c|c|c|c|c|}
\hline & \multicolumn{2}{|c|}{$\mathrm{T} 2 \mathrm{~K}$} & \multicolumn{2}{|c|}{ NOVA } & \multicolumn{2}{|c|}{$\overline{\mathrm{T} 2 \mathrm{~K}+\mathrm{NOVA}}$} & \multicolumn{2}{|c|}{ LBNE } & \multicolumn{2}{|c|}{ T2HK } \\
\hline & $\mathrm{NH}$ & $\mathrm{IH}$ & $\mathrm{NH}$ & $\mathrm{IH}$ & $\mathrm{NH}$ & $\mathrm{IH}$ & $\mathrm{NH}$ & $\mathrm{IH}$ & $\mathrm{NH}$ & $\mathrm{IH}$ \\
\hline $\mathrm{MH}-\Delta \chi^{2}$ for $100 \%$ of $\delta_{C P} \mathrm{C}(\%)$ & 0.0 & 0.0 & 0.1 & 0.1 & 1.7 & 1.8 & 21.0 & 28.6 & 1.3 & 1.0 \\
\hline $\mathrm{MH}-\Delta \chi^{2}$ for $100 \%$ of $\delta_{C P} \mathrm{D}(\%)$ & 0.0 & 0.0 & 0.1 & 0.1 & 1.5 & 1.6 & 20.3 & 27.6 & 0.9 & 0.7 \\
\hline MH - $\Delta \chi^{2}$ for $0 \%$ of $\delta_{C P} \mathrm{C} \mathrm{( \% )}$ & 1.7 & 1.7 & 7.4 & $\overline{77.4}$ & 8.9 & 8.9 & 209.4 & 235.1 & 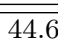 & 51.0 \\
\hline $\mathrm{MH}-\Delta \chi^{2}$ for $0 \%$ of $\delta_{C P} \mathrm{D}(\%)$ & 1.4 & 1.4 & 6.5 & 6.6 & 7.8 & 7.9 & 192.4 & 218.7 & 43.7 & 44.8 \\
\hline $\mathrm{MH}-2 \sigma \delta_{C P}$ coverage $\mathrm{C} \mathrm{( \% )}$ & 0 & 0 & 31 & 31 & 31 & 31 & 100 & 100 & 53 & 53 \\
\hline $\mathrm{MH}-2 \sigma \delta_{C P}$ coverage D (\%) & 0 & 0 & 28 & 28 & 31 & 31 & 100 & 100 & 47 & 53 \\
\hline MH - $3 \sigma \delta_{C P}$ coverage $\mathrm{C}(\%)$ & 0 & 0 & 0 & 0 & 0 & 0 & 100 & 100 & 28 & 31 \\
\hline $\mathrm{MH}-3 \sigma \delta_{C P}$ coverage D $(\%)$ & 0 & 0 & 0 & 0 & 0 & 0 & 100 & 100 & 28 & 31 \\
\hline "MH - $5 \sigma \delta_{C P}$ coverage $\mathrm{C}(\%)$ & $\overline{0}$ & $\overline{0}$ & 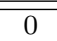 & $\overline{0} 0$ & 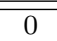 & $\overline{0}$ & 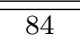 & 100 & 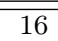 & 22 \\
\hline $\mathrm{MH}-5 \sigma \delta_{C P}$ coverage D (\%) & 0 & 0 & 0 & 0 & 0 & 0 & 84 & 100 & 9 & 16 \\
\hline
\end{tabular}




\section{APPENDIX D}

\section{Computational Resources}

This appendix gives a brief overview of the computational resources used in computing the various sensitivities in this thesis.

For a typical minimization where all four samples $\left(\nu_{e}\right.$ appearance, $\bar{\nu}_{e}$ appearance, $\nu_{\mu}$ disappearance, and $\bar{\nu}_{\mu}$ disappearance) are included, the oscillation parameters are profiled, the mass hierarchy is profiled, and normalization systematics are included on the signal and background the minimization of the $\Delta \chi^{2}$ takes approximately 20 seconds per point. Table D.1 lists estimates for a representative set of the types of jobs run.

TABLE D.1. Estimates of the computational time for various types of jobs run to make the sensitivities in this thesis.

\begin{tabular}{lccc} 
Plot Type & Grid Size & $\Delta \chi^{2}{ }^{\prime}$ s Minimized & Processing Time (hours) \\
\hline$\delta_{C P}$ vs $\sin ^{2}\left(2 \theta_{13}\right)$ & $80 \times 80$ & 12,800 & 7 \\
\hline$\delta_{C P}$ vs $\sin ^{2}\left(\theta_{23}\right)$ & $80 \times 80$ & 12,800 & 7 \\
\hline Significance vs $\delta_{C P^{-}}$CP Violation & 37 & 148 & 0.1 \\
& & & 0.1
\end{tabular}

All of the processes are single core processes as the $\Delta \chi^{2}$ minimization is intrinsically a single core process. When necessary, parallelization was achieved by splitting up the runs with respect to the oscillation parameter being scanned. For example, when scanning over multiple true values of $\delta_{C P}$, the $\delta_{C P}$ values were split into 37 separate processes each with a different true value of $\delta_{C P}$ considered. 